\title{
The Behaviour of Integral Bridges under Vertical and Horizontal Earthquake Ground Motion
}

\author{
Masrilayanti \\ School of Science, Computing and Engineering \\ University of Salford, United Kingdom
}

Submitted in Partial Fulfilment of Requirements of the Degree of Doctor of Philosophy, December 2013 


\begin{abstract}
Integral bridges are monolithic and are known to possess good earthquake resistance when founded on a stable soil. One important consideration is the relative displacements which can occur at the support points on structures where there is significant spacing between, i.e. bridges. Factors such as soil, foundation types etc. can all influence the dynamic response, and the stiffness of the bridge can influence how relative displacements affect the internal force actions within the structure. In this study, the effect of earthquakes on integral bridges built on several different soil types is examined, through computer simulation of an integral abutment bridge. The study is made based on Eurocode 8 recommendations, which provides data for different types of soil to be used for earthquake analysis. A symmetrical medium length integral bridge obtained from an existing structure is used for the analysis. Artificial EC8 spectrum compatible time histories (with a $0.35 \mathrm{~g}$ peak ground acceleration) are applied to the structure for a range of soil stiffnesses. In conjunction with this, both static and dynamic relative displacement studies are carried out to develop insight as to the significance or dominance of either dynamic or relative displacement effects.
\end{abstract}

The final aim of this study is to propose a simplified approach for design/appraisal which can allow predictions of dynamic response based on the results of static relative displacement studies coupled with simple computer models, without having to resort to full nonlinear integration time-history analysis. Synthetic time histories for 5 different types of soil were created using Mathcad. The synthetic acceleration time history was validated using Seismospect (by Seismosoft). The time histories were then used to carry our full integration time history analyses in ANSYS (engineering simulation software) to simulate the dynamic response of the bridge.

The results show that relative displacements play an important role in overall structural response of the integral bridge, compared to the pure dynamic response. The results also confirm that lower stiffness soils suffer a more detrimental effect of the earthquake compared to a soil of higher stiffness.

\title{
Keywords:
}

Integral bridge, dynamic load, relative displacements. 


\section{Acknowledgement}

I am greatly indebted to my supervisor, DR. Laurence Weeks, who is always my reliance throughout the research and study. His timely encouragement, specialist knowledge and invaluable professional suggestions have guided me through all the difficulties, whether that's academic, financial, or emotional. The most amazing supervisor and lecturer I have had in all my life. His efforts can never and will never be forgotten.

I am also grateful to all staff of School of Computing, Science, and Engineering and the former chairman of Civil Engineering Department, Prof. Clive Melbourne, for his kind encouraging before retiring from the job. DR. Jinyan Wang, who supports me in the difficult time and understands my feeling as a 'single’ mom. Thank for Prof Miklas Scholtz for being a good supporter for us in Room 244, DR. Leach as a nice examiner for me in internal assessment and gave so valuable feedbacks. Big thanks for Mr. Haynes, Neil Currie, Sonia Faisal, DR. Victoria Sheppard, Nathalie, Catriona, and other University Staffs who have helped me to go through this study.

I would especially also like to thank all of my research friends in Newton Building Room 244, for their continuous supports to my study, their care, laugh and share fun throughout the years had increased my motivation and spirit in finalization this study.

I own a debt of gratitude to many advisors, both formal and informal, and my colleagues and friends who provided valuable advices and supports along the way. My highest thank for my colleagues in University of Andalas, Civil Engineering Department, Padang, Sumatra Barat, for their supports and encouragements. Indonesian and Malaysian community for having a good time together. And I also thanks to the Education Ministry of Republic of Indonesia, for their funding and scholarship in this study.

Finally I would like to thank my parents, my brothers, my husband Zulkarnaen, and my beloved three children, for being so patient and support me lots for this study. You are the greatest gifts from the Creator to me. 
Dedication

I lovingly dedicate this thesis to my husband who supported me each step of the way

iv 


\section{List of Content}

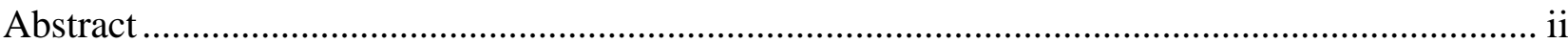

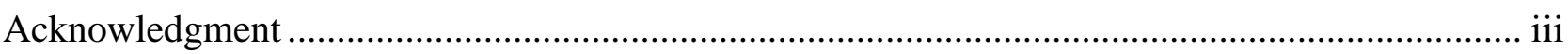

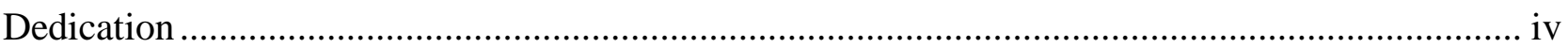

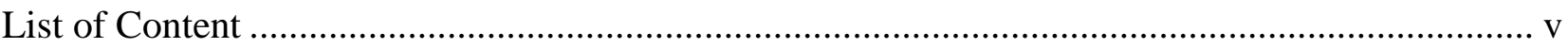

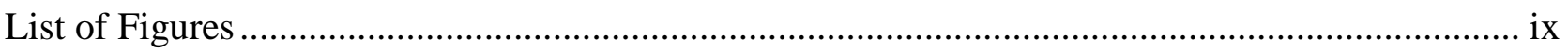

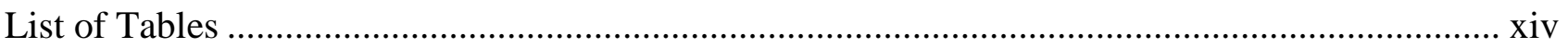

Chapter I INTRODUCTION...................................................................................1

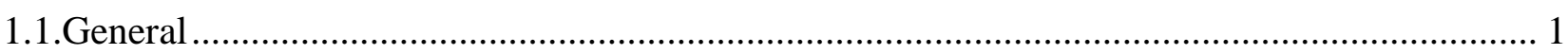

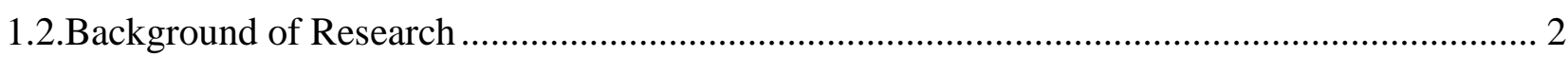

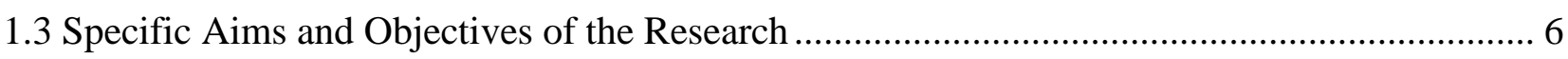

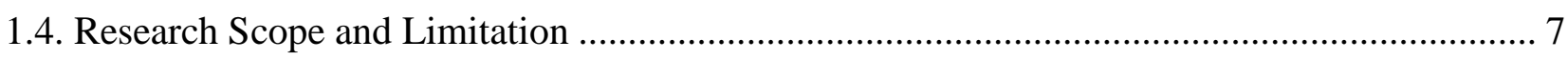

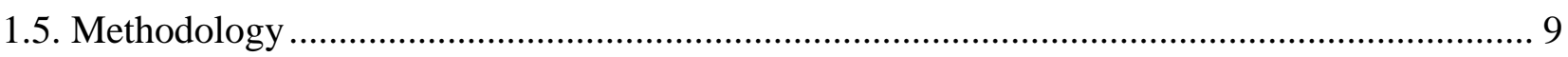

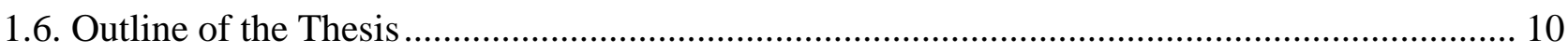

Chapter II REVIEW OF INTEGRAL BRIDGES ....................................................12

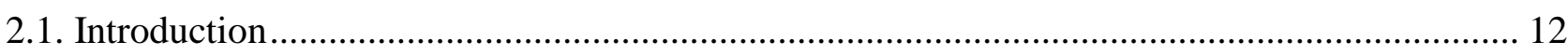

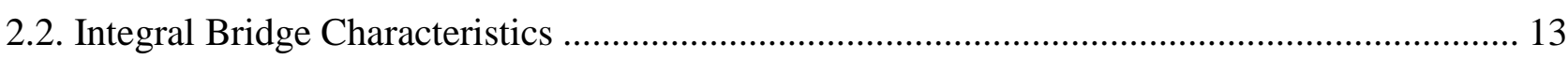

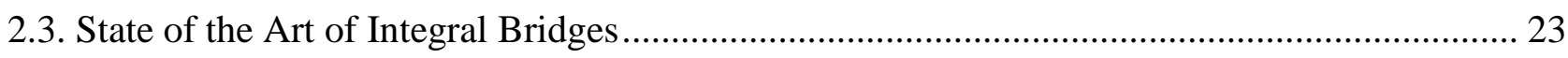

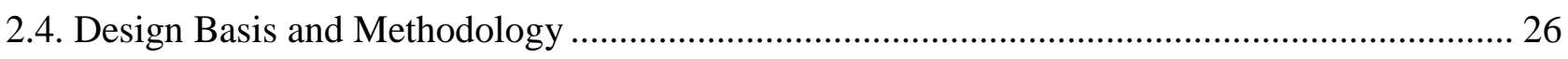

2.5. Substructures for Integral Bridges under Earthquake Loads ................................................. 29

2.6. Failure Mechanism of Concrete Integral Bridges ............................................................. 35

Chapter III EARTHQUAKE GROUNDMOTION EFFECTS - REVIEW.........37

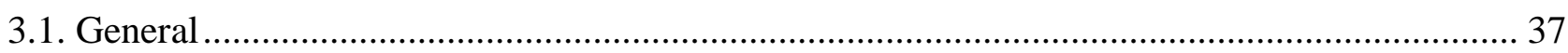

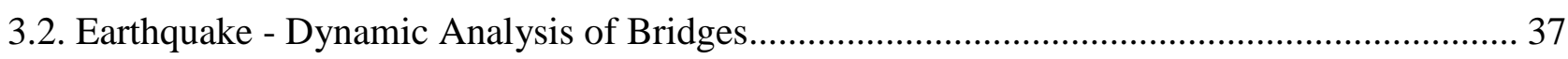


3.2.1. Fundamentals of Dynamic Analysis 39

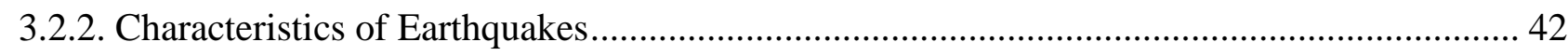

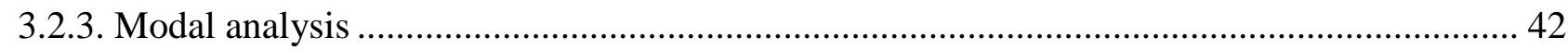

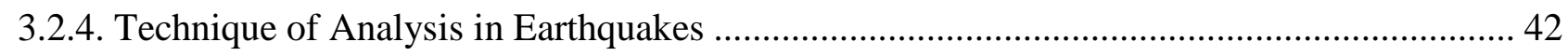

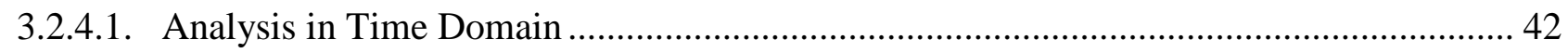

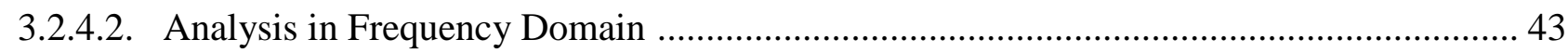

3.2.4.3. Performance based Design for Bridges ................................................................... 46

3.2.4.4 Eurocode 8 Approach to Earthquake Engineering ….................................................. 47

3.2.5. Horizontal and Vertical Ground Motion Studies .............................................................. 48

3.2.6. Effects of Vertical Ground Motions on Bridges ................................................................ 51

3.3. Behaviour of Integral Bridges Subject to Seismic Loads .................................................... 53

3.3.1. Structural Response of Integral Bridges due to Seismic Loads .......................................... 53

3.3.2. Earthquake History of Integral Bridges .......................................................................... 54

3.3.3. Previous Research on Integral Bridges under Seismic Loads ........................................... 56

3.4. Dynamic and Static Response of Bridges under Seismic Load ............................................ 57

3.5. Relative Displacements Effects on Bridges ..................................................................... 58

Chapter IV RESEARCH METHODOLOGY .......................................................61

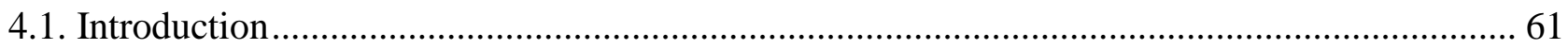

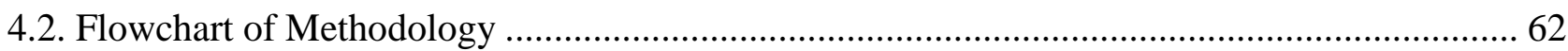

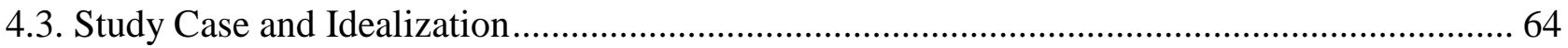

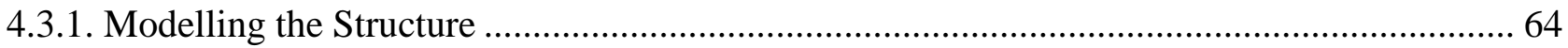

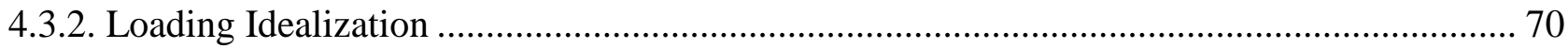

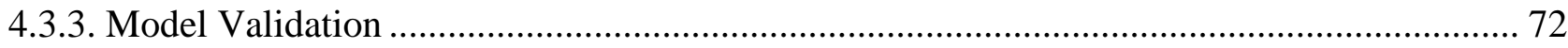

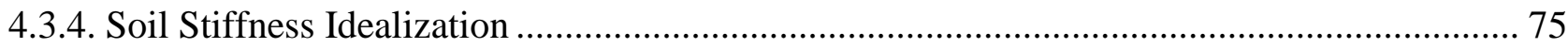

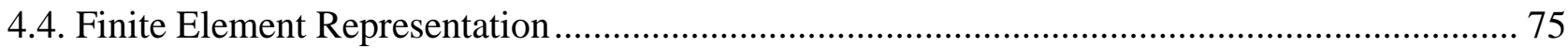

4.5. Synthetic Earthquake Accelerogram in Eurocode 8 .............................................................. 78

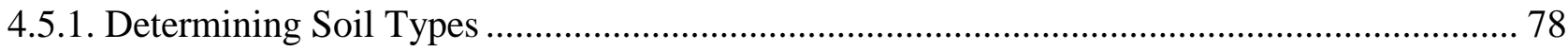

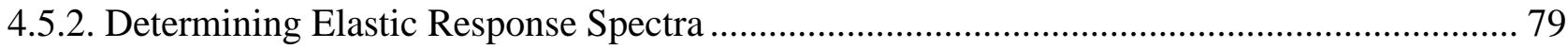

4.5.3. Determining a Series of Synthetic Earthquake Accelerations ............................................ 83

4.5.4. Determining Relative Displacement on the Supports ........................................................ 84

4.5.5. Creating Relative Displacement Moment Bending Diagram ............................................... 85 


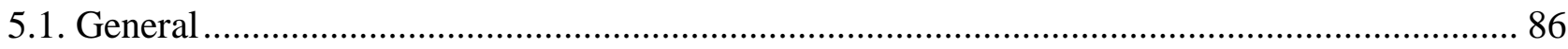

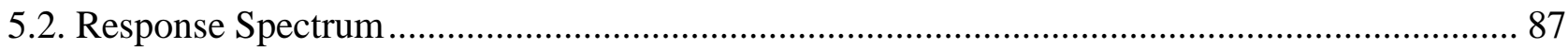

5.3. Synthetic Ground Motion Time Histories............................................................................. 96

5.4. Baseline Correction for Earthquake Ground Motion.................................................................. 103

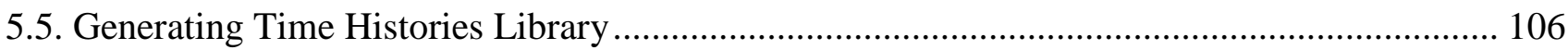

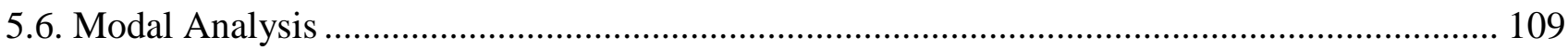

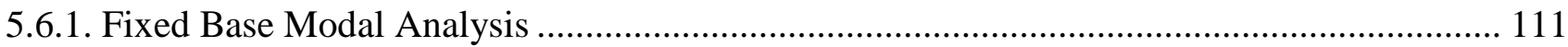

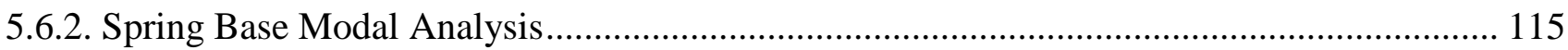

5.7. 3D Modelling for Frequency Analysis ................................................................................. 121

5.8. Time History (Transient) Analysis ...................................................................................... 124

\section{Chapter VI RELATIVE DISPLACEMENT EFFECTS ON THE BRIDGE ......139}

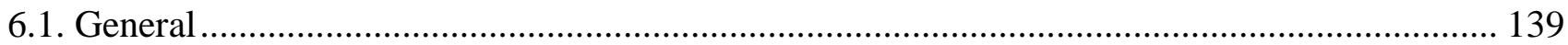

6.2. Effects of Relative Displacement on Bending Moments in the Bridge ..................................... 142

6.2.1. Case 1 (Fixed Base, Without Springs) .............................................................................. 143

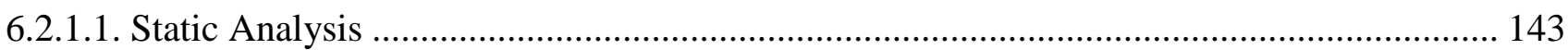

6.2.1.2. Relative Displacements as a Sensitive Study for Fixed Base Bridge .................................. 149

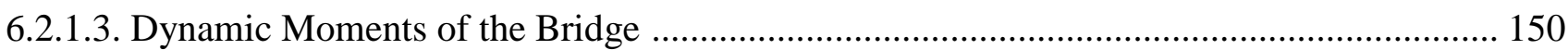

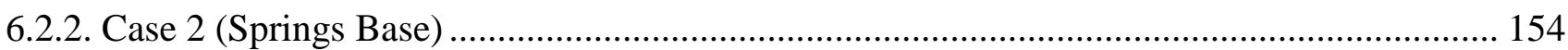

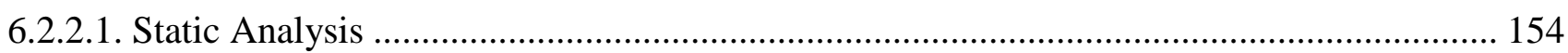

6.2.2.2. Relative Displacements as a Sensitive Study for Fixed Base Bridge ................................. 156

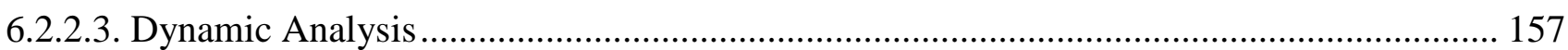

6.2.2.4. Dynamic Moments Response of the Bridge ………………………………………….... 159

6.3. Bending Moment Pattern of the Bridge ……………………................................................ 162

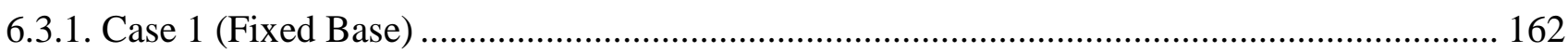

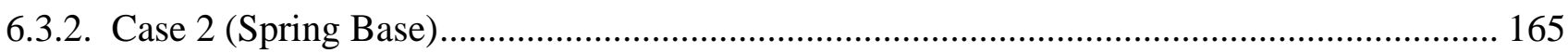

Chapter VII CORRELATION BETWEEN RELATIVE DISPLACEMENT

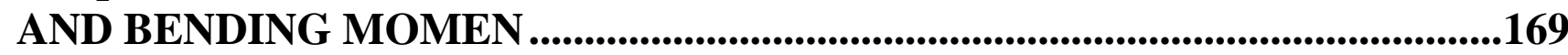

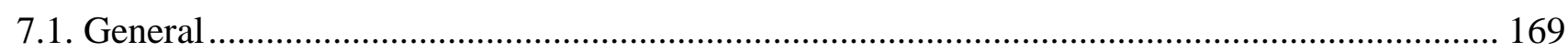

7.2. Generating the relative displacement and bending moment relationship diagram .................... 171

7.3. Bridge response under horizontal and vertical relative displacements.................................... 178 
7.4. The contributions of relative displacement to the overall moment distribution of the bridges.

7.5. The responses of the bridge under differing soil conditions 184

7.6. The responses of the bridge under a fixed base support condition ..... 187

7.7. Static and Dynamic Relative Displacement - Moments Relationship 191

7.7.1. Static-Dynamic Bending Moment Ratio..... 191

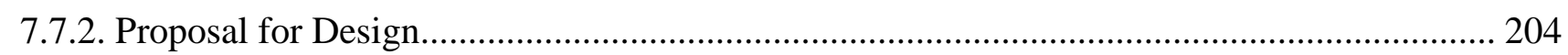

7.7.3. Using the Relative Displacement-Bending Moment Diagram for Design and Analysis..... 206

\section{Chapter VIII Conclusion} 208

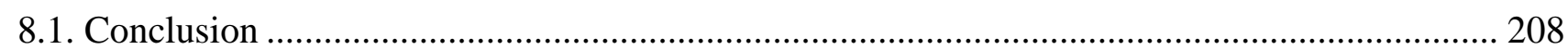

8.2. Recommendations and Future Work ............................................................................. 214

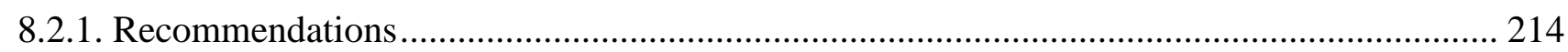

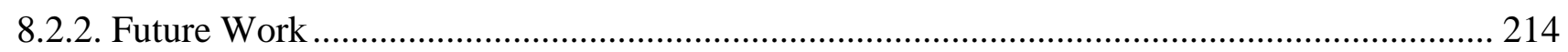

References .........................................................................................................................216

\section{Appendices}




\section{List of Figures}

Figure II-1: Fundamental differences between conventional bridge (left) and integral bridge construction (right)........14

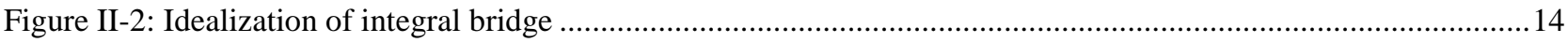

Figure II-3: Structural arrangement of integral bridge and conventional bridge construction.....................................15

Figure II-4: Comparison of (a) conventional bridge, (b) integral bridge, and (c) semi -integral bridge ......................17

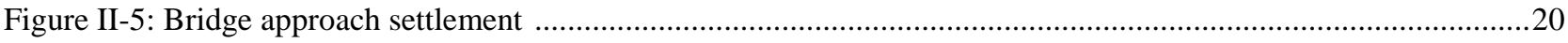

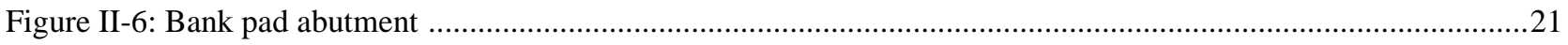

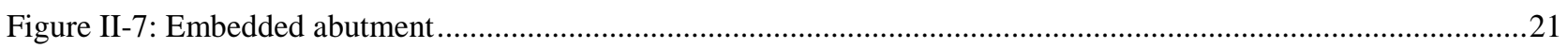

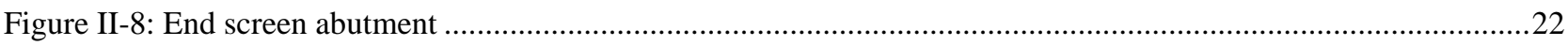

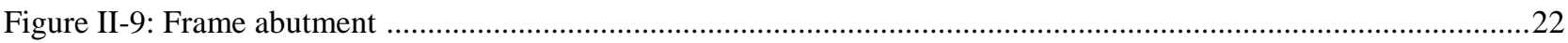

Figure II-10: Trends of bridge construction in steel material from pre-2000 to 2004 in United Kingdom (Illes,

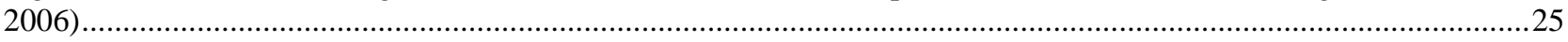

Figure II-11: Deformation of integral bridge due to temperature and traffic load................................................28

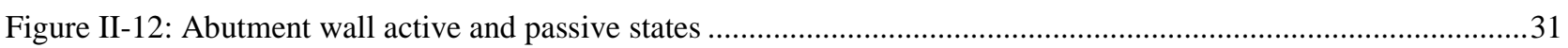

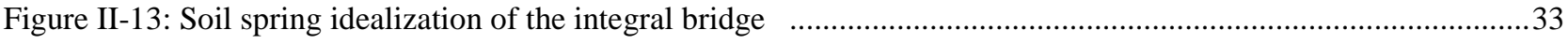

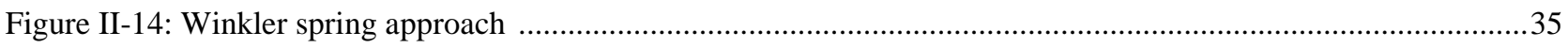

Figure III-1: Comparison of the dimension of the structure related to frequency ......................................................39

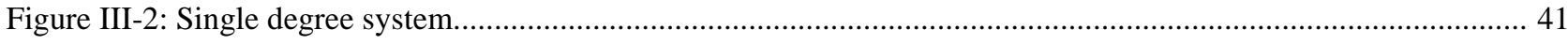

Figure III-3: Idealization of single degree of freedom system........................................................................41

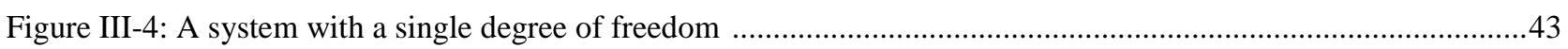

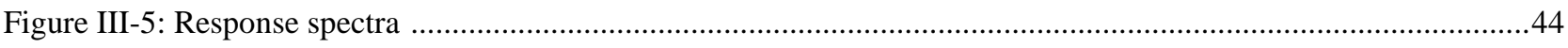

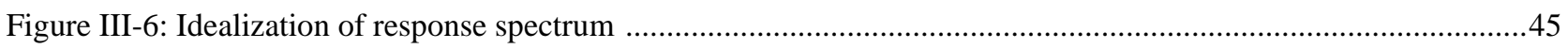

Figure III-7: Spectral acceleration in accordance with natural period and mode shape (Austin, 1996) .......................46

Figure III-8: Well designed structures dissipate seismic energy by elastic . ........................................................47

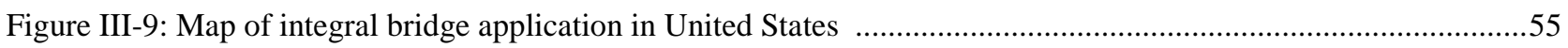

Figure III-10: Map of earthquake prones areas in Canada http://atlas.nrcan.gc.ca/site/english/maps/environment/naturalhazards/earthquakes/majorearthquakes [accessed:

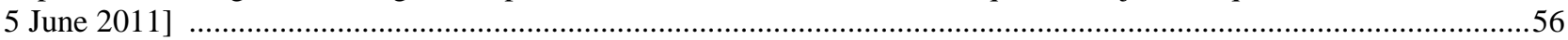

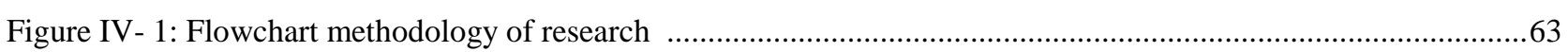

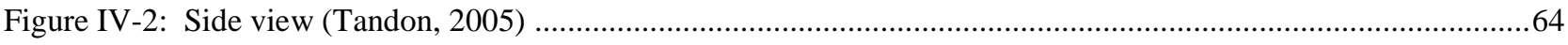

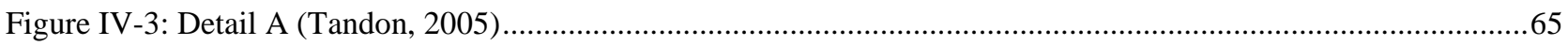

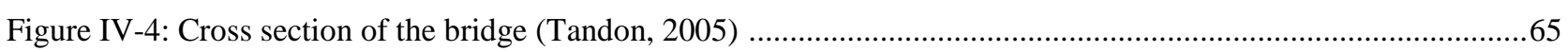

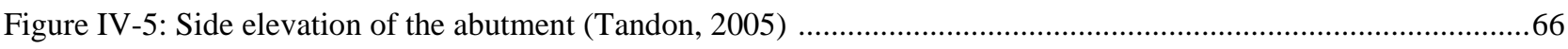

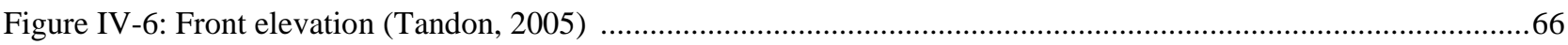

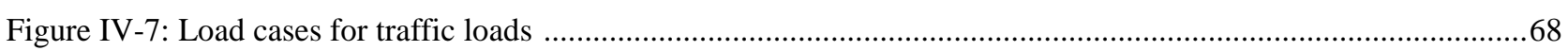

Figure IV-8: Standard value of vehicle load from AASHTO .........................................................................69

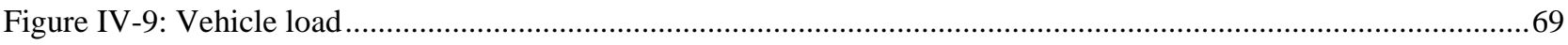




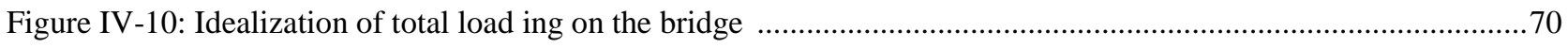

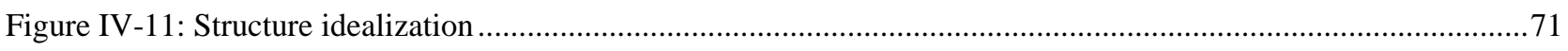

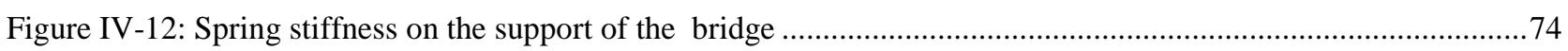

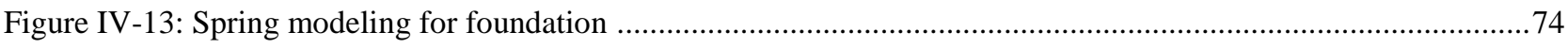

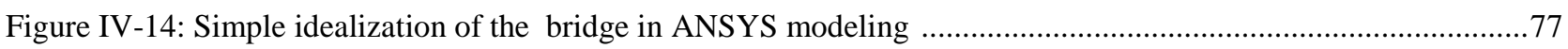

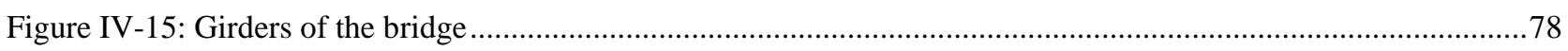

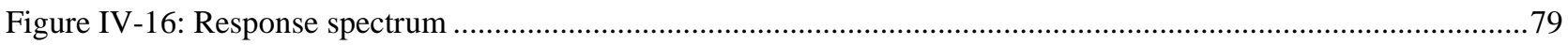

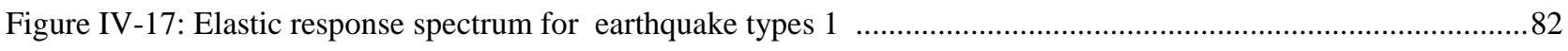

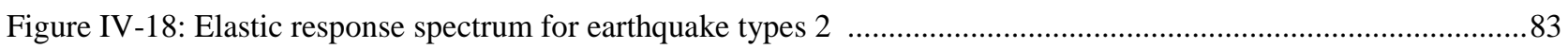

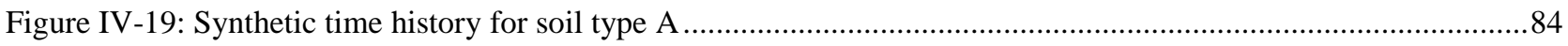

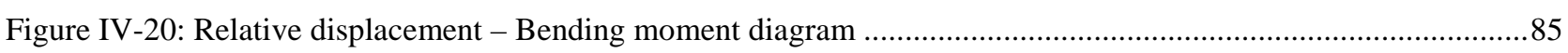

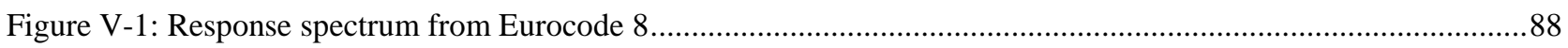

Figure V-2: Synthetic acceleration spectra type 1 earthquake to Eurocode 8, a = 0.35g ......................................89

Figure V-3: Three different data from same specific ground motion ....................................................................90

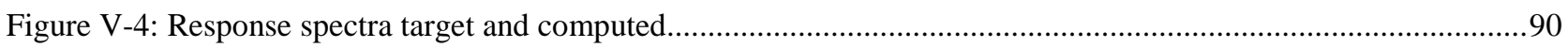

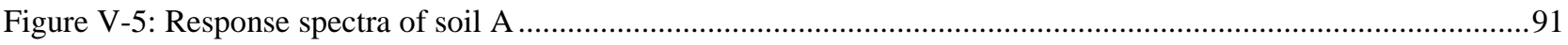

Figure V-6: Response spectra in different sensitive region (Lumantarna, et al., 2012) ............................................93

Figure V-7: Response spectrum for soil type A from seismosoft (a) acceleration, (b) velocity, and (c)

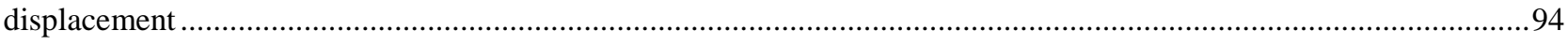

Figure V-8: Region classification of spectral response acceleration .....................................................................95

Figure V-9: Horizontal earthquake ground-motion applied to the bridge structure ................................................99

Figure V-10: Vertical earthquake ground-motion applied to the bridge structure ................................................100

Figure V-11: Synthetic acceleration earthquake ground motion .......................................................................100

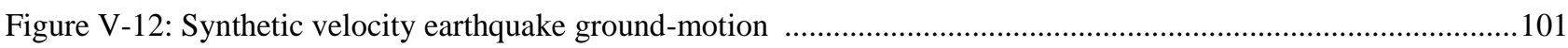

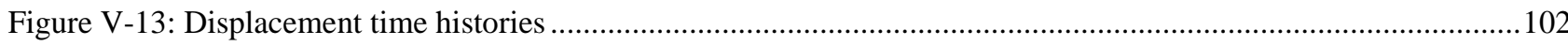

Figure V-14: Horizontal displacement time-histories comparison of 5 soil types....................................................103

Figure V-15: Data comparison after and before applying baseline correction for horizontal and vertical ground-

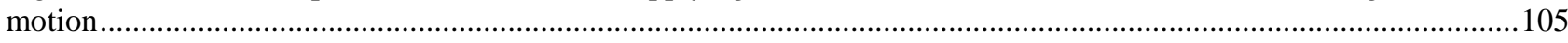

Figure V-16: Displacement time histories after and before applying baseline correction for horizontal direction .........106

Figure V-17: Three different time histories resulted from the same spectra earthquake for acceleration, velocity, and displacement 108

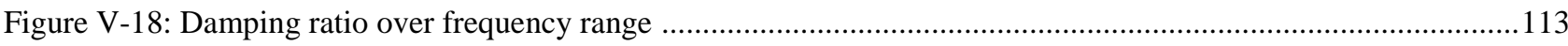

Figure V-19: Mode shape and frequencies of the structure for fixed base structure ..................................................115

Figure V-20: Mode shape and frequencies of the structure for spring base structure ...........................................116

Figure V-21: Co-relation between mode shape and frequency of 2 types of soil .................................................118

Figure V-22: Mode shapes comparison for fixed base structure in deck ...............................................................119

Figure V-23: Mode shapes comparison for spring base structure in deck .......................................................120

Figure V-24: Comparison of ratio effective mass to total mass in $x$ and $y$ direction ............................................121

Figure V-25: Frequencies and mode shapes for transversal deck direction $(3 D)$................................................123

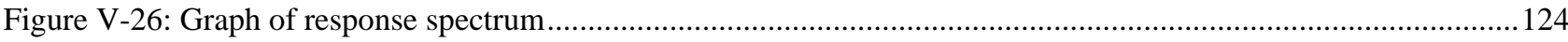

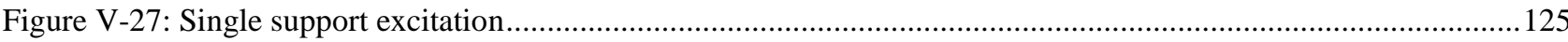


Figure V-29: Displacement on the supports of the bridge for same acceleration input; horizontal (left), and vertical (right).

Figure V-30: Displacement on the supports of the bridge for creating relative displacement; horizontal (left), and vertical (right)

Figure V-31: Simulation of (a) horizontal relative displacement, and (b) vertical relative displacement

Figure V-32: Modeling of earthquake acceleration time histories on the supports of the bridge (same acceleration)

Figure V-33: Two synthetic horizontal accelerations applied to the supports of the bridge ......................................132

Figure V-34: Relative displacement for fixed base (a) horizontal and (b) vertical ...............................................134

Figure V-35: Relative displacement for spring base (a) horizontal and (b) vertical ...............................................135

Figure V-36: ANSYS procedures to subtract the two displacement time histories for obtaining relative

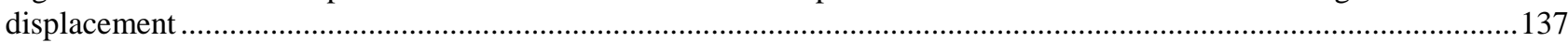

Figure VI-1: Freebody diagram for equation of motion (Sucuoglu \& Akkar, 2011).............................................140

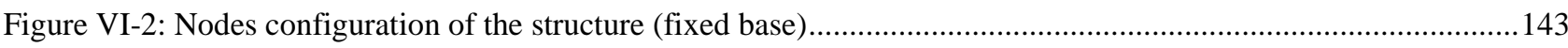

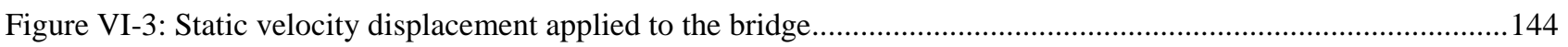

Figure VI-4: Bending moment diagram for different support beam ................................................................144

Figure VI-5: Comparison between $\mathrm{M}-\varphi$ Curves for rigid, semi rigid and simple / flexible connection .....................145

Figure VI-6: Bending moment diagram for gravity load (fixed base) ...............................................................146

Figure VI-7: Bending moment diagram for beam with relative displacement .....................................................147

Figure VI-8: Bending moment diagram for relative displacement loading ........................................................147

Figure VI-9: Bending moment diagram for fixed base bridge with relative displacement (a) horizontal relative displacement, (b) vertical relative displacement

Figure VI-10: Static moments-displacements diagram for fixed base(a) middle and (b) joints nodes in $x$ direction.

Figure VI-11: Dynamic moment for a fixed base: (a) dynamic only without relative displacement, (b) dynamic with relative displacement - horizontal direction, (c) dynamic with relative displacementb - vertical direction ............151

Figure VI-12: Dynamic bending moment diagram for horizontal relative displacement (fixed base) ..........................152

Figure VI-13: Dynamic moments behaviour in vertical relative displacement (fixed base) ......................................153

Figure VI-14: Nodes configuration of the structure (spring base) …................................................................. 154

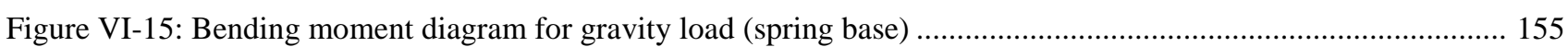

Figure VI-16: Bending moment diagram for spring base bridge with (a) horizontal relative displacement, (b) vertical relative displacement

Figure VI-17: Static moments-displacements diagram for spring base (a) middle nodes and (b) joints nodes for horizontal relative displacement.....

Figure VI-18: Comparison between fixed base support and spring base support ..................................................158

Figure VI-19: Relative displacement for (a) fixed base and (b) spring base ..........................................................158

Figure VI-20: Dynamic moment for the spring base (a) dynamic only without relative displacement, (b) dynamic with horizontal displacement-horizontal dir, (c) dynamic with vertical relative displacement........................159

Figure VI-21: Horizontal relative displacement effect to bending moment (spring base) ........................................160

Figure VI-22: Vertical relative displacement effect to bending moment (spring base) .............................................162

Figure VI-23: Dynamic bending moment without relative displacement (fixed base) ............................................162

Figure VI-24: Dynamic bending moment after applying relative displacement (fixed base) .....................................163 
Figure VI-25: Bending moments for dynamic load only and dynamic load plus relative displacement at node 1 (left support-for fixed base).

Figure VI-26: Bending moments for dynamic load only and dynamic load plus relative displacement at node 31 (mid span-for fixed base).

Figure VI-27: Dynamic bending moment before applying relative displacement (spring base) ...............................165

Figure VI-28: Dynamic bending moment after applying relative displacement (spring base) .....

Figure VI-29: Bending moments for dynamic load only and dynamic load plus relative displacement at node 1 (mid span- spring base)

Figure VI-30: Bending moments for dynamic load only and dynamic load plus relative displacement at node 31 (mid span-spring base). .168

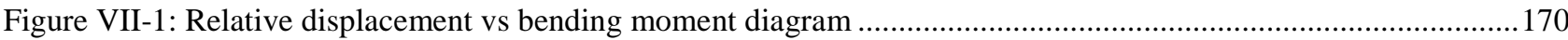

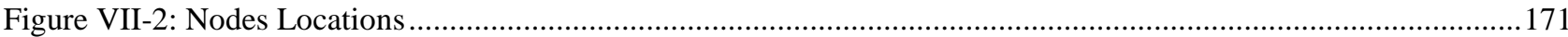

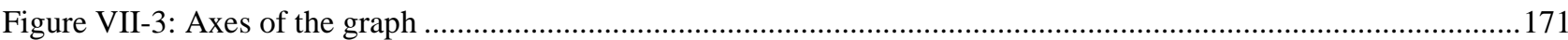

Figure VII-4: Linear relationship between bending moment and relative displacement ........................................172

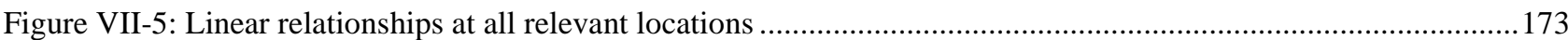

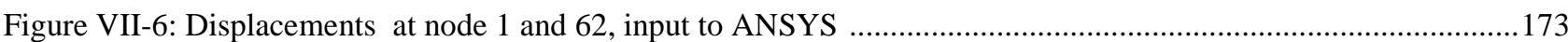

Figure VII-7: Relative displacement between node 1 and the other support locations............................................174

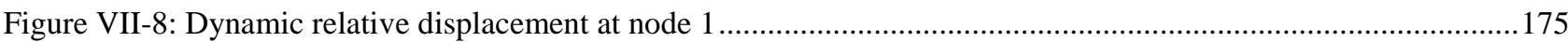

Figure VII-9: Dynamic relative displacement and static bending moment line .....................................................175

Figure VII-10: Correlation between linear bending moment to max relative displacement. ......................................176

Figure VII-11: Graph for analyzing bending moment relationship to relative displacement in joint nodes ..................177

Figure VII-12: Bending Moments under horizontal relative displacements (deck).................................................178

Figure VII-13: Bending Moments under vertical relative displacements (deck) ..................................................179

Figure VII-14: Bending Moments under horizontal relative displacements (piers) .................................................179

Figure VII-15: Bending Moments under vertical relative displacements (piers) .....................................................180

Figure VII-16: Pattern of Bending Moments under horizontal relative displacements ..........................................181

Figure VII-17: Pattern of Bending Moments under vertical relative displacements .................................................182

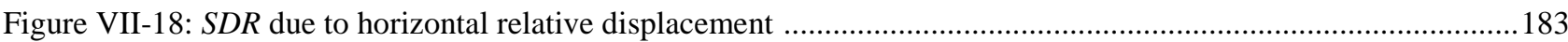

Figure VII-19: Pattern of bending moments under vertical relative displacements ................................................184

Figure VII-20: Effect of soil stiffness differences in static bending moment (horizontal RD)....................................185

Figure VII-21: Effect of soil stiffness differences in dynamic bending moment (horizontal relative displacement) ......185

Figure VII-22: Bending moment for different vertical and horizontal soil stiffness due to horizontal relative displacement (deck)

Figure VII-23: Bending moment for different vertical and horizontal soil stiffness due to vertical relative displacement (deck)

Figure VII-24: Static Dynamic Moment ratio for fixed base (horizontal relative displacement) .................................187

Figure VII-25: Pattern of bending moments under horizontal relative displacements (fixed base) ............................188

Figure VII-26: Bending moment for fixed base bridge due to horizontal relative displacement (deck)......................188

Figure VII-27: Bending moment for fixed base bridge due to horizontal relative displacement (piers) .......................189

Figure VII-28: Static Dynamic Moment ratio for fixed base (vertical relative displacement) ....................................189

Figure VII-29: Pattern of bending moments under vertical relative displacements (fixed base)................................190

Figure VII-30: Bending moment for fixed base bridge due to vertical relative displacement (deck)..........................190 
Figure VII-31: Bending moment for fixed base bridge due to horizontal relative displacement (deck).

Figure VII-32: Moment distribution value for deck for horizontal direction .........................................................192

Figure VII-33: Moment distribution value for piers for horizontal direction ............................................................192

Figure VII-34: Static dynamic moment ratio for fixed base for horizontal relative displacement .............................193

Figure VII-35: Moment distribution value for deck for vertical direction ..............................................................194

Figure VII-36: Moment distribution value for piers for vertical direction . ...............................................................194

Figure VII-37: Static dynamic moment ratio for vertical direction ..................................................................195

Figure VII-38: Static and dynamic style of supports and middle nodes for fixed base for horizontal direction .............196

Figure VII-39: Moment distribution value for deck for horizontal direction .........................................................197

Figure VII-40: Moment distribution value for piers for horizontal ..................................................................198

Figure VII-41: Static dynamic moment ratio for horizontal direction ..................................................................198

Figure VII- 42: Static and dynamic moment range of supports and middle nodes for hard soil .................................199

Figure VII-43: (a) Static and, (b) dynamic extreme moment values comparison for hard soil in horizontal

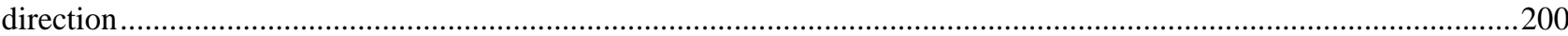

Figure VII-44: Static dynamic moment ratio distribution for horizontal direction ................................................200

Figure VII-45: Static dynamic moment ratio for vertical direction .................................................................201

Figure VII-46: Static and dynamic style of supports and middle nodes for hard soil for vertical direction ...................202

Figure VII-47: (a) Static, and (b) dynamic extreme moment values comparison for hard soil in vertical direction .......202

Figure VII-48: Static dynamic moment ratio distribution for vertical direction .......................................................203

Figure VIII-1: Dynamic bending moment in node 1 (deck) and node 62 (piers) ....................................................210 


\section{List of Tables}

Table II-1: Indiana limitation for integral abutment bridges (Frosch \& Lovell, 2011)............................................29

Table II-2: Types of soil constitutive model (David \& Forth, 2011) ......................................................................30

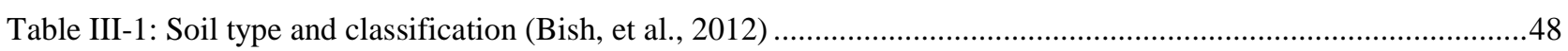

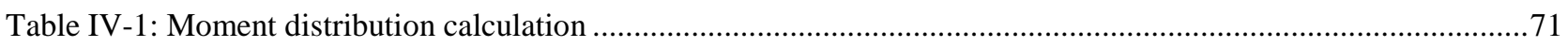

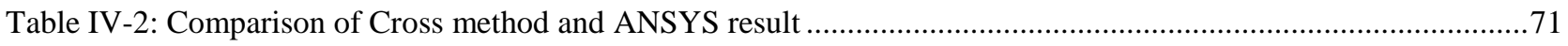

Table IV-3: Typical Elastic Moduli of soils based on soil type and consistency (density) .........................................72

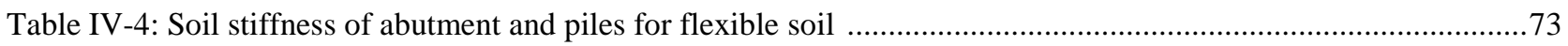

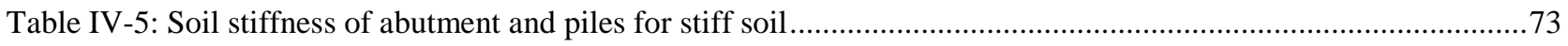

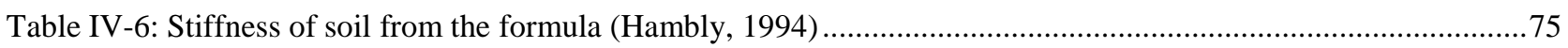

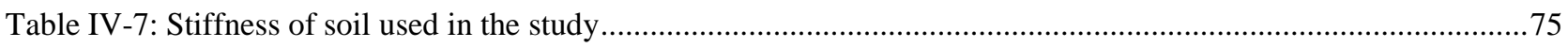

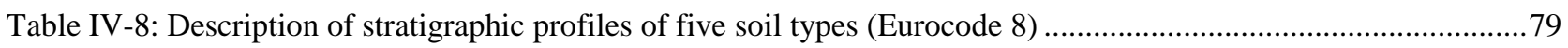

Table IV-9: Recommended values of parameters describing the vertical elastic response spectra .............................81

Table IV-10: Values of the parameters describing the recommended type 1 elastic response spectra.........................82

Table V-1: Participation factor calculation from modal analysis in the $\mathrm{x}$ direction (fixed base) ..................................111

Table V-2: Participation factor calculation from modal analysis in the y direction (fixed base) ..................................112

Table V-3: Participation factor calculation from modal analysis in the $\mathrm{x}$ direction (spring base) ...............................117

Table V-4: Participation factor calculation from modal analysis in the y direction (spring base) ................................117

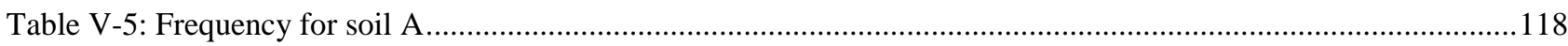

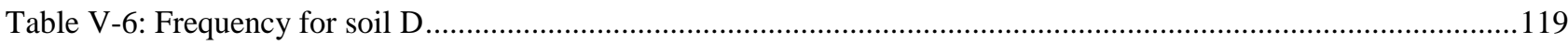

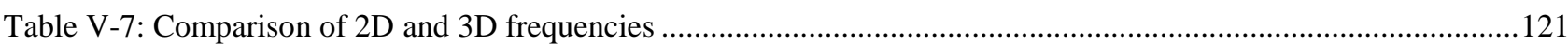

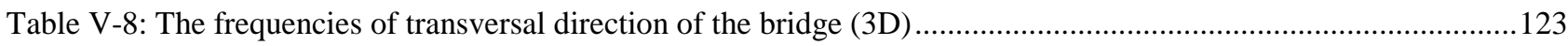

Table VII-1: The ' $k$ ’ factors for horizontal relative displacement ......................................................................204

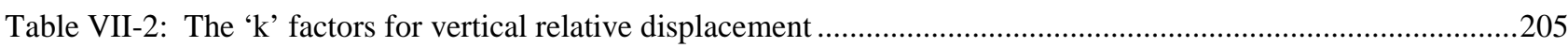

Table VIII-1: Multiplying factors ' $k$ ’ obtained from the study.......................................................................212 


\section{Chapter I INTRODUCTION}

\subsection{General}

An integral bridge is a bridge, which is built monolithically as one structure. These bridges are also known as integral abutment bridges, jointless bridges and rigid frame bridges. Different with a conventional bridge, which is constructed with the incorporation of movement joints, integral bridges are built without movement joints and connections. As a result, compared to conventional bridges, integral bridges will be stiffer as this provides greater redundancy. In conventional bridges, when the superstructure and substructure are effectively separated, it will allow some differential movement caused by settlement, temperature and so forth. For integral bridges, this differential movement is addressed by the abutments and then transferred to the ground. Therefore, abutments in integral bridges will have an important role compared to the conventional bridges.

There are two types of integral bridges, namely fully integral bridges and semi-integral bridges (Burke, 2009). Fully integral bridges are constructed rigidly without any expansion joints or bearings, whereas semi-integral bridges typically have sliding bearings but no expansion joints. Fully integral bridges are really monolithic structures. Meanwhile semi-integral bridges have a continuous deck across the length of the bridges. Therefore, it can be said that semi-integral bridges are a combination of conventional bridges and fully integral bridges.

Integral bridges have some advantages and disadvantages when compared to conventional bridges. The first advantage of this kind of bridge is an improved cost regarding 
construction and future maintenance. The reason behind this is the fact that integral bridges do not have expansion joints and bearings so that the process of the construction can be relatively simple compared to traditional bridges. Joints and bearings can account for a significant portion of the cost when compared to traditional bridges because they need to be maintained properly, regarding their vulnerability in fatigue and corrosion. Therefore, by eliminating joints and bearings the cost can be significantly reduced. Secondly, integral bridges are simple in their design because the designers do not need to design the bearings and joints. Thirdly, these bridges can be constructed rapidly due to the absence of bearings and joints. The obvious structural advantage is the increased degree of redundancy and this leads to improved structure. There are some disadvantages, however. The first is that due to its high rigidity, there is a propensity for cracks to form in certain locations, especially at the connections between the deck and piers or abutments. Therefore, integral bridges require more complex analysis for this loading. The second disadvantage is that this type of a bridge is not recommended for long spans, due to its lack of flexibility. The third disadvantage is that settlement in the approach slabs will have a significant effect on the structural integrity of the bridge.

When compared to other types of structures, integral bridges are considered to possess good dynamic load (earthquake) resistance (Burke, 2009). The reason for this is that integral bridges, as rigid and monolithic structures, have a high redundancy, smaller displacements, can eliminate the possibility of unseating, and have a larger damping due to soil structure interaction. Therefore, it is interesting and useful to explore the behaviour of integral bridges under dynamic loads, especially in correlation with relative displacements due to vertical and horizontal ground motion in supports, which can be an issue for rigid structures like integral bridges.

\subsection{Background of Research}

The consideration that integral bridges have been found to have good resistance to earthquake loads has been acknowledged by many researchers. Tandon (2005) has stated that integral bridges can be made earthquake resistant more conveniently than bridges with bearings. His reason for this is the fact that in traditional bridges, bearings commonly become a big problem because usually the damage in bridges for earthquake loading is the result of bearing failures. The statement of Tandon (2005) can be accepted because bearings are the place where the service loads transferred before coming to the abutments. 
Burke (2009) also claims that an integral bridge should be able to survive unusual ground displacements without a collapse. He has also stated that when integral bridges are constructed on stable embankments and subsoil, they should have an adequate response to most earthquakes. It is because for seismic forces, the soil behind the abutment provides additional damping to the overall system. This leads to good structural integrity with regards to earthquake loads.

As a monolithic structure, integral bridges are affected by temperature changes and secondary stress occurred. However, according to Burke (2009), the effect of temperature on the integral bridge is not significant for a bridge less than $300 \mathrm{ft}$ (91 metres) of span and can be ignored. This simplification is permitted because design specifications permit higher stresses when secondary stresses (shrinkage, creep, passive pressure, etc.) are combined with primary stresses (dead load, live load, and impact), to determine maximum allowable stresses. In addition, Burke (2009) said it should be remembered that secondary stresses do not alter the ultimate load capacity of structures. Burke's statements are also supported by Indoria (2010) who stated that secondary effects may not need to be considered when designing short single span or multiple span continuous bridges. Indoria (2010) has also said that these considerations are possible because design specifications usually permit higher primary stresses than these secondary stresses.

Relating to the earthquake, the principal cause of earthquake induced damage is ground shaking. As the earth vibrates, all structures on the ground surface including a bridge, will respond to that vibration in varying degrees. Earthquake induced accelerations, velocities and displacements can damage or destroy a bridge structure unless it has been designed and constructed or strengthened to be earthquake resistant. Therefore, the effect of ground shaking on structures is a principal area of consideration in the design of earthquake resistant structures. Seismic design loads are extremely difficult to determine due to the random nature of earthquake motions. However, experiences from past strong earthquakes have shown that reasonable and prudent practices can keep a structure safe during an earthquake.

Generally, earthquake ground motions have different directions, namely horizontal and vertical direction. Behaviour of integral bridges under earthquake loading has already been studied by many scholars. Rodriguez, et al. (2011) stated that for an earthquake in the transverse direction, the central piers of an integral bridge tend to carry greater portions of 
the global load, but the fact remains that all piers and the two abutments contribute to transmitting the inertial load to the ground, avoiding the hard points and stress concentrations of conventional bridges.

Many works also have been published with regards to a structural design that resists horizontal earthquake loads. Rodriguez, et al. (2011) made a conclusion that integral bridges display a very good response during earthquakes, particularly in the longitudinal direction. Plastic yielding and other non linearities tend to reduce progressively the effects of any differences caused by pre-existing displacements.

However, the vertical component of earthquake ground motion has commonly been underestimated in the earthquake design of structures. Papazoglou and Elnashai (1996) state that it is commonly argued that vertical strong-motion acceleration peaks are insignificant for damage potential due to their low energy content. It is indeed the case that the energy content of the vertical component is significantly less than that of the corresponding horizontal component. Nevertheless, such a basis for dismissing the vertical component is inadequate, since the horizontal energy content is dominated by long period pulses, which are non-existent in vertical strong-motion records. The significance in earthquake response of the structure is the relationship between structural and excitation periods (resonance). Hence, the strong-motion energy stored in this frequency range is the absolute criterion that decides on the importance of the components of an earthquake. Furthermore, it is argued that a large safety factor against gravity loads exists in properly engineered structures and hence, the probability against failure from vertical earthquake forces is low.

Meanwhile, Veletzos and Restrepo (2008) have reported their study on the effect of vertical earthquake ground motion. They stated that the vertical component of ground motion significantly affected the segment joint response and the magnitude of the response can vary dramatically depending on the pre-earthquake stress-state in the superstructure.

The difference between the displacements of two distant supports (known as relative displacements), has been long interesting to engineers due to its importance in designing expansion joints and support length of long span structures such as bridges and pipelines. Kasai et al. (1994) also remarked in their paper on the complex nature of the problem, with an emphasis on the relative displacement characteristics of the bridge. 
Nowadays, integral bridges have been already well constructed all over the world. Starting in the USA, this type of bridge is now employed in Europe, Australia, New Zealand, Japan and other Asian countries. The United Kingdom has even adopted integral bridges in its regulations, as any bridges less than $60 \mathrm{~m}$ should be constructed integrally. However, because the standards of integral bridge constructions have traditionally been based on engineering judgement, the design practices of integral bridges vary. Therefore, not many design codes and provisions apply to this type of bridges. Hence, studies are necessary to simplify the design or appraisal of integral bridges.

While considerable work has been accomplished to date, many questions remain regarding the behaviour of integral bridges, specifically regarding the deformation demand for the supporting piles. In particular, a large gap exists in the understanding of the static and dynamic behaviour of integral bridges. In other words, although many studies have been carried out with regards to the structural dynamic behaviour of integral bridges, comprehensive studies are still required. It is hoped that further research can provide insight into mitigation against earthquake loading, and demonstrate that integral bridges are an attractive design option where such dynamic loading is an issue.

It is wise to study the effect of vertical and horizontal earthquake ground motions on the integral bridge structures as vertical earthquake components do not get much attention in the analysis and bridge construction. In addition, since the integral bridge will be more greatly affected by the displacement of its supports, the study should also incorporate the effects of soil types. However, this study is more focused on the structural behaviour of integral bridges rather than the geotechnical aspect of it.

In this research, the behaviour of integral bridges subject to biaxial translational earthquake ground motion was analysed. The observation was conducted by assessing the static response of the bridges which was then compared to the dynamic response of the structure due to seismic loading. When the bridges are subject to an earthquake, both dynamic response due to resonance effects and relative displacements of the founding supports are required, as the supports usually have significant distance between them. The contribution of static relative displacements of the bridges to overall moment distribution of the structure is required first. Following on from this, the dynamic analysis attempted to identify the contribution of resonance effects and dynamic relative displacement to the overall structural response. In addition, since the integral bridges have a rigid structure, the 
effect of relative displacements occurring in their supports due to earthquake loads needs to be considered. As a rigid structure, relative displacements are an important problem for this type of bridges because these can disturb the system overall.

It is envisaged that recommendations for design approaches can be made for the use of this type of bridge construction for use in areas where there is a high risk of major seismic events occurring. The analysis will be carried out primarily using the finite element software ANSYS.

\subsection{Specific Aims and Objectives of the Research}

The advantages offered by integral bridges leads to a desire for their extended use and applicability. However, the relative displacement effects on integral bridges, especially those from earthquake loads, should be understood. As bridges have several supports in their structures which are built on different areas, different displacements may occur for each of the supports, especially when earthquakes occur. This leads to relative displacements which may cause additional structural loading of the bridge. Integral bridges, due to their rigidity, are prone to this problem, and it is becoming increasingly difficult to ignore the relative displacement effects of integral bridges.

Therefore, the objective of this study is to develop an understanding of the behaviour of integral bridges under earthquake ground motion, especially in relative displacements problems, such that their use and applicability can be extended and a rational design can be developed. The final purpose is to propose a simplified approach to design/appraisal of the bridge, which can produce a conservative estimate of the dynamic behaviour based on static relative displacement analysis results for a range of soils.

Hence, several questions need to be addressed in this research, namely:

a. How does this particular bridge construction respond to relative displacements due to earthquake ground motion that are applied to it vertically and horizontally?

b. How do the contributions of relative displacement at the supports of the bridges compare to the overall moment distribution of the bridges? 
c. What are the responses of the bridge under differing soil conditions applied to it and how does this differential displacement of the supports affect the loads experienced within the structure?

d. What are the responses of the bridge under a fixed base support condition and springs base support condition?

The primary aim of the research is to carry out a finite element analysis to provide insights into the behaviour of integral bridges under dynamic or seismic loading. Features inherent in bridges for seismic mitigation were to be examined statically and dynamically, and modifications suggested where appropriate to improve seismic performance.

\subsection{Research Scope and Limitation}

Studies of this nature can quickly lead to complex models where many parameters can affect the results. In the majority of cases the task of interpreting the influence of these parameters becomes impossible. Engineers require simple and appropriate solutions to design problems, and this also applies to any analysis models adopted. In this instance, dynamics, soil characteristic, soil structure interaction all form part of the study, yet each of these provides a myriad of issues on their own. Thus, as every research has its own limitation, this research also has several limitations. Hence, the following general assumptions are adopted to enable a sensible interpretation of how parameters affect the results:

a. The model will be 2D linear elastic. 2D analysis is chosen for simplification and by considering that the bridge geometry which is used in this research is not complicated. It is also assumed that the stiffness in the cross deck direction is large and therefore distribution of loading will be uniform. In addition, buckling is not considered in the study because bridge decks are commonly configured to control or eliminate the problem, so the bridge may be modelled in 2D.

b. Both static and dynamic direct integration transient analyses will be conducted. Static analysis is used by applying static relative displacement to one node of the bridge. The relative displacements are applied at the left end support of the bridge (node 1, as can be seen in Figure VI-3). 
c. Dynamic direct integration transient analysis is conducted in order to obtain relative displacement at one node (the left end node as the static relative displacement applied). Both static and dynamic analyses give bending moments which are then compared.

d. Relative displacement is only applied to one support of the bridge. How this relative displacement affects the behaviour of the bridge structure is then analysed by comparing the bending moment. Although in reality the relative displacements are considered to occur not only in one node or one area, however for this simplified approach is acceptable as a basic principle.

e. A range of synthetic horizontal and vertical earthquakes time histories will be constructed for various soil types (in accordance with Eurocode- 8 spectra). These different synthetic horizontal and vertical earthquake time histories are needed to create different displacements of the supports which causes relative displacements at the left end support of the bridge.

f. A range of soil springs (as well as a fixed base scenario) will be used at the bridge supports. Four cases are considered; the first case applies high stiffness to the supports vertically and horizontally, the second case is applies low stiffness to the support vertically and horizontally, and the third case is by applying high stiffness horizontally and low stiffness vertically, and the fourth case is applies low stiffness horizontally and hard stiffness vertically. The results are compared and analyzed.

g. Static relative displacement studies are conducted, to be compared with the dynamic case whereby different earthquake time histories are applied at the supports.

h. To determine the soil types used in this research, Eurocode 8 is used (Kolias, 2008). However, since this study is more about structural behaviour, focus will be more on the structural subject and not on soil and geotechnical problems. Moreover, this study purpose has been looking more at qualitative behaviour rather than quantifying structure integrity.

i. Effects of cracking are not considered as the analysis is done linearly. Temperature and secondary effects also have not been considered in this study, since the length of the bridge is less than $300 \mathrm{ft}$ or $91 \mathrm{~m}$ (Burke, 2008). 
j. Therefore, only earthquake loads, traffic loads and self-weight loads will be considered in this research, since other loads are assumed constant and do not impact the analysis. Variable loads considered are the earthquake loads and variable material properties considered are different type of soil conditions.

\subsection{Methodology}

In general, finite element analysis will be employed to conduct two analyses. The first is a series of static analyses and the second is a series of transient dynamic analyses (seismic analysis). Since this research is to develop an understanding of the behaviour of integral bridges under earthquake ground motion, the following research steps will be conducted:

a. Evaluate the effect of relative displacement resulted from supports settlements horizontally and vertically. This static analysis will be conducted by using ANSYS ver. 12 .

b. Generate synthetic earthquake ground motions based on Eurocode 8 by using Clough and Penzien procedures (Clough \& Penzien, 2003) . A Dynamassist, which is a derivation of Mathcad software is used for this purpose (Larsen, 2001).

c. Create different acceleration time histories that come from the same earthquake ground acceleration. For this purpose, Seismosoft ver. 1.3 is used for obtaining the baseline corrected version of the results from Dynamassist.

d. Evaluate the effect of earthquake ground motions to the structures. Acceleration time histories are applied to every support of the 2D bridge model by using ANSYS ver. 12.

e. Develop analysis procedures that properly yield the composition of moment from relative displacements and moments from earthquake loading. A graph which describes the relationship between bending moment and relative displacement is constructed. From this graph, the contribution of static moment due to relative displacement in relation to the overall dynamic bending moment can be distinguished.

f. Develop appraisal recommendations that define dynamic response based on static response of this bridge type. The relative displacement - bending moment diagram is used as a core material to develop the recommendation. 
As a study case, a concrete integral bridge with $3 \times 20 \mathrm{~m}$ spans is used. The bridge is an existing bridge in India, as India is known to have a high seismic risk. The bridge will be initially modelled in 2D using beam elements through the finite element software, ANSYS ver.12.

From static analysis, a pattern of static moments is going to be achieved and these patterns will be used as a trend line to recognize the static relative moments from dynamic moments of the structure. Meanwhile, for the dynamic analysis, a transient analysis is done by applying a series of synthetic acceleration time histories of $0.35 \mathrm{~g}$. This analysis will give results of dynamic bending moments of the structure from which the contribution of dynamic and static moments may be analysed and compared.

Model validation is conducted using simple moment distribution method. Addressing different soil types in same steps and methods is also a validation strategy in this research. Similar patterns and trend lines are assumed to be a good sign that the results of the analysis are acceptable.

\subsection{Outline of the Thesis}

The dissertation has been organised in the following way. It begins by introducing the background of the research, specific aims, scope and limitations, and the methodology in Chapter I. The next two chapters (Chapter II and III) address the literature review of integral bridges and earthquakes. The advantages and disadvantages of integral bridges are presented and how earthquakes influence the loading system in a structure is also performed. Chapter IV addresses the methodology applied in this research including the study case of the research. Procedures in generating synthetic earthquakes ground motions also conveyed.

Chapter V describes the design, synthesis, characterization and evaluation of dynamic analysis of the research. Response spectra created are also discussed in this chapter. The response spectrum is then adopted in the synthetic earthquake accelerations which are then applied to the supports of the bridge model. This chapter also assesses the baseline corrections which are considered to the displacements in order to provide the accurate displacements data. Modal and transient analyses also are discussed in this section. 
Chapter VI contains the results and discussion of the analysis. It discusses the relationship between bending moment and relative displacements. How the relationship is presented in a graph is also explained in this section. Finally a conclusion and summary of findings are presented in Chapter VII, along with suggestions for future works and recommendation. 


\section{Chapter II REVIEW OF INTEGRAL BRIDGES}

\subsection{Introduction}

Bridges are conventionally designed as structures with bearings and roller connections at their supports. This is for several reasons, such as severity of the weather (temperature), movements of the earth, traffic loading, and the material used in construction, any of which could cause expansion and contraction to the bridge superstructure. For conventional bridges, expansion and contraction stresses are relieved by adding expansion joints in the structure. However, bearings and expansion joints are recognized as a reason for the high maintenance cost of bridges. The weak points in the bridges are also considered to occur at these bearings and joint connections, especially because they are vulnerable to environmental problems such as corrosion from humidity or ice and snow ( Burke, 2009).

An alternative bridge construction is the integral bridge, which does not have expansion joints or bearings. Instead, integral bridges have a rigid structure and all of the loads, including stresses induced by expansion and contraction, are transferred directly to the abutments and substructures. However, for short- and medium-span integral bridges, these secondary effects of loading can be ignored (Burke, 2009).

In recent decades, integral bridges have become an alternative choice for bridge engineers for short and medium spans to overcome the maintenance problems previously mentioned. In America and some countries in Europe, integral bridges have been constructed and used for a considerable time. Considering that integral bridges can be constructed in earthquake 
zones, there has been an increase in the interest of bridge engineers to conduct research and expand the possibilities of building them in other parts of the world. Although much research has been carried out on this subject, and much remains to be explored, with a deeper understanding of earthquakes and integral bridges. The unique characteristics, such as higher redudancy structure and rigid connections, encourage researchers to explore further.

Regarding earthquakes, codes of practice and provisions from many countries do not adequately explain vertical earthquake ground motions. Vertical earthquake loads are assumed to be equivalent to only two-thirds of horizontal earthquake loads (Newmark, 1973). This means that current codes of practice assume that vertical earthquake loads will not exceed the values of horizontal earthquake loads. Unfortunately, histories and field evidence show that much structural damage is caused by vertical earthquake ground motion (Papazoglou \& Elnashai, 1996).

\subsection{Integral Bridge Characteristics}

Integral abutment bridge construction has become an increasingly popular alternative in recent years and has been applied and constructed worldwide. These kinds of bridge have shown good structural performance due to their redudance and durability. In the USA, more than a thousand integral bridges have recently been built in several states, including Tennessee and California. For the US as a whole, a survey indicates that there are over 13,000 integral abutment bridges in service in the country (Frosch, et al., 2009). In addition, many integral bridges have been built in the United Kingdom, Germany and other European countries. This method of bridge construction has become especially popular in the UK, where Highway Agency regulations now state that for a new bridge with a length of less than 60 metres, where possible it must be in the form of an integral bridge. Advice note BA 42/96 The design of integral bridges published by the Highway Agency 'states that in principle all bridges should be continuous over intermediate supports, and bridges with overall lengths not exceeding $60 \mathrm{~m}$ and skews not exceeding $30^{\circ}$ are to be integral with their abutments (Iles, 2006).

As already mentioned, there are some significant differences between integral and conventional bridges. The most important is that integral bridges are rigid in their structure and conventional bridges are not. These differences are illustrated in Figure II-1. 

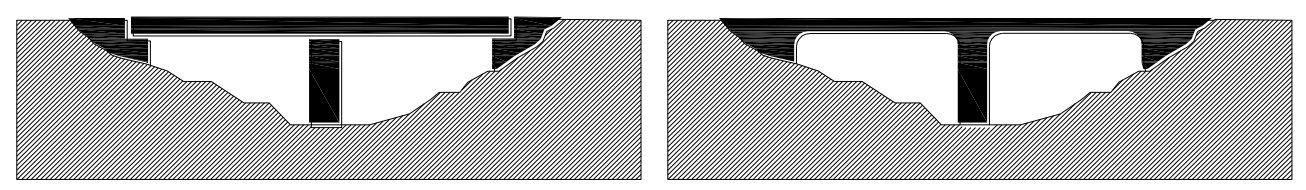

Figure II-1: Fundamental differences between conventional bridge (left) and integral bridge construction (right)

It can be seen than the conventional bridges have expansion joints and bearings in their structure, while integral bridges are built monolithically without bearings or expansion joints.

The idealization of the bridge in simple form is shown in Figure II-2. Such bridges are the solution for small and medium-length bridges where bearings and expansion joints can either be eliminated altogether or reduced to a minimum. Their decks are continuous and connected monolithically to the abutment with a moment-resistant connection. This leads the structure to act as one unit. So far, this type of bridge has had a good record of initial cost savings, with economical use of material and maintenance. The absence of expansion joints at the abutment and bridge deck leads to reduced construction and maintenance costs. Engineers are therefore increasingly interested in using integral bridges, although there are still many problems to be overcome, such as soil-structure interaction and cracking.

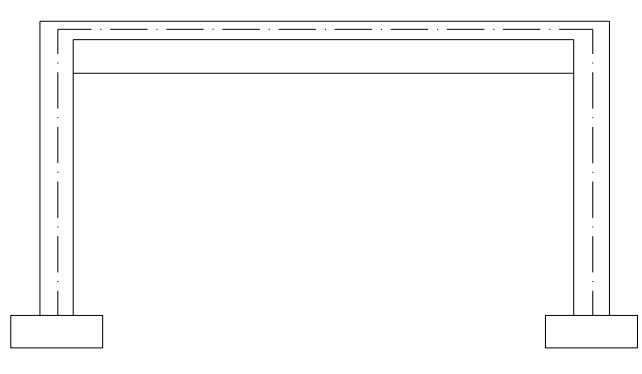

(a) Integral Bridge

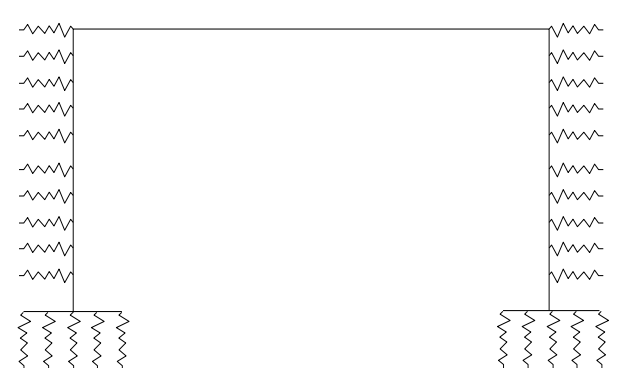

(b) Integral Bridge Idealization

Figure II-2: Idealization of integral bridge

In Figure II-2 above, soil is modelled as a series of springs acting horizontally and vertically to support the structure.

In comparison to conventional bridges, there are two important differences in the structural arrangements of integral bridges, as shown in Figure II-3 below, namely: 
1. The road transition between the bridge and the adjacent embankment.

Integral bridges have to be provided with approach slabs to prevent vehicular traffic from causing the adverse effect of consolidating backfill adjacent to the abutments. This approach slab must be anchored to the bridge, otherwise continual bridge movements and joint infiltration may shift the slab towards the approach pavement.

2. The connection between the superstructure and abutments or piers.

The main difference between conventional bridges and integral bridges is the connection between the superstructure and abutment or piers. It is clear from Figure II-3 that the connection for the integral bridge is fixed, but for the conventional bridge it is not fixed.
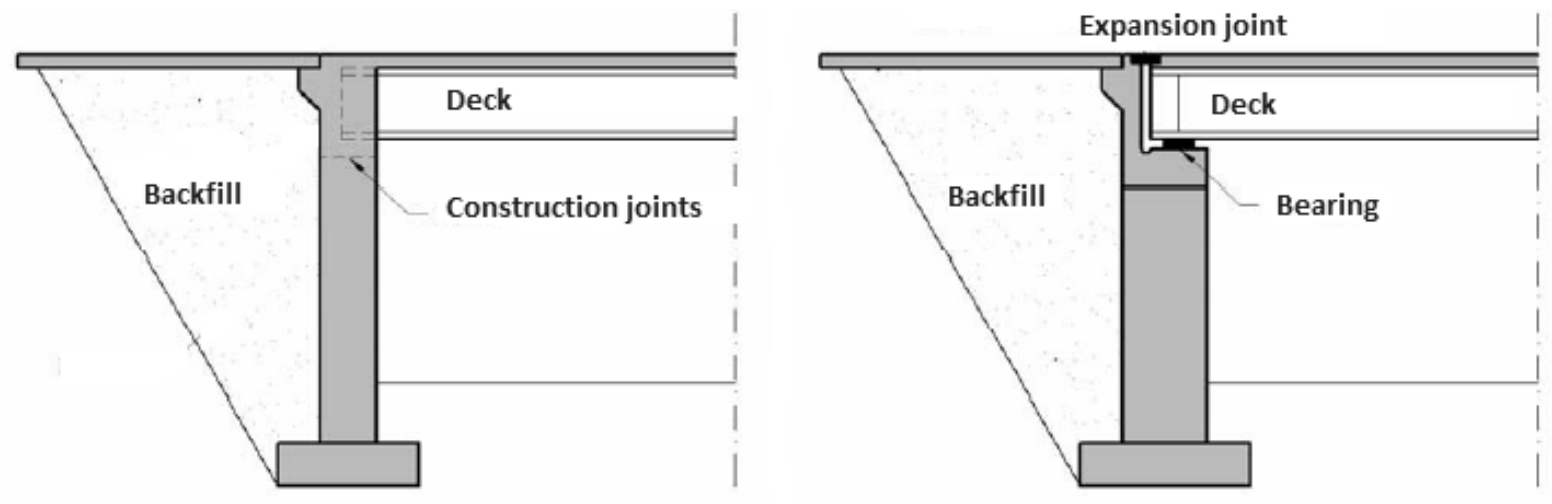

Figure II-3: Structural arrangement of integral bridge and conventional bridge construction

Bearings in conventional bridges, as shown in Figure II-3, will allow free rotation of the bridge ends; the bearings are thus considered to be hinged supports, independent of the geometry of the abutment. Meanwhile, the expansion joints are usually applied to allow free expansion in the longitudinal and transverse direction of the bridge, thereby reducing stress from temperature changes which have secondary effects on the structure.

However, for relatively short bridges, neglecting the effects of the passive pressures may be acceptable (Arsoy, et al., 1999). Burker (2009) agrees that the secondary effects on short integral bridges are insignificant.

There are two types of integral bridge, namely fully integral bridges and semi-integral bridges. The former is a structure without expansion joints and bearings, whereas the latter is a rigid structure without expansion joints but with bearings. In the semi-integral configuration, the deck is continuous and bearings are put between the deck and the piers; 
hence, this formulation creates a structure which is not strictly an integral bridge, although it does eliminate the conventional expansion joint details between the bridge deck and abutment.

Figure II-4 compares conventional bridges, fully integral bridges and semi-integral bridges. Conventional bridges use expansion joints to accommodate the effects of temperature change, such as expansion in the summer heat. With their absence of joints, integral bridges have more soil-bridge interaction than other types of bridge. Combined with their structural continuity, this provides redundancy and resilience and improves their ability to sustain overloads.

As stated earlier, integral bridges cost less to construct and require less maintenance than equivalent bridges with expansion joints. In addition to reducing construction and future maintenance costs, integral bridges provide for additional efficiencies in the overall structural design.

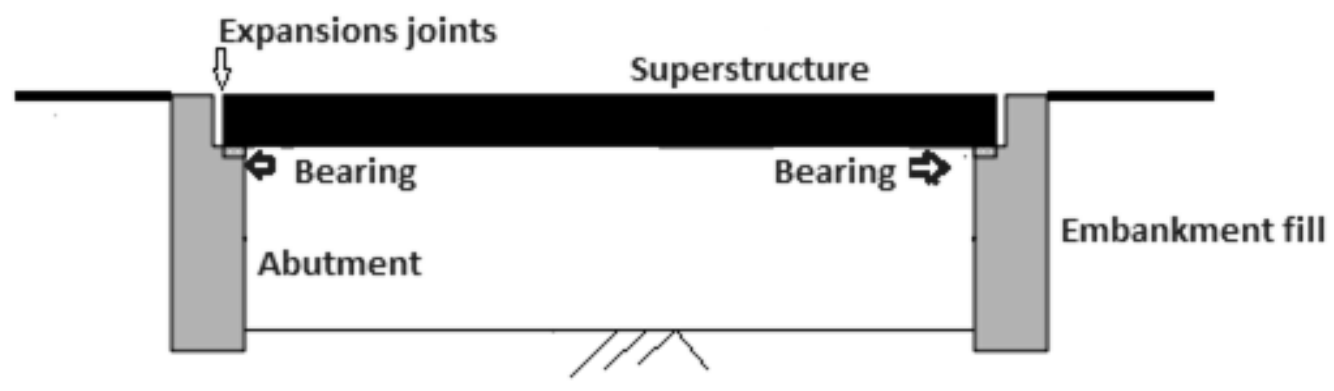

a. Conventional bridge

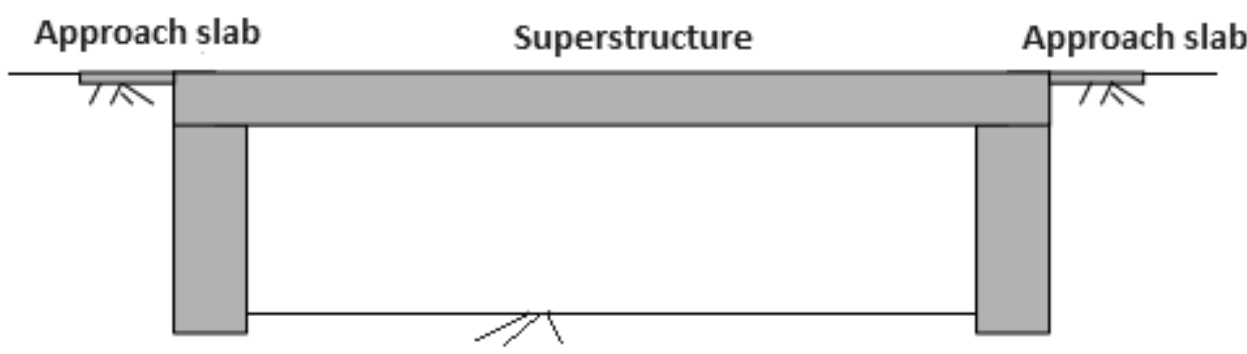

b. Fully integral bridge 


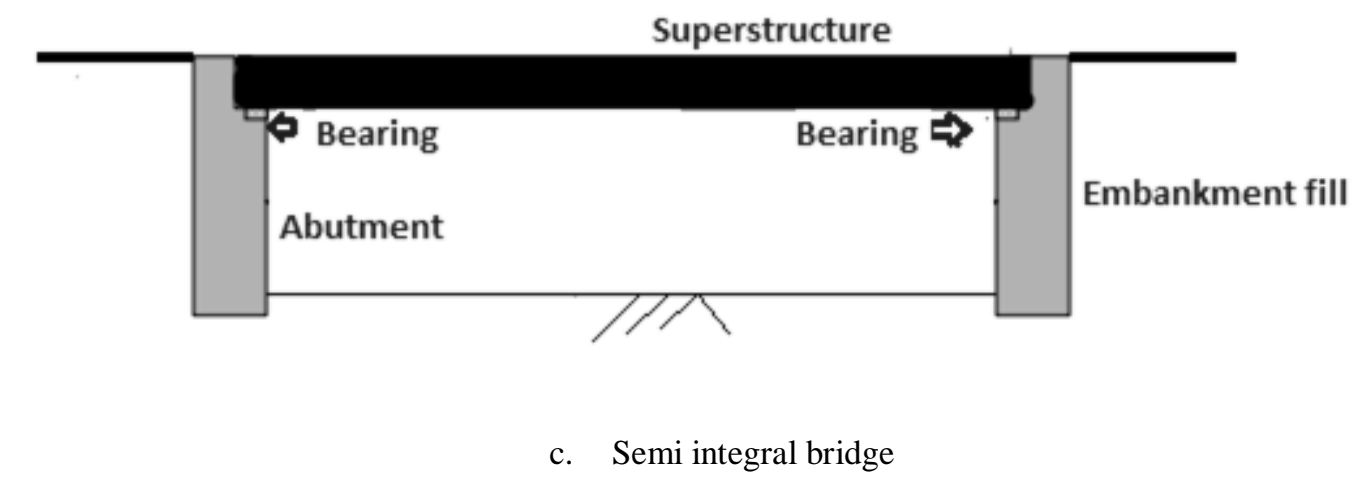

Figure II-4: Comparison of: (a) conventional bridge, (b) integral bridge, and (c) semi integral bridge

Integral bridges have numerous attributes and few limitations. Some of the most important attributes are summarized below.

i. Simple Basic Design

Integral bridges are simply framed structures. They can be designed as continuous beams because the piers' flexural stiffness is considerably less than deck stiffness. This simplicity gives an advantage to the structural concept and also to the designer because it will not consume so many resources. However, it is also important to create a structural model including both the superstructure and substructure in order to obtain an economic design. The fact that the stiffness of the abutments influences the distribution of internal forces in superstructure and in the abutment itself cannot be ignored. Therefore, since the soil stiffness interaction should be considered, the whole design process of an integral bridge can be more complex than for a conventional bridge.

ii. Rapid construction

The simplicity of the integral bridge in design and characteristics leads to rapid and economical construction.

iii. Lower construction costs and future maintenance costs

Since an integral bridge has no bearings or joints, the design will benefit from savings in initial costs. The absence of joints and bearings also leads to reduced maintenance cost and effort. Additionally, current deck joint-sealing devices have been shown to have only a short effective service life. 
iv. Built-in abutments can be designed to accommodate some bending moment capacity, reducing end-span bending moments with possible minor savings in endspan girders.

v. If end spans are part of a flexurally continuous superstructure, integral abutments can provide capacity against uplift if necessary.

vi. Bridge bearings usually require a reasonably close degree of tolerance in their assembly. This precision can be avoided with integral abutments.

vii. A jointless bridge with integral abutments will have a greater degree of redundancy that may be beneficial in earthquake zones; Jointless design is actually preferable, since it reduces the most common cause of seismic structural damage. Joints are identified as a potentially high collapse mechanism in the performance of the overall structure (Dexter \& Connor, 1997). Integral bridges show consistently good performance during actual seismic events.

Despite the significant advantages of integral abutment bridges, there are some problems and uncertainties associated with them. The primary concern in the design of integral bridges is that high stresses can develop in the superstructure and substructure as a result of secondary loads, because of the continuity of connection between the superstructure and the substructure. These stresses are the result of restrained thermal expansion and contraction, creep, shrinkage and settlement.

The other uncertainties related to the design and performance of integral abutment bridges are:

1. The elimination of intermediate joints in multiple spans results in a structural continuity that may induce secondary stresses in the superstructure. These forces are due to shrinkage, creep, thermal gradients, differential settlement, differential deflections, and earth pressure, which can cause cracks in concrete bridge abutments. Wing walls can crack due to rotation and contraction of the superstructure. Also, differential settlement of the substructure can cause more damage to integral bridges than to traditional bridges.

2. Integral bridges should be provided with approach slabs to prevent vehicular traffic from consolidating backfill adjacent to abutments, to eliminate live load surcharging of backfill, and to minimize the adverse effect of consolidating backfill 
and approach embankments on the movement of vehicular traffic. For bridges with closed decks (kerbs, barriers, etc.), approach slabs should be provided with kerbs to confine and carry deck drainage across backfill to the approaches and prevent erosion, or saturation and freezing of the backfill.

3. The piles that support the abutments may be subjected to high stresses as a result of cyclic elongation and contraction of the bridge structure. These stresses due to lateral load can cause deformation of plastic hinges in the piles and may reduce their axial load capacities.

4. Other limitations to integral bridges include the fact that they cannot be used with weak embankments or subsoil, and they can only be used for limited lengths, although the maximum length is still somewhat unclear. Integral bridges are suitable if the expected temperature-induced moment at each abutment does not exceed the value specified by national authorities, and somewhat larger moments can be tolerated (Burke, 2009).

There are several specific features of integral bridges, as follows:

\section{Approach Slab}

The approach slab, as shown in Figure II-5, is a gradual transition between the roadway and the bridge deck. Although the approach slab does not have a significant effect up on the magnitude of the differential settlement that will ultimately develop, it reduces the local settlement quite significantly. The bridge approach consists of two kinds of settlement, namely global and local settlement. Global settlement is a consolidation of the underlying natural foundation soils, and is evidence of possible long-term differential settlement between the bridge structure and the bridge embankment. Local settlement consists of compression of fill materials directly beneath the approach pavement. It is the combination of global and local settlements adjacent to the bridge end piers that forms the characteristic 'bump'in the pavement at the bridge ends. The purpose of the bridge approach slab is to induce a smooth grade transition between the bridge and the roadway (Burker, 2009). 


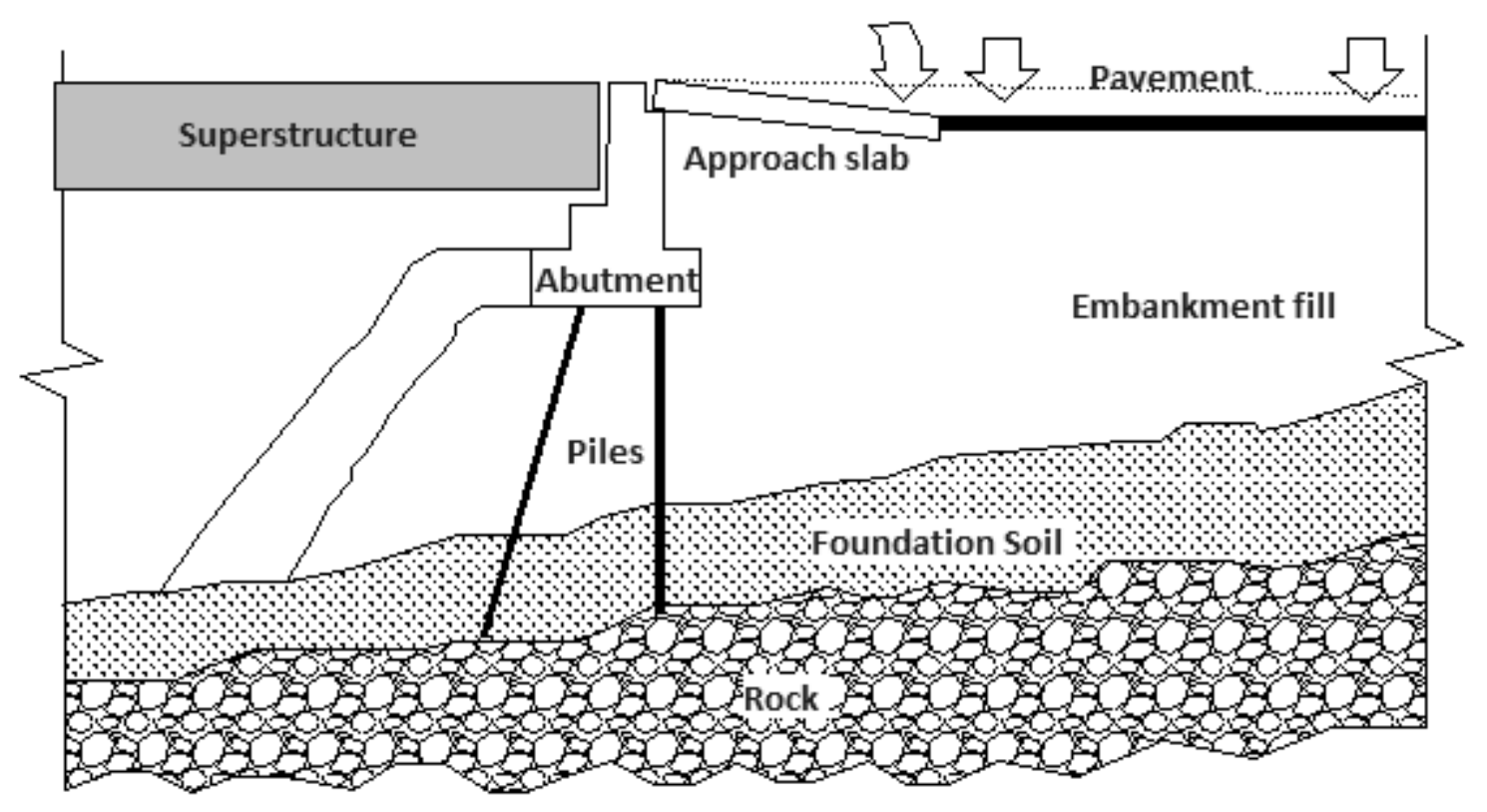

Figure II-5: Bridge approach settlement

\section{Abutment}

The abutment is an important support for a bridge, because the functions of the abutments are to transmit the loads from the superstructure to the foundation or to the earth, and to withstand the earth underneath and adjacent to the approach roadway. For an integral bridge, abutments are very important because this element is one of the main supports of the structure to resist the loads, thermal movements, breaking forces and seismic forces.

There are several types of abutment for integral bridges, namely:

\section{a. Bank pad abutment}

This is the simplest form of abutment an integral bridge can have. As shown in Figure II-6, it is constructed integrally with the deck, acting as a shallow foundation for the end span and as a shallow retaining wall for adjoining pavements and embankment. This shallow abutment is only applicable to a condition where the foundation is very stiff and no settlement problem can occur. During thermal expansion and contraction of the deck, this abutment will move horizontally. However, it must have adequate weight, and the end span have adequate flexibility, to avoid uplift from live loads or from differential settlement. 


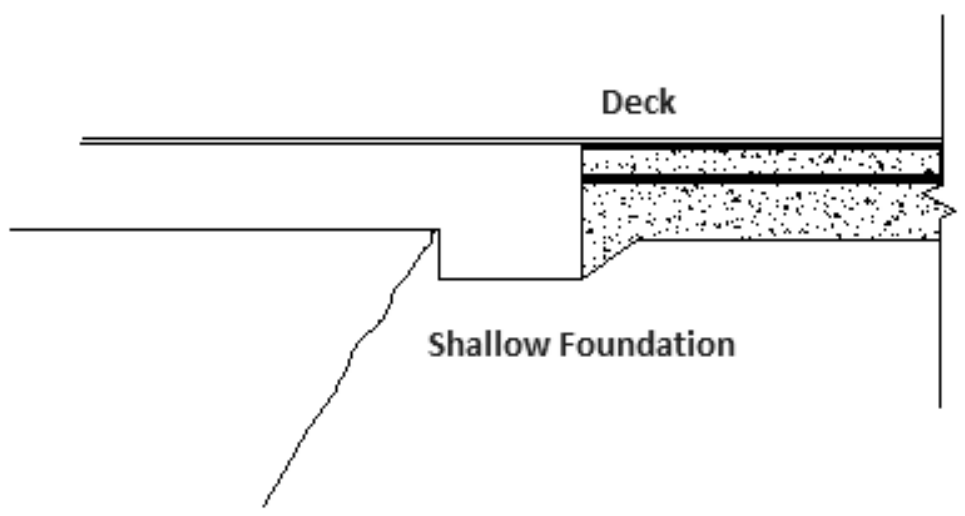

Figure II-6: Bank pad abutment

\section{b. Embedded Abutment}

This is a configuration of end support comprising a diaphragm wall, including contiguous secant or sheet pile walls, with the toe embedded in the ground below the lower ground surface. This type of abutment is also more suitable when used for short-span bridges. It can be seen in Figure II-7.

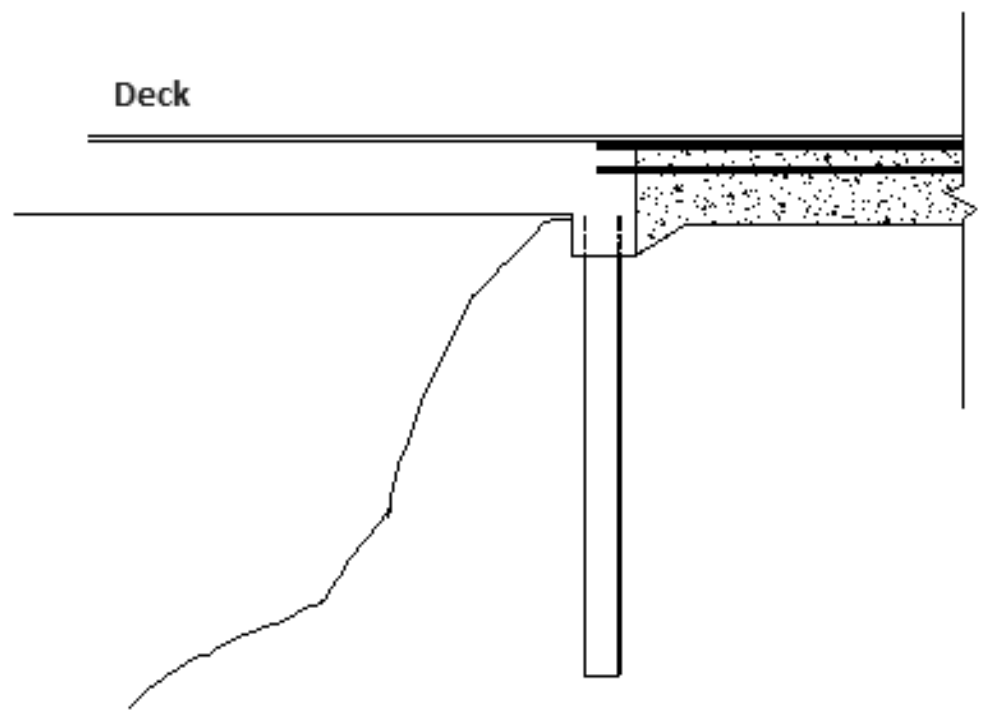

Figure II-7: Embedded abutment

\section{c. End Screen Abutment}

This type of abutment, as shown in Figure II-8, is a wall structure cast monolithically with, and supported off, the end of the bridge deck, providing a retaining wall for adjoining ground, but not acting as a support for vertical loads. It is only a retaining wall for embankment earth pressures and transfer of longitudinal loads. The vertical loads on the 
deck are supported by separate supports, located several metres off the end screen in order to limit the vertical movement of the end screen when the end span deflects. The end supports may be isolated structurally from horizontal movements of the end screen, or they may be connected to the deck, in which case they must be able to resist, or avoid, the earth pressures arising from their movement relative to the embankment.
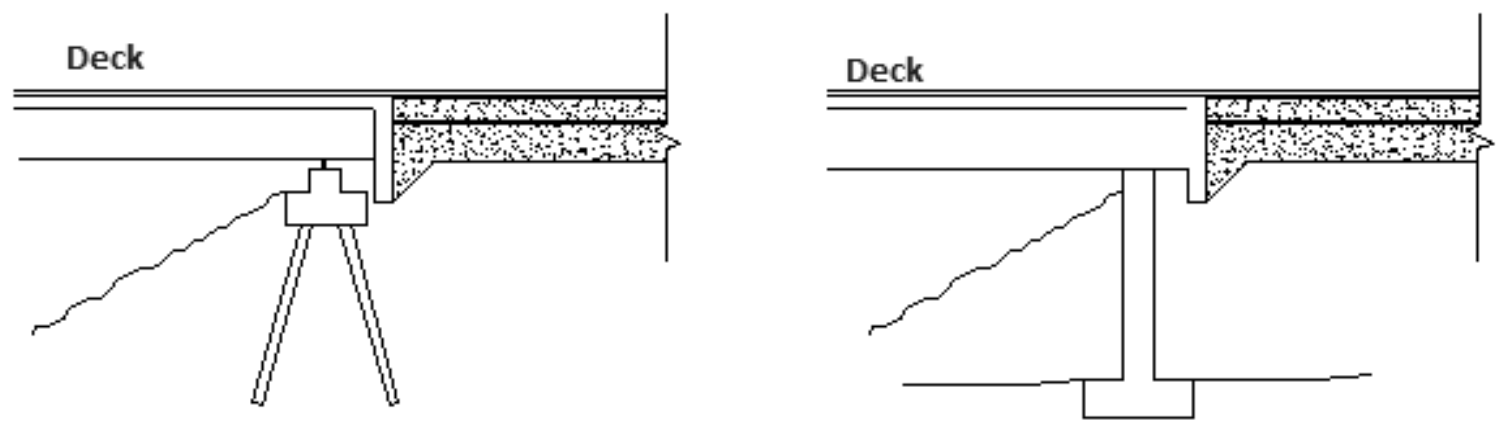

Figure II-8: End screen abutment

\section{d. Frame Abutment}

This is the end support for bridges constructed integrally with the deck and acting as a retaining wall for adjoining pavement and ground below. Figure II-9 shows the configuration of this abutment.
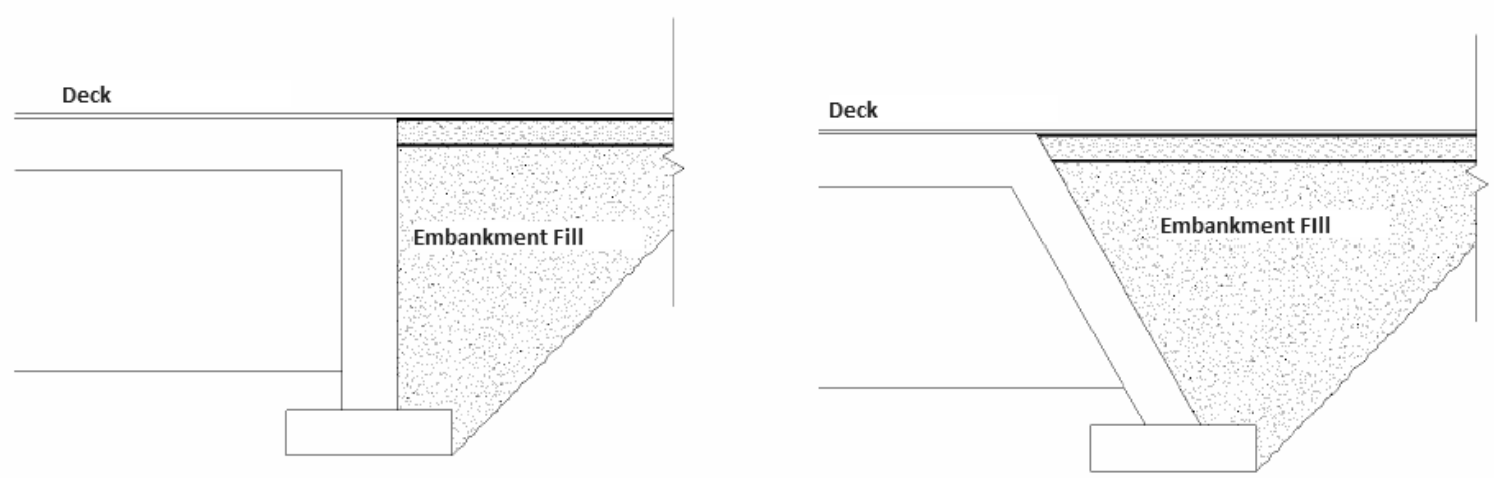

Figure II-9: Frame abutment

\section{Granular Backfill}

Granular backfill is selected granular material placed adjacent to the abutment wall and forming the sub-grade for the adjoining pavement construction. 


\section{Piers}

Piers are the supports placed in between the ends of the integral bridges; they can be designed to move horizontally with the superstructure or with a bearing which allows lateral movement beneath the deck (Burke, 2009).

\subsection{State of the Art of Integral Bridges}

The principle of the integral bridge has long been known, and used by the Romans. Since then and until the mid-1920s, integral bridges were constructed as arch bridges.

According to Mourad and Tabsh (1999), the use of this kind of structure in the modern era was first considered after analyzing the successful performance of old bridges without joints. Hence, and because conventional bridges suffer from the use of joints and connections, jointless bridges have recently been built in several countries (Mourad \& Tabsh, 1999). In 1940, a brief 10-page paper written by Professor Hardy Cross, entitled Analysis of continuous frames by distributing fixed end moments, led to the widespread application of framed structures, although it initially The provoked serious discussion among academics Cross had described a simple and quick method for the analysis of a continuous frame or beam structure, and this method has since been widely used by bridge engineers, in what amounts to a revolution in bridge design and construction. Previously, many multiple span bridges were built as a series of simple statically determinate spans.

Since the 19th century, engineers have used expansion joints in bridge design as stone, the traditional construction material, was replaced by steel and concrete. Expansion joints and bearings separate the superstructure from the substructure and allow their relative displacement. However, the lifespan of expansion joints and bearings is significantly lower than the lifespan of the structure itself. Hence, the bearings and expansion joints are often thought of as a problem because of their maintenance and cost. The effort to eliminate maintenance and operating costs led back to a structure which no longer used expansion joints and bearings, the integral bridge. Because the superstructure and substructure are usually a framed connection, the integral bridge is also termed a frame bridge.

In the USA, there are currently more than 9,000 fully integral bridges and 4,000 semiintegral bridges. The USA started to apply the use of integral abutments tied to the bridge superstructure in 1930. Burke (2009) said that minimization of movable deck joints at 
piers began in the late 1920s. Tennessee, Missouri, Ohio and Kansas are several of the early states which built this type of bridge. In 1990, 11 states had already constructed continuous integral bridges with lengths of up to 300 feet $(91 \mathrm{~m})$, while Tennessee and Missouri had even longer bridges. Most reports state that the bridges exhibit a good and effective performance, since they have remained in service for a long time with only simple maintenance and repairs. By 2001, 35 of the 50 states had built integral bridges. Burke also describes how the Tennessee Department of Transportation appears to be leading the way in the construction of continuous bridges. For example, the Long Island Bridge at Kingsport was constructed in 1980 using 29 continuous spans without a single intermediate movable deck joint. The total length of this bridge is some 2,700 ft. (823 m), centre to centre of abutment bearings. Movable deck joints and movable bearings were furnished, but only at the two abutments.

Virginia has reported more than 10 years of satisfactory performance with their more than 25 integral bridges. California, Kansas, Tennessee, Washington and Wyoming have each constructed over 1,000 integral bridges. All have had at least satisfactory experiences. Kansas and Tennessee rate their best experiences as being very good. Arizona discontinued the use of integral bridges because of the expensive repairs to all the approaches of their more than 50 integral bridges. Alaska also had problems with integral bridges, as frozen soil adhered to integral back walls and caused hairline cracking.

Several Canadian provinces have embraced integral bridge construction. Alberta, Quebec, Nova Scotia and Ontario have jointless bridges, and most have reported from good to satisfactory experiences with their use. Nova Scotia built its first integral bridge in 1986, and Quebec in 1988. Ontario limited its integral bridge span to less than 325 feet $(100 \mathrm{~m})$ and a 20-degree skew angle. Ontario's recommendations for their integral bridges are typical of those adhered to by many US states.

Like the USA, the UK applied this bridge concept at the beginning of its development, and integral bridges have become increasingly popular over recent years. Furthermore, because of the great success in its application, the UK now recommends that any new bridge less than $60 \mathrm{~m}$ in length should be constructed as an integral bridge. Overall cost efficiencies and longer life expectancy of integral bridges compared with jointed bridges are the main reason for this recommendation. 
A graph presented by Iles in his 2006 paper and reproduced in Figure II-10 describes the trend for bridge construction in the UK using steel material pre-2000 to 2004.

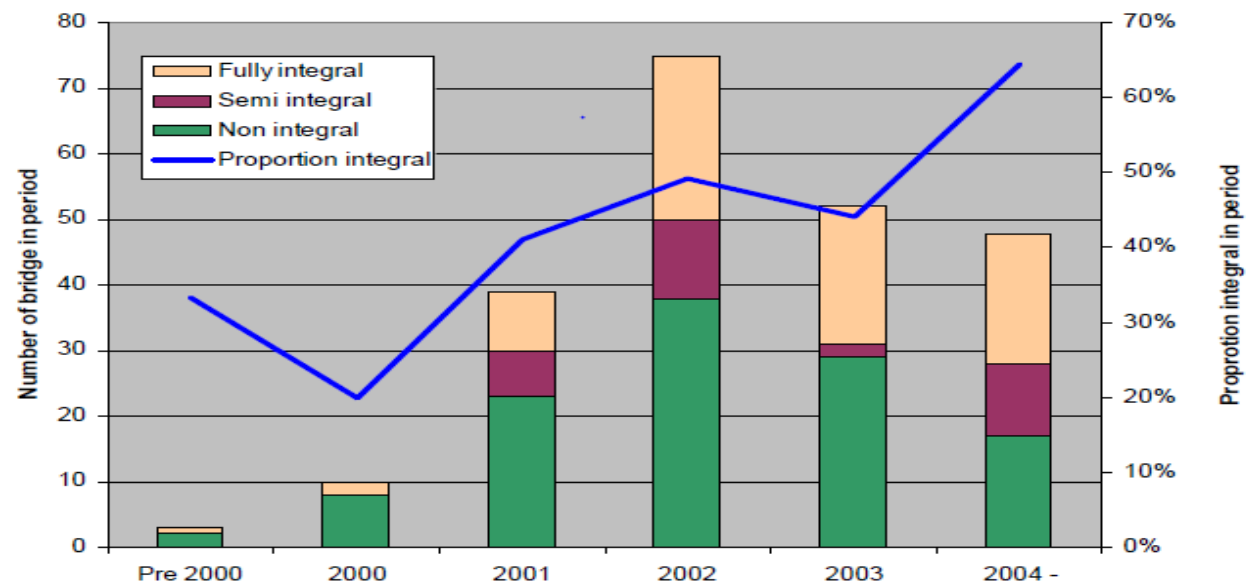

Figure II-10: Trends of bridge construction in steel material from before 2000 to 2004 in the UK (Iles, 2006)

Figure II-10 also shows that the construction of conventional bridges (non-integral bridges) has decreased since 2002, replaced by integral bridges, fully and semi-integral. The blue line illustrates the proportion of integral bridges, and it is clear that their proportion increased significantly during this period, accounting for about half of the total bridge construction by 2004 .

According to a European survey carried out in 2007, only Britain, Finland, Ireland and Sweden were building semi-integral bridges. Although they did not respond to the survey, Norway is reported to be employing semi-integral bridges, but not fully integral bridges. Flener (2004) wrote that in Sweden, integral bridge construction had been in use for over 70 years. He stated that integral bridges are the most common bridge types, representing 8,000 of the 14,000 Swedish Road Administration-owned bridges. Germany also reports the construction of integral bridges throughout the country.

White (2007) believed that the European experience with integral abutments was significantly less, but that the experience gained to date had been positive. Consequently, integral bridges will form a larger proportion of planned and newly constructed bridges across Europe.

The typical Australian practice for most medium to short span bridges is to minimize the use of expansion joints generally by adopting link slabs over piers for simply supported spans. The Australian Bridge Design Code contains no particular reference to integral 
abutment bridges or jointless bridge decks (Connal, 2005). Designers must refer to the general design requirements contained in the code and to relevant specialized literature.

In Japan, integral bridges have not been as popular as in the United States, Canada or the United Kingdom. Nevertheless, construction of this type of bridge has started to increase rapidly, because of cost efficiency and ease of maintenance. The first integral bridge, the Naibekoshinai River Bridge, which is 110 m in length, was built in 1996. South Korea started to apply this type of construction in 2002.

To summarize, different countries have different reasons for adopting integral bridges, ranging from earthquake resistance to an urgent need to reduce the cost of maintenance and inspection, even when increased resistance to sudden loads has to be taken into account.

\subsection{Design Basis and Methodology}

In the design of integral bridges, forces are induced in the structure by:
a. Dead and live loads
b. Temperature changes and gradients in the superstructure
c. Moisture content and shrinkage of concrete bridge decks
d. Differential settlement of foundations
e. Earth pressures
f. Pavement pressures.

An integral bridges can be analyzed and designed as a continuous frame with a single horizontal member and two or more vertical members. Burke (2009) stated that because the vertical members are more flexible than the horizontal members, for this kind of a bridge it can also be assumed that the horizontal member has simple supports. Hence, the frame action of an integral bridge can be ignored when considering the vertical load effects on the superstructure, except for the design of continuity connections at the abutments. Further, for a short integral bridge with flexible piers constructed monolithically, a further simplification is that piers and abutments do not need to be designed to resist either lateral or longitudinal loads. This is because the longitudinally and laterally rigid concrete deck 
slabs are rigidly connected to the abutments and to one or more piers, whereas abutments are rigidly restrained by confining embankments. As a consequence, all lateral and longitudinal loads applied to the superstructure will be directly transmitted to the abutment embankment. Hence, piers and abutments do not need to be designed for resisting horizontal loads applied to the superstructure. Meanwhile, the design of abutment and superstructure continuity connection and transverse wing wall can be standardized for a wide range of bridge applications. In overcoming the loads, including secondary effects such as shrinkage, creep and passive pressure, a nominal amount of reinforcement will be suitable. This reinforcement is also important for transverse wing walls to resist the maximum anticipated passive pressure. Once these configuration details have been established, each bridge abutment can be configured and reinforced for vertical reactions associated with various roadway width and span lengths. Basically, only the determination of appropriate pile loads and spacing, and pile cap reinforcement, is required.

Piers are designed in a similar way. The horizontal superstructure loads are transferred to approach embankments. The moments regarding the piers or the superstructure continuity connection are also usually negligible. As a consequence, the piers of integral bridges need to be designed just as a vertical superstructure and pier loads, and for lateral loads that could be applied directly to the piers such as stream flow, stream debris, earth pressure, wind and earthquake loads. These lateral pier loads are usually small and therefore, most piers, like abutments, can basically be designed for vertical loads alone.

For piers that receive much of their lateral support from their connection to the superstructure, attention should be given to the construction procedures to ensure that these piers are not laterally loaded until the continuity connection between superstructure and abutments has been accomplished. Because the superstructure and abutments resist all primary lateral loads, piers including piles, columns, footings and foundation can be reduced to minimum size and dimensions. To summarize, piers can be simplified to the extent that standard designs can be developed for a wide range of roadway widths and span length (Burke, 2009).

Integral and conventional bridges are subject to the same loadings. The difference is, as integral bridges are designed integrally and monolithically, they are more likely to have a number of secondary effects that are difficult to quantify. However, with a proper approach to the integral bridge with some limitations and simplification, the secondary 
effects can be moderate and controlled to such an extent that the resulting design will be not only be efficient and durable, but will also be cost effective.

Regarding the secondary effects, integral bridges are actually subjected to shrinkage, creep, thermal gradients and differential settlement. They also have to withstand passive pressure when the abutment backfill is compressed during superstructure elongation. This process can be seen in Figure II-11. However, Burke (2009) stated that these secondary effects do not need to be considered when designing single or multiple span continuous bridges that are not more than $300 \mathrm{ft}(91 \mathrm{~m})$ long.
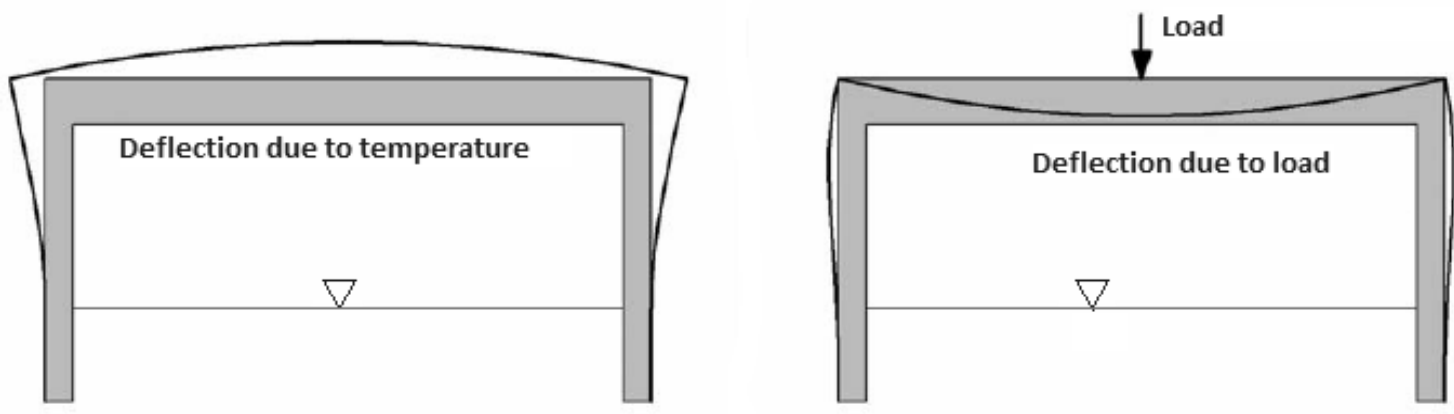

Figure II-11: Deformation of integral bridge due to temperature and traffic load

Again according to Burke (2009), the effect of temperature on the integral bridge is not significant for a bridge with a span of less than $300 \mathrm{ft}(91 \mathrm{~m})$, and can be ignored. This simplification is possible because design specifications permit higher stresses when secondary stresses (shrinkage, creep, passive pressure, etc.) are combined with primary stresses (dead load, live load, and impact) to determine maximum allowable stresses. In addition, Burke has said it should be remembered that secondary stresses do not alter the ultimate load capacity of structures.

Burke (2009) also stated that an examination of a number of trial designs has shown that, except for the design of single spans and the continuity connections of continuous spans, the sum of these secondary effects is small in comparison to the usual dead and live load effects after establishing a nominal amount of continuity reinforcement. Therefore, secondary effects can usually be ignored for most bridges less than $300 \mathrm{ft}(91 \mathrm{~m})$ long. Burke's statements agree with Indoria (2010), that secondary effects need not necessarily be considered when designing short single span or multiple span continuous bridges. This simplification is possible because design specifications usually permit stresses that are greater than these secondary stresses. 
A suitable structural model of an integral bridge is a statically indeterminate frame, where internal forces are influenced by support settlements. In the case of foundations, it is appropriate to represent soil flexibility by elastic springs located in the vertical and horizontal direction on the footings (Burke, 2009).

To take one example of specifications for maximum integral bridge length, see the figures for Indiana listed in Table II-1, as presented by Frosch and Lovell (2011).

Table II-1: Indiana limitation for integral abutment bridges (Frosch \& Lovell, 2011).

\begin{tabular}{|c|c|c|}
\hline Structure Type & $\begin{array}{l}\text { Maximum Skew } \\
\text { (degrees) }\end{array}$ & $\begin{array}{l}\text { Maximum Bridge } \\
\text { Length (ft) }\end{array}$ \\
\hline Reinforced Concrete Slab & No restriction & 500 \\
\hline Structural Steel & 30 & 500 \\
\hline Prestressed Concrete & 30 & 500 \\
\hline
\end{tabular}

\subsection{Substructures for Integral Bridges under Earthquake Loads}

As previously explained, the basic difference between integral and conventional bridges is the inclusion of the superstructure, the substructure and the surrounding soil in a single structural model. In the case of conventional bridges, the expansion joints and bearings allow relative movements between the superstructure and the substructure, and thus these two elements can be analyzed separately. However, in the case of the integral bridge, the superstructure has a necessary and direct impact on the substructure, so it needs to be modelled as a unit. For the substructure itself, it is important to incorporate the influence of the backfill behind the abutments into the structural model. The influence of the backfill is addressed by soil springs, which are applied in the abutment. These soil springs are applied horizontally, in other words transverse to the back of the abutment. This soil interaction with the abutments and superstructure as a unity is called soil-structure interaction. As an integral bridge is a rigid structure, then this soil-structure interaction is important and needs to be considered in designing this type of bridge.

Some five modelling approaches are employed by researchers in the area of soil-structure interaction, as shown in Table II-2. 
Table II-2: Types of soil constitutive model (David \& Forth, 2011)

\begin{tabular}{|c|c|}
\hline Constitutive model & Description \\
\hline Winkler model & $\begin{array}{l}\text { Idealizes the soil medium as linear or non-linear elastic springs. It } \\
\text { is considered as oversimplification of soil medium. However, it is } \\
\text { adequate and suitable for computational purpose for its reasonable } \\
\text { performance and simplicity. }\end{array}$ \\
\hline $\begin{array}{l}\text { Mohr-Coulomb } \\
\text { model }\end{array}$ & $\begin{array}{l}\text { An elastic perfectly plastic model. The model stress strain } \\
\text { behaves linearly in the elastic range. Friction angle and cohesion } \\
\text { of the soil define the failure criteria. }\end{array}$ \\
\hline $\begin{array}{l}\text { Cam-Clay model } \\
\text { (modified) }\end{array}$ & $\begin{array}{l}\text { An elastic plastic hardening model where the non-linear } \\
\text { behaviour is modelled by means of hardening plasticity. It is } \\
\text { reported that this model is suitable to describe deformation } \\
\text { compared to failure for consolidated soft soil. }\end{array}$ \\
\hline Duncan-Chang model & $\begin{array}{l}\text { A stress-dependent model which could represent the non-linear } \\
\text { behaviour of soil. Also know as the Hyperbolic model. It can } \\
\text { describe non-linearity, stress-dependent and inelastic behaviour of } \\
\text { both cohesive and cohesionless soil. Its soil parameter can be } \\
\text { obtained from the standard triaxial test. }\end{array}$ \\
\hline $\begin{array}{l}\text { Elastic Continuum } \\
\text { model }\end{array}$ & $\begin{array}{l}\text { A conceptual approach to dealing with boundary distances and } \\
\text { loaded areas. It is an infinite soil media representation. It has been } \\
\text { found that this idealization may provide more information on the } \\
\text { stresses and deformations within the soil mass than the Winkler } \\
\text { model, but it often fails to represent the physical behaviour of the } \\
\text { soil very closely. }\end{array}$ \\
\hline
\end{tabular}

An integral bridge, especially when its main span is long, is subjected to longitudinal displacements due to daily and seasonal temperature fluctuations. This effect, which is usually expressed as expansion and contraction of the bridge, will be restrained by the abutment backfill and interactive substructure, as seen in Figure II-12. 


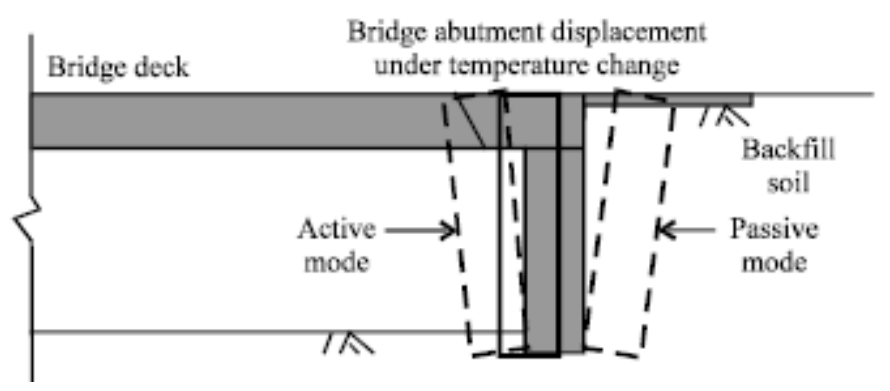

Figure II-12: Abutment wall: active and passive states (Alizadeh, et al., 2010)

Figure II-12 explains the process of fluctuations in the length of the bridge due to temperature changes. An increase in temperature drives the expansion and makes the back soil denser. When the structure contracts, the abutment will move away from the back soil and it may reduce the density of the soil. This condition will cause sliding over the wall and create an active earth pressure behind the abutment wall. Meanwhile, when the structure is elongated, the abutment will move toward the back soil, causing passive earth pressure (Alizadeh, et al., 2010). However, Burke (2009) has said that this effect is not significant for a short integral bridge of up to 300 feet.

Most civil engineering structures involve some type of structural element with direct contact with the ground. The concept of soil-structure interaction refers to static and dynamic phenomena mediated by a compliant soil and a stiffer superstructure. This area of expertise includes the amplification of seismic waves in the soil even before any structure has been erected, so it includes the complex dynamic interactions that arise spontaneously in soil layers. When external forces, such as earthquakes, act on these systems, neither the structural displacements nor the ground displacements are independent of each other. The process in which the response of the soil influences the motion of the structure and the motion of the structure influences the response of the soil is an example of soil-structure interaction. The structure imposes stresses and forces to the ground, and this will give additional force and deformation to the bridge as a consequence. This process continues until the structure and the soil reach their equilibriums or fail (in the case of excessive loading and deformations of the system). However, in general, structural analysis simplifies soil behaviour, while geotechnical analysis simplifies structural behaviour (Tandon, 2005).

Conventional structural design methods neglect the soil-structure interaction effects. This neglect is reasonable for light structures in relatively stiff soil, such as simple short-span 
bridges or simple rigid retaining walls. However, the effect of soil-structure interaction becomes prominent for heavy structures resting on relatively soft soils, for example nuclear power plants, high-rise buildings and elevated highways (Wolf, 1985).

Lateral earth pressure is mainly influenced by the soil's properties and responses. Modelling the soil is an important aspect in analysis of integral bridges. This is because the performances of the integral abutment bridges are known to be affected by the interaction between the backfill soil and the abutment, as shown in Figure II-13; this involves the relative displacement and soil stress-strain behaviour due to the lateral earth pressure. Therefore a reasonable soil constitutive model needs to be used to represent the soil properties in an analysis. Soil constitutive models are drastic idealizations of soil characteristics and a requirement for practical applications.

Damage sustained in the Kobe earthquake of 1995 also highlighted the fact that the seismic behaviour of a structure is strongly influenced not only by the response of the superstructure, but also by the response of the foundation and the ground (Mylonakis et al., 2000). Hence, modern seismic design codes stipulate that the response analysis should be conducted by taking into consideration the whole structural system including superstructure, foundation and ground.

How the structures and the soils become one rigid structure is also described in Figure II13. It is clear that the soils are connected to the structure and assumed as a series of soil springs. 


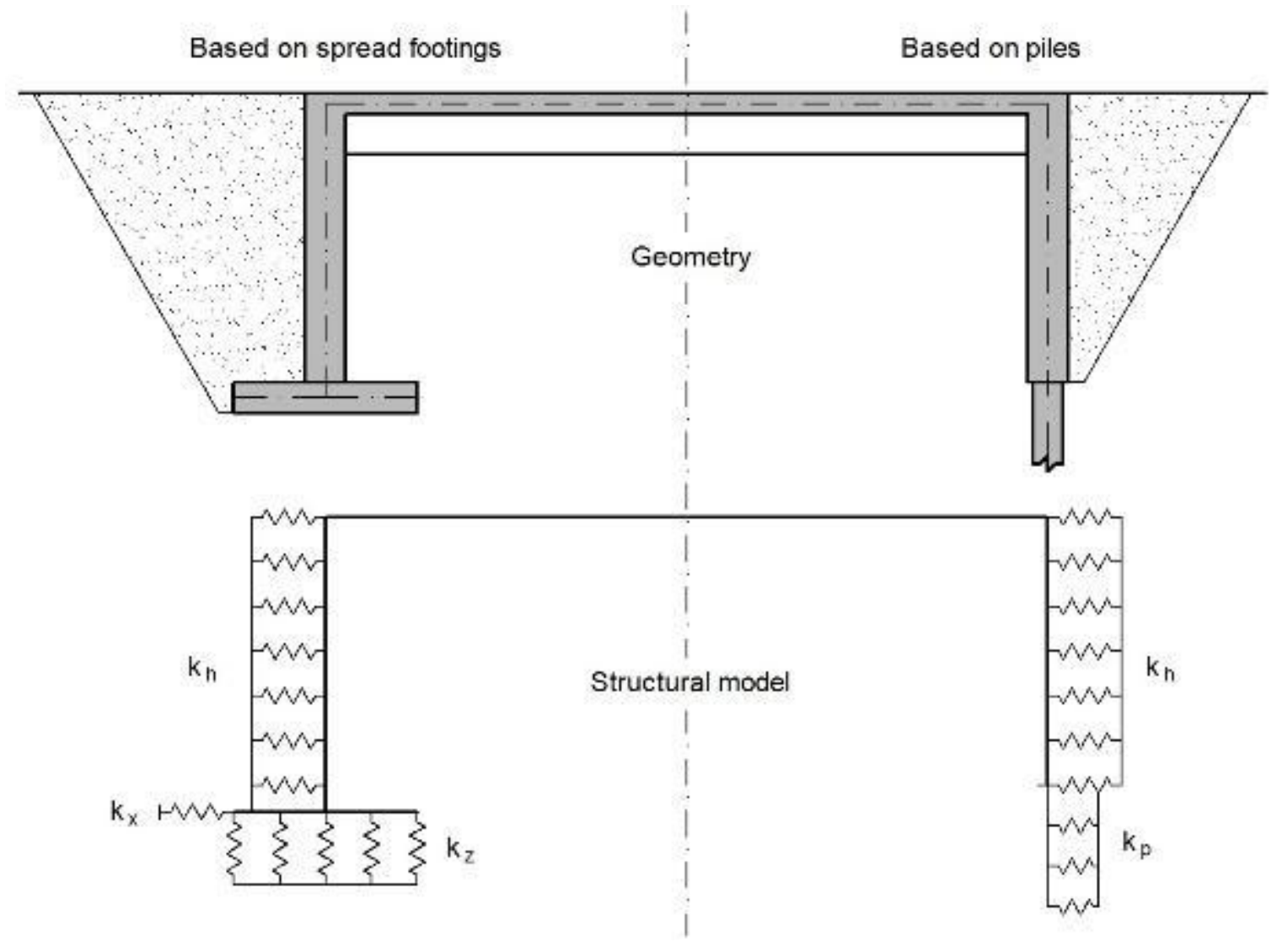

Figure II-13: Soil spring idealization of the integral bridge

It is a conventional belief that soil-structure interaction has a beneficial effect on the seismic response of a structure. However, many design codes have suggested that the effect of soil-structure interaction can reasonably be neglected for the seismic analysis of structures (NEHRP, 2004). This myth about soil-structure interaction apparently stems from the false perception that it reduces the overall seismic response of a structure, and hence leads to improved safety margins. Most of the design codes use oversimplified design spectra, which attain constant acceleration up to a certain period, and thereafter decrease monotonically with period. Moreover, considering the soil-structure interaction effect increases the effective damping ratio of the system. The smooth idealization of the design spectrum suggests smaller seismic response with the increased natural periods and effective damping ratio, due to soil-structure interaction. With this assumption, it was traditionally considered that soil-structure interaction can conveniently be neglected for conservative design. In addition, neglecting soil-structure interaction significantly reduces complexity in the analysis of structures, which has tempted designers to neglect the effect of soil-structure interaction in the analysis. 
This conservative simplification is valid for certain classes of structure and soil conditions, such as light structures in relatively stiff soil. Unfortunately, the assumption does not always hold true. In fact, soil-structure interaction can have a detrimental effect on the structural response, and neglecting it in the analysis may lead to unsafe design for both the superstructure and the foundation.

Basically, when a structure is subjected to an earthquake excitation, it interacts with the foundation and the soil, and thus changes the motion of the ground. Earthquake ground motion causes soil displacement known as free-field motion. However, the foundation embedded into the soil will not follow the free-field motion. This inability of the foundation to match the free-field motion causes kinematic interaction. On the other hand, the mass of the superstructure transmits an inertial force to the soil causing further deformation in the soil; it is termed inertial interaction.

Observations from recent earthquakes have shown that the response of the foundation and soil can greatly influence the overall structural response. At low levels of ground shaking, the kinematic effect is dominant, causing the lengthening of the period and increasing the radiation damping. However, with the onset of stronger shaking, near-field soil modulus degradation and soil-pile gapping limits radiation damping, and the inertial interaction becomes predominant, causing excessive displacements and bending strains concentrated near the ground surface, resulting in pile damage near the ground level (Wolf, 1985).

Since integral abutment analysis is a typical soil-structure interaction problem, a very realistic and reliable modelling approach has to be adopted. The approach employed to model the soil-structure interaction should be able to provide reliable and accurate analysis results.

The search for a physically close and mathematically simple model to represent the soil media in the soil-structure interaction problem has produced two classic approaches, namely the Winklerian and the Continuum approaches. The earliest mathematical idealization of the foundation medium is due to Winkler, who assumed a linear load versus settlement relation (Chandra et al., 1985). The model is thus referred to as the Winkler method; it can be seen in Figure II-14. 


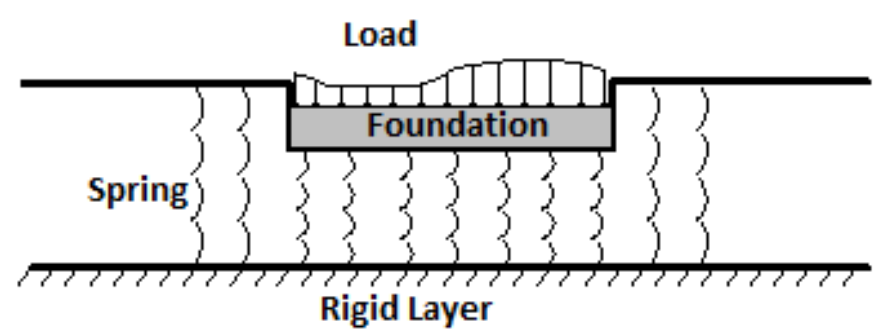

Figure II-14: Winkler spring approach

Winkler represented the case of a finite soil layer resting on basement rock by a family of linear springs resting on a rigid base, as shown in the figure above; a Winkler model requires only one parameter, the elastic spring constant. A number of researchers have modified the original model in an effort to make it more realistic. To do this, an additional parameter is needed, and these modified models are usually referred to as two-parameter elastic models.

\subsection{Failure Mechanism of Concrete Integral Bridges}

Structural failure refers to loss of the load-carrying capacity of a component or member within a structure, or of the structure itself. Structural failure is initiated when the material is stressed to its strength limit, thus causing fracture or excessive deformation. In other words, failure of a structure can be defined as any behaviour that renders it unsuitable for its intended function. Static loading can result in unacceptable deflection and elastic instability, as well as fracture.

Structures prone to distortion failure are classed as ductile, and those prone to fracture without significant prior distortion as brittle. Unfortunately, there is an intermediate grey area wherein a given material can fail in either a ductile or a brittle manner, depending on circumstances. If brittle failure occurs, it is because the conditions of loading and environment are such that they cause an almost instantaneous propagation to failure of one or more of the original cracks. If there is fatigue (cyclic) loading, the initial cracks may grow very slowly until one of them reaches a critical size, at which time total fracture occurs. However, stress is much greater at the base of a crack. Fatigue failure might better be described as progressive fracture under fluctuating or repeated loading. Fatigue fractures begin with a minute (usually microscopic) crack at a critical area (Millais, 1997). 
Wei et al. (2008) studied the patterns and failure mechanisms of bridge pile foundations under earthquake loading. They stated that with regard to the earthquake, the failure mechanism of piles is related to the force conditions under earthquake loading, so the failure mechanism can be subcategorized into three types:

(1) The failure caused by additional dynamic stress that is induced by vibration. This failure mode generally occurs when the ground motion level is high, the quality of the pile is poor and the soil layer is weak. Under such conditions, the reaction forces to piles from the surrounding soil are relatively small, the deformation of piles is relatively large, and relatively big additional dynamic stresses are generated in the pile shaft.

(2) The failure caused by additional static stress that is induced by soil lateral movement. This failure mode usually occurs at a bank-side site. Two conditions are required for this failure mode to take place:

a. The soil of a bank-side site is usually very weak, and it is easy to produce large permanent deformation under earthquake loading;

b. Due to the slope of a bank-side site, when an earthquake occurs soil elements will produce static shear stress whose direction will be the same as the bank slope; thus, lateral soil movement will be generated along the stress direction. The statistics from previous earthquake damage indicate that such failure modes tend to take place at relatively low ground-motion levels.

(3) The length of pile penetrating into the steady soil layer is not enough or the pile tip does not arrive at a steady soil layer, so a pile foundation can easily lose bearing capacity due to liquefaction of sandy soil under earthquake loading. 


\section{Chapter III EARTHQUAKE GROUNDMOTION EFFECTS - REVIEW}

\subsection{General}

Basically, earthquakes do not kill people, but bad structures do. This sentence is quite familiar to the designers in structural engineering. Usually earthquakes only cause death by the damage they induce in structures such as buildings, bridges and other works of man. In other words, damage caused by earthquakes most commonly relates to man-made structures.

Earthquakes, as time-dependent loadings, will affect structures dependent on time. The extent to which the loads affect the structures needs to be known, and a proper calculation and analysis conducted in order to reach the correct answer. For this reason, the characteristics of earthquakes need to be fully understood.

\subsection{Earthquake - Dynamic Analysis of Bridges}

\subsubsection{Fundamentals of Dynamic Analysis}

Every structure acts statically and dynamically when subject to displacements or loads. In dynamic responses, the structures will have additional inertial forces which, according to the second law of Newton, are equal to their mass multiplied by acceleration. Thus, if the loads or displacements are enforced very slowly, the inertia forces can be ignored since the 
time can be assumed as zero and a static load analysis can be justified. Hence, dynamic analysis is a simple extension of static analysis.

Wilson (2002) stated that the force equilibrium of a multi-degree-of-freedom lumped mass system as a function of time can be expressed by the following relationship:

$F(t)_{I}+F(t)_{D}+F(t)_{S}=F(t)$ .....Equation III-1

in which the force vectors at time $t$ are:

$\mathrm{F}(t)_{I} \quad$ : is a vector of inertia forces acting on the node masses

$\mathrm{F}(t)_{D} \quad$ : is a vector of viscous damping, or energy dissipation, forces

$\mathrm{F}(t)_{s} \quad$ : is a vector of internal forces carried by the structure

$\mathrm{F}(t) \quad$ :is a vector of externally applied loads

Equation III-1 is based on physical laws and is valid for both linear and non-linear systems if equilibrium is formulated with respect to the deformed geometry of the structure.

For many structural systems, the approximation of linear structural behaviour is made to convert the physical equilibrium statement, Equation III-1, to the following set of secondorder, linear, differential equations:

$$
M \ddot{u}(t)_{a}+C \dot{u}(t)_{a}+K u(t)_{a}=F(t)
$$

in which $M$ is the mass matrix (lumped or consistent), $C$ is a viscous damping matrix (which is normally selected to approximate energy dissipation in the real structure) and $K$ is the static stiffness matrix for the system of structural elements. The time-dependent vectors $u(t)_{a}, \dot{u}(t)_{a}$ and $\ddot{u}(t)_{a}$ are the absolute node displacements, velocities and accelerations, respectively.

For seismic loading, the external loading $F(t)$ is equal to zero. The basic seismic motions are the three components of free-field ground displacements $u(t)$ that are known at some point below the foundation level of the structure. Therefore, equations can be written in terms of the displacements $u(t), \dot{u}(t)$ velocities and accelerations $\ddot{u}(t)$ that are relative to the three components of free-field ground displacements (Wilson, 2002). 


\subsubsection{Characteristics of Earthquakes}

An earthquake has several characteristics, which should be examined for a better understanding of their effects on structures. The most important earthquake ground motion characteristics of structures are: the duration (period), the amplitude of the displacement velocity-accelerations, and frequency of the ground motions. Frequency is measured in Hertz and defined as the number of complete waves or cycles of vibrations per second. A ground motion at the site of a structure is basically a combination or superposition of many and complex different vibration frequencies. Earthquakes have a random acceleration, velocity, displacement and frequency and have three different directions subject to the structure. There is a frequency around 0 - 33 Hertz as input motion to the equations, and the structure should be analysed to avoid resonance.

Vibration of the structure tends to one particular frequency, defined as the natural frequency or the fundamental frequency. The shorter the structure, the higher is its natural frequency; and the taller the structure, the lower is its natural frequency, as described in Figure III-1. This can be explained by the fact that frequency is related to stiffness and mass which have a correlation with the dimensions of the structures.

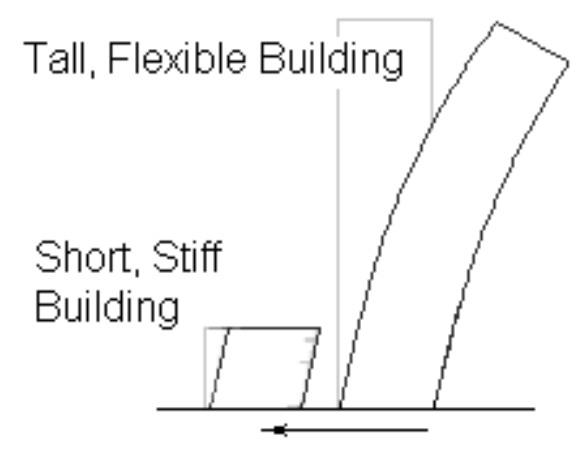

Figure III-1: Comparison of the dimension of the structure related to frequency

Period is the time for the structures to make one cycle of vibration. It is the inverse of the frequency and their relationship can be explained in simple maths:

$f=\frac{1}{T}$, and $T=\frac{1}{f}$ Equation III-3

where:

$\mathrm{f}=$ frequency

$\mathrm{T}=$ Period 
Natural frequencies are very important for the analysis of a structure's behaviour under a dynamic load, because it has a strong relationship with resonance. Resonance is a phenomenon where the structure absorbs more energy when the frequency of its oscillations matches the system's natural frequency of vibration. In other words, when the frequency contents of the ground motion are centred around the structure's natural frequency, it is said that the building and the ground motion are in resonance with one another. Resonance tends to increase or amplify the structure's response. For this reason, structures suffer the greatest damage from ground motion at a frequency close or equal to their own natural frequency, and will be subject to more shaking (acceleration) than if the frequencies were more different. However, how much shaking and damage is caused depends on the strength, flexibility, geometry, masses, and damping of the components of the structures, and the characteristics of the earthquake (Clough \& Penzien, 2003).

Resonance is often the reason why one structure collapses and other structures do not. It can explain why some shorter buildings or bridges suffer a worse impact than taller buildings or longer bridges, if their frequencies are similar to the frequency range of the earthquake.

The main purpose of response analysis in dynamic analysis, especially in earthquake loading, is the estimation of earthquake-induced forces and deformations in structures under the action of earthquake ground motions. It will start with developing response analysis procedures for the simplest dynamic system, called the single degree of freedom system. The degrees of freedom (dof) of a dynamic system gives an approximate indication of the complexity of the system. It describes the number of ways in which a body can move. The number of modes of vibration in which the structure can respond is equal to the number of degrees of freedom of the structure. 


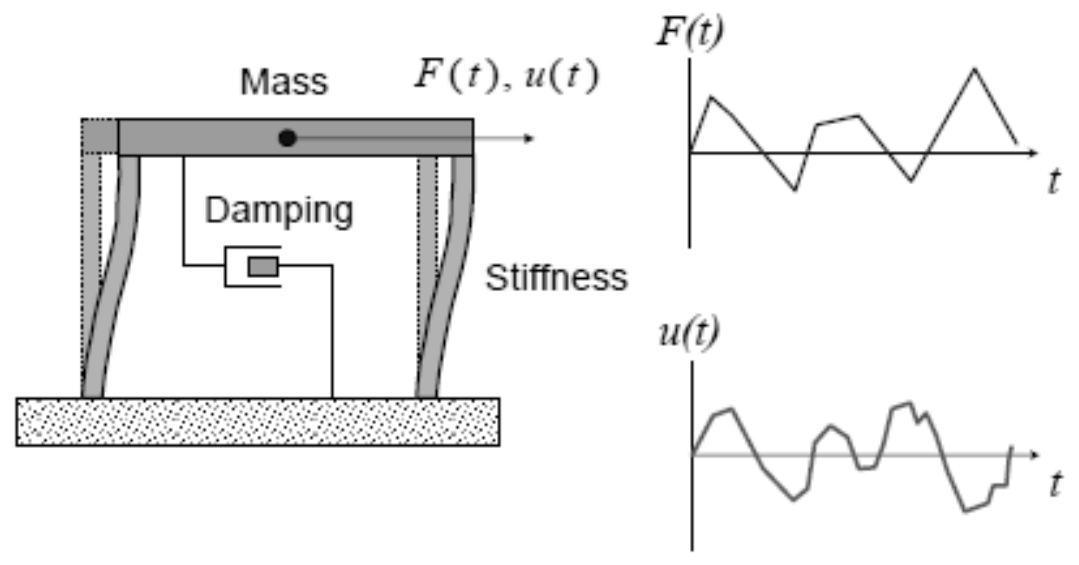

Figure III-2: Single degree system

The simple frame in Figure III-2 above is idealized as a Single Degree of System (SDOF) mass-spring-dashpot model with a time-varying applied load. The function $u(t)$ defines the displacement response of the system under the loading $F(t)$. From Figure III-2 it can also be seen that there is a positive correlation between loading $(F(t))$ and displacement $(u(t))$. The properties of the structure can be completely defined by the mass, damping and stiffness, as shown. The idealization assumes that all of the mass of the structure can be lumped into a single point and that all of the deformation in the frame occurs in the columns with the beam staying rigid. The equation of the SDOF system above can be represented in Figure III-3.

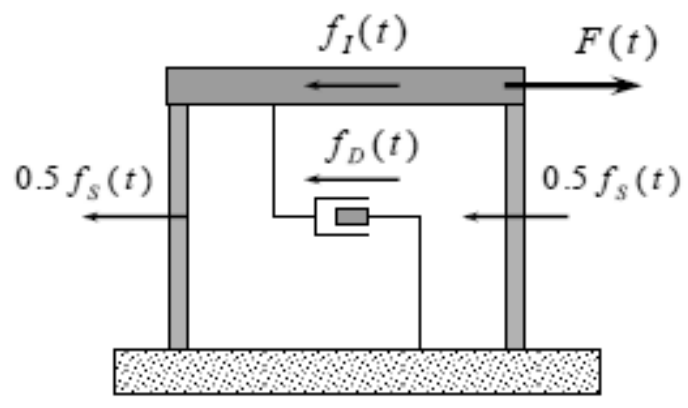

Figure III-3: Idealization of single degree of freedom system

where:

$\mathrm{F}_{(\mathrm{t})} \quad=$ Total force

$\mathrm{f}_{I(t)} \quad=$ Force due to inertia $\left(m \ddot{u}_{(t)}\right)$

$\mathrm{f}_{D(t)} \quad=$ Force due to damping $\left(c \dot{u}_{(t)}\right)$

$\mathrm{f}_{S(t)} \quad=$ Force due to stiffness $\left(k u_{(t)}\right)$ 
For Multiple Degrees of Freedom (MDOF), the system is more complicated but has a similar principle to the SDOF. The differences are in the variables of the equations that relate to the number of $d o f$ of the system.

\subsubsection{Modal analysis}

Modal analysis is a convenient method of solution of the forced vibration problem when the elements of the stiffness matrix are constant, i.e. the structure is linear. Modal analysis provides an understanding of the dynamic behaviour of a component, whether a structure or an equipment component, as it provides insight into how we might expect the structure or component to behave. It can also be a starting point for another more detailed dynamic analysis, such as a transient dynamic analysis, a harmonic response analysis, or a spectrum analysis. Modal analysis is used to determine the vibration characteristics (natural frequencies, mode shapes and mode participation factors). The natural frequencies and mode shapes are important parameters in the design of a structure for dynamic loading conditions. The mode participation factor is used to acknowledge how much a given mode participates in a given motion direction of a structure.

This analysis solves the equation:

$$
M \ddot{u}(t)+C \dot{u}(t)+K u(t)=0
$$

where $M$ is the mass of the structure, $C$ is the damping of the structure, $k$ is the stiffness of the structure, $\ddot{u}$ is the acceleration, $\dot{u}$ is velocity and $u$ is the displacement.

\subsubsection{Technique of Analysis in Earthquakes}

\subsubsection{Analysis in Time Domain}

Analysis in the time domain is commonly known as time history analysis or transient analysis. Because of the dynamic nature of seismic loads, the actual displacements and forces in the structure are time dependent. In other words, the displacements and the forces resulting are a function of time $(t)$. To increase the reliability of the method, a set of artificial accelerograms that represent the seismicity of a particular region is usually generated. This procedure, however, renders the method very expensive.

A system which is considered as having a single degree of freedom is shown in Figure III4. 


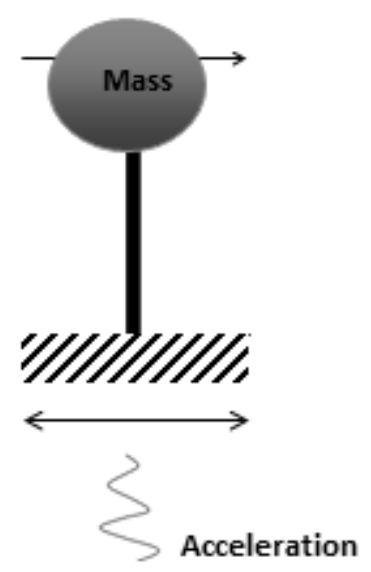

Figure III-4: A system with a single degree of freedom.

The motion equation for a $S D O F$ structure subject to a time varying force $\mathrm{F}(t)$, can be seen in equation III-2.

To solve this equation, the Duhamel integration can be used (Clough and Penzien, 2003). Duhamel is a general method for obtaining solutions to evolution equations. The results of this equation can be plotted in displacement, velocity or accelerations against time or period.

\subsubsection{Analysis in Frequency Domain}

Analysis in the time domain does not provide any information about the frequency or system damping effect. Therefore, another analysis can be done, namely response spectra. Basically, this analysis will give a description of the maximum response (acceleration, velocity or displacement) of a single degree of freedom system with a defined damping and various frequencies of the system. This is a very important tool in earthquake engineering. Response spectra are useful in quantifying the demands of earthquake ground motion on the capacity of buildings to resist earthquakes. Data on past earthquake ground motion is generally in the form of time-history recordings obtained from instruments placed at various sites that are activated by sensing the initial ground motion of an earthquake. The amplitudes of motion can be expressed in terms of acceleration, velocity and displacement. The first data reported from an earthquake record is generally the peak ground acceleration which expresses the tip of the maximum spike of the acceleration ground motion (Freeman, 2007). 
The amount of acceleration a structure undergoes during an earthquake is a critical factor in determining how much damage it will suffer. The spectra in Figure III-5 provide some indication of how acceleration is related to frequency characteristics, which shows one way in which response spectra can be useful, since identifying the resonant frequencies at which a structure will undergo peak acceleration is a very important step in designing a structure to resist earthquakes.

Response spectra plot maximum dynamic responses such as displacement, velocity, and acceleration of a range natural frequency of single degree of freedom system under a certain earthquake loading. In other words, response spectra do not describe the response of the actual ground motion, but show the response of a single degree of freedom system connected to the ground and showing only the peak response.
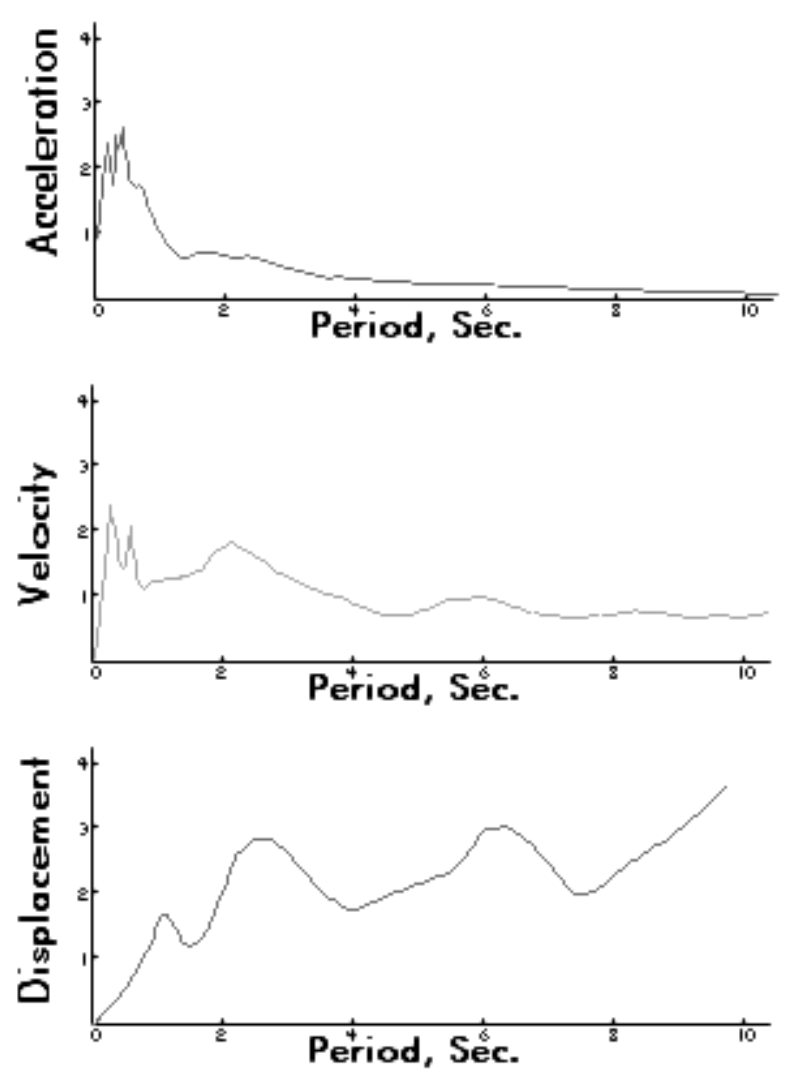

Figure III-5: Response Spectra

By definition, the response spectra analysis procedure involves the evaluation of the maximum value of structure responses such as displacements and member forces for each mode of vibration, using a spectrum of earthquake records. 
The response spectrum provides the required information for design purposes and at the same time, simplifies the analysis by reducing the problem to a static problem of the estimated maximum responses. The response spectrum is defined, based on a single degree of freedom system of varying frequency excited by a specific earthquake, as the maximum response of the system, ignoring the particular time of its occurrence. If the response is the displacement of the system then the displacement spectrum is formed. For velocity and acceleration, they are pseudo spectra and derived by multiplying displacement spectra by $\omega$ and $\omega^{2}$ respectively, where $\omega$ is the natural frequency of a single degree of freedom system.

Spectral acceleration, $S_{a}$, is the most commonly used intensity measure in practice today for analysis of structures. This value represents the maximum acceleration that a ground motion will cause in a linear oscillator with a specified natural period and damping level. In fact, the true measure is pseudo-spectral acceleration, which is equal to spectral displacement times the square of the natural frequency, but the difference is often negligible and the name is often shortened to 'spectral acceleration' (Baker \& Corner, 2006).

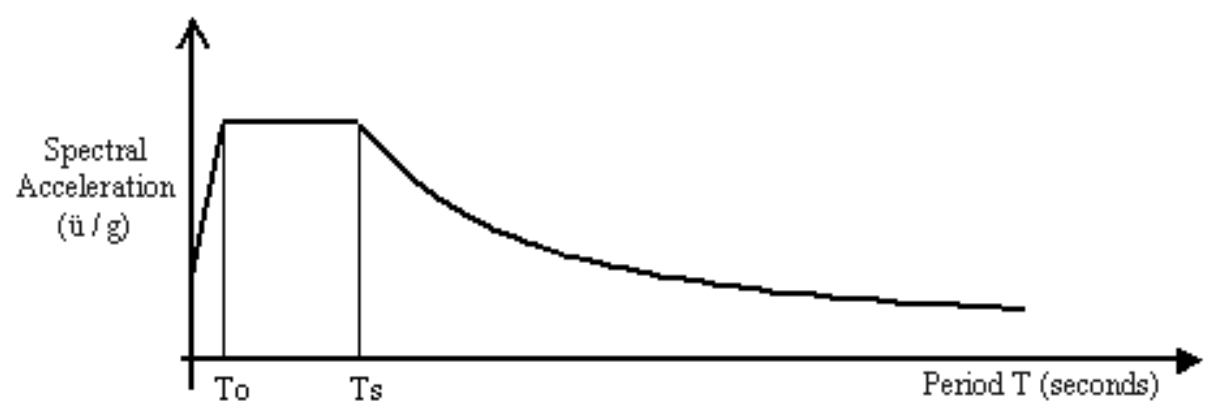

Figure III-6: Idealization of response spectrum

Figure III-6 presents the basic shape of a response spectrum in acceleration and period base. $T_{\mathrm{o}}$ is a point where spectral acceleration is constant until $T_{\mathrm{s}}$, where the acceleration begins to decrease significantly. These conditions are in the peak responses of a single degree of freedom system of the structure, due to ground motion acceleration, as can be seen in Figure III-7. 


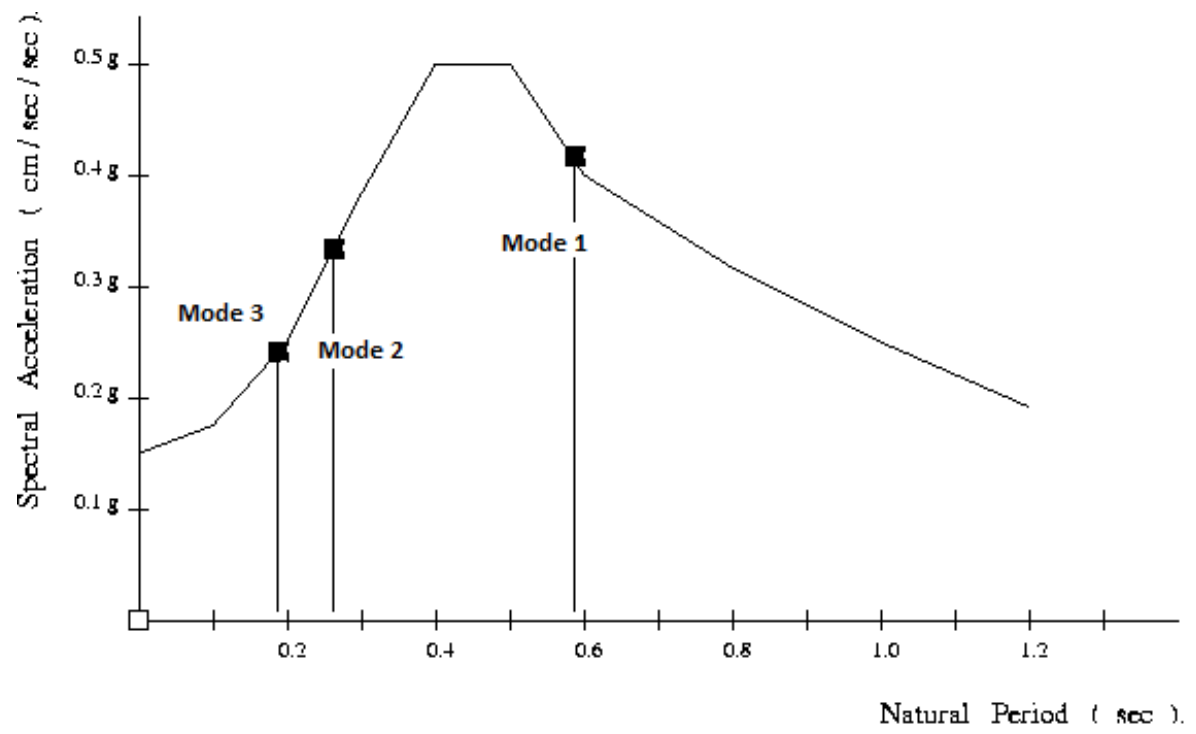

Figure III-7: Spectral acceleration in accordance with natural period and mode shapes (Austin, 1996)

Relating to the spectra, Ansary et al. (2000) stated that maximum amplitude of the acceleration spectra decreases as the soil type changes from soft to hard. For larger periods, it is evident that soft soil spectral acceleration is greater than rock spectral acceleration. They also observed that the largest amplifications occur near the natural time period of the soil, and suggested that the increase in damping results in a corresponding decrease in the spectral amplification.

\subsubsection{Performance-based Design for Bridges}

Performance-based design provides an insight into the expected performance of a designed structure during an earthquake. Over recent decades, considerable development in the performance-based design of buildings has taken place, but studies regarding the performance-based design of bridges are limited (Priestley, 2000).

Most of the current codes for seismic design of structures follow the force-based design approach in which force actions in the members are the governing parameters. Forces developed in the members due to external loads are checked against the strength capacity of the member. It is, basically, an elastic method where the effect of inelastic deformations is considered indirectly, through the response reduction factor (or behaviour factor). On the other hand, in the performance-based design approach, estimates of non-linear deformations and the damage state of the structure are obtained at multiple levels of earthquake excitation. 
In bridges, the superstructures (piers and abutments) are the main structural elements which provide resistance to seismic action (Tandon, 2005). For energy dissipation, ductile behaviour is necessary during flexure of these structural elements under lateral seismic loads. This essentially means that the formation of plastic hinges or flexural yielding is allowed to occur in these elements during severe shaking to bring the lateral design forces down to acceptable levels. Since yielding would lead to damage, plastic hinges are localized by design at points accessible for inspection and repair, i.e. parts of the substructure that lie from the foundation upwards, as can be seen in Figure III-8. No plastic hinges are, of course, allowed to occur in the foundations or in the bridge deck.

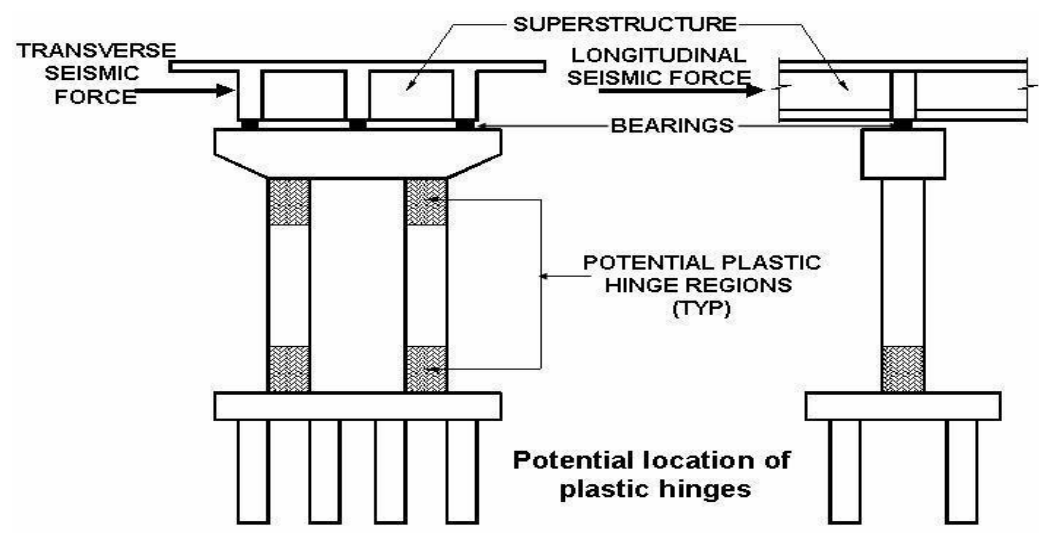

Figure III-8: Well designed structures dissipate seismic energy by inelastic deformations in localized zones of selected members (Tandon, 2005).

\subsubsection{Eurocode 8 Approach to Earthquake Engineering}

Eurocode 8, denoted in general by EN 1998: "Design of structures for earthquake resistance”, applies to the design and construction of buildings and civil engineering works in seismic regions. It covers common structures and, although its provisions are of general validity, special structures, such as nuclear power plants, large dams or offshore structures are beyond its scope. The seismic action to be considered for design purposes should be based on the estimation of the ground motion expected at each location in the future; for example, it should be based on the hazard assessment (Bisch et al., 2012).

In Eurocode 8, design spectra based on two different types of seismic action are specified, namely type 1 for regions with higher and type 2 for regions with lower seismicity. This means design spectra of type 2 are applicable for Central European earthquake regions (Schott \& Schwarz, 2004). This code also provides a subsoil classification scheme. 
Compared to type 1 spectra for soft soil sites in particular, high soil factor $S$ is defined that results in high amplifications, but in a narrow range of constant spectral acceleration.

The background of Eurocode 8 is to consider the two types of spectrum shape (type 1 and type 2) for varying seismicity conditions. In this regard, provisions of Eurocode 8 state: 'If the earthquakes that contribute most to the seismic hazard defined for the site for the purpose of probabilistic hazard assessment have a surface wave magnitude, $\mathrm{M}_{\mathrm{s}}$, not greater than 5,5 , it is recommended that the Type 2 spectrum is adopted.'

In Eurocode 8, five different subsoil classes (A-E) are defined, as shown in Table III-1:

Table III-1: Soil type and classification (Bisch, et al., 2012)

\begin{tabular}{|l|l|}
\hline \multicolumn{1}{|c|}{$\begin{array}{c}\text { Soil } \\
\text { type }\end{array}$} & \multicolumn{1}{c|}{ Classification } \\
\hline A & $\begin{array}{l}\text { Rock or other rock-like geological formation, including at most } 5 \mathrm{~m} \\
\text { of weaker material at the surface. }\end{array}$ \\
\hline B & $\begin{array}{l}\text { Deposits of very dense sand, gravel, or very stiff clay, at least several } \\
\text { tens of metres in thickness, characterised by a gradual increase of } \\
\text { mechanical properties with depth. }\end{array}$ \\
\hline C & $\begin{array}{l}\text { Deep deposits of dense or medium dense sand, gravel or stiff clay } \\
\text { with thickness from several tens to many hundreds of metres. }\end{array}$ \\
\hline D & $\begin{array}{l}\text { Deposits of loose-to-medium cohesionless soil (with or without some } \\
\text { soft cohesive layers), or of predominantly soft-to-firm cohesive soil. }\end{array}$ \\
\hline E & $\begin{array}{l}\text { A soil profile consisting of a surface alluvium layer with vs values of } \\
\text { type C or D and thickness varying between about } 5 \text { m and } 20 \mathrm{~m}, \\
\text { underlain by stiffer material with } v_{\mathrm{s}}>\text { 800 m/s. } v_{s}=\text { shear wave } \\
\text { velocity }\end{array}$ \\
\hline
\end{tabular}

\subsubsection{Horizontal and Vertical Ground Motion Studies}

Legeron and Sheikh (2009) presented a theoretical approach to calculate support reactions of bridges under vertical earthquake ground motion. Based on this theoretical approach, an efficient method has been developed that enables the calculation of the support reactions with less computational effort. The method can be easily adopted in the seismic design of 
highway bridges. They said that, generally, three methods are used in practice for the calculation of support reactions:

1. Rayleigh method: recommended by the American and Canadian bridge design codes for irregular ordinary multi-span bridges and regular essential or emergencyroute bridges. The Rayleigh method is generally not recommended for vertical ground motion since the choice of deflection shape for a complex system is not straightforward and does not provide results close to the exact calculation in most cases.

2. Modal analysis: most suitable for structures with irregular geometry, mass and stiffness. This method does not take into account non-linear effects. However, nonlinear effect is not considered significant for vertical earthquake ground motion, as mentioned earlier. Moreover, the ductility of bridge piers under vertical earthquake ground motion is not well known and is usually considered to be low.

3. Time history analysis: very complex and time consuming, and usually carried out only for critical structures in high seismic zones. The choice of representative ground motion may add complexity in applying this method. Hence, elastic modal analysis has been considered sufficient for the calculation of the support reactions under the vertical earthquake ground motion in this study.

The need for definable levels of reliability in structures increases with time, as society becomes increasingly dependent upon infrastructure. This is exacerbated in safety-critical structures such as nuclear installations, particularly those constructed in seismic regions of the world. For this reason there is international interest and participation in the production and dissemination of research regarding earthquake-resistant structures. Consideration of vertical earthquake motion has often been neglected in the past, although nowadays it has begun to take priority amongst the engineering community.

Measurements of ground motions during past earthquakes indicate that the vertical acceleration can reach, or even exceed, values comparable to horizontal accelerations. Furthermore, measurements of structural response show the possibility of significant amplification in the response of bridges in the vertical direction that can be attributed to the vertical component of ground motion (Saadeghvariri \& Foutch, 1991). Analyses of actual bridges indicate that, in general, the vertical motion will increase the level of response and the amount of damage sustained by a highway bridge. Vertical motion 
generates fluctuating axial forces in the columns, which cause instability of the hysteresis loops and increase the ductility demand.

Kim et al. (2008) presented a paper concerning analytical assessment of the effect of vertical earthquake ground motion on reinforced concrete (RC) bridge piers. A bridge structure damaged during the Northridge earthquake and a Federal Highway concept bridge design were examined. The effects of a suite of earthquake ground motion records with different vertical-to-horizontal peak acceleration ratios on the two bridges were presented and the results compared with the case of horizontal-only excitation. The effects of the arrival-time interval between horizontal and vertical acceleration peaks were also reported and compared to the case of coincident motion. It was observed that the inclusion of the vertical component of ground motion had an important effect on the response at all levels and on all components. It was therefore concluded that vertical motion should be included in analysis for assessment and design, especially when there are no particular challenges impeding its inclusion.

Many researchers in the field of earthquake engineering are now recommending further study regarding vertical earthquake ground motion. Saadeghvariri and Foutch (1990) described how vertical motion can produce high magnitude forces in abutments and foundations that are not accounted for by the current seismic design guidelines. Therefore, it is important to consider this ground motion component in the design of highway bridges, especially for those located in regions near seismic faults where the effect is greatest.

Collier and Elnashai (2001) stated that the vertical component of earthquake ground motion has generally been neglected in the earthquake-resistant design of structures. Elnashai and Papazoglou (1996) described some field evidence and results from dynamic analysis on possible structural effects of strong vertical ground motion. These collectively confirm that structural failure may ensue due to direct tension or compression as well as due to the effect of vertical motion on shear and flexural response.

In many earthquakes, the vertical component of the ground motion was found to exceed the horizontal component, which directly contradicts the current general code provision that assumes the value of the vertical ground motion to be between one- and two-thirds of the horizontal component. After almost every destructive earthquake some engineers postulate that structural damage was due to strong vertical ground motion. Therefore, 
seismic design of the structure without the consideration of the vertical ground motion component may result in unquantifiable risk of collapse, especially those in constructed in close proximity to the fault. However, there seems to be little consensus as to the methods of quantifying the contribution of vertical motions in overall damage caused. Moreover, little has been learned from the recent earthquakes in Loma-Prieta, Northridge or Kobe, which indicate conclusively that damage to structures was predominantly caused by vertical motions (Shrestha, 2009).

\subsubsection{Effects of Vertical Ground Motions on Bridges}

Much evidence has now been published stating that vertical earthquake motion has a detrimental effect on structures. Papazoglou and Elnashai (1996) have stated that the Greek earthquake of 1986 and the Northridge earthquake both generated serious failures attributed to high vertical to horizontal ratios of ground motion.

A vertical earthquake motion may have significant energy content, which is very dangerous to substructures such as retaining walls. Siddharthan et al. (1992) found that vertical accelerations can have a significant effect on the displacement of gravity walls, considering both sliding and overturning. They assessed likely displacements using records from five earthquakes, considering both vertical and horizontal accelerations. They found that the displacements varied considerably even though the horizontal accelerations were all scaled to $0.3 \mathrm{~g}$, and attributed this to the frequency content of the records; hoever, this could also be due to different levels of concurrency between vertical and horizontal motions.

Cai and Bathurst (1996) assessed modular block-faced geosynthetic-reinforced soil retaining walls and showed that high vertical accelerations would give a lower critical horizontal acceleration, which would imply a larger displacement. Ling and Leshchinsky (1998) used pseudo-static limit equilibrium analyses to carry out a parametric study and suggested that vertical accelerations would be significant where the horizontal acceleration is greater than $0.2 \mathrm{~g}$. They also presented a case study of a $6.0 \mathrm{~m}$ high geosyntheticreinforced wall that displaced $100 \mathrm{~mm}$ at the base during the 1995 Hyogoken-Nanbu earthquake, and attributed the displacement to the vertical ground shaking recorded in the area. 
Kunnath and Zhao (2010), Khunnath (2008) and Karantzikis (2000) stated that superstructures can also suffer unduly from vertical earthquake motion. Many severe failures during the Northridge earthquake of 1994 are attributed to vertical motion. In 2003, Rahai presented a model of an existing four-span RC bridge, analysed using the finite element method. A dynamic linear and non-linear analysis of the model was carried out using different accelerograms and time-history analysis to study the longitudinal displacements, axial and shear forces in the piers. Results showed important variation in axial force values, which could produce circumferential cracks in the RC piers (Rahai, 2004).

However, contrary to the current view, Berenev et al. (2002) found that horizontal motion dominates vertical motion at frequencies up to approximately $10 \mathrm{~Hz}$.

Most typical short- and medium-span bridges are very stiff in the vertical direction. Therefore, the first modes which contribute most to the seismic response have periods below that at which the design acceleration response spectrum starts to ascend. Hence, $S_{a}$ can be considered as the maximum response spectral acceleration $\left(S_{a, \max }\right)$ for rigid bridges. This will provide a slightly conservative estimate of the support reactions, as some of the contributing higher modes, although not significant, may fall in the initial ascending branch of the response spectrum (period $<0.05 \mathrm{~s}$ ), when such an ascending branch exists in code.

Kim et al. (2011) have studied the effect of vertical ground motion on bridge piers for various ratios of peak vertical to horizontal $(\mathrm{V} / \mathrm{H})$ ground accelerations and the time lag between the arrival of the peak horizontal and vertical accelerations. The most important findings are summarized below. As the $\mathrm{V} / \mathrm{H}$ ratio increases, significant increases in axial force variation within the piers are observed. The studies also show that although the moment pier varies due to vertical ground motion, the moment demand in some records increases due to high axial force variation even if the lateral displacement is reduced. It was also observed that the significant increase of axial force variation due to vertical ground motion leads to an observed reduction of shear capacity of up to $30 \%$. For the structures considered and the motion set used, the arrival time has a minimal effect on the axial force variation and shear demand. Taking into account the above observations, RC bridge piers subjected to the combined horizontal and vertical components of earthquakes could be more vulnerable than those subjected to horizontal ground motion only. 
Therefore, including vertical ground motion in the analysis is recommended for reliable seismic assessment of RC bridges in the vicinity of active faults, where $\mathrm{V} / \mathrm{H}$ is likely to be high.

In the seismic design of bridges, designers do not usually consider the effect of vertical motion. However, measurements of ground motion during past earthquakes indicate that the vertical acceleration may exceed the horizontal acceleration. Rahai and Andi (2008) report analyses of actual bridges which indicate that, in general, the vertical motion will increase the level of response and the amount of damage sustained by a highway bridge. Vertical motion generates fluctuating axial forces in the columns, which cause instability of the hysteresis loops. Furthermore, vertical motion can generate forces of high magnitude in the abutments and foundations that are not accounted for by the current seismic design guidelines. Also, the impulsive vertical motion induces circumferential cracks on reinforced piers. In their study of linear and non-linear time history analysis, namely the Tabas, Northridge and Kobe scaled records, Rahai and Andi consider two cases of loading. In the first case, the bridge was subject to horizontal motion of earthquakes, whereas in the second case the bridge was subject to both the horizontal motion of case 1 and to vertical motion. Analyses indicate vertical motion will increase the axial loads, whereas axial and shear strain and variation of longitudinal displacement and shear force are negligible.

Papazoglou and Elnashai (1996) attempted to collate field evidence and results from dynamic analysis on possible structural effects of strong vertical ground motion. They recorded observational evidence from three earthquakes and assessed them with regard to failure modes of buildings and bridges attributable to high vertical earthquake forces. They confirmed that structural failure may ensue due to direct tension or compression as well as to the effect of vertical motion on shear and flexural response.

\subsection{Behaviour of Integral Bridges Subject to Seismic Loads}

\subsubsection{Structural Response of Integral Bridges due to Seismic Loads}

As monolithic structures, integral bridges are usually more rigid than conventional bridges. Consideration should be given to the behaviour of piles because of the significance of soil 
structure interaction in this type of bridge. When piles are subjected to shaking during an earthquake, they move back and forth horizontally almost in union with the movement of the surrounding ground. During the main shaking, sandy soils in a deposit have not yet softened significantly due to liquefaction, so that the relative movement between the piles and the ground would be small. However, it is possible that the piles will be damaged if the ground motion is sufficiently large and consequent development of bending moment within the piles is great enough to become equal to a limiting value. It should be noted that the damage to the piles under the above loading conditions takes place in the upper portion of the pile near the head because of the bending moment reaching its maximum around this portion. Since the load comes from the inertial displacement of the superstructure, the influence of seismic loading may be referred to as a top-down effect (Arsoy, 1999).

In summary, the effects of seismically induced loading for foundations such as piles are twofold. One is associated with the dynamic loads at the time of the main shaking coming from the inertia force of superstructures, which are applied to the top of the pile. As a result of such a top-down load transfer, the maximum bending moment and consequent damage tend to occur near the pile head. The other effect is related to the static loads emerging from the liquefaction-induced lateral movement of the surrounding soil deposit. These loads are applied to the middle portion of the pile during the period of time after the main shaking of an earthquake is over. As a result of this bottom-up mode of load application, the maximum bending moment and consequent damage tends to occur in the middle portion of the pile at a certain depth.

\subsubsection{Earthquake History of Integral Bridges}

In the United States, the design of integral bridges in California, Washington State, Oregon and Nevada, where seismic loading is correlated with seismic activities, appears to be similar. Burke commented that the suitability of semi-integral bridges in this region is confirmed by the results of the Northridge earthquake in California, during which many of these structures provided adequate performance while portions of bridges with movable deck joints collapsed. 


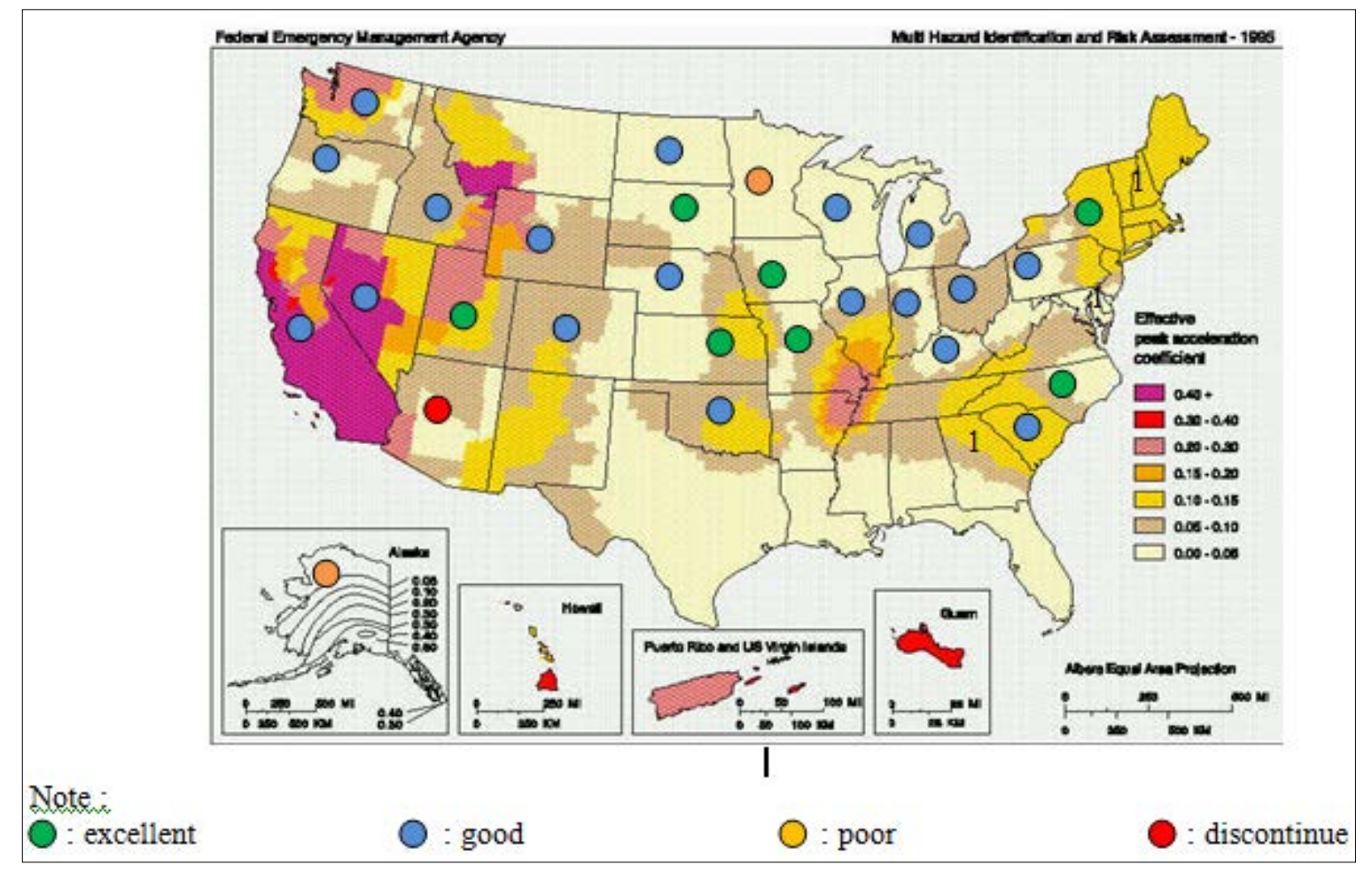

Figure III-9: Map of integral bridge application in United States

Figure III-9 above illustrates the application of integral bridges in areas with different earthquake risk in the United States. California and Nevada have a high risk of earthquakes, but reports suggest that they have good performance from their integral bridges. Green and blue bullets on the map show excellent and good applications of integral bridges, while yellow and red bullets indicate poor and discontinued applications of integral bridges (Burke, 2009).

Quebec stands in a high risk area of earthquakes compared to other areas in Canada, as can be seen in Figure III-10, and reports good performance of its integral bridges. Red areas of the map below indicate bad disasters resulting from earthquakes, while the green stars are the places where earthquakes have occurred in the past. It can be concluded that even though Quebec is in a high risk area, integral bridges constructed there give good performance. 


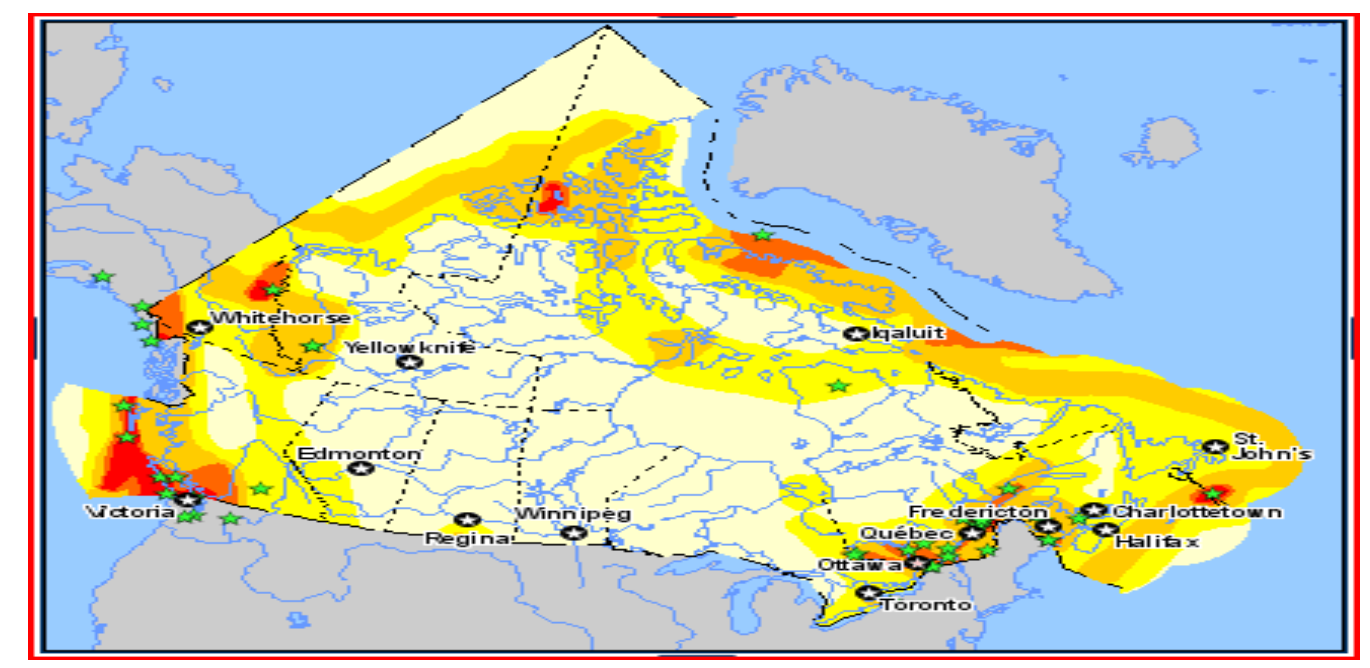

Figure III-10: Map of earthquake-prone areas in Canada http://atlas.nrcan.gc.ca/site/english/maps/environment/naturalhazards/earthquakes/majorearthquakes [accessed: 5 June 2011]

\subsubsection{Previous Research on Integral Bridges under Seismic Loads}

There has been a significant amount of research conducted on bridges and their response to earthquakes, but not necessarily on integral bridges, considering that many bridge experts say that integral bridges possess good structural resistance to dynamic loading. Some studies of integral bridges were presented in 2006 by Shamsabadi and Kapuskar, focusing on the behaviour of skewed and non-skewed bridges under seismic loading. They stated that the bridges with skew on their abutments had significant rotations about the vertical axis during seismic ground shaking, whereas the non-skewed bridges showed little or no rotation.

Karantzikis and Spyrakos (2000) discussed the effects of soil-abutment interaction on the seismic analysis and design of integral bridges. They concluded that the abutments attract a large proportion of the seismic forces, particularly in the longitudinal direction. Consequently, it is important to consider the participation of backfill soil at the abutments. Pekcan et al. (2011) have also made a study of seismic design recommendations for steel girder bridges with integral abutments. Non-linear pushover analyses were conducted to assess primarily the load path, and hence the distribution of abutment forces to various components. The results of these analyses indicate that the piles play a detrimental role in the longitudinal response of bridges with integral abutments. The piled abutments present an area of concern when subject to seismic loading. 
Mitoulis and Tegos (2011) also conducted research relating to integral bridges and found that their seismic performance was strongly influenced by the dynamic contribution of the abutment and the backfill soil. They recommend that two new abutments be utilized in possible future design of integral bridges. The comparisons were conducted in terms of dynamic (periods and response accelerations) and seismic (displacements of the deck and bending moment of the piers) response-critical parameters. The response of unconventional bridge models was compared to that of conventional bridge design. The bridge models which employed the new abutments were found to be stiffer in comparison to the conventional bridge. The reductions were almost $50 \%$ and $65 \%$ in the longitudinal and the transverse modal periods respectively.

\subsection{Dynamic and Static Response of Bridges under Seismic Load}

Structure can suffer under loading statically and dynamically. Studies into these static and dynamic behaviours have been conducted by many researchers. For example, Issa and Shahawy (1993) performed dynamic and static tests on prestressed concrete girder bridges in Florida. The results indicated an increase in the strain and deflection amplitudes with an increase in vehicle speed. A linear relationship was also found to exist between the applied load and the measured strains and deflections. Studies of static behaviour of bridges have been undertaken for many years. Lavelle (1969) developed a procedure for analysing curved girders using a stiffness matrix, and Wu and Clarke (1966) developed a method of determining normal stress caused by warping.

An earthquake is categorized as a dynamic load because its magnitude and direction can change over time. Many studies have been conducted on this subject. For example, Burdette and Elnashai (2011) examined the effect of asynchronous earthquake motion on complex bridges, demonstrating the importance of asynchronous analysis in the design of long and complex bridge structures. Burdette and Elnashai (2011) stated that in cases of moderate incoherence that are likely to occur in practice, asynchronous response can be almost twice that of a synchronous response. Wave travel speed, reflection and refraction, and heterogeneous soil conditions all cause deviations from synchronous excitation for distributed foundation structures such as bridges, creating a synchronous condition. For simplification, asynchronous motion is commonly divided into three components: wave passage effect; geometric incoherence of the input; and local site conditions. The first 
component results from the finite travel speed of seismic waves, resulting in progressive excitation of each support point as a wave front passes. The second component concerns the reflection and refraction of seismic waves as they pass through the ground, changing their signal content between support points. The final component considers situations where structures are founded on different soil types, such as a bridge that spans a geologic divide. Although the asynchronous motion observed during earthquakes is a complex interaction of all three components, for analytical simplicity researchers have divided this phenomenon into these three sources (Burdette \& Elnashai, 2011). Zanardo et al. (2002) stated that analyses of different cases showed that the spatial variation properties of the earthquake ground motion can drastically change the structural response of the bridge. Sextos et al. (2004) stated that asynchronous motion cannot easily be replaced by an alternative reference uniform motion.

\subsection{Relative Displacements Effects on Bridges}

A displacement is the shortest distance from the initial to the final position of a point, and relative displacement is defined as a differential displacement which is compared between two displacements occurring in a structure. There are two types of relative displacement based on their causes, namely static relative displacements and dynamic relative displacements. Static relative displacement is a series of relative displacements applied to a structure due to static load, for example abutment settlements. Dynamic relative displacement is defined as relative displacements occurring to bridges because of dynamic load, such as earthquake or traffic vibration load.

An earthquake causes shaking of the ground, so a bridge or building will experience motion at its base. From Newton's first law of motion, even though the base of the structure moves with the ground, the rest of the structure which is not connected directly to the ground has a tendency to stay in its original position. For bridges, with their connectivity between superstructure and substructure, the structure is dragged along with the ground movement. The tendency to remain in the previous position is known as inertia.

During an earthquake, bridge segments often vibrate out of phase due to their different dynamic characteristics as well as support conditions that allow the relative motions between the segments. The out-of-phase motion of the bridge leads to two types of relative displacement problem. First, when the distance between the segments increases and 
exceeds the range of support provided by either abutment, column or hinge seat, the bridge can fall. Many of the catastrophic span-type failures of bridges in past earthquakes have been attributed to this effect. Second, when the distance between the segments decreases, pounding (i.e. collision) of the segments can occur and a severe impact force develop at the contact region. This typically results in localized damage to the bridge (Kasai et al., 1992).

Accelerations which are applied to the abutment and piers of a bridge are influenced by, for example, the magnitude of the earthquake, type of soil and distance from the epicentre. The members of a bridge which have the same acceleration as its abutments and piers will move in the same direction. However, if a series of different accelerations is applied to the abutments and piers, different displacement responses are obtained. This discrepancy of displacements among the supports of the bridge will create a relative displacement, which in turn results in more forces to the structure, in addition to seismic loading (Attewel \& Taylor, 1984).

If a structure is deformed by earthquake loading, this will result in inertia force, as previously mentioned. This force will be transferred to the ground through abutments and piers. During earthquake shakings, the piers and the deck undergo relative movement between their ends. In the straight vertical position, the elements carry no earthquake forces inside them. A straight vertical position means the displacement occurrs in a vertical direction by the same amount. However, when forced to bend, they establish internal forces. The stiffer the structures are, the larger is this force. For this reason, these internal forces in the elements are called stiffness forces. In fact, the stiffness force in an element is the element stiffness times the relative displacement between its ends (Attewel \& Taylor, 1984).

Earthquakes cause shaking of the ground in all three directions along the two horizontal ( $x$ and $y$ ) and vertical ( $z$ ) axes. The ground shakes randomly back and forth along each of these $x, y$ and $z$ directions. All structures are primarily designed to carry gravity loads, which means they are designed for a force equal to the mass $M$ times the acceleration due to gravity g acting in a vertical downward direction. The vertical acceleration during ground shaking either adds to or substracts from the acceleration due to gravity. Since factors of safety $(>1.0)$ are used in the design of structures to resist the gravity loads, most structures tends to be adequately protected against vertical shaking. For example in 
Eurocode 1 , the safety factors are $1.35 \mathrm{G}_{\mathrm{k}}+1.5 \mathrm{Q}_{\mathrm{k}}$ for general load combination, and $1.0 \mathrm{G}_{\mathrm{k}}$ $+1.0 \mathrm{Q}_{\mathrm{k}}$ for earthquake accidental combination. $\mathrm{G}_{\mathrm{k}}$ is gravity load and $\mathrm{Q}_{\mathrm{k}}$ is earthquake load.

However, structures designed only for gravity loads may not be able to sustain the effects of horizontal earthquake shaking. Hence, it is necessary to ensure the adequacy of the structures against horizontal earthquake effects.

The fact that ground motion is commonly more emphatic along some axes than others has been long understood. John Milne, one of the European scientists in Japan in the last half of the 1800s who helped lay the groundwork for Japan's subsequent prominence in seismic research, found a contemporary account of an ancient (136 AD) Chinese seismoscope, which elegantly illustrates the point that inertial force may be exerted in any direction (Arnold \& Reitherman, 1982).

Considering that integral bridges can be constructed in earthquake zones, bridge engineers are becoming increasingly interested in conducting research and expanding the possibilities of building integral bridges in other parts of the world. Despite much research, more knowledge and understanding about earthquakes and integral bridges are required. The unique characteristics of this combination, such as higher redundancy, rigid connections, strong relation to soil behind the abutments, encourage researchers to explore further.

To summarize the literature review chapters (Chapters II and III), integral bridges are considered to exhibit good earthquake resistance. For this reason, integral abutment bridges are becoming highly attractive to designers, constructors and road administration. Additionally, these bridges tend to be less expensive to build, easier to maintain and more economical to own over their lifetime. Bearings and joints are the main sources of maintenance costs during a bridge's lifetime, and these costs vanish because the bridges are joint- and bearing-free. However, this very advantage makes the design more complex than that of conventional bridges in some crucial aspects. Combined with the fact that most countries have only limited experience with integral bridges to date, has led to reluctance on the part of some road administrations to use this bridge type. More research into this kind of bridge will be advantageous to bridge construction in general. 


\section{Chapter IV RESEARCH METHODOLOGY}

\subsection{Introduction}

There are several methodologies used in research such as simulation, case study, and experiment. In this research, the methodology used is to conduct a case study and carry out a series of simulation analyses using a simple model. A procedure is generated to obtain a graph which is then used to study the effect of relative displacement on the behaviour of a bridge.

Case study research excels at bringing an understanding of a complex issue or object and can extend experience or add strength to what is already known through previous research. Case studies emphasize detailed contextual analysis of a limited number of events or conditions and their relationships. Researchers have used the case study research method for many years across a variety of disciplines.

In this research, the behaviour of integral bridges subject to bi-axial translational earthquake ground motion will be analyzed. Theoretical evaluation of anticipated seismic performance mostly requires a structural simulation which is based on the concept of structural likeness. Simulation of bridge, soil and earthquakes in this research is carried out using a variety of computer software programs, namely ANSYS ver. 12, Mathcad ver. 14 and Seismosoft. For the simulation of earthquake and soil conditions, reference to Eurocode 8 is made. According to Burke (2009), for an integral bridge which has a length less than 91 metres (300 ft), the secondary load effects and temperature can be neglected; this is the case in this study. 
The main purpose of this research is to obtain knowledge and insight into the structural response to earthquakes of an integral bridge. A series of horizontal and vertical earthquake ground motions will be applied to the bridge and the results will be analyzed and compared. For this purpose, Eurocode 8 is used to arrange the series of earthquake ground motions. The other aim of the study is to acquire insight into the behaviour of the bridge subject to different soil stiffness, since the monolithic construction of integral bridges means that the effect of soil-structure interaction cannot be ignored.

\subsection{Flowchart of Methodology}

The research uses finite element methods to gain insight into the seismic behaviour of an existing bridge with simplicity and idealization. Beginning with the generation of synthetic earthquake accelerations, these are then applied to the structure boundaries, also incorporating relative support displacements when different time histories are used in combination in the same model. Hence the responses of the structure are analyzed. The flowchart in Figure IV-1 describes these steps.

Data collection was conducted by observing integral bridges which are suitable for this research. An integral bridge in India was found to be a suitable case study, as India is known as a high-risk earthquake region. The details of the bridge are presented in the following sub-chapter; some modifications have been made to comply with the limited scope of the research. Data for soil and earthquakes were obtained from Eurocode 8.

Modelling the structure was carried out using the computer software ANSYS ver. 12. Checks and validation were conducted by simple hand calculation (moment distribution method) since the model is in $2 D$. 


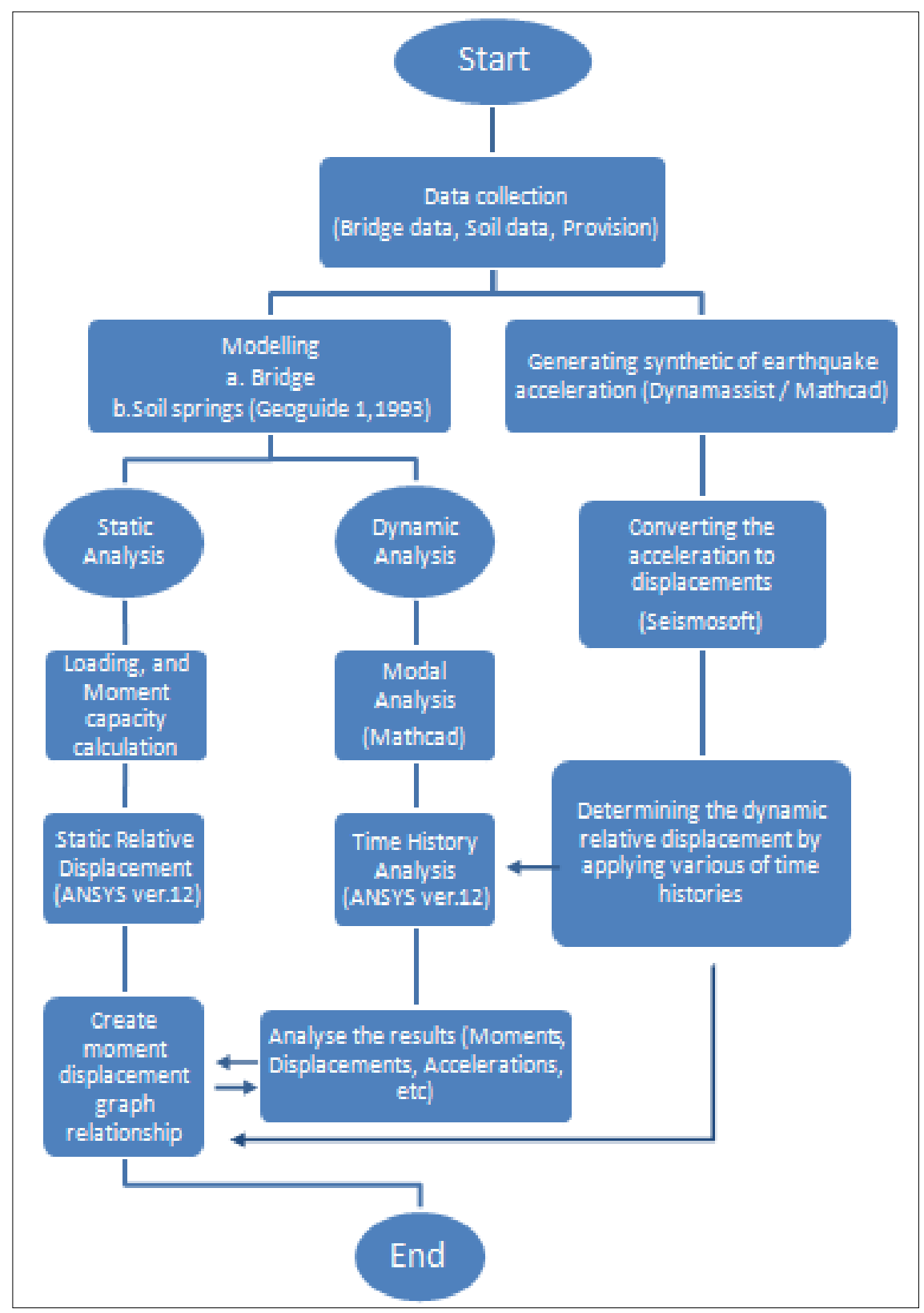

Figure IV-1: Flowchart methodology of the research

Two kinds of analysis are conducted in this study. The first is a normal static analysis which comprises a series of static relative displacements on one abutment at the left side of the bridge. The results are later compared with the second, dynamic, analysis. Dynamic analysis is carried out using transient analysis in ANSYS ver 12. This requires the creation and generation of a synthetic earthquake acceleration using procedures described by Clough and Penzien (2003). Dynamassist Mathcad software is used after applying some modifications to the data from Eurocode 8. 
The final step is to determine the ratio of static and dynamic bending moment by comparing the results of the static and dynamic analyses.

\subsection{Study Case and Idealization}

\subsubsection{Modelling the Structure}

For the case study, a three-span pre-stressed concrete bridge located in India (Tandon, 2005) was used as the basis for a parametric study to determine the influence of variables encountered in integral bridges such as relative displacements, different earthquake spectra and soil spring conditions. India is a high-risk earthquake region, and it is suitable to adopt one of its existing integral bridges to be analyzed in this study.

The bridge is an existing pre-cast concrete integral bridge; some modifications and assumptions have been made to fit it into the study. A side view of the bridge and other detailed drawings of the bridge superstructure can be seen in Figures IV-2, IV-3 and IV-4.

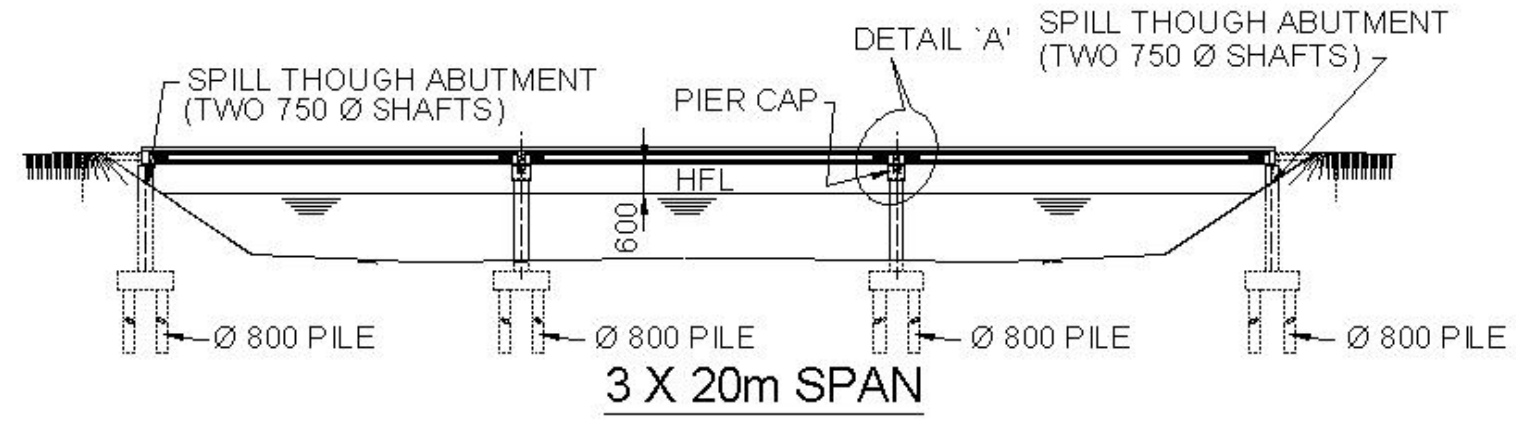

Figure IV-2: Side view (Tandon, 2005) 


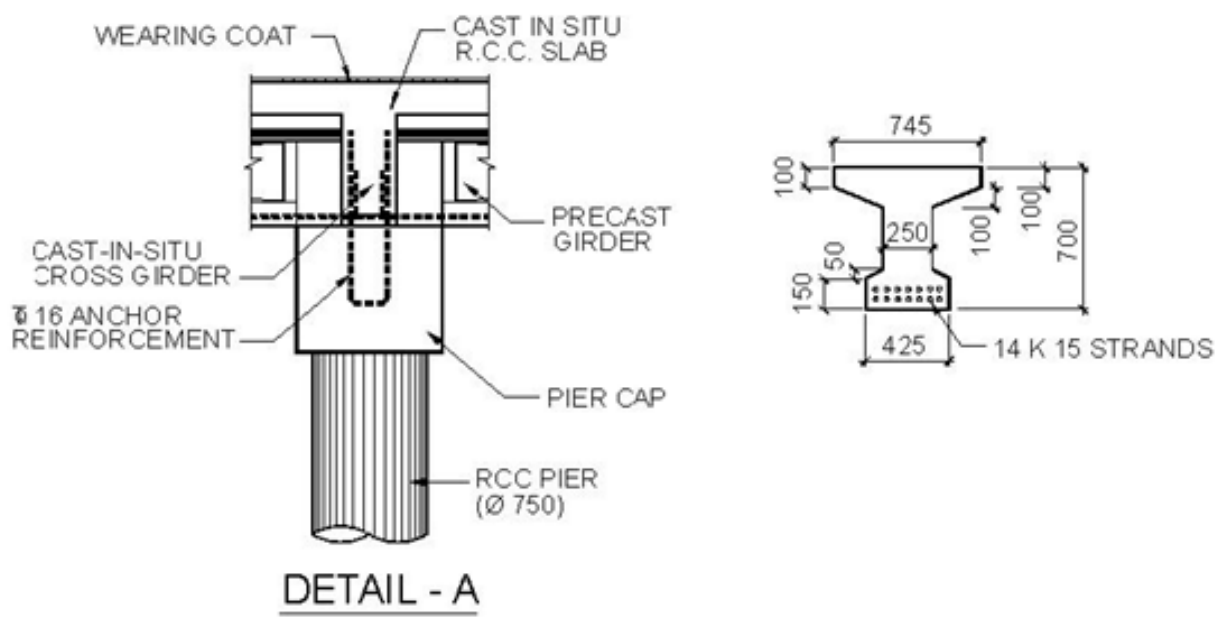

Figure IV-3: Detail A (Tandon, 2005)

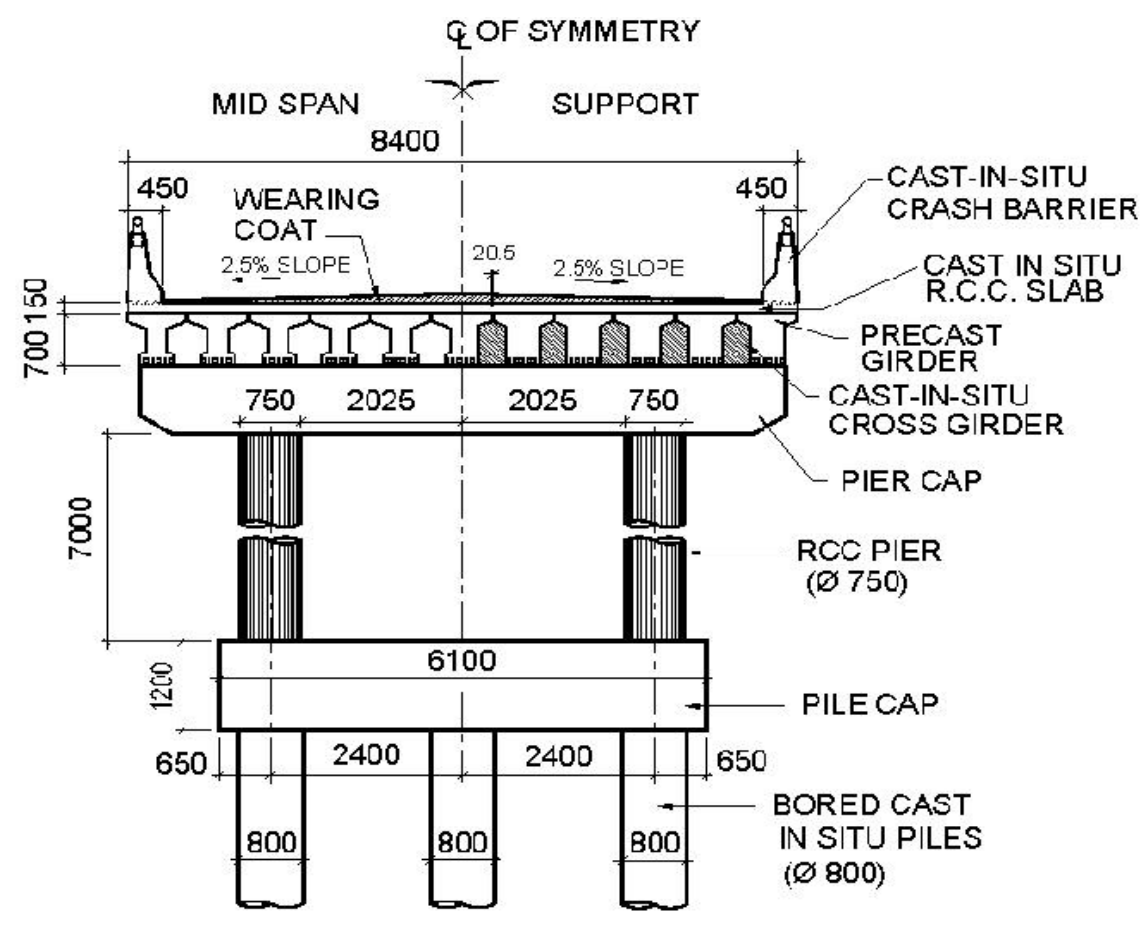

Figure IV-4: Cross section of the bridge (Tandon, 2005) 
The abutment of the bridge is shown in Figures IV-5 and IV-6.

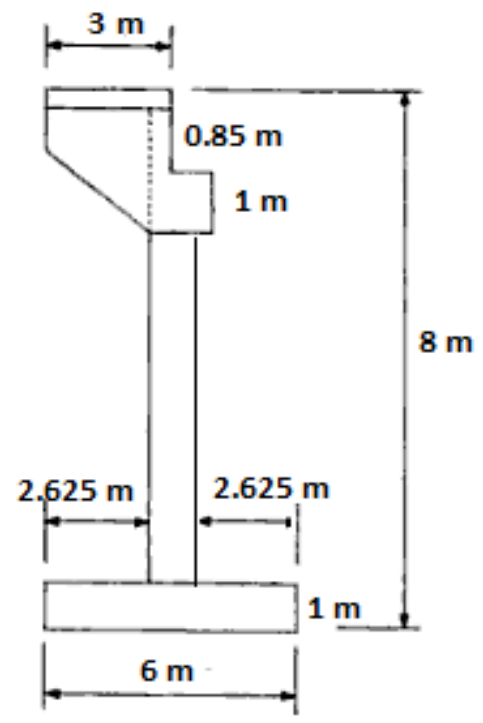

Figure IV-5: Side elevation of the abutment (Tandon, 2005).

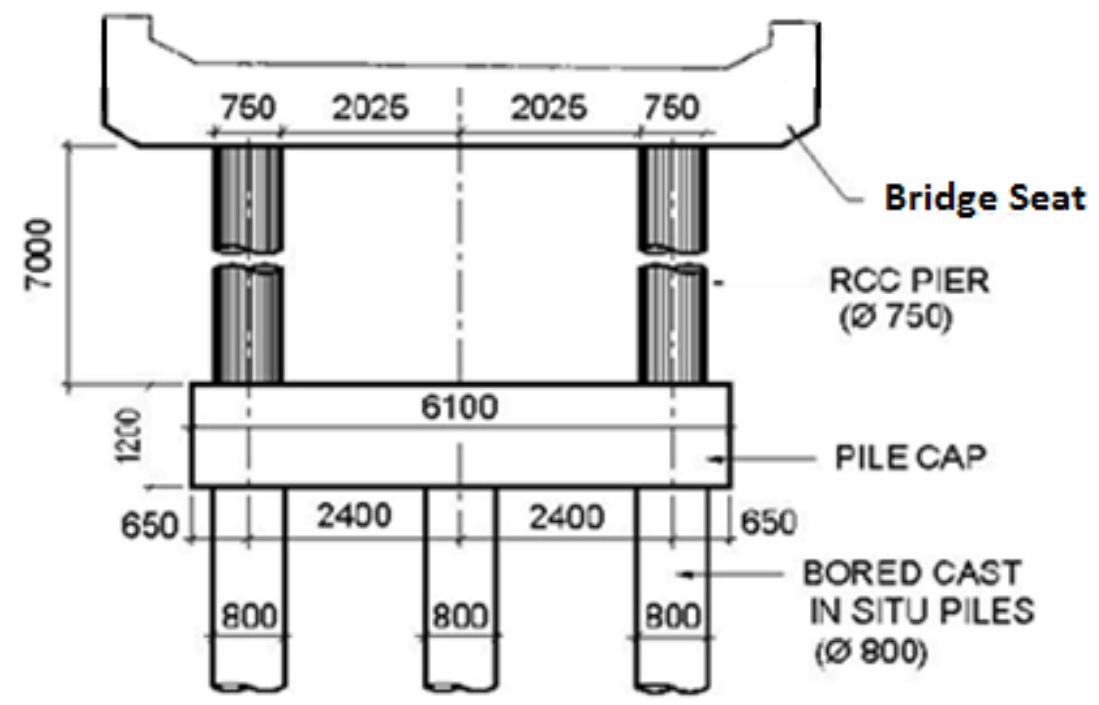

Figure IV-6: Front elevation (Tandon, 2005)

The bridge consists of three 20-metre spans which are supported by reinforced concrete piers. The piers are constructed on a pile cap which has a thickness of 1.2 metres. The size of the piers is 7 metres high and $750 \mathrm{~mm}$ in diameter. There are six cylindrical concrete piles with an $800 \mathrm{~mm}$ diameter. The concrete material density is $2400 \mathrm{~kg} / \mathrm{m}^{3}$.

The superstructure consists of eleven concrete I girders and a concrete slab. The bridge depth deck and width are $150 \mathrm{~mm}$ and $8400 \mathrm{~mm}$ respectively. 
A $2 D$ study of the bridge was chosen for a number of reasons. First, this would provide a simple approach capturing the 'in-plane' longitudinal response. Regarding behaviour relating to the bridge width, it was judged that bridge width and capacity would tend to provide adequate strength in most cross-deck loading scenarios. Torsion may be of concern, however; at locations where the bridge deck is supported by piers, the torsional stiffness would be larger and also be likely to possess adequate torsional capacity in most scenarios. Only in the deck spans could potential torsional displacements be larger, and the deck section used was judged to possess sufficient deformation and forced capacity to withstand this.

\subsubsection{Loading Idealization}

The purpose of this study is to analyze the effect of horizontal and vertical earthquake ground motion on integral bridges. The focus is on the variations of earthquake loading rather than the gravity load. Loading considers the gravity load (self-weight, superimposed load and traffic load) and earthquake loading. Secondary effect loadings are not considered because the length of span is less than $300 \mathrm{ft}$.

The dead load of the deck is distributed to the beams based on their respective tributary widths. Superimposed dead loads (wearing course, future wearing surface, railings, barriers), with the exception of footpath loads, are to be distributed equally to all beam lines. Superimposed load is achieved by multiplying the density of material by the area of the elements. The calculation of the superimposed load is as follow:

$$
\begin{array}{lllll}
\text { a. } & \text { Parapet } & : 1.00 \mathrm{~m} \times 24 \mathrm{kN} / \mathrm{m}^{3}=24 \mathrm{kN} / \mathrm{m}^{2} \times 0.2 \times 2 & =9.6 \mathrm{kN} / \mathrm{m} \\
\text { b. } & \text { Pavement } & : 0.02 \mathrm{~m} \times 22 \mathrm{kN} / \mathrm{m}^{3}=\frac{0.44 \mathrm{kN} / \mathrm{m}^{3} \times 7 \mathrm{~m}}{} & =3.08 \mathrm{kN} / \mathrm{m}
\end{array}
$$

The load will be transformed to mass inertia load, because for further analysis such as modal analysis, the loading considered is only inertia load.

$$
\begin{aligned}
\text { Mass inertia load } \quad & =(\text { Load } / \text { area / gravity }) \times \text { converter } \\
& =(12.68) \mathrm{kN} / \mathrm{m} \times 1000 / 9.81 /(0.5785 \mathrm{~m} \times 8 \mathrm{~m}) \\
& =279.2765 \mathrm{~kg} / \mathrm{m}^{3}
\end{aligned}
$$

It is important to obtain the optimum configuration of the bridge for this traffic load, since this study requires the maximum moments of the bridge. 
For this purpose, there are four cases of patterned loading to be analyzed, as shown in Figure IV-7. This study adopts case number 3 (Figure IV-7c) to be applied to the bridge structure.
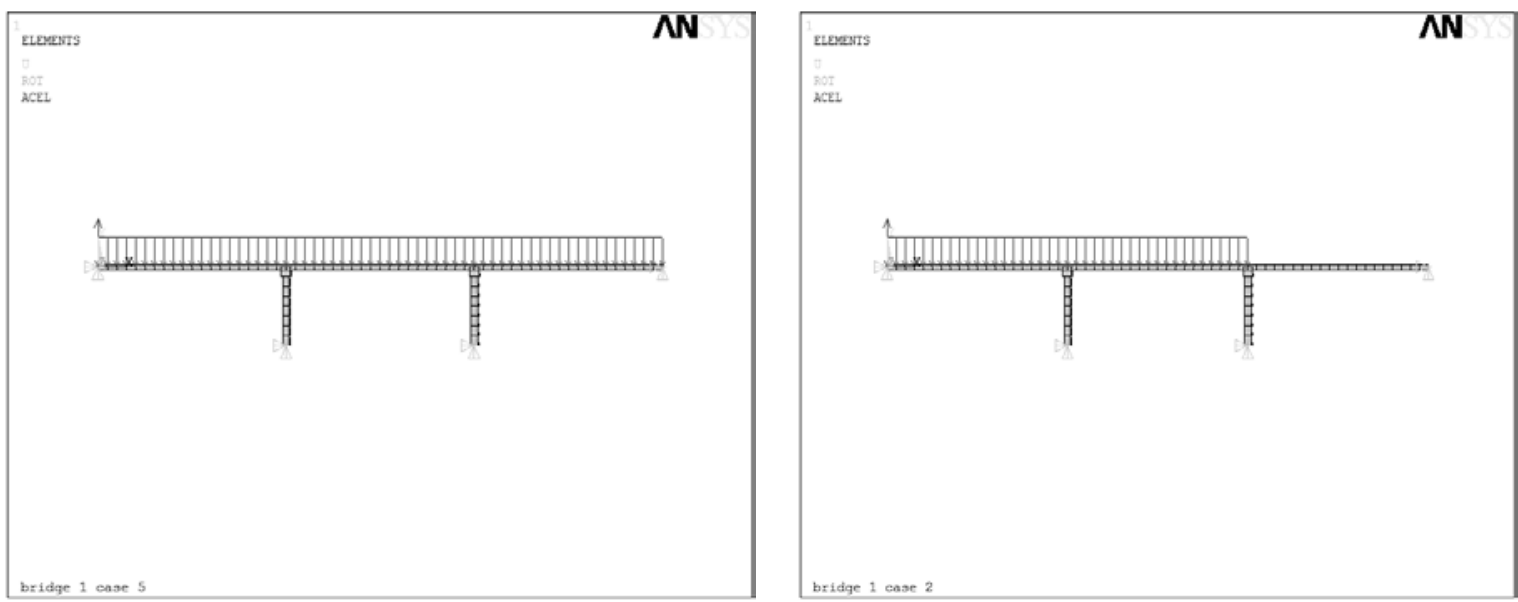

a. Case 1

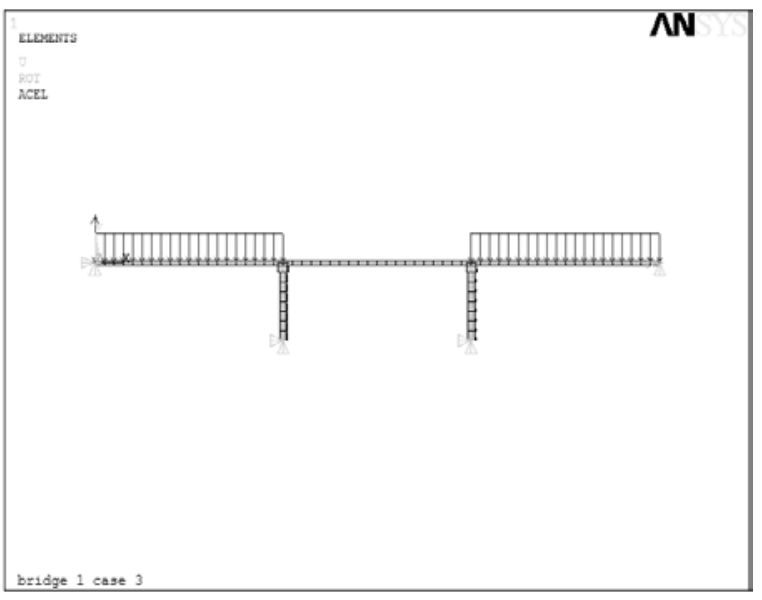

b. Case 2

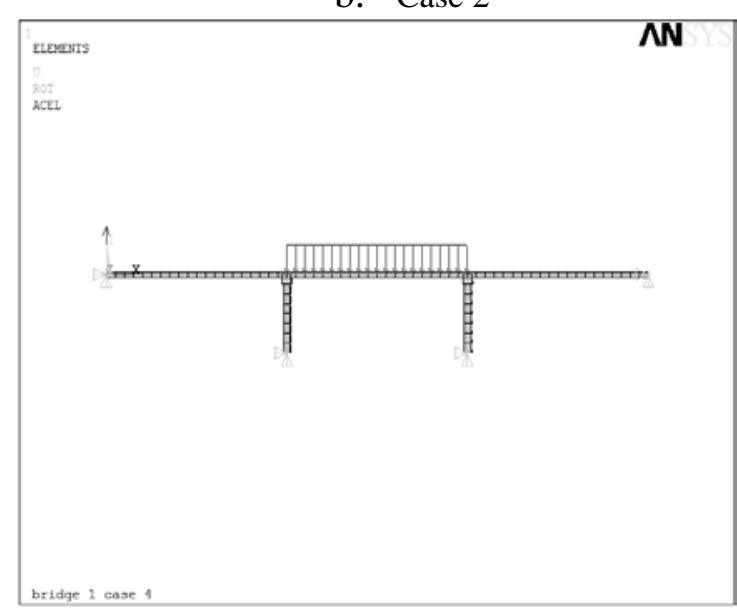

c. Case 3

d. Case 4

Figure IV-7: Load cases for traffic loads

The traffic loading resulting from the analysis is converted to an inertial load for dynamic analysis by the ANSYS software.

The traffic load in this research is based on the standard value from AASHTO; it can be seen in Figure IV-8. AASHTO is considered a mature standard and is simple and easy to use. 


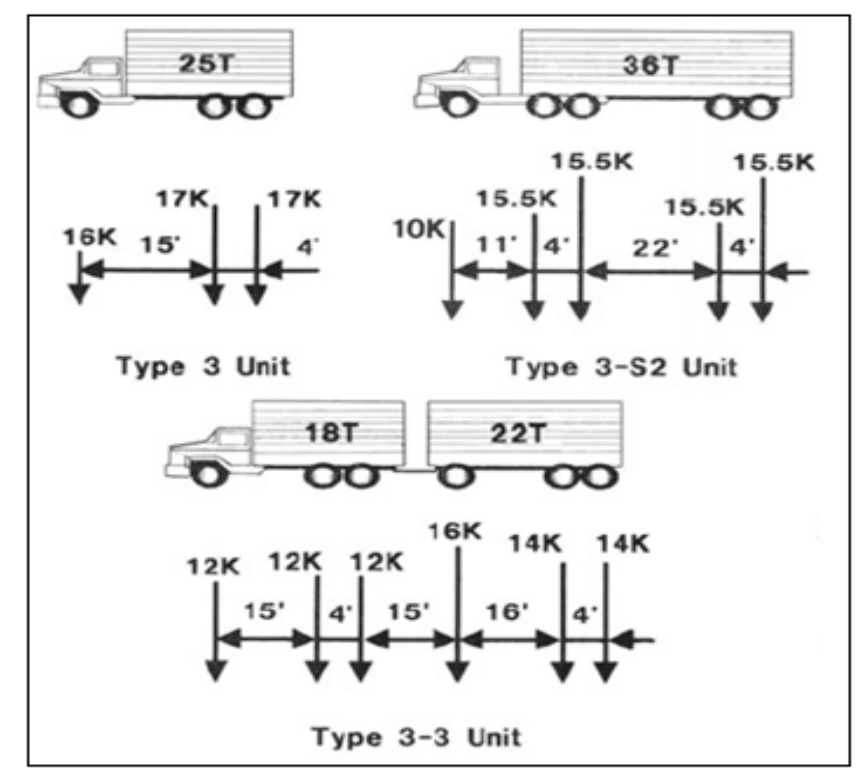

Figure IV-8: Standard value of vehicle load from AASHTO

(Source: http://www.fhwa.dot.gov/publications/publicroads/05jul/09.cfm)

The illustrations show AASHTO legal loads, which control weight limits for short (type 3 unit), medium (type 3-S2 unit), and long (type 3-3 unit) span bridges, respectively. The “ $T$ " refers to weight in tons, and " $K$ " refers to weight in kips (1000 pounds or 907 metric tonnes). In this research, traffic load is assumed to be for type 3 unit, which means the load is as in Figure IV-9.

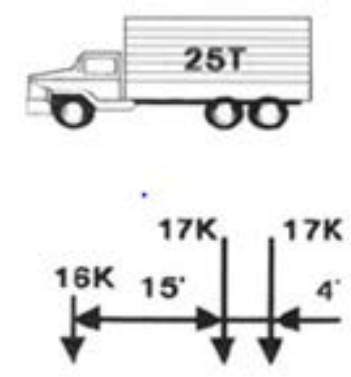

Figure IV-9: Vehicle load

The vehicle has a length of about 20 feet (6 metres). The length of spans is 20 metres, therefore each span will accommodate two vehicles. The load for one vehicle is about $222.5 \mathrm{kN}$ and for simplification it is distributed within each span as $11.125 \mathrm{kN} / \mathrm{m}$. This load will be applied to the bridge as inertia load of $245 \mathrm{~kg} / \mathrm{m}^{3}$.

There will be three kinds of mass used in modelling the traffic loading, namely: mass 1 which only considers the mass of self-weight $\left(2400 \mathrm{~kg} / \mathrm{m}^{3}\right)$; mass 2 which only considers 
the mass of self-weight and superimposed load $\left(2679.276 \mathrm{~kg} / \mathrm{m}^{3}\right)$; and mass 3 which considers the mass of self-weight, superimposed load and traffic load $\left(2924.304 \mathrm{~kg} / \mathrm{m}^{3}\right)$. The resulting model of the bridge is shown in Figure IV-10.

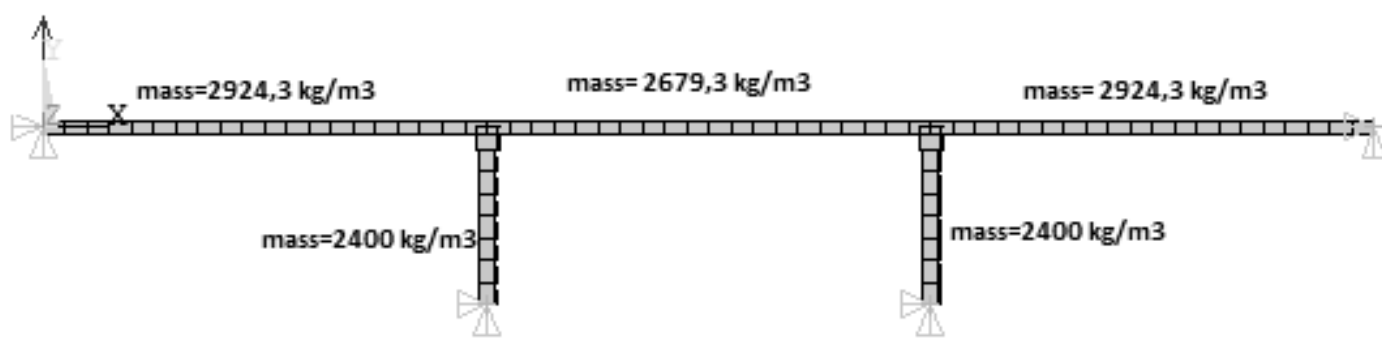

Figure IV-10: Idealization of total loading on the bridge

\subsubsection{Model Validation}

For validation, support reactions are calculated using moment distribution (Crosss method). A simplified version of the model of the structure can be seen in Figure IV-11.

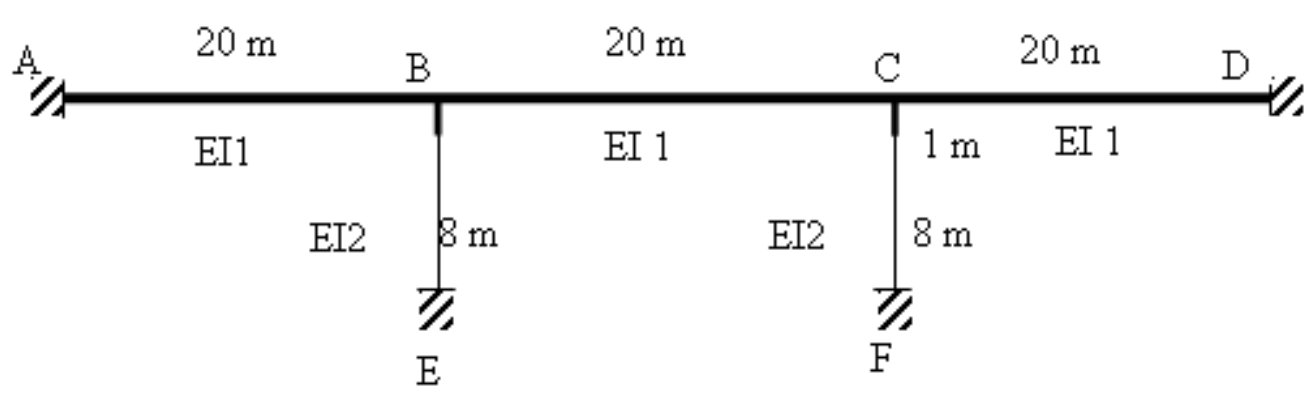

Figure IV-11: Structure idealization

The results are obtained as follows:

$A_{\mathrm{AB}}=A_{\mathrm{BC}}=A_{\mathrm{CB}}=4.6282 \mathrm{~m}^{2}$

$A_{\mathrm{BE}}=A_{\mathrm{CF}}=0.883 \mathrm{~m}^{2}$

$I_{\mathrm{AB}}=I_{\mathrm{BC}}=I_{\mathrm{CB}}=0.3076 \mathrm{~m}^{4}$

$I_{\mathrm{BE}}=I_{\mathrm{CF}}=0.0031 \mathrm{~m}^{4}$

The density of the total deck plus superimposed load is $2924.304 \mathrm{~kg} / \mathrm{m}^{3}$.

From moment distribution, the results are summarized in Table IV-1. 
Table IV-1: Moment distribution calculation

\begin{tabular}{|c|c|c|c|c|c|c|c|c|c|c|c|}
\hline \multirow{2}{*}{ ITEM } & $\mathrm{A}$ & \multicolumn{3}{|c|}{ B } & \multicolumn{3}{|c|}{$c$} & \multirow[b]{2}{*}{$D C$} & \multirow{2}{*}{$E$} & \multirow{2}{*}{\begin{tabular}{|l|}
$\mathrm{F}$ \\
$\mathrm{FC}$ \\
\end{tabular}} & \\
\hline & $A B$ & BA & $B C$ & $\mathrm{BE}$ & $C B$ & $C D$ & CF & & & & \\
\hline \multirow{2}{*}{ SF } & \multicolumn{2}{|c|}{$4 E I / L$} & $4 E \mathrm{I} / \mathrm{L}$ & \multirow{2}{*}{\begin{tabular}{|l|}
$4 E 1 / 2$ \\
$4.66 E+0 g$
\end{tabular}} & \multirow{2}{*}{$\begin{array}{l}4 E \mathrm{E} / \mathrm{L} \\
1.85 \mathrm{E}+09\end{array}$} & \multirow{2}{*}{$\begin{array}{l}4 \mathrm{E} / \mathrm{L} \\
1.85 \mathrm{E}+09\end{array}$} & \multirow{2}{*}{$\begin{array}{c}4 E \mid / 2 \\
4.66 E+08\end{array}$} & \multirow{2}{*}{\begin{tabular}{|l|}
$4 E \mathrm{E} / \mathrm{L}$ \\
$1.95 \mathrm{E}+09$
\end{tabular}} & $4 E V / L$ & \multicolumn{2}{|l|}{$4 E V L$} \\
\hline & \multicolumn{2}{|c|}{$1.85 E+09$} & $\begin{array}{r}4 \mathrm{E} / \mathrm{L} \\
1.85 \mathrm{E}+0 \mathrm{~g}\end{array}$ & & & & & & $4.66 \mathrm{E}+0.8$ & $4.66 \mathrm{E}+0 \mathrm{~g}$ & \\
\hline DF ratio & 0 & 4.44E-01 & \begin{tabular}{|l|}
$4.44 \mathrm{E}-01$ \\
\end{tabular} & $1.12 \mathrm{E}-01$ & 4.44E-01 & 4.44E-01 & $1.12 \mathrm{E}-01$ & $\begin{array}{l}0 \\
\end{array}$ & 0 & 0 & \\
\hline FEM & 4425.70 & -4425.70 & 4054.87 & 0.00 & -4054.87 & 4425.70 & 0.00 & -4425.70 & 0.00 & 0.00 & siklus 1 \\
\hline Dist 1 & & 164.65 & 164.65 & 4154 & -164.65 & -164.65 & -41.54 & 0.00 & & & Intose 1 \\
\hline $\mathrm{CO} 2$ & 82.32 & $0 . \infty$ & -8232 & 0.00 & 82.32 & $0 . \infty$ & 0.00 & -82.32 & 20.77 & -20.77 & siklu: \\
\hline Dist. 2 & & 36.55 & 36.55 & 9.22 & -36.55 & -36.55 & -9.22 & 0.00 & & & आरा \\
\hline $\mathrm{CO} 3$ & 18.28 & $0 . \infty$ & -18.28 & 0.00 & 18.28 & $0 . \infty$ & 0.00 & -18.28 & 4.61 & -4.61 & siklus 3 \\
\hline Dist. 3 & & 8.11 & 8.11 & 2.05 & -8.11 & -8.11 & -2.05 & 0.00 & & & \\
\hline $\mathrm{CO4}$ & 4.06 & $0 . \infty$ & -4.06 & 0.00 & 4.06 & $0 . \infty$ & 0.00 & -4.06 & 1.02 & -1.02 & siklus 4 \\
\hline Dist. 4 & & 1.80 & 1.90 & 0.45 & -1.80 & -190 & -0.45 & 0.00 & & & 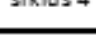 \\
\hline $\cos$ & 0.90 & $0 . \infty$ & -0.90 & 0.00 & 0.90 & $0 . \infty$ & 0.00 & -0.90 & 0.23 & -0.23 & \\
\hline Dist. 5 & & 0.40 & 0.40 & 0.10 & -0.40 & -0.40 & -0.10 & 0.00 & & & \\
\hline $\cos$ & 0.20 & $0 . \infty$ & -0.20 & 0.00 & 0.20 & $0 . \infty$ & 0.00 & -0.20 & 0.05 & -0.05 & siklus 6 \\
\hline Dist. 6 & & 0.09 & 0.09 & 0.02 & -0.09 & -0.09 & -0.02 & 0.00 & & & 2101020 \\
\hline $\mathrm{CO} 7$ & 0.04 & 0.00 & -0.04 & 0.00 & 0.04 & $0 . \infty$ & 0.00 & -0.04 & 0.01 & -0.01 & siklus 7 \\
\hline Dist. 7 & & 0.02 & 0.02 & 0.00 & -0.02 & -0.02 & 0.00 & 0.00 & & & 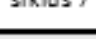 \\
\hline TEM & 453151 & -4214.08 & 4160.70 & 53.39 & -4160.70 & 4214.08 & -53.39 & -4531.51 & 26.69 & -26.69 & \\
\hline
\end{tabular}

Moment $A=4.5 \mathrm{MNm}$

Moment $D=-4.5 \mathrm{MNm}$

Moment $E=26.7 \mathrm{kNm}$

Moment $F=-26.7 \mathrm{kNm}$

The results are close to the ANSYS results, which can be seen in Appendix II.

Table IV-2: Comparison of Cross method and ANSYS result

\begin{tabular}{|c|c|c|c|}
\hline Node & Cross Method (N) & Ansys Calculation (N) & Persentage \\
\hline 1 & $4.5 \times 10^{6}$ & $4.6 \times 10^{6}$ & $98 \%$ \\
\hline 61 & $-4.5 \times 10^{6}$ & $-4.6 \times 10^{6}$ & $98 \%$ \\
\hline 62 & 26,690 & 27,843 & $96 \%$ \\
\hline 70 & 26,690 & $-27,843$ & $96 \%$ \\
\hline
\end{tabular}

Table IV-2 shows the comparison between the Cross method and ANSYS results. It can be seen that the results are close with the percentages of the comparison above $96 \%$. 


\subsubsection{Soil Stiffness Idealization}

The stiffness of the supports under a structure can have a significant influence on the distribution of forces. Most analysis models will assume the supports are rigidly positioned in space (the classical assumptions of pinned or fixed supports). This is clearly inaccurate as all soil and rock is compressible and changes will be exacerbated by the transient nature of vehicle loadings and the flexibility of superstructure connections. It is therefore worthwhile exploring the effects of soil stiffness by including a crude representation of soil as an elastic spring and undertaking a series of increasingly complex analyses which model the structure supported on soil springs. Young's moduli of soils considers soil type and the magnitude and duration of loading; they have to be determined by tests for specific conditions (Hambly, 1994).

In this study, soil stiffness will be modelled using simple linear springs. The spring stiffnesses will be varied for each analysis to encompass a broad range of soil types. Soil stiffness as shown in Table IV-3 is used as the basis of analysis. However, the incorporation of springs to represent specific soil-structure interaction analyses involves further analysis (time) and software beyond the scope of the project budget.

The value for the stiffness $(k)$ is computed using a formula which is based on the recommendation of Terzaghi (2009).

Table IV-3: Typical Elastic Moduli of soils based on soil type and consistency (density)

\begin{tabular}{|c|c|}
\hline Soil & Es (tsf) \\
\hline Very soft clay & $5-50$ \\
Soft clay & $50-200$ \\
Medium clay & $200-500$ \\
Stiff clay, silty clay & $500-1000$ \\
Sandy clay & $250-2000$ \\
Clay shale & $1000-2000$ \\
Loose sand & $100-250$ \\
Dense sand & $250-1000$ \\
Dense sand and gravel & $1000-2000$ \\
Silty sand & $250-2000$ \\
\hline
\end{tabular}

tsf $=$ tons per square foot (1tsf equals $95.76 \mathrm{kPa}$ ) 
Terzaghi and Sisk (2009) gave guidance on calculating the soil spring of an integral bridge, as noted in the range:

$$
K=0.8 E s \sim 1.8 E s
$$

Equation $I V-1$

Based on the figures in Table IV-3, Es is 250 tsf $(\approx 24000 \mathrm{kPa})$ for loose (flexible) sand and 650 tsf ( $\approx 62000 \mathrm{kPa}$ ) for dense (hard) sand. The range of stiffnesses used is as shown in Tables IV-4 and IV-5.

Table IV-4: Soil stiffness of abutments and piles for flexible soil.

\begin{tabular}{|l|r|r|r|r|r|r|}
\hline & \multicolumn{7}{|c|}{$\mathrm{K}(\mathrm{kN} / \mathrm{m})$} \\
\hline Es $\left(\mathrm{kN} / \mathrm{m}^{3}\right)$ & 20000 & 25000 & 30000 & 35000 & 40000 & 45000 \\
\hline Abutment & 160000 & 200000 & 240000 & 280000 & 320000 & 360000 \\
\hline Pile & 96000 & 120000 & 144000 & 168000 & 192000 & 216000 \\
\hline
\end{tabular}

Table IV-5: Soil stiffness of abutments and piles for stiff soil.

\begin{tabular}{|l|r|r|r|r|r|r|}
\hline & \multicolumn{7}{|c|}{ Stiffness k (kN/m) } \\
\hline Es $\left(\mathrm{kN} / \mathrm{m}^{3}\right)$ & 50000 & 55000 & 60000 & 65000 & 70000 & 75000 \\
\hline Abutment & 400000 & 440000 & 480000 & 520000 & 560000 & 600000 \\
\hline Pile & 240000 & 264000 & 288000 & 312000 & 336000 & 360000 \\
\hline
\end{tabular}

The spring stiffness is applied on the supports of the bridge, as shown in Figure IV-12. 


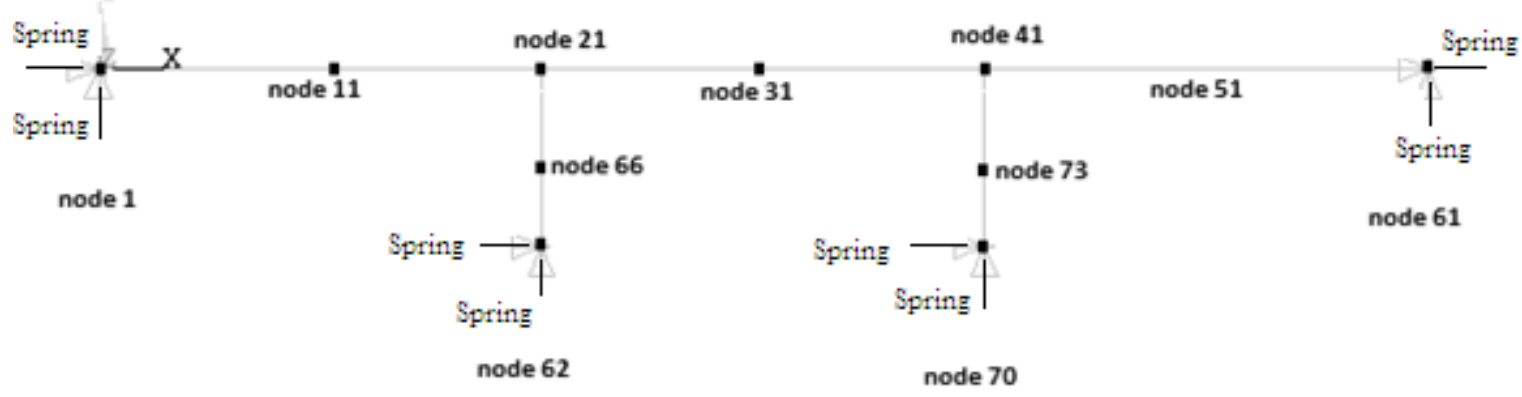

Figure IV-12: Spring stiffness on the support of the bridge.

Three different cases relating to soil stiffness are conducted in the study. The first case applies the stiffness for hard soil, the second for soft soil, and the third applies different soil stiffness in vertical and horizontal springs.

The range of the soil stiffness can be seen in Tables IV-4 and IV-5. For validating the spring stiffness applied to the structure, simple analyses are conducted using the formula suggested by Hambly (1994).

A $2 D$ analysis of a spread foundation of width $w$ and breadth $b$ (into the page), subject to vertical and horizontal forces, is modelled as shown in Figure IV-13.

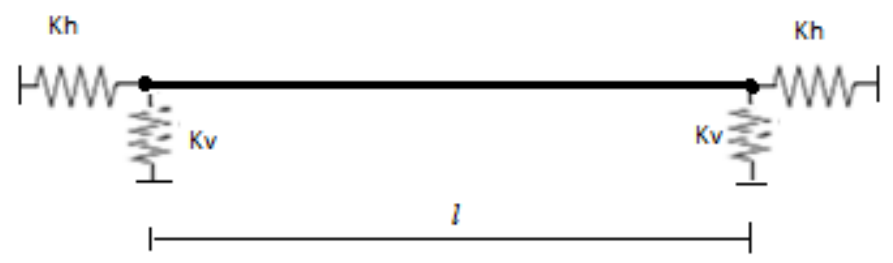

Figure IV-13: Springs modelling for foundation

$$
\begin{aligned}
& l=0.82 \times b^{0.25} b_{w^{0.75}}{ }^{0.75} \\
& k_{v}=\left(0.25 G \times b_{w}{ }^{0.25}\right) /(1-v) \\
& k_{h}=2 G\left((1+v)\left(b_{w}{ }^{0.5}\right)\right) \\
& G=\frac{E}{2(1+v)} \\
& \mathrm{k}_{\mathrm{v}} \quad=\text { vertical stiffness, } \\
& \mathrm{k}_{\mathrm{h}} \quad=\text { horizontal stiffness, } \\
& \mathrm{b} \quad=\text { breadth } \\
& \mathrm{b}_{\mathrm{w}} \quad=\text { width } \\
& \mathrm{E} \quad=\text { Modulus Young } \\
& \mathrm{G} \quad=\text { shear modulus }
\end{aligned}
$$


$v \quad=$ Poison's ratio, for soil generally lie in range $0.3-0.5$

From the formula, the spring stiffnesses for the foundation are as shown in Table IV-6, and the soil stiffnesses are as in Table IV-7.

Table IV-6: Stiffness of soil from the formula (Hambly, 1994)

\begin{tabular}{|c|c|c|}
\hline Stiffness & Flexible soil $\left(\mathrm{E}=15000 \mathrm{kN} / \mathrm{m}^{2}\right)$ & Stiff soil $(250000 \mathrm{kN} / \mathrm{m})$ \\
\hline Vertical & $26,000 \mathrm{kN} / \mathrm{m}$ & $436,000 \mathrm{kN} / \mathrm{m}$ \\
\hline Horizontal & $36,000 \mathrm{kN} / \mathrm{m}$ & $612,000 \mathrm{kN} / \mathrm{m}$ \\
\hline
\end{tabular}

Table IV-7: Stiffness of soil used in the study

\begin{tabular}{|l|c|c|}
\hline \multicolumn{1}{|c|}{ Stiffness } & Lowest stiffnest & Highest stiffness \\
\hline Abutment & $160,000 \mathrm{kN} / \mathrm{m}$ & $600,000 \mathrm{kN} / \mathrm{m}$ \\
\hline Pile & $96,000 \mathrm{kN} / \mathrm{m}$ & $360,000 \mathrm{kN} / \mathrm{m}$ \\
\hline
\end{tabular}

It can be seen from these two tables that the stiffnesses used in the study lie within the conservative range obtained from the formulas suggested by Hambly.

\subsection{Finite Element Representation}

The total length of the integral bridge is $60 \mathrm{~m}$ and the width is $8 \mathrm{~m}$. The bridge deck consists of eleven equally spaced ' $I$ ' pre-stressed concrete girders, 150 mm-thick concrete deck and 20 mm-thick asphalt concrete, resting on $6 \mathrm{~m}$ high and $1.56 \mathrm{~m}$ thick abutments. The abutments are supported by two $800-\mathrm{mm}$ diameter bored piles equally spaced. These dimensions and the geometry of the bridge were selected based on a typical bridge drawing with some minor changes in dimensions to simplify the analysis.

The analysis of bridges and structures is a mixture of science and engineering judgement. In most cases, simple models with conservative assumptions can be used to arrive at the design forces for various elements. The impacts of creep, shrinkage, and relaxation will 
not be considered in this study since they are insignificant for deck lengths not more than $91 \mathrm{~m}$. Satisfying force equilibrium and identifying a load path to adequately transfer the loads are important considerations.

The bridge generally consists of three main parts, namely: deck, superstructure and substructure. The deck is the part which carries traffic loads. The superstructure supports the deck, and becomes the second part of load transfer after the deck. In other words, the deck is a plate which is cast monolithically on the superstructure, and will transfer the traffic loads to the superstructure then to the substructure and the soil. Current design practice tends to avoid inelastic action in the bridge deck; therefore it is considered to respond elastically. The substructure or foundation of the bridge is the part which supports the whole structure of the bridge and usually consists of piers and abutments which carry the superimposed load of the superstructure to the underlying soil or rock.

The bridge is assumed to be a $2 D$ portal and consists of three equal spans, as can be seen in Figure IV-14. Idealization is made in $2 D$ by assuming that the stiffness in the cross-deck direction is large and therefore distribution of loading will be uniform. In addition, buckling is not considered in the study, so the bridge may be modelled in $2 \mathrm{D}$. It is assumed the bridge sits on a simple abutment. The superstructure is fixed on cap beams at the top of the piers and at the abutment. The joints and the abutment have fixed connection. Rotation is assumed zero in the connection of deck and abutment since the abutments are stiff and relative displacement occurs in the abutments. 


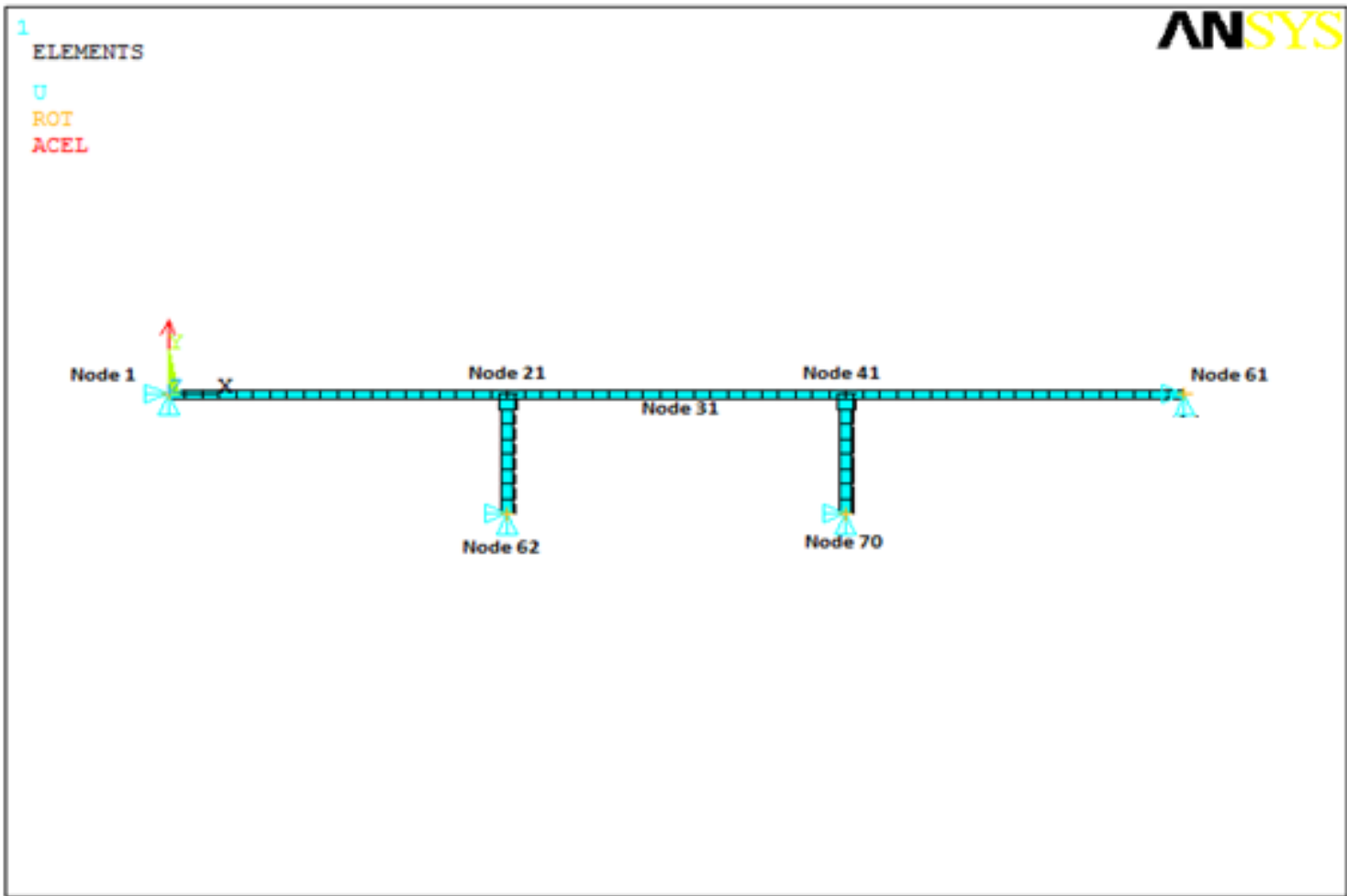

Figure IV-14: Simple Idealization of the bridge in ANSYS modelling

The bridge has three spans and two piers. The deck and the girder are modelled as a single unit and represented as a beam. The piers are treated as columns and stand on soil which has a range of conditions. The bridge is made of high-strength concrete, modulus elasticity $(\mathrm{E})=30 \mathrm{GPa}$.

Loading in this study is considered constant, except for earthquake loading. The focus is not the loading, but the behaviour of the structure due to earthquake loading. The variable will not be the structure's dimensions, or primary load such as gravity loads and traffic load, but the intensity and condition of the earthquake load. This is because the purpose of the study is not to analyze the ability of the structure in resisting the load, but to see its behaviour under different variations of the earthquake loading.

The bridge in this study consists of a deck and 11 'I' girders, as can be seen in Figure IV15. The deck is idealized as a part of the girder to simplify the calculation. The deck and I girders are then modelled using beam elements. 


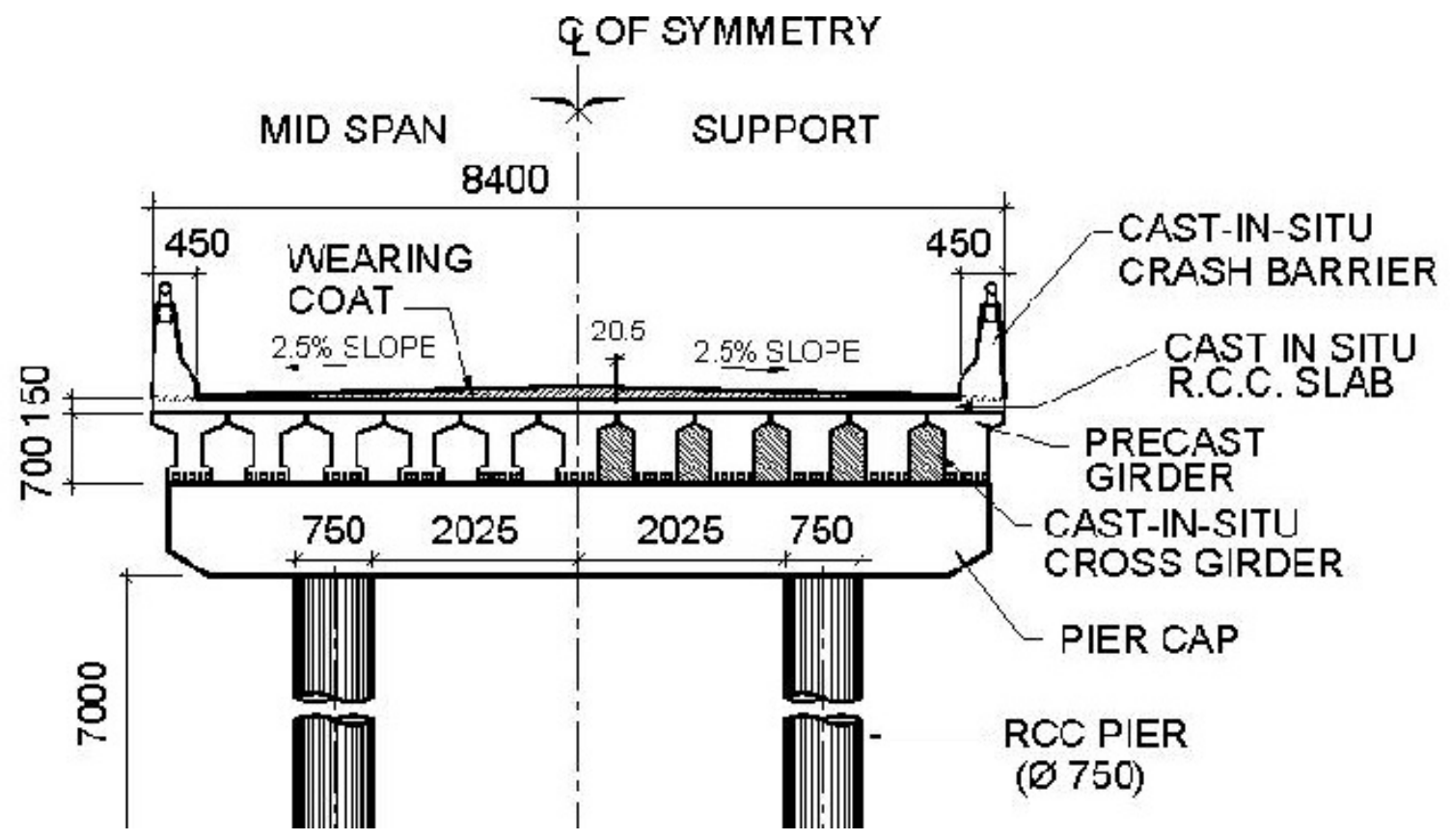

Figure IV-15: Girders of the bridge

For finite element analysis, the deck and the girders are simplified as two-dimensional concrete beam elements. The dimension of the idealization is width $8000 \mathrm{~mm}$ and thickness $580 \mathrm{~mm}$. Pier caps are modelled as $1 \mathrm{~m}$ x $8 \mathrm{~m}$ beams.

The moment capacity of the bridge will be compared to the moments resulting from the analysis. The calculation of moment capacity of the bridge can be seen in Appendix III.

\subsection{Synthetic Earthquake Accelerogram in Eurocode 8}

\subsubsection{Determining Soil Types}

As the objective of this research is to obtain relevant knowledge about the behaviour of the bridge constructed on different types of soil, the soil types need to be determined. For this purpose, the study refers to Eurocode 8 which identifies five ground types. These are A, B, $\mathrm{C}, \mathrm{D}$ and $\mathrm{E}$, described by the profiles and parameters given in Table IV-8; they are used to account for the influence of local ground conditions on the seismic action. Analysis of the bridge behaviour uses soil type A for strong soil and soil type D for flexible soil; A is assumed to be the strongest soil and D the weakest. However, for generating the response spectrum, all of the soil types are considered to compare them with the Eurocode response spectrum. 
Table IV-8: Description of stratigraphic profiles of five soil types (Eurocode 8)

\begin{tabular}{|l|l|}
\hline \multicolumn{1}{|c|}{ Type } & \multicolumn{1}{|c|}{ Description of stratigraphic profile } \\
\hline A & $\begin{array}{l}\text { Rock or other rock-like geological formation, including at most } 5 \text { m of weaker } \\
\text { material at the surface }\end{array}$ \\
\hline B & $\begin{array}{l}\text { Deposits of very dense sand, gravel, or very stiff clay, at least several tens of } \\
\text { metres in thickness, characterized by a gradual increase of mechanical properties } \\
\text { with depth. }\end{array}$ \\
\hline C & $\begin{array}{l}\text { Deep deposits of dense or medium-dense sand, gravel or stiff clay with thickness } \\
\text { from several tens to many hundreds metres. }\end{array}$ \\
\hline D & $\begin{array}{l}\text { Deposit of loose to medium cohesionless soil (with or without some soft } \\
\text { cohesive layers), or of predominantly soft to firm cohesive soil. }\end{array}$ \\
\hline E & $\begin{array}{l}\text { A soil profile consisting of a surface alluvium layer with vs values of type c or d } \\
\text { and thickness varying between about } 5 \mathrm{~m} \text { and } 200 \mathrm{~m} \text {, underlain by stiffer } \\
\text { material with } \mathrm{v}>800 \mathrm{~m} / \mathrm{s}\end{array}$ \\
\hline
\end{tabular}

\subsubsection{Determining Elastic Response Spectra}

A response spectrum is defined as a graphical representation of the variation with natural frequency or natural period and damping ratio of the absolute value of the maximum response of a single degree of freedom system to a given ground acceleration history; this is shown in Figure IV-16 (Clough \& Penzien, 2003). 


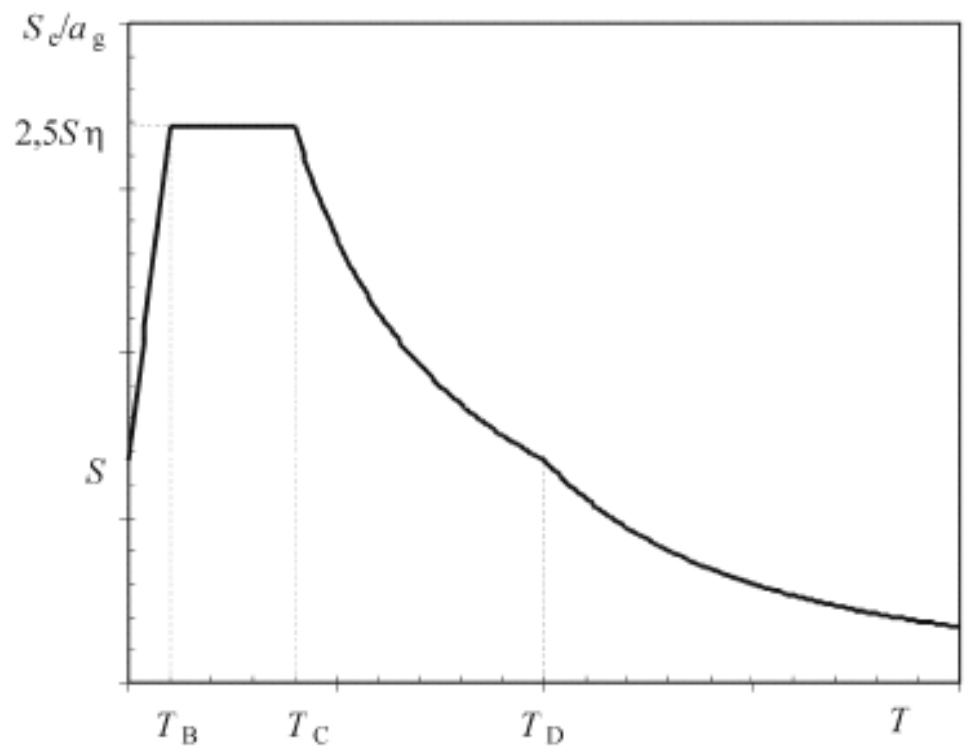

Figure IV-16: Response spectrum

Eurocode 8 is used to determine the elastic response spectra, based on the five different types of soil already mentioned.

Eurocode 8 provides horizontal and vertical elastic design spectra which are generated from several equations.

The equation for response spectra, $S \mathrm{e}(T)$, is derived using expressions:

$$
\begin{gathered}
0 \leq T \leq T_{B}: S_{e}(T)=a_{g} \cdot S \cdot\left[1+\frac{T}{T_{B}} \cdot(\eta \cdot 2,5-1]\right. \\
T_{B} \leq T \leq T_{C}: S_{e}(T)=a_{g} \cdot S \cdot \eta \cdot 2,5 \\
T_{C} \leq T \leq T_{D}: S_{e}(T)=a_{g} \cdot \eta \cdot 2,5 \cdot\left[\frac{T_{C}}{T}\right] \\
T_{D} \leq T \leq 4 s: S_{e}(T)=a_{g} \cdot \eta \cdot 2,5\left[\frac{T_{C} \cdot T_{D}}{T^{2}}\right]
\end{gathered}
$$

Where, $\mathrm{Se}(\mathrm{T})$ is the elastic spectral acceleration, $T$ is the vibration period of a linear SDOF system, $a_{g}$ is the design ground acceleration on soil type ground, $T_{B}$ is the lower limit of the period of the constant spectral range, $T_{C}$ is the upper limit of the period of the constant spectral acceleration, $T_{D}$ is the period at the beginning of the constant spectral 
displacement range, $S$ is the soil factor and $\eta$ is a correction factor for damping ratio $\zeta$ (Fardis, et al., 2005).

The equations are similar for both vertical and horizontal earthquakes. For vertical earthquake motion, the 2.5 factor is replaced by 3 and the soil factor, $S$, is 1 .

Table IV-9: Recommended values of parameters describing the vertical elastic response spectra

\begin{tabular}{|c|c|c|c|c|}
\hline Spectrum & $\mathbf{a}_{\mathbf{v g}} / \mathbf{a}_{\mathbf{g}}$ & $\mathbf{T}_{\mathbf{B}} \mathbf{( s )}$ & $\mathbf{T}_{\mathbf{C}} \mathbf{( s )}$ & $\mathbf{T}_{\mathbf{D}} \mathbf{( s )}$ \\
\hline Type 1 & 0.90 & 0.05 & 0.15 & 1.0 \\
\hline Type 2 & 0.45 & 0.05 & 0.15 & 1.0 \\
\hline
\end{tabular}

Values of vertical parameters for elastic response spectra are presented in Table VI-9. As the response spectra have been obtained from this analysis, the next step is to derive a series of spectrum-compatible synthetic earthquake accelerograms for use in a full integration time-history analysis. Each elastic response spectrum has three different acceleration results and these results will be applied to the structure to calculate relative displacements of the supports.

Using the equations IV-2 to IV-5 above, elastic design spectra for different seismicity conditions and subsoil classes can be created. The range between corner periods $T_{B}$ and $T_{C}$ constitutes the branch of constant spectral acceleration, whereas periods $T_{C}$ and $T_{D}$ are the limits of the constant spectral velocity branch. In addition, constant spectral displacement starts at control period $T_{D}$. The values of the periods $T_{B}, T_{C}$ and $T_{D}$ and of the soil factor $S$ describing the shape of the elastic response spectrum depend upon the ground type.

Regarding the types of earthquake, this analysis refers to the type 1 earthquake defined in Eurocode 8. Design spectra are based on two different types of seismic action (specified type 1 for regions with higher and type 2 for regions with lower seismicity), and a new subsoil classification scheme (Schott \& Schwarz, 2004). Type 1 represents earthquakes of magnitude Ms $\geq 5.5$, and the type 2 spectrum is recommended only for regions where the design earthquake has a surface magnitude of $\mathrm{Ms} \leq 5.5$. The values of soil characteristics are presented in Table IV-10. 
Table IV-10: Values of the parameters describing the recommended Type 1 elastic response spectra

\begin{tabular}{|c|c|c|c|c|}
\hline $\begin{array}{c}\text { Ground } \\
\text { Type }\end{array}$ & $\mathbf{S}$ & $\mathbf{T}_{\mathbf{B}}(\mathbf{s})$ & $\mathbf{T}_{\mathbf{C}}(\mathbf{s})$ & $\mathbf{T}_{\mathbf{D}}$ (s) \\
\hline A & 1.0 & 0.15 & 0.4 & 2.0 \\
\hline B & 1.2 & 0.15 & 0.5 & 2.0 \\
\hline C & 1.15 & 0.2 & 0.6 & 2.0 \\
\hline D & 1.35 & 0.2 & 0.8 & 2.0 \\
\hline E & 1.4 & 0.15 & 0.5 & 2.0 \\
\hline
\end{tabular}

The two horizontal design spectra from Eurocode 8 can be seen in Figures IV-17 and IV18.

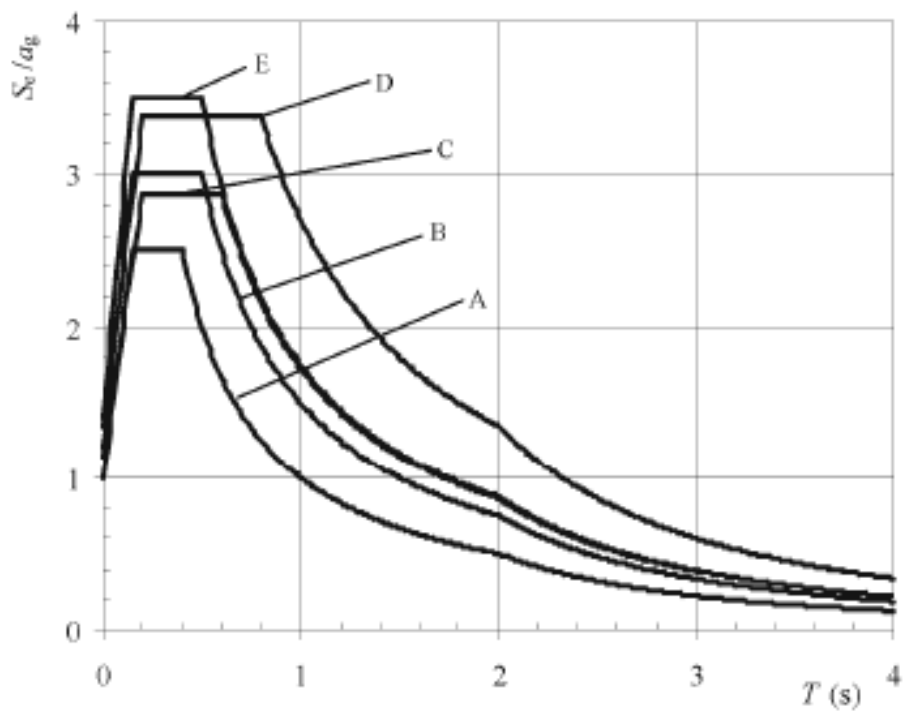

Figure IV-17: Elastic response spectra for earthquakes of type 1 


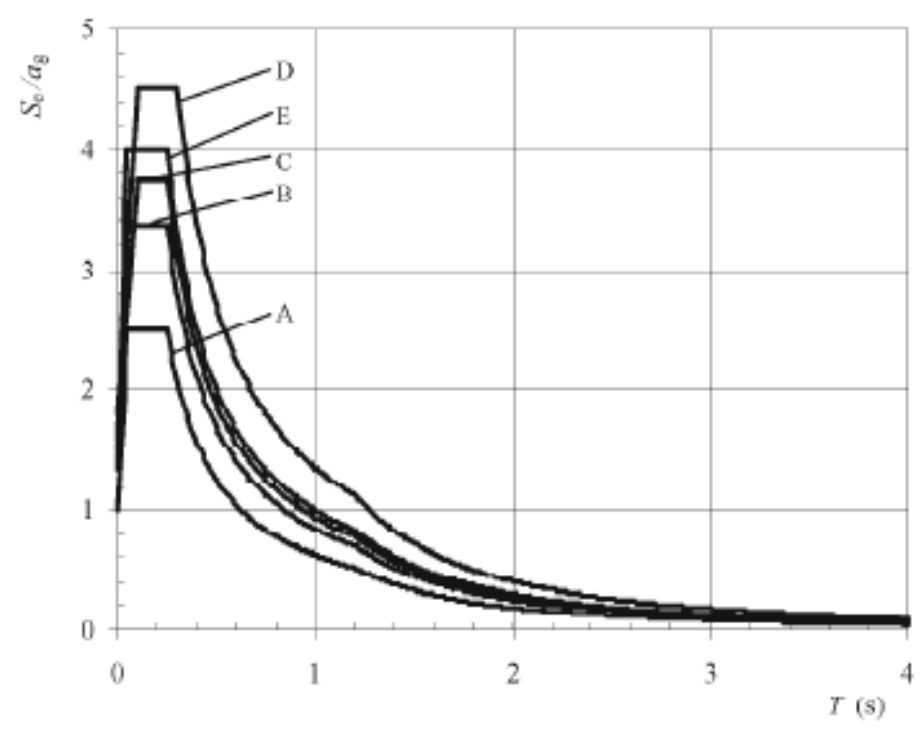

Figure IV-18: Elastic response spectra for earthquakes of type 2

The horizontal displacement spectra are not directly defined in the codes. They are calculated by multiplying the elastic acceleration response spectra with the square of the circular natural frequency (Clough \& Penzien, 2003). Applying the principle of equal displacements, the elastic displacement spectra determined in this way match the design spectra.

\subsubsection{Determining a Series of Synthetic Earthquake Accelerations}

For each response spectrum, three artificial acceleration time histories are derived from which displacement time histories are produced, and these displacements are then applied as ground motions to the structure.

This study uses Clough and Penzien's (2003) procedure to create the synthetic earthquake time histories for each of the soil types. Five types of synthetic acceleration time history of different soil characteristics are arranged by using a modification of Dynamassist in Mathcad ver. 14. Dynamassist is a suite of Mathcad programs developed to help in analyzing dynamic problems in structural analysis. Hence, a bank of spectrum-compatible acceleration time histories can be built. The example of a synthetic acceleration time history compatible with the spectra of soil type A, earthquake type 1, can be seen in Figure IV-19. 


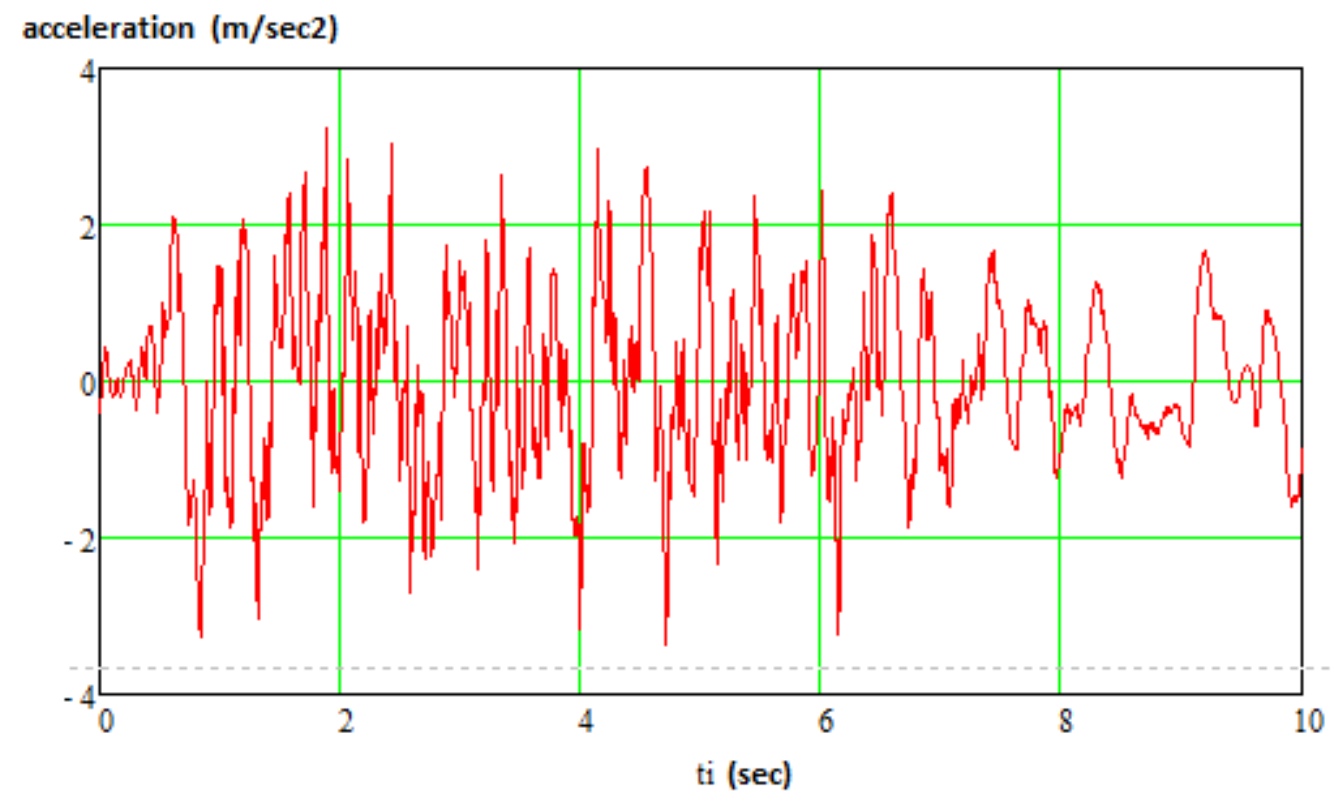

Figure IV-19: Synthetic time history for soil type A

The data accelerations to be applied to the supports of the bridge can be seen in Appendix VIII.

As previously mentioned, the synthetic displacements in this analysis are obtained from different response spectra based on the type of soil and earthquake.

\subsubsection{Determining Relative Displacement on the Supports}

Relative displacements are a significant term in this study, since the focus is on the behaviour of the integral bridge under earthquake ground motion horizontally and vertically, especially in the context of relative displacements. The source of relative displacements which are then applied to the structure is an exisiting bank series of artificial earthquake accelerations. In other words, the bridge will suffer from different earthquake accelerations, each resulting in a different displacement on the supports. This condition will result in relative displacements.

Determining the relative displacements in this research is conducted statically and dynamically, and considered in one node of the bridge, that is at the left end of the deck. Static relative displacements are applied by allowing displacement loads to the left end support of the bridge. Dynamic relative displacements are obtained by applying different accelerations to the supports of the bridge, so that the supports have different displacements which then lead to dynamic relative displacements. 


\subsubsection{Creating Relative Displacement Moment Bending Diagram}

Having different displacements on the supports of the bridge will produce a relative displacement between the two supports, which in turn will create an additional bending moment in the structure.

This study aims to understand the correlation between the relative displacement of the supports and the bending moment of the bridge. Therefore, graphs to describe the relationship between bending moments and relative displacements are constructed. This is discussed in detail in Chapter VI.

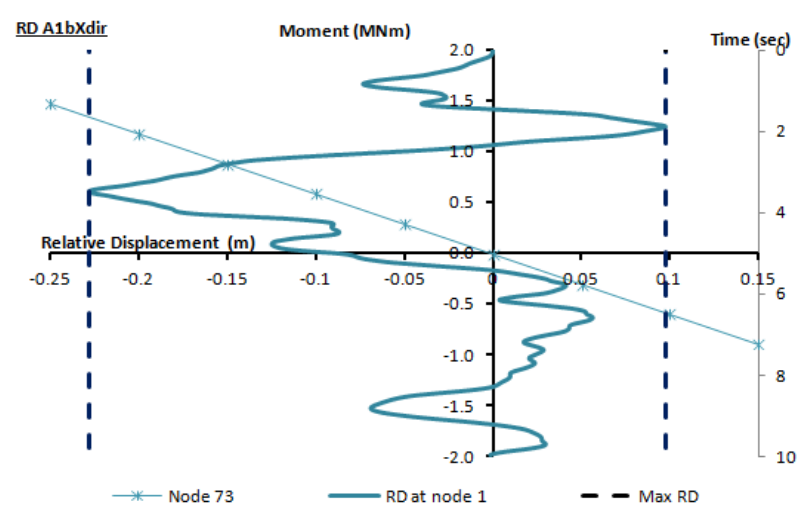

Figure IV-20: Relative displacement-bending moment diagram

Figure IV-20 shows the relative displacement-bending moment relationship. From this diagram, the contribution of the relative displacement in one support to the overall bending moment of the bridge can be obtained. 


\section{Chapter V \\ DYNAMIC ANALYSIS}

\subsection{General}

The analysis of a structural system to determine the deformation and forces induced by applied loads or ground excitation is an essential step in the design of a structure to resist earthquakes. A structural analysis procedure requires: modelling the structure, generating a representation of the earthquake's effects on the ground motion, and choosing a method of analysis. A range of methods from plastic analysis to sophisticated non-linear, dynamic analysis of a detailed structural model is available, depending on the purpose of the analysis in the design process (Filippou \& Fenves, 2004).

The parameters are measures of free-field ground motion or that reveal some basic ground motion characteristics, independent of any structural systems and models. These include peak ground motion values (acceleration, velocity and displacement), strong-motion duration and Fourier spectra of the ground motion (Bozorgni \& Campbell, 2004).

Earthquakes are by nature dynamic, with a rate of change of loading. A dynamic load is defined as a load which is dependent on time. In static analysis the structure is governed by its internal elastic properties, whereas for dynamic analysis, the accelerations produce additional inertia forces. These inertia forces form a significant portion of load equilibrium by the internal elastic forces of the structure. In addition, a damping factor contributes significantly to the structural response. 
In this chapter on the the dynamic analysis covered in this study, the response spectrum, synthetic time-history generation, modal analysis and transient analysis are discussed.

\subsection{Response Spectrum}

The first consideration when conducting a seismic analysis of any structure is to ensure that the dynamic response does not exceed the allowable forces, deflections and so forth. Therefore, the way the structure behaves under this dynamic load must be ascertained. The measure of acceleration is commonly used to indicate the possible destructive power of an earthquake in relation to a structure such as a bridge, in accordance with Newton's law, but it cannot provide dynamic amplification through resonance unless a response spectrum is available.

The design response spectrum does not represent the particular acceleration response from a single ground motion time-history, but rather it is intended to be representative of general characteristics for a reasonable range of expected ground motions at a given site.

The response spectrum is a useful tool in earthquake engineering as it enables the analysis of structures especially in earthquakes, when many systems behave as a single degree of freedom $(S D O F)$. If the natural frequency of the structure can be found, then the peak response of the structure can be estimated by reading the value of the ground response spectrum for the appropriate frequency. In most structural codes in seismic regions, this value forms the basis for calculating the forces that a structure must be designed to resist, especially in seismic analysis.

Many multi-degree of freedom systems can be simplified to a single degree of freedom system for each natural frequency mode. A response spectrum is defined as a plot of the peak values of the response such are displacement, velocity, or acceleration of a number of SDOF systems with different natural vibration periods subjected to the same seismic input. Therefore, an acceleration response spectrum represents the peak accelerations that a suite of SDOF systems with a range of natural periods may exhibit when subject to a given ground motion component. This response spectrum will depend on the damping ratio and the ground motion selected. The dynamic response of any given structure relies on its mass and stiffness distributions. For example, stiff structures will experience low accelerations, by their nature. 
As can be seen in Figure V-1, Eurocode 8 addresses this by providing a series of response spectra, which are determined by soil condition, including soil stiffness. The equations to obtain these response spectra have been detailed in Chapter IV. Figure V-1 describes five different response spectra representing five different soil types (A, B, C, D, and E) for 4second periods.

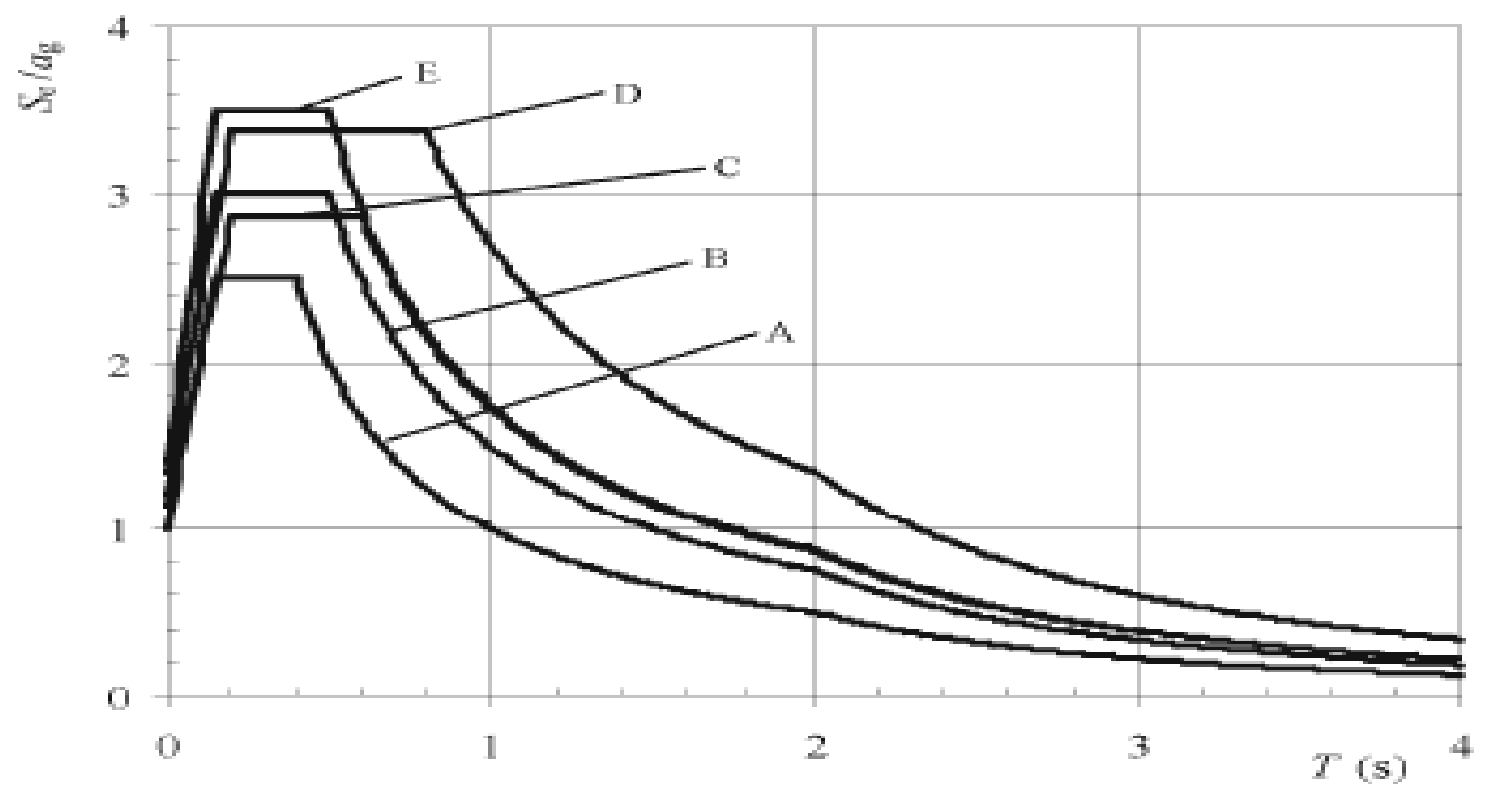

Figure V-1: Response spectra from Eurocode 8

In this study, as mentioned in Chapter 4, the Eurocode 8 spectra family is derived from the artificial time histories. A bank of accelerations was obtained and these were analysed using the Seismosoft program. Seismosoft then validated and reproduced the response spectra for five different kinds of soil accelerograms, as shown in Figure V-2 below. Each of the response spectra has three different time histories: the displacement time history, velocity time history and acceleration time history. Design response spectrum models used in contemporary engineering standards and codes of practice are typically of the flat hyperbolic form in the acceleration format and parameterized by spectral ordinates at natural points in the period range, which are in the acceleration and velocity controlled region, respectively, of a conventional response spectrum model.

Although there are strict criteria in ASCE 4-98 for modelling target and input spectra, the output spectra were sufficient to ensure that the time histories were acceptable for use in this study. Moreover, the aim of this study is to investigate qualitative behaviour rather than quantifying structural integrity. 


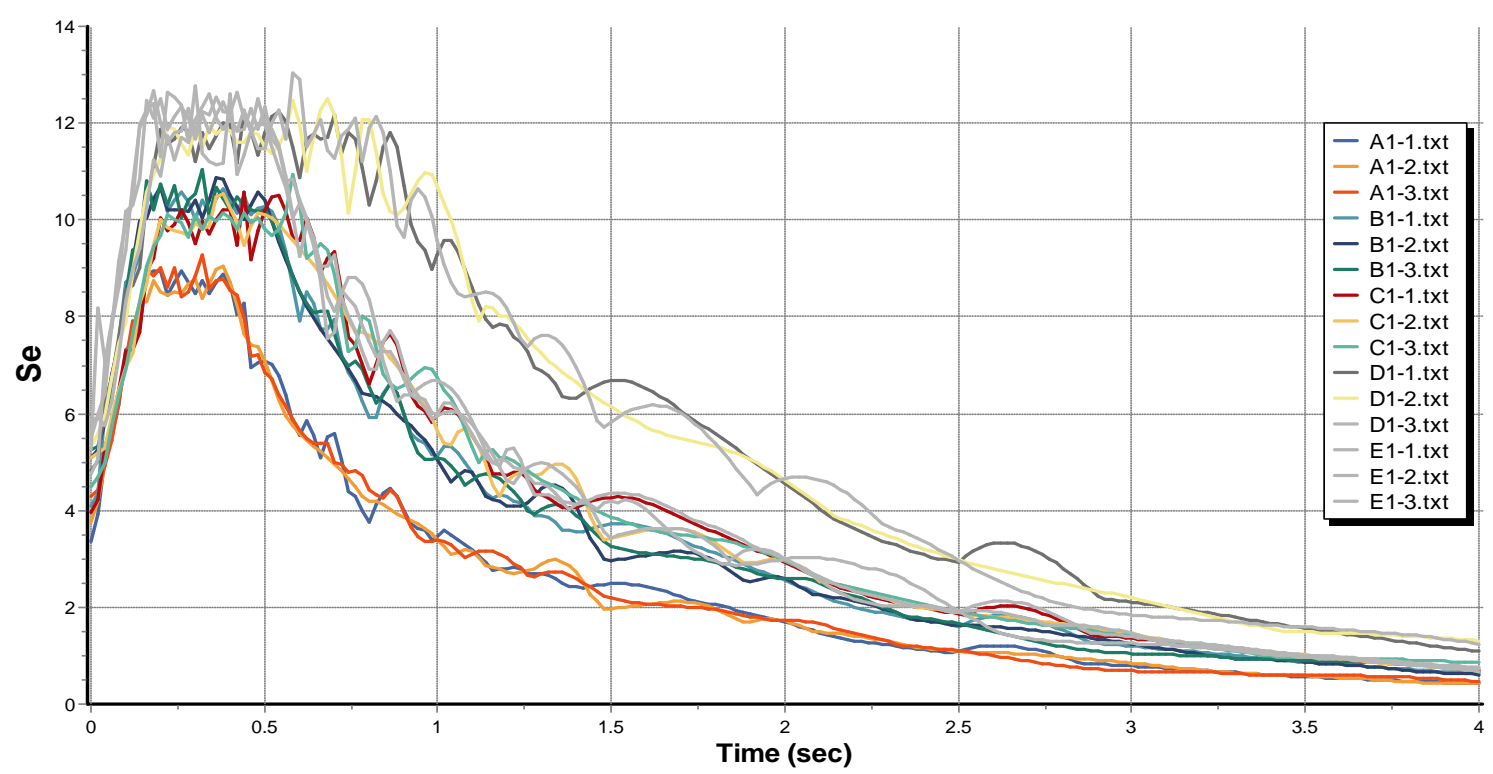

Figure V-2: Synthetic Acceleration Spectrum type 1 Earthquake According to Eurocode 8, a $=0.35 \mathrm{~g}$

Figure V-2 contains graphs of the acceleration response spectrum for five types of soil obtained from the artificial time histories. It shows the different response spectrum for each soil condition. From these graphs, it can be seen that soil A (the lowest lines, i.e. A11, A1-2 and A1-3) has the shortest peak range of period, while the longest range of period occurs in the response spectra for soil D. Graizer and Kalkan (2009) state that in addition to magnitude and distance dependence, the spectral shape depends on site conditions, which are the strengths of the soil. The predominant period of the spectral shape from a rocky site is generally lower than that of a soil site. By referring to Figures V-1 and V-2, the target response spectra for the study are a good match to the response spectra from Eurocode 8.

Acceleration time histories were then applied to the bridge structure in the time history (transient) analysis phase, as explained in Section 5.7. Acceleration time histories were applied to the structure in order to obtain the dynamic response of the bridge, with or without relative displacements. This was achieved by producing a number of independent time histories with the same spectral envelope, as can be seen in Figure V-3. 


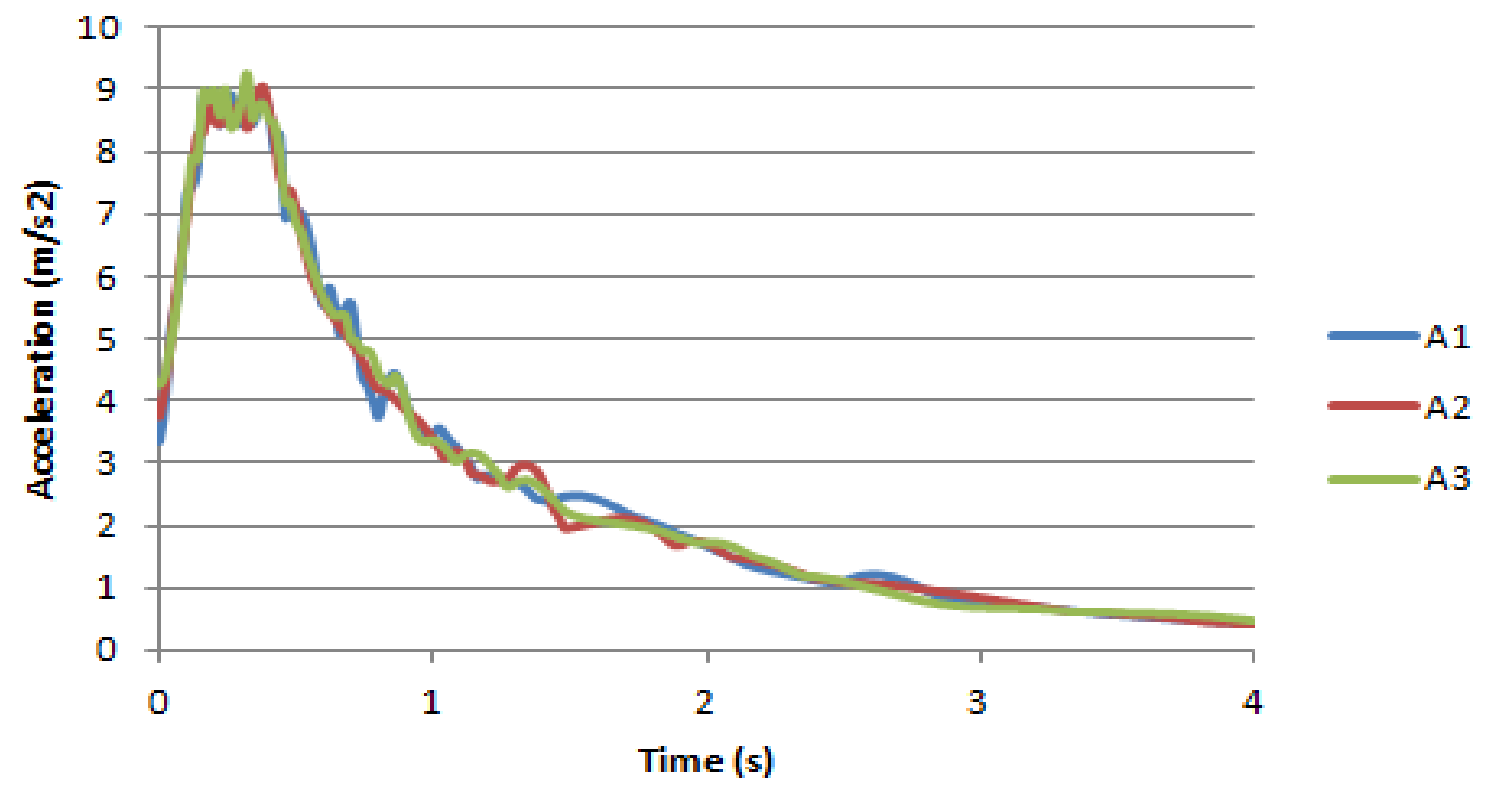

Figure V-3: Three different sets of data from the same specific ground motion

Figure V-3 shows three different response spectra, obtained from the same design response spectrum. It describes the variation of the maximum response acceleration for soil A with the natural period of a single degree of freedom system subject to a specific ground motion. Based on a certain seismic characteristic, different time histories can be created that possess the same response spectrum. For creating a relative displacement that will be used in the analysis, different time histories with the same spectral shape were generated.

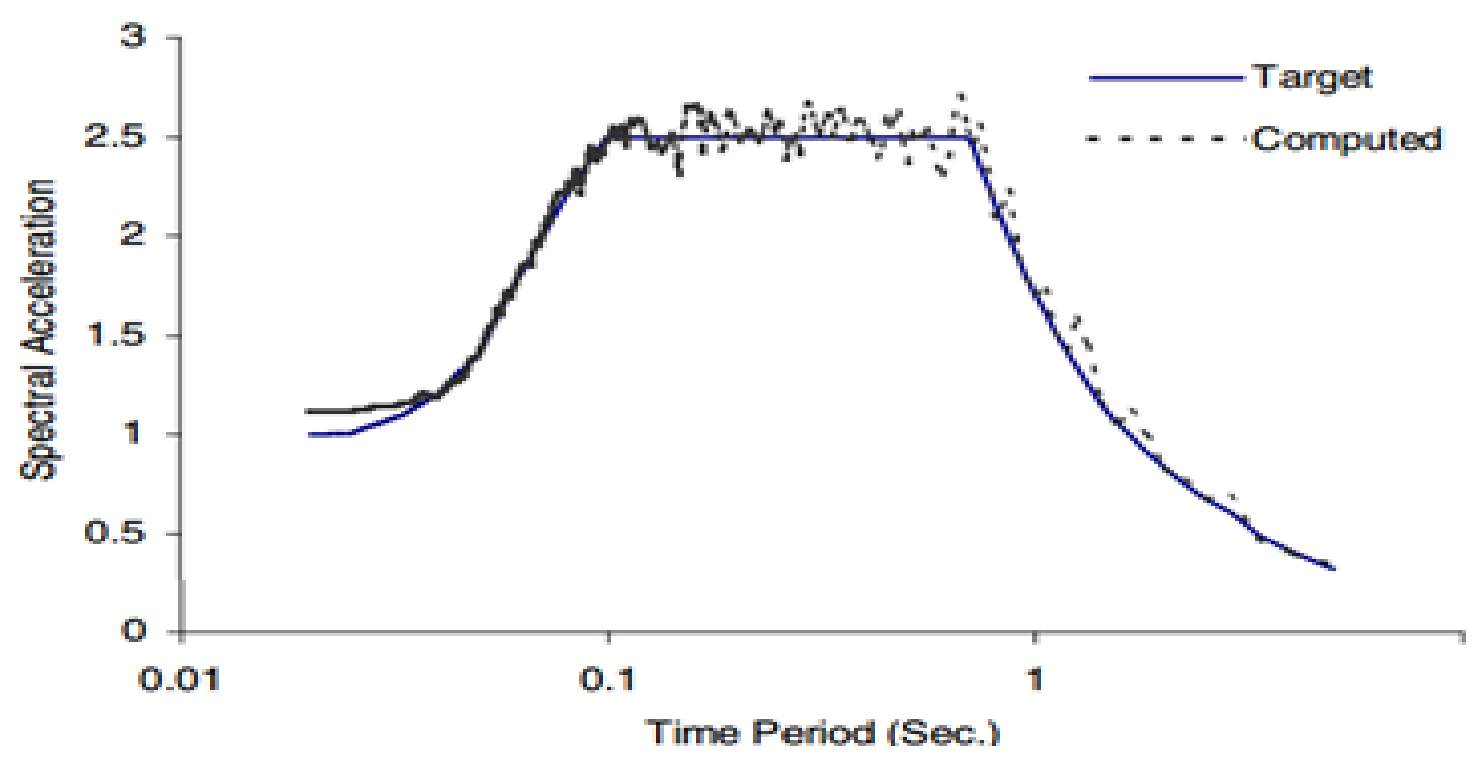

Figure V-4: Response spectrum: target and computed 
Although the data for these three response spectra are different, they have the same design response spectrum, or target response spectrum, as can be seen in Figure V-4. For the particular spectra there exist any number of compatible time histories. In this study, statistically independent time histories were produced, which are born from the same spectra.

These different response spectra, which refer to the same design response spectrum, then derived a series of different acceleration time histories, velocity time histories and displacement time histories. In other words, by creating different time histories from the same design response spectrum, the specific earthquake data input such as peak ground acceleration or soil characteristic would not change. This is called the design spectrum compatible time histories approach, explained in the next section. The response spectra of the acceleration for soil A are shown in Figure V-5.

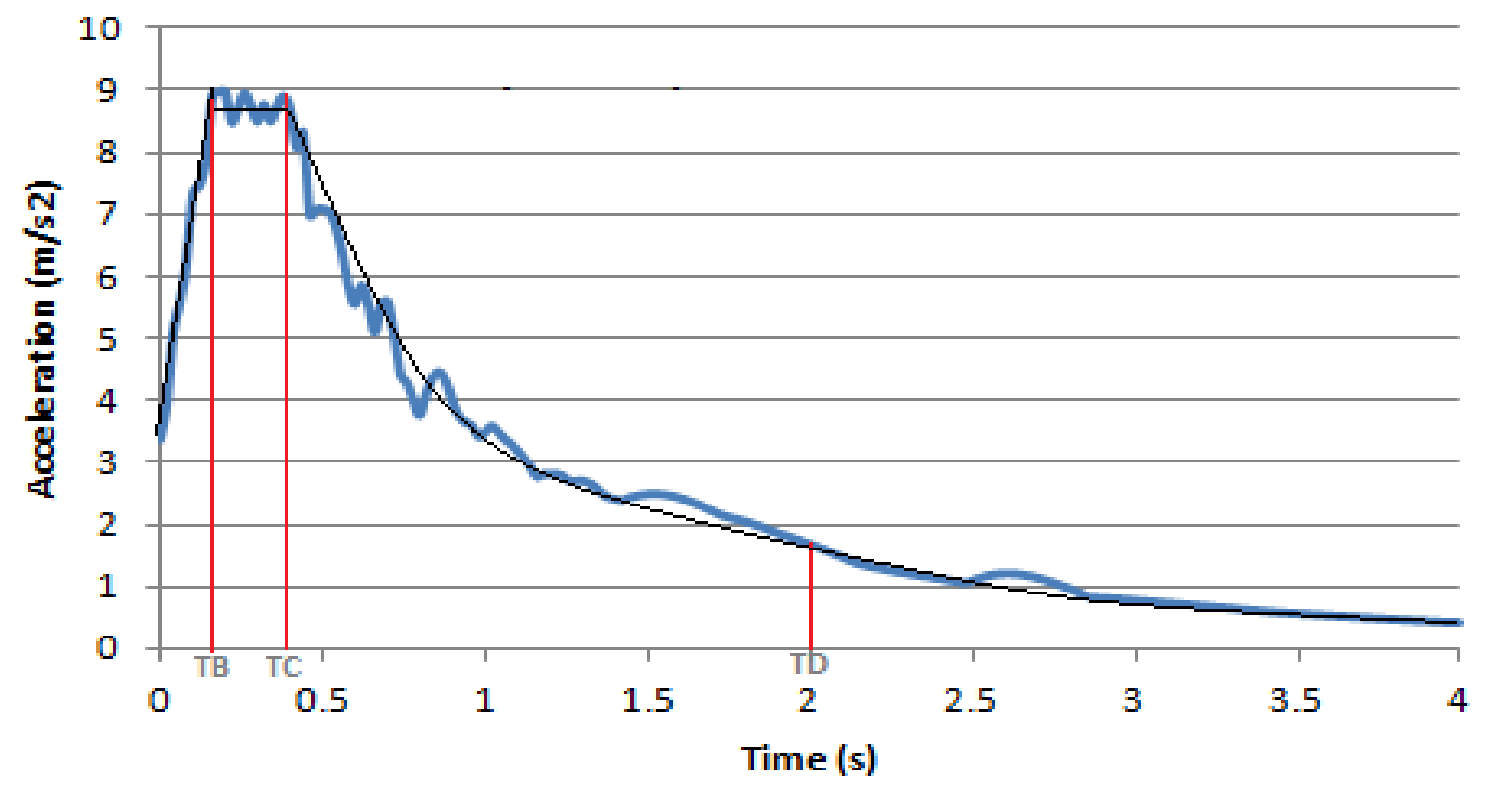

Figure V-5: Response spectra of soil A

From the graph in Figure V-5 it can be seen that the acceleration at $t=0$ is $3.4 \mathrm{~m} / \mathrm{s}^{2}$, as the magnitude of the earthquake is $0.35 \mathrm{~g}$. The peak of the acceleration lies between $T_{\mathrm{B}}$ and $T_{\mathrm{C}}$, namely between 0.15 and 0.4 seconds. By considering the natural frequency is $1 T^{-1}$, it can be calculated that the peak of the acceleration occurs with a frequency of $2.5 \mathrm{~Hz}$ to 6.666 Hz. The acceleration for these frequencies is about 8.3 to $8.7 \mathrm{~m} / \mathrm{s}^{2}$. These numbers are useful when analysing a structure relating to the resonance problems, as every structure has a series of natural frequencies of vibration. Since the response spectrum describes the 
acceleration of the ground motion associated to the period of the ground motion, this can be used to analyse whether a structure or a bridge will have a resonance by comparing the natural frequency of the structure or bridge to the frequency described in the response spectrum. For example, if the natural frequency of a bridge is $20 \mathrm{Hertz}$, then this corresponds to the natural period of the bridge, i.e. 1/20 Hertz, which equals 0.05 seconds. From Figure V-5 it can be predicted that the acceleration for that period occurs near the peak of the spectra where the maximum acceleration occurs. This is more onerous for this structure.

Response spectra are often idealized as divided into period ranges, or regions in which structural responses are best related to a particular ground motion parameter. Figure V-6 shows the sensitivity regions of the response spectra for acceleration, velocity and displacement. Sensitivity regions are useful in determining the maximum response spectrum for each category. The displacement sensitivity region is the longest period region (part (a) of Figure V-6), the velocity sensitivity region is the medium period region, shown in part (b), and the acceleration sensitive region is the shortest period region (part (c)). The effect of the damping on the response spectrum is greatest in the velocity region, and smallest in the acceleration sensitive and displacement sensitive regions. It is known that the spectral ordinates for all damping levels increase with the period from zero to some maximum value and then descend to converge at the value of the peak ground displacement at long periods (Bommer \& Elnashai, 1999).

Lumantarna et al. (2012) divides the response spectrum into three regions, based on the sensitivity of the acceleration, velocity and displacements, as seen in Figure V-6. Figure V-6 describes $T_{1}$ as the first corner period and $T_{2}$ as the second corner.

By synchronizing Figure V-5 and Figure V-6, it can be seen that the sensitivity region for acceleration is defined from $T_{\mathrm{B}}$ to $T_{\mathrm{C}}$. Meanwhile for velocity, the sensitive region is from $T_{C}$ to $T_{D}$. On the other hand, the sensitivity region for displacement is in the region after $T_{\mathrm{D}}$. Relating to these sensitivity regions, based on Eurocode 8, the sensitive areas for each item of earthquake property of the soil types in this study are not the same and the magnitudes of acceleration, velocity and displacement are also different. 


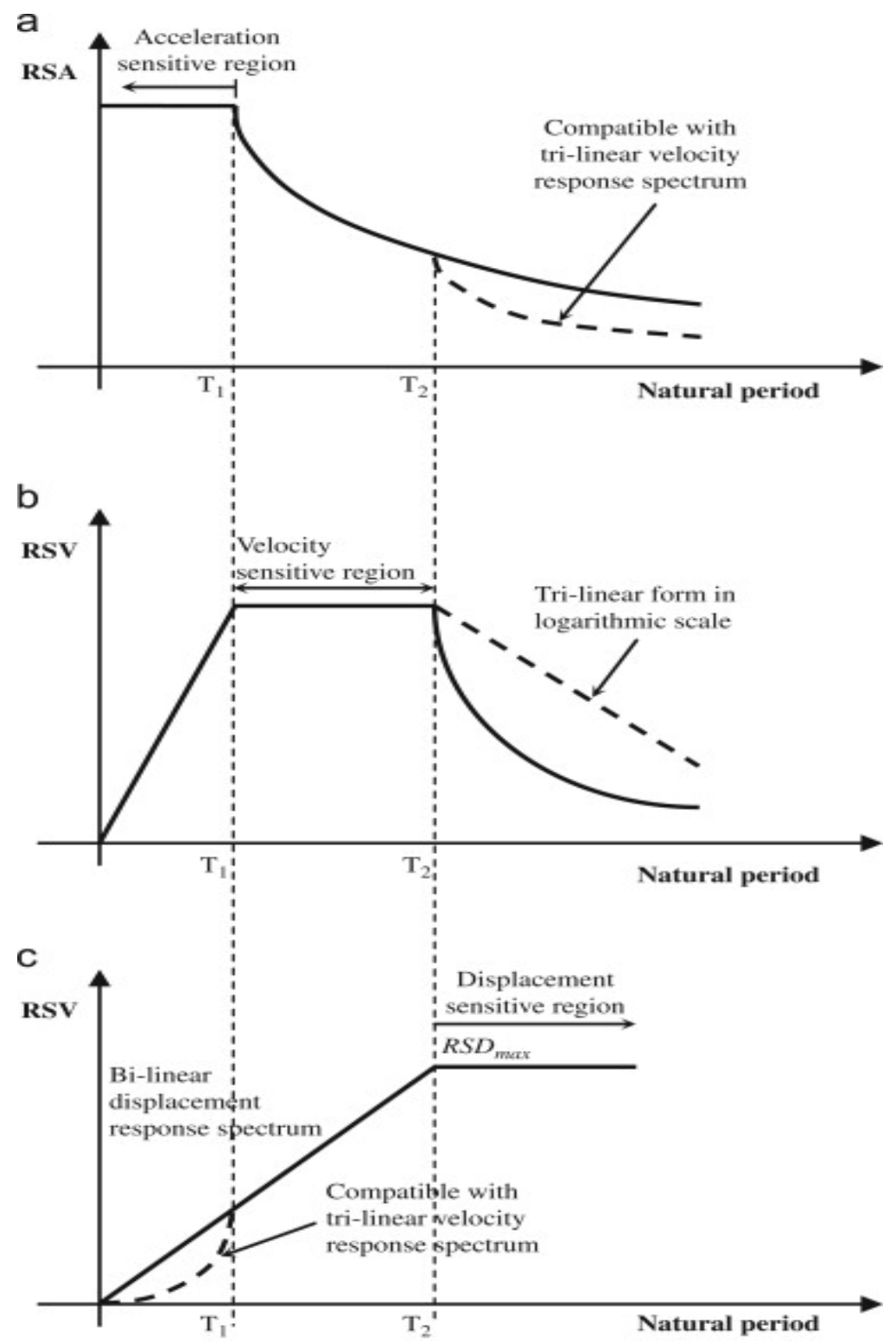

Figure V-6: Response spectra in different sensitive regions (Lumantarna, et al., 2012)

Examples of response spectrum acceleration, velocity and displacement sensitivity for this study are shown in Figure V-7 below. The rests are in the Appendix 4. The figure consists of three graphs, namely response spectrum acceleration ( $r s a)$, response spectrum velocity (rsv), and response spectrum displacement ( $r s d$ ) for soil type A. It can be concluded that for soil A, in this study, the sensitive region for acceleration occurs at the peak condition of the acceleration response spectrum, namely from 0.15 to 0.4 seconds. For displacement, on the other hand, the sensitive region will happen after 2 seconds.

The plots in Figure V-7 show that the sensitive region for acceleration spans from $0.0 \mathrm{~s}$ to $0.4 \mathrm{~s}$; for velocity the sensitive region started at $0.4 \mathrm{~s}$ and lasted to $2.0 \mathrm{~s}$. 


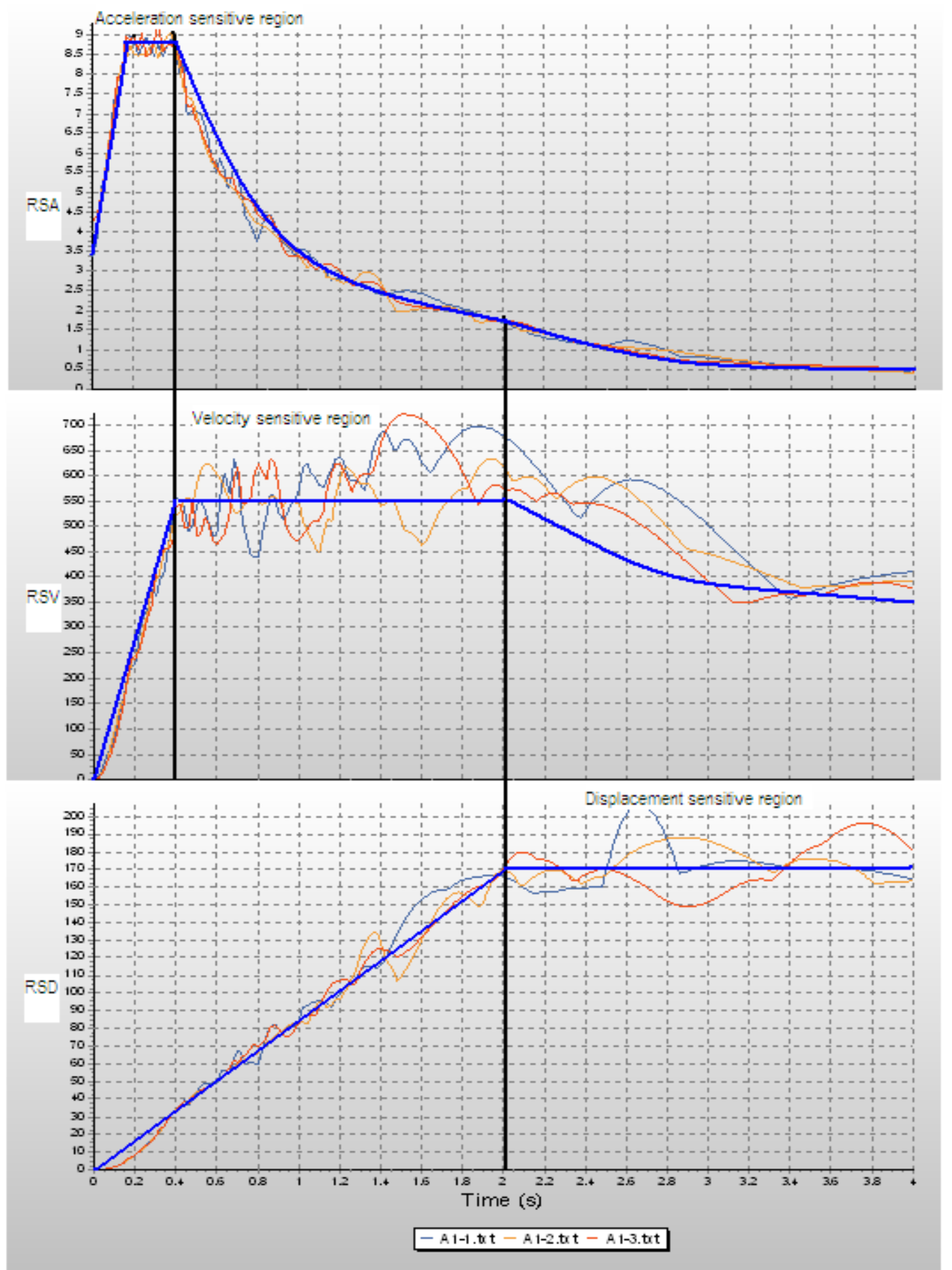

Figure V-7: Response spectrum for soil type A (from Seismosoft): (a) Acceleration, (b) Velocity, (c) Displacement

The most sensitive regions for displacement started at $2.0 \mathrm{~s}$. As previously mentioned, the sensitivity regions can predict the most affected region for damping effects, so it can be said that the area most affected by the damping effects is from time 0.4 to $2.0 \mathrm{~s}$. The rest of the period range is the least affected by the damping of the response spectrum. 
Figure V-8 describes the other classification of spectral response acceleration according to the period range. The purple area indicates the short period range representing constant spectral response acceleration and is known as region 1 . Region 2 is the area for the long period range representing constant spectral response velocity and depicted in green. Finally, the very long period range representing constant spectral response displacement is symbolized by the blue area, region 3 .

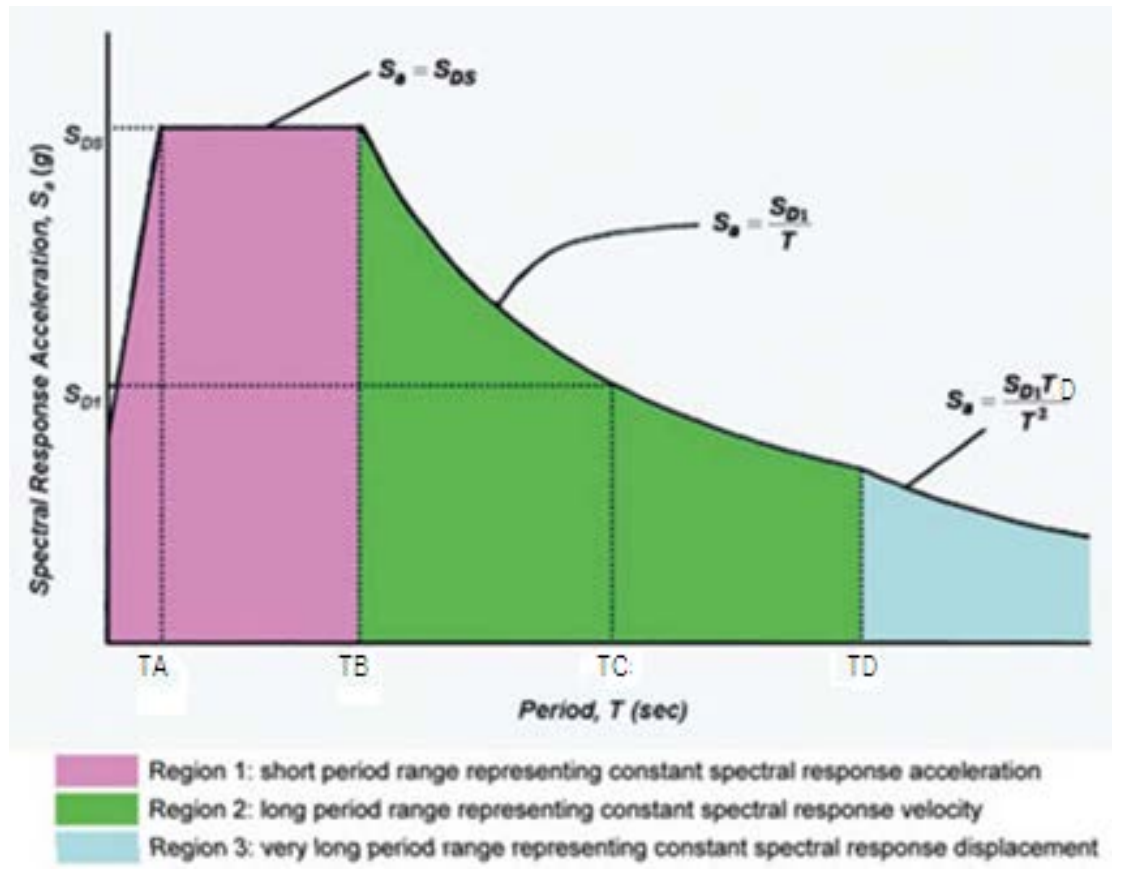

Figure V-8: Region classification of spectral response acceleration.

In fact, the true measure for structural analysis is a pseudo-spectral acceleration, which is equal to spectral displacement times the square of the natural frequency, but the difference is often negligible and the name is often shortened to simply spectral acceleration.

Spectral acceleration, $S a$, is the most commonly used intensity measure in practice today for the analysis of structures, including bridges. This value represents the maximum acceleration that a ground motion will cause in a linear oscillator with a specified natural period and the damping level of a structure. In other words, spectral acceleration is approximately what would be experienced by a structure, as modelled by a particle with a mass-less, single degree of freedom object, having the same natural period of vibration as the structure, whereas peak ground acceleration is what is experienced by a particle on the ground. Thus, spectral acceleration $(S a)$, with a value related to the natural frequency of 
vibration of the structure, is used in seismic engineering and gives a closer approximation to the motion of a structure in an earthquake than does the peak ground acceleration value.

\subsection{Synthetic Ground Motion Time Histories}

Dynamic responses of the structure, or dynamic internal forces, are a function of time for a specific ground motion. To determine the dynamic response of a structure, it is required to have the accelerograms of the design earthquake or to have several representative accelerograms of the earthquakes as the accelerogram provides an intuitive representation of ground shaking. An accelerogram is defined as a graphic record in chart form, produced by an accelerograph in response to seismic ground motion, or produced by a series of calculations for creating a synthetic earthquake ground motion.

It can be agreed then that besides response spectra, if a more detailed analysis is required, then ground motion time histories are needed for the design and analysis of the structure. Moreover, in an earthquake, actual damage to bridges and other infrastructure is more closely related to ground motion than to the magnitude of the earthquake since the magnitude represents the energy released at the epicentre. Therefore, for the analysis and design of earthquake-resistant structures, realistic measurement of ground motion is required. Unfortunately, in most cases reliable motion records at any given site are unavailable, and there are limited historical records. Even if such recordings are available, there is no reason to expect that a future earthquake might generate the same or similar ground motion. Thus, for earthquake-resistant time-history analysis of a structure, synthetic time histories must be generated for specific sites and cases.

Ground accelerations during strong-motion earthquakes are generally extremely irregular, resembling random time functions; however, these irregularities have some common features. The motion is highly oscillatory and periodic; it is initiated with small amplitudes that rapidly build up until they reach an intensity that remains almost stationary for a certain time; these then decay, steadily, until the end of the record. Acceleration is the rate of change of velocity and when it is multiplied by mass, it will result in the inertial force $(F=m a)$ which the structure must resist. From the acceleration, velocity and displacements can then be obtained by using integration methods with respect to time. Meanwhile, velocity is measured in inches or centimetres per second and refers to the rate of ground 
motion. Displacement is usually measured in inches or metres and refers to the distance an object is removed from its rest position.

To provide the necessary data for modelling artificial earthquake ground motion, various methods of creating artificial records have been developed. These should match the target design response spectrum for the project.

There are several difficulties associated with artificial ground motions, the most basic being that they are not real ground motions. Because of the way they are generated, their duration, which is important for structural response analysis, is not taken into account. In addition, the target spectrum used to generate the motions may represent an average or mean plus one standard deviation event. In other cases, the target spectra are also smoothed. Generating synthetic motions to match these targets will not capture the uncertainty in estimating the actual ground motion at a site (Bozorgni \& Campbell, 2011).

Generating ensembles of artificial non-stationary earthquake accelerograms compatible with a given (target) response/design spectrum has a stochastic basis. The design spectrum of Eurocode 8 seismic code provisions for various soil conditions and damping ratios is used as a paradigm of a design/target spectrum (Fardis et al., 2005).

Field observations on ground motions from recent earthquakes imply that current knowledge is limited with regard to relating vertical and horizontal motions at liquefiable sites. The transformation of vertical motions in the deposit differs considerably from the transformation of horizontal motions. Both the amplitude and frequency content of the horizontal motions are strongly dependent on the shaking level or the associated soil behaviour (Yang \& Sato, 2002). Therefore, more research is required regarding earthquake ground motion in order to gain more knowledge of the effects of earthquakes on structures.

The effects of earthquake ground motions on structures have also been studied by Silva (1997). He concluded that the variations in vibrational properties of a structure influence the response analysis of the structure. Three of these considerations are:

1. The amplitude and spectral content of earthquake motion vary with the magnitude and distance of the earthquake. However, the relative amplitude of vertical motion is higher compared to horizontal motion for sites in an epicentral region. 
2. When analysing the response to vertical motion, variation of the amplitude and frequency content is to be considered.

3. For vertical members, axial force commonly becomes the cause of vertical vibration while for horizontal response it is from shear and bending. Axial stiffness and strength is beyond that of the flexure/shear, so response in the vertical direction would be expected to be rigid, whilst in flexure the response may be larger due to resonance.

The mass of a structure, along with its size and shape, and its configuration, partially determine the forces generated by earthquake motion and also partially determine how well these forces can be resisted. A more significant measure is that of the acceleration combined with duration. This is not hard to visualize intuitively and it is important to understand that a number of cycles of moderate acceleration may be much more difficult to withstand than a single peak of a much higher value. On the other hand, frequency is another major parameter of ground motion for design purposes as this governs the magnitude of acceleration and, hence, the forces to which a structure is subjected.

To generate synthetic ground motions simple Fourier analysis methods such as those found in Dynamassist Mathcad software can be used. One way of doing this is to take a real ground motion and decompose it into its Fourier amplitude and phase angle spectra. By randomizing the phase angle spectrum, re-convolution produces a descendant with similar frequency content but differing temporal characteristics. Similar methods can be used to generate a ground motion that will have a particular response spectrum. This is often done when creating motions to fit a target spectrum.

Earthquake properties in this research are defined as the characteristics of the synthetic earthquake related to the acceleration, velocity and displacements of the ground motion. In this study, these ground motion characteristics are considered for each type of soil as outlined in Eurocode 8, namely A, B, C, D and E. In order to correct non-physical shifts in velocity and displacement, the generated time histories were corrected using baseline correction in the Seismosoft software. Seismosoft constitutes a simple and efficient platform that allows the creation of a personal library of ground motion records and saves them all in a single file, making it easy to handle and share large numbers of records. Seismosoft also provides baseline corrections to the data so that any disturbance 
of the data can be avoided. It is important to apply baseline correction before using displacement earthquake data. This is done by adding polynomials to the generated time history in an attempt to ensure that the structure end point (in terms of displacement) is not inordinately disparate from its starting location, whilst maintaining the characteristics of the time history.

Thus, for horizontal and vertical earthquake ground motions, a series of synthetic accelerations were applied to the bridge substructures. For each of the soil types outlined in Eurocode 8, synthetic accelerograms were generated, to be used as input to the finite element model. At the end of the study, the response of the structure under various conditions of the horizontal earthquake ground motions, applied to the substructure, will be discussed.

Additional conditions were introduced to improve the time histories by taking into account the recent recommendations of Eurocode 8. For generating the synthetic earthquake ground motion, based on Eurocode 8, it was decided to choose an earthquake type 1 with a peak ground acceleration of $0.35 \mathrm{~g}$.

There are three results of generating synthetic time histories: acceleration, velocity and displacement time histories. However, only acceleration earthquake ground motion is required as an input to the transient analysis. Displacement time histories are required for the analysis of relative displacements created from the specific earthquake input.

Structural idealization of the horizontal and vertical earthquake ground motion can be seen in Figures V-9 and V-10.

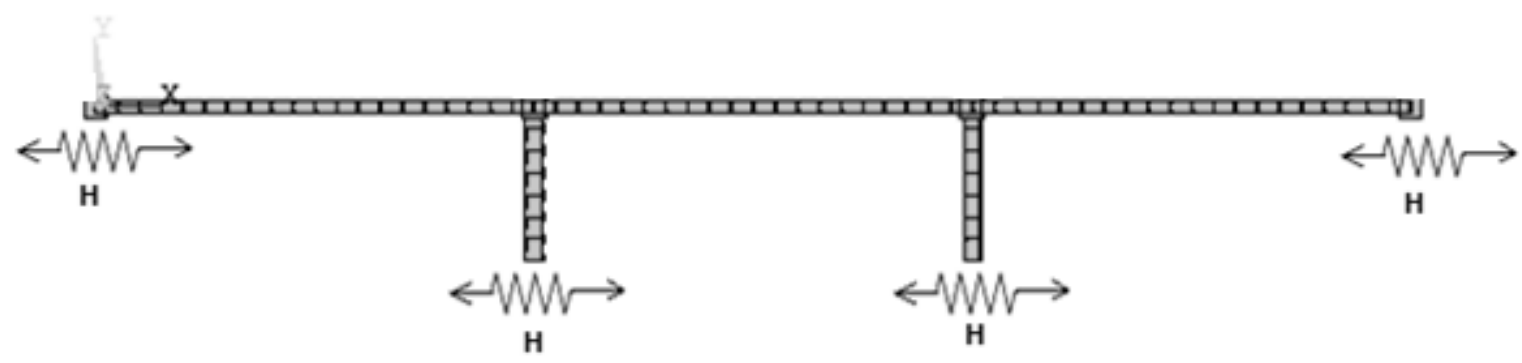

Figure V-9: Horizontal earthquake ground motion applied to the bridge structure 


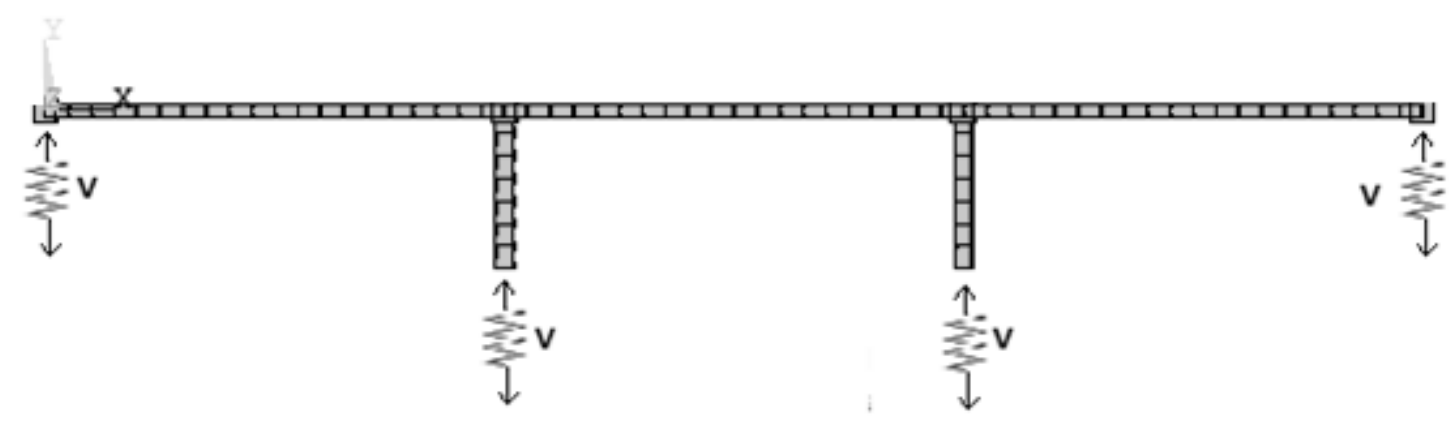

Figure V-10: Vertical earthquake ground motion applied to the bridge structure

For assessing the effects of vertical earthquake ground motion in this research, the earthquake force is logically applied to the structure in a vertical direction. As with horizontal earthquake ground motion, the vertical earthquake ground motion is analyzed by using the artificial earthquake ground motion obtained from Clough and Penzien's (2003) procedures. The horizontal and vertical earthquake ground motions are applied as shown in Figures V-11(a) and (b) respectively.

Figure V-11 describes the synthetic acceleration that was applied to the bridge's supports. This artificial acceleration is for soil type A, while the other four soil types are illustrated in Appendix VI of this thesis.

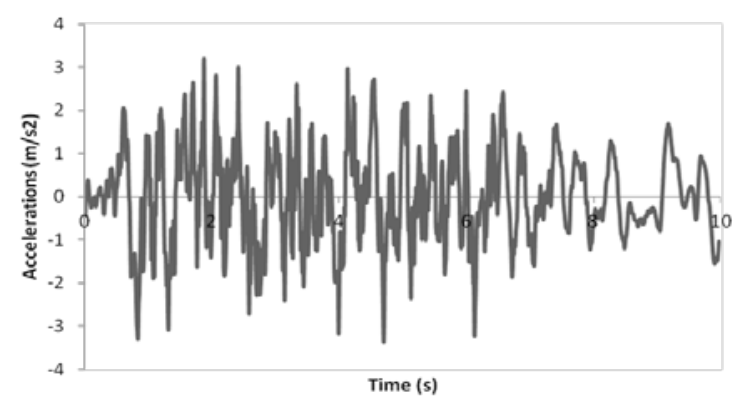

(a) Horizontal Synthetic Acceleration

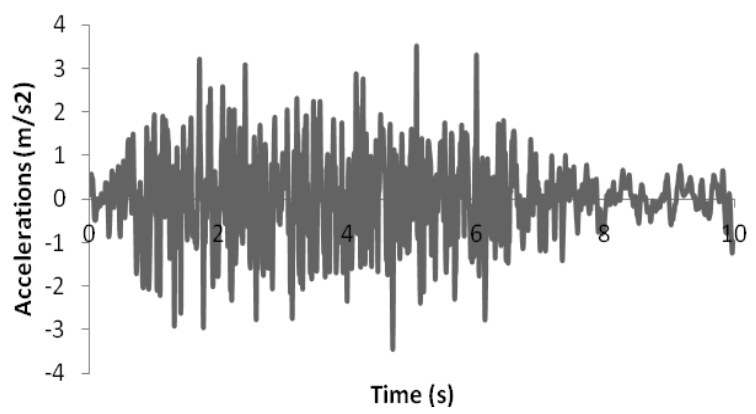

(b) Vertical Synthetic Acceleration

Figure V-11: Synthetic Acceleration Earthquake Ground Motion

The data series of synthetic earthquake acceleration were applied to the supports of the bridge vertically and horizontally. It can be seen from Figure V-11 that the frequency of the horizontal acceleration is smaller than that of the vertical acceleration. This is as expected, because Elnashai (2001) noted that the wavelength of vertical earthquake waves is shorter than that of horizontal earthquake waves, which means that the former are associated with higher frequencies. 
The acceleration time series of soil A for horizontal components is described in Figure V11 above. The maximum amplitudes of the acceleration range between $-3.3718 \mathrm{~m} / \mathrm{s}^{2}$ and $+3.2043 \mathrm{~m} / \mathrm{s}^{2}$. This data was applied to the substructures of the integral bridge in order to obtain the structural response of the bridge. ANSYS ver. 12 software was used in a full integration time-history transient analysis, which then provided results for the structural response of the bridge.

It is noted from the current literature that recent studies indicate that the average amplitude of peak ground acceleration at soil sites is about 1.4 times greater than that at rock sites ( $\mathrm{Si}$ \& Midorikawa, 2000). However, the main focus of this research is not on soil analysis but on the structural response of the integral bridge.

Figure V-12 shows the first artificial acceleration time history, namely the artificial velocity time history. As previously mentioned, the velocity of the ground motion comes from $0.35 \mathrm{~g}$ earthquake ground motion. Therefore, as in the acceleration analysis, the frequency of the vertical earthquake velocity is also higher than the frequency of the horizontal earthquake velocity, and for the same reason.

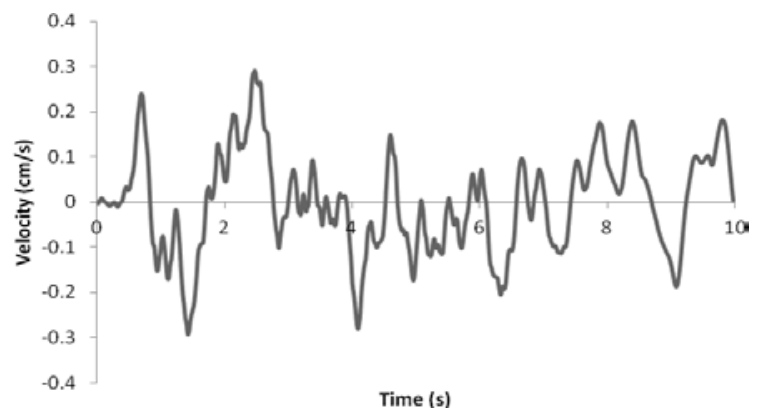

(a) Horizontal Synthetic Velocity

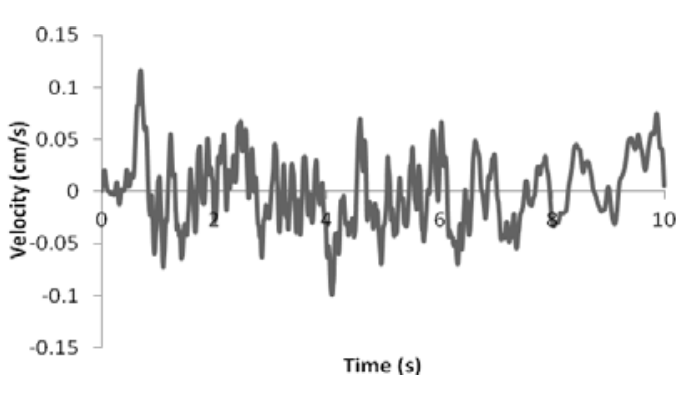

(b) Vertical Synthetic Velocity

Figure V-12: Synthetic velocity earthquake ground motion

Peak ground velocity has received much less attention in the technical literature than more widely used parameters such as peak ground acceleration (pga) and response spectral ordinates (Bommer \& Alarcon, 2006). These authors also stated that although peak ground velocity is not considered as important a parameter as peak ground acceleration, there are many examples of the use of peak ground velocity in earthquake engineering, including a 
parameter from which to estimate structural damage. They also note that one of the most important uses of peak ground velocity is in the construction of elastic response spectra.

The graphs in Figure V-13 compare the results of horizontal and vertical earthquake ground motion for soil A. The horizontal displacement time history comes from the double integration of synthetic acceleration time history after applying baseline correction. Compared to Figure V-12, the content of displacement time history is less dense than the velocity and acceleration time series.

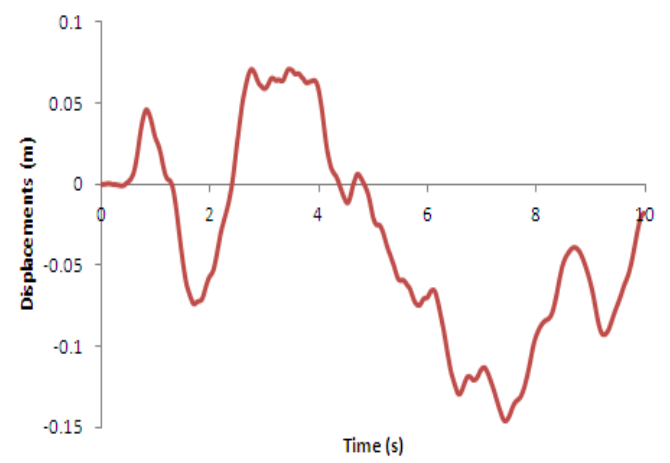

(a) Horizontal displacement time history

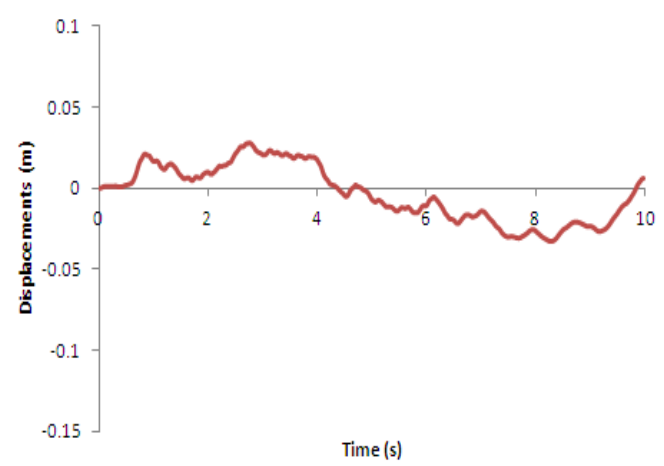

(b) Vertical displacement time history

Figure V-13: Displacement time-series

Figure V-13 generally explains the displacement of the ground due to the $0.35 \mathrm{~g}$ acceleration peak ground motion of the earthquake for a period of ten seconds. It can be seen that the displacement of the ground varies from zero to positive and negative amplitudes. The maximum ground displacement for the horizontal direction is $0.1458 \mathrm{~m}$ in the negative direction and occurs at $7.42 \mathrm{~s}$, while the maximum positive value is about $0.0709 \mathrm{~m}$ at $2.26 \mathrm{~s}$. Meanwhile, for the vertical displacement, the plot in Figure V-13 shows that the maximum value is $0.0328 \mathrm{~m}$ in the negative direction (downwards) and $0.0278 \mathrm{~m}$ in the positive direction (upwards). The vertical displacement ground motion increases until almost 4 seconds, declines until it reaches 8 seconds, and then increases again until the end of the time range. 
The displacement time series for all five soil types is shown Figure V-14.

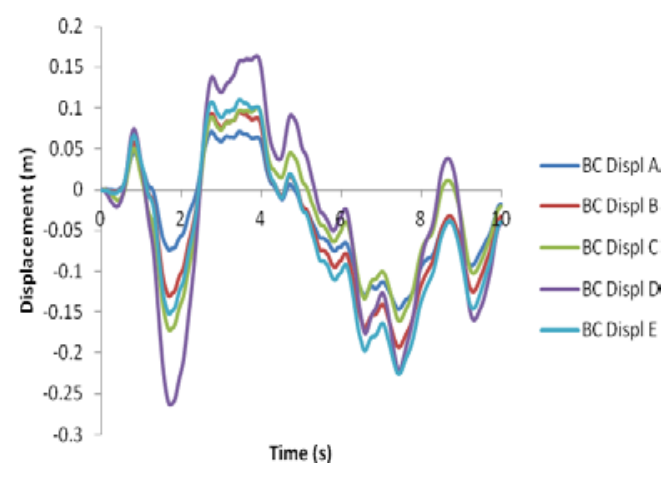

Figure V-14: Horizontal displacement time-series’s comparison of 5 soil types.

The patterns of the peak ground displacements are similar, due to way in which Mathcad inputs data. However, Figure V-14 shows that there is a noticeable attenuation in the displacement pattern of soil type D. It can also be seen that soil A has the smallest amount of peak ground displacement, whereas soil D has the largest displacement. The reason is that soil A is the stiffest soil, and soil D the weakest. Thus, it can be said that the stiffer the soil is, the smaller the peak ground displacements.

\subsection{Baseline Correction for Earthquake Ground Motion}

Synthetic earthquake ground motion for dynamic analysis can be used in two ways. The first method is by applying the acceleration time history to the bridge, and the second is by applying the displacement time history instead of the acceleration time series.

When deriving a displacement time history from an accelerogram, the integration procedures tend to cause a drift in the data set such that the beginning and end displacement may be unduly disparate. Hence, a structure may end up at some unrealistically large distance away from where it started. This issue can be addressed using baseline correction. The velocities and displacements were obtained by performing integration on the accelerations. Boore (2001) stated that the displacements reach residual values which are essentially constant, and the velocities oscillate around zero after the end of the shaking. This behaviour of the velocities is a reasonable constraint on any groundmotion data unless the recording is from the presumably rare earthquake that has a large amount of after-slip immediately following the strong shaking. 
The baseline correction is a procedure that flattens the baseline. It is employed to correct for drift in a data set. This drift is usually attributed to inaccuracies in the scanning of a waveform or problems with calculation. For the analysis, it is usually best to work with a ground velocity time history that oscillates about zero. This is a spectral manipulation technique used to correct spectra with sloped or varying baselines. It is used to ensure that the starting points of the time histories are not too different from the ending points of the spectra. For this study, baseline correction was applied to the data spectrum using Seismosoft.

The baseline correction of short vibration records consists of finding a function that approximates the long period offset from the line axis (baseline error) of a vibration record. The error could be caused by digitization of the analogue record or other reasons. In uncorrected, the baseline error may cause erroneous values of ground velocity and displacement when they are integrated in time from the acceleration record. The best fit of the record average values (baseline) is then subtracted from the record considered to produce a desirable adjustment. Different criteria may be used to define the best fit. Sometimes, the least square approach is used, for example for the minimization of the sum of squared differences between the uncorrected and corrected records. In other cases, it may be required to achieve zero end velocity. The baseline correction can provide reasonable results for records of short duration. Depending on the shape of the recorded average values, different functions can be considered, from linear to polynomials of a higher order and other function (Srbulov, 2011).

The graphs in Figure V-15 explain the differences between baseline correction data and non-baseline correction data. 


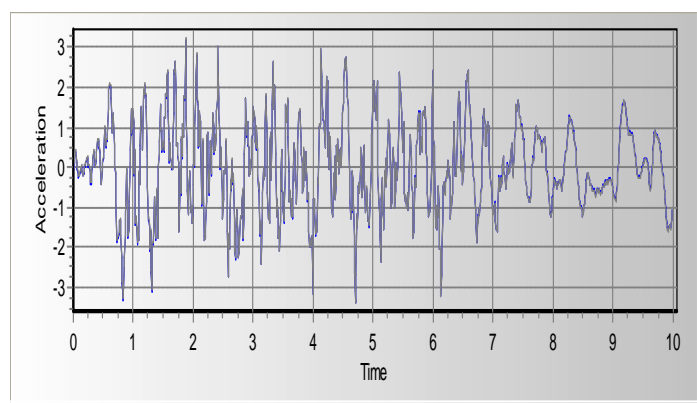

(a) Horizontal Acceleration

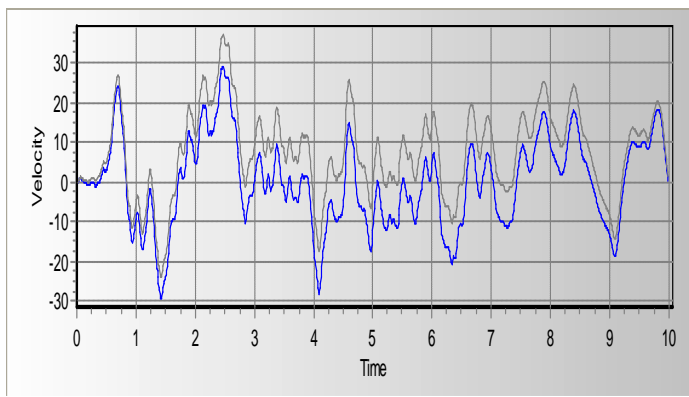

(c) Horizontal Velocity

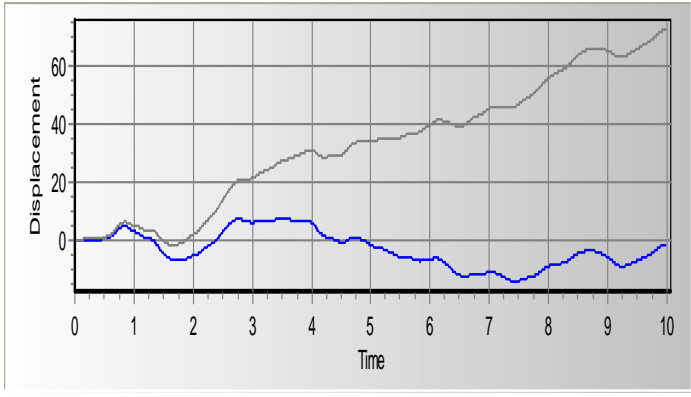

(e) Horizontal Displacement

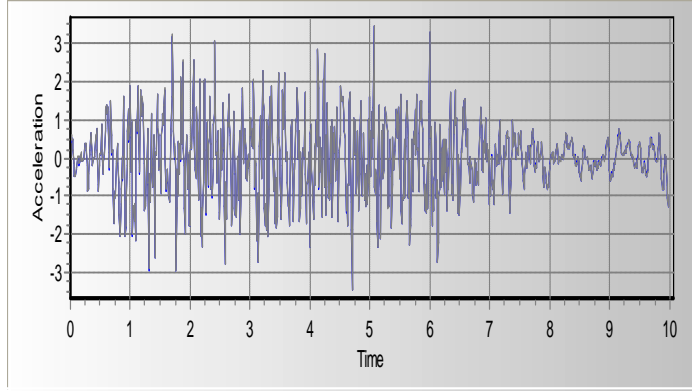

(b) Vertical Acceleration

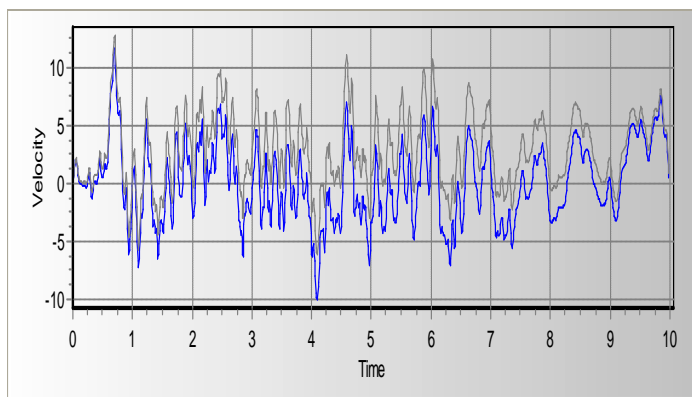

(d) Vertical Velocity

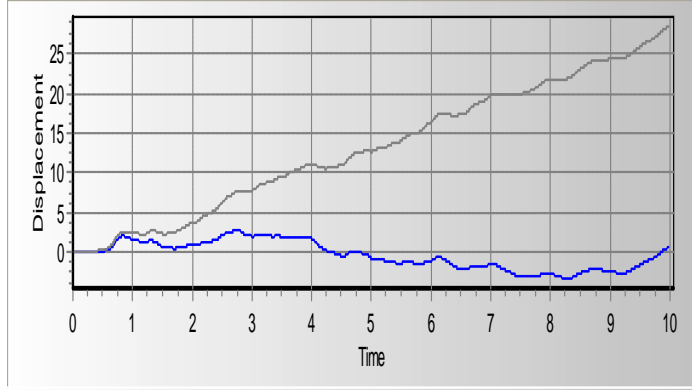

(f) Vertical Displacement

Figure V-15: Data comparison before and after applying baseline correction for horizontal and vertical ground motion

The grey lines are the data without applying baseline correction, and the blue lines are the data after applying baseline correction. There is clearly a significant difference between the grey and blue lines for the displacement time history (bottom set), a small difference for velocity time history (middle) and negligible differences between the grey and blue lines for acceleration (top).

Figure V-16 shows more clearly the importance of applying baseline correction for time histories, especially for displacements. The blue line is the displacement time history without baseline correction, and the purple line is the displacement time history after applying baseline correction. It is clear that without applying baseline correction, the 
displacement data is illogical, as the graph would indicate a constant shift in displacement which is not physically possible.

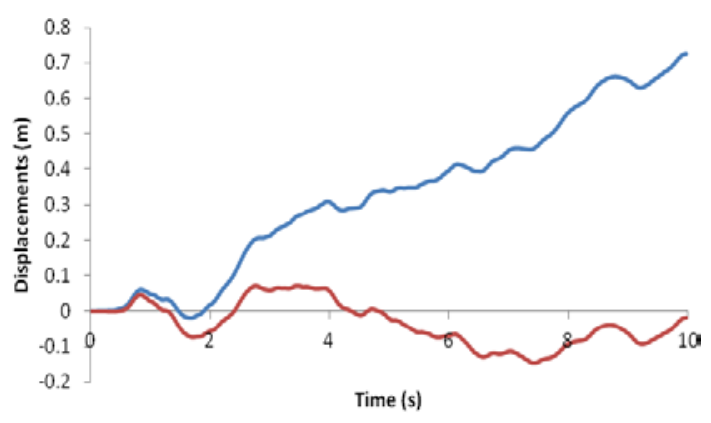

(a) Horizontal displacement series

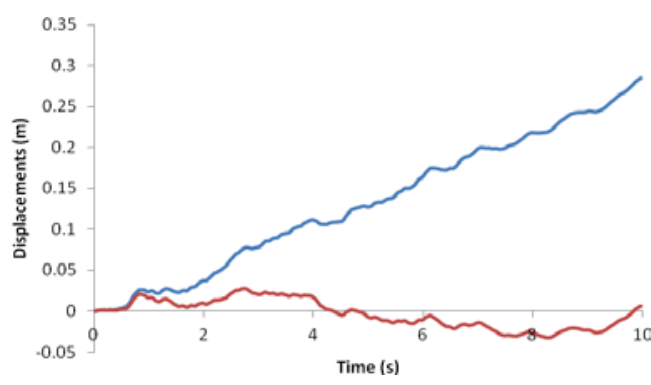

(b) Vertical displacement series

Figure V-16: Displacement time history before and after applying baseline correction.

\subsection{Generating Time Histories Library}

As mentioned in Chapter I, the aim of this research is to study the behaviour of the integral bridge under vertical and horizontal ground motion. More specifically, this study is conducted to see the effects of vertical and horizontal earthquake ground motion regarding the relative displacement occurring in the supports of the bridge. For this purpose, dynamic relative displacements need to be explored.

The method for creating relative displacement for the bridge under dynamic load is applies two different dynamic forces to the supports of the structure. In other words, different earthquake accelerations or displacements should be applied to the different supports of the bridge. If one support has a certain earthquake excitation property that is different from the other supports', relative displacement may occur because the displacements between the supports are different.

Applying multi-support excitations to the supports of the bridge has been widely applied, although usually only for designing long bridges, since the excitation that is imparted to the supports of very long bridges must be different as the supports are far apart.

For this research, different earthquake excitations were applied in order to see the effect of these relative displacements.

Thus, a bank of different earthquake excitations was created for use in transient analysis, to determine the effects of relative displacements as compared to dynamic response, which 
is the main focus of this study. Separate horizontal and vertical earthquake ground motion observations were made.

In some provisions, such as $U B C$, vertical earthquakes $(E v)$ are defined as the load effects resulting from the vertical component of the ground motion. For strength design, vertical earthquake ground motion has the effect of increasing compression and tension or uplift effects on vertical load-carrying systems. Commonly, and similarly to horizontal earthquakes, the vertical earthquake comes to the bridge through the substructure and transfers the energy to the rest of the bridge. Recent studies clearly show the importance of including the vertical component of earthquake ground motion in seismic analysis and design. In addition, pioneering studies, such as Elnashai and Papazoglou's (1997), have explored and documented the characteristics of available near-field vertical ground motion records.

Figure V-17 illustrates three synthetic earthquake ground motions for different excitations (acceleration, velocity and displacement), generated from the same specific earthquake (0.35 g) and specific soil (type A), and possessing the same response spectra.
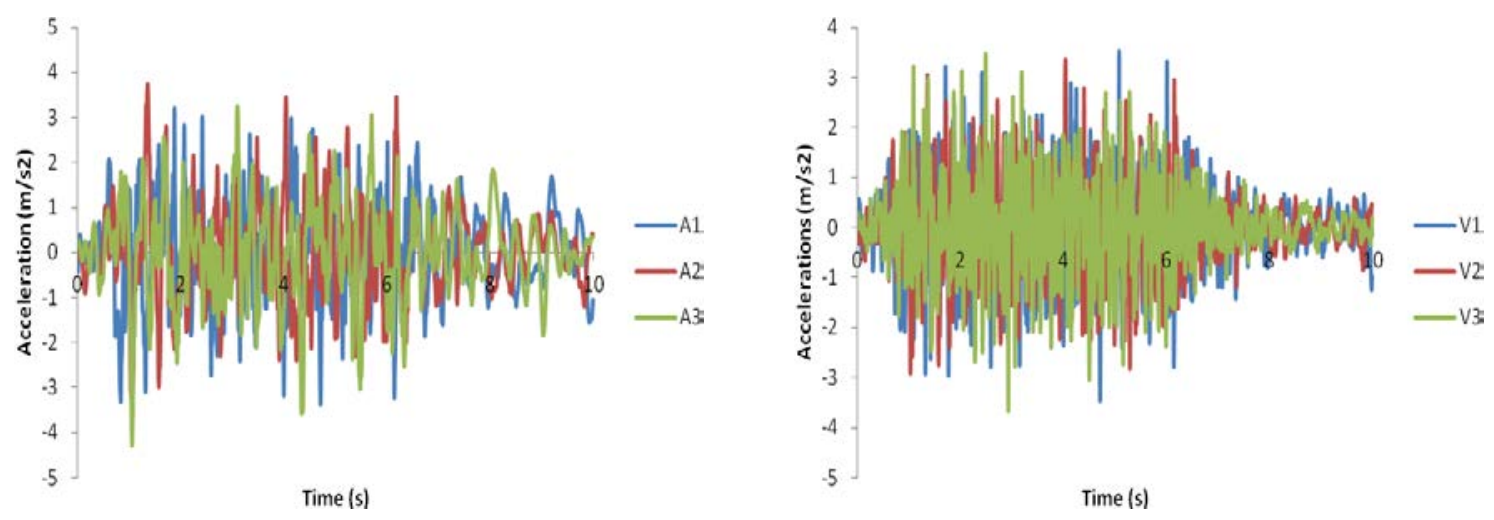

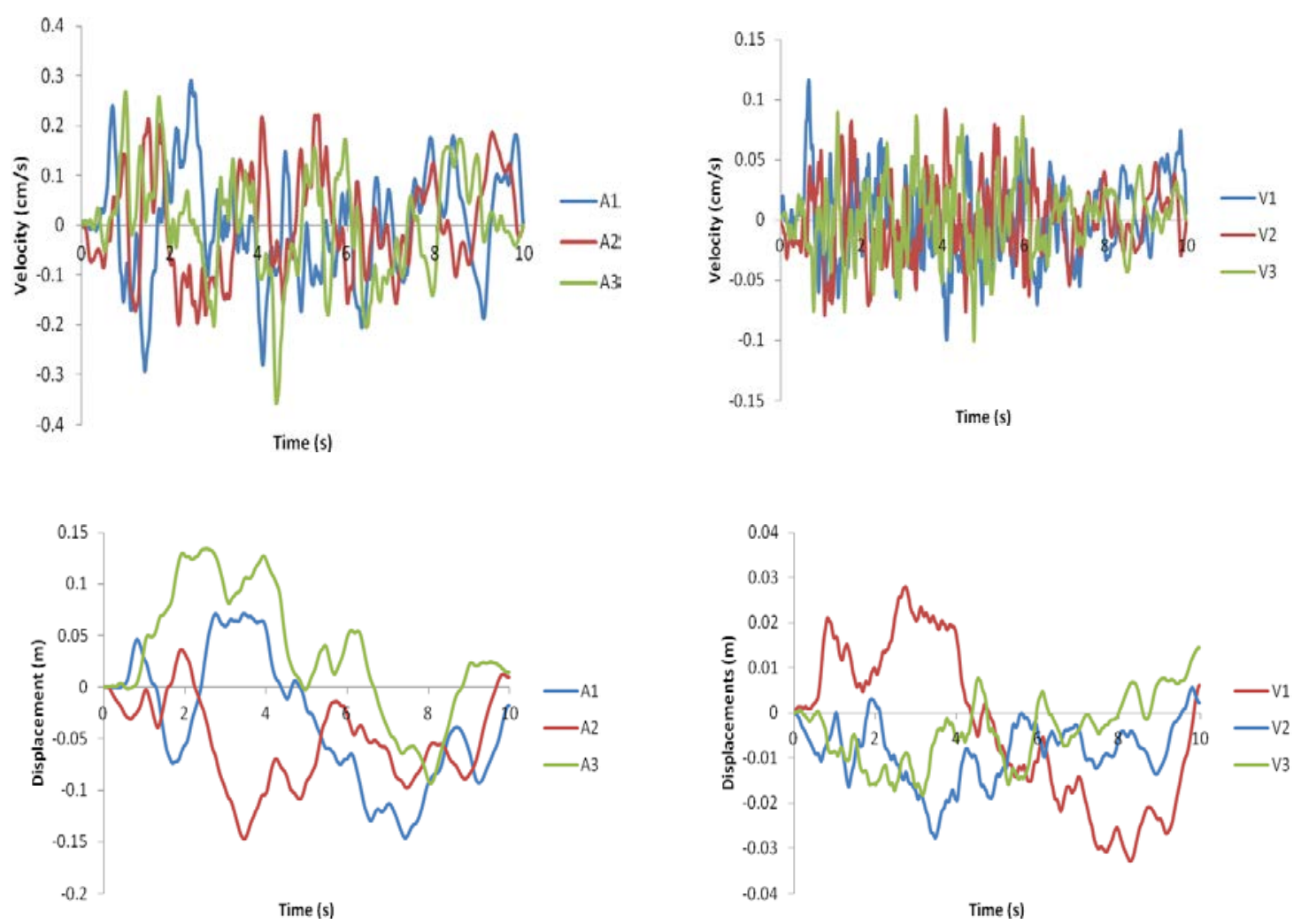

Figure V-17: Three different time histories resulting from the same specific earthquake for acceleration, velocity and displacement.

It can be seen that although the magnitude of the earthquake and the source of soil were the same, the resulting time series are different. This is due to the random data input process of the Mathcad or Seismosoft software. It is in contradiction to Figure V-14, which shows similar patterns for the displacement time histories.

Of the three different time histories, acceleration time histories are used as a series of input data to be applied to the transient analysis. For the horizontal direction, 15 synthetic earthquake accelerations for five different soil types were produced in this research. This means that for each soil type three different earthquake excitations are generated. Additionally, three synthetic earthquake acceleration time histories for the vertical direction and for each soil type were produced. Of these, two different acceleration time histories were chosen for creating relative displacements to the support of the integral bridge, as the structure is in $2 D$. The remaining synthetic earthquake ground motions generated can be seen in Appendix VIII of this thesis. 


\subsection{Modal Analysis}

Modal analysis is another important part of this research. It is usually conducted before applying the other steps of dynamic analysis.

Modal analysis was conducted using the ANSYS ver.12 software. It can be done by the simple lumped mass and lumped spring approach, or it can be conceptualized as a mass/spring system. This modelling process simply reduces the complicated structure into many mass or spring systems. The analytical problem then solves an eigenvalue problem to get the frequency, mass and mode shape of each mode for the assumed mass and stiffness distribution. Earthquake analysis of bridges requires that the period and damping ratio be determined for each significant mode of vibration (Goel, 1997).

Frequency is determined by two factors, namely the amount of mass and the stiffness of the structure, in this case a spring. The frequency of the fixed base structure's stiffness is larger than that of the spring base structure, because the frequency has a direct correlation to the stiffness: the stiffer the structure, the greater its frequency. This is formulated as shown in Equation V-1.

$$
f_{\mathrm{n}}=\frac{1}{2 \pi} \sqrt{\frac{k}{m}},
$$

Equation $V-1$

where $f_{n}$ is the natural frequency of the system, $k$ is the stiffness, and $m$ is the mass of the system (Clough \& Penzien, 2003).

Frequencies in the structure have a large correlation to resonance. Each structure has its own natural frequency, at which the structure will vibrate without any force applied to it. That is, if a structure is set to vibrate on its support and left free, it will vibrate at its natural frequency. If the frequency of forced vibration happens to be the natural frequency, then resonance will be set up. The frequency implies the resonance of the structure is the natural frequency. Resonance is the tendency of a system to oscillate with great amplitude at some specific frequencies but less at others. This is dangerous for a structure such as bridge, therefore resonance should be avoided by structural engineers.

Modal analysis will give the eigen or characteristic frequencies of the structure. After obtaining the eigen values, the next step is to run the dynamic analysis where the synthetic ground acceleration is applied in the horizontal $(x)$ and vertical $(y)$ directions. The effective 
mass in the $y$ or $x$ direction must be higher than $90 \%$ of the total mass, and most codes use this as a requirement for dynamic analysis, as mentioned in ASCE-4-98.

For the most basic problem involving a linear elastic material which obeys Hooke's Law, the matrix equations take the form of a dynamic spring mass system. The generalized equation of motion is given as:

$$
[M][\ddot{U}]+[C][\dot{U}]+[K][U]=[F]
$$

Equation $V-2$

where $[\mathrm{M}]$ is a mass matrix; $[\ddot{U}]$ is the second time derivative of the displacement $[\mathrm{U}] ;[\dot{U}]$ is the velocity, $[\mathrm{C}]$ is a damping matrix and $[\mathrm{F}]$ is the force vector.

The general problem, with non-zero damping, is a quadratic eigenvalue problem. However, for vibrational modal analysis, the damping is generally ignored, leaving only the first and third terms on the left hand side:

$$
[M][\ddot{U}]+[K][U]=[0]
$$

Equation $V-3$

This is the general form of the eigen system encountered in structural engineering using finite element analysis. To represent the free vibration solutions of the structure, harmonic motion is assumed, so that $[\ddot{U}]$ is taken to equal $\lambda[U]$ where $\lambda$ is the eigenvalue. The equation reduces to:

$$
[M] \lambda[U]+[K][U]=[0]
$$
Equation $V-4$

In contrast, the equation for a static problems is

$$
[\mathrm{K}][\mathrm{U}]=[\mathrm{F}]
$$
Equation $V-5$

Mass participation factors are one way, to measure which modes are important and which are not. From the ANSYS modal analysis, the results show that for a fixed base analysis, the $y$ direction gives a higher ratio (about 90\%) of effective mass to total mass compared to the $x$ direction (about 80\%). Moreover, it is seen that two of the modes (mode 1 and mode 10) are contributing more than $90 \%$ of the effective mass and consequently it can be expected that the earthquake response will be dominated by these modes. Therefore, for Rayleigh damping, the modes considered in the analysis are modes 1 to 10 . 
It can be seen from the data in Tables V-1 and V-2 that the frequencies in the fixed base structure are higher than in the spring base structure. For mode shape 1, the fixed base structure's frequency is $4.61 \mathrm{~Hz}$, and for the spring base structure it is $4.15 \mathrm{~Hz}$. The frequencies of the fixed base structure range from $4.61 \mathrm{~Hz}$ to $32.62 \mathrm{~Hz}$, and of the spring base structure from $4.15 \mathrm{~Hz}$ to $22.39 \mathrm{~Hz}$.

\subsubsection{Fixed Base Modal Analysis}

Table V-1: Participation Factor Calculation from modal analysis in the $x$ direction

\begin{tabular}{|c|r|r||r|}
\hline Mode & Frequency & \multicolumn{1}{|c|}{ Period } & $\begin{array}{c}\text { Ratio Eff Mass to } \\
\text { Total Mass }\end{array}$ \\
\hline \hline 1 & 4.61 & 0.22 & $1.39 \mathrm{E}-32$ \\
\hline 2 & 6.15 & 0.16 & $8.36 \mathrm{E}-05$ \\
\hline 3 & 7.19 & 0.14 & $2.67 \mathrm{E}-32$ \\
\hline 4 & 15.17 & 0.07 & $1.11 \mathrm{E}-03$ \\
\hline 5 & 17.43 & 0.06 & $4.44 \mathrm{E}-29$ \\
\hline 6 & 18.16 & 0.55 & $2.93 \mathrm{E}-05$ \\
\hline 7 & 26.13 & 0.04 & $8.11 \mathrm{E}-01$ \\
\hline 8 & 28.66 & 0.03 & $1.71 \mathrm{E}-22$ \\
\hline 9 & 31.98 & 0.03 & $1.09 \mathrm{E}-03$ \\
\hline 10 & 32.62 & 0.03 & $3.59 \mathrm{E}-21$ \\
\hline \hline Sum & & & 0.81 \\
\hline
\end{tabular}

Table V-1 lists the modal participation factors of the structure in the $x$ direction. Many codes are concerned that a structure has a ratio of effective mass to total mass of more than $90 \%$ for dynamic loads. In this case, the dynamic analysis should be more significant in that direction. In Table V-1, the effective mass ratio to total mass in the $x$ direction is only $81 \%$ percent, meaning that this direction is not significant in design analysis. However, because this study is not about the design itself, but to see the behaviour of the bridge under the $x$ (horizontal) direction, analysis in the $x$ direction is still conducted. 
Table V-2: Participation factor calculation from modal analysis in the $y$ direction

\begin{tabular}{|c|r|r|r|}
\hline Mode & Frequency & \multicolumn{1}{|c|}{ Period } & $\begin{array}{r}\text { Ratio Eff Mass to } \\
\text { Total Mass }\end{array}$ \\
\hline \hline 1 & 4.61 & 0.22 & 0.00 \\
\hline 2 & 6.15 & 0.16 & $2.30 \mathrm{E}-32$ \\
\hline 3 & 7.19 & 0.14 & 0.68 \\
\hline 4 & 15.17 & 0.07 & $1.18 \mathrm{E}-28$ \\
\hline 5 & 17.43 & 0.06 & 0.01 \\
\hline 6 & 18.16 & 0.55 & $2.78 \mathrm{E}-28$ \\
\hline 7 & 26.13 & 0.04 & $1.98 \mathrm{E}-23$ \\
\hline 8 & 28.66 & 0.03 & 0.15 \\
\hline 9 & 31.98 & 0.03 & $6.78 \mathrm{E}-21$ \\
\hline 10 & 32.62 & 0.03 & 0.07 \\
\hline \hline Sum & & & 0.91 \\
\hline
\end{tabular}

Contrary to the $x$ direction, Table V-2 shows that the ratio of effective mass to total mass for the $y$ direction is more than $90 \%$, so this direction affects the structure more during an earthquake. The frequencies used are in mode 1 and 10.

Results show that the frequency in mode $1\left(f_{a}\right)$ is $4.61 \mathrm{~Hz}$ and for mode $10\left(f_{b}\right)$ is $32.62 \mathrm{~Hz}$. The damping ratio $\zeta$ is $5 \%$. Hence, Alpha and Beta for determining Rayleigh damping can be calculated by using the formulas:

$$
\omega_{a}=2 \pi f_{a}
$$

Equation $V-6$

and,

$$
\omega_{b}=2 \pi f_{b}
$$

Equation $V-7$

Meanwhile,

$$
\alpha=\frac{2 \times \omega_{a} \times \omega_{b}\left(\zeta_{b} \times \omega_{a}-\zeta_{b} \times \omega_{a}\right)}{\omega_{a}^{2}-\omega_{b}^{2}}
$$

And,

$$
\begin{array}{r}
\beta=\frac{2\left(\zeta_{a} \times \omega_{a}-\zeta_{b} \times \omega_{b}\right)}{\omega_{a}{ }^{2}-\omega_{b}{ }^{2}} \\
\alpha=2.53062 / \mathrm{sec}
\end{array}
$$




$$
\beta=0.000435 \mathrm{sec}
$$

Alpha ( $\alpha$ ) defines the mass matrix multiplier for damping and beta $(\beta)$ defines the stiffness matrix multiplier for damping. One form of the viscous damping matrix $[C]$ is given by $\alpha[M]+\beta[K]$, where $[M]$ is the mass matrix and $[K]$ is the stiffness matrix. The damping ratio will determine how much the system oscillates as the response decays toward steady state. So, from the result it is known that $\alpha$ is 2.53062 and $\beta$ is 0.000435 .

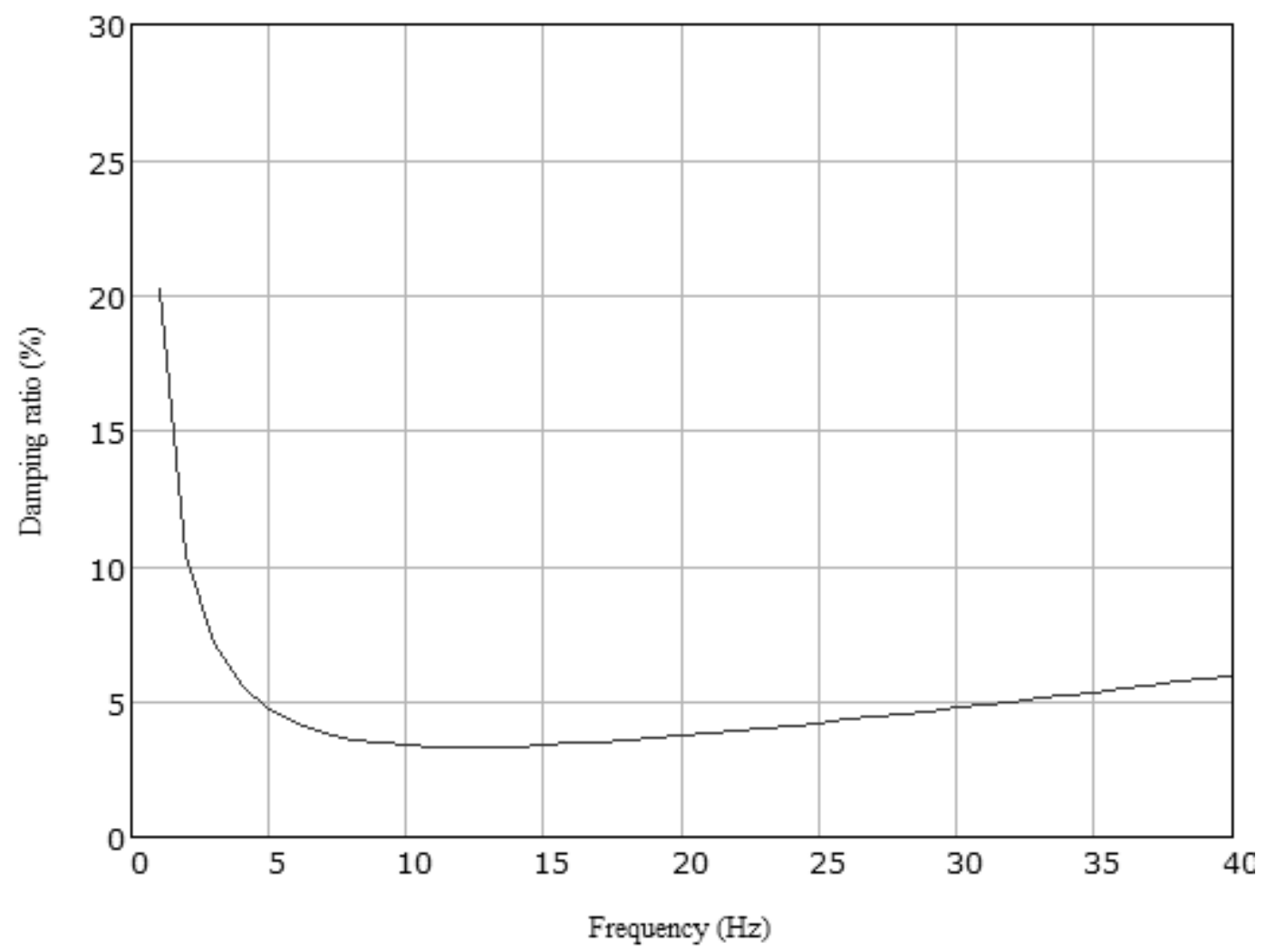

Figure V-18: Damping ratio over frequency range

Figure V-18 describes the ratio of damping for the range of frequency. This study assumes the ratio of the system is 5\%, and for that percentage, the frequencies are in the range 4.6 and 33 Hertz. For frequency less than 4 and more than 33, the damping ratio becomes more than 5\% (over damping).

Mode shapes of the structure are shown in Figure V-19. 

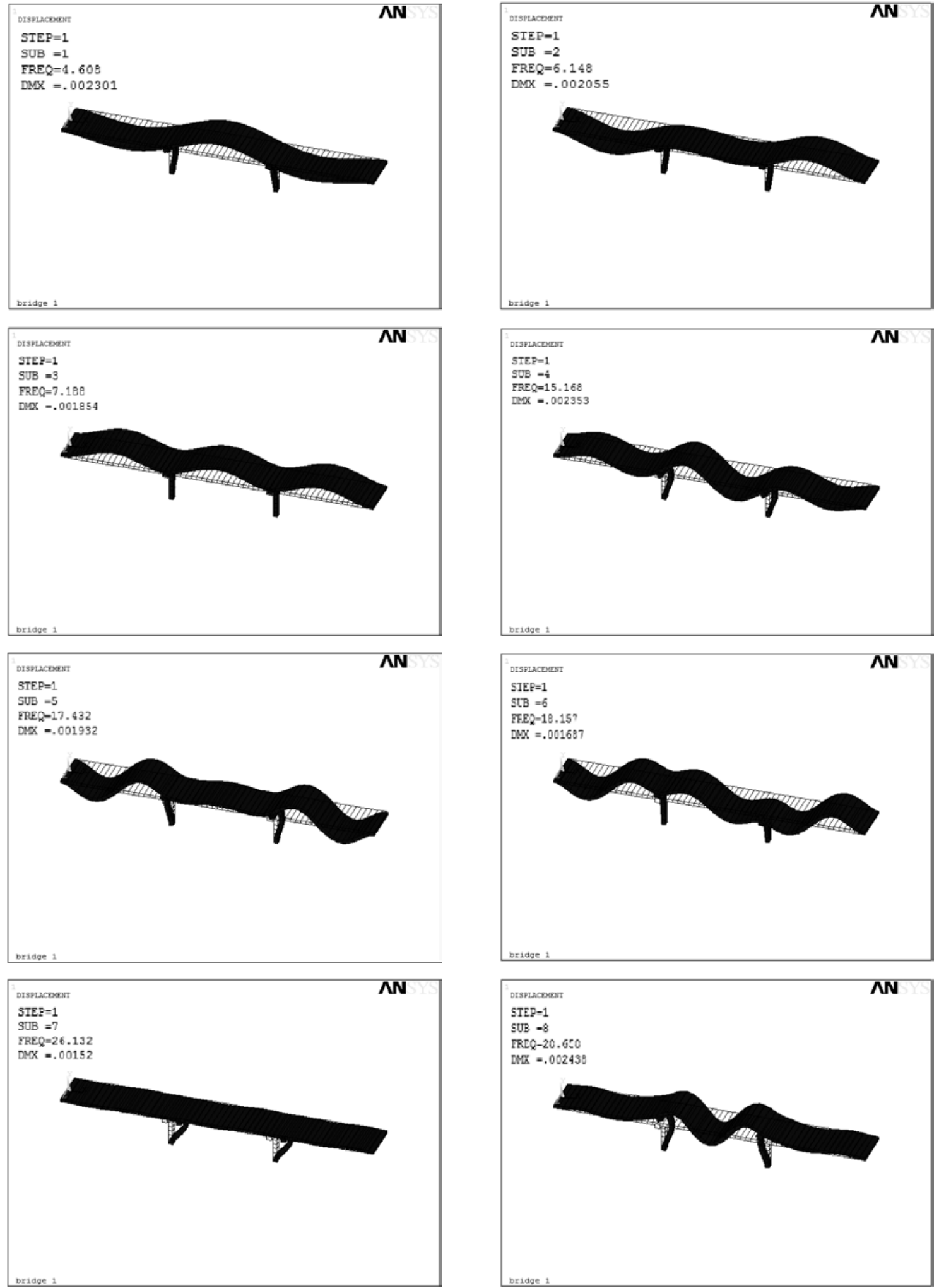

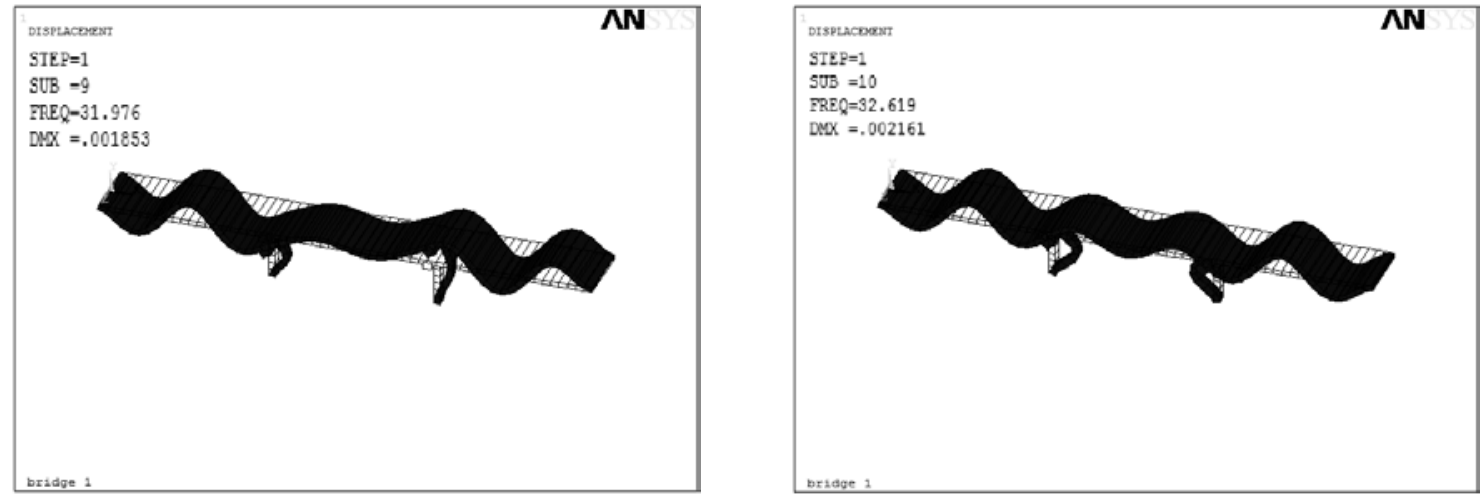

Figure V-19: Mode shapes and frequencies of the structure for fixed base structure

Figure V-19 shows 10 mode shapes and frequencies of the structure. The frequency of the fixed base structure is in the range $4.61 \mathrm{~Hz}$ to $32.62 \mathrm{~Hz}$. By referring to Figure V-18, it can be seen that this range is acceptable for damping.

The effects of soil stiffness on the frequency of the bridge structure were also studied in this research. A range of stiffnesses for flexible soil and for stiffer soil was presented in Tables IV-2 and IV-3.

\subsubsection{Spring Base Modal Analysis}

In this phase, a series of springs was given to the bridge at the supports, and the results of mode shapes can be seen in Figure V-20.
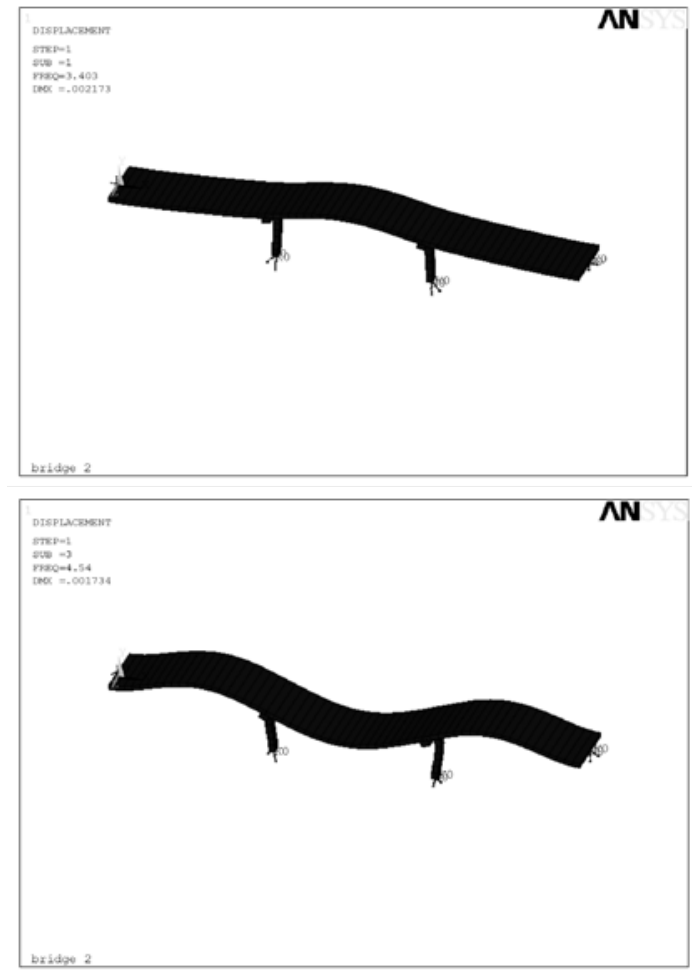
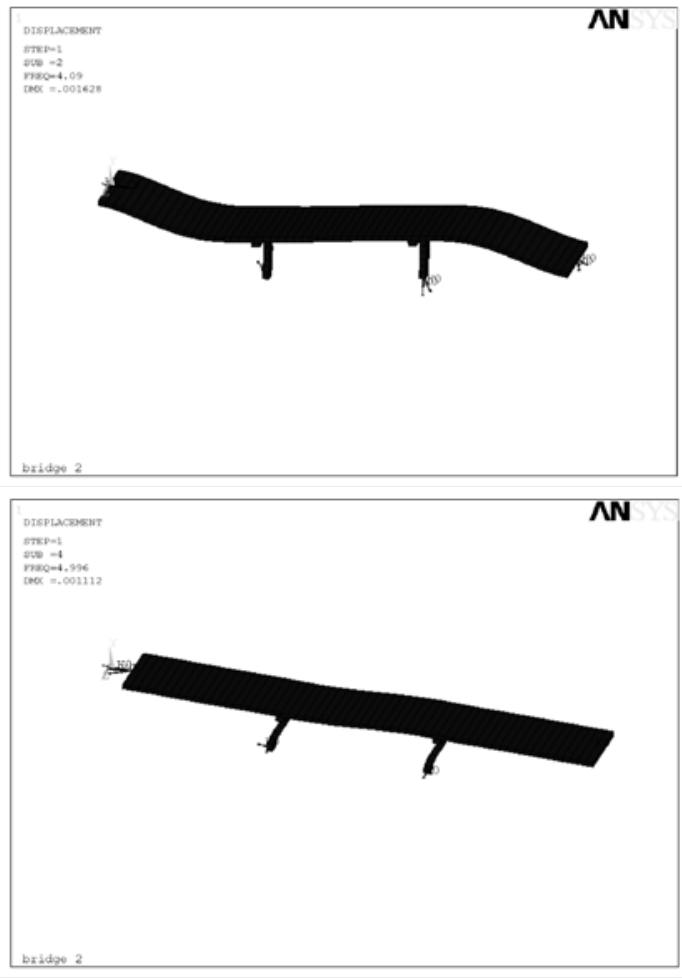

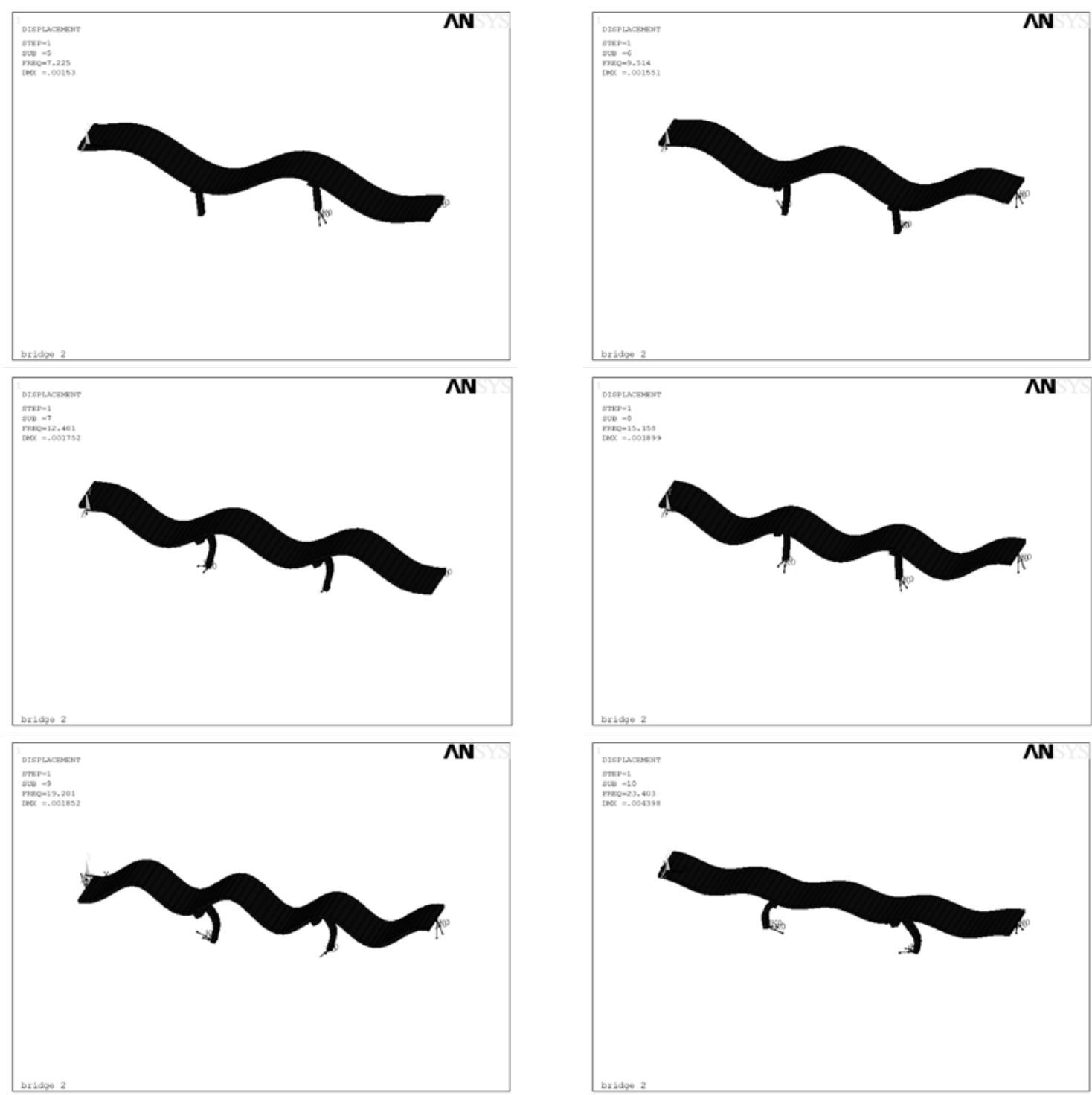

Figure V-20: Mode shapes and frequencies of the structure for the springs base structure

Mode shapes are useful because they represent the shape in which the bridge will vibrate in free motion. Figure V-20 illustrates ten mode shapes which tend to dominate the motion during an earthquake.

Generally, the first mode of the vibration is the primary interest. It usually makes the largest contribution to the structure's motion, and its period is the longest. Higher-order modes also exist in the structure but then the period becomes much shorter. 
The significance of a mode is indicated by the mass participation. This factor indicates the amount of the total structural mass that is activated by a single mass. If all the modes of a structure are considered, the cumulative mass participation will be $100 \%$.

For the spring base, as mentioned above, a series of spring stiffness values is applied to the supports of the bridge. There are two groups of spring stiffness series of the bridge, namely dense spring stiffness and loose spring stiffness. The results show that the frequency changes as the spring stiffness is changed. The natural frequencies are higher when the stiffness is higher, as expected.

Table V-3: Modal participation factor for $x$ direction (spring base)

\begin{tabular}{|c|r|r||r|}
\hline Mode & \multicolumn{1}{|c|}{ Frequency } & \multicolumn{1}{|c|}{ Period } & $\begin{array}{c}\text { Ratio Eff Mass to } \\
\text { Total Mass }\end{array}$ \\
\hline \hline 1 & 3.15 & 0.32 & 0.0002 \\
\hline 2 & 3.19 & 0.31 & 0.0000 \\
\hline 3 & 3.50 & 0.29 & 0.0000 \\
\hline 4 & 4.88 & 0.20 & 0.9924 \\
\hline 5 & 6.53 & 0.15 & 0.0001 \\
\hline 6 & 8.79 & 0.11 & 0.0000 \\
\hline 7 & 12.01 & 0.08 & 0.0002 \\
\hline 8 & 15.13 & 0.07 & 0.0000 \\
\hline 9 & 18.94 & 0.05 & 0.0003 \\
\hline 10 & 22.39 & 0.04 & 0.0000 \\
\hline \hline Sum & & & 0.9933 \\
\hline
\end{tabular}

Table V-4: Modal participation factor for $y$ direction (spring base)

\begin{tabular}{|c|r|r|r|}
\hline Mode & Frequency & \multicolumn{1}{|c|}{ Period } & $\begin{array}{c}\text { Ratio Eff Mass to } \\
\text { Total Mass }\end{array}$ \\
\hline \hline 1 & 3.15 & 0.32 & 0.0000 \\
\hline 2 & 3.19 & 0.31 & 0.9024 \\
\hline 3 & 3.50 & 0.29 & 0.0345 \\
\hline 4 & 4.88 & 0.20 & 0.0000 \\
\hline 5 & 6.53 & 0.15 & 0.0000 \\
\hline 6 & 8.79 & 0.11 & 0.0053 \\
\hline 7 & 12.01 & 0.08 & 0.0000 \\
\hline 8 & 15.13 & 0.07 & 0.0433 \\
\hline 9 & 18.94 & 0.05 & 0.0000 \\
\hline 10 & 22.39 & 0.04 & 0.0070 \\
\hline \hline Sum & & & 0.9925 \\
\hline
\end{tabular}

Tables V-3 and V-4 describe the modal participation factor for spring bases for $x$ and $y$ directions, respectively. It can be seen that for both directions the effective mass ratio to 
total mass ratio is more than $90 \%$. This suggests that the structure has the same risk for dynamic response in both orthogonal directions.

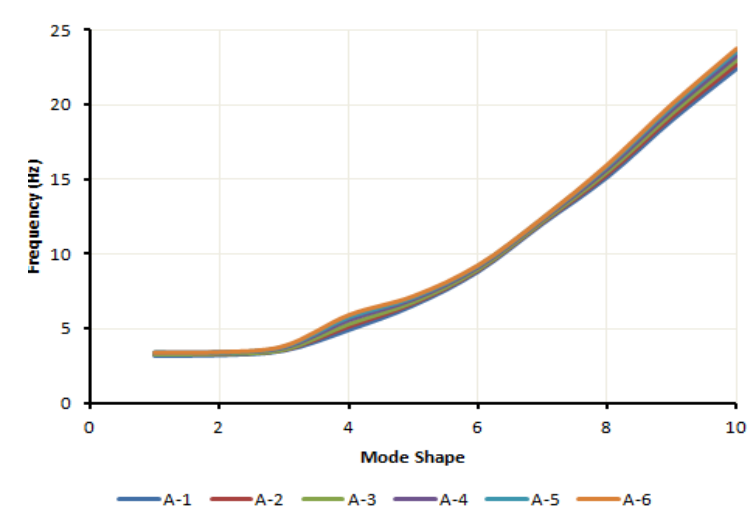

(a) For soil A (hard soil)

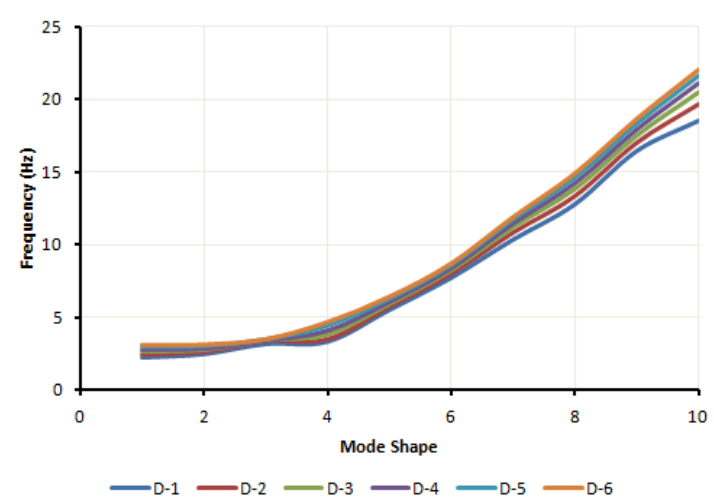

(b) Soil D (flexible soil)

Figure V-21: Co-relation between mode shape and frequency of two types of soil

In analysing the relation between mode shape and frequency for two of the soil types, a graph describing the trend line of mode shape and soil type is presented in Figure V-21 (a) and (b). The stiffer the soil applied to the structure, the higher frequencies will be obtained. In addition, for stiffer soil, it can be shown that the frequencies are more dense, while for looser soil the frequencies become less dense.

Table V-5: Frequencies for soil A

\begin{tabular}{|c|c|c|c|c|c|c|}
\hline \multirow{2}{*}{ MS } & \multicolumn{6}{|c|}{ Frequency } \\
\cline { 2 - 7 } & A-1 & A-2 & A-3 & A-4 & A-5 & A-6 \\
\hline 1 & 3.15331 & 3.22333 & 3.28537 & 3.34063 & 3.38478 & 3.39919 \\
\hline 2 & 3.19306 & 3.27494 & 3.32961 & 3.36343 & 3.39008 & 3.43451 \\
\hline 3 & 3.49793 & 3.54535 & 3.60927 & 3.68472 & 3.76448 & 3.84392 \\
\hline 4 & 4.8794 & 5.10635 & 5.32237 & 5.52875 & 5.72656 & 5.91664 \\
\hline 5 & 6.52964 & 6.66775 & 6.80171 & 6.93199 & 7.05894 & 7.18277 \\
\hline 6 & 8.79251 & 8.89788 & 8.99578 & 9.08784 & 9.17522 & 9.25874 \\
\hline 7 & 12.0052 & 12.1223 & 12.22 & 12.3024 & 12.3729 & 12.4336 \\
\hline 8 & 15.1261 & 15.3478 & 15.5416 & 15.7126 & 15.8648 & 16.0015 \\
\hline 9 & 18.9398 & 19.2173 & 19.4655 & 19.6878 & 19.8874 & 20.067 \\
\hline 10 & 22.3869 & 22.7255 & 23.0298 & 23.3042 & 23.5522 & 23.7766 \\
\hline
\end{tabular}


Table V-6: Frequencies for soil D

\begin{tabular}{|c|c|c|c|c|c|c|}
\hline \multirow{2}{*}{ MS } & \multicolumn{7}{|c|}{ Frequency } \\
\cline { 2 - 7 } & D-1 & D-2 & D-3 & D-4 & D-5 & D-6 \\
\hline 1 & 2.21007 & 2.43596 & 2.63193 & 2.80375 & 2.95451 & 3.07385 \\
\hline 2 & 2.44981 & 2.61707 & 2.75823 & 2.87894 & 2.98316 & 3.08493 \\
\hline 3 & 3.14906 & 3.35658 & 3.38812 & 3.41389 & 3.43795 & 3.46424 \\
\hline 4 & 3.31297 & 3.50266 & 3.82169 & 4.11428 & 4.38577 & 4.6399 \\
\hline 5 & 5.50337 & 5.72324 & 5.91099 & 6.08015 & 6.23748 & 6.38663 \\
\hline 6 & 7.71935 & 8.00738 & 8.22574 & 8.40115 & 8.54869 & 8.6773 \\
\hline 7 & 10.354 & 10.825 & 11.1867 & 11.4669 & 11.6871 & 11.8628 \\
\hline 8 & 12.7916 & 13.3419 & 13.8181 & 14.2255 & 14.5731 & 14.8704 \\
\hline 9 & 16.4627 & 17.0034 & 17.4753 & 17.9004 & 18.2843 & 18.6298 \\
\hline 10 & 18.5503 & 19.6496 & 20.4548 & 21.0724 & 21.5763 & 22.0075 \\
\hline
\end{tabular}

Tables V-5 and V-6 describe the correlation between mode shape and the frequencies of the structure for each series of spring stiffness. For dense soil, mode shapes 1 and 2 have frequencies from $3.14 \mathrm{~Hz}$ to $3.40 \mathrm{~Hz}$, and 3.19 to $3.43 \mathrm{~Hz}$, respectively. The significant differences among the five soil stiffnesses occur in mode shape 4 and mode shapes 8 to 10 . On the other hand, for loose soil, significant variation can be seen from mode shape 4, continuing to mode shapes 7, 8, 9 and 10 .

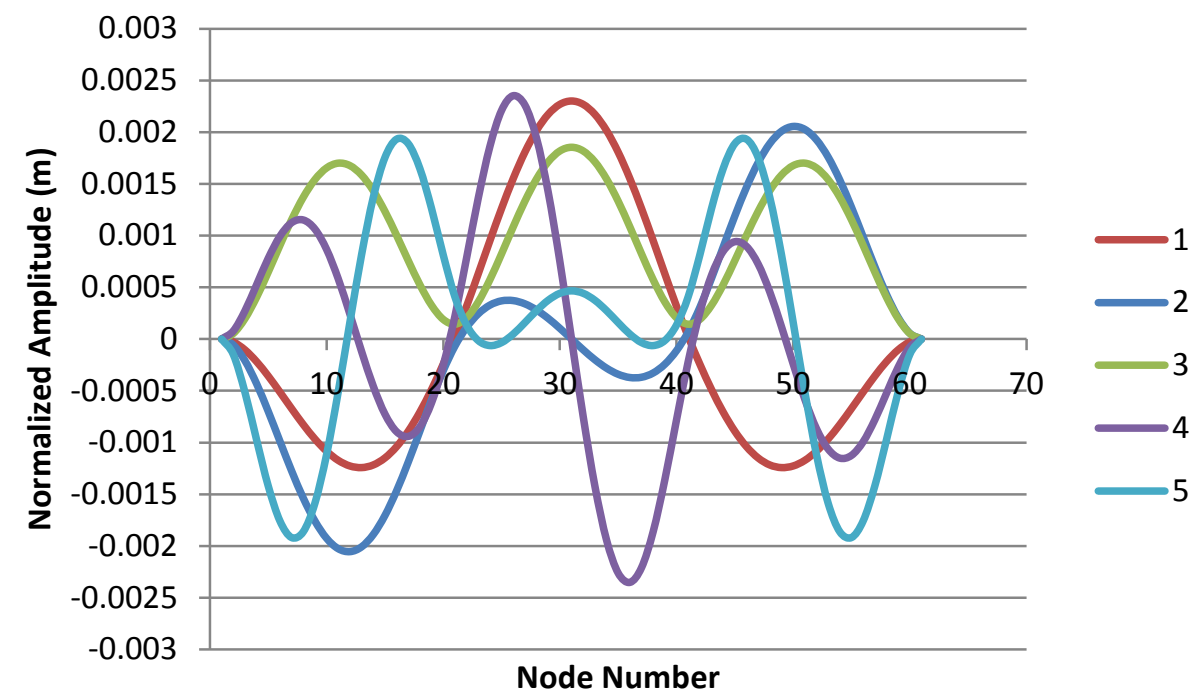

Figure V-22: Mode shape comparison for fixed base bridge in deck 


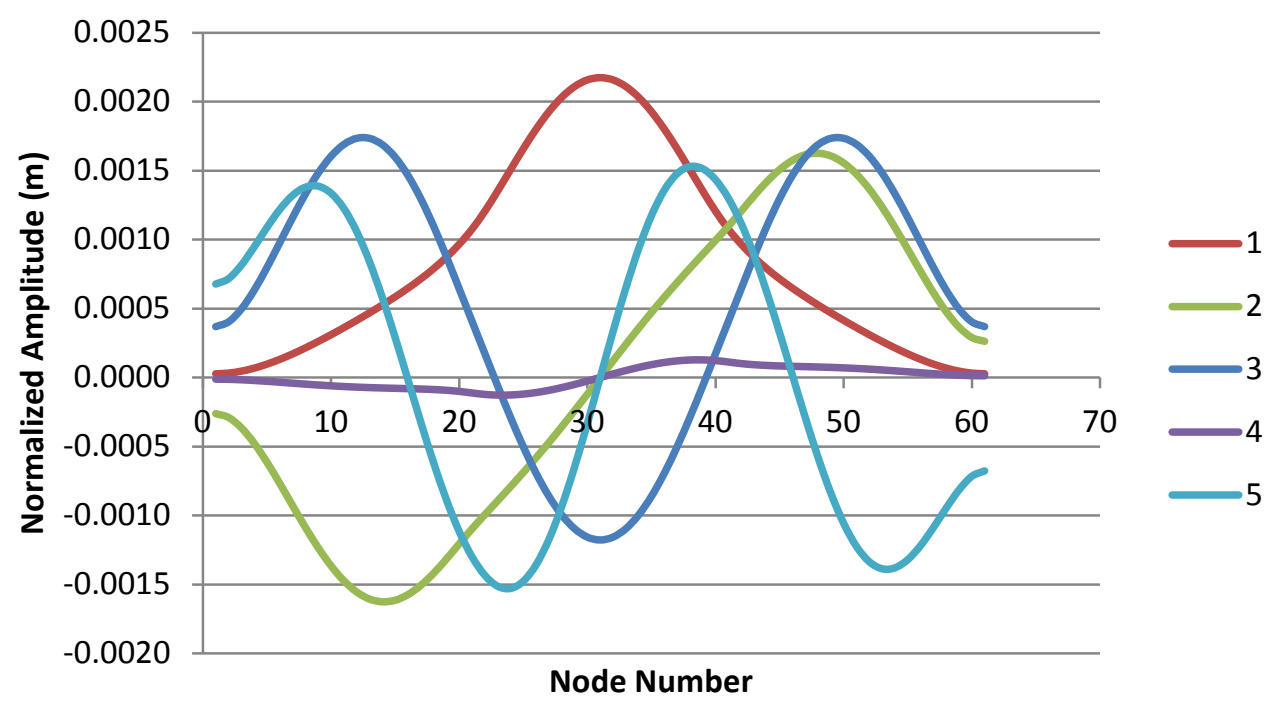

Figure V-23: Mode shape comparison for spring base bridge in deck

Figure V-22 shows the five first mode shapes of a fixed base bridge in this study and Figure V-23 for a spring base bridge. For both, the first mode seems to be important since it has high amplitude. From the graph in these figures it can be seen that the deck in mode shape 1 fluctuates harmonically; similarly, in mode shape 3 the deck also fluctuates but there is also an increase in the frequency.

It also can be observed from Figures V-22 and V-23 that the springs have reduced the fluctuation of the deck and the frequency of the structure becomes lower.

To sum up, it is clear that for a stiff soil, which has the same range of spring stiffness as the loose soil, the discrepancy in the frequencies among the soils are less than for the loose soils. The variation of the frequency value is not especially significant for the stiffer soil, compared to the flexible soil with the same range of soil stiffness variation.

Relating to the ratio of effective mass to total mass in the $x$ and $y$ directions, the spring base structure gives a result of more than $90 \%$ for both directions. Thus, the significant modes have been captured.

From the graph in Figure V-24, it can be deduced that the gradient of the ratio of effective mass to total mass in the $x$ direction is larger than that in the $y$ direction, decreasing sharply from soil stiffness 1 to 6 . 


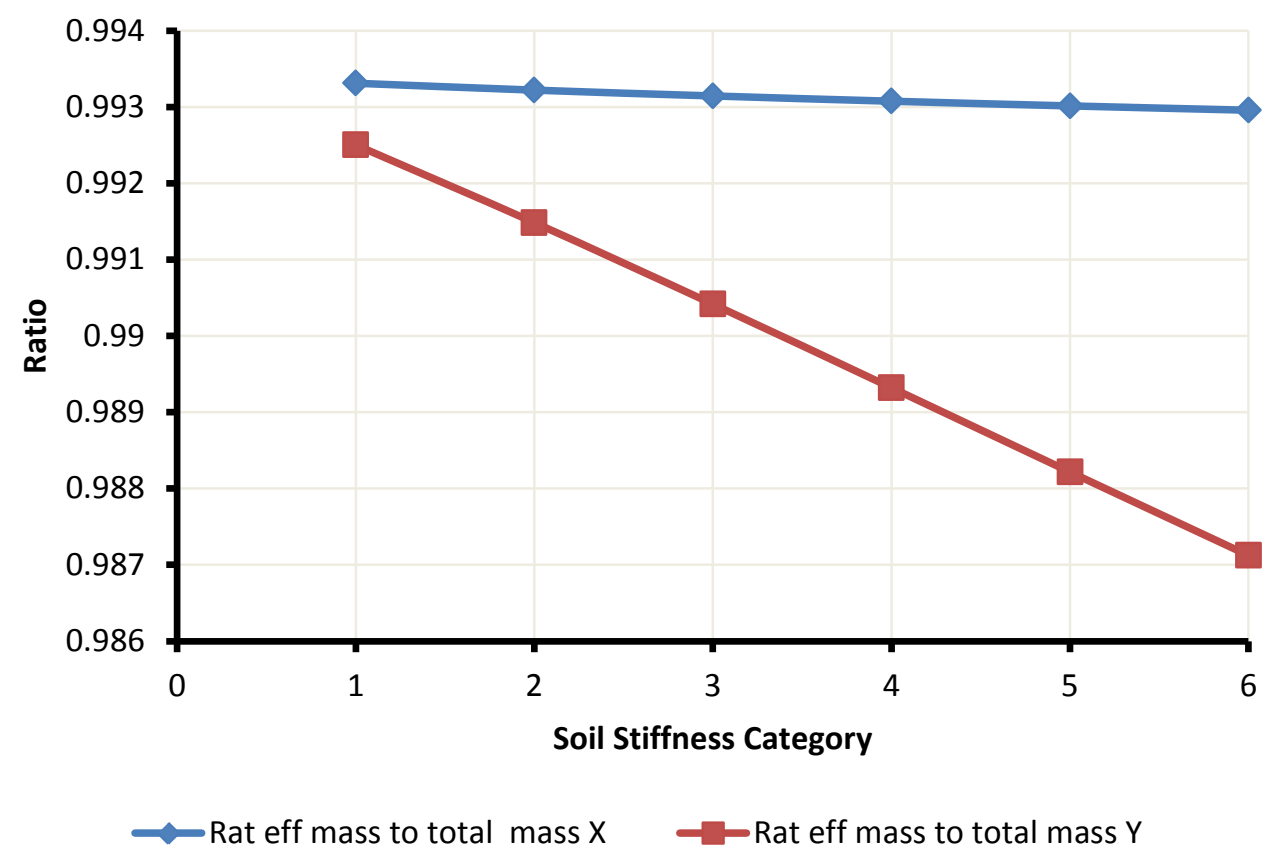

Figure V-24: Comparison of ratio effective mass to total mass in $x$ and $y$

\subsection{D Modelling for Frequency Analysis}

The main study was conducted in $2 D$; however, a simple $3 D$ analysis was also carried out to confirm that modelling the bridge in $2 D$ was acceptable. An analysis using a $3 D$ beam element was conducted to examine any modes which could mobilize 'across deck' or torsional response. Theoretically, greater stiffness will result in higher frequency, so it was predicted that the modes would be high frequency and hence away from the spectral peak, hopefully to be confirmed by the Finite Element analysis.

Comparison of the $2 D$ and $3 D$ mode shape and frequencies are given in Table V-7.

Table V-7: Comparison of 2D and 3D frequencies.

\begin{tabular}{|c|c||c|c||}
\hline \multicolumn{2}{|c||}{ Mode shape } & \multicolumn{2}{c|}{ Frequency (Hz) } \\
\hline 2D & 3D & 2D & 3D \\
\hline \hline 1 & 1 & 4.61 & 4.60 \\
\hline
\end{tabular}




\begin{tabular}{|c|c|c|c|}
\hline 2 & 2 & 6.14 & 6.08 \\
\hline 3 & 3 & 7.19 & 6.93 \\
\hline 4 & 5 & 15.17 & 14.63 \\
\hline 5 & 7 & 17.43 & 16.49 \\
\hline 6 & 6 & 18.16 & 16.48 \\
\hline 7 & 9 & 26.13 & 24.44 \\
\hline 8 & 10 & 28.65 & 26.32 \\
\hline 9 & 11 & 31.98 & 29.00 \\
\hline 10 & 12 & 32.62 & 32.72 \\
\hline
\end{tabular}

For the transversal deck direction, the mode shapes and the frequencies are shown in Figure V-25.

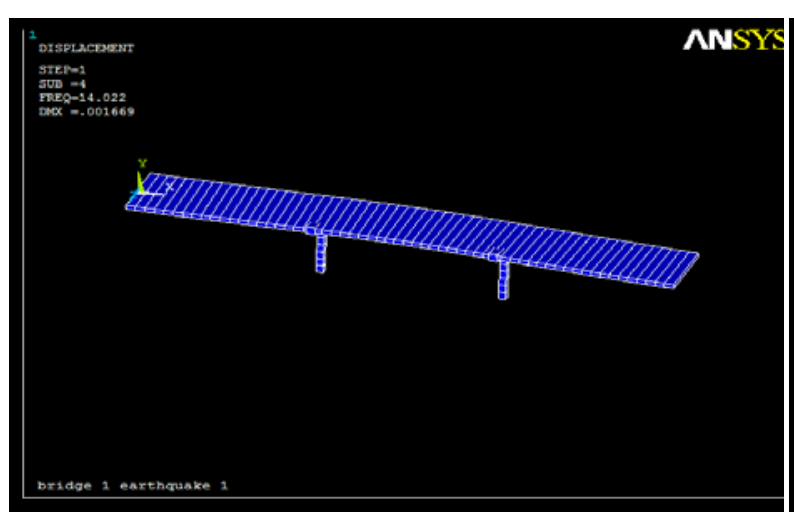

Mode shape 4, $\mathrm{f}=14.022 \mathrm{~Hz}$

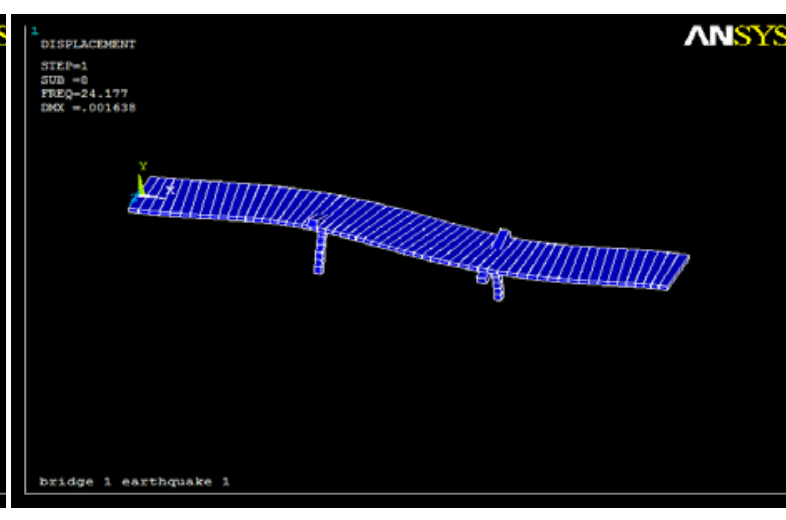

Mode shape 8, $\mathrm{f}=24.177 \mathrm{~Hz}$ 


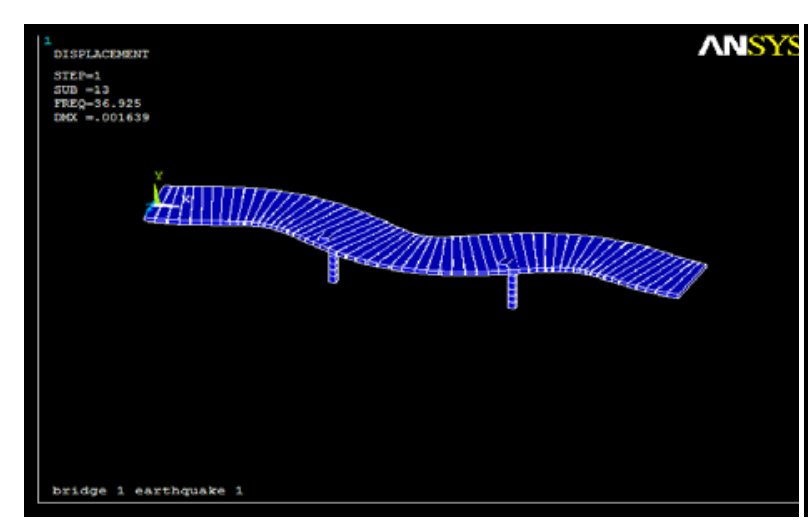

Mode shape 13, $\mathrm{f}=36.925 \mathrm{~Hz}$

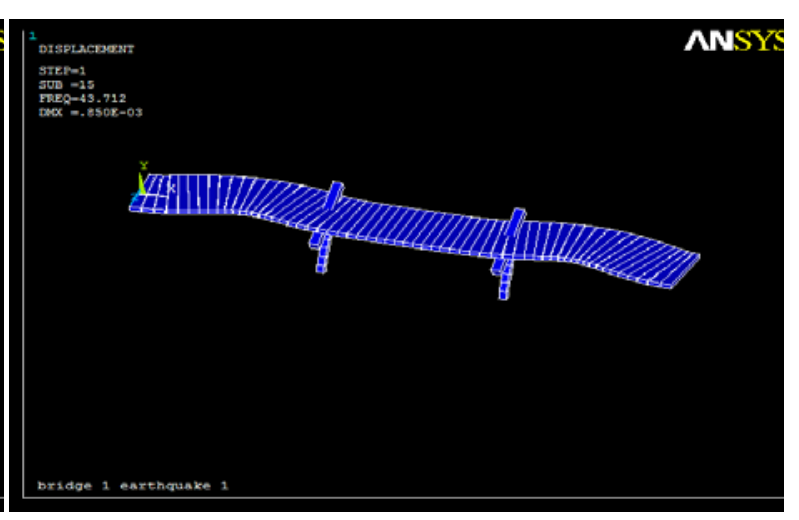

Mode shape 15, $\mathrm{f}=43.925 \mathrm{~Hz}$

Figure V-25: Frequencies and mode shapes for transversal deck direction (3D)

The frequencies are given in Table V-8.

Table V-8: The frequencies of transversal direction of the bridge (3D)

\begin{tabular}{||c||c|c||}
\hline $\begin{array}{c}\text { Mode } \\
\text { shape }\end{array}$ & Frequency (Hz) & Period (secs) \\
\hline \hline 4 & 14.02 & 0.0713 \\
\hline 8 & 24.18 & 0.0414 \\
\hline 13 & 36.93 & 0.0271 \\
\hline 15 & 43.712 & 0.0228 \\
\hline
\end{tabular}

The lowest frequency is $14.02 \mathrm{~Hz}$; translating to a period of $1 / 14.02$ equals 0.074 sec. From the response spectrum graph in Figure V-26, it can be seen that 0.07 secs was half of the peak period, i.e. from 0.15 to 0.4 for soil A. The peak periods of soils B, C, D and E were even larger.

Since the highest period of the transversal direction (mode shape 4) was about 0.074 secs, so from the response spectrum it can be seen that the periods in deck direction (transversal 
direction in $3 D$ ) were below the peak. Acceleration of these mode shapes was lower than the mode shape in the $2 D$ analysis.

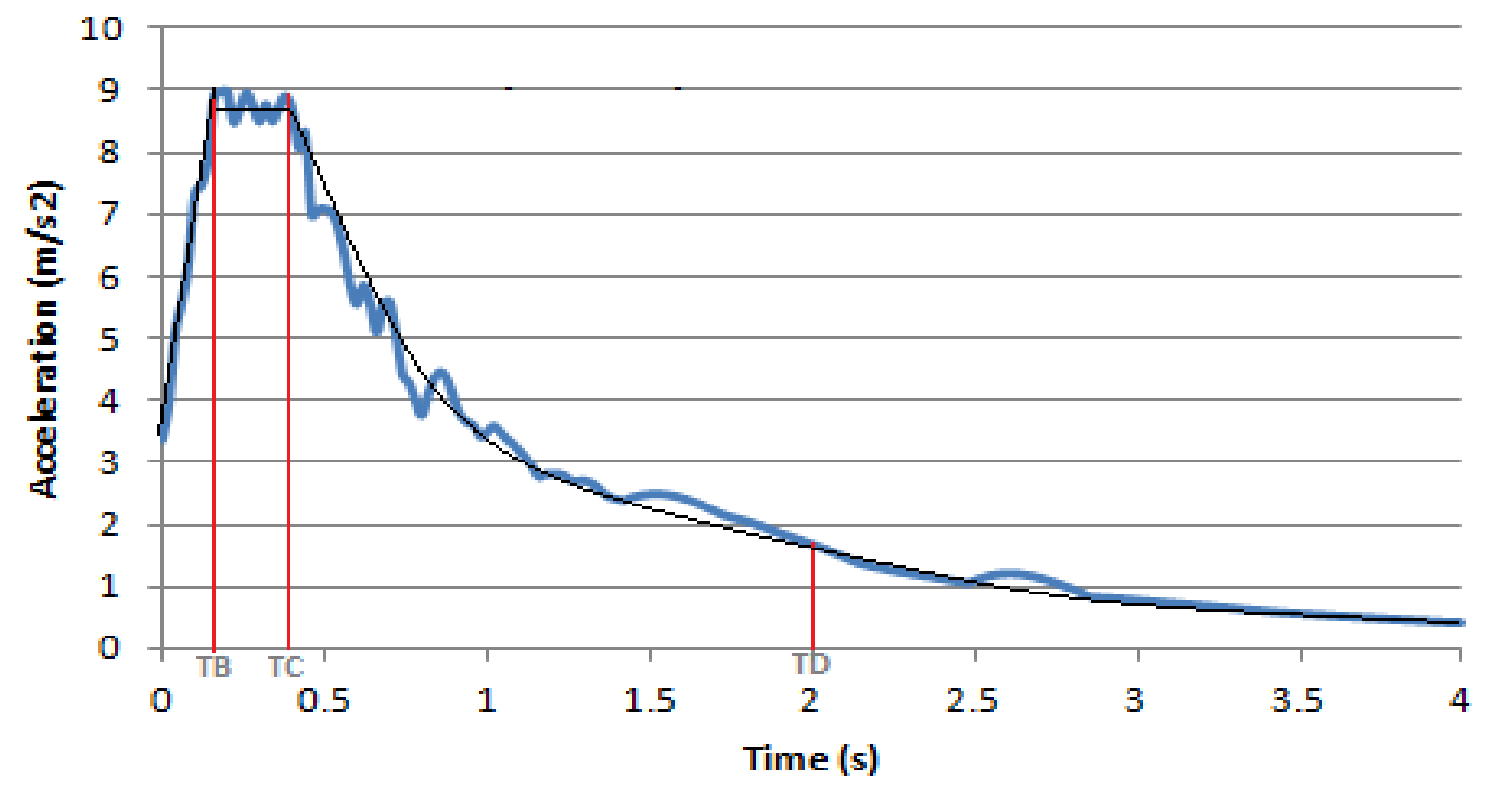

Figure V-26: Graph of response spectrum

This analysis supports the assumption that $3 D$ modelling is not required, and that the dominant response is likely to be in the 'plane' of the bridge, i.e. $2 D$ analysis is justified.

\subsection{Time History (Transient) Analysis}

Transient dynamic analysis or time history analysis is a technique used to determine the dynamic response of a structure under the action of any general time-dependent load, i.e. earthquake loading. It can be used to determine the time-varying displacements, strains, stresses and forces in a structure as it responds to any combination of static and transient loads. As transient dynamic analysis is more likely to be a repetitive method, involving more complex calculations than static analysis, it generally requires more computer resources in terms of time To save a significant amount of time and resources, preliminary work can be carried out to understand the physics of the problem, for example by doing some simpler modelling.

There are two different ways to apply earthquake ground motion to the bridge in the computer program: first, to input acceleration to the supports of the bridge; and second, to input the displacement earthquake ground motion. To manually input the ground motion at 
specific supports it is necessary to first convert the acceleration record into its corresponding displacement time-history record.

The basic equation of motion solved by transient dynamic analysis is:

$$
(M)\{\ddot{u}\}+(C)\{\dot{u}\}+(K)\{u\}=\{F(t)\}
$$

Where $\mathrm{M}$ is the mass matrix, $\mathrm{C}$ is the damping matrix, $\mathrm{K}$ is the stiffness matrix, $\{\ddot{u}\}$ is the nodal acceleration vector, $\{\dot{\mathrm{u}}\}$ is the nodal velocity vector, $\{u\}$ is the nodal displacement vector and $\{\mathrm{F}(\mathrm{t})\}$ is the load vector.

At a given time $t$, these equations can be thought of as a set of static equilibrium equations that also take into account inertia and damping forces. The ANSYS ver. 12 program uses a Newmark time integration method to solve the equations at discrete time points.

The support-induced vibrations cause deformations and stresses in the structural system. To achieve the aim of this research, it is important to perform a dynamic analysis on the structures subjected to earthquake-induced ground motion.

The support excitations can be divided into two types: single support excitation and multisupport excitation.

In a single support excitation, it is assumed that all the supports undergo an identical or uniform ground motion. In other words, the supports move as one rigid base, as shown in Figure V-27. Hence, the mass attached to dynamic degrees of freedom are excited by the ground motion.

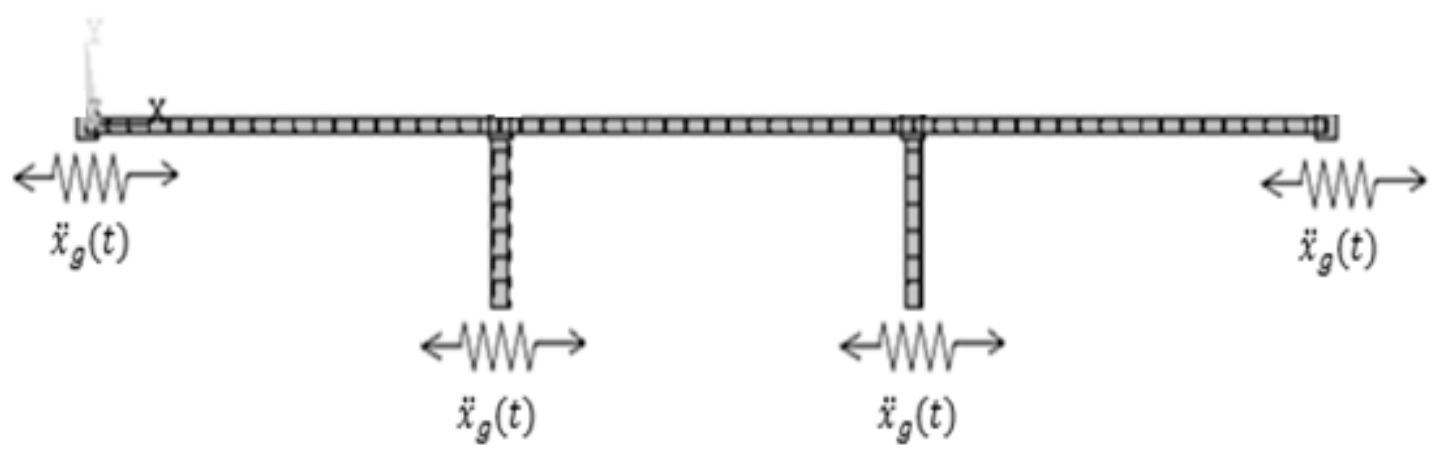

Figure V-27: Single support excitation 
In multi-support excitations, the ground or support motions (ground excitations) are dissimilar at different supports, as shown in Figure V-28. For the same travelling wave of an earthquake, the time histories of the ground motion at two supports could be different if the two supports are separated by a large distance. This is because the travel time of the wave between any two supports is not sufficiently negligible to allow the assumption that the ground motions are the same at the two supports. Symbol $\ddot{u}_{g 1}(t)$ refers to a certain acceleration occurring on the ground, with the resulting displacement $x_{1}$. Meanwhile the symbol $\ddot{u}_{g 2}(t)$ means the other acceleration occurred at a different support of the structure, which results in another displacement, namely $x_{2}$. This behaviour will lead to the creation of relative displacements among the supports of the bridge structure.

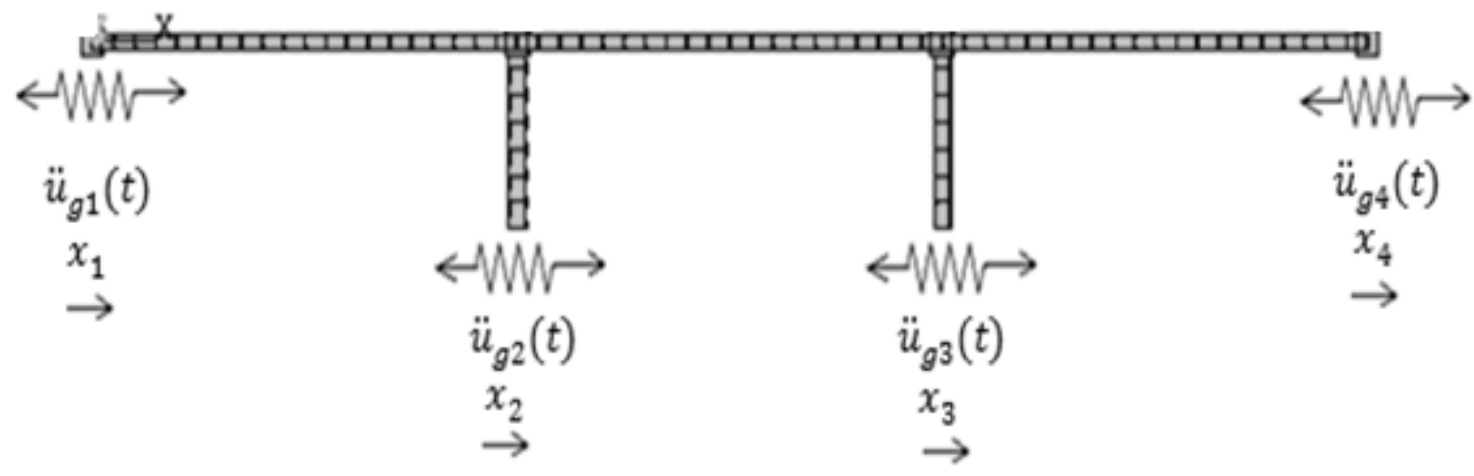

Figure V-28: Multi-support excitation

To examine the behaviour of integral bridges under pure seismic loads without considering relative displacement, the same acceleration was applied to the supports of the structure. In this case, all the responses of the bridge are caused purely by the dynamic loads, which are acceleration, velocity and displacement. Acceleration affects the bridge due to inertia, velocity affects it because of damping, and displacement causes the internal response through stiffness.

Two further cases were applied to accommodate the horizontal and vertical earthquake ground motion to the bridge. Applying different earthquake excitations to the structure will also produce different results in the internal forces of, because of relative displacement. Referring to the equation of motion, applying a relative displacement to the structure should be correlated with its stiffness. When a linear elastic structure is supported at more than one point and is subjected to different input ground motions, the formulation of the 
response to each input component is different from that of a system having uniform support excitation. The difference is that when the supports move independently of each other, they induce quasi-static stresses that must be considered in addition to the dynamic response effects resulting from inertial forces.

Thus, there are several analyses related to the acceleration time histories in this study:

1. Applying the same time histories to the supports.

2. Applying different time histories to the supports.

In the first analysis, the same acceleration time histories were applied to the four supports of the bridge. Figure V-29 (left) shows how the horizontal synthetic earthquake ground motion waas applied to the bridge supports. $\mathrm{D}_{\mathrm{h} 1}$ and $\mathrm{H}_{1}$ in Figure V-29 refer to the displacement due to horizontal acceleration 1. In this case, all the supports will have the same synthetic horizontal acceleration, which will result in the same displacement value in the supports, namely $\mathrm{D}_{\mathrm{h} 1}$.
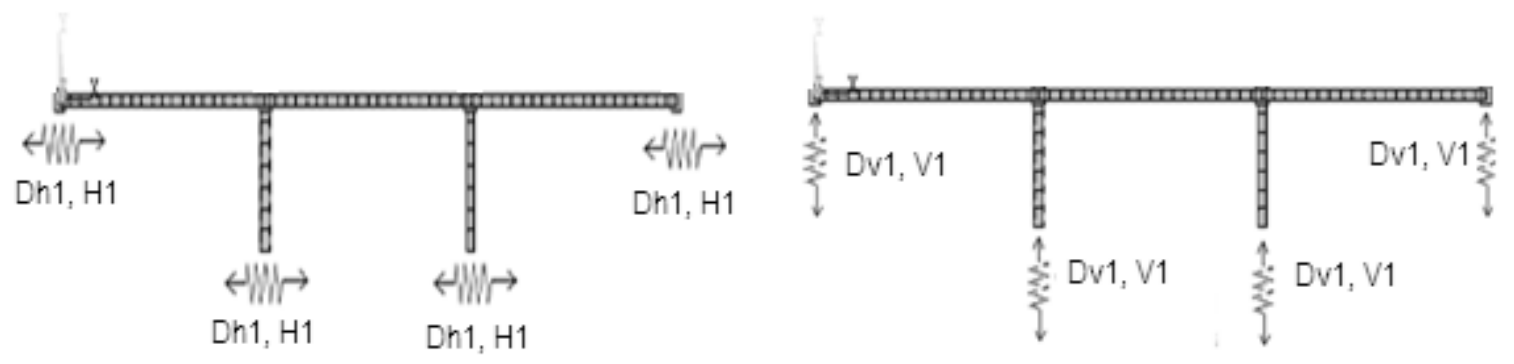

Figure V-29: Displacements on the supports of the bridge for the same acceleration time histories input: horizontal (left), vertical (right)

Similar conditions were conducted for the vertical earthquake acceleration, as can be seen in Figure V-29 (right). $D_{\mathrm{v} 1}, V_{1}$ denote the vertical displacements that were generated from the vertical synthetic acceleration in the supports.

For the second analysis, different acceleration time histories with the same target response spectra were applied to the supports of the bridge. This condition created relative displacements between the supports, because the displacement occurring in each support was different. These relative displacements were caused by the displacements created from acceleration time history 1 , subtracted from acceleration time history 2. 
In this research, the relative displacement analysis was carried out in two phases. In the first phase, the horizontal relative displacements were applied only to the left beam and column support. In the second phase, the vertical relative displacements were applied only to the left beam and the column support. The differences between these two phases are shown in Figure V-30.
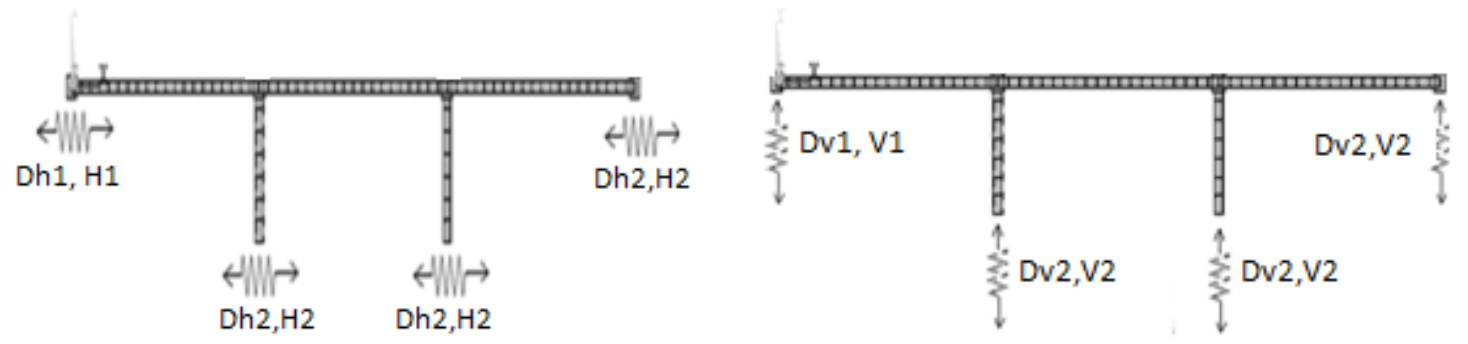

Figure V-30: Displacements on the supports of the bridge for creating relative displacement; horizontal (left) and vertical (right).

Figure VI-30 shows two different displacements occurred in the supports of the bridge for both horizontal direction (left) and vertical (right). $D_{h 1}$ is the first displacement due to the first acceleration time history $H_{1}$ and $D h_{2}$ are the second displacement resulting from a different acceleration, that is the second acceleration time history $\mathrm{H}_{2}$. As in the vertical case, $D_{v 1}$ was the first displacement, resulting from the first acceleration time history $V_{1}$; and $D_{v 2}$ is the result of the second acceleration in the vertical direction, $V_{2}$.

The two different acceleration excitations caused different internal forces and thus resulted in different displacements to the supports where the accelerations were applied. The simulation of these different time histories can be seen in Figure V-31(a) and (b), for horizontal and vertical displacements, respectively.

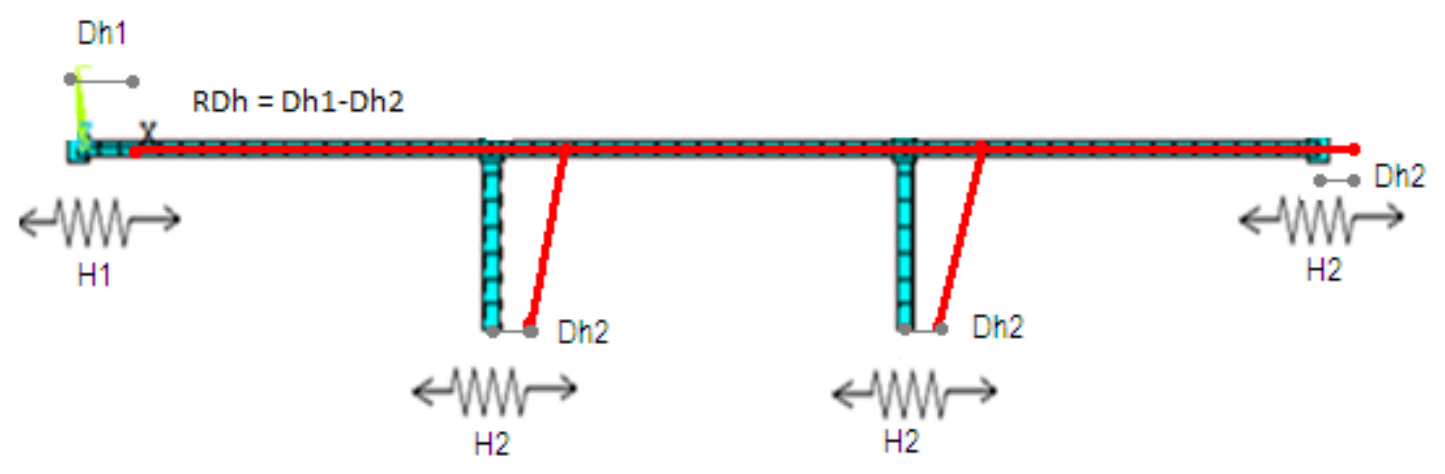

(a): Horizontal Relative Displacement 


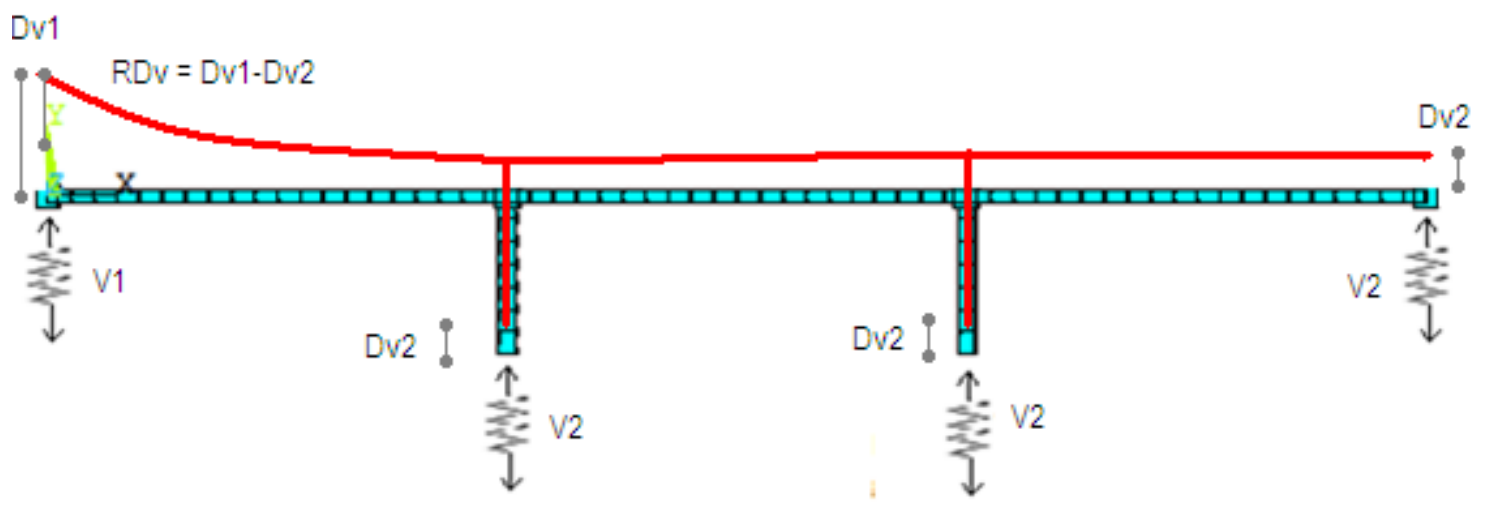

(b): Vertical Relative Displacement

Figure V-31: Simulation of (a) Horizontal relative displacement and (b) Vertical relative displacement.

In other words, acceleration time history 1 caused displacement in the area where it was applied. Acceleration time history 2 also created displacement to the supports where it was applied; a relative displacement then occurred and the value equalled the first synthetic acceleration $\left(H_{1}\right)$ minus the relative displacement due to the second synthetic acceleration $\left(H_{2}\right)$.

ANSYS modelling for applying the synthetic acceleration time histories, for three different cases (for the same time history, and for different time histories; for horizontal and for vertical) can be seen in Figure V-32. This figure also describes the configurations of the nodes for each analysis. 


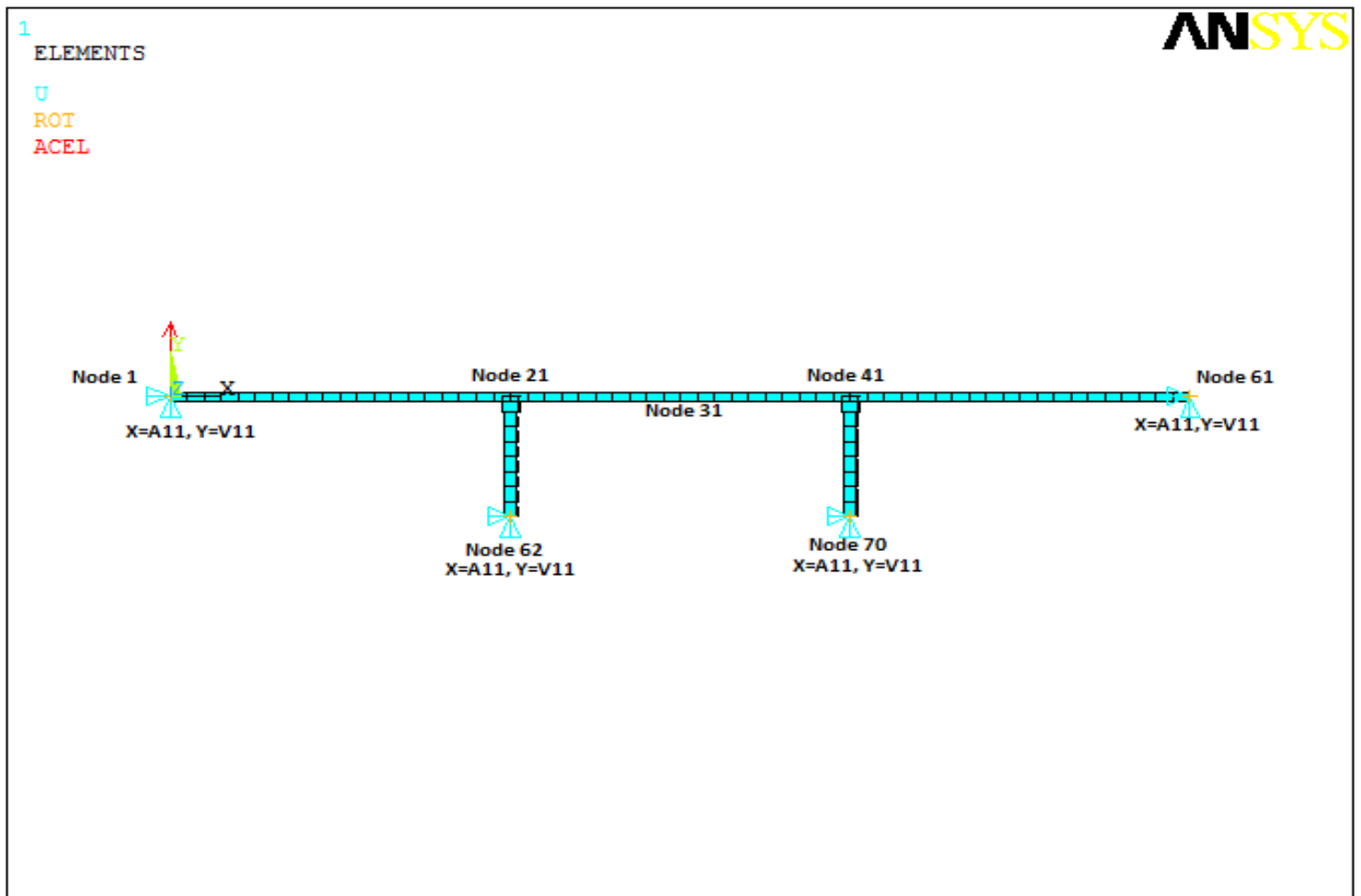

(a). Same synthetic acceleration

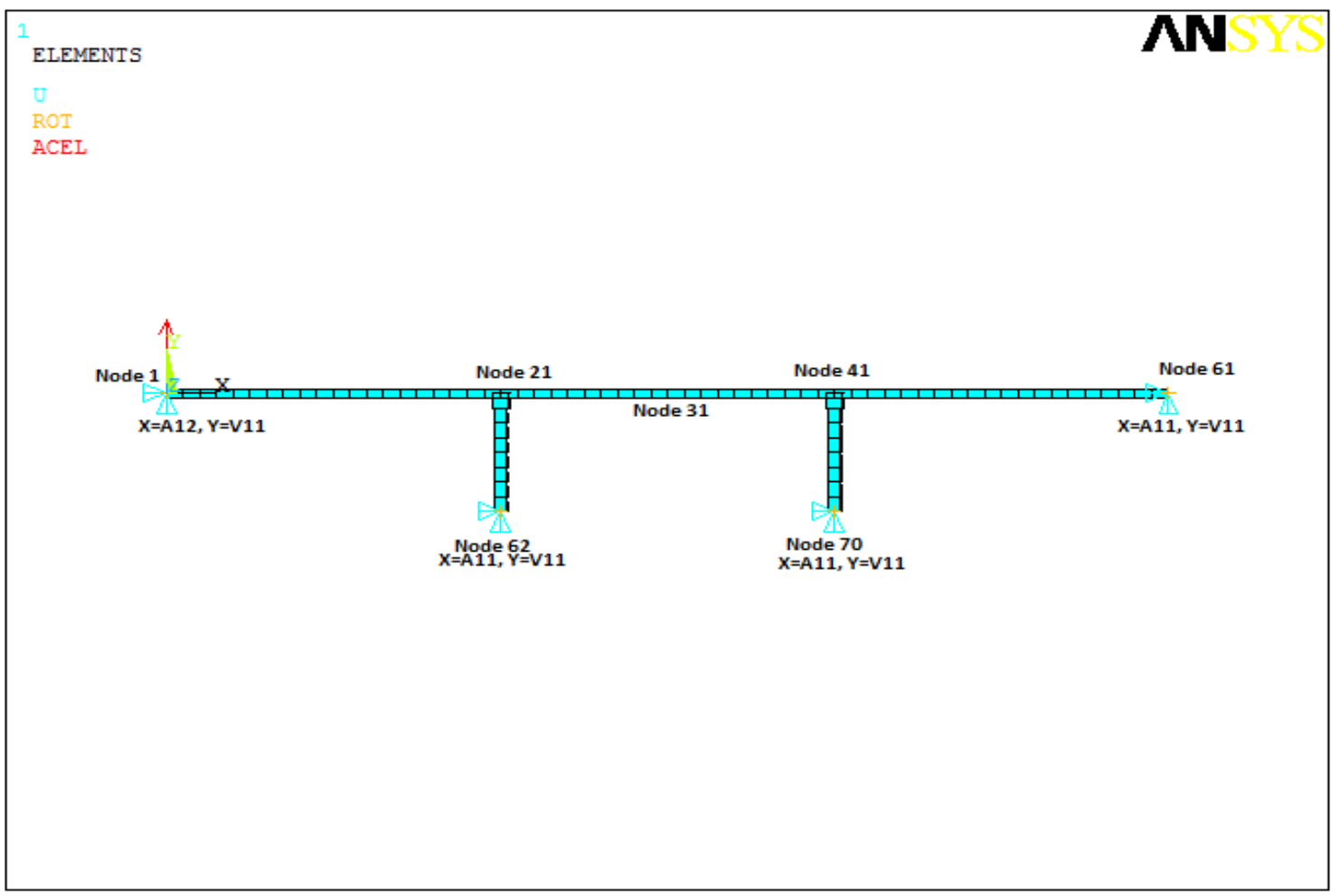

(b). Different acceleration for horizontal 


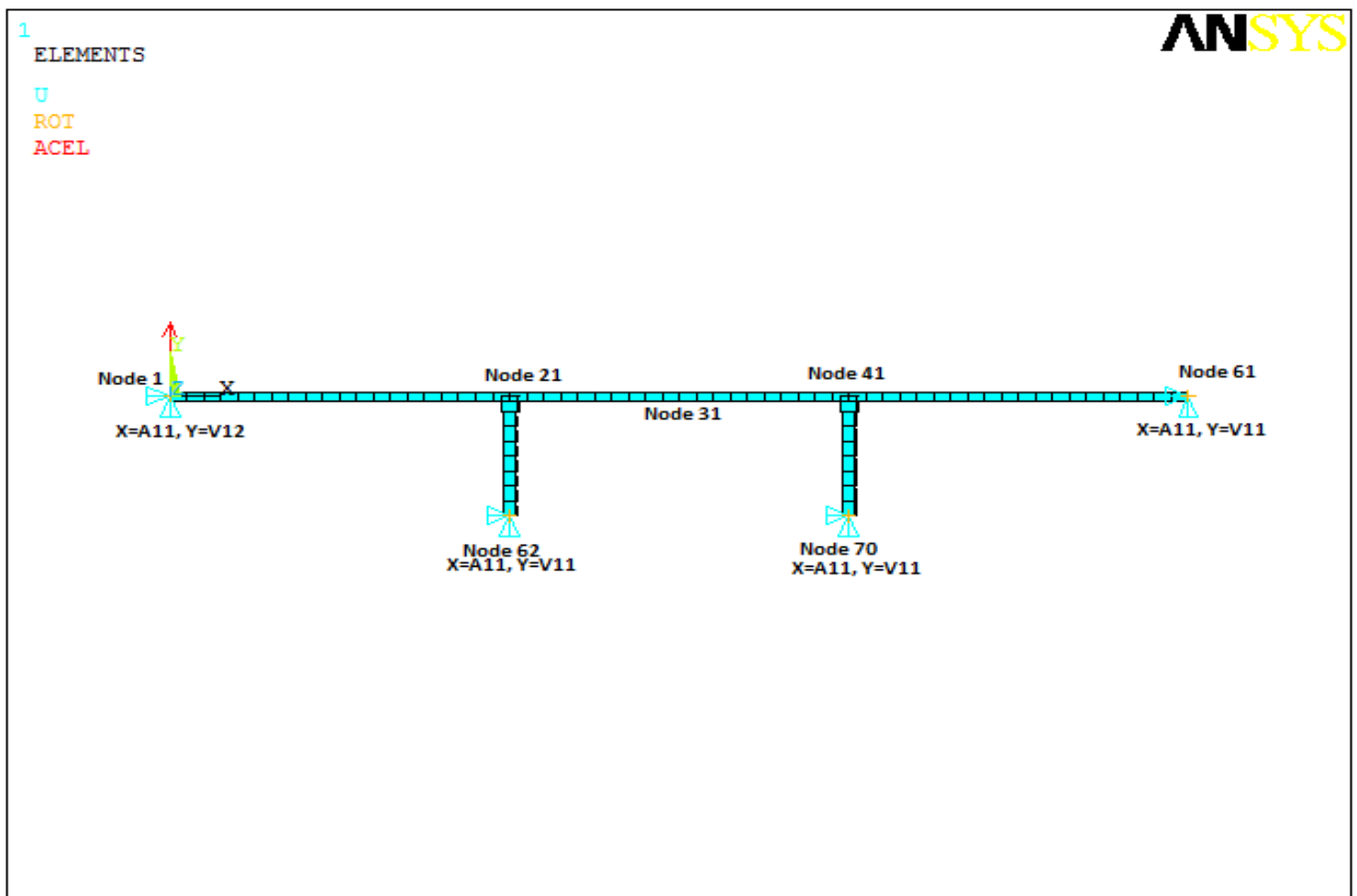

(c). Different acceleration for vertical direction

Figure V-32: Modelling of earthquake acceleration time histories on the supports of the bridge (same acceleration).

As can be seen in Figure V-32 (a), all supports in the bridge structure have the same time history, $V_{11}$ for vertical and $A_{11}$ for horizontal directions. $V_{11}$ refers to the vertical earthquake and random excitation number $1 . A_{11}$ means acceleration time history for soil $\mathrm{A}$ and random spectrum number 1 . These accelerations then created the same displacement to the supports of the bridge. No relative displacements occurred. In other words, in this case, because the acceleration applied to the supports was the same, the reactions and the response of the bridge structure were dynamic only, without a relative displacement contribution.

Figure V-32 (b) shows that the support at node 1 was applied with a time history $V_{11}$ for vertical and $A_{12}$ for horizontal directions. $V_{11}$ refers to the vertical acceleration earthquake and random excitation number $1 . A_{12}$ refers to the acceleration time history for soil $\mathrm{A}$ and random spectrum number 2 . This condition produced the same displacement in the vertical direction, but a different displacement in the horizontal direction. Because of this, a relative displacement in the horizontal direction was achieved. 
It can be seen from in Figure V-32 (c) that, instead of applying the same synthetic time history in a vertical direction, a similar synthetic acceleration was applied in the horizontal direction. A time history $\mathrm{V}_{11}$ was applied to the support at node 1 for the vertical and $A_{11}$ for the horizontal direction. Meanwhile, for other supports, the time histories applied were $V_{12}$ for vertical and $A_{11}$ for horizontal directions, respectively. This is in contrast to the previous horizontal case where other supports had the same acceleration time history for the horizontal $(x)$ direction, but a different acceleration time history in the vertical $(y)$ direction. From this procedure, a vertical relative displacement for the support was achieved by substracting the time history 2 from time history 1 .

Summarizing the above results, two different kinds of treatment were carried out. First, the synthetic acceleration applied was the same for both vertical and horizontal directions, thus there were no relative displacements in the bridge supports. Second, however, the synthetic accelerations applied to the supports were different vertically and horizontally. An example of a synthetic acceleration applied to the bridge (soil type A) can be seen in Figure V-33.
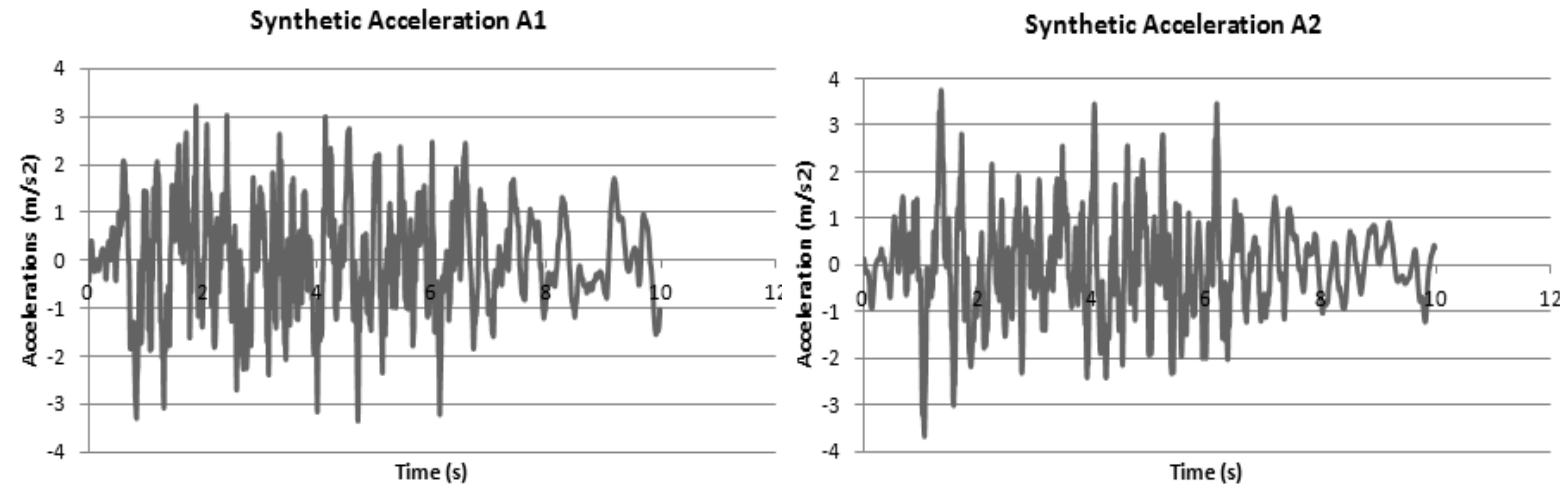

Figure V-33: Two synthetic horizontal accelerations applied to the supports of the bridge

Figure V-33 shows the difference between synthetic acceleration 1 and synthetic acceleration 2. The patterns of excitation for the two accelerations were not the same in either frequency or amplitude. These discrepancies induced variations in the displacements occurring, and relative displacements resulted.

The relative displacements obtained can be seen in Figure V-34. The figure shows two graphs: the left and right plots describe the relative displacement which occurs on the left end of beam and column horizontally and vertically, respectively. This figure is applicable to a fixed base bridge in the horizontal direction. The blue line in the figure is the relative 
displacement occurring in the support of the bridge. Meanwhile, the blue dashed line represents the maximum value of the relative displacement. This uses the example of soil type A; the remaining relative displacement time histories are in Appendix VII.

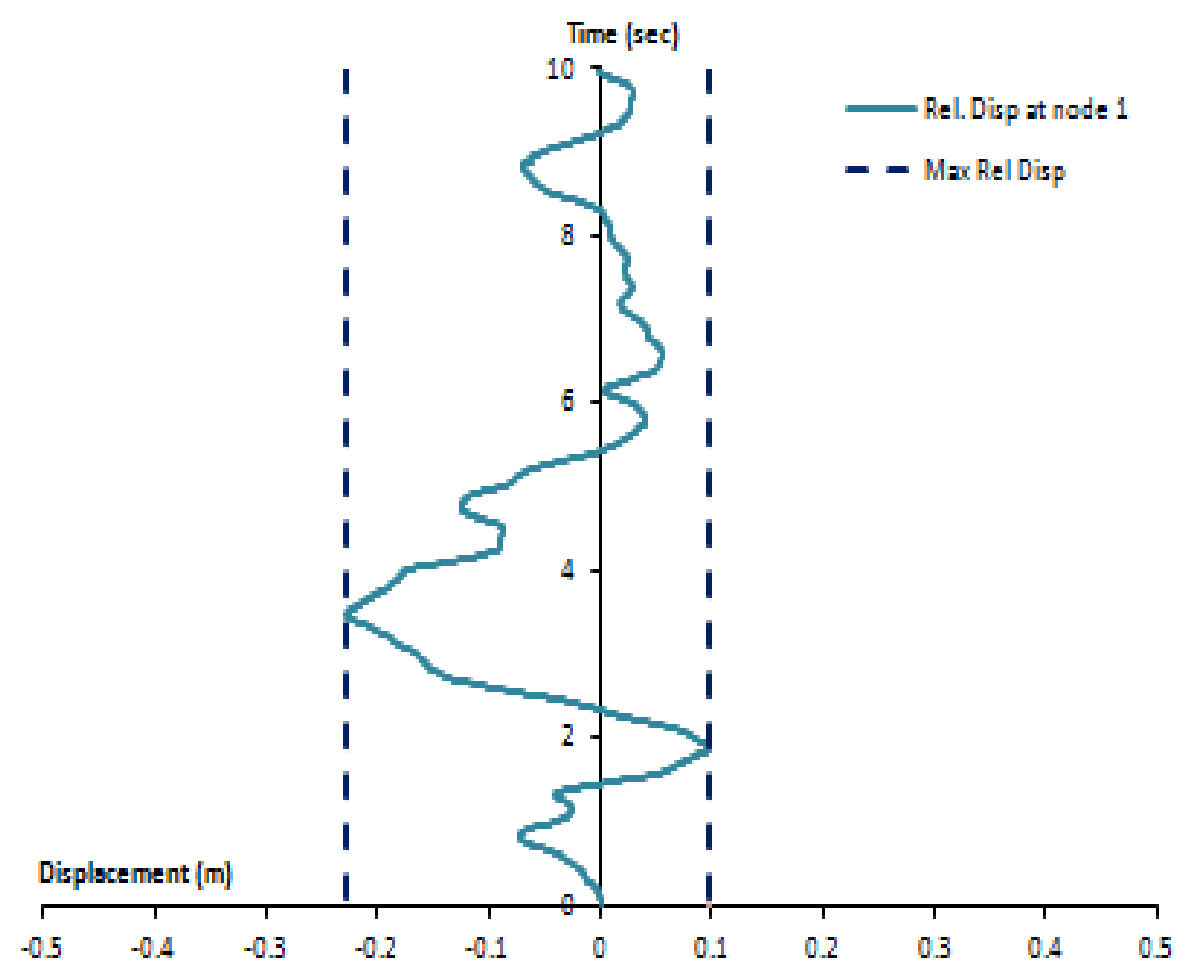

(a) 


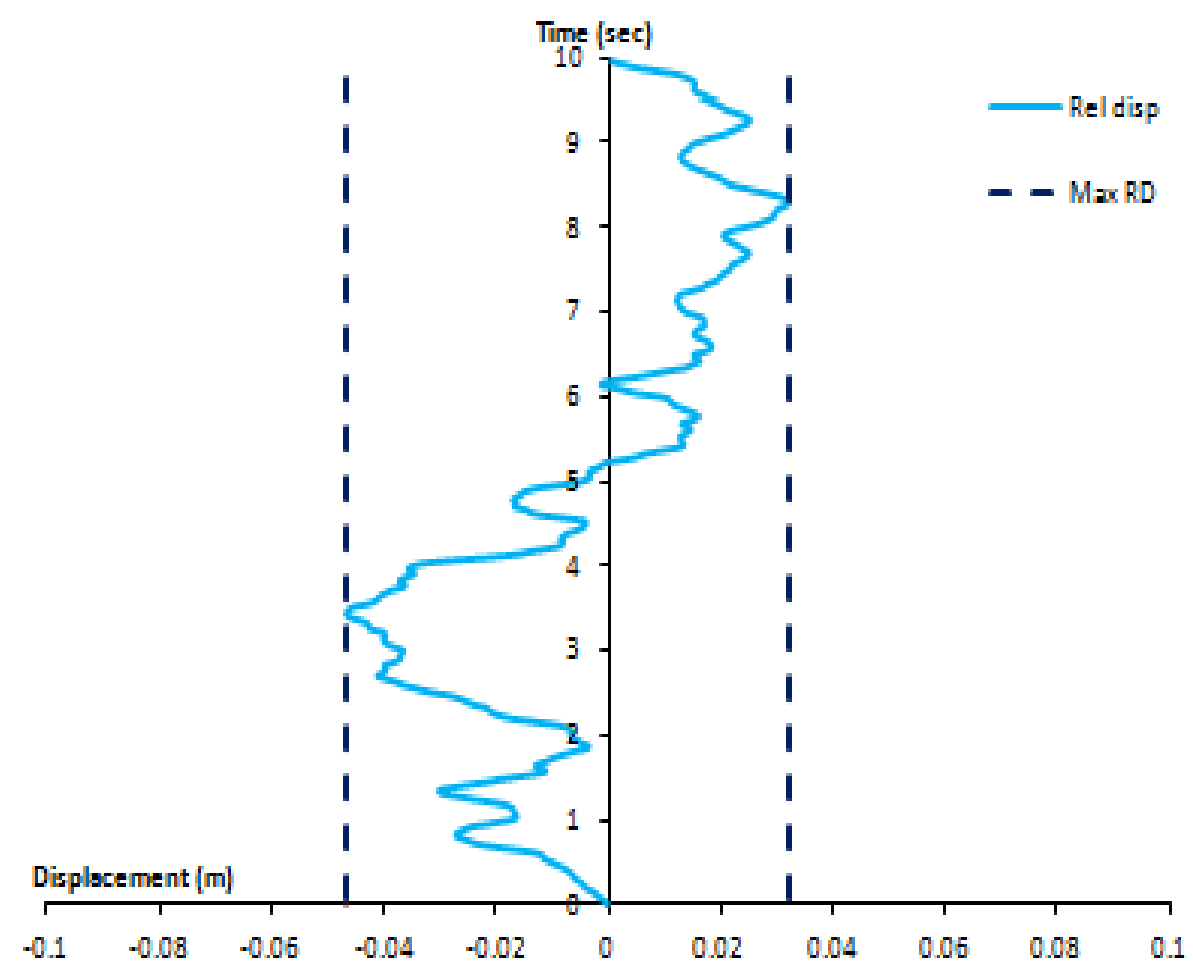

(b)

Figure V-34: Relative displacement for fixed base (a) horizontal and (b) vertical

For spring base, the relative displacements are shown in Figure V-35.

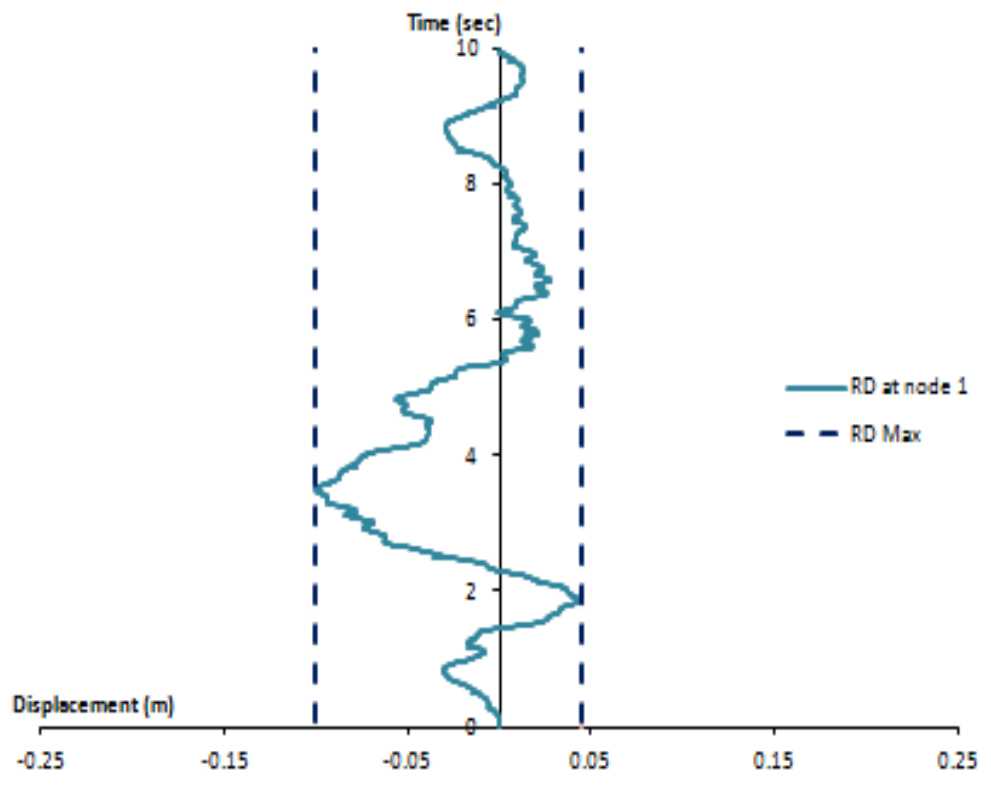

(a) 


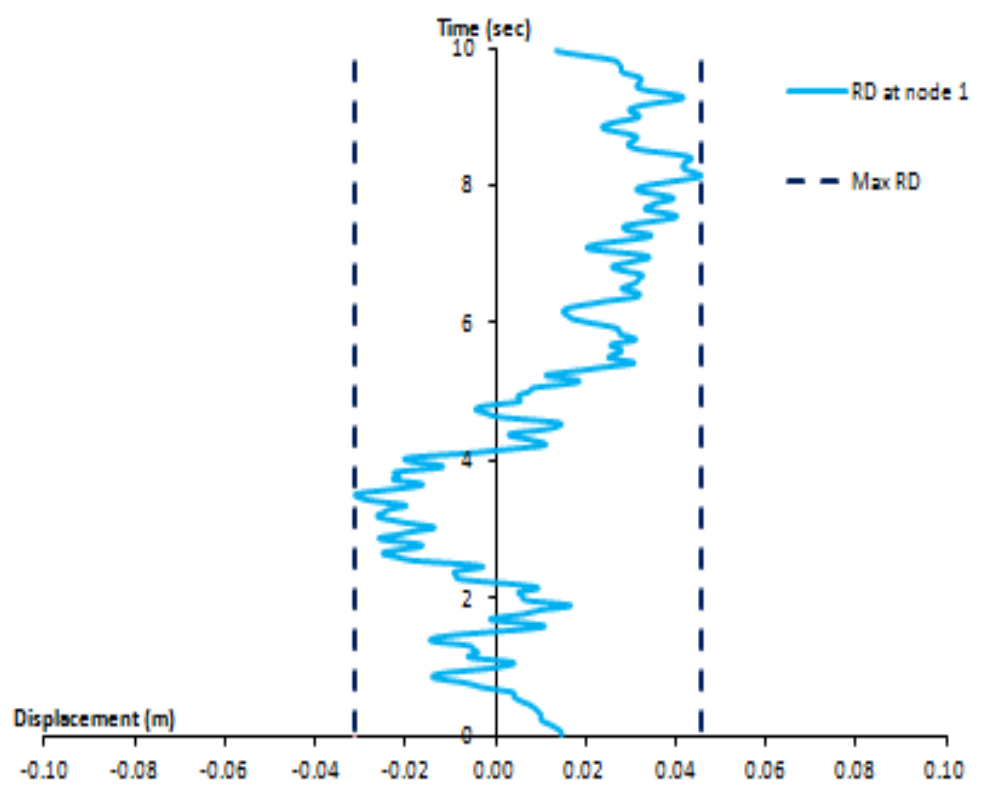

(b)

Figure V-35: Relative displacement for spring base (a) horizontal and (b) vertical

The relative displacement in Figure V-35 has a similar pattern to corresponding displacements in Figure V-34. The difference between the two relative displacements in these figures is that the one for the fixed base is smoother than the relative displacement for the spring base. This is because the stiffness of the structure standing on a fixed base is less than the stiffness of the structure standing on a spring base.

From Figures V-34 and V-35, it can be seen that for the fixed base structure, the relative displacements occurred dynamically on the left support, from $0.0977 \mathrm{~m}$ (right) to $0.228 \mathrm{~m}$ (left), whereas the relative displacement occurred vertically from $0.03 \mathrm{~m}$ (up) to $0.04 \mathrm{~m}$ (down). For the spring base bridge structure, the horizontal relative displacement occurred in the range 0.045 (right) to 0.101 (left) for the largest stiffness, and 0.04 (right) to 0.112 (left) for the smallest stiffness. For vertical relative stiffness in the spring base structure, the relative displacement is in the range of $0.031 \mathrm{~m}$ (right) and $0.046 \mathrm{~m}$ (left) for the largest stiffness, and $0.034 \mathrm{~m}$ (right) and 0.041 (left) for the smallest. The data of these relative displacements are detailed in Appendix VII.

These graphs resulted from the ANSYS transient analysis, by subtracting the displacement that occurred at node 1, which resulted from acceleration time history 1, from the displacement occurring at node 62, which resulted from acceleration time history 2. ANSYS provides a menu to directly subtract the results of one time history from another. 
ヘ ANSYS Multiphysics Utility Menu Eile Select List plot Plotctrls WorkPlane Parar

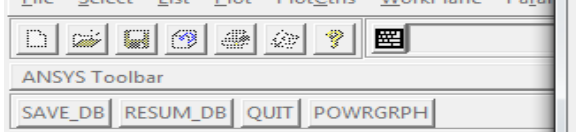
\begin{tabular}{l|l|l|}
\hline SAVE_DB & RESUM_DB & QUTT \\
\hline ANSYSMain Menu
\end{tabular} \begin{tabular}{|c|}
\hline ANSYS Main Mer \\
\hline \hline Preferences \\
$\square$ Preprocessor \\
P Element Typ
\end{tabular} ⿴囗十 Element Type
Theal Constants Material Props 田 Sections

田 Modeling

田 Meshing

田 Checking Ctrls

Numbering Ctris

田 Coupling/Ceq

TIOTRAN Set Up

田 Multi-field Set U

田 Loads

田 Path Operations

田 Solution

田 General Postproc

⿴囗十 TimeHist Postpro

田 Topological Opt

田 ROM Tool

$\boxplus$ Design Opt

Prob Design

Tun-Time Stats

Session Editor

Finish

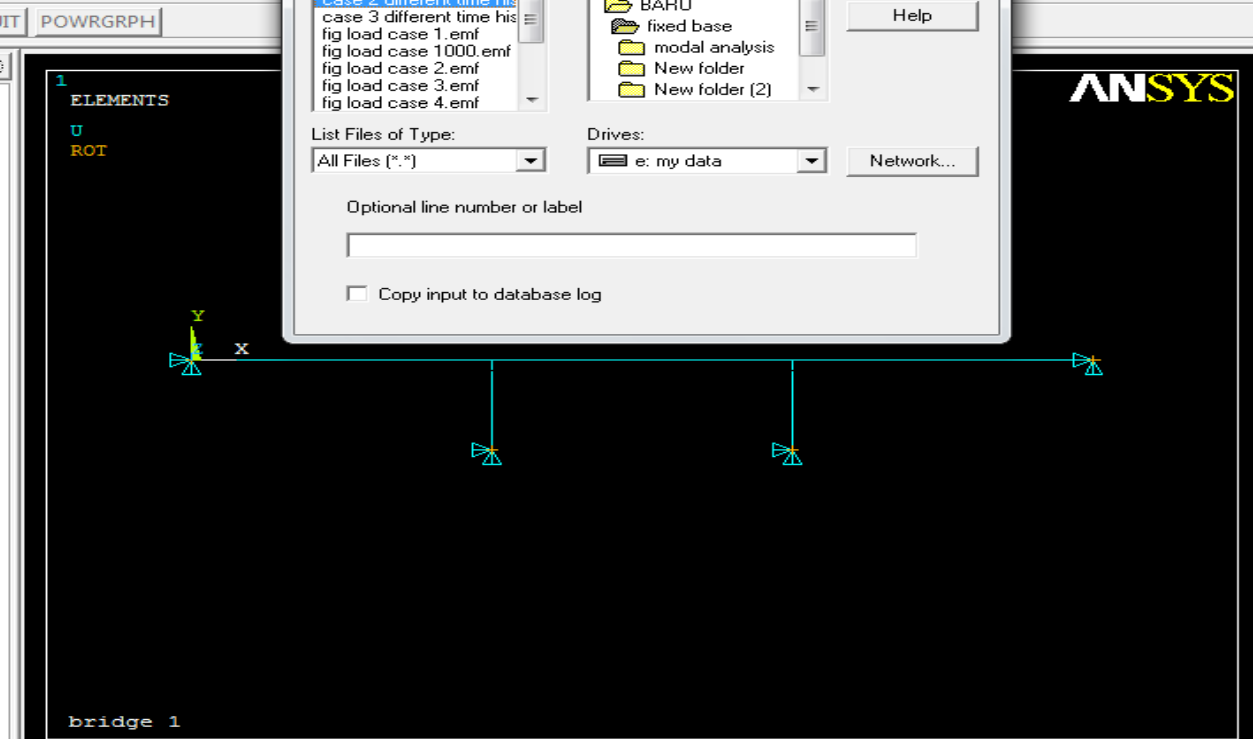

(a)

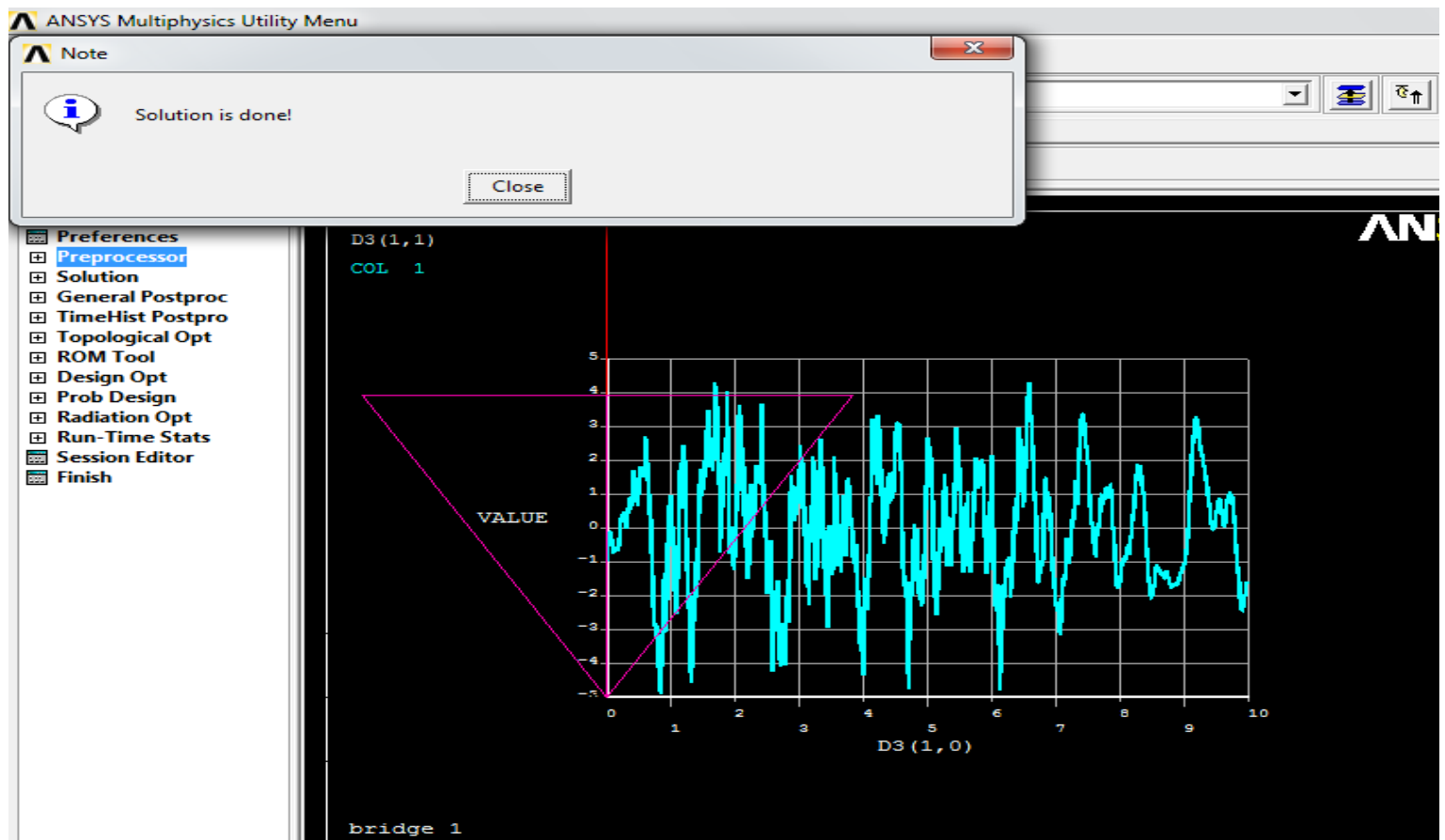

(b) 


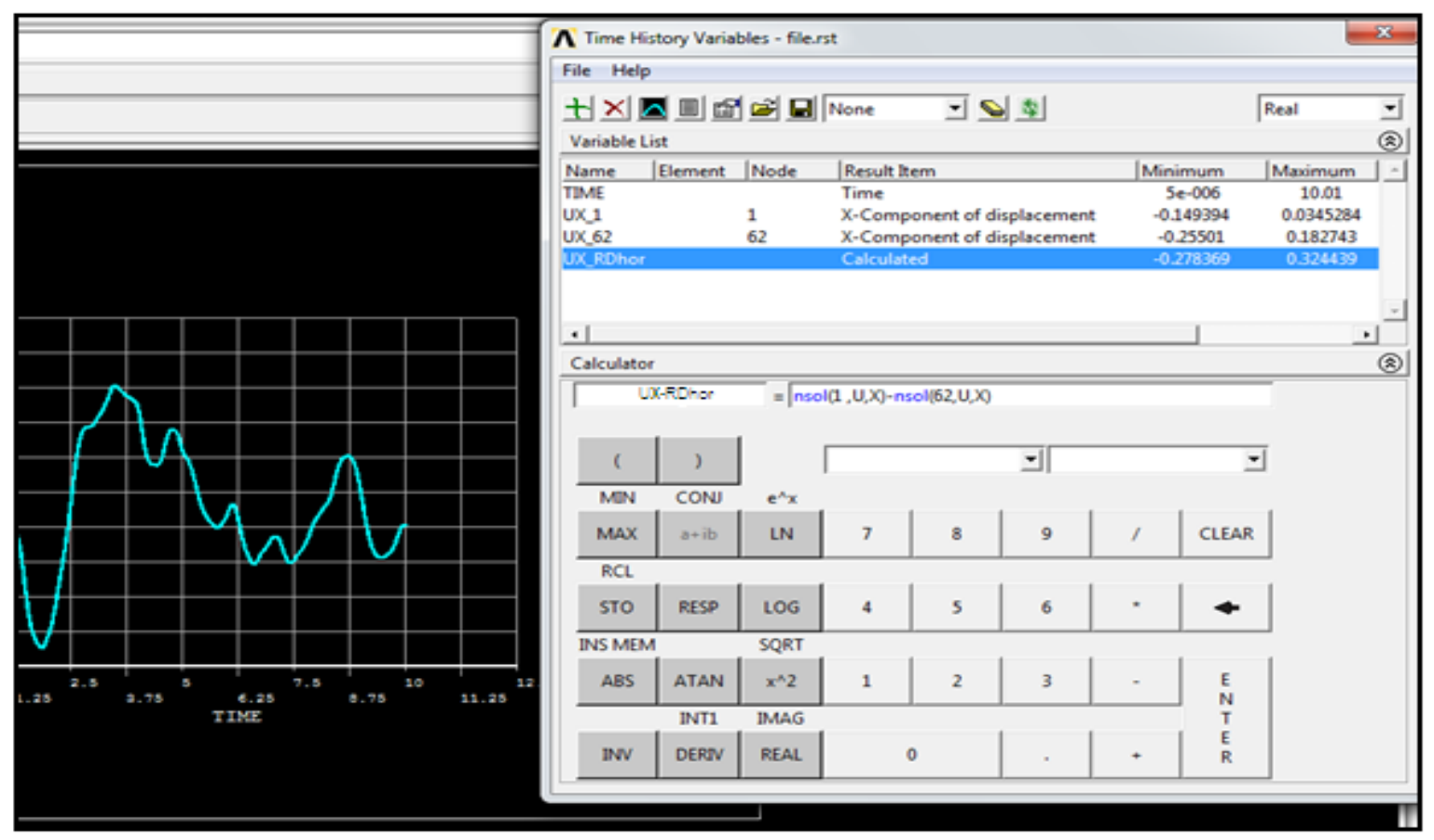

(c)

Figure V-36: ANSYS procedures to subtract the two displacement time histories for obtaining relative displacement.

Figure V-36 shows the ANSYS procedure to obtain the relative displacement at a particular node; the displacements of two adjacent supports can be determined. These two displacements then were analyzed to obtain the relative displacements between the two supports. Figure V-36 (a) shows how to input the transient analysis code, which was written in a text extension and stored in a folder. The codes used in all the analysis are presented in Appendix V. Figure V-36 (b) describes the ANSYS performance while processing the input code and data, and Figure V-36 (c) uses the analysis facility of ANSYS to subtract the two displacement time histories as shown.

To summarize the content of this chapter, generating synthetic time histories is an important step in this research. These artificial time histories can be stored in a bank of time-series, which proves useful in recognizing the earthquake ground motion which is then applied to the supports of the bridge structure. Modal analysis also plays a significant role in the study, as it is a very useful tool in dynamic analysis. By conducting modal analysis, the characteristics of the structure, such as natural frequency and period, can be determined. These characteristics are crucial to the next step of dynamic analysis. Relative displacements are achieved by varying the earthquake ground motions applied to the 
supports. After these prerequisites have been achieved, analysis to determine the response of the bridge under vertical and horizontal earthquake ground motion can be conducted. 


\section{Chapter VI \\ RELATIVE DISPLACEMENT EFFECTS ON THE BRIDGE}

\subsection{General}

The deformation of a bridge which occurs during a seismic excitation is due to the forced motion of its foundations, resulting in the oscillation of the structure. Penelis and Kappos (1997) outline a procedure during which an amount of kinetic energy is imparted to the structure in the form of elastic deformation. Therefore, while the main consideration in the design of a structure for static loading is strength, in seismic design equally important factors are the flexural stiffness of the structural elements, their ability to deform (ductility), and the mass of the structure. Thus, there is an important correlation between bending as an internal force and displacement in the bridge. This correlation is used to explain the contributions of static relative displacement to the overall bending moments as a result of the earthquake ground motion.

The base of the structure moves with the ground during an earthquake with a ground displacement of $u_{g}(t)$, as shown in Figure VI-1 (Sucuoglu \& Akkar, 2011). In equation VI$1, \ddot{u}^{\text {total }}$ is the sum of ground acceleration and the acceleration of the mass relative to the ground.

$$
\ddot{u}^{\text {total }}=\ddot{u}_{g}+\ddot{u}
$$

Equation. VI-1

There is no direct external force $F(t)$ acting on the mass when the ground moves, but inertial force develops on the mass according to the second law of Newton $(F=m a)$, where $a$ is the total acceleration of the mass $m$.

The free body diagram of the mass is shown in Figure VI-1. 


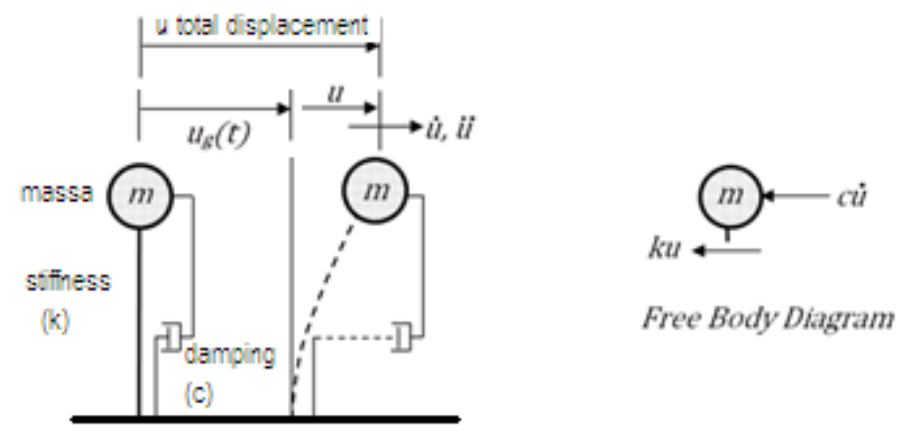

Figure VI-1: Free body diagram for equation of motion (Sucuoglu \& Akkar, 2011)

Then, by considering the second law of Newton, $\Sigma F=m \ddot{u}^{\text {total }}$ yields:

$$
\begin{aligned}
& -c \dot{u}-k u=m \ddot{u}^{t o t a l}, \text { or } \\
& m\left(\ddot{u}_{g}+\ddot{u}\right)+c \dot{u}+k u=0 .
\end{aligned}
$$

Equation. VI-3

Thus, the equation of motion for a single degree of freedom can be written as:

$$
m \ddot{u}+c \dot{u}+k u=-m \ddot{u}_{g}(t),
$$

where $m$ is the mass of the structure, $\ddot{u}$ is the acceleration of the structure, $c$ is the damping factor, $\dot{u}$ is the velocity, $k$ is the stiffness of the structure and $u$ is the displacement relative to the ground.

The equation consists of four basic forces: inertial, damping, stiffness and external forces. Inertial forces represent the forces that come from the acceleration of the system. The damping force acts when there is a relative velocity between the mass and the ground. For linear viscous damping, this force is directly proportional to the velocity, and the constant of proportionality is the damping coefficient. The stiffness force acts on the elements of the structure when there is a displacement of the mass. For a linear system, this force is directly proportional to the relative displacement of the top and bottom ends of the supports. Therefore, it can be seen that there are three components involved in a motion of a structure, namely mass, damping factor and stiffness factor. Other terms involved are acceleration, velocity, and displacement of the structure.

For relative displacement, the equation can be written as: 


$$
\begin{gathered}
m \ddot{u}+c \dot{u}+k\left(u_{1}-u_{2}\right)=-m \ddot{u}_{g}(t), \text { or } \\
m \ddot{u}+c \dot{u}+k \Delta u=-m \ddot{u}_{g}(t) .
\end{gathered}
$$

Different displacements are imparted to the different supports of the bridge and this condition results in relative displacements.

Theoretically, for a system with a single support excitation the total displacement of the structure is achieved by adding the input ground motion with respect to the support; however, for multi-support excitation the total displacements of the superstructure can be expressed as the summation of the relative displacements $\left(x_{s}\right)$ that will result from a static support displacement. Static support displacement is the displacement produced by quasistatic motions on the support.

The equation is as follows:

$$
x^{t}(t)=x(t)+x_{s}(t)
$$

The quasi-static displacements can conveniently be described by an influence coefficient vector $\Gamma$, which represents the displacements resulting from the unit support displacements.

Therefore,

$$
x_{s}=x(t)+\{1\} x_{g}(t)
$$

For a multi-degrees of freedom system, the equation of motion can be expressed as follows:

$$
\left[\begin{array}{ll}
M_{s S} & M_{s g} \\
M_{g s} & M_{g g}
\end{array}\right]\left\{\begin{array}{l}
\ddot{x}^{t} \\
\ddot{x}_{g}
\end{array}\right\}+\left[\begin{array}{ll}
C_{s s} & C_{s g} \\
C_{g s} & C_{g g}
\end{array}\right]\left\{\begin{array}{l}
\dot{x}^{t} \\
\dot{x}_{g}
\end{array}\right\}+\left[\begin{array}{ll}
K_{s S} & K_{s g} \\
K_{g s} & K_{g g}
\end{array}\right]\left\{\begin{array}{l}
x^{t} \\
x_{g}
\end{array}\right\}=\left\{\begin{array}{c}
0 \\
F_{g}
\end{array}\right\}, \quad \text {....... Equation. VI-9 }
$$

where:

$\mathrm{M}_{\mathrm{ss}} \quad$ = the mass matrix corresponding to superstructure (non-support) degrees of freedom.

$\mathrm{M}_{\mathrm{gg}} \quad$ = the mass matrix corresponding to the support degrees of freedom.

$\mathrm{M}_{\mathrm{sg}}$ and $\mathrm{M}_{\mathrm{gs}} \quad=$ the coupling mass matrices that express the inertia forces in superstructure degrees of freedom due to the motions of the supports. 
The term damping and stiffness matrices are defined in similar ways:

$$
\begin{array}{ll}
\mathrm{x}^{\mathrm{t}} & =\text { the vector of the total displacements corresponding to superstructure } \\
& \text { degrees of freedom. } \\
& =\text { the vector of the input ground displacements at the supports. } \\
\mathrm{x}_{\mathrm{g}} & \dot{x}^{t}, \dot{x}_{g}, \ddot{x}^{t}, \ddot{x}_{g} \\
\mathrm{~F}_{\mathrm{g}} & =\text { the velocity and acceleration vectors defined in similar ways. } \\
& =\text { the vector of forces generated at the support degrees of freedom. }
\end{array}
$$

Two cases were carried out in this study, case 1 for a fixed base study, and case 2 for a spring base study. The same synthetic acceleration data was applied to the two cases. Applying springs to the supports of the bridge reduces the stiffness of the bridge, and how this stiffness affects the structure behaviour can then be distinguished. Modelling of the system also has differences. For the fixed base structure, the synthetic accelerations are applied at the support nodes, whereas for the spring base structure, the synthetic accelerations are applied to the ends of the soil springs (which are essentially the support nodes of the bridge).

\subsection{Effects of relative displacement on bending moments in the bridge}

When a bridge suffers consistent seismic excitation, the motion at different pier/abutment bases is the same because of the basement rigidity, and the internal structural force is caused by its only dynamic response. It is known that every supporting point of one structure moves in the same way as base does, therefore the structure itself does not suffer external dynamic loads during an earthquake. However, when the bridge suffers to real seismic excitation, this condition will create relative displacements on the bridge.

In other words, when a bridge is subject to equal displacement at its supports, the dynamic moments and forces within the structure are due solely to dynamic resonance effects. Due to the distance between the supports, it is likely that relative displacement will occur, resulting in additional bending moments and forces to that produced by the dynamic.

This study is based on single node relative displacement. In reality the displacement are likely to be different at each node. The amount of scenarios in this procedures is 
considerable, and so it is logical to reduce this to a set of scenarios which hopefully represent a worst enveloping case. By displacing one node only relative to the others, insight is gained as to its effect and this can be predicted by simple hand calculation.

By observing resonant mode shapes of importance enquiries judgement can exercised as to the wost case scenarios of relative support displacement. In practice, it would be expected that a 'design' relative displacement would be chosen between nodes and applied statically to a structure.

Regardless of location of the application of relative displacement, the effects of moment growth at supports and joints would be observed, whilst mid-span moments would tend to be least affected. So, by applying relative displacement to each node in turn, provides enveloping cases of moments and forces which would ever be likely to develop in the structure.

\subsubsection{Case 1 (Fixed Base, Without Springs)}

The first case to be analyzed is a structure with fixed end supports. All four supports of the integral bridge are fixed in translation and rotation. The modelling and node configuration for case 1 are as shown in Figure VI-2.

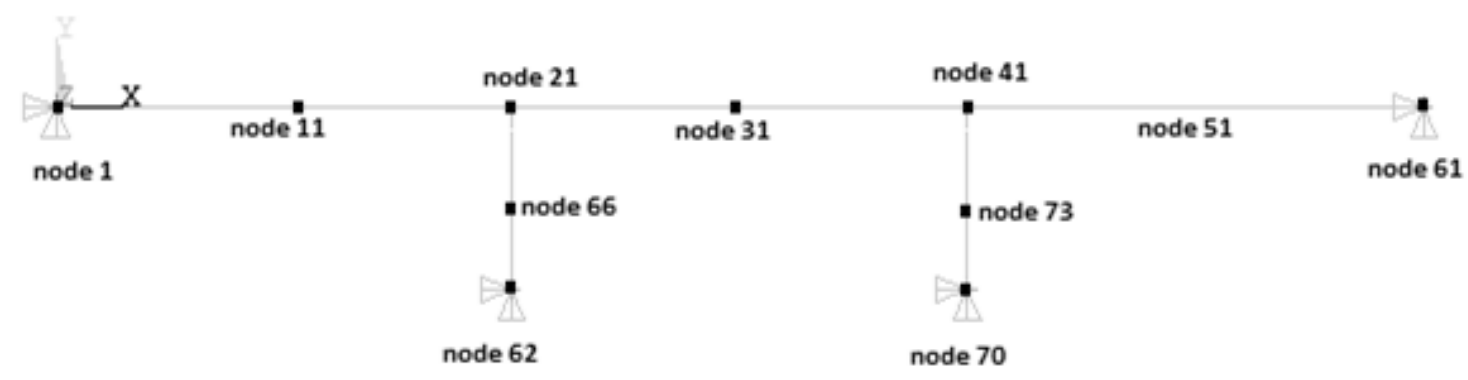

Figure VI-2: Nodes configuration of the structure (fixed base)

\subsubsection{Static Analysis}

One of the purposes of this research is to investigate the behaviour of integral bridges under vertical and horizontal earthquake ground motions, and the final aim is to propose a simplified design or appraisal of this type of bridge under dynamic loads. Therefore the static relative displacement analysis will be compared to a dynamic analysis.

In addition to normal static analysis under gravity alone, relative displacement analyses were carried out. Static analysis of the bridge was conducted by applying relative 
displacements to the left end of the beam and column of the structure (node 1). The relative displacements are in the range between $-0.25 \mathrm{~m}$ to $0.25 \mathrm{~m}$ in the $x$ (horizontal) and $y$ (vertical) directions respectively. The idealization of relative displacement in the $x$ and $y$ direction can be seen in Figures VI-3 (a) and (b).

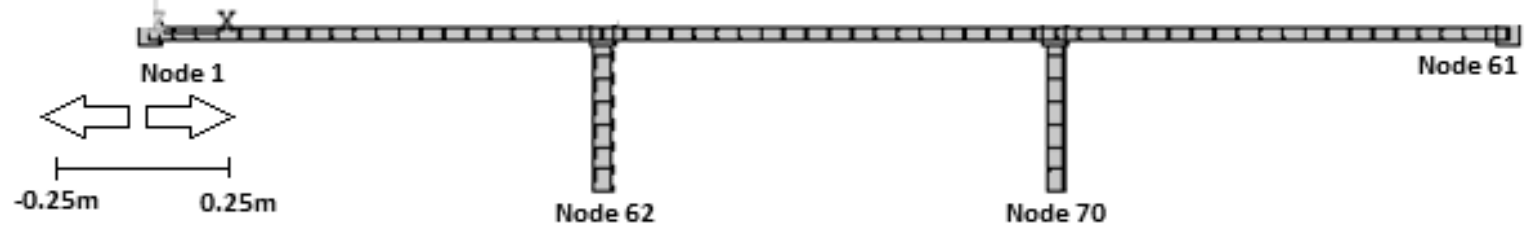

(a) Static relative displacements applied to the bridge in the $x$ direction

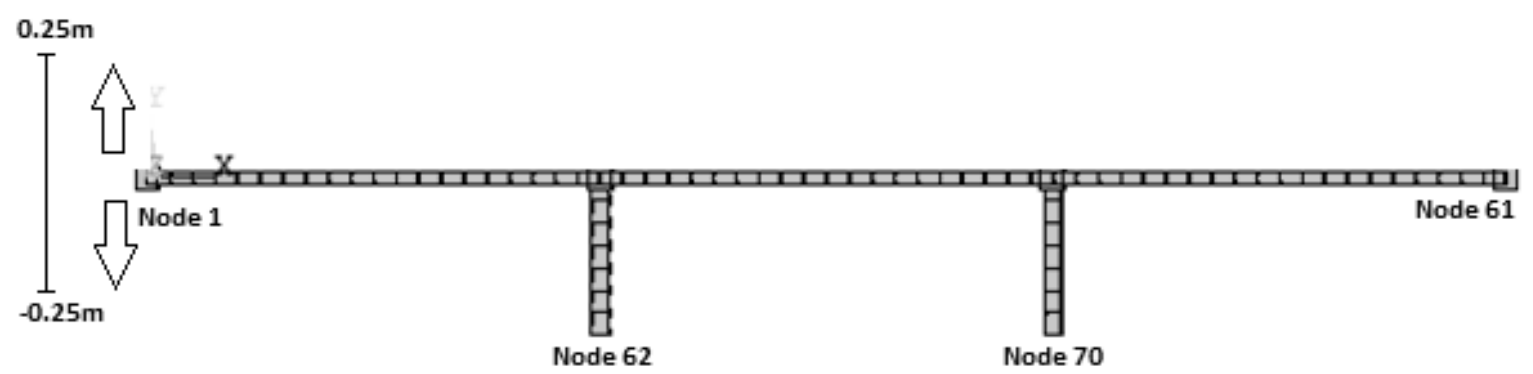

(b) Static relative displacements applied to the bridge in the $y$ direction

Figure VI-3: Static relative displacements applied to the bridge

Figure VI-3 shows how the relative displacements were applied statically to the structure from a range of $-0.25 \mathrm{~m}$ to $0.25 \mathrm{~m}$ horizontally and vertically. Because the loads are static, so the patterns of the moments governed by this static analysis are also expected to be linear. Hence, when the relative displacement applied to node 1 is increased, the moment resulting in node 1 also rises linearly, as the analysis and the load are also linear. For other nodes, the moments will either increase or decrease depending on the position of the nodes and the relative displacements.

Attaching a support to a free body will create reaction forces. The supports that prevent translation will create a force opposite to the direction of translation; meanwhile, supports that prevent rotation will yield coupled moments opposite to the direction of rotation. 
There are differences in the bending moment diagrams for flexible support, semi-rigid support and rigid support, as can be seen from Figure VI-4.

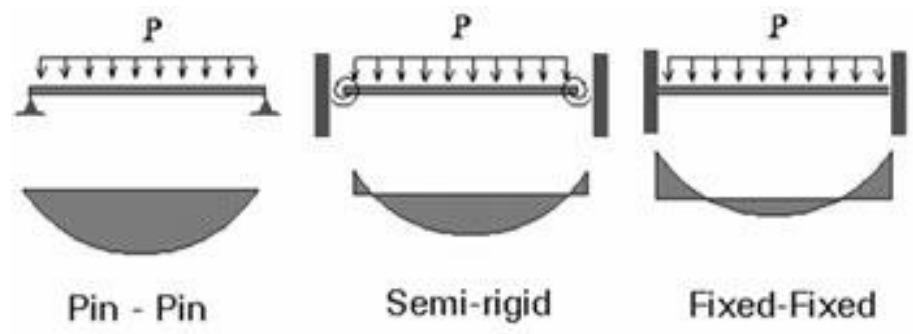

Figure VI-4: Bending moment diagram for different support beams

Rigid supports induce bending moments in them because the deflection and rotation is restricted there. The more rigid the supports, the greater are the bending moments. Thus, a semi-rigid support will create less bending moments than full rigid supports.

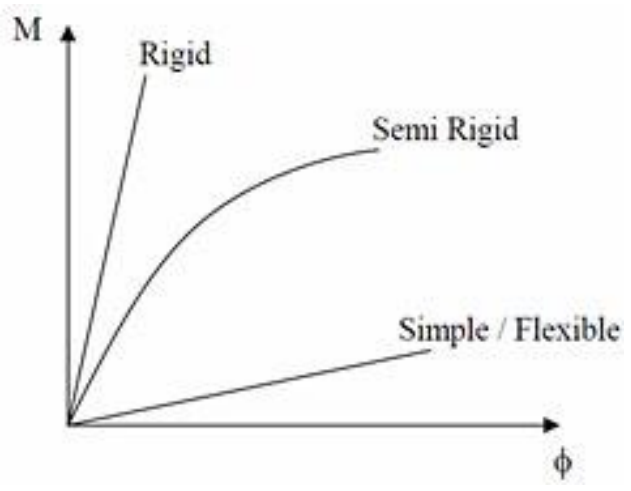

Figure VI-5: Comparison between $\mathrm{M}$ - $\varphi$ Curves for rigid, semi-rigid and simple / flexible connection

To sum up, Figure VI-5 describes the relation between moments $M$ and the degree of rotation $\varphi$ of the supports. A rigid support structure will create high bending moments and small rotation, whereas a simple or flexible support will create fewer bending moments and large rotation.

Figure VI-6 shows the behaviour of a fully symmetrical rigid bridge under a static load. A similar moment has occurred at the end of the bridge, in size and in direction. This is the pattern of static moment of the structure system. It is also the pattern of the dynamic moment occurring in the structure. 


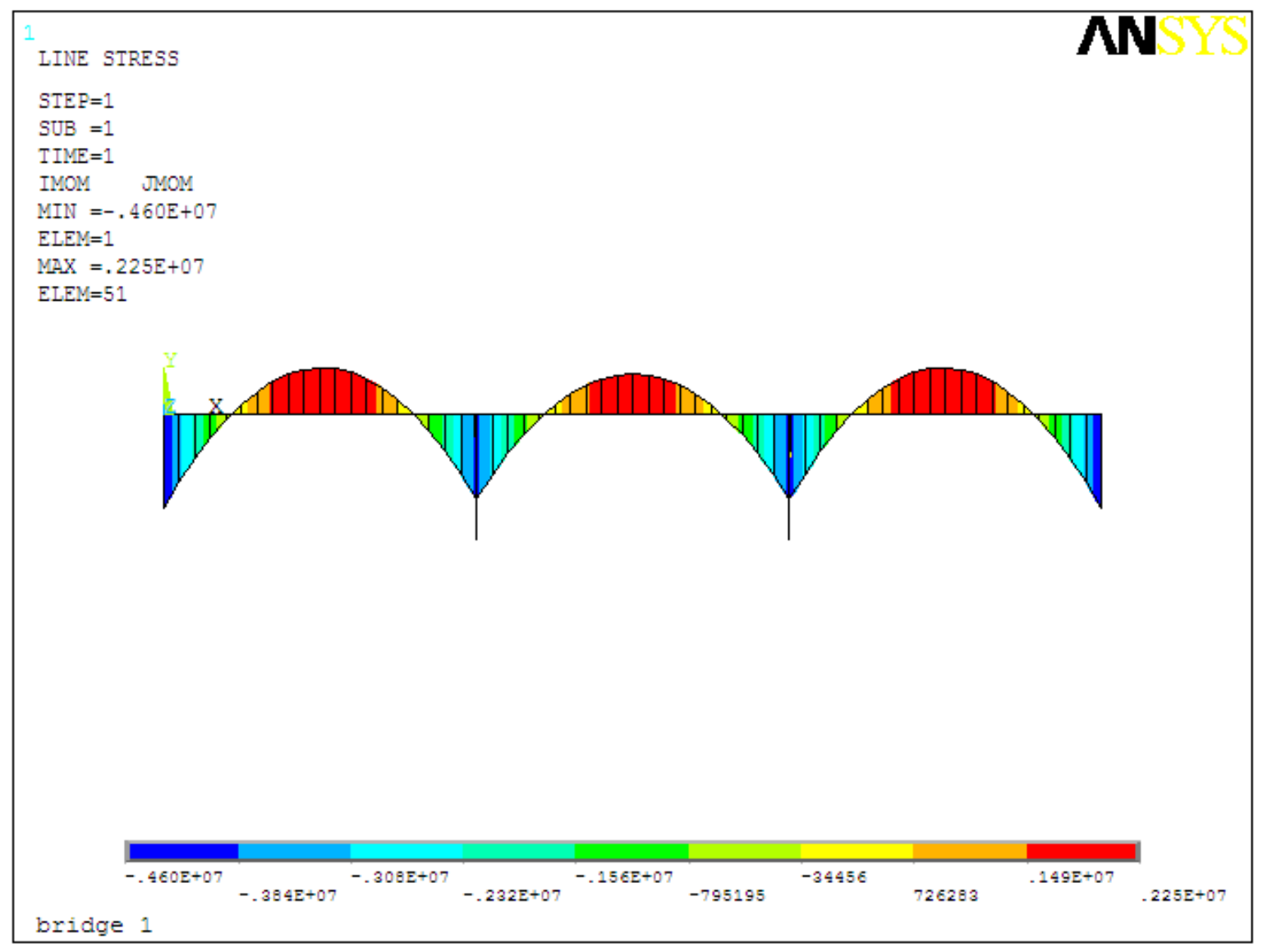

Figure VI-6: Bending moment diagram for gravity load (fixed base)

The bending moment diagram in Figure VI-6 shows how the gravity loads to an element of a bridge structure created a moment variation along the length of the structure and give rise to the location of the critical section. The bending moment at a section through a structural element may be defined as the sum of the moments about that section of all external forces acting to one side of that section. The forces and moments on either side of the section must be equal in order to counteract each other and maintain a state of equilibrium, so the same bending moment will result from summing the moments, regardless of which side of the section is selected.

Applying a relative displacement to a given support of the structure will also change the magnitude of the bending moment along the element. 

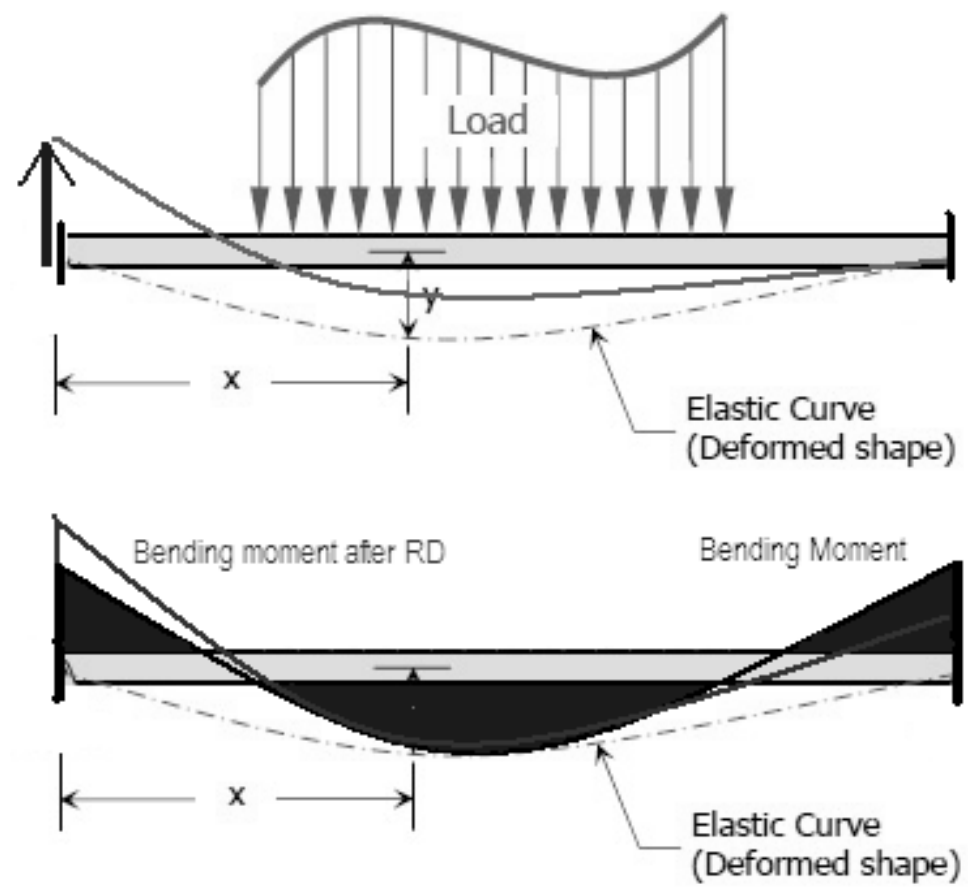

Figure VI-7: Bending moment diagram for beam with relative displacement

Figure VI-7 shows how the symmetrical beam's bending moment changes the pattern and the magnitude of the beam before and after applying the relative displacement on the support. The relative displacement obviously also accommodates the elastic curve of the beam. The elastic curve is defined as the curve assumed by the longitudinal axis of an originally straight elastic beam element bent within its elastic limits by any system of forces.

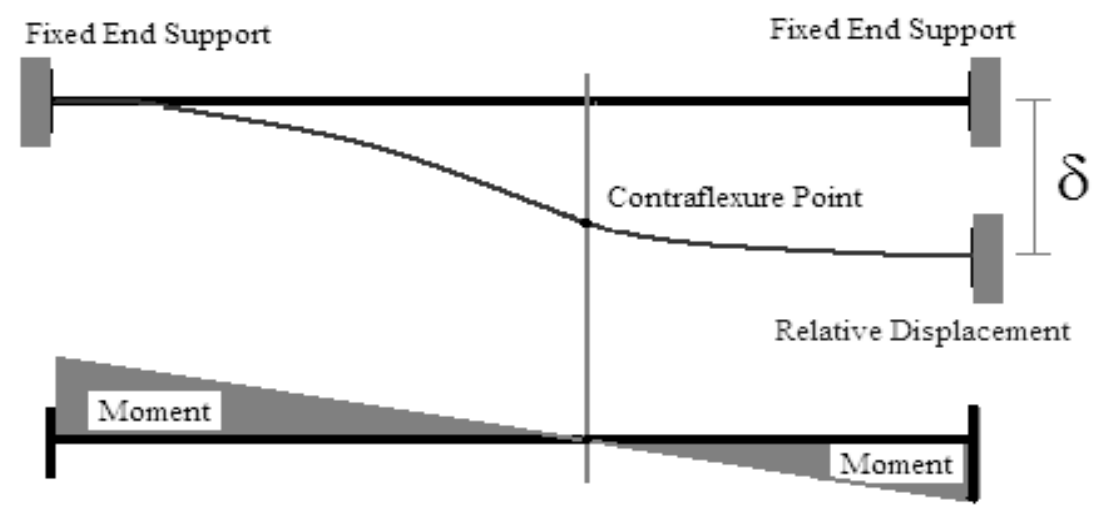

Figure VI-8: Bending moment diagram for relative displacement loading 
It can be seen from Figure VI-8 that supports or joints will be most affected from vertical relative displacements related to bending moments. Meanwhile, in the middle of the element, relative displacement has less effect.

For the bridge structure in this study, Figure VI-9 describes the bending moment patterns occurring in the structure due to self-weigh loads and relative displacements at one support, referred to in Figure VI-3. Figure VI-9 shows the differences in the bending moment diagram for both horizontal and vertical directions.

It can be seen from Figure VI-9 (a) that for horizontal relative displacement, the largest bending moments occur in the left pier support (node 62), because this area has the larger moment from the force applied. The moments are then distributed along the members. Meanwhile, for vertical relative displacement, Figure VI-9 (b), the largest bending moment occurs in node 1 , which is the area where the vertical relative displacement is applied. Remote from the node at which relative displacement has occurred, the moments are reduced due to the distribution of stiffness of multiple members framing into the adjacent joints.

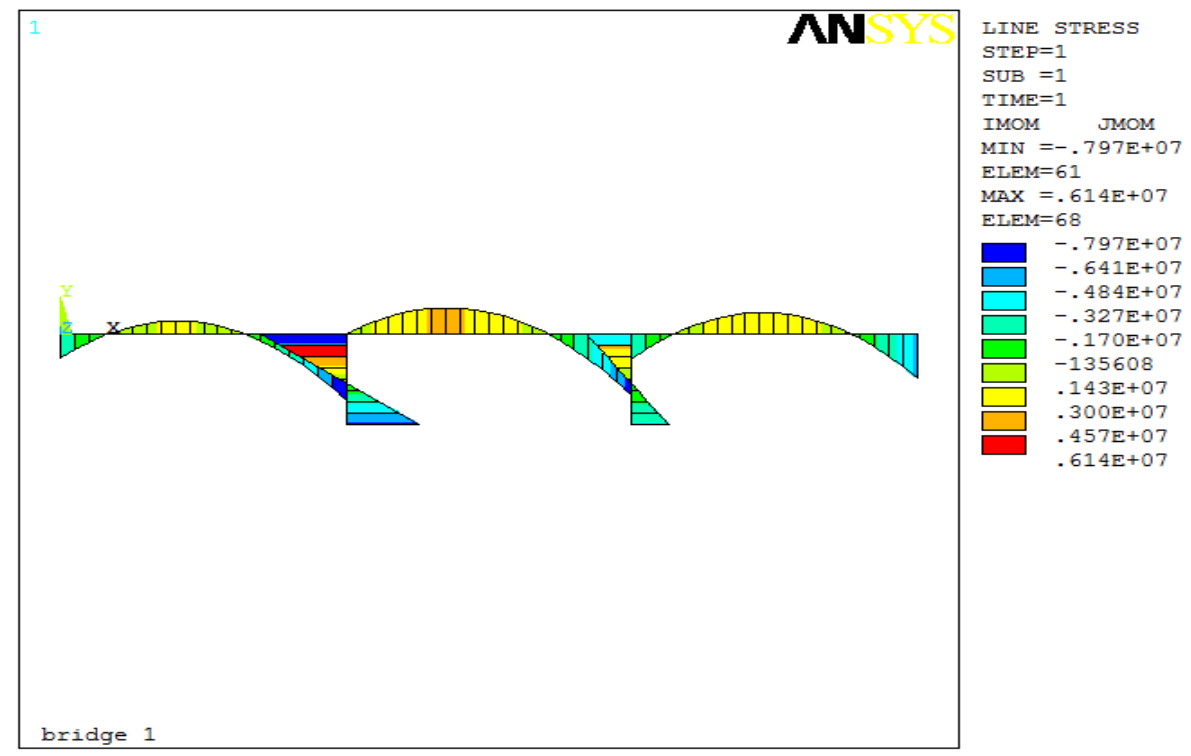

(a) 


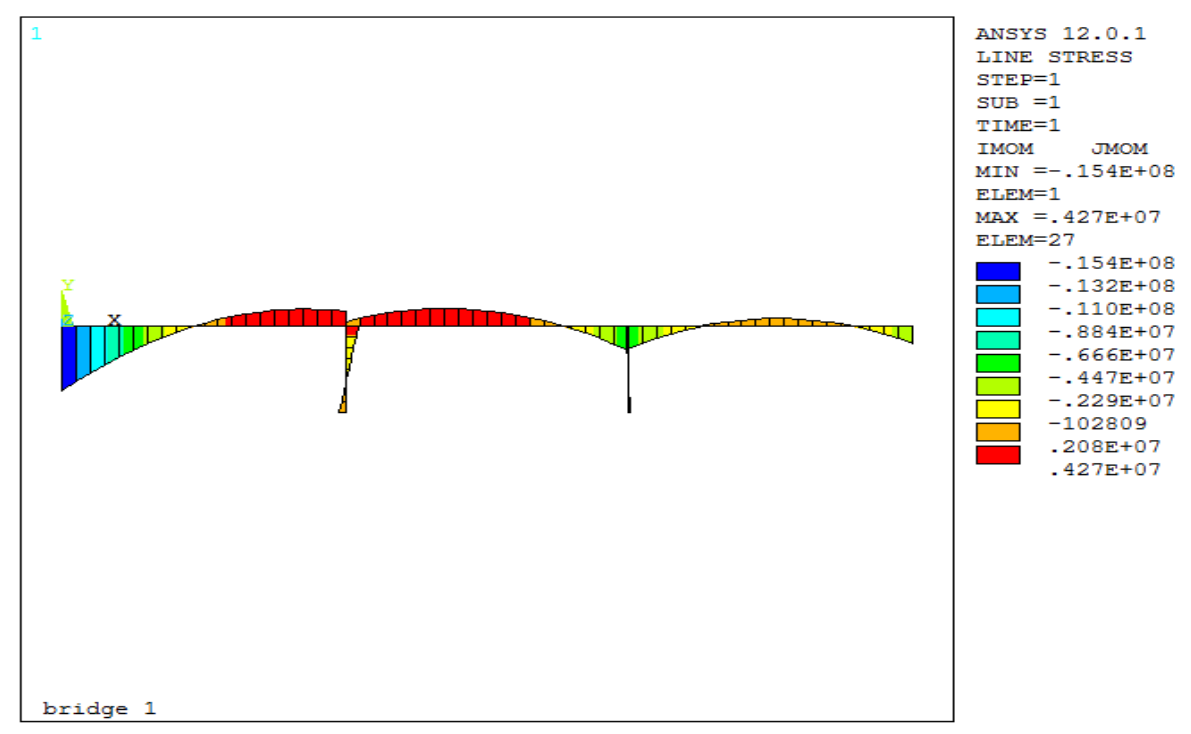

(b)

Figure VI-9: Bending moment diagram for fixed base bridge with relative displacement (a) horizontal relative displacement, (b) vertical relative displacement.

There are two reasons for the static analysis. First, the behaviour of the structure in general due to static load (self-weight, superimposed load, traffic load, and relative displacements) can be observed. Secondly, it yields basic reference graphs which are useful in describing the relationship between moment and displacement. The reference graphs can be used to synchronize the moments resulting from dynamic analysis with the moments resulting from static analysis relating to the static and dynamic relative displacement. It can be predicted logically that dynamic bending moments must be larger compared to static bending moments. This is because static bending moments only have one component $(\mathrm{ku})$ : stiffness and displacements to be transferred as moments; whereas dynamic bending moments have two additional components besides $(k u)$, namely mass time-dependent component acceleration $(m \ddot{u})$, and damping time-dependent component velocity $(c \dot{u})$.

\subsubsection{Relative Displacements as Sensitivity Study for Fixed Base Bridge}

The support of a highway bridge may have to cater for the effects of differential vertical displacements and differential horizontal movements longitudinally and transversely, depending on the loadings producing the movements. Horizontal ground movements alter the moment acting on the elements of the bridge, such as the deck. Due to these movements, the moments occurring in the bridge structure may decrease or increase, 
depending on the position of the observed areas. They may produce failure as a result of bending.

To learn how the bridge behaves due to static relative displacements, static analysis was conducted to observe the pattern of the bending moments at certain locations in the bridge. Relative displacement static analysis was carried out to obtain the trend line of structural moments. These trend lines are then used as the baseline of static moments resulting from dynamic relative displacement in the dynamic analysis.

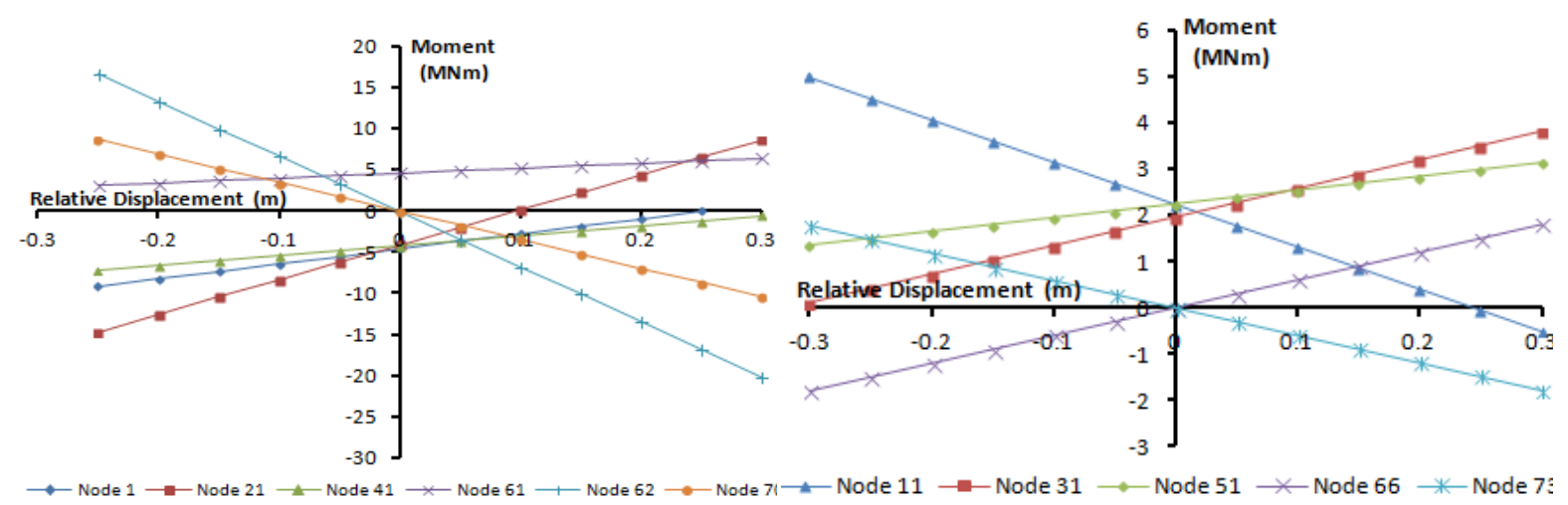

Figure VI-10: Static moments-displacements diagram for fixed base (a) middle and (b) joints nodes in $x$ direction.

The graphs in Figure VI-10 describe the patterns of moments resulting from static relative displacement for node 1 in the horizontal direction. As previously mentioned, a series of relative displacement were applied to node 1. The two graphs show the trend lines of bending moments of certain nodes of the bridge superstructure. The graphs show that the correlation between bending moments and displacements is linear, as expected, since the displacement applied is also static.

Due to horizontal (longitudinal) relative displacements, the most extreme moment value occurs in node 62, shown as the pattern seen in Figure VI-10. Meanwhile the least moment value is in nodes 66 and 73 . Nodes 66 and 73 are nodes in the pier supports of the bridge.

\subsubsection{Dynamic Moments of the Bridge}

Because the loading applied to the structure is a dynamic load (earthquake acceleration), the internal forces occurring in the bridge are also dynamic, including moment. These results of the dynamic moments in the bridge are shown in Figure VI-11. 


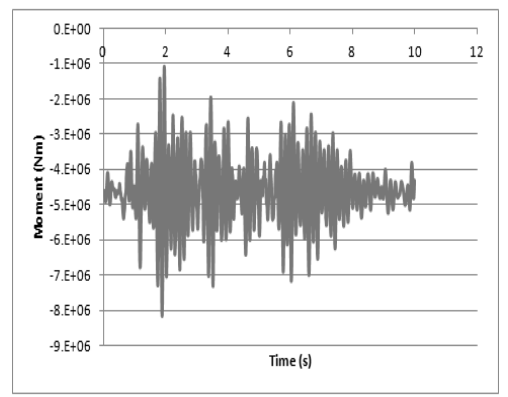

(a)

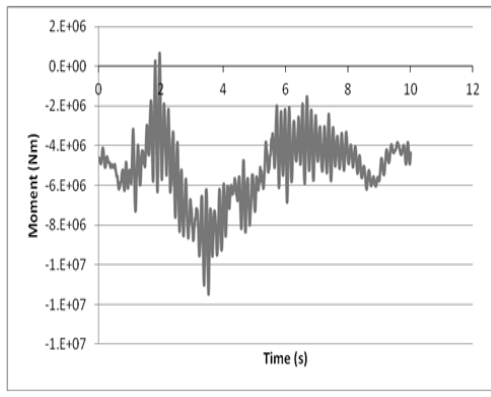

(b)

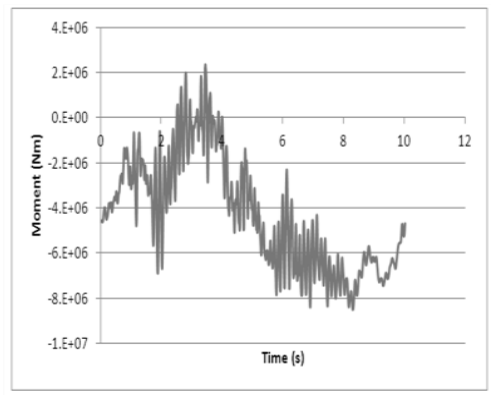

(c)

Figure VI-11: Dynamic moment for a fixed base: (a) dynamic only without relative displacement, (b) dynamic with relative displacement - horizontal direction, (c) dynamic with relative displacement - vertical direction

Figure VI-11 describes the dynamic moment due to earthquake accelerations applied to it. A dynamic moment occurs in the structure depending on time.

In Figure VI-11 (a), the dynamic moment is the result of a dynamic response when the same time histories are applied to the structure. In this case, no relative displacements occur in the supports of the bridge since the time histories applied are identical. It can be seen in Figure VI-11 (a), (b) and (c) that all of the moments started with the same magnitude as in the static moment, namely $4.6 \times 10^{6} \mathrm{Nm}$.

Meanwhile, in (b) and (c), two different time histories are applied and this leads to different displacements of the structural supports. In Figure VI-11 (b), the relative displacement which occurs in node 1 is in the $x$ direction because the ground motion accelerations are also applied in the $x$ or horizontal direction. It can be seen from the graph that there is a fluctuation in dynamic moments when a relative displacement is applied to it; meanwhile, in a pure dynamic loading analysis, the dynamic moment happens without fluctuation. It can be clearly seen that there is a correlation between a bending moment and the relative displacements of the structure. The same behaviour can be observed in Figure VI-11 (c), but the relative displacements are in the $y$ or vertical direction. 


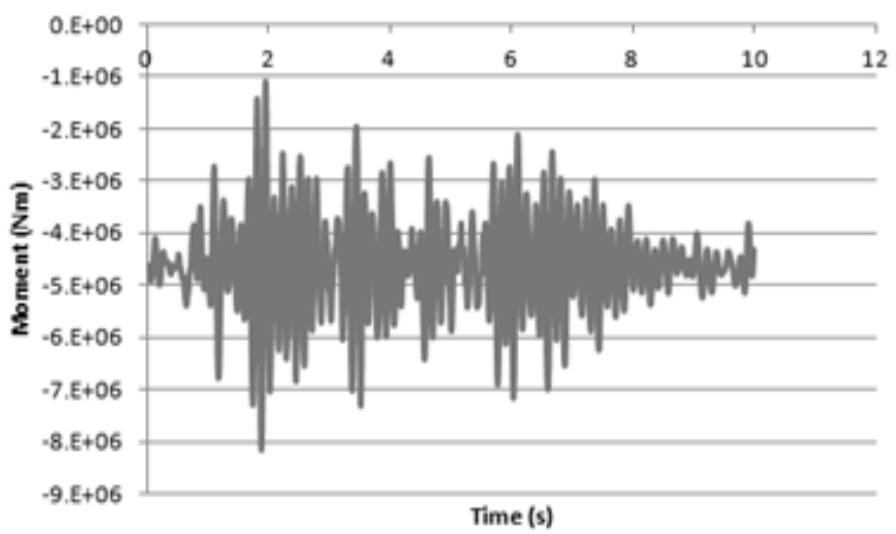

(a). Dynamic Noments for Bridge without RD

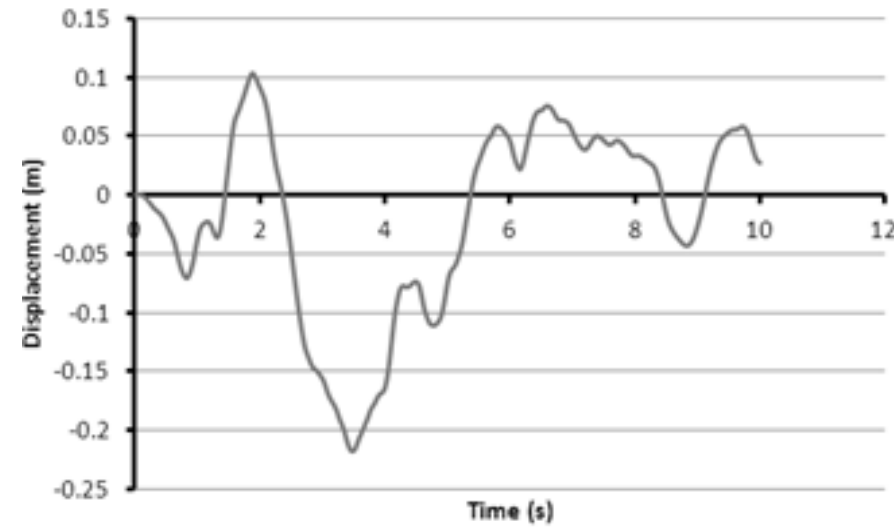

(b). Relative Displacements Occurred

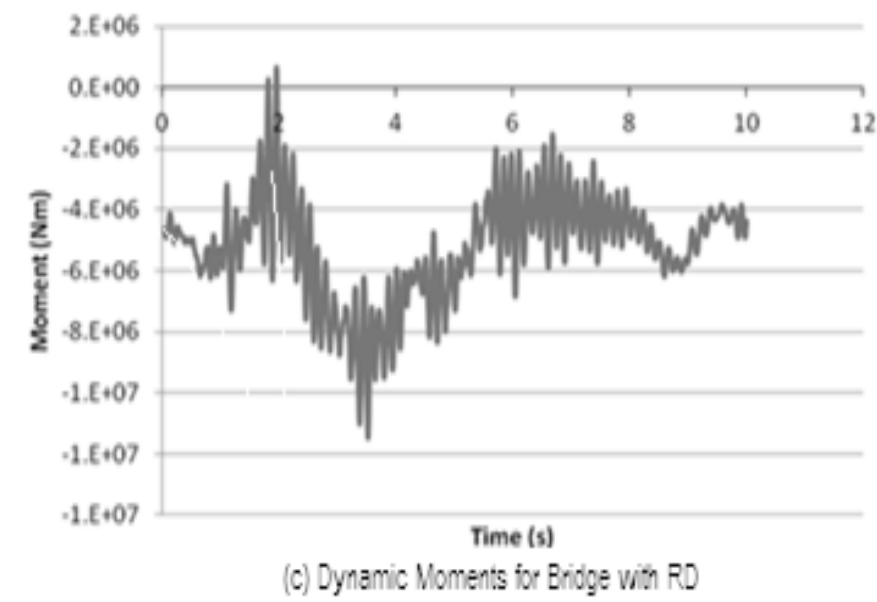

Figure VI-12: Dynamic bending moment diagram for horizontal relative displacement (fixed base)

Figure VI-12 shows the correlation between relative displacements and bending moments of the structure. Figure VI-12 (a) shows the dynamic moments without relative displacements in the bridge, and (b) shows the relative displacements occurring in the area where the moments are observed. Again, it can be seen that there is a positive correlation between the dynamic moments and the relative displacement, because the third figure (c) 
shows that the dynamic moments patterns are now following the pattern of the relative displacements.

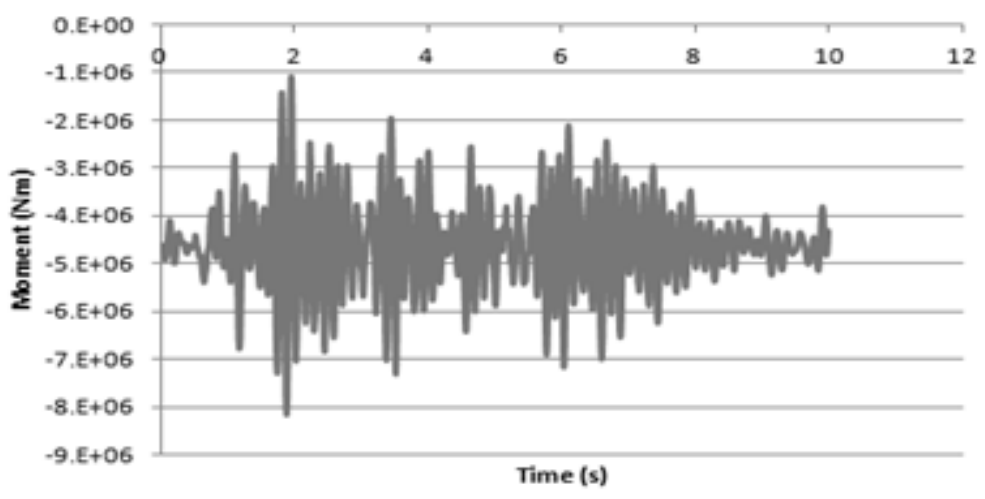

(a). Dynamic Moments for Bridge vithout RD

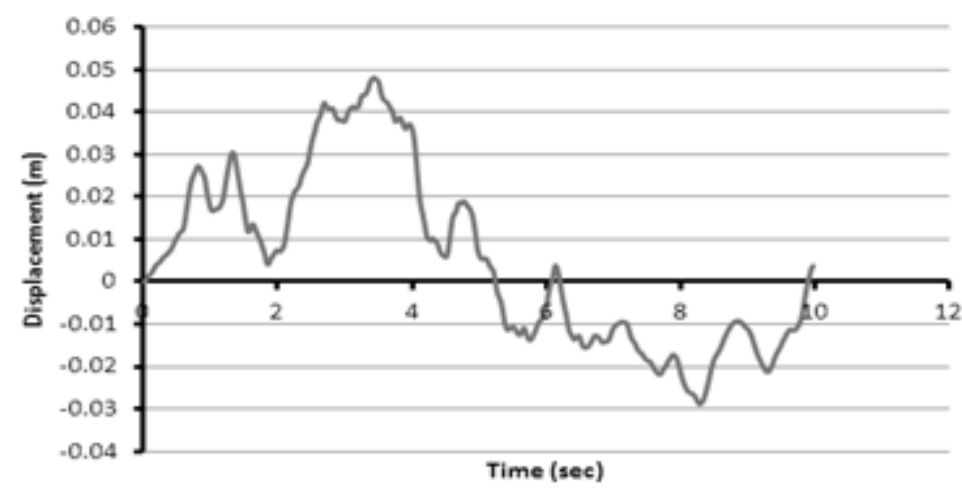

(b). Relative Displacements Occurred

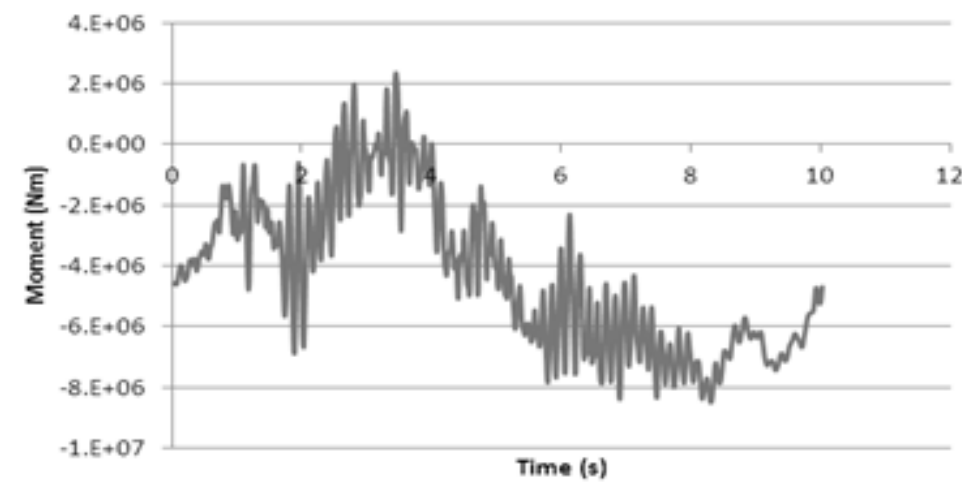

(c). Dynamic Moments for Bridge with RD

Figure VI-13: Dynamic moments behaviour in vertical relative displacement (fixed base)

Vertical relative moments are shown in Figure VI-13, where similar behaviour is observed. Relative displacement plays an important role in distinguishing the bending moment values of the system. The pattern of the bending moment correlates with the pattern of relative displacements. 


\subsubsection{Case 2 (Springs Base)}

There were two different performances for this case, those are: same stiffness for both vertical and horizontal stiffness and different stiffness for vertical and horizontal as can be seen in Appendix X. For different stiffness performances, there were also two different circumstances. The first circumstance was conducting a higher vertical stiffness, and the second circumstance was conducting a lower vertical stiffness.

\subsubsection{Static Analysis}

The stiffness of soil in this study is based on soil type and consistency/density, as explained in Chapter 4. The range of soil stiffness used can be seen in Tables IV-2 and IV3. The node configuration is shown in Figure VI-14.

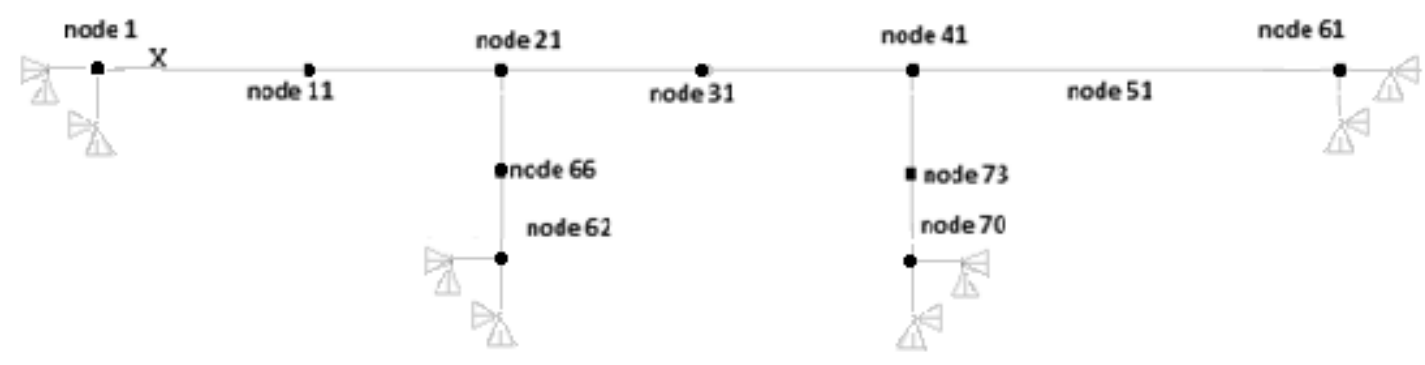

Figure VI-14: Node configuration of the structure (spring base)

The node configuration is the same as in the fixed base system. The difference is that two springs are applied in the supports in both vertical and horizontal directions. Node 1 is assumed to be rotation constrained but released in translation. Moreover, the internal forces studied in this research are the bending moment only.

Figure VI-15 shows the bending diagram moment under static gravity load, without relative displacements applied to it. . For this system, the maximum bending moment is $6.07 \times 10^{6} \mathrm{Nm}$.

The patterns of bending moment distribution can be observed in Figure VI-16. The distribution of the moments has a similar pattern to that in the fixed base structure case. As with the fixed base, horizontal relative displacement (a) creates more moment in the middle of the spans. Meanwhile, vertical relative displacements (b) induce more moment in the joints of the spans. 


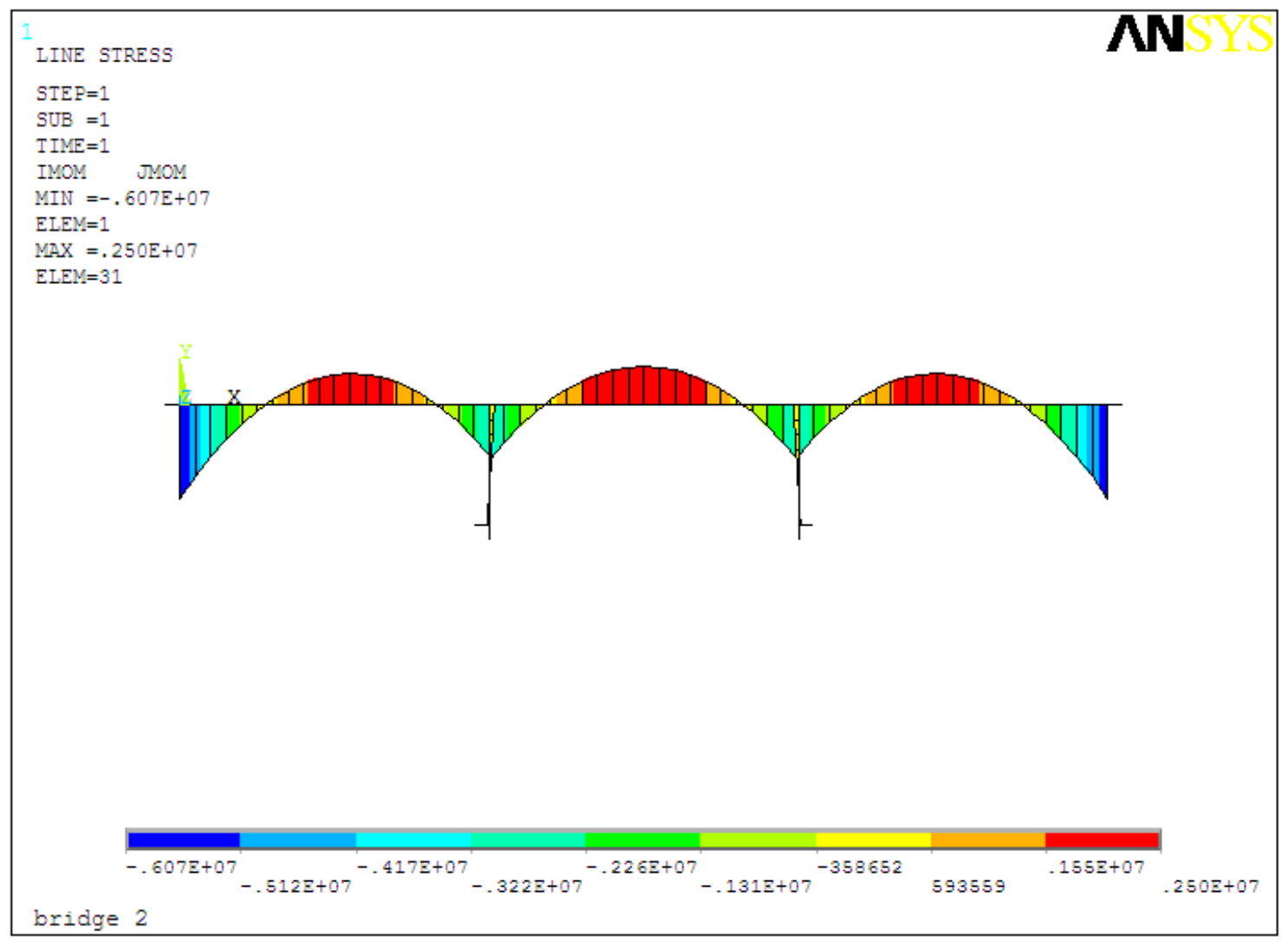

Figure VI-15: Bending moment diagram for gravity load (spring base)

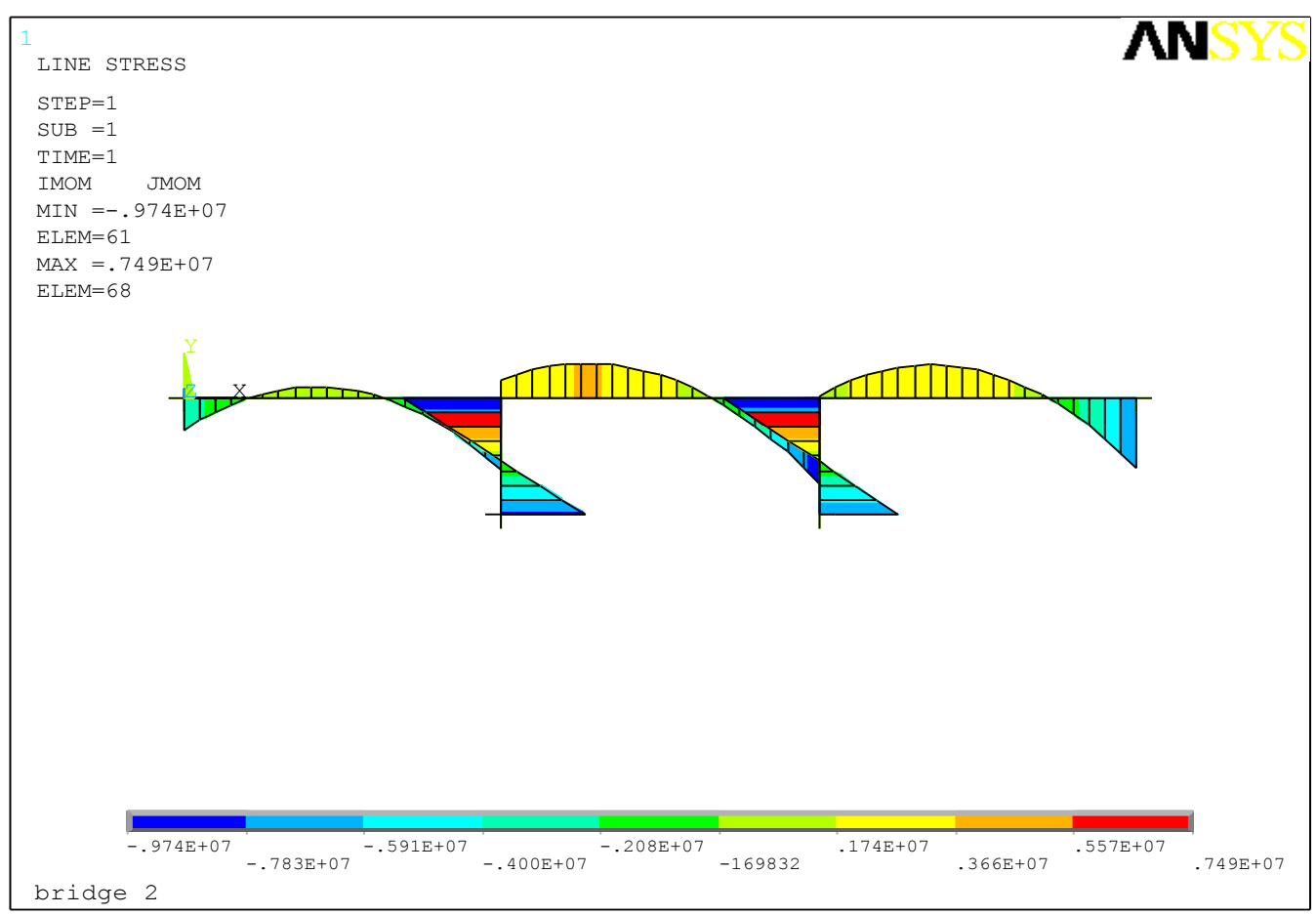

(a) 


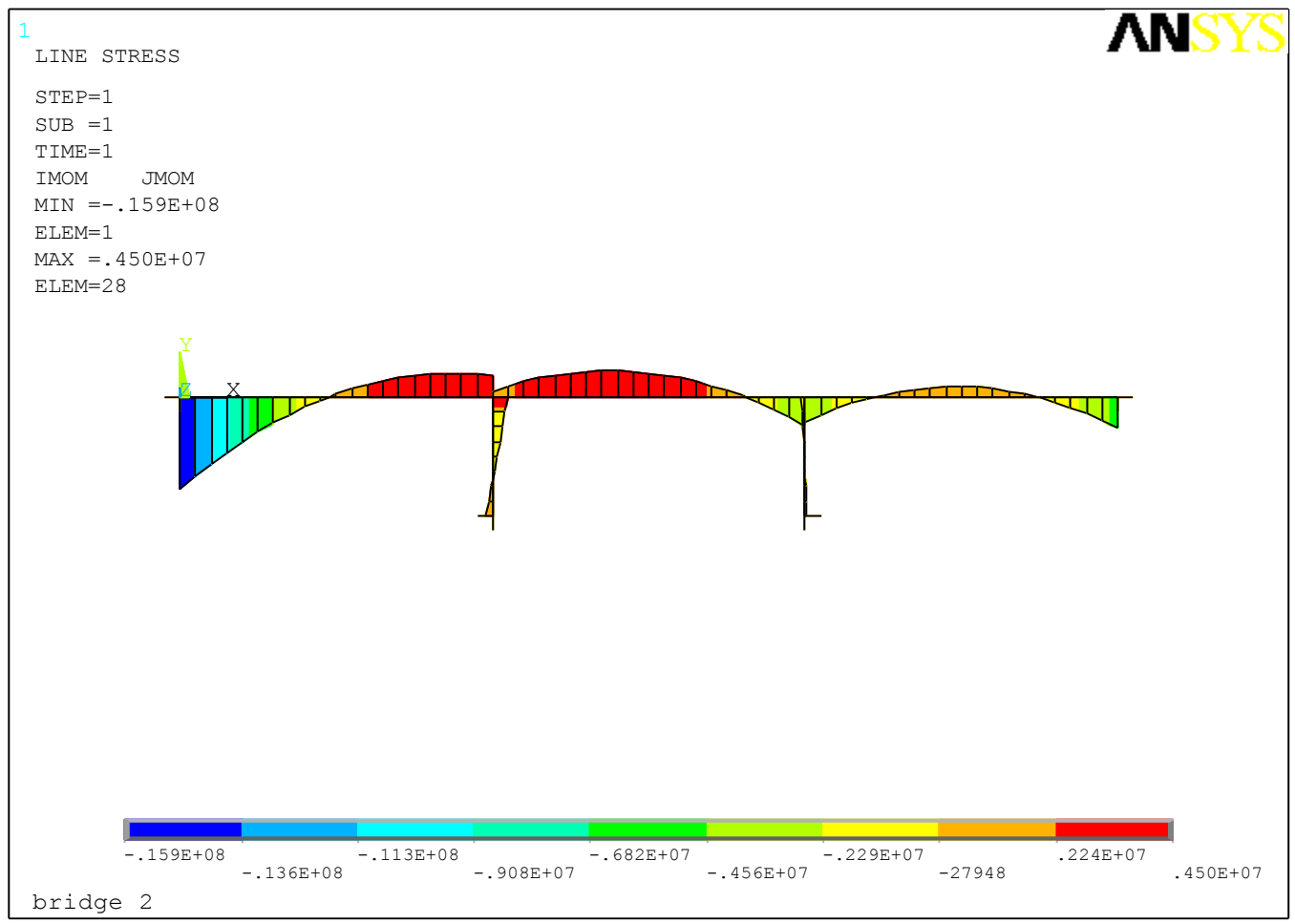

(b)

Figure VI-16: Bending moment diagram for spring base bridge with (a) horizontal relative displacement, (b) vertical relative displacement.

\subsubsection{Relative Displacements as a Sensitivity Study for Spring Base Bridge.}

Relative displacements for the spring base structure are also similar to the previous fixed base description. The graphs in Figure VI-17 are the reference for accessing dynamic relative displacements, as previously mentioned.

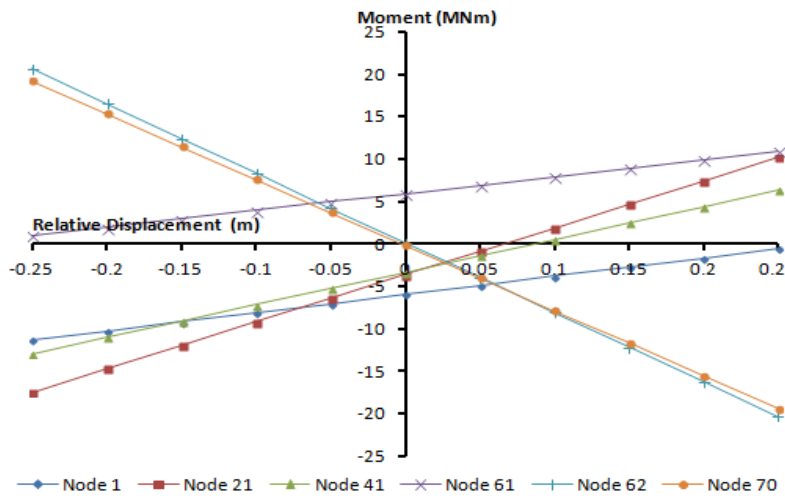

(a)

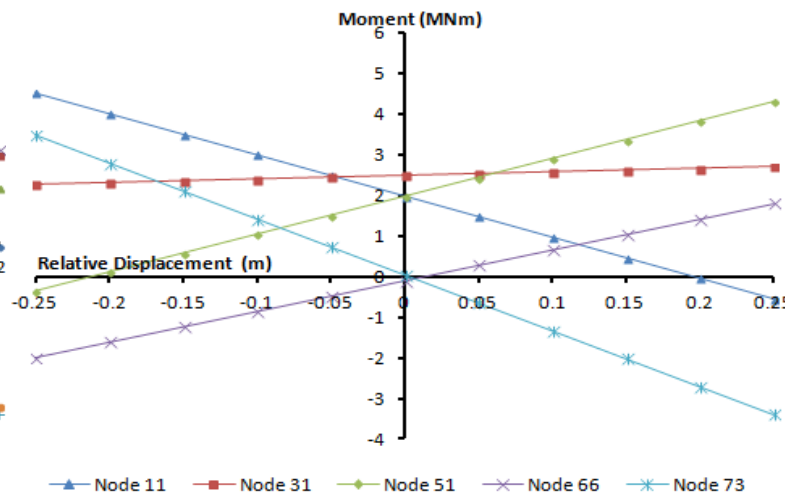

(b)

Figure VI-17: Static moments-displacements diagram for spring base (a) middle nodes and (b) joints nodes for horizontal relative displacement. 
The graphs (a) and (b) in Figure VI-17 describe the trend lines of the static moments of the structure in relation to the relative displacements. These trend lines will be used to correlate the dynamic moment which is obtained from earthquake ground motion. Different time histories applied to the different supports of the structure will cause dynamic relative displacements, from where the amount of static moment resulting from this relative displacement can be observed.

\subsubsection{Dynamic Analysis}

Figure VI-18 (a) and (b) shows the difference between supports of the fixed base bridge and spring base bridge. It can also be seen why the fixed base supports structure resulted in a smoother dynamic relative displacement than the spring base supports structure. The springs contribute to the flexibility of the global system providing a significantly modified response. How the springs affect the stiffness of the global system is thus explained in Figure VI-18.

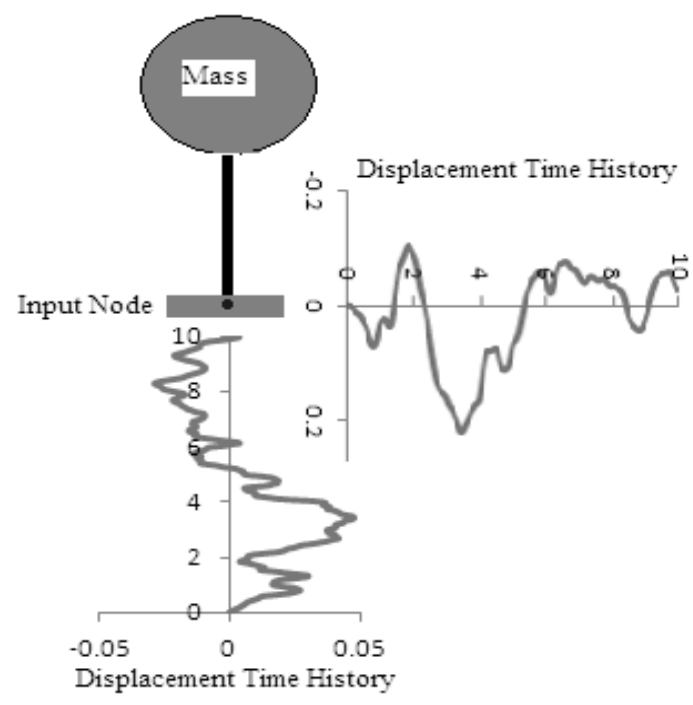

(a) 


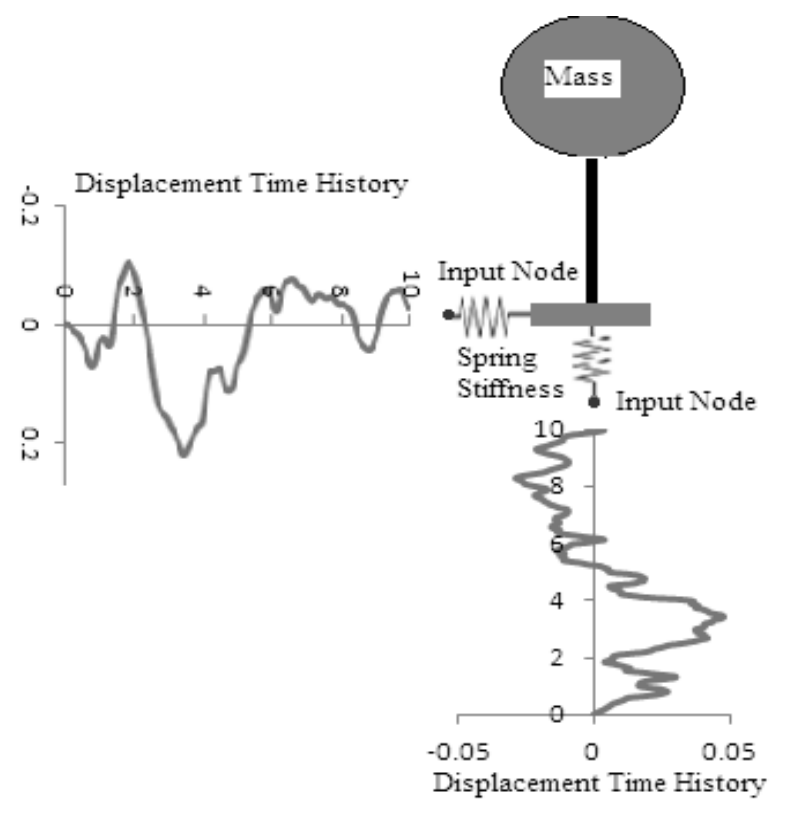

(b)

Figure VI-18: Comparison between fixed base support and spring base support.

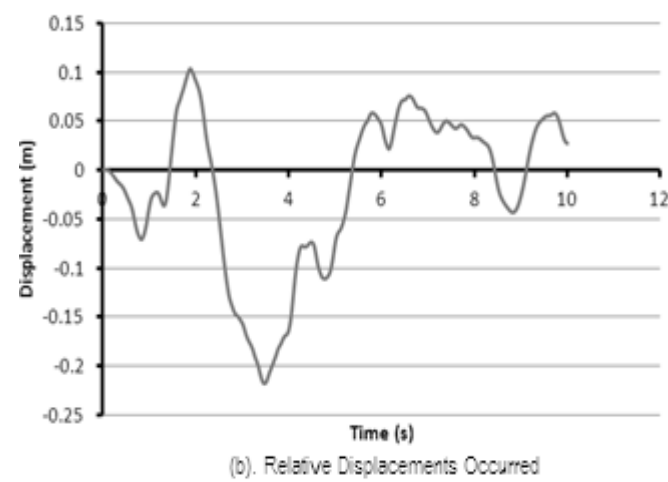

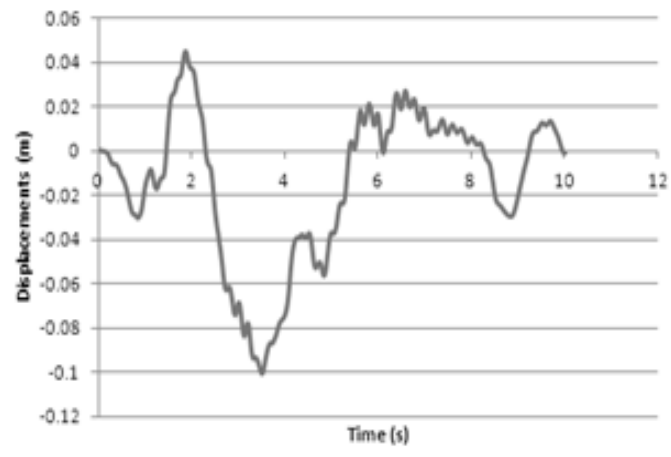

(b). Relative Disolacements Occumed

Figure VI-19: Relative displacement for (a) fixed base and (b) spring base.

Figure VI-19 (a) shows the relative displacement occurring in a structure with fixed base supports, and Figure VI-19 (b) shows the relative displacement occurring in the structure with spring base supports. It can be seen that there is a significant difference between the spring base structure and the fixed base structure regarding their dynamic relative displacements. The figure shows that the wavy lines of relative displacements for both vertical and horizontal directions are not smooth. There is a cogent fluctuation in the in the spring base system, but in the fixed base system this fluctuation does not occur. The reason for this is the presence of springs on the supports of the structure, which results in a sway or relatively unstable condition on the the springs, providing the structure with a mode which is being excited to some degree by the earthquake. 
When the acceleration time histories are applied to the input nodes of the structure, two different responses are observed. The difference is due to the varying global stiffness of the structure. Springs that are attached to the structure have reduced the stiffness of the structure globally, and this has a significant effect on the response of the structure.

\subsubsection{Dynamic Moments Response of the Bridge}

Dynamic bending moments resulting from different conditions of acceleration time histories are presented in Figure VI-20.

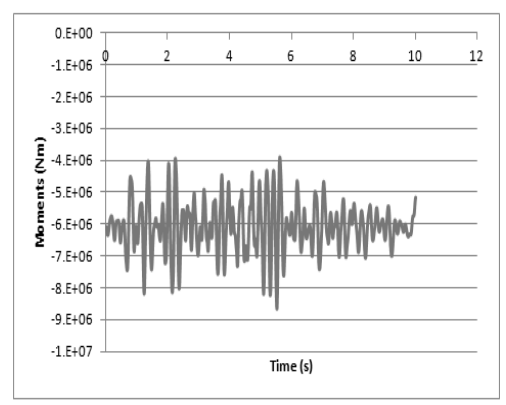

(a)

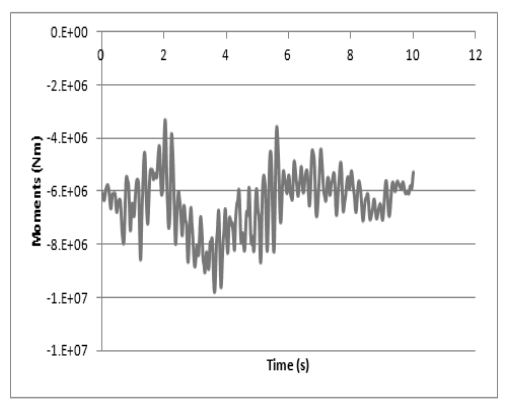

(b)

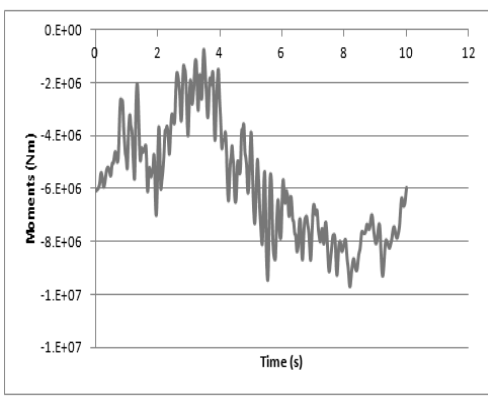

(c)

Figure VI-20: Dynamic moment for the spring base (a) dynamic only without relative displacement, (b) dynamic with horizontal displacement, (c) dynamic with vertical relative displacement

The three graphs in Figure VI-20 show different results for three different conditions of the input of transient analysis. Figure VI-20 (a) describes the bending moment of node 1 (left end support). This bending moment occurs after applying the same acceleration time histories to all supports of the bridge. The pattern of this type is similar to that of the fixed base support structure. However, the frequency (density) of the bending moment change is lower. It can be seen from Figure VI-20 (a) that the bending from this spring base structure is less dense than in Figure VI-11 (a), which shows the fixed base structure. The structure is reacting more flexibly, i.e. its natural frequency is less. Hence, the response is such that the structure oscillates with lower frequency.

Figure VI-20 (b) shows the dynamic bending moment after applying a relative displacement in the horizontal direction. As can be predicted from the previous results, the pattern of this dynamic bending moment is similar to the fixed base structure's bending moment. However, the frequency of the bending moment created is different. As explained 
before, the spring stiffness applied in the support of the bridge causes a reduction in the internal forces and it yields a less dense dynamic bending moment compared to the dynamic bending moment resulting from the fixed base structure. The same results and explanations are applicable to Figure VI-20 (c), which shows the dynamic bending moments of the spring base structure under the application of vertical relative displacements. The effect of relative displacements can also be seen in this spring base case. Figure VI-21 describes the correlation of bending moment and relative displacements of the spring base structure.

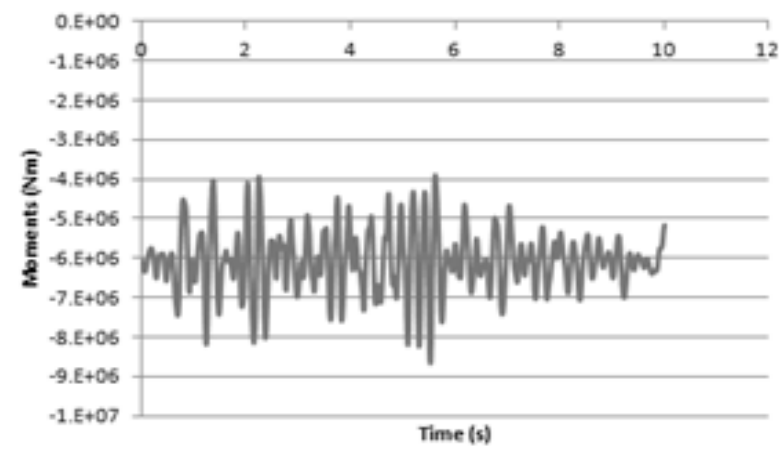

(a). Dynamic Moment for Spring Based Bridge without RD

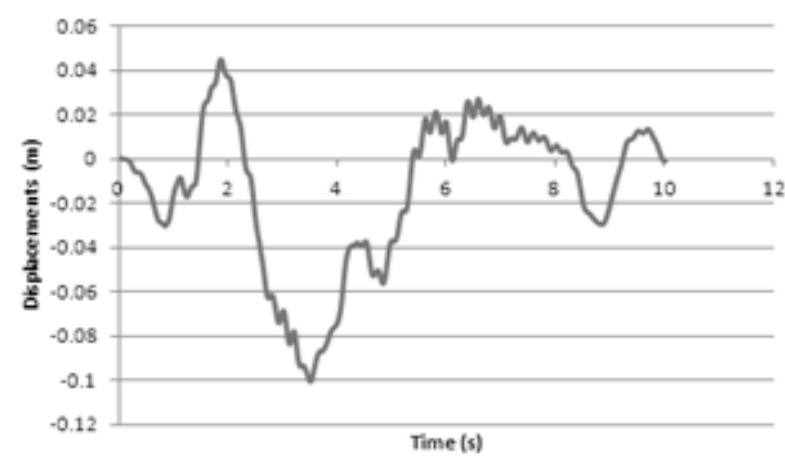

(b). Relative Displacements Occurred

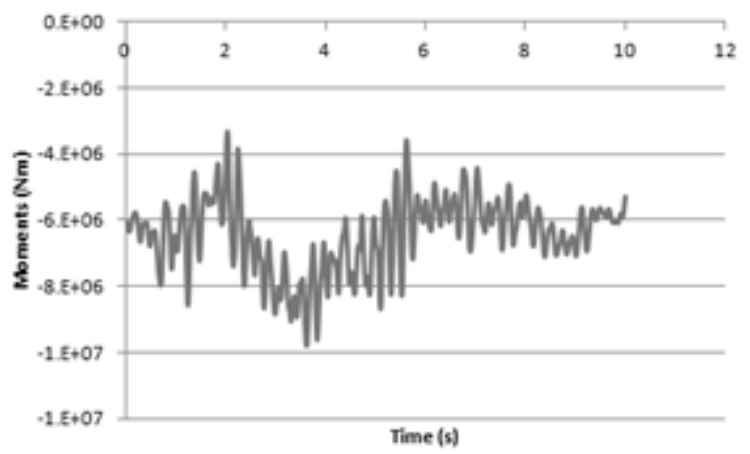

(c). Dynamic Moment for Spring Based Structure vith RD

Figure VI-21: Horizontal relative displacement effect to bending moment (spring base) 
Figure VI-21 (a) shows the dynamic bending moment of the system without applying the horizontal relative displacement to the system, whereas Figure VI-21 (b) describes the dynamic relative displacement occurring in the support. Figure VI-21 (c) is the dynamic bending moment of the spring base structure under a vertical relative displacement. It is clear that there is a strong correlation between the bending moments due to only dynamic load (without relative displacement) and the relative displacement occurring in the support. The pattern of the dynamic bending moment after applying a relative displacement follows the pattern of the dynamic relative displacement. It can be also noted from Figure VI-21 that the dynamic bending moment occurring in the spring system is less dense than that in the fixed base system, as expected, and the previous explanation applies.

Figure VI-22 below can be explained in a similar manner as for Figure VI-21, as it illustrates the vertical relative displacement applied to the spring system.

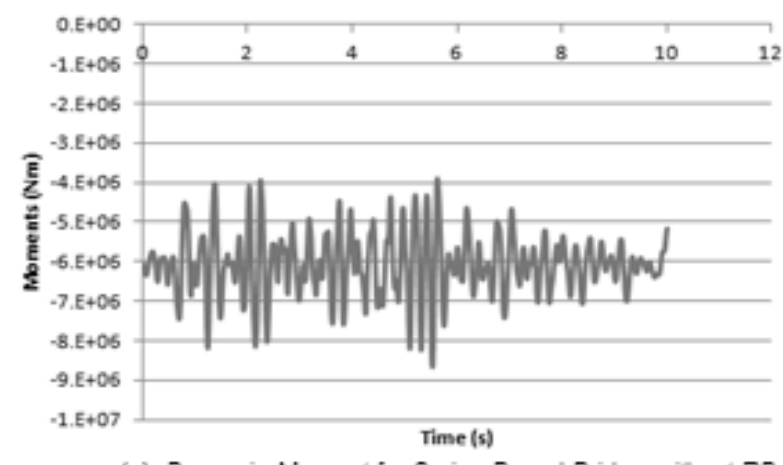

(a). Dynamic Moment for Spring Based Bridge vithout RD

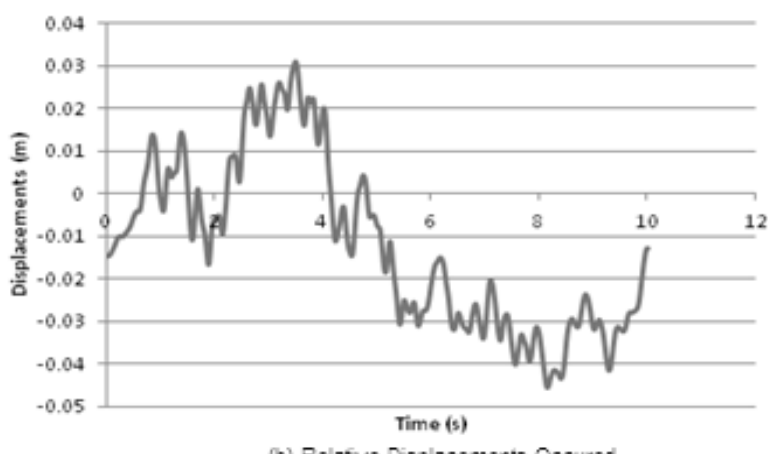

(b) Relative Displacements Occured 


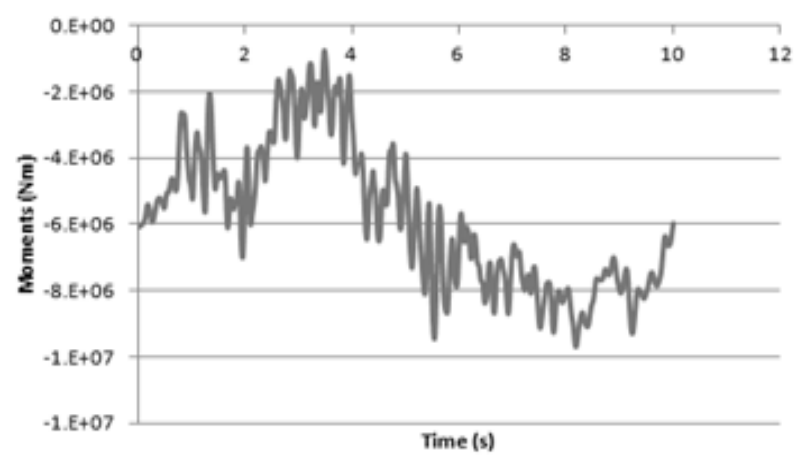

(c). Dynamic Moments for Spring Base with RD

Figure VI-22: Vertical relative displacement effect to bending moment (spring base)

In summary, the patterns of dynamic bending moment of a structure to which a relative displacement has been applied follow the relative displacement patterns. In other words, the pattern of the moments occurring in the structure are a superposition of dynamic moments only (without the relative displacement) and the relative displacement which has resulted from different time histories.

\subsection{Bending Moment Pattern of the Bridge}

\subsubsection{Case 1 (Fixed Base)}

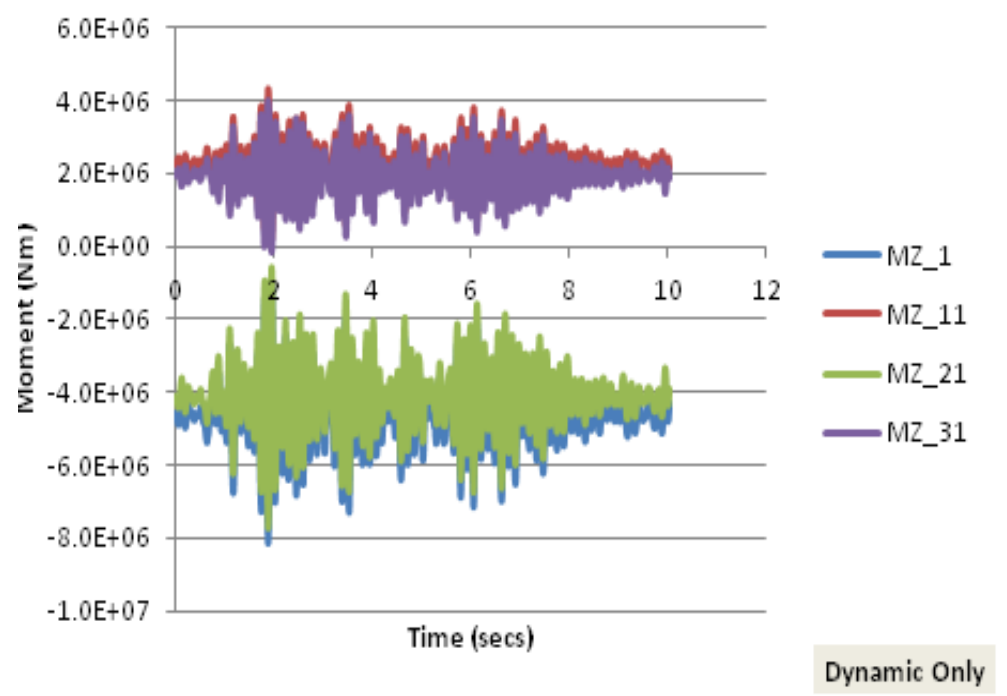

Figure VI-23: Dynamic bending moment without relative displacement (fixed base)

Figure VI-23 illustrates four different dynamic bending moments. Nodes 1 and 21 are the nodes on the joints of the bridge structure and have similar bending moments. The same 
behaviour occurs in nodes 11 and 31, which are the nodes in the middle of the spans of the bridge. From this bending moment, the deflection shape of the structure can also be predicted, since the bending moments in the joints are in hogging mode and the moments in the middle of the spans are in sagging mode. It can be seen from the figure that the bending moments fluctuate due to the dynamic loading applied. No other secondary fluctuation occurs in the pure dynamic-only case. Secondary fluctuation has been created due to the fluctuation of the dynamic relative displacements.

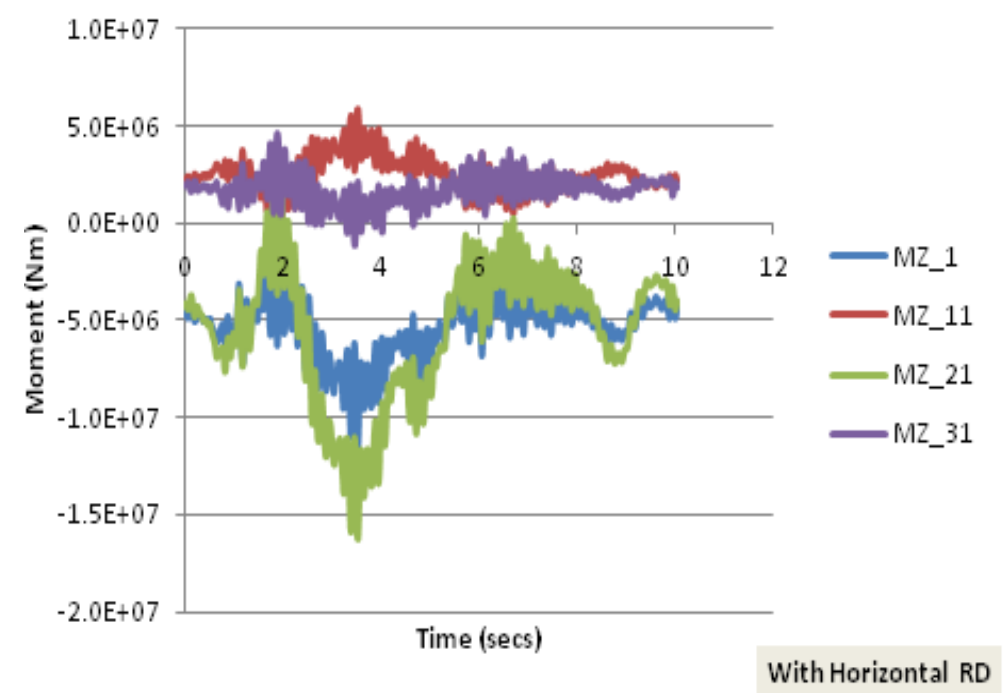

(a) After applying horizontal relative displacement

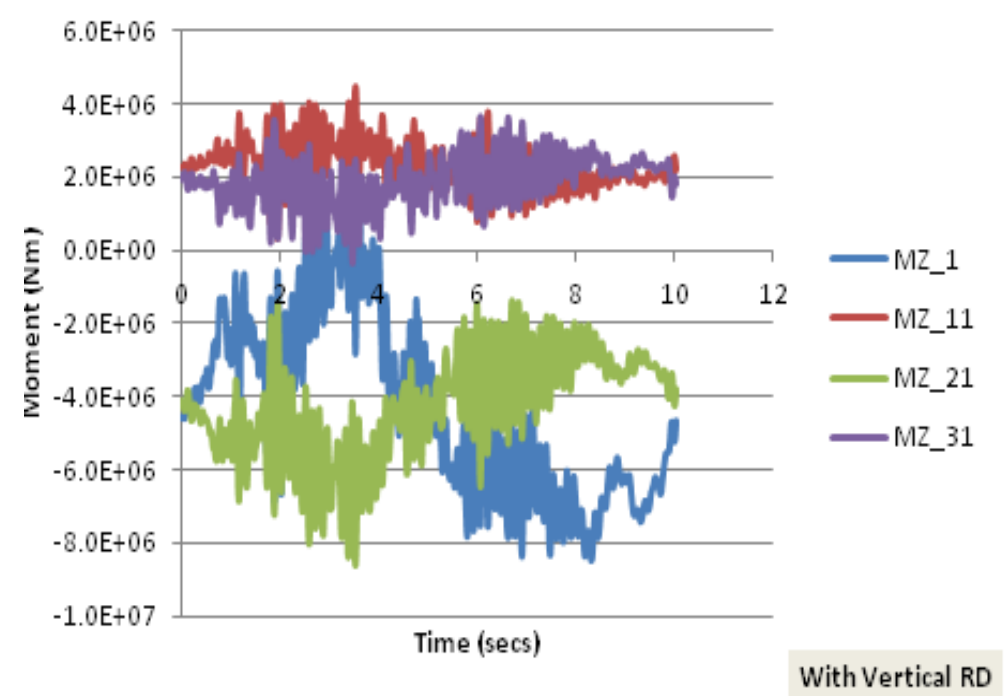

(b) After applying vertical relative displacement

Figure VI-24: Dynamic bending moment after applying relative displacement (fixed base)

Figure VI-24 (a) and (b) describes the dynamic bending moment after applying relative displacement to the support of the structure. Both Figure VI-24 (a) and Figure VI-24 (b) 
show that the bending moments have two kinds of fluctuation. The first fluctuation is due to the dynamic load, and the second to the dynamic relative displacements.

The different bending moments between dynamic load only, dynamic load plus horizontal relative displacement, and dynamic load plus vertical relative displacement are presented in Figure VI-25. It can be seen that there is a significant difference in the pattern and value of these bending moments.

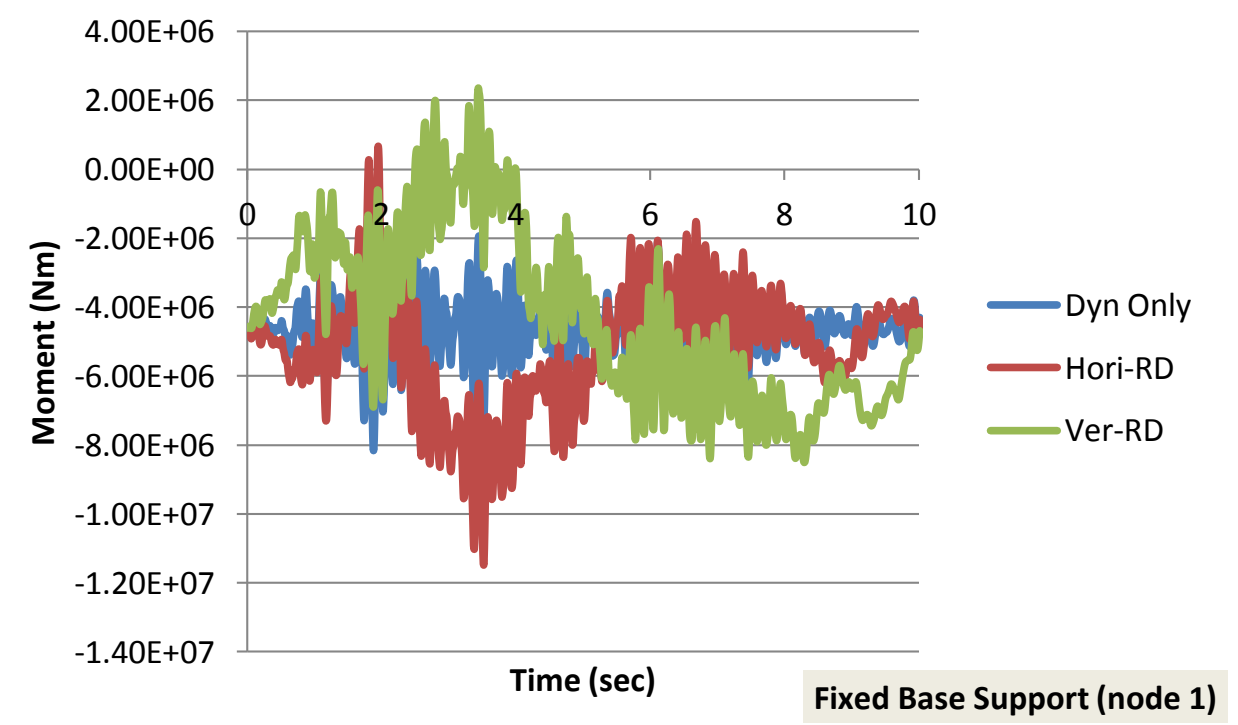

Figure VI-25: Bending moments for dynamic load only and dynamic load plus relative displacement at node 1 (left support-for fixed base).

Figure VI-26 shows the bending moment patterns and values for a mid-span node, i.e. node 31 . 


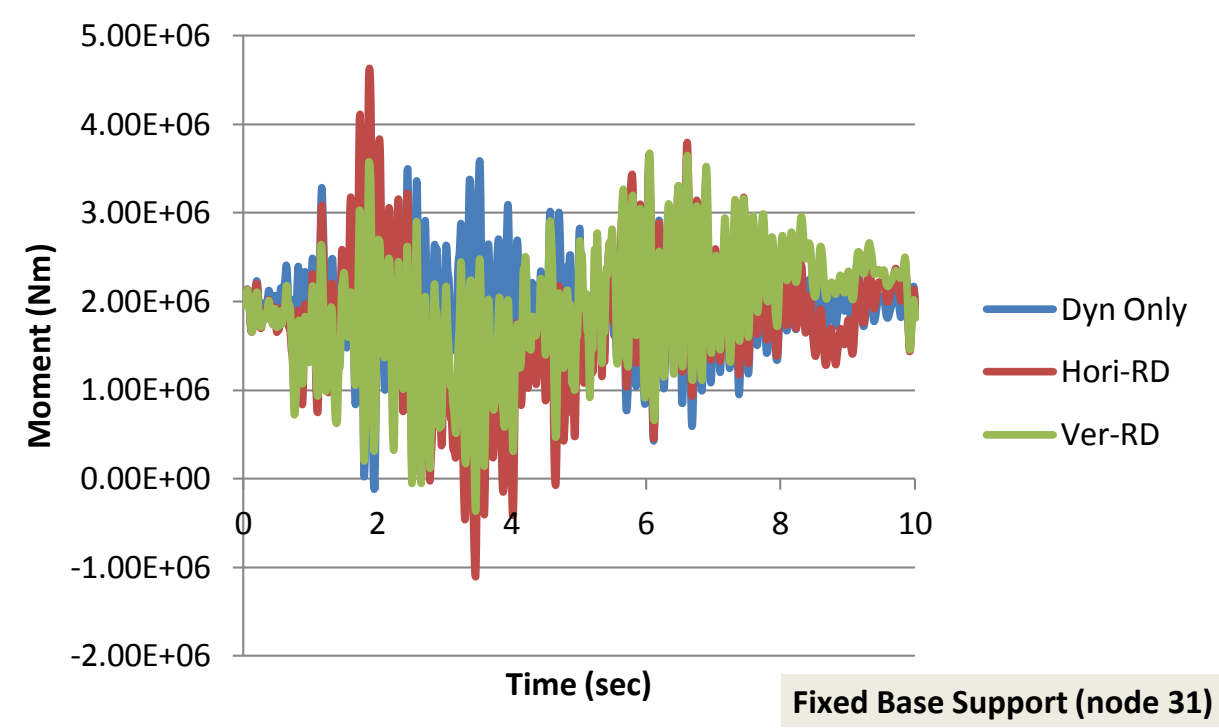

Figure VI-26: Bending moments for dynamic load only and dynamic load plus relative displacement at node 31 (mid-span for fixed base).

\subsubsection{Case 2 (Spring Base)}

For the spring base bridge structure, the dynamic bending moments can be found in Figures VI-27 and VI-28. Figure VI-27 shows the response of the structure to dynamic loads in the absence of relative displacement. The dynamic bending moments shown in the figures are for one half of the bridge deck, i.e. for nodes 1, 11, 21 and 31.

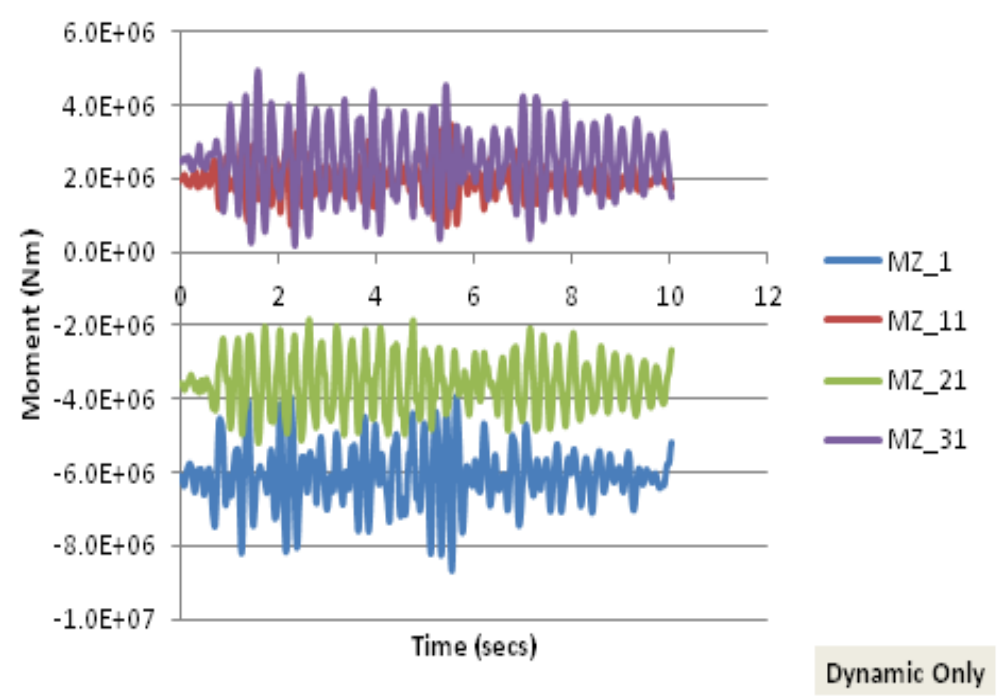

Figure VI-27: Dynamic bending moment before applying relative displacement (spring base)

It can be seen from the figure that the dynamic bending moment for node 1 is larger than that for node 21 , and both of them are in the hogging position (negative bending moment). 
The magnitude of dynamic bending moment for nodes 11 and 31, which are the nodes for mid-spans, are in the sagging position and node 31 has a greater magnitude than node 11. None of the moments has secondary fluctuation as there is no relative displacement. The fluctuations occurring in the bending moments are only caused by the characteristic and nature of the dynamic load applied to the structure.

Figure VI-27 also shows that the intensity of the wave occurring in those dynamic bending moments are not the same as the intensity of dynamic bending moments for the fixed base bridge structure. In this spring base case, the bending moments created are less dense than in the fixed base structure. The reason is as previously mentioned, that the global stiffness of the system is not the same. Springs attached to the structure have changed the global stiffness so that the static and dynamic responses of the structure are also different.

Figure VI-28 (a) and (b) describes the dynamic bending moments occurring in the spring base integral bridge. There are two fluctuations occurring in the dynamic bending moments. The first is created by the dynamic loads, and the second by the dynamic relative displacements. The patterns of these dynamic bending moment fluctuations also follow the patterns of the relative displacements fluctuations. As in Figure VI-24, the intensity of these dynamic bending moments is not as dense as the dynamic bending moments of the fixed base bridge structure.

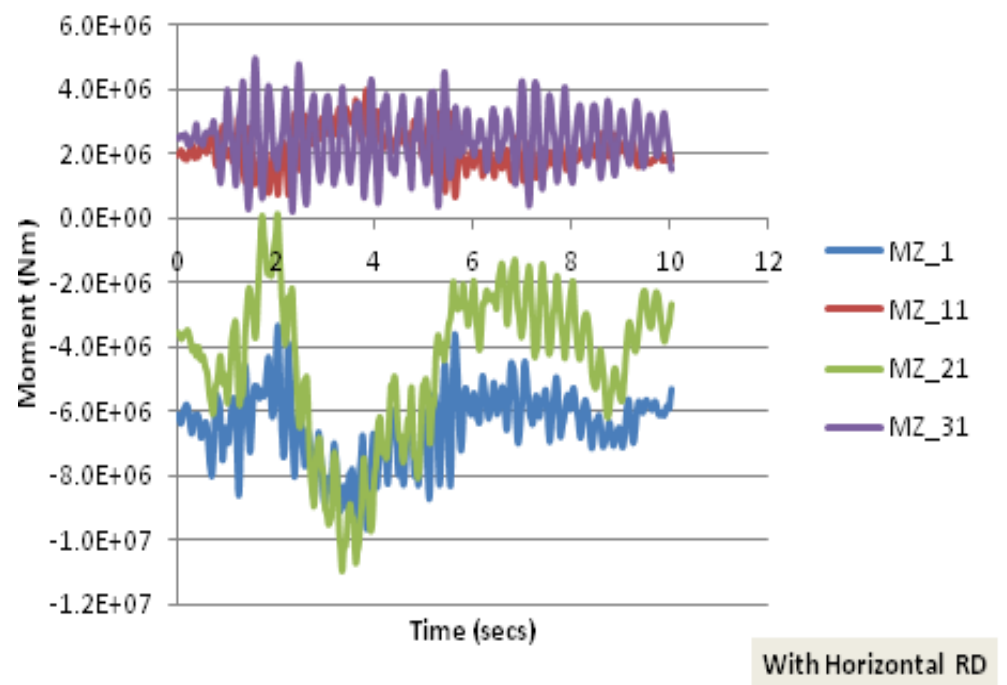

(a) For horizontal relative displacement 


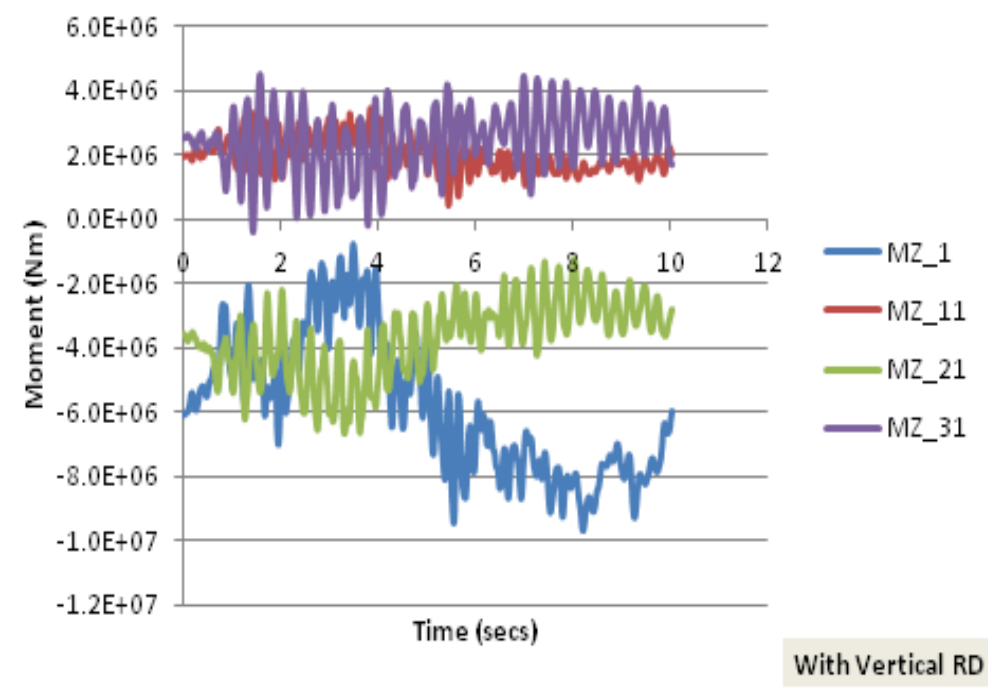

(b) For vertical relative displacement

Figure VI-28: Dynamic bending moment after applying relative displacement (spring base)

The difference in bending moments between dynamic load only, dynamic load plus horizontal relative displacement, and dynamic load plus vertical relative displacement for the spring base bridge structure is presented in Figure VI-29. As in the fixed base structure, it can be seen from the figure that there is a significantly different pattern and value of those bending moments.

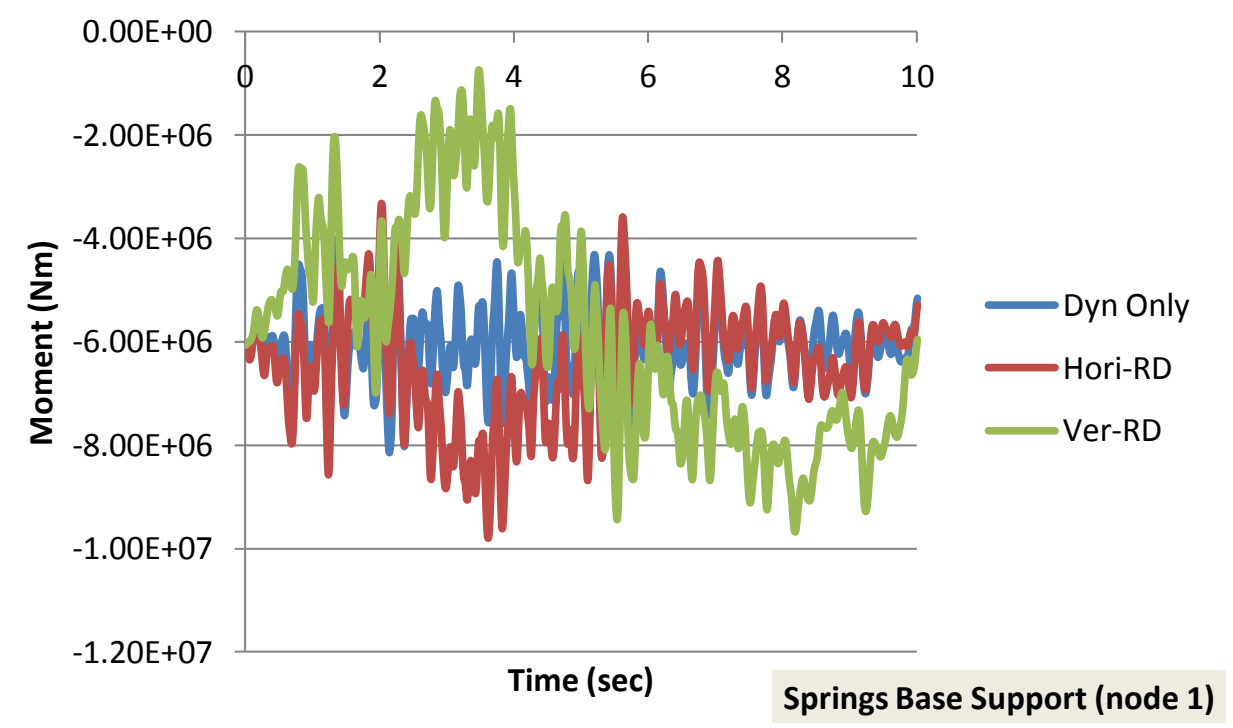

Figure VI-29: Bending moments for dynamic load only and dynamic load plus relative displacement at node 1 (mid-span spring base).

For node 31 , which is a mid-span node, the bending moment patterns and values can be seen in Figure VI-30. 


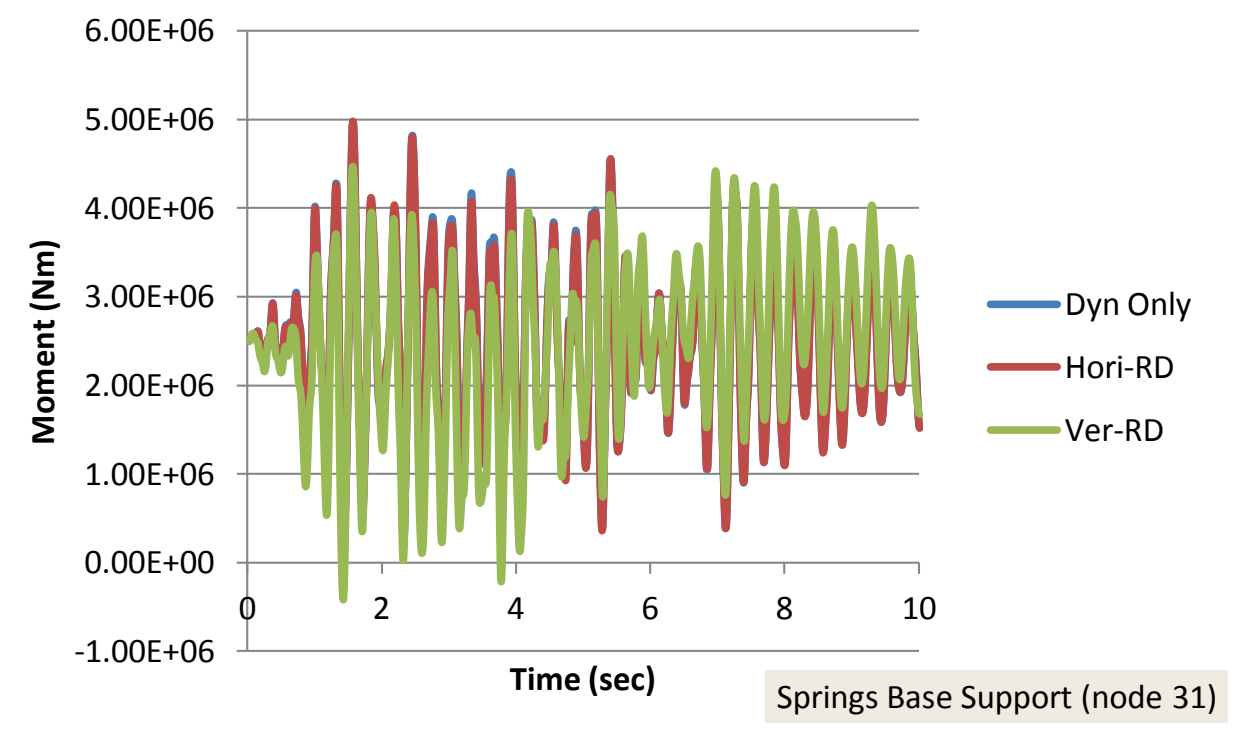

Figure VI-30: Bending moments for dynamic load only and dynamic load plus relative displacement at node 31 (mid-span spring base)

For bridge with different stiffness in vertical and horizontal supports, the patterns are same as can be seen in the appendix IX. 


\section{Chapter VII CORRELATION BETWEEN RELATIVE DISPLACEMENT AND BENDING MOMENT}

\subsection{General}

As explained in Chapter I, the aim of this research is to present a simplified appraisal of the dynamic behaviour of integral bridges, taking into account the results of static analyses only. To achieve this, static relative displacement analyses of the bridge supports are carried out and are then compared to dynamic transient analyses which incorporate relative displacements. The dynamic response is dominated by components from relative support displacement and inertia effects. Comparison of static and dynamic analyses provides insight into the significance of the effects of each component. In this study, only bending moments in the structure are considered as the criteria for structural capacity. In general terms, it can be seen that relative displacement effects dominate the composition of the bending moment at connection locations.

Bending moments are an important part of this problem. They exist in a structural element when a loading is applied to the element such that the element bends. This usually occurs when loads with a component transverse to the element are applied, creating a moment. Additionally, the external forces can be applied statically and dynamically, and the resulting moments in the bridge's structure will also be static and dynamic. There are two kinds of bending to be analysed in this study. The first is the moment that occurs to the structure statically and the second is the moment that occurs to the structure dynamically. In the former case, a structure such as a bridge will respond as to a static load and the 
internal forces will act statically. On the other hand, if a load is applied dynamically, the action and structural response will also be dynamic. This study tries to explain the influence of a structure's static response in the overall response, compared to dynamic response.

The dynamic transient analyses consist of acceleration/displacement time histories applied to each support. Although these time histories had mutual target response spectra, one of them was different, so as to create a relative displacement between support points.This would provide both dynamic relative displacement effects plus dynamic inertia effects in the response.

To examine the relative contributions of these two effects, a graphical construction was produced to illustrate/compare static and dynamic relative displacement effects. This graph is shown in Figure VII-1, and its construction and use are described below.

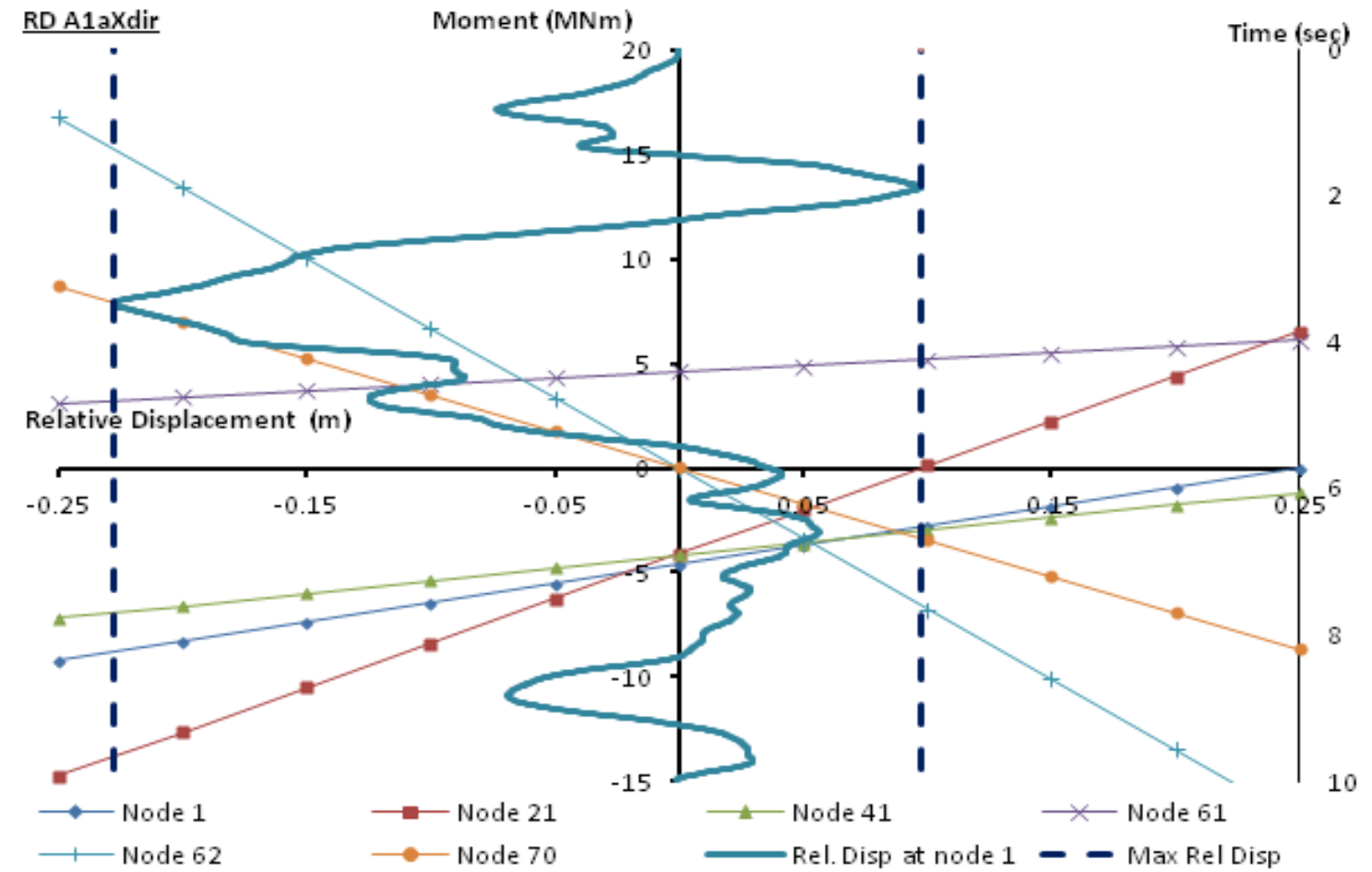

Figure VII-1: Relative displacement vs bending moment diagram

The graph in Figure VII-1 relates static relative displacement effects to dynamic relative displacement effects and provides an estimate of the bending moments at various key locations around the structure. 


\subsection{Generating the relative displacement and bending moment relationship diagram}

First, critical locations where the designer might be interested in bending moment magnitude were identified in the finite element model, usually at joints and in the middle of the span. The node locations (numbering) for those critical points are shown in Figure VII-2.

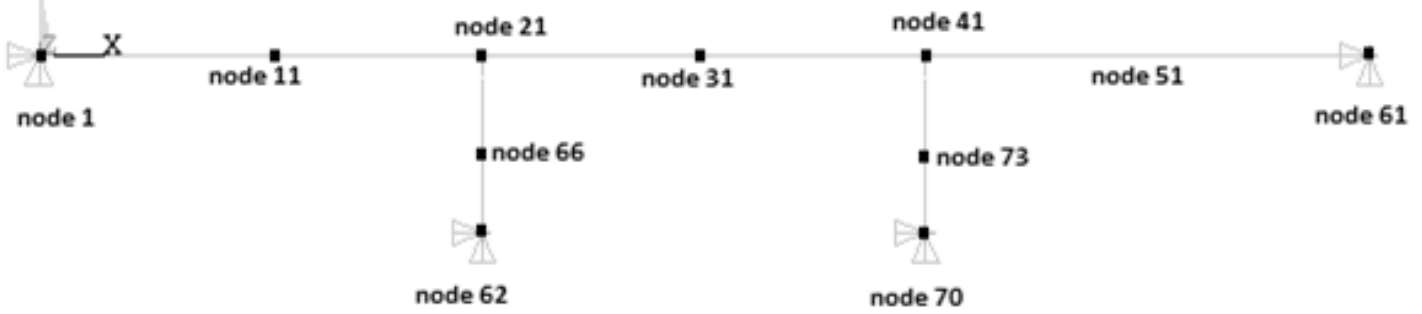

Figure VII-2: Node locations

Figure VII-3 presents the axes of the graph. The abscissa ( $x$ axis) represents relative displacement. Two vertical axes are shown, one for bending moment (which will be used for both static and dynamic analysis results), the other for time (which will be used for the dynamic relative displacement time history).

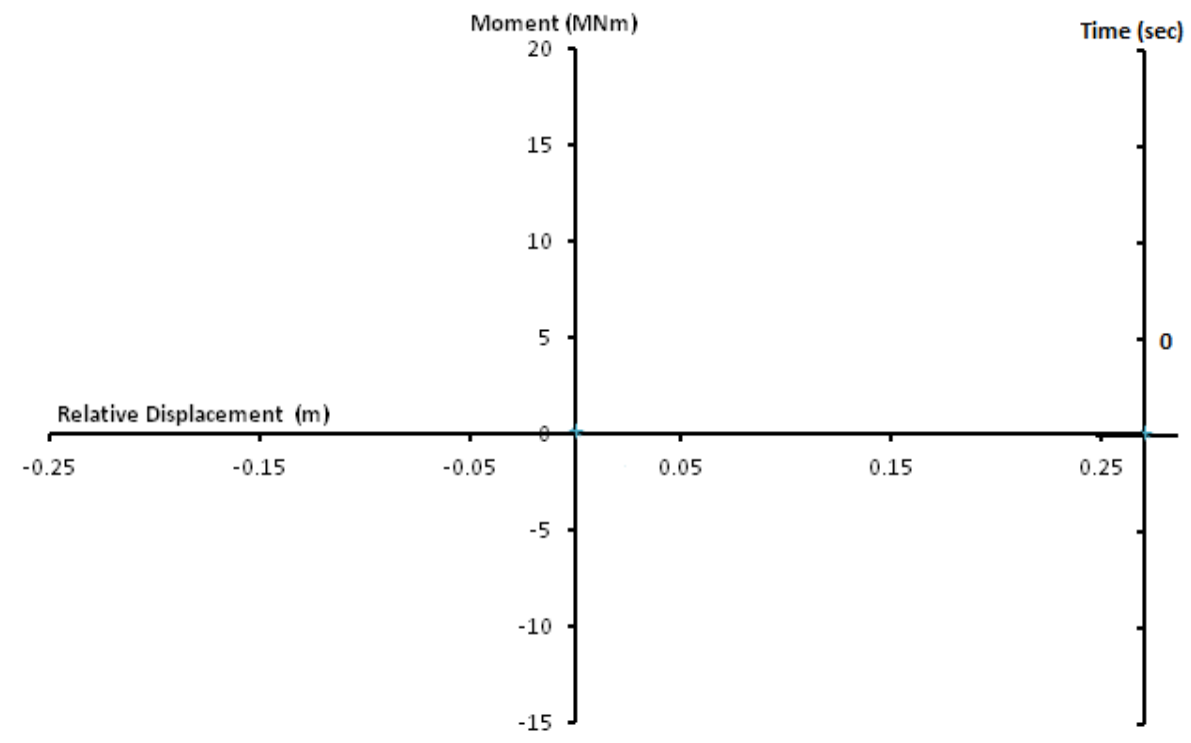

Figure VII-3: Axes of the graph 
Considering only the static relative displacement analysis, one support node is moved in the direction of either the $x$ or $y$ coordinate. This generates a set of additional bending moments in the structure (in addition to gravity/normal loading) at the coordinate locations shown in Figure VII-1. As the structure is linear elastic, it follows that the relationship between relative displacement and bending moment at any one location will also be linear.

Hence for any one location, a straight line can be drawn on the graph, as shown in Figure VII-4, in this instance for node 1. Similarly for all the locations shown in Figure VII-1, all of the linear relationships between bending moment and relative displacement can now be drawn on the graph, as shown in Figure VII-5.

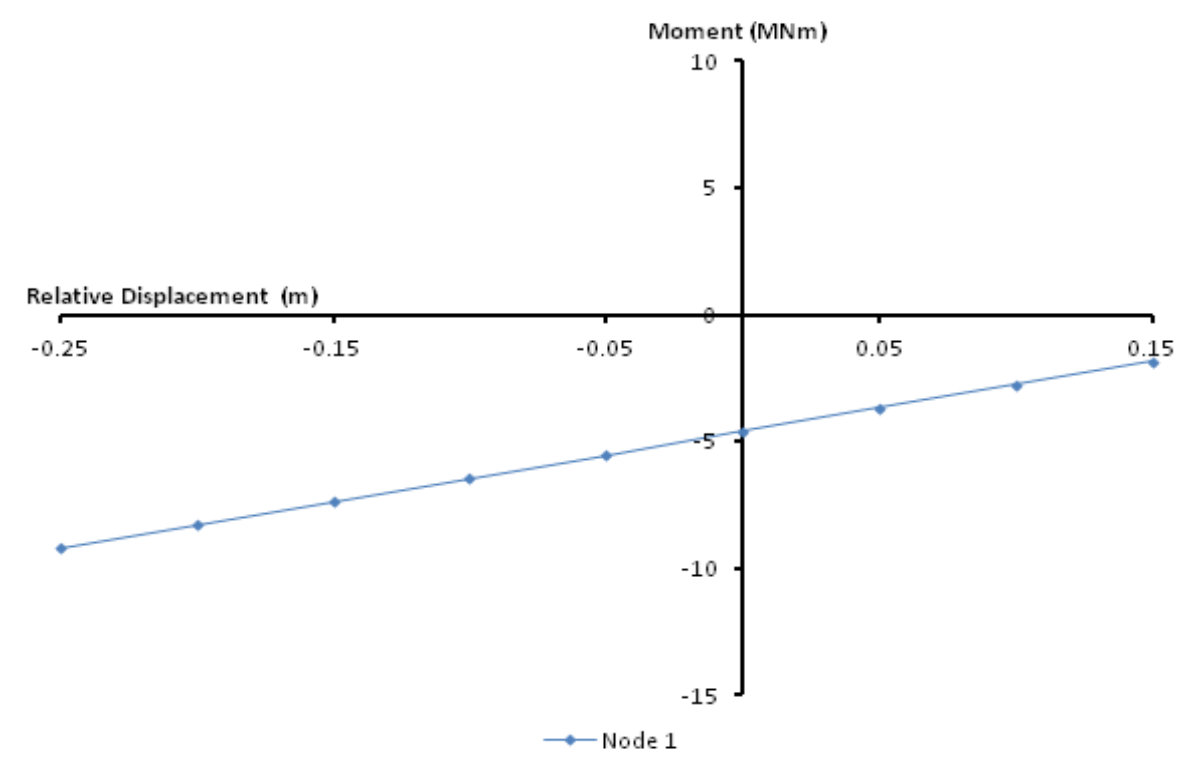

Figure VII-4: Linear relationship between bending moment and relative displacement 


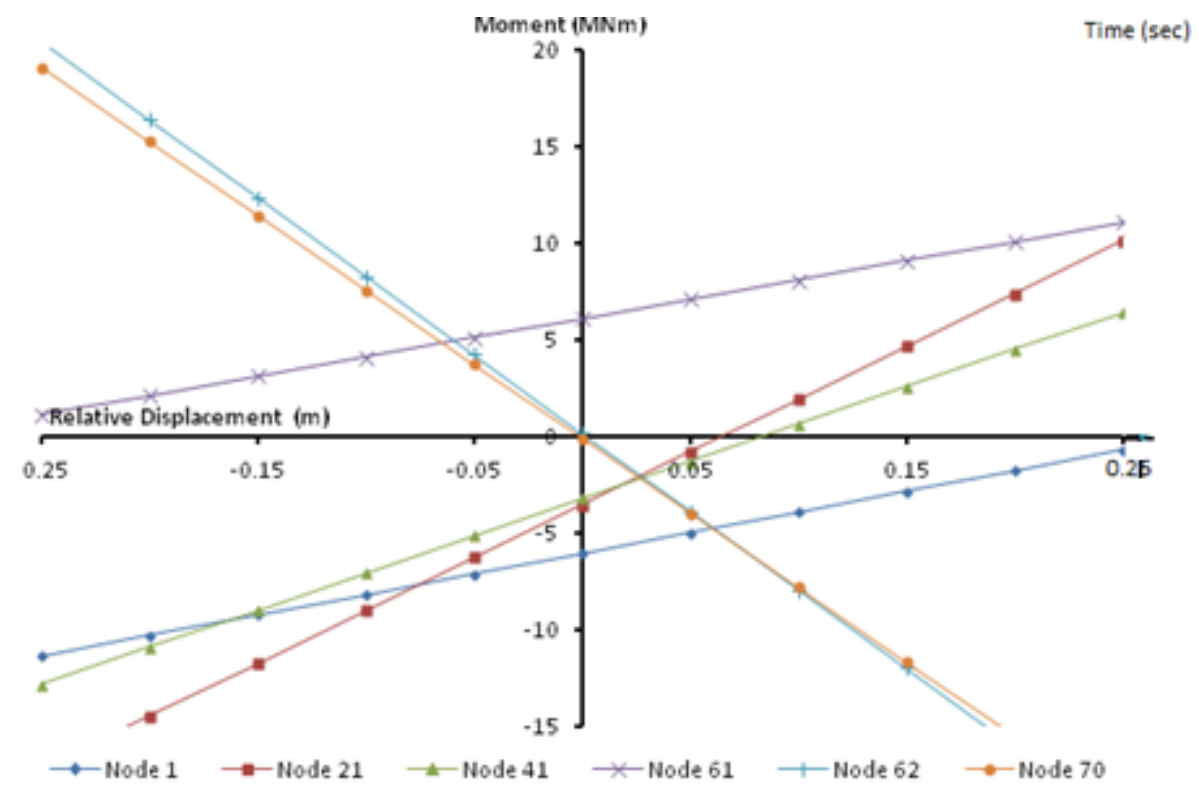

Figure VII-5: Linear relationships at all relevant locations

Having constructed the static linear $R D$-bending moment relationships, attention now turns to the dynamic analyses. For the transient dynamic analyses, the displacement time history input at one of the four supports was altered, thereby creating a relative displacement between this and the other three supports. Figure VII-6 shows these different time histories (specifically for nodes 1 and 62), and Figure VII-7 shows the relative displacement between them.

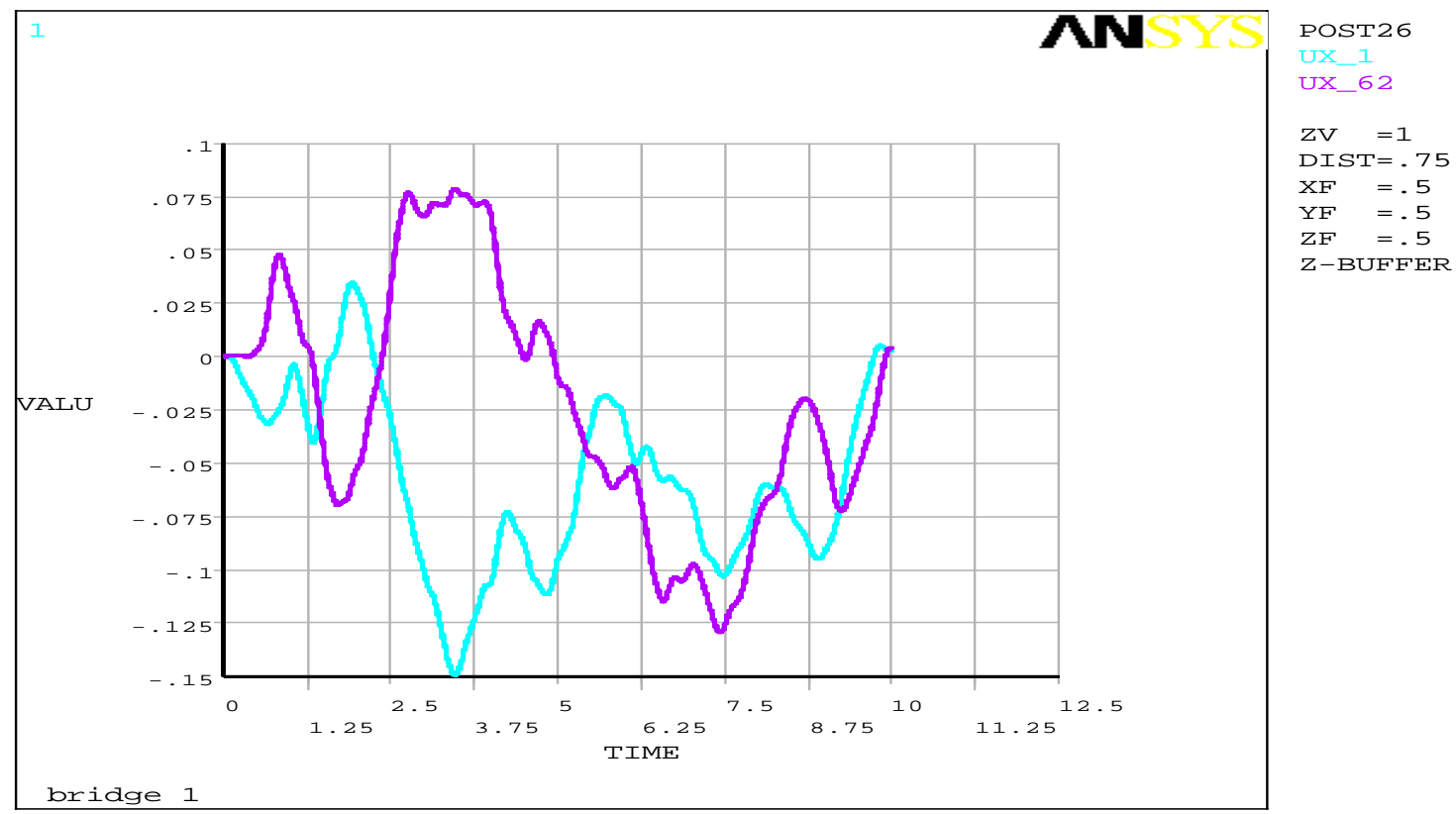

Figure VII-6: Displacements at nodes 1 and 62, input to ANSYS. 


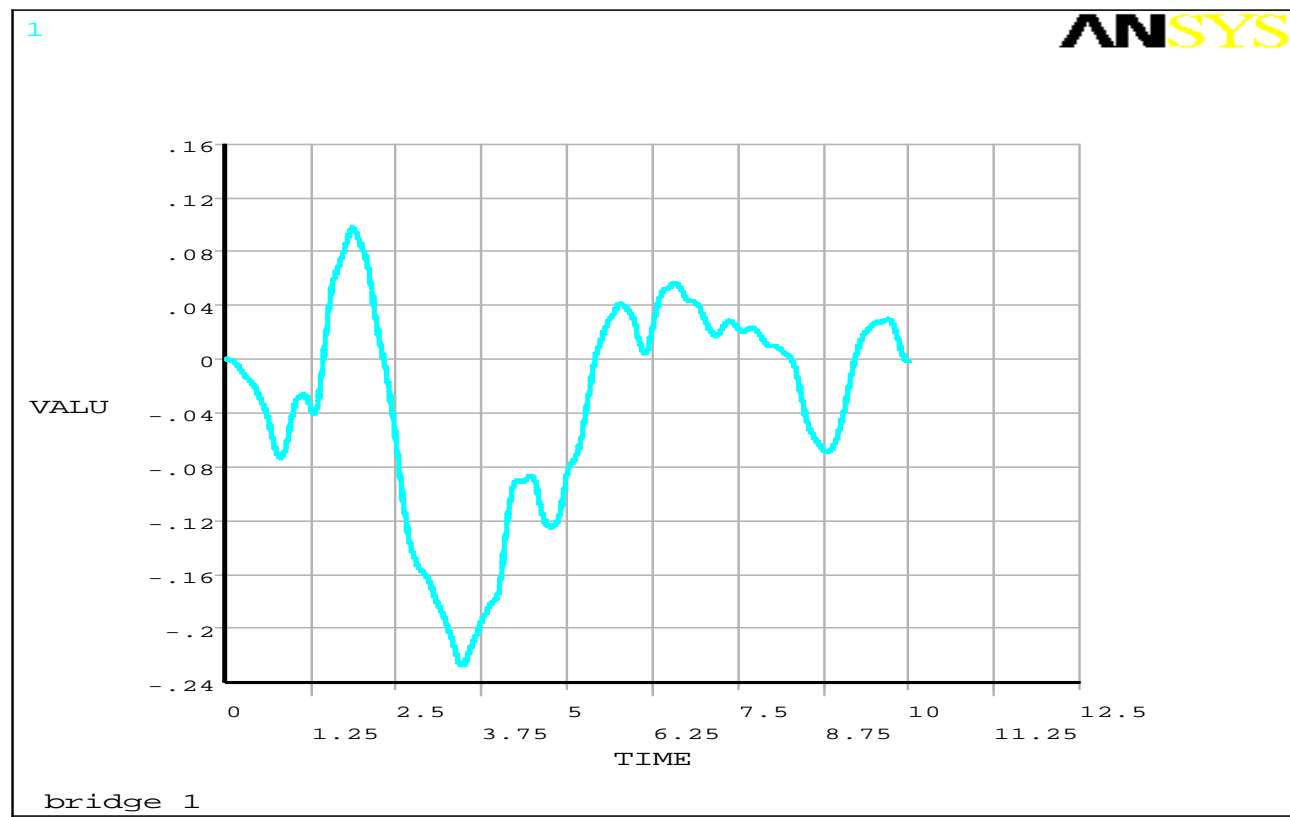

Figure VII-7: Relative displacement between node 1 and the other support locations

The relative displacement shown in Figure VII-7 can then be reproduced on Figure VII-3. Note that the time axis runs vertically on the graph in Figure VII-3, hence the relative displacement $(R D)$ time history is effectively rotated through 90 degrees clockwise. This is shown in isolation in Figure VII-8. The points of importance on the graph are now effectively the minimum and maximum values of relative displacement, so the time element is ignored for now. Two vertical dashed lines can now be drawn at these maximum and minimum peaks. 


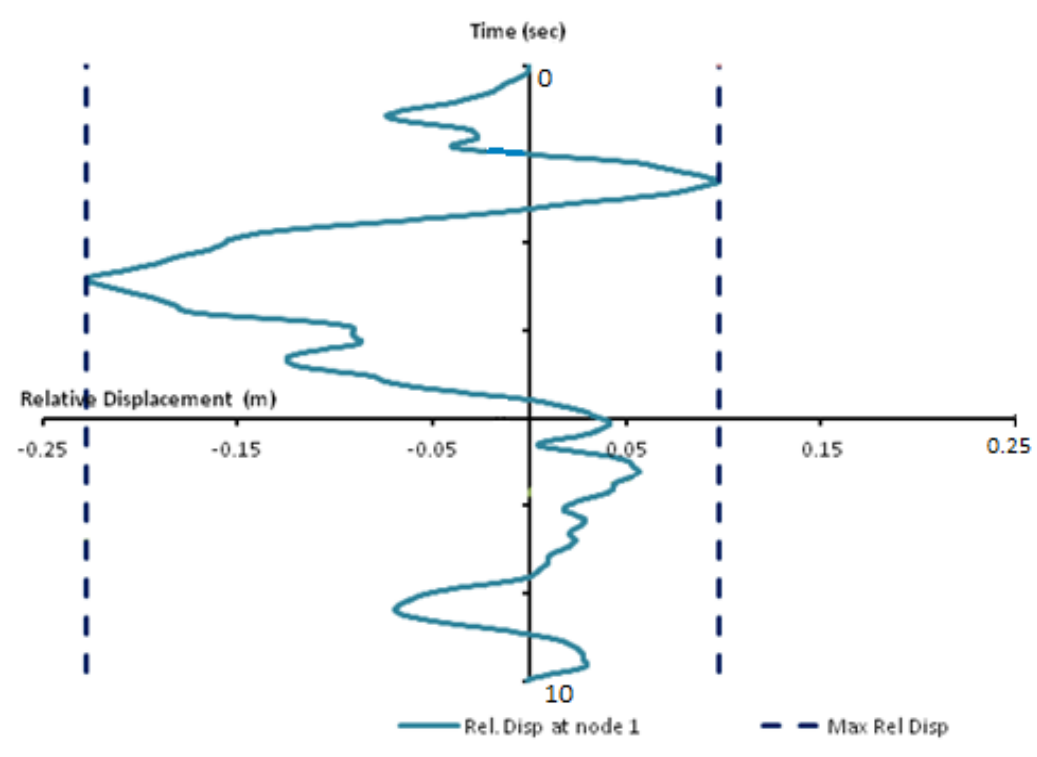

Figure VII-8: Dynamic relative displacement at node 1

Figure VII-9 re-introduces one of the static bending moment / $R D$ lines at a location, for example node 1. It then follows that the intersection of the vertical dashed lines and the static bending moment lines indicates the extreme bending moment values which would be obtained if the $R D$ was applied statically.

Figure VII-10 shows how these 'static' bending moments can then be obtained from the intersection point between the dashed 'max-min' lines and the node / location in question.

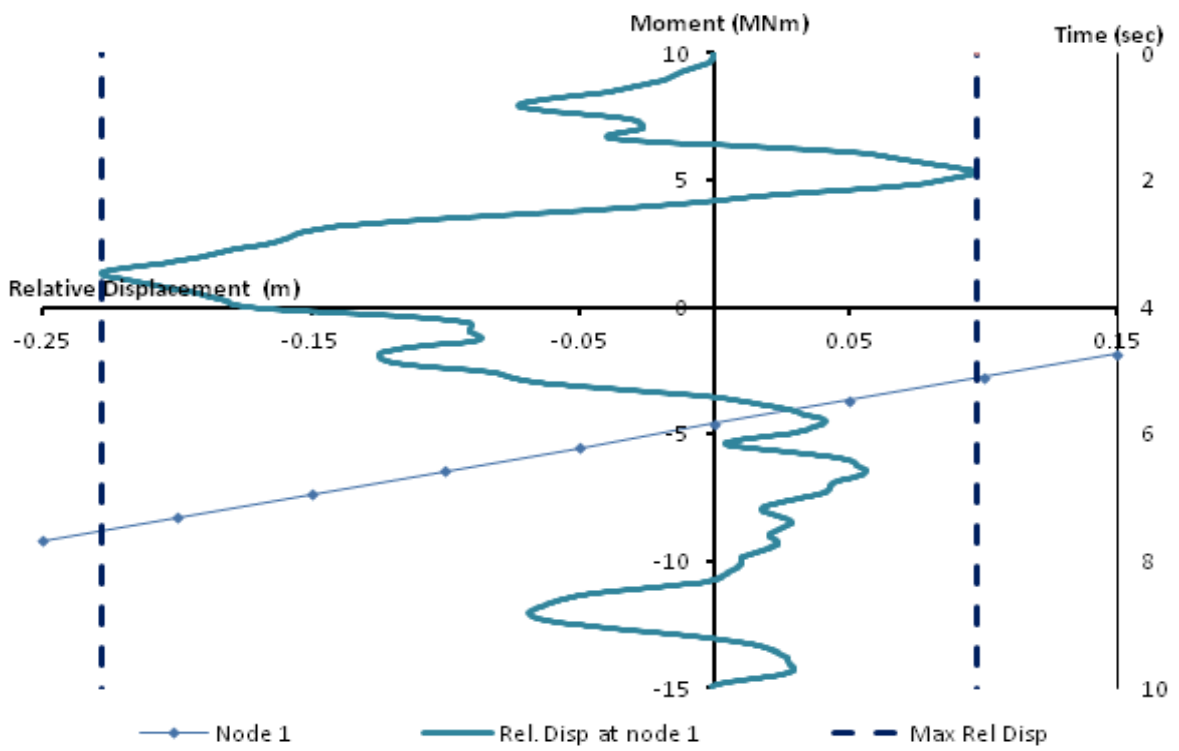

Figure VII-9: Dynamic relative displacement and static bending moment line. 


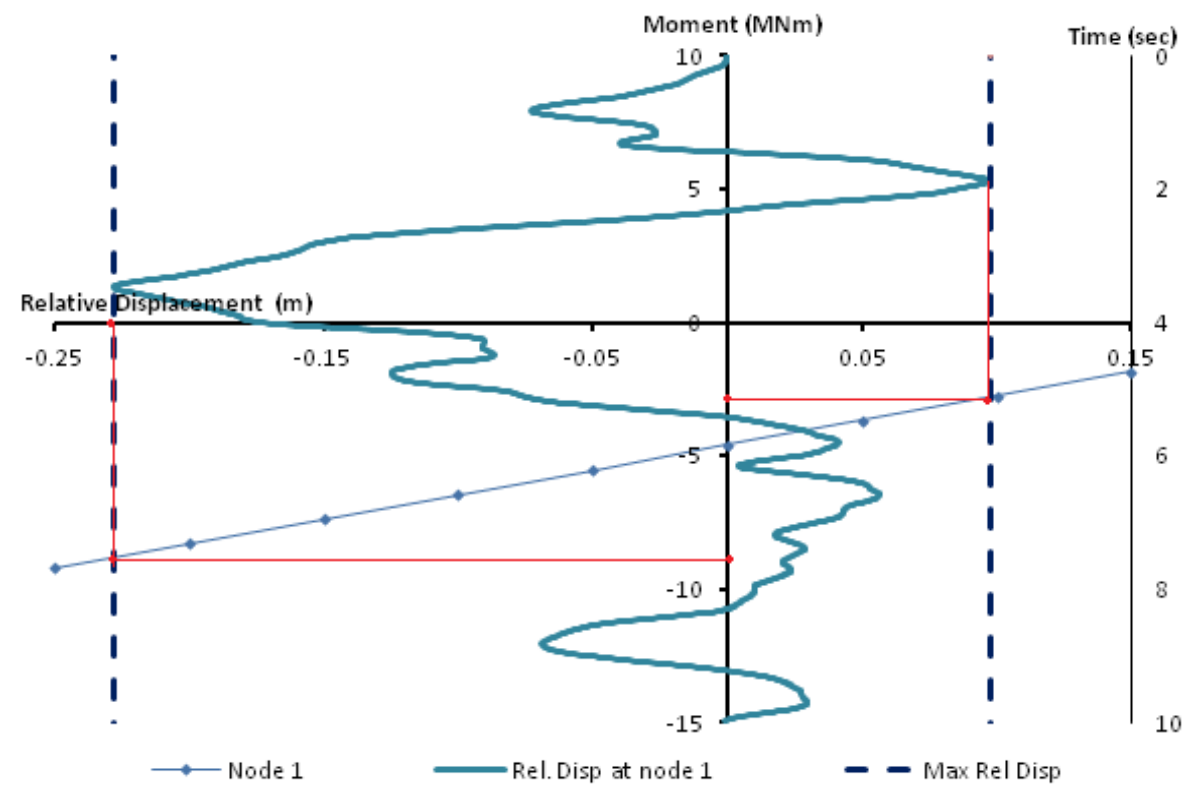

Figure VII-10: Correlation between linear bending moment to max relative displacement.

For example it can be seen from the graph that the maximum dynamic relative displacements occur at points $-0.22 \mathrm{~m}$ and $+0.097 \mathrm{~m}$ along the horizontal coordinate. The gradient line can be represented by the following equation:

$y=1.84 \times 10^{7} x-4.6 \times 10^{6}$ (Equation VII.1)

where:

$y=$ bending moment $(\mathrm{MNm})$

$x=$ relative displacement $(\mathrm{m})$

Thus, from this equation, the magnitude of the bending moment due to static relative displacement can be determined. In this example, for node 1 , the static bending moments due to relative displacement are $-2.80 \mathrm{MNm}$ and $-8.79 \mathrm{MNm}$. For the dynamic moment, transient analysis gives the magnitude of the dynamic bending moments as $0.67 \mathrm{MNm}$ and $-11.5 \mathrm{MNm}$.

Maximum static and dynamic values are compared to give the ratio. In this case, the maximum static moment is $8.79 \mathrm{MNm}$ and the maximum dynamic moment is $11.5 \mathrm{MNm}$.

The comparison of static bending moments due to relative displacement and dynamic bending moment, is the Static Dynamic Ratio $(S D R)$. Thus the SDR for node 1 is 8.79/11.5, which equals 0.76 . 
An SDR of 0.76 means the proportion of static moment due to relative displacement is $76 \%$ of the dynamic bending moment. Thus, the pure dynamic bending moment is about $24 \%$ of the dynamic result.

This confirms that at node 1, relative displacement plays an important role in the structural response, especially for bending moments.

These procedures are applied for nodes at the locations of interest around the bridge structure. The chosen nodes in this case are joint nodes, mid-span nodes and support nodes. The whole graphical construction can be seen in Figure VII-11.

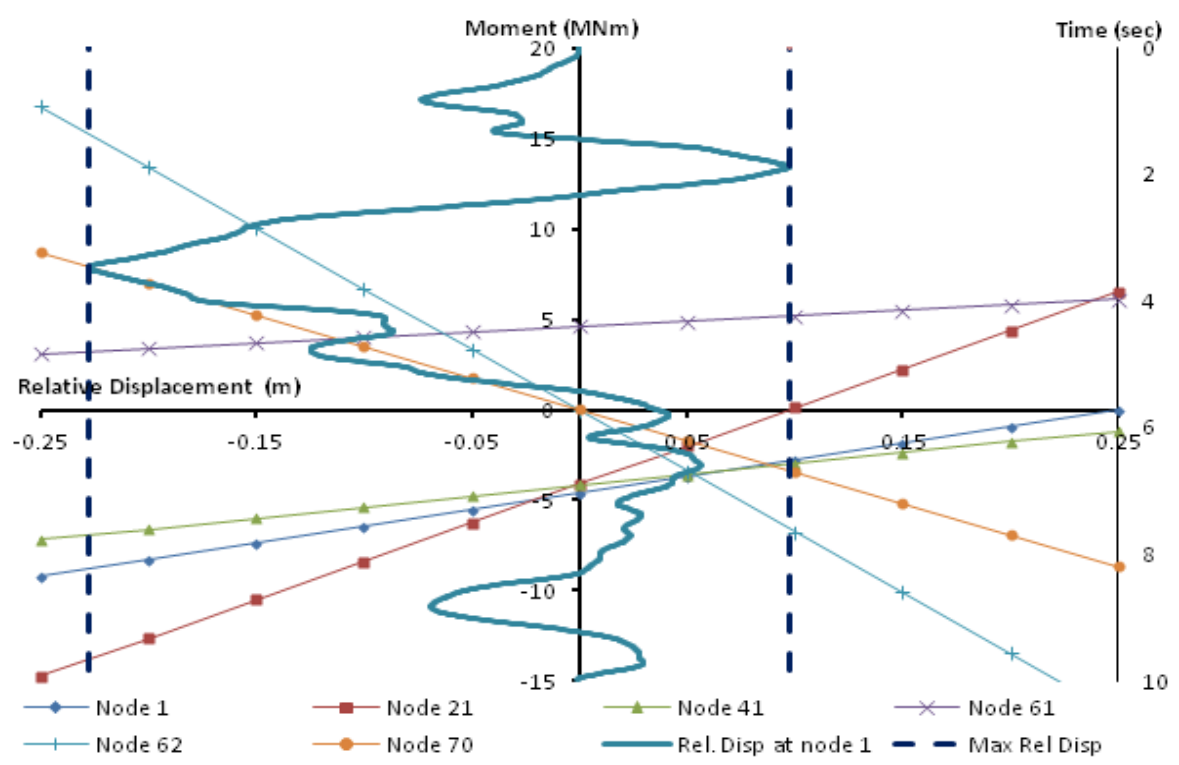

Figure VII-11: Graph for analyzing bending moment relationship to relative displacement in joint nodes. 


\subsection{Bridge response under horizontal and vertical relative displacements.}

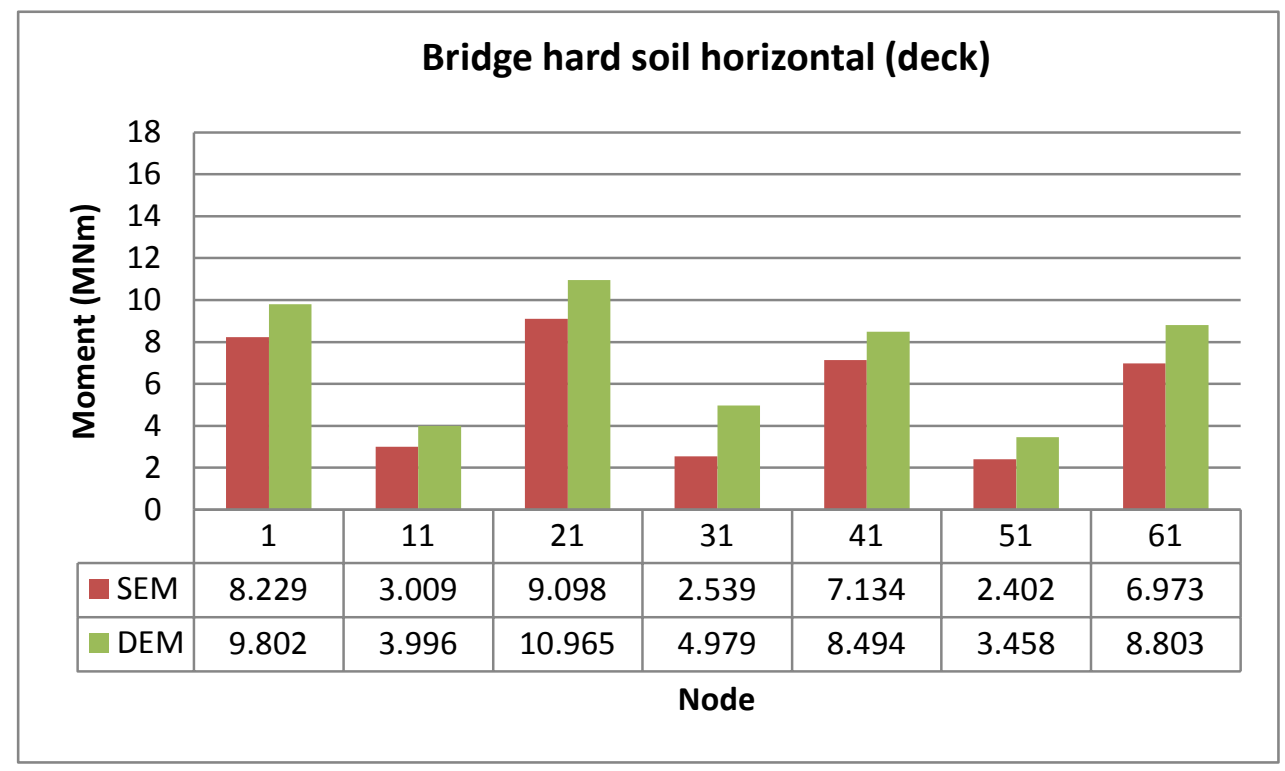

Figure VII-12: Bending moments under horizontal relative displacements (deck).

Applying horizontal relative displacement to node 1 (left end support) of this bridge may create a bending moment in the structure. By using the relative displacement and bending moment diagram, the magnitude of bending moments can be distinguished.

Figure VII-12 describes the magnitude of the bending moment of the deck under horizontal relative displacement. The red bars refer to static moments and the green bars to dynamic moments. The static bending moment is the moment obtained by considering relative displacement occurring in node 1 (left end node). Hence, this moment comes from the loads applied to the bridge without considering the dynamic load; these are gravity load and relative displacement. Meanwhile, the dynamic bending moment is the moment obtained from transient analysis by considering the different accelerations to the support, which then leads to relative displacement to node 1 . Therefore, dynamic bending is the accumulative bending moment of the bridge which consists of the moment from gravity load, dynamic load and relative displacement. Thus, the graph shows that dynamic bending moments are larger than static bending moments.

Figure VII-12 represents support nodes (nodes 1 and 61) and joint nodes (nodes 21 and 41)., while the rest (nodes 11, 31 and 51) are in mid-span. The mid-span nodes clearly suffer a less bending moment than support and joint nodes under horizontal relative displacement. 


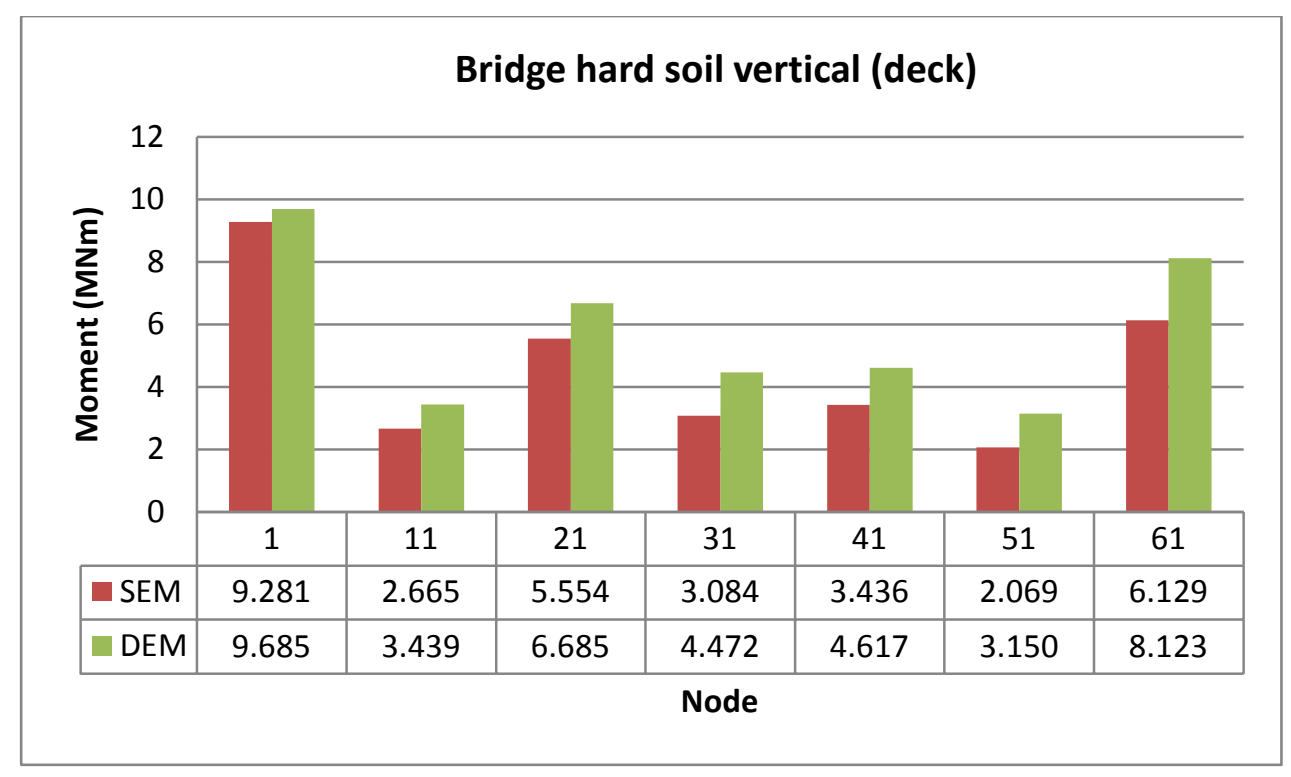

Figure VII-13: Bending moments under vertical relative displacements (deck).

Figure VII-13 describes the patterns of bending moment due to relative displacement applied vertically. As with the horizontal relative displacement, in the case of vertical relative displacement, the patterns of the comparison between static and dynamic bending moment are relatively the same. Mid-spans have smaller values of bending moment than support and joint nodes. In addition, the dynamic bending moment is larger rather than the static bending moment.

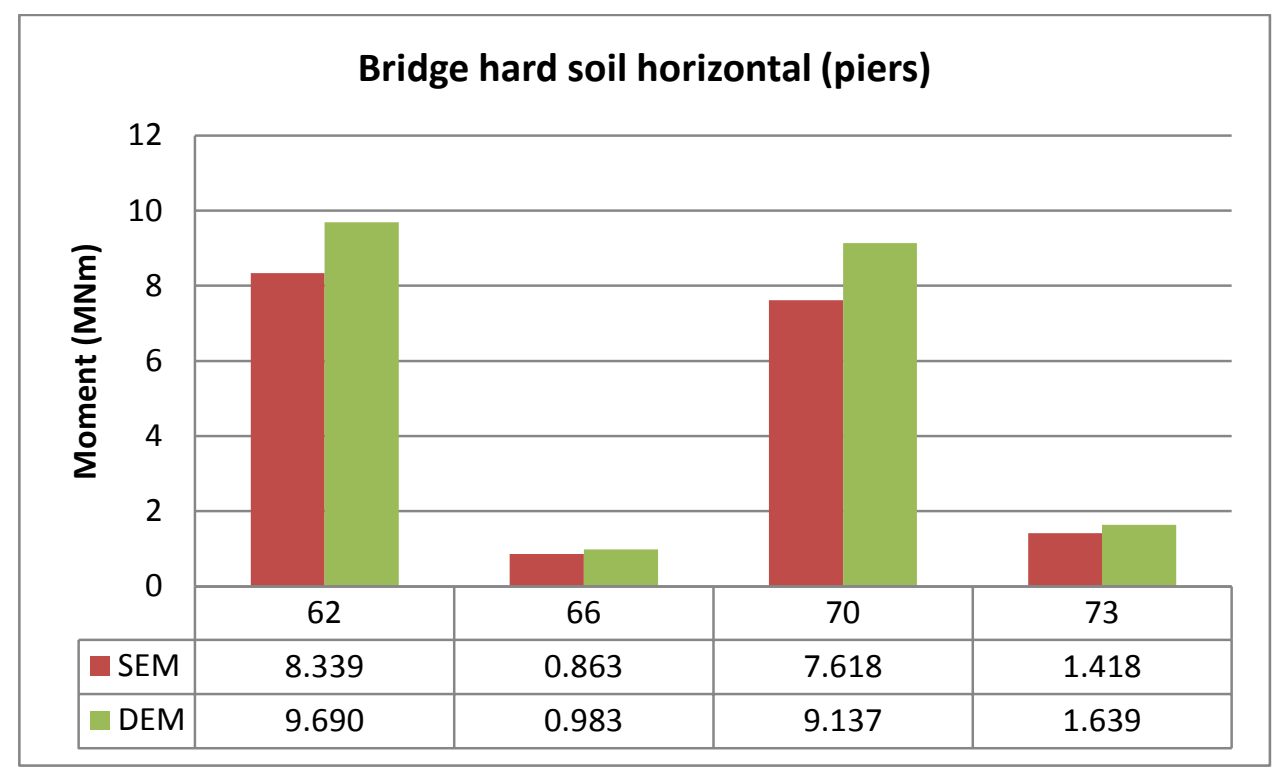

Figure VII-14: Bending moments under horizontal relative displacement (piers). 
The magnitude of the bending moment in piers under horizontal relative displacements is shown in Figure VII-14. In this case, the support nodes are 62 and 70, and the mid-span nodes are 66 and 73 . Figure VII-15 shows the magnitude of the bending moment due to vertical relative displacement. It can be seen that, similar to horizontal relative displacement, vertical relative displacement will create more bending moments in the bridge supports than in the mid-spans.

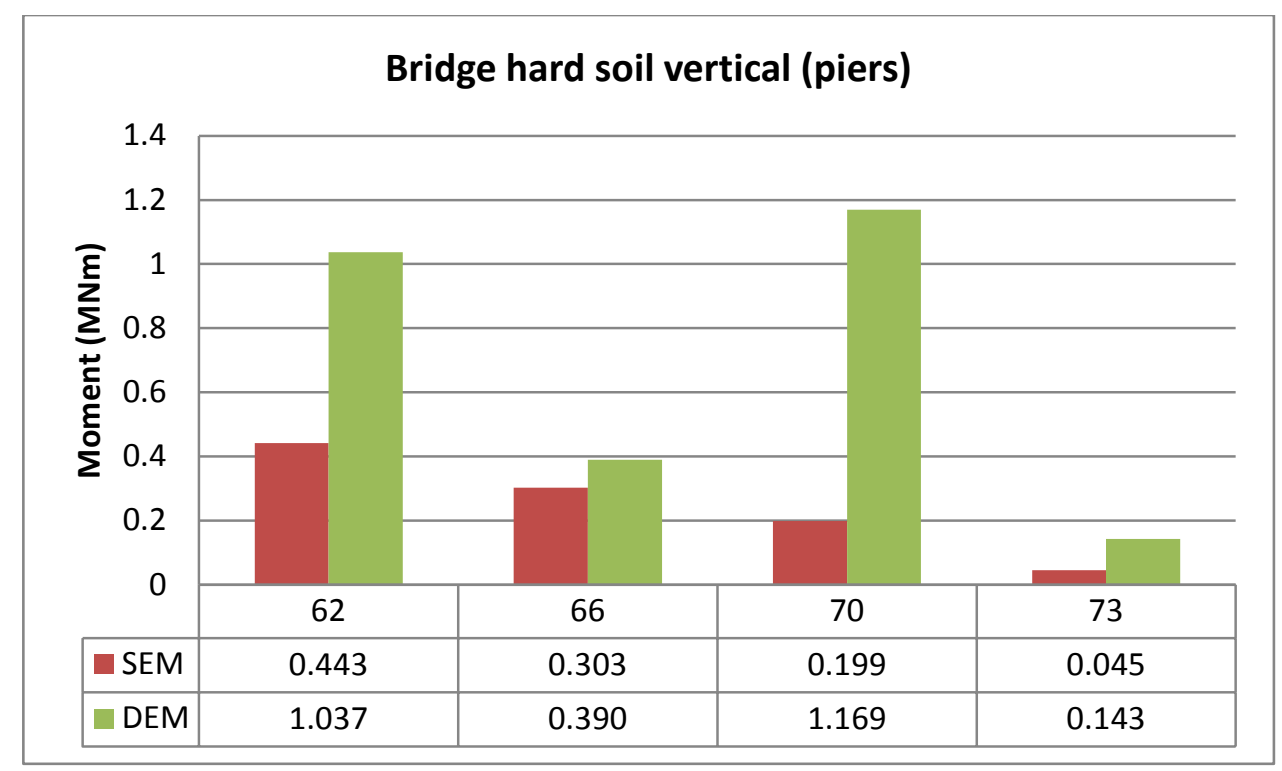

Figure VII-15: Bending moments under vertical relative displacements (piers).

Figure VII-15 represents the behaviour of the bridge under vertical relative displacement. As in the previous figures, the support nodes have larger bending moments than the midspan nodes. The difference between relative displacement applied vertically and horizontally is that in vertical relative displacement, node 70 (right pier support) has a larger bending moment than node 62 (left pier support); however, in horizontal relative displacement, node 62 has the larger bending moment. 


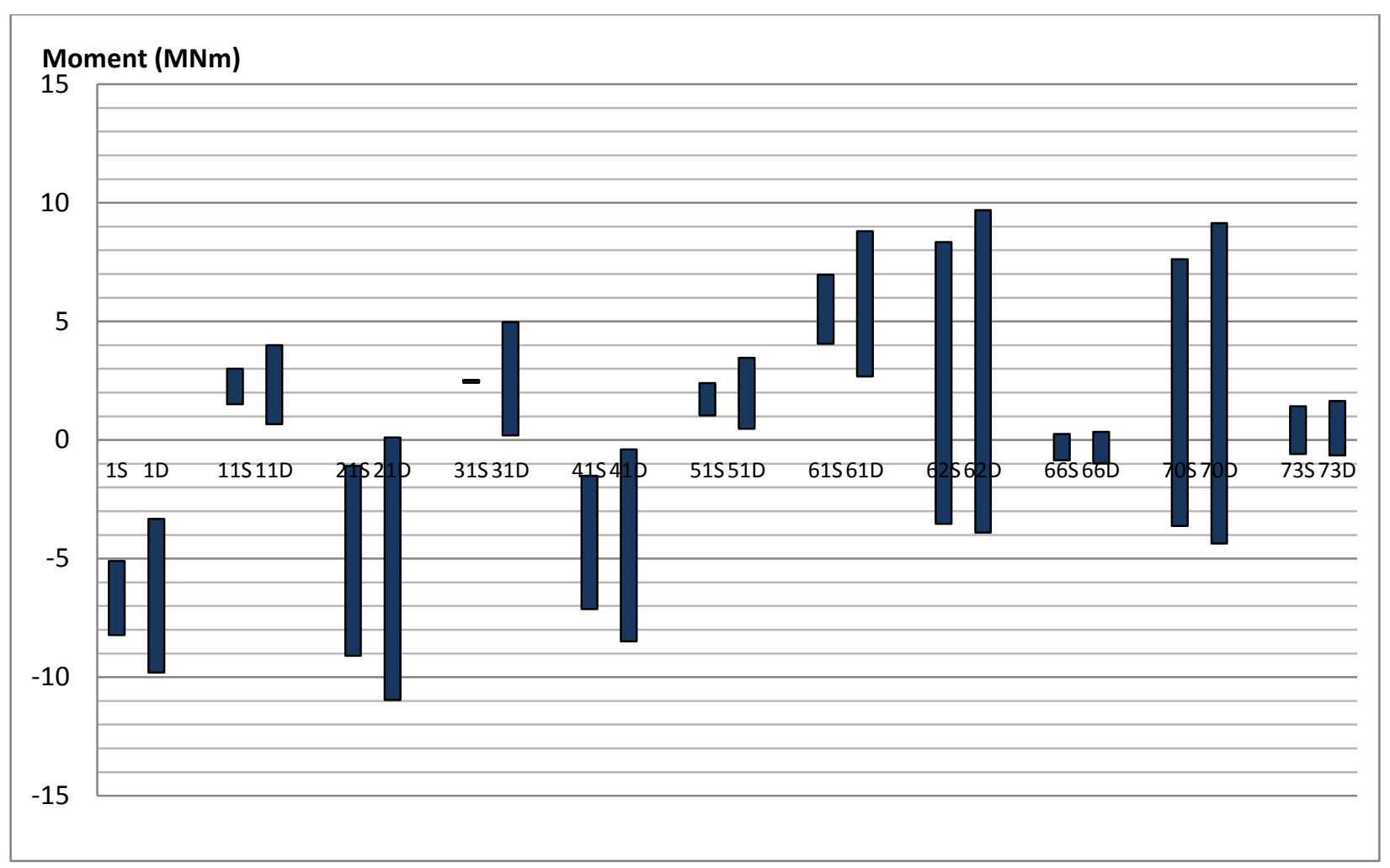

Figure VII-16: Pattern of bending moments under horizontal relative displacements .

Figure VII-16 presents the map of bending moments occurring in the bridge under horizontal relative displacement. The $x$ coordinate refers to the nodes, $S$ for static and $D$ for dynamic. The $y$ coordinate refers to the magnitude of bending moments in MNm. This figure identifies the condition of the bending moment, whether sagging $(+)$ or hogging $(-)$.

It can be seen that under horizontal relative displacement, mid-spans nodes suffer from sagging moments whereas joint and support nodes suffer from hogging moments. It also can be seen that static bending moments are smaller than dynamic bending moments. The difference between these two bending moments is greater in the deck area (nodes 1 to 61) than in the piers area (nodes 62 to 73 ). 


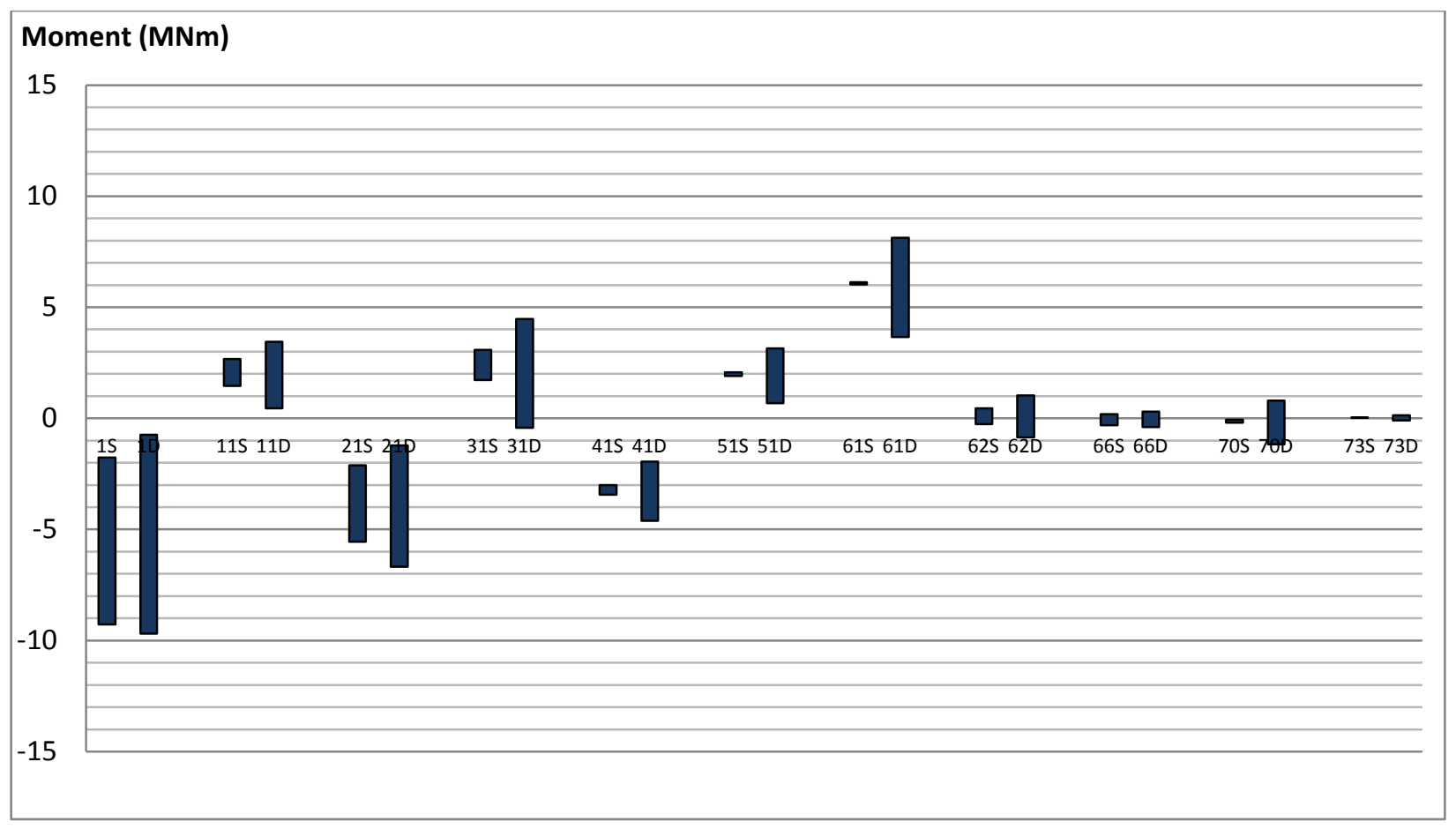

Figure VII-17: Pattern of bending moments under vertical relative displacements.

Figure VII-17 similarly explains the behaviour of the bridge under vertical relative displacement. The largest moment is in node 1 (left end support), where the relative displacement occurs. The piers have smaller moments than the other nodes.

\subsection{The contributions of relative displacement to the overall moment distribution of the bridges.}

The contribution of the relative displacements to the overall moment distribution is observed by using ratios. The bending moment due to relative displacement is compared with the bending moment resulting from transient analysis. This is the $S D R$, as explained above. From the SDR, the composition of bending moment (resulting from static relative analysis) in a total bending moment due to total loadings (resulting from dynamic analysis) can be obtained. By using this ratio, the effect of relative displacement on the overall bending moment of the bridge can be analyzed. The higher the ratio, the greater is the effect of relative displacement on that area or node.

The value of the $S D R$ can be used to estimate the constant factor to be applied to the design procedure in order to assess the magnitude of dynamic analysis using static relative displacement analysis. 


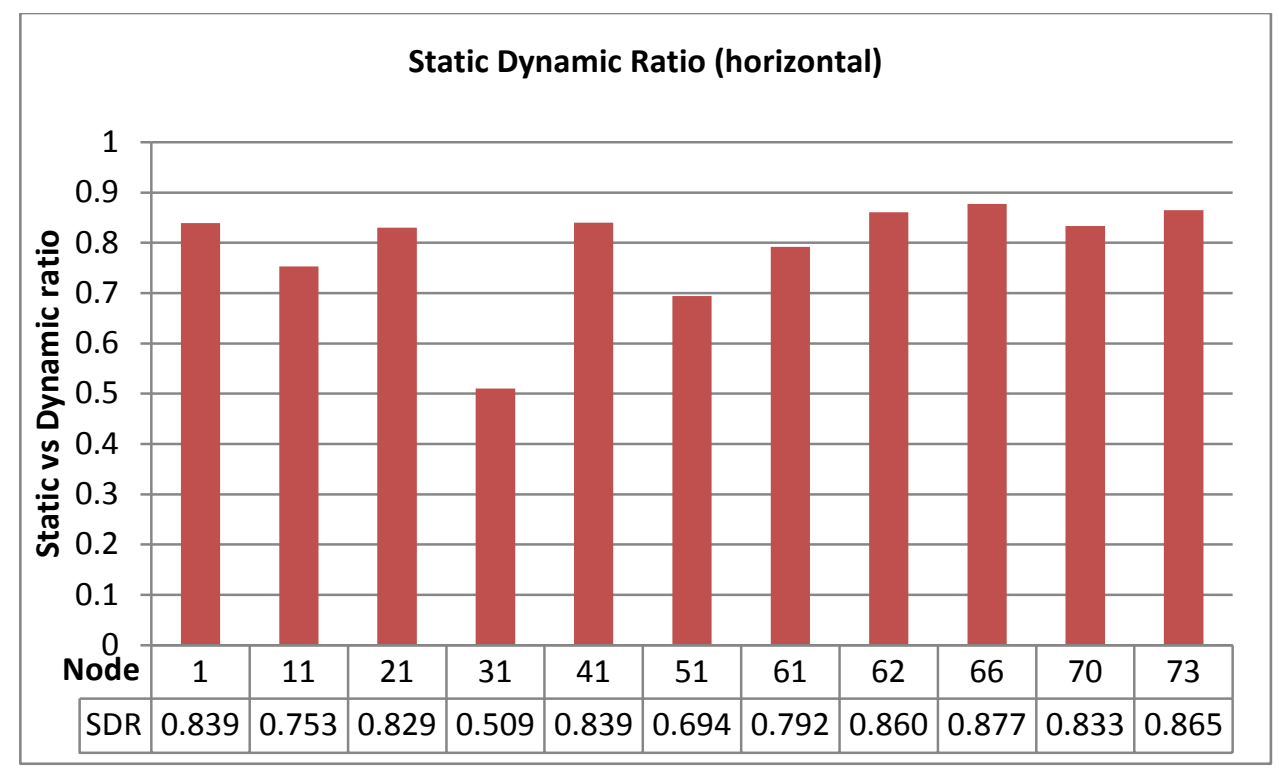

Figure VII-18: SDR due to horizontal relative displacement.

Figure VII-18 gives the $S D R$ values for the bridge under horizontal relative displacement. It can be seen that the ratios for the deck (nodes 1 to 61 ) lie between 0.5 and 0.85 . For the piers, the ratios lie between 0.8 and 0.9 .

The mid-span node (node 31) has the lowest ratio of static and dynamic bending moment. It means relative displacement has the lowest effect on this area. Piers, however, are considered to have the highest $S D R$, which means that these areas show the biggest effect of horizontal relative displacement. 


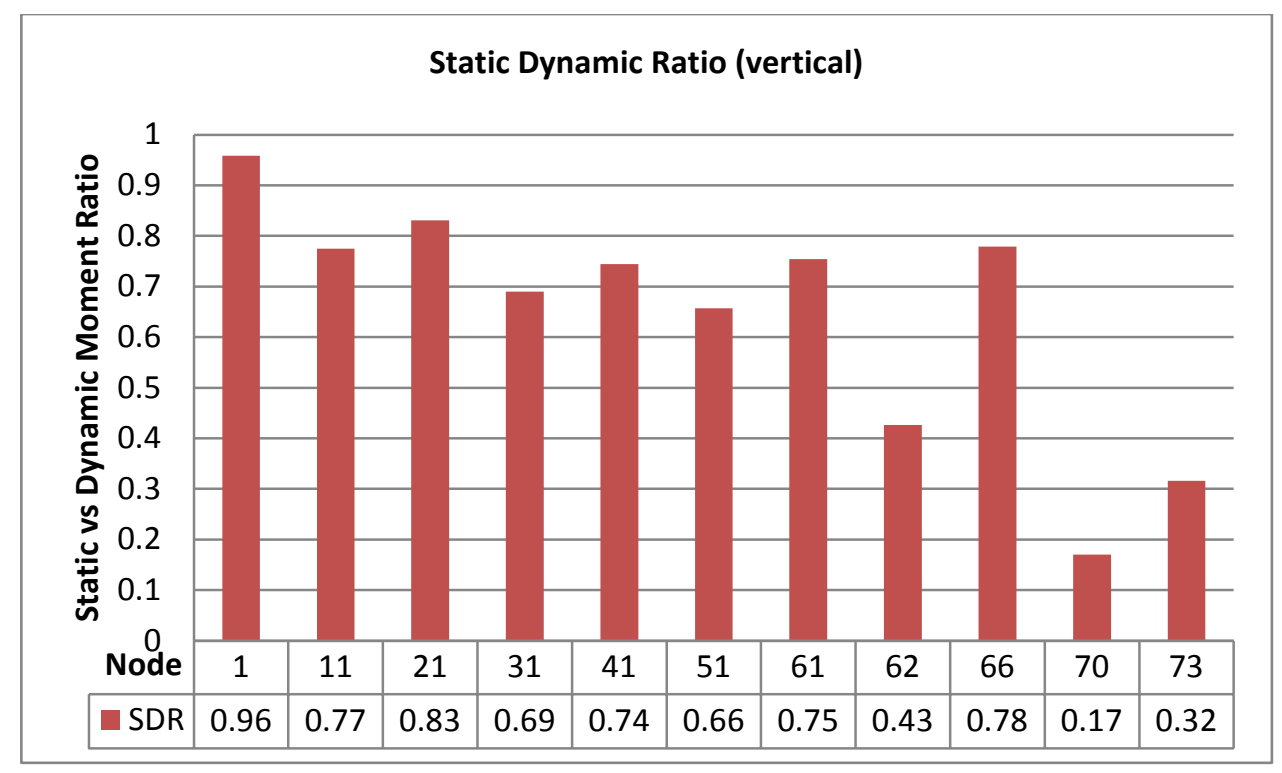

Figure VII-19: Pattern of bending moments under vertical relative displacements.

Figure VII-19 shows the ratio of static and dynamic bending moments for vertical relative displacements. Node 1 (end left support) has the highest ratio. On the other side, piers have smaller values, excluding node 66 (mid-span of left piers). Unlike horizontal relative displacement, vertical relative displacement yields larger ratio for the deck area than the piers area.

\subsection{The responses of the bridge under differing soil conditions}

In this study, several soil conditions are considered, in order to examine the effects differences in soil stiffness on the relative displacements to the bridge. Although the study does not focus on the geo-technical aspects of soil and the bridge, assessing the bridge with different soil stiffness conditions can be used to estimate its behaviour under different soil types.

The magnitudes of bending moment due to static relative displacements on different soil stiffness under horizontal relative displacements are described in Figure VII-20. At several nodes the value of bending moments have increased, while at others they have decreased. Overall, the stiffness of the soil does affect the bending moments of the structure. 


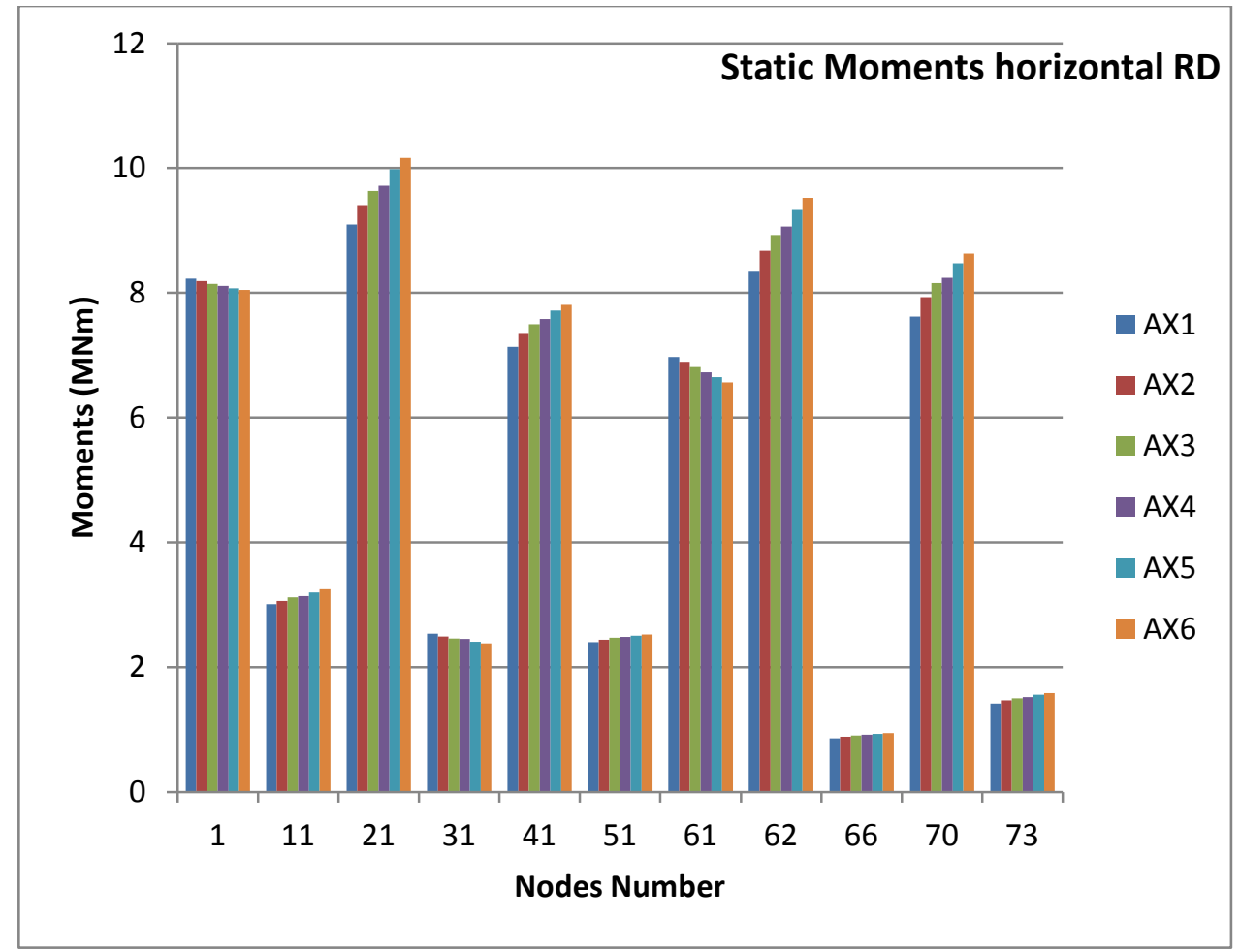

Figure VII-20: Effect of soil stiffness differences in static bending moment (horizontal RD)

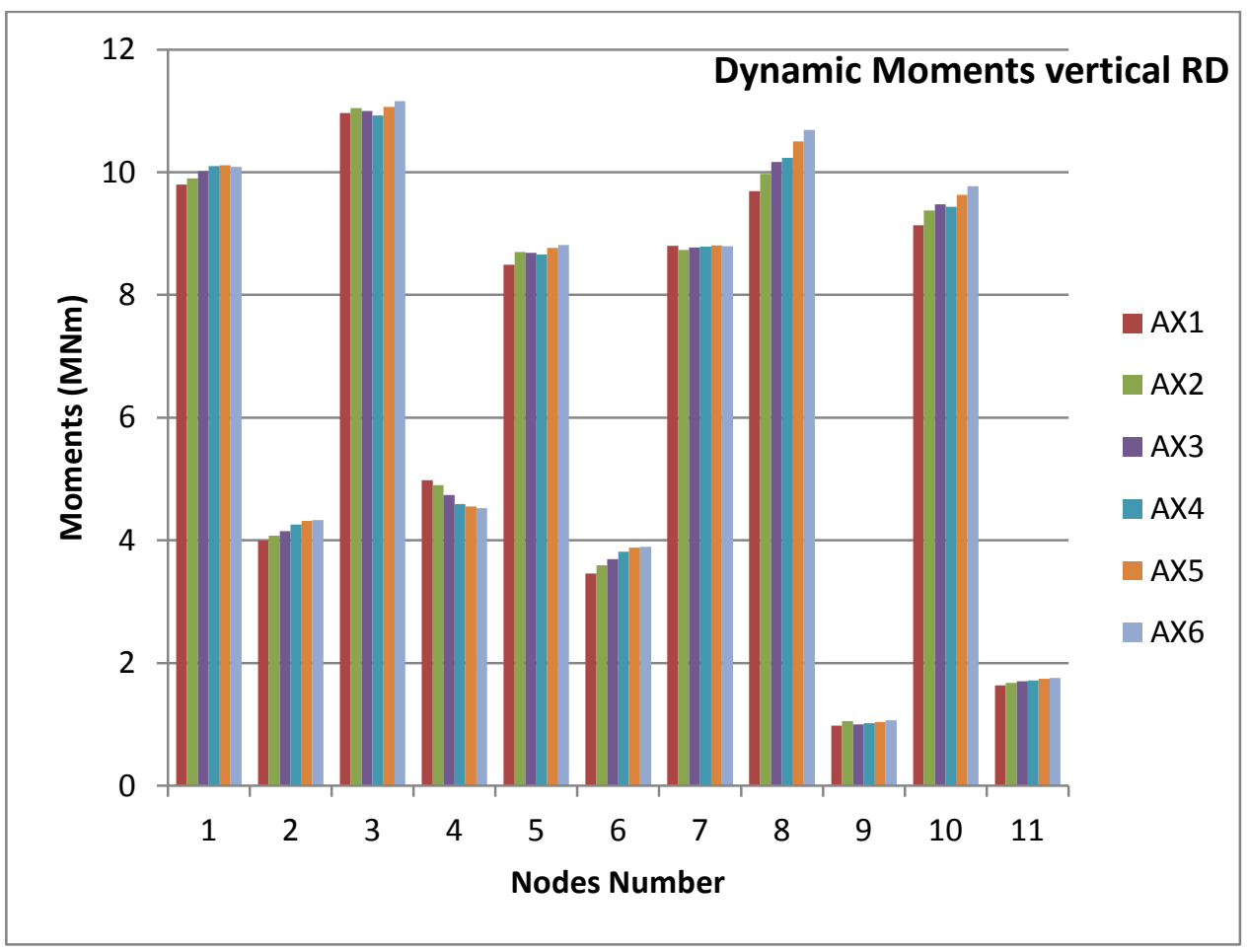

Figure VII-21: Effect of soil stiffness differences in dynamic bending moment (horizontal relative displacement) 
Figure VII-21 shows the magnitude of bending moment due to dynamic loadings, for different soil stiffness. The results are obtained from transient analysis. In general, the patterns of the increase in moments are similar to the static bending moments.

The two figures VII-22 and VII-23 show the behaviour of the bridge under different soil conditions for horizontal and vertical stiffness. The patterns of the graphs in these figures are similar to the patterns for a bridge which has the same soil stiffness vertically and horizontally. It can be seen from these two figures, that when horizontal and vertical relative displacement is applied, supports and joints experience greater effects than the mid-span area.

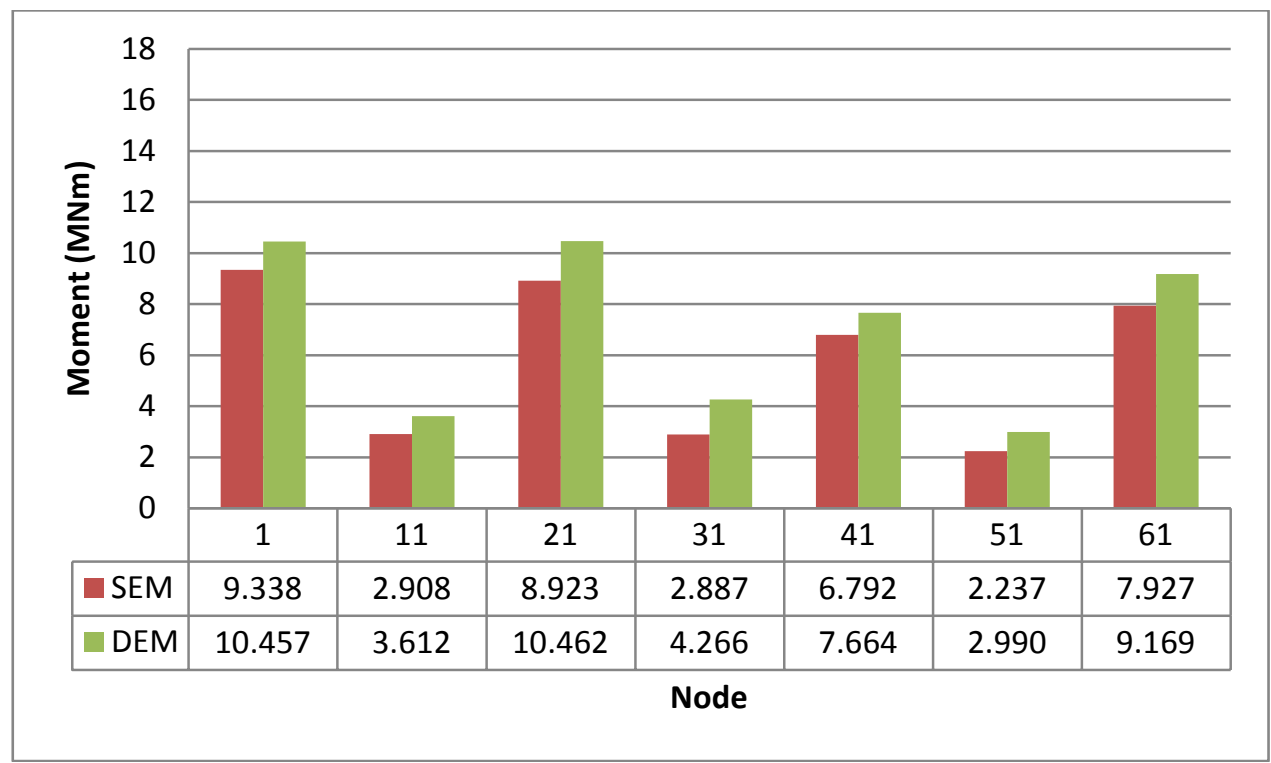

Figure VII-22: Bending moment for different vertical and horizontal soil stiffness due to horizontal relative displacement (deck). 


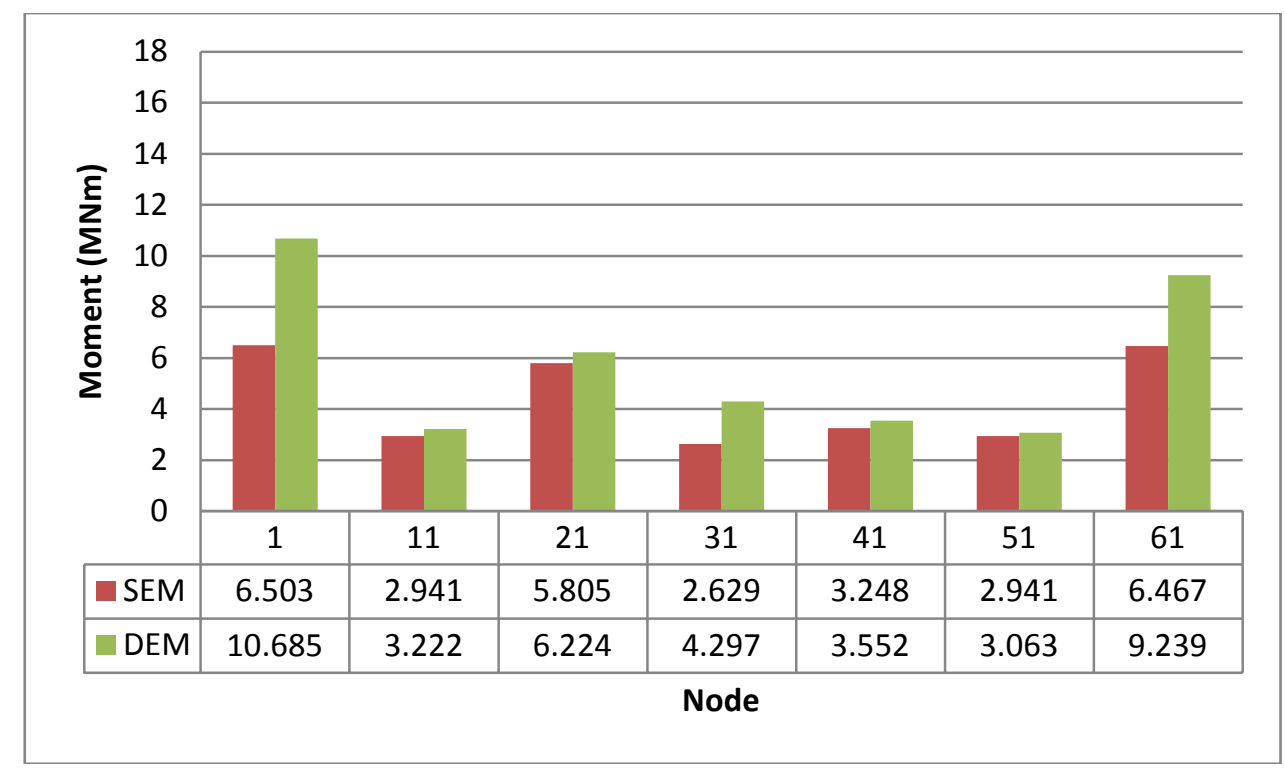

Figure VII-23: Bending moment for different vertical and horizontal soil stiffness due to vertical relative displacement (deck).

\subsection{The responses of the bridge under a fixed base support condition.}

Figure VII-24 is the ratio of static and dynamic bending moments of the fixed based bridge under horizontal relative displacement. It can be seenthat the piers have a higher ratio than the deck nodes, and that mid-span bridge nodes have a lower ratio than the joints.

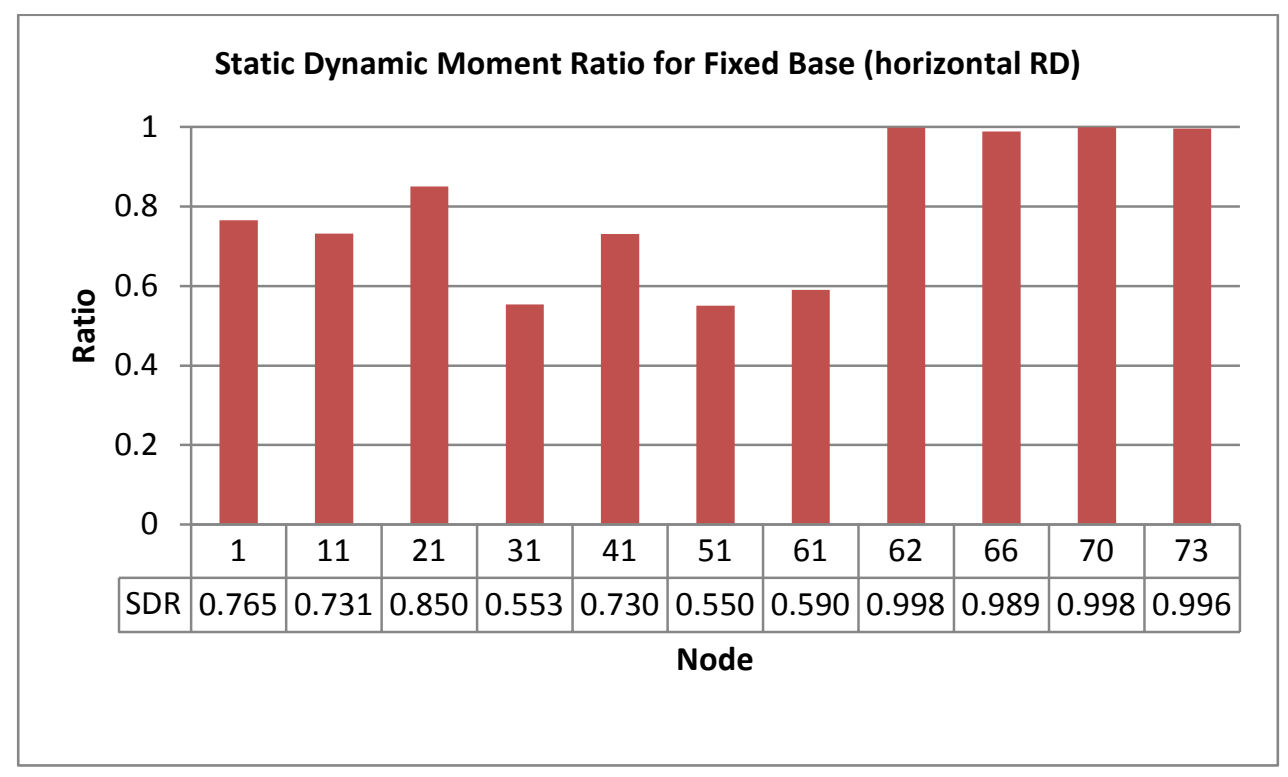

Figure VII-24: Static Dynamic Moment ratio for fixed base (horizontal relative displacement) 


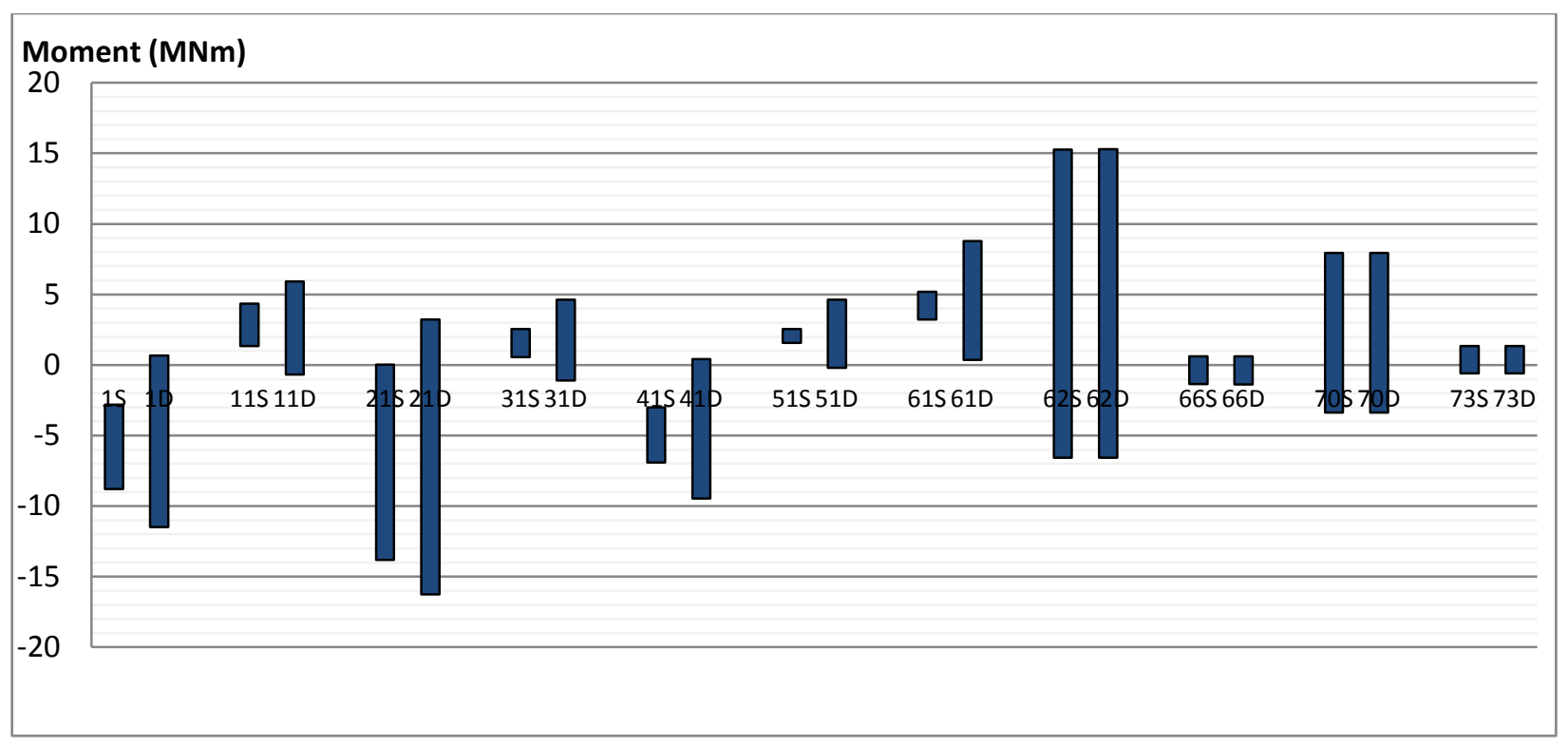

Figure VII-25: Pattern of bending moments under horizontal relative displacements (fixed base).

Figure VII-25 describes the map of bending moments in the bridge. It can be seen that static moments in the deck area (nodes 1 to 61 ) are smaller than the dynamic moments. Meanwhile, in the piers the static bending moments are relatively close to the dynamic bending moments. It also can be seen that horizontal relative displacement creates significant bending moments in piers. Figures VII-26 to VII-31 illustrate the characteristic behaviour of fixed based bridges under horizontal and vertical relative displacement loadings; the discussion parallels that above.

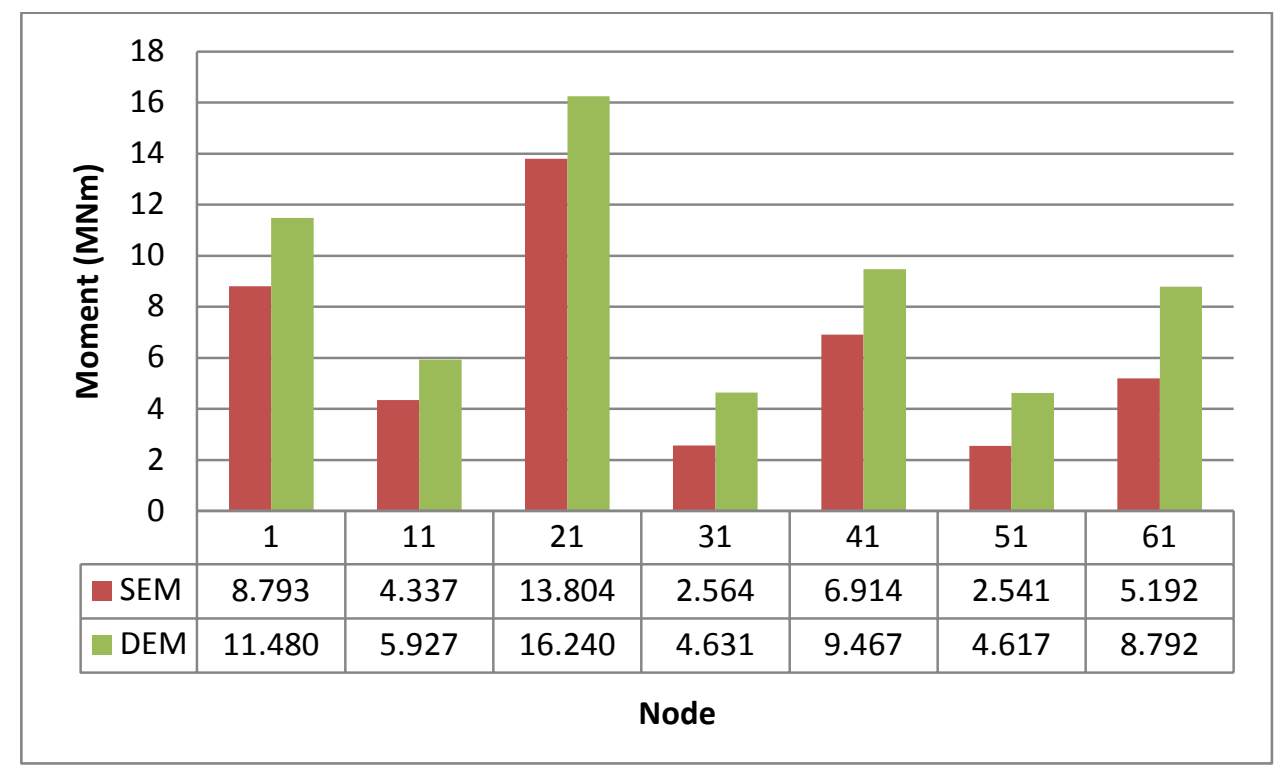

Figure VII-26: Bending moment for fixed base bridge due to horizontal relative displacement (deck). 


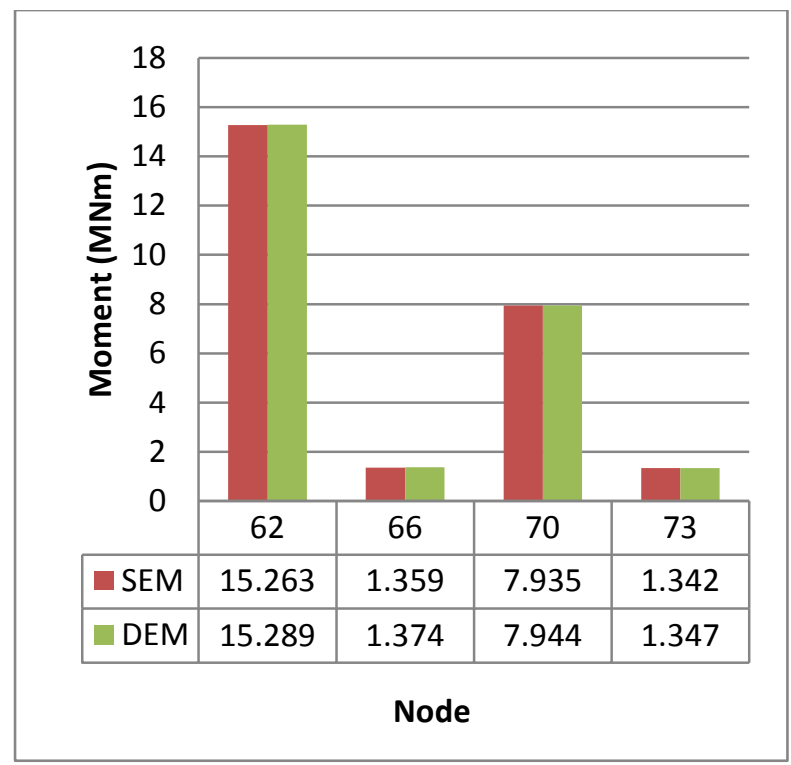

Figure VII-27: Bending moment for fixed base bridge due to horizontal relative displacement (piers).

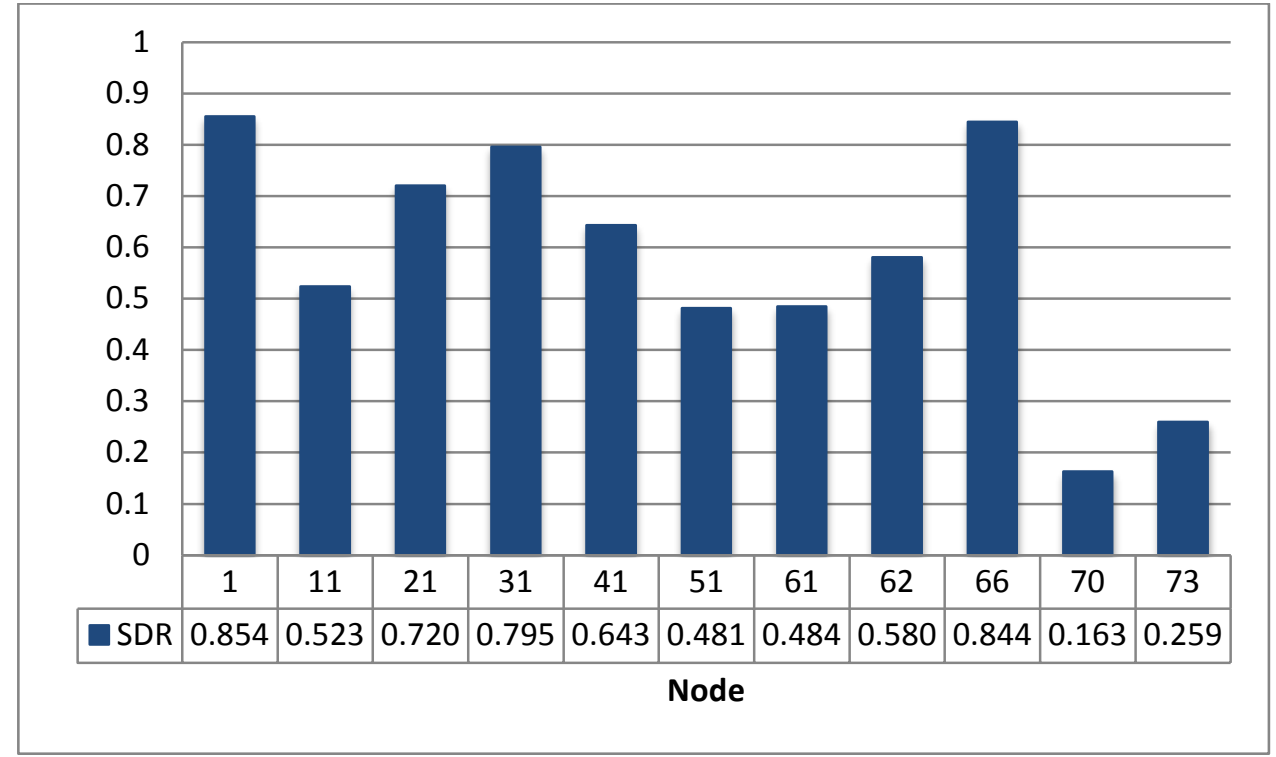

Figure VII-28: Static Dynamic Moment ratio for fixed base (vertical relative displacement) 


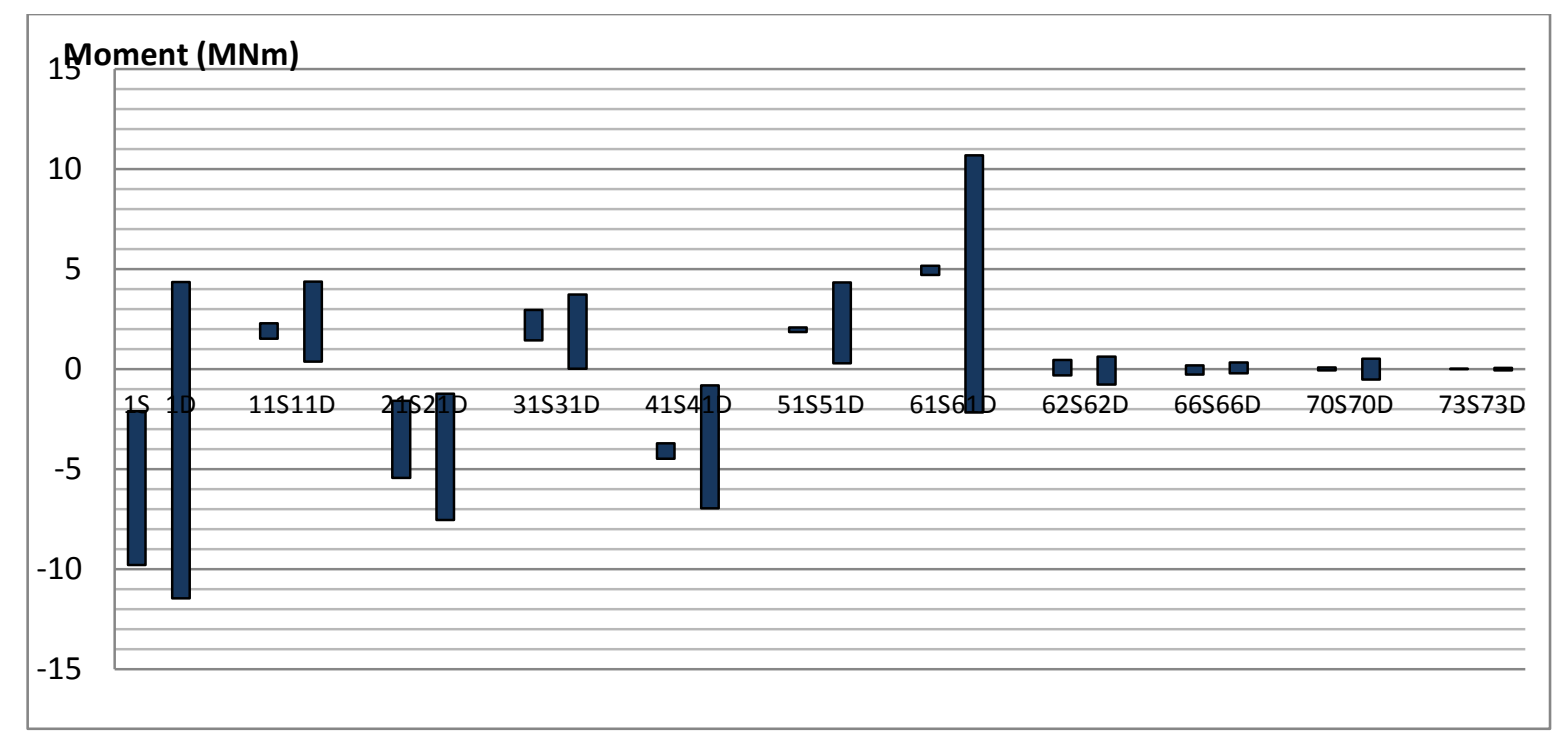

Figure VII-29: Pattern of bending moments under vertical relative displacements (fixed base).

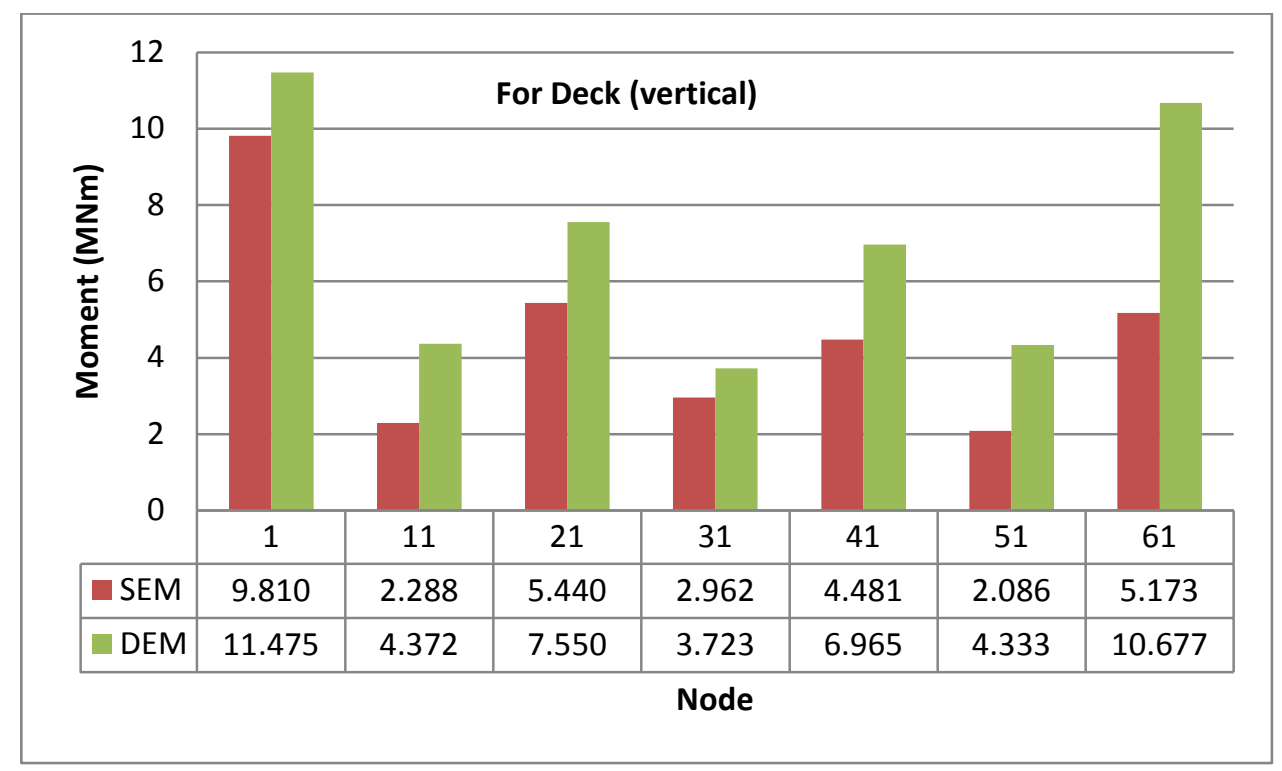

Figure VII-30: Bending moment for fixed base bridge due to vertical relative displacement (deck). 


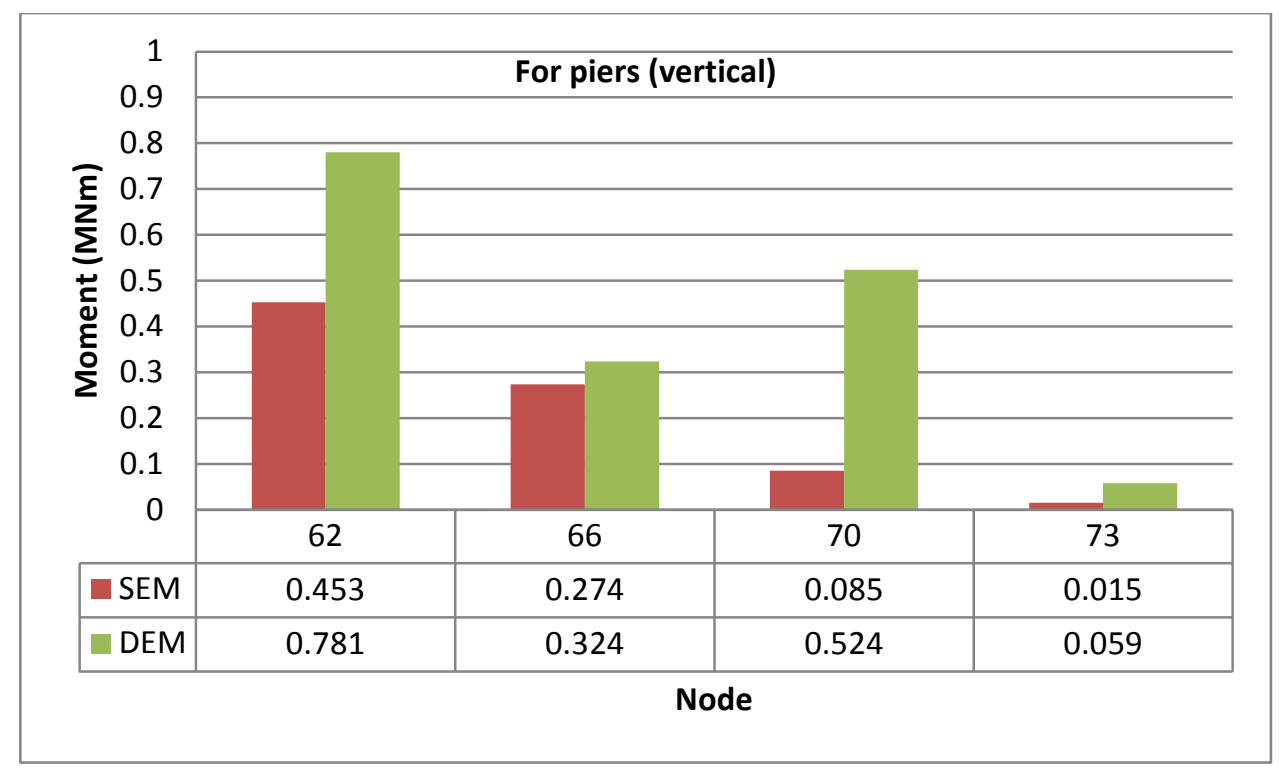

Figure VII-31: Bending moment for fixed base bridge due to horizontal relative displacement (deck).

\subsection{Static and Dynamic Relative Displacement - Moments Relationship}

\subsubsection{Static-Dynamic Bending Moment Ratio}

The static-dynamic bending moment ratio is defined in this study as a comparison of the static and dynamic bending moments of the bridge structure.

$$
S D R=\frac{\text { Static Bending Moment }}{\text { Dynamic Bending Moment }}
$$

Static bending moments are identified as described previously, and dynamic bending moments through transient analysis. The greater the ratio, the greater is the contribution of static relative displacement to the dynamic bending magnitude of the element.

Discussion is separated into two the different cases under consideration in this research, namely the fixed base (case 1) and the spring base (case 2). 


\subsubsection{Case 1 (Fixed Base)}

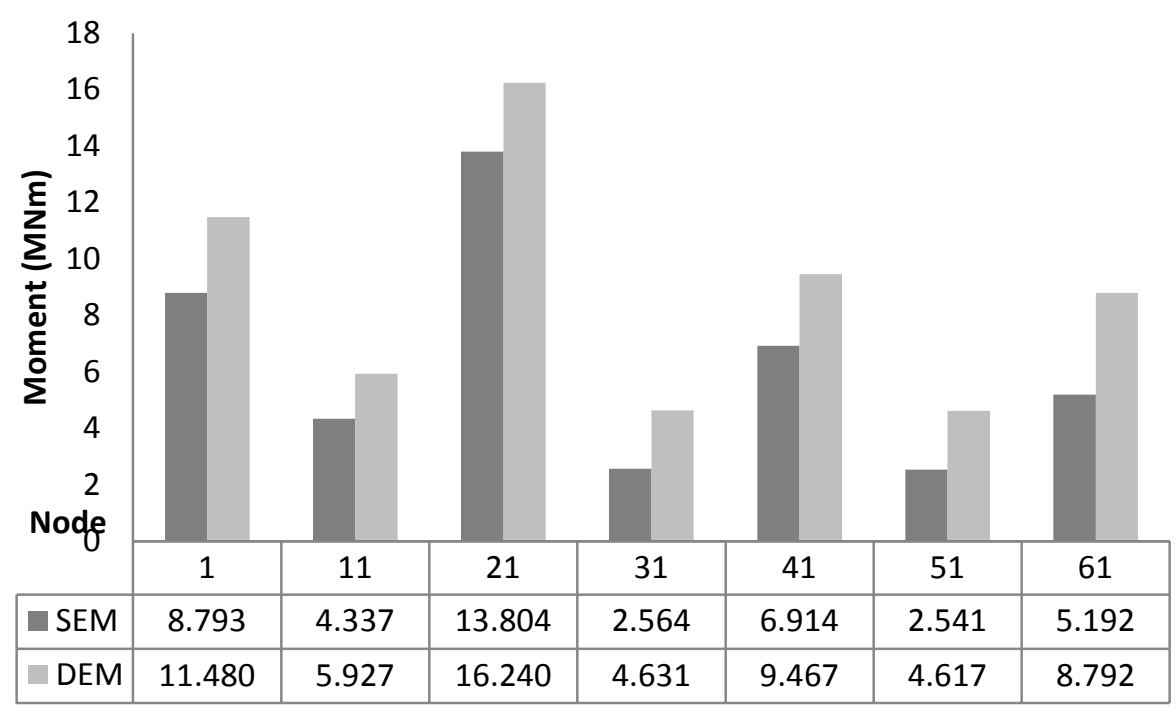

Figure VII-32: Moment distribution value for deck for horizontal direction

Figures VII-32 and VII-33 summarize the extreme static and extreme dynamic moments of nodes 1 to 61 with fixed base supports. The darker bar refers to static moments and the lighter to dynamic moments. Loads applied to the structure are relative displacements, gravity load and earthquake load. It can be seen from Figure VII-32 that dynamic moments are always higher than static moments, especially in the deck of the bridge. In the piers the static and dynamic moments are almost the same(Figure VII-33).

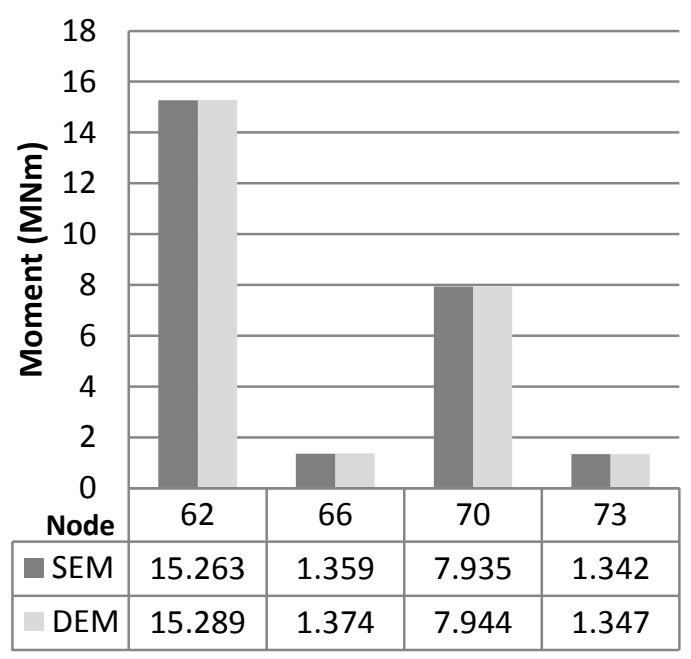

Figure VII-33: Moment distribution value for piers for horizontal direction 
The next step is to determine the ratio of static and dynamic moments of the structure. The results can be seen in Figure VII-34.

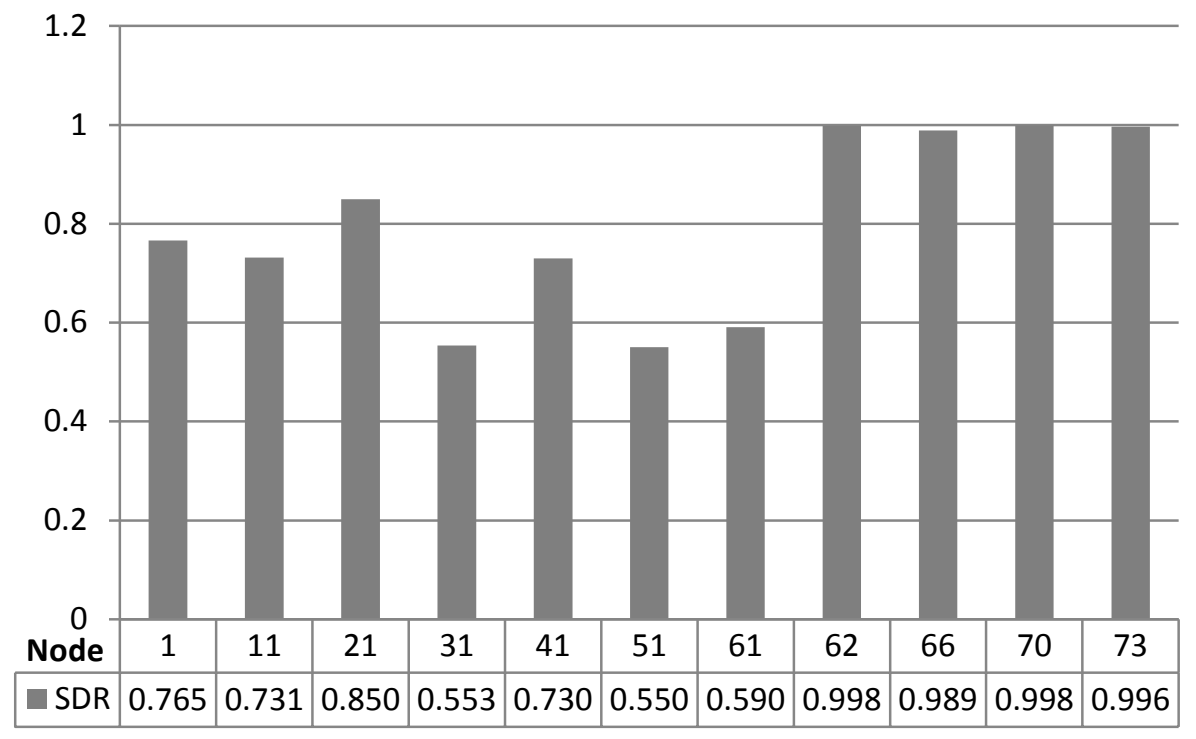

Figure VII-34: Static dynamic moment ratio for fixed base for horizontal relative displacement

The graph shows that the ratio between static and dynamic moments $(S D R)$ at all nodes is more than 0.5. In other words, relative displacements of the supports have more impact on the structure than dynamic alone. For the piers, the static ratio is almost $100 \%$, which means that the relative displacements are considered significant here. In the deck region, the effect of horizontal relative displacement is less significant for the mid-spans area than for the joints area. In other words, the area most affected by horizontal relative displacement is the joints. 


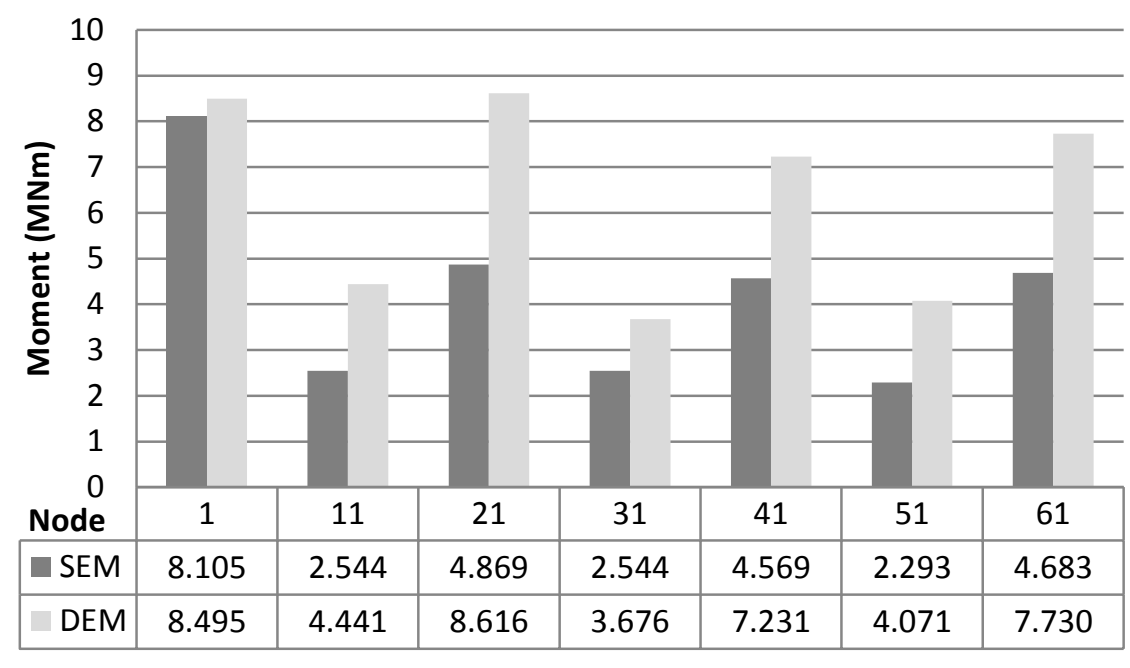

Figure VII-35: Moment distribution value for deck for vertical direction

The bar chart in Figure VII-35 shows the effect of vertical relative displacements on moment distribution in the deck area. It can be seen that nodes in joints (i.e nodes 1, 21, 41 and 61) have higher values than those in middle nodes (i.e nodes 11, 31 and 51).

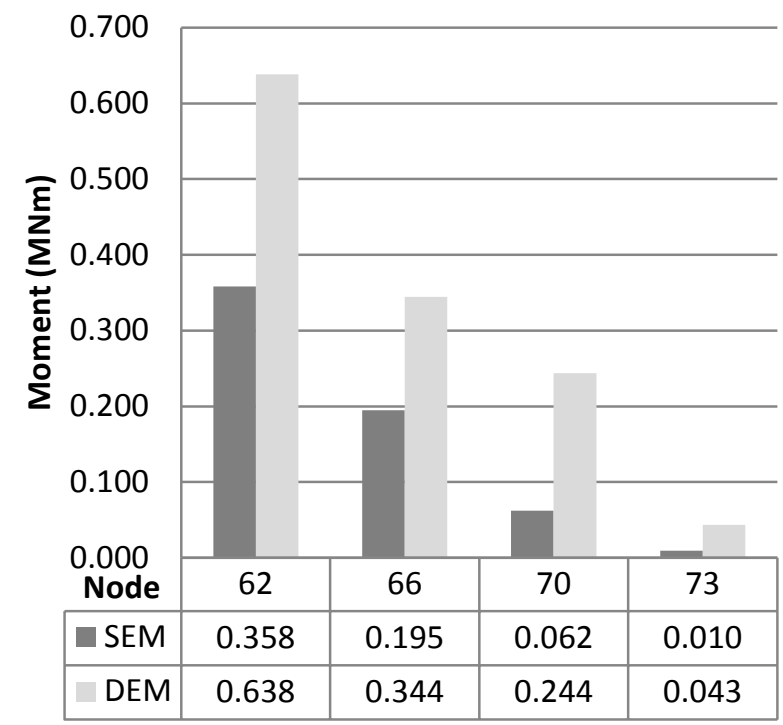

Figure VII-36: Moment distribution value for piers for vertical direction

Similarly, in the piers, the moment values become lower as the nodes are further from the relative displacements (see Figure VII-36). Relative displacement occurs in node 1, and it affects node 62 more than nodes 66,70 and 73, which are not as close as node 62 to node 1. 


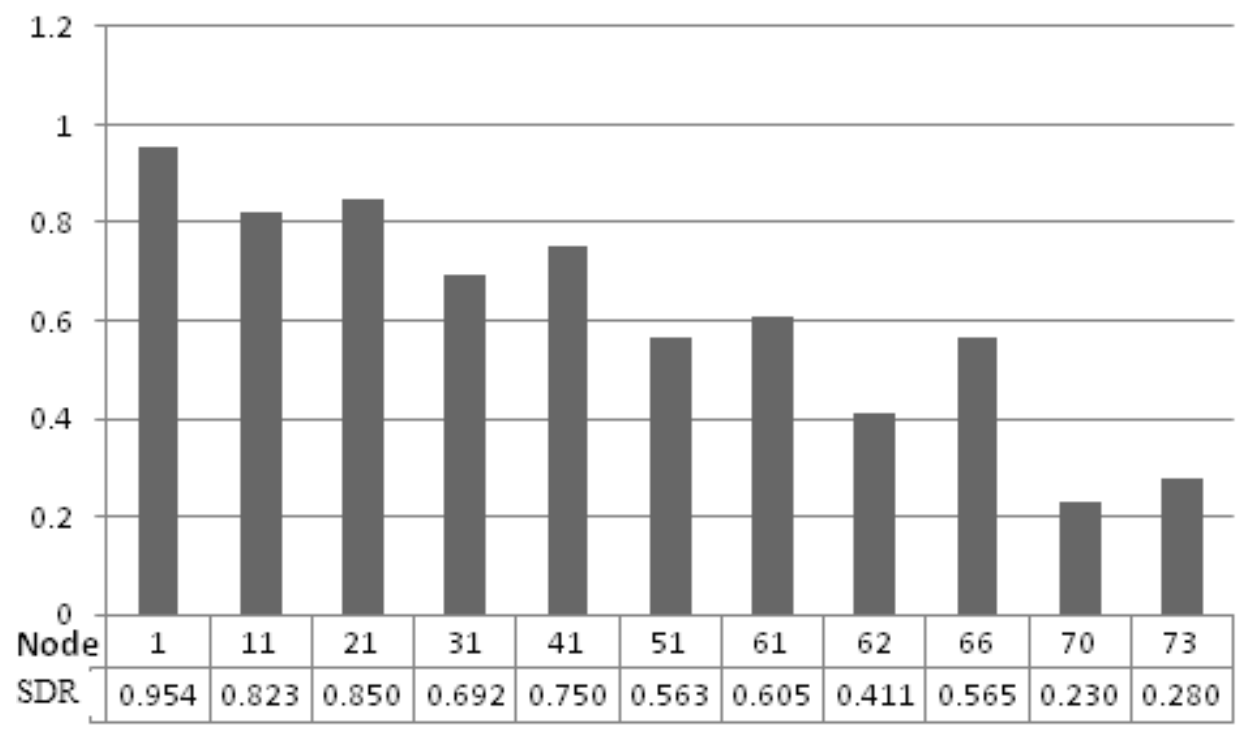

Figure VII-37: Static dynamic moment ratio for vertical direction

In Figure VII-37, it can be seen that the static dynamic moment ratios in the vertical direction are more than 0.5 except for node 70 and node 73 (node for right pier). However, the difference with the horizontal direction is that node 1 has the highest ratio, which means that in that node (the node where relative displacement occurs), the static relative displacement moment has a more important effect than the dynamic moment. In nodes 62, 66, 70 and 73 (node for piers), the ratio is lower than for other nodes.

Figure VII-38 compares static and dynamic moments regarding the range of moments in the structure. The negative (hogging) and positive (sagging) moments can be seen from this figure, as they affect the behaviour of the bridge in general. 


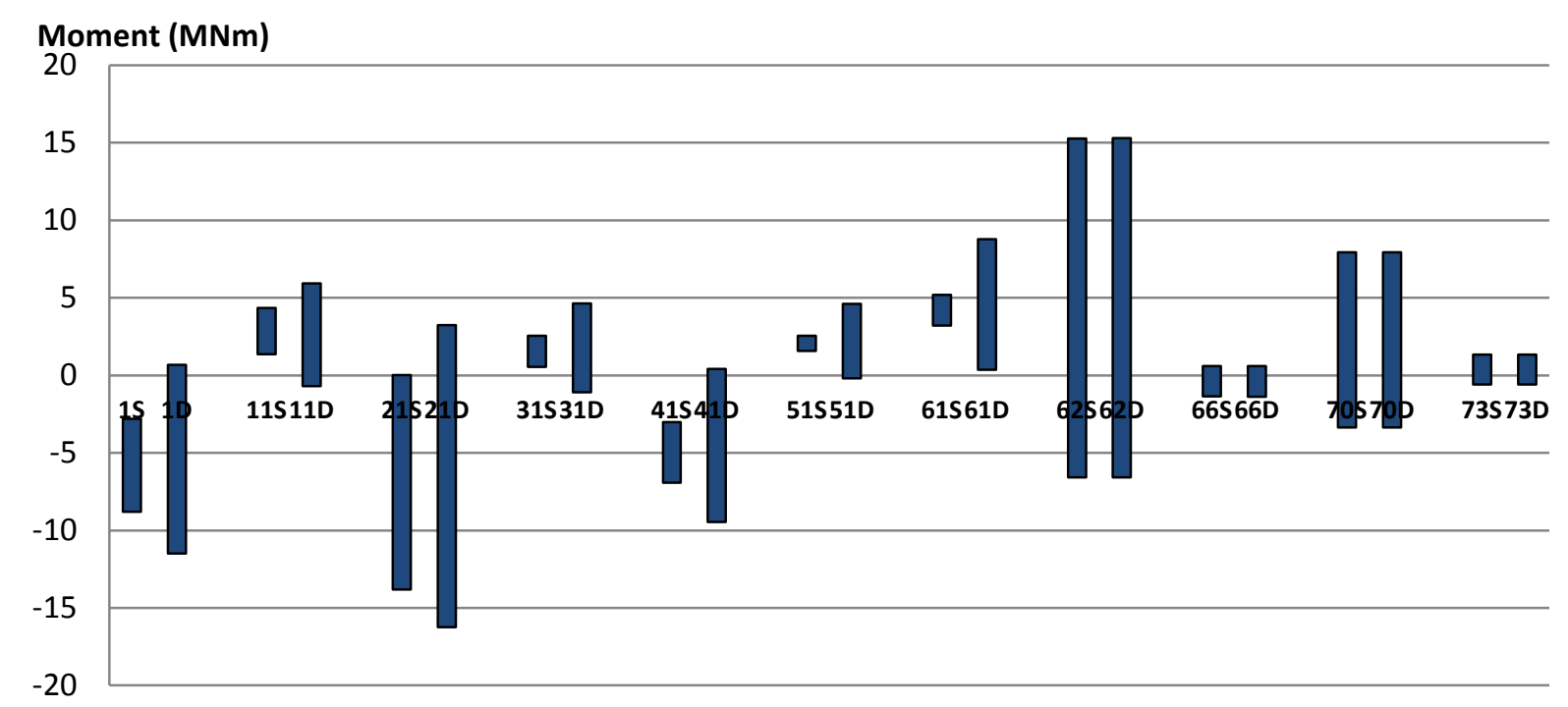

Figure VII-38: Static and dynamic style of support and middle nodes for fixed base for horizontal direction

Figure VII-38 describes the bending moments from static and dynamic loads. The bars refer to the range of magnitude of the moments occurring in the nodes of the bridge. The static moment for node 1 is described as $1 \mathrm{~S}$ and the dynamic moment for node 1 is symbolized as $1 \mathrm{D}$, and so forth. Therefore, the contribution of static relative displacement moments to the dynamic moments of the structure can be analyzed: in the piers, the static moments are almost the same. This means that moments from the relative displacement play an important role in this area. 


\subsubsection{Case 2 (Spring Base)}

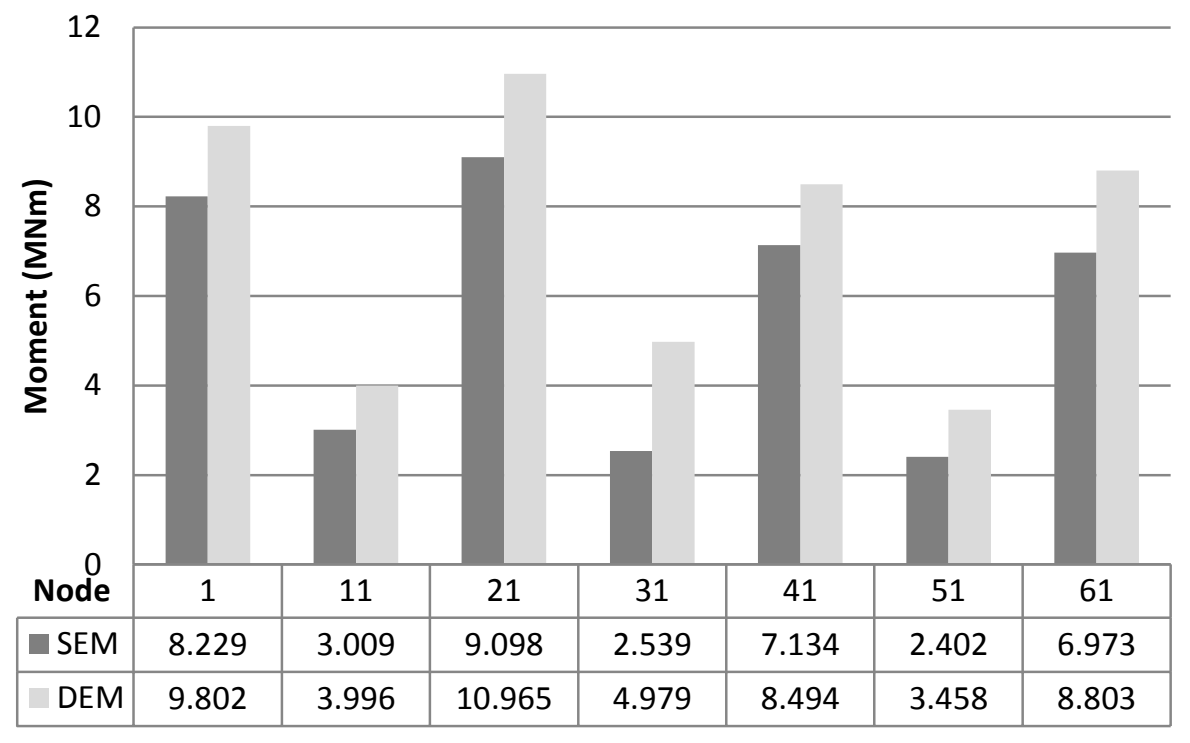

Figure VII-39: Moment distribution value for deck for horizontal direction

Figure VII-39 describes almost the same condition as Figure VII-32. The difference is that in Figure VII-39 the springs are attached to the supports of the structure, whereas in Figure VII-32 the supports are in a fixed base. The general trend line is similar in both, but for the fixed base support, this indicates that the gap between dynamic moments and static moments is larger than for the springs base supports. It can be predicted, then, that the effect of relative displacement is more significant in a spring base structure. This is logically and theoretically acceptable, because the relative displacements created on the spring base structure are larger than on the fixed base structure. The greater the relative displacement, the greater the effect on the structure. 


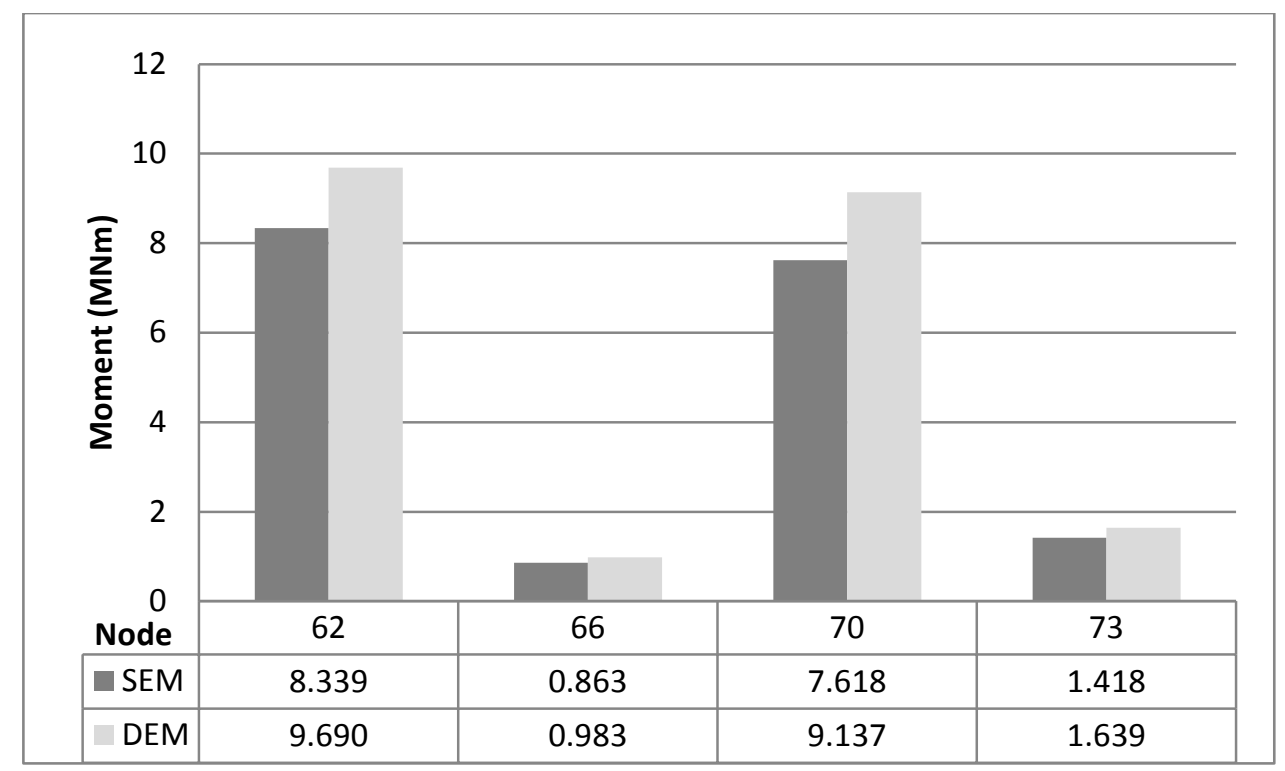

Figure VII-40: Moment distribution value for piers for horizontal direction

From Figure VII-40, it can be seen that there is a large moment at the base of piers, while in the middle of the piers the moment is small. The supports of the piers have larger moments than the body of the piers for both static and dynamic moments. This is because the supports are restrained from rotation although released from the deformation.

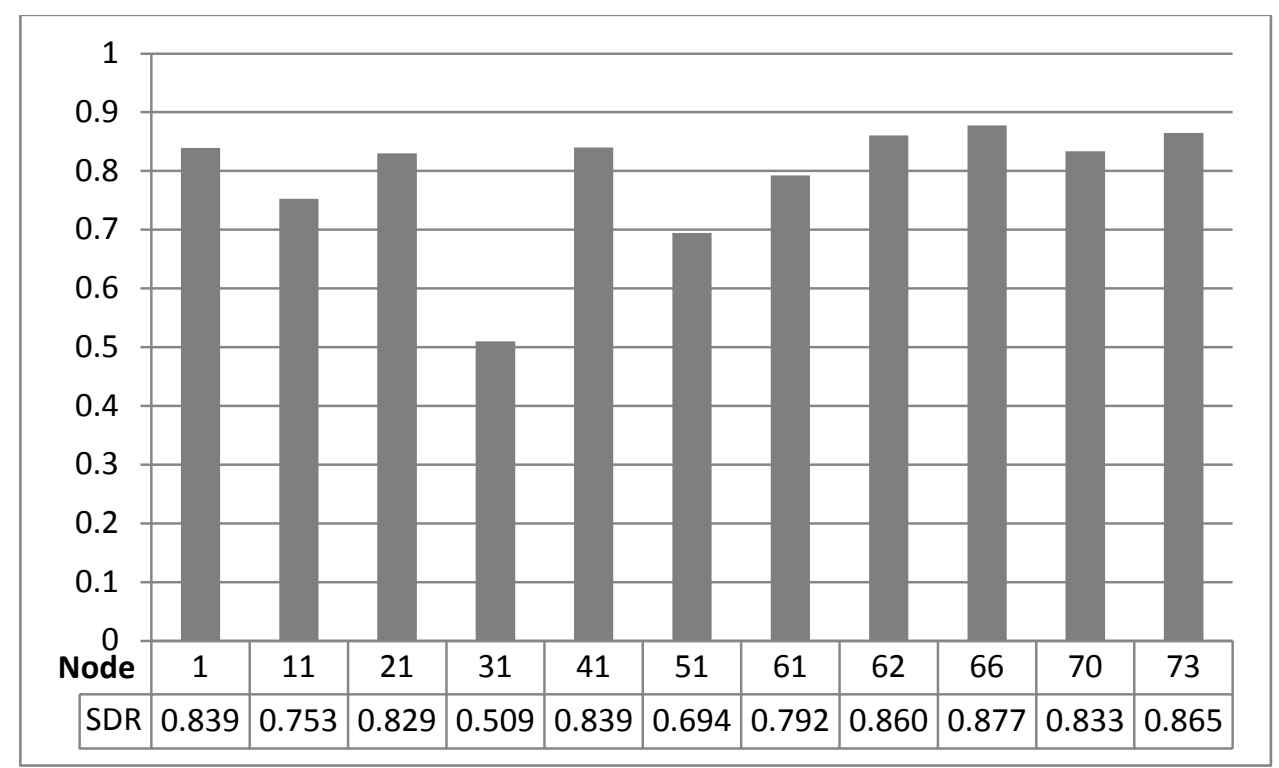

Figure VII-41: Static dynamic moment ratio for horizontal direction

The bar chart in Figure VII-41 illustrates the static dynamic ratio for the horizontal direction, for support with spring bases. It can be seen that node 31, which is the middle node of the deck spans, has the lowest ratio of all, at 0.51 . Also, joint nodes have a larger static dynamic ratio than the middle nodes. Therefore it can be said that relative 
displacement has a greater effect on the joints area of the structure. The reason is that relative displacements does not actually cause a significant bending moment in the middle area of a beam element. By referring to Figure VI-8 in Chapter VI, it can be seen that there is a clear theoretical and logical explanation why the middle section of a beam element does not conduct a significant bending moment resulting from relative displacement.

Meanwhile, in the piers, it can be seen from Figure VII-42 that the static and dynamic ratio of the moments is also high. The relative displacements applied there make an important contribution in the area of the piers.

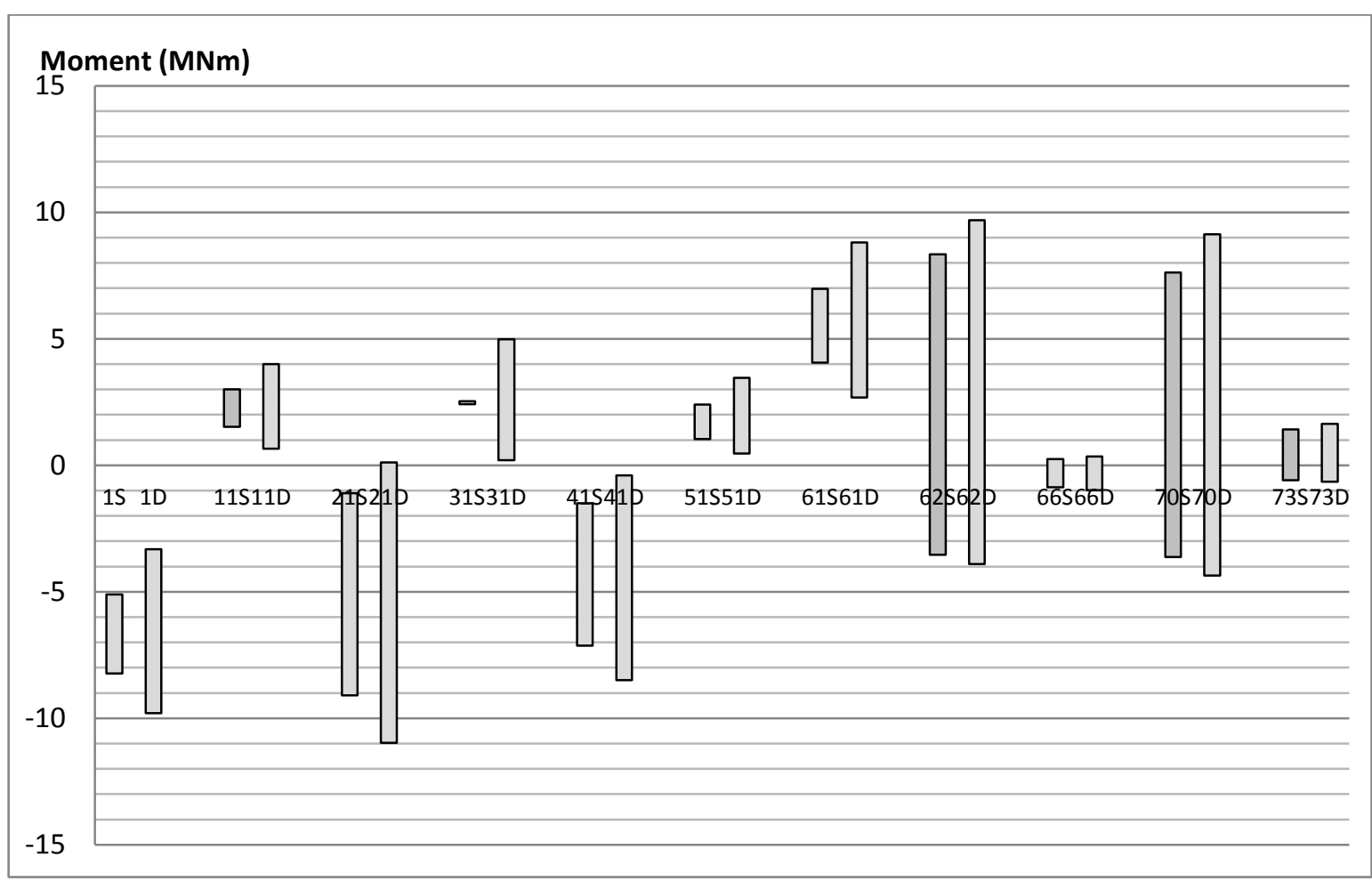

Figure VII-42: Static and dynamic moment range of supports and middle nodes for hard soil

Figure VII-42 shows the ranges of moments due to relative displacements and dynamic loading of the bridge if applied in the horizontal direction. It can be seen that the dynamic moments are always larger than the moments caused by relative displacement. The figure also shows that areas in the middle of the spans have a small static moment compared to the area at the joints. This means that the relative displacement does not give a significant impact on the overall moments in the middle of the spans. 

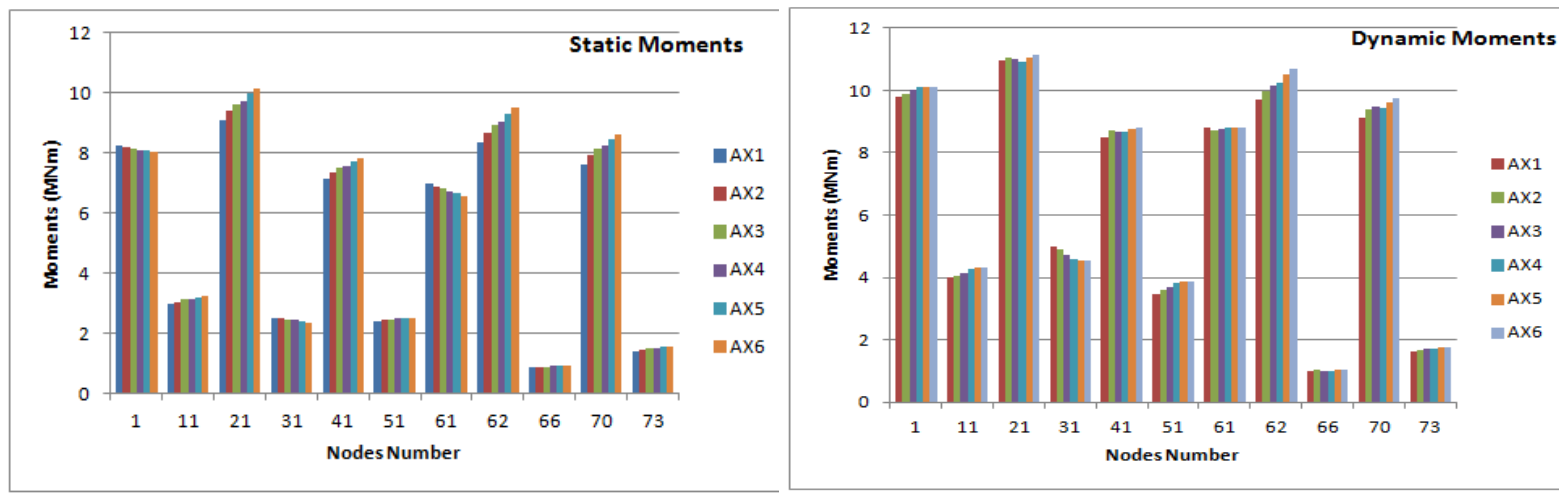

Figure VII-43: Comparison of (a) static, and (b) dynamic extreme moment values for hard soil in horizontal direction

Figure VII-43 (a) illustrates the static extreme moment for different stiffness of soil. In the deck support area (nodes 1 and 61), the static extreme moments become larger if the stiffness of the soil decreases. This happens because lower stiffness will result in larger relative displacements, causing the moments to become higher too. On the other hand, the structure with more stiffness will have smaller relative displacements, leading to small moment values. For dynamic extreme values, as can be seen in Figure VI-43 (b), it appears that the stiffness of the soil increases the moment in the structure, although this is not applicable in all cases.

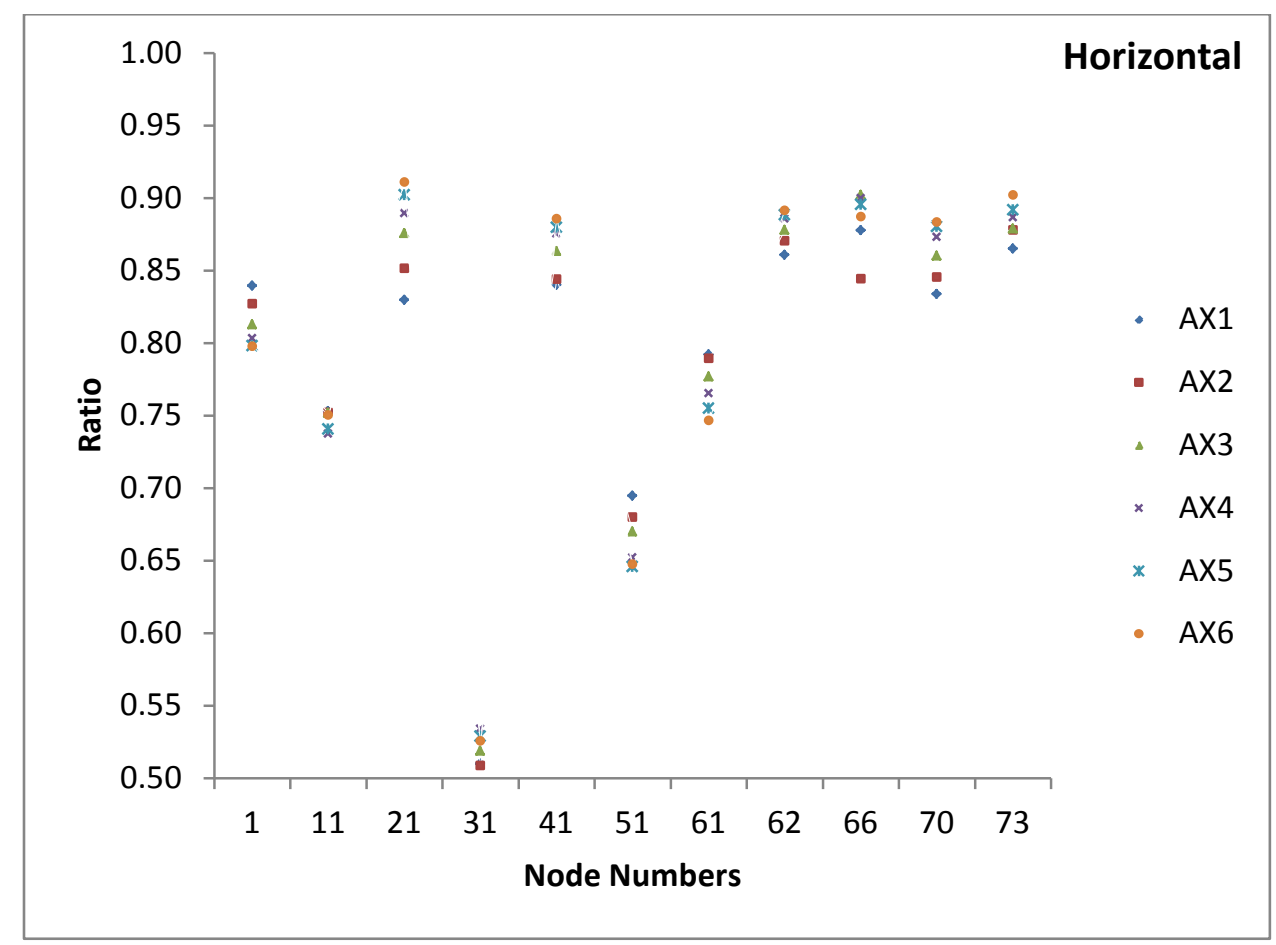

Figure VII-44: Static-dynamic moment ratio distribution for the horizontal direction 
Figure VII-44 shows the distribution of the static-dynamic moment ratio in the horizontal direction. There is correlation between the stiffness of the soil and the ratio. Figure VII-44 also shows that node 31 (the node in the middle of the deck) has the lowest ratio of static and dynamic moments.

For vertical relative displacement, as can be seen in Figure VII-50, it is apparent that the $S D R$ in joints nodes in the deck region is significantly larger than in the middle nodes.I In the piers, the supports have a larger static dynamic bending moment ratio than the nodes in the middle of the spans.

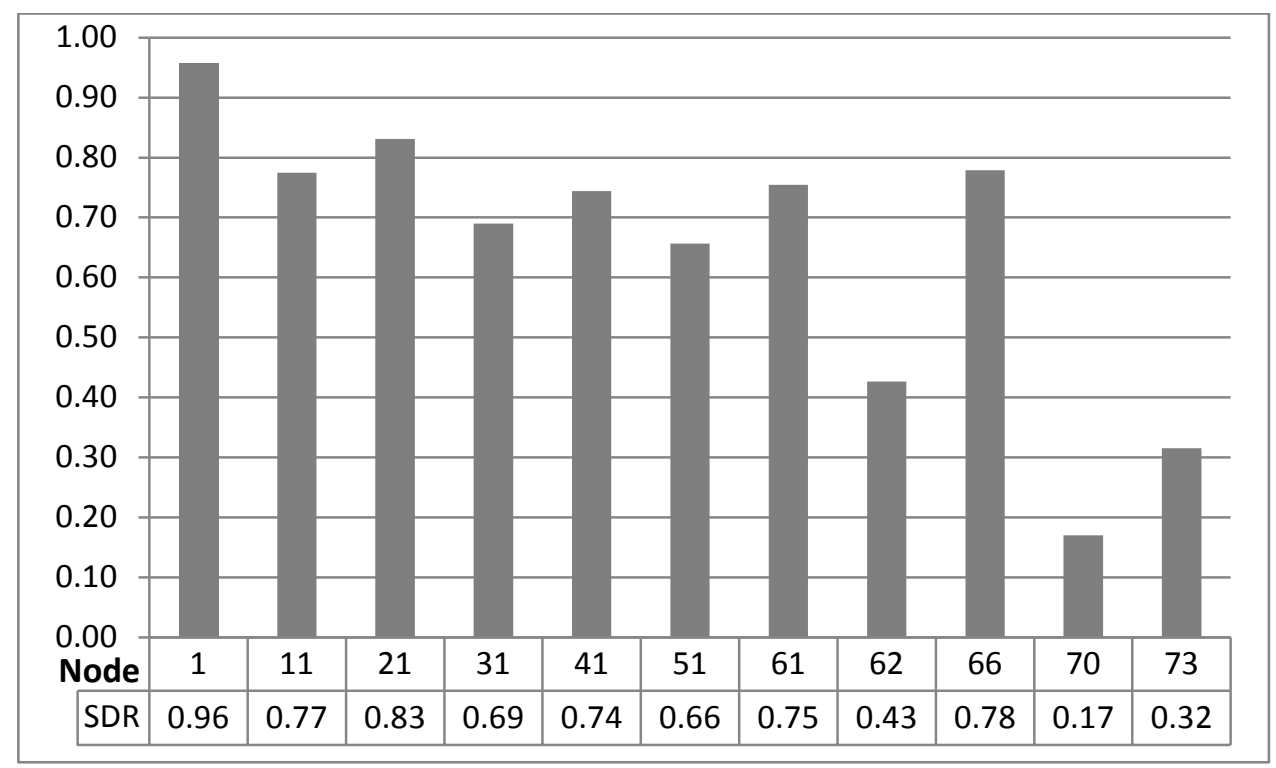

Figure VII-45: Static dynamic moment ratio for vertical direction

Figure VII-45 describes the static dynamic moment ratio when relative displacements are applied vertically. It shows that, for the deck section, nodes in joints have a larger moment ratio than the ratio of nodes in the middle of elements. For piers, however, the middle nodes have a larger moment value than the nodes in the joints. 


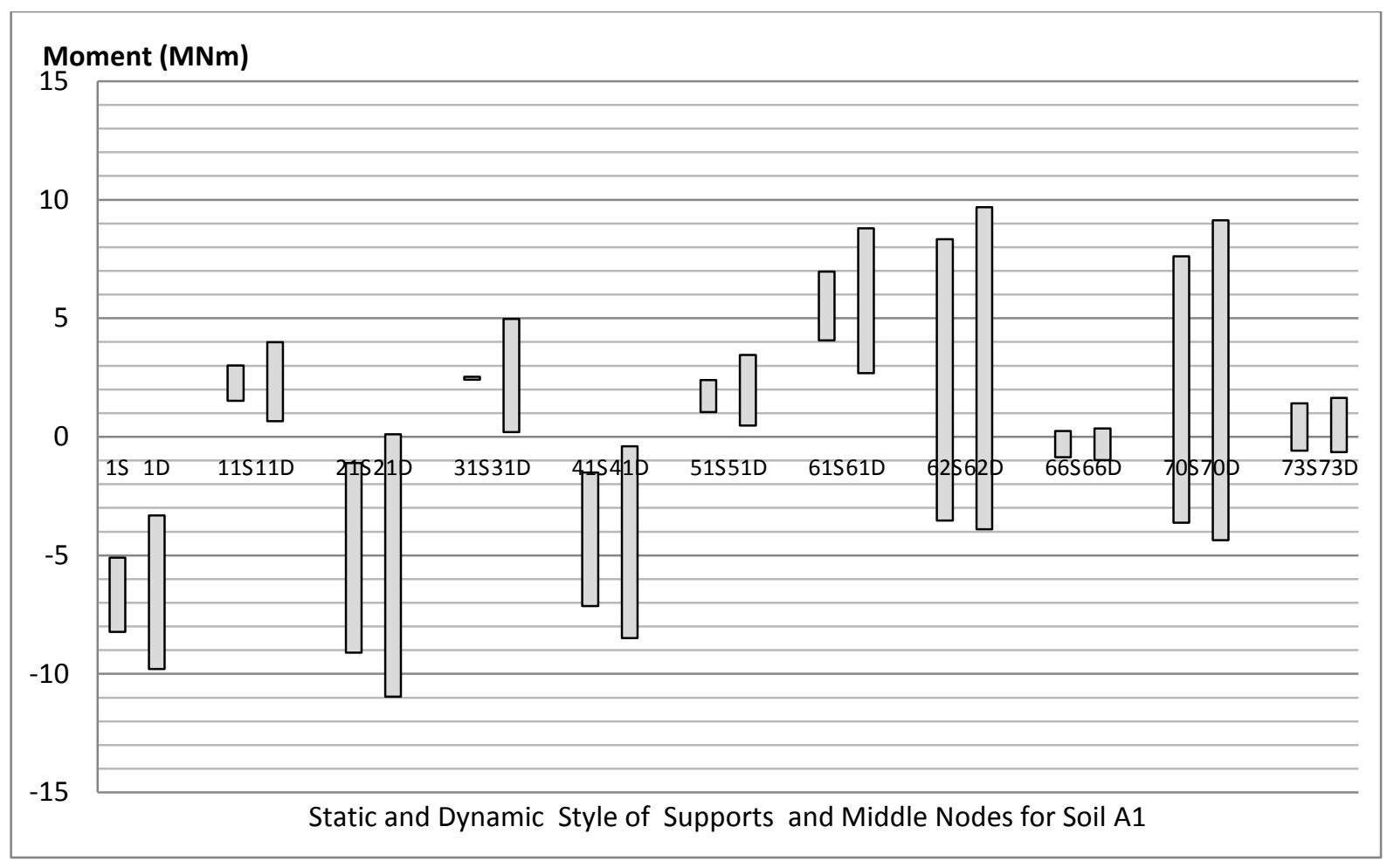

Figure VII-46: Static and dynamic style of supports and middle nodes for hard soil for vertical direction

Figure VII-46 compares the dynamic and static moments. In node 31 (the node in the middle of the deck) the difference between static and dynamic is significant.

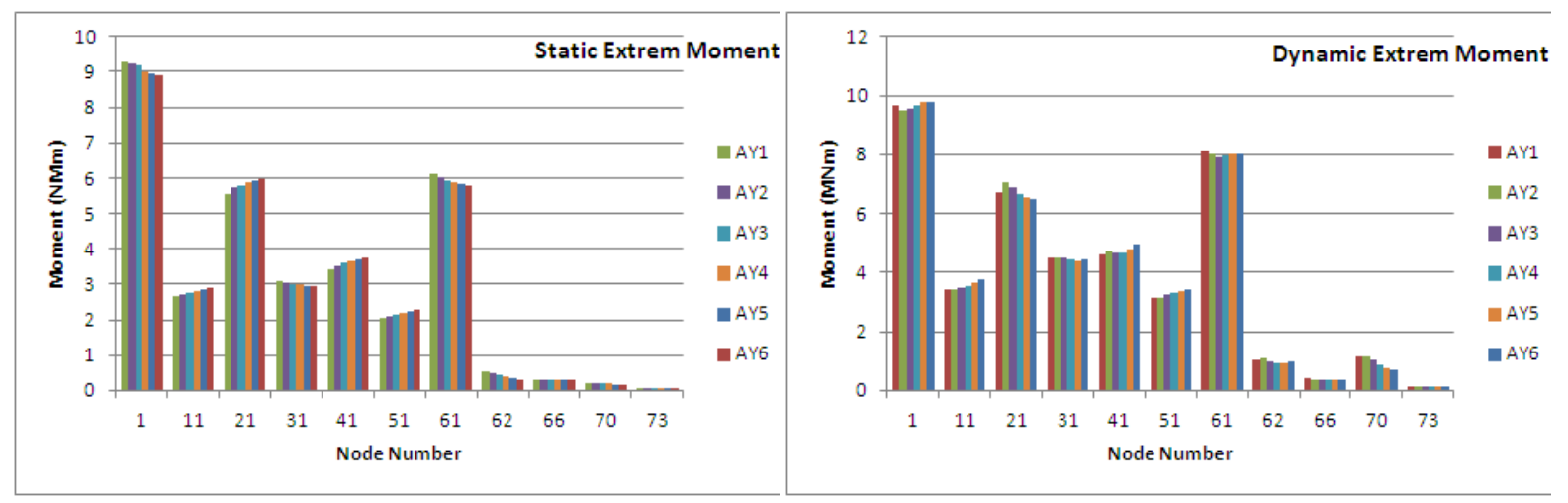

Figure VII-47: Comparison of (a) static, and (b) dynamic extreme moment values for hard soil in the vertical direction

Figure VII-47 shows the static moment value comparison for soil types in the vertical direction. Significant moment values occur in the deck, although in the piers the value is not significant. Moment values are still high in the joints area. 
It also can be seen from Figure VII-47 that the area of the piers, nodes 62, 66, 70 and 73, has smaller moment values than the deck. This can be understood theoretically, since piers are stiffer than the deck, which means they will be less vulnerable to vertical dynamic actions.

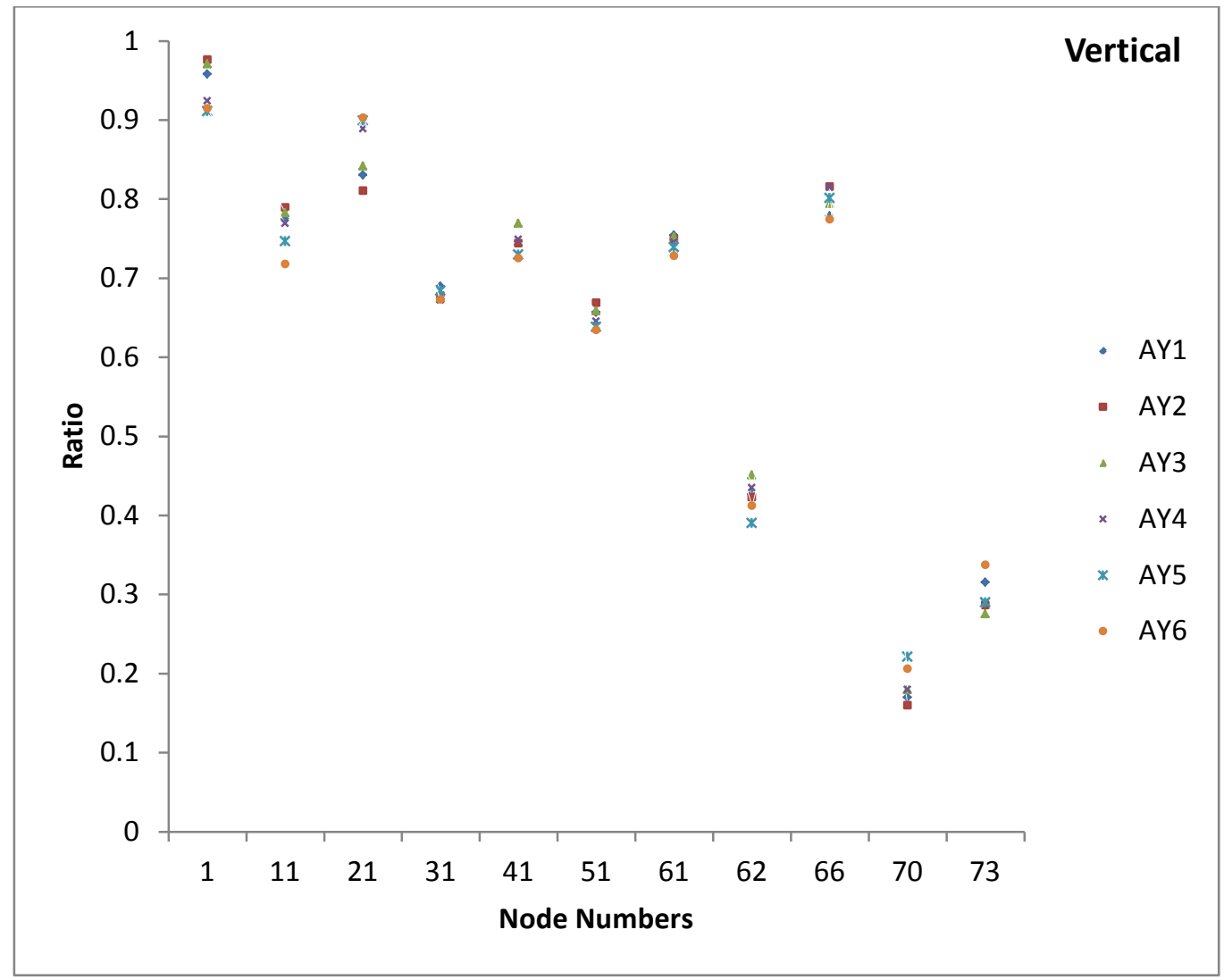

Figure VII-48: Staticdynamic moment ratio distribution for vertical direction

Figure VII-48 shows the distribution of the static-dynamic moment ratio in the vertical direction. There is a correlation between stiffness of soil and the ratio. The figure also shows that node 31 (the node in the middle of the deck) has the lowest ratio of static and dynamic moments.

The static and dynamic relative displacement moment relationship is the approach used in this analysis to study the correlation between static and dynamic bending moments to the bridge. It is well known that, from static relative displacements, the structure will have static bending moments, while from dynamic relative displacements the structure obtains its dynamic bending moments. These two bending moments are compared to see the ratio between and the contribution of static and dynamic moments in the structure. 


\subsubsection{Proposal for Design.}

This study explored the contribution of static and dynamic moments to the structures, and analyzed the differences between fixed base and spring base structural responses. The effect of different stiffness was also be examined. As a result, recommendations can be made regarding design scenarios for an integral bridge.

A simple design procedure is therefore proposed relating design bending moments to those from static relative displacement. From the results already discussed, it can be seen that there is a correlation between static bending moments (due to static relative displacement) and dynamic bending moments (due to transient analysis). A direct relationship was observed by finding a load factor ' $k$ ' through analyzing the results of the SDR.

The two tables below list the ' $k$ ' factors for certain elements of the bridge. Table VII-1 shows the ' $k$ ' factors due to horizontal relative displacement, and Table VII-2 those due to vertical relative displacement.

The factor ' $k$ 'is proposed as a multiplier for the bending moments due to static relative displacement, to produce design moments which envelope both the static and dynamic load cases.

Table VII-1: The ' $k$ 'factors for horizontal relative displacement

\begin{tabular}{|c|c|c|c|c|c|c|c|c|}
\hline \multicolumn{6}{|c|}{ Horizontal RD } & \multirow{3}{*}{$\begin{array}{c}\text { Average } \\
S D R\end{array}$} & \multirow{3}{*}{$\begin{array}{c}k= \\
1 / S D R\end{array}$} & \multirow{3}{*}{$\begin{array}{l}k \text { for } \\
\text { used }\end{array}$} \\
\hline \multirow[b]{2}{*}{ Node } & \multirow{2}{*}{$\begin{array}{l}\text { Fixed } \\
\text { Base }\end{array}$} & \multicolumn{4}{|c|}{ Spring Base } & & & \\
\hline & & $\begin{array}{l}\text { Hard } \\
\text { Soil }\end{array}$ & $\begin{array}{l}\text { Flexible } \\
\text { Soil }\end{array}$ & $\begin{array}{l}\text { Different } \\
\text { Stiff }(\mathrm{V}+)\end{array}$ & $\begin{array}{l}\text { Different } \\
\text { Stiff (V-) }\end{array}$ & & & \\
\hline 1 & 0.77 & 0.84 & 0.83 & 0.89 & 0.89 & 0.89 & 1.12 & 1.2 \\
\hline 11 & 0.73 & 0.75 & 0.66 & 0.81 & 0.87 & 0.84 & 1.19 & 2 \\
\hline 21 & 0.85 & 0.83 & 0.74 & 0.85 & 0.94 & 0.89 & 1.12 & 1.2 \\
\hline 31 & 0.55 & 0.51 & 0.61 & 0.68 & 0.41 & 0.55 & 1.83 & 2 \\
\hline 41 & 0.73 & 0.84 & 0.75 & 0.89 & 0.91 & 0.90 & 1.12 & 1.2 \\
\hline 51 & 0.55 & 0.69 & 0.63 & 0.75 & 0.71 & 0.73 & 1.37 & 2 \\
\hline 61 & 0.59 & 0.79 & 0.83 & 0.86 & 0.81 & 0.84 & 1.20 & 1.2 \\
\hline 62 & 1.00 & 0.86 & 0.68 & 0.85 & 0.95 & 0.90 & 1.11 & 1.2 \\
\hline 66 & 0.99 & 0.88 & 0.69 & 0.83 & 0.95 & 0.89 & 1.12 & 1.2 \\
\hline 70 & 1.00 & 0.83 & 0.67 & 0.85 & 0.96 & 0.90 & 1.11 & 1.2 \\
\hline 73 & 1.00 & 0.87 & 0.68 & 0.85 & 0.95 & 0.90 & 1.11 & 1.2 \\
\hline
\end{tabular}


From Table VII-1 it can be seen that for the joints in the deck, ' $k$ ' can be taken as 1.2; for mid-span the constant factor is 2; and for the piers it can be assumed as 1.2.

Table VII-2: The ' $k$ 'factors for vertical relative displacement

\begin{tabular}{|c|c|c|c|c|c|c|c|c|}
\hline \multicolumn{6}{|c|}{ Vertical } & \multirow{3}{*}{$\begin{array}{c}\text { Average } \\
\text { SDR }\end{array}$} & \multirow{3}{*}{$\begin{array}{c}k= \\
1 / S D R\end{array}$} & \multirow{3}{*}{$\begin{array}{l}k \text { for } \\
\text { used }\end{array}$} \\
\hline \multirow[b]{2}{*}{ Node } & \multirow[b]{2}{*}{$\begin{array}{l}\text { Fixed } \\
\text { Base }\end{array}$} & \multicolumn{4}{|c|}{ Spring Base } & & & \\
\hline & & $\begin{array}{l}\text { Hard } \\
\text { Soil }\end{array}$ & $\begin{array}{l}\text { Flexible } \\
\text { Soil }\end{array}$ & $\begin{array}{l}\text { Different } \\
\text { Stiff }(\mathrm{V}+)\end{array}$ & $\begin{array}{l}\text { Different } \\
\text { Stiff (V-) }\end{array}$ & & & \\
\hline 1 & 0.95 & 0.96 & 0.99 & 0.61 & 0.97 & 0.88 & 1.14 & 1.5 \\
\hline 11 & 0.57 & 0.77 & 0.67 & 0.91 & 0.60 & 0.73 & 1.37 & 1.5 \\
\hline 21 & 0.57 & 0.83 & 0.67 & 0.93 & 0.74 & 0.75 & 1.34 & 1.5 \\
\hline 31 & 0.69 & 0.69 & 0.82 & 0.61 & 0.77 & 0.70 & 1.42 & 1.5 \\
\hline 41 & 0.63 & 0.74 & 0.66 & 0.91 & 0.44 & 0.74 & 1.35 & 1.5 \\
\hline 51 & 0.56 & 0.66 & 0.57 & 0.96 & 0.58 & 0.69 & 1.45 & 1.5 \\
\hline 61 & 0.61 & 0.75 & 0.74 & 0.70 & 0.95 & 0.70 & 1.43 & 1.5 \\
\hline 62 & 0.56 & 0.43 & 0.26 & 0.27 & 0.26 & 0.49 & 2.02 & 3 \\
\hline 66 & 0.57 & 0.78 & 0.88 & 0.74 & 0.93 & 0.67 & 1.49 & 1.5 \\
\hline 70 & 0.26 & 0.17 & 0.12 & 0.27 & 0.12 & 0.21 & 4.70 & 5 \\
\hline 73 & 0.22 & 0.32 & 0.27 & 0.37 & 0.13 & 0.27 & 3.72 & 4 \\
\hline
\end{tabular}

From Table VII-2, under vertical relative displacements, the factor is higher than for horizontal relative displacements, especially for the piers. The factor ' $k$ ' can be summarized as 3 to 5; meanwhile for the deck (mid and joint) the constant factor can be assumed as 1.5 to 2 . The higher values of ' $k$ ' mean that the moments due to relative displacement are lower.

The steps taken by the designer in using the SDR method are:

1. Select bridge code (Ultimate Limit State $(U L S)$ and Serviceability Limit State (SLS) approach).

2. Carry out static relative displacement analysis.

3. Note the relative displacements results - multiplying support moments and midspan moment by ' $k$ ' factors. Check against ULS.

4. Compare step 3 with design in step 1 and adjust the design accordingly. 


\subsubsection{Using the Relative Displacement-Bending Moment Diagram for Design and}

Analysis.

The relative displacement-bending moment diagram can be used to determine the proportion of relative displacement when compared to the whole dynamic response of the bridge. The proportion of static and dynamic bending moment (or SDR) can be investigated to obtain a relationship between the static bending moment and overall dynamic response so at to understand their relative contributions. In this way a factor may be formulated to allow the designer to use the results of a static analysis to incorporate a degree of overload due to potential dynamic response.

To summarize this chapter:

1. A simple method to analyze the static relative displacement effects to dynamic loading was proposed. By considering the bending moment resulting from static relative displacement, the contribution of this kind of load to the overall dynamic moment can be obtained. It is envisaged that this simple method can give easier and simpler guidance to designers of earthquake-resistant structures.

2. There are significant differences between the fixed base bridge structure and the springs base bridge structure relating to the structural response of the system. Structures with spring base supports have a lower frequency on the dynamic moment change compared to fixed base structures.

3. By comparing the three different bending moments resulting from the different models, it can be seen that the relative displacement changes and increases the magnitude of the bending moments.

4. Applying a relative displacement to the structure makes an important change in the behaviour of its moment fluctuation. Without considering relative displacement to the bridge support, the bridge has a steady fluctuation regarding the bending moment. However, if relative displacement is applied, another fluctuation occurs in the bending moment and this new fluctuation is similar to the relative displacement. 
5. The stiffness of the system has a significant influence on the response of the structure. A structure with lower stiffness will have larger relative displacements, causing the moments to become higher. On the other hand, a stiffer structure will have smaller relative displacements, leading to a smaller moments overall.

6. Piers have a significant effect on relative displacement in dynamic action. For horizontal relative displacement, the moments in supports and joints (for deck and piers) are larger than the moments in the mid-spans. Meanwhile, for vertical relative displacements, there is a significant difference between the supports and mid-spans of the piers. The mid-spans have a larger static-dynamic ratio than the supports. 


\section{Chapter VIII CONCLUSION AND RECOMMENDATIONS}

\subsection{Conclusion}

An important consideration in designing a good earthquake-resistant structure is the relative displacements which can occur at support points with significant spacing between them, i.e. bridges. Integral bridges are monolithic structures and are known to possess good earthquake resistance when founded on stable soil. Factors such as soil, foundation types, the length of the bridge and the type of bridge can all influence the dynamic response. The stiffness of the bridge also influences how relative displacements affect the internal force actions within the structure.

In this study, the effect of earthquakes on integral bridges built on several different soil stiffnesses is examined, through computer simulation of an integral abutment bridge. The study was based on Eurocode 8 recommendations, which provide data for different types of soil to be used for earthquake analysis. A symmetrical medium-length integral bridge based on an existing structure was used for the analysis. Artificial Eurocode 8 spectrumcompatible time histories (with a $0.35 \mathrm{~g}$ peak ground acceleration) were applied to the structure for a range of soil stiffness. In conjunction with this, both static and dynamic relative displacement studies were carried out to develop investigate the significance or dominance of dynamic or relative displacement effects.

Synthetic time histories for five different types of soil were created using Mathcad. The synthetic acceleration time history was validated using Seismospect (by Seismosoft), and 
the time histories produced were then used to carry out full integration time history analysis in ANSYS (engineering simulation software) to simulate the dynamic response of the bridge.

The aim of this study was to propose a simplified approach for a design / appraisal which can allow predictions of dynamic response based on the results of static relative displacement studies coupled with simple computer models, without having to resort to full non-linear integration time-history analysis. In other words, the final purpose of this study is to propose a simplified approach to design / appraisal of the bridge which can conservatively predict dynamic behaviour based on static analysis results for a range of soils.

The results show that relative displacements play an important role in overall structural response of the integral bridge, compared to the pure dynamic response in the absence of relative displacement. The results also confirm that earthquakes have a more detrimental effect on lower stiffness soils than on stiffer soils.

To conclude, this research has investigated the behaviour of an integral bridge under horizontal and vertical earthquake ground motion. Four research questions were posed initially, and based on the previous sections and chapters, they have been discussed.

The first research question to be addressed was the response of integral bridges to earthquake loads applied horizontally and vertically. The answers are as follow:

1. The horizontal earthquake creates larger moments in joints than in the mid-spans.

2. Piers have smaller moments than the deck moments, as can be seen in Figure VIII1.

3. The fluctuation of the dynamic moments is only due to the transient condition of the load. 


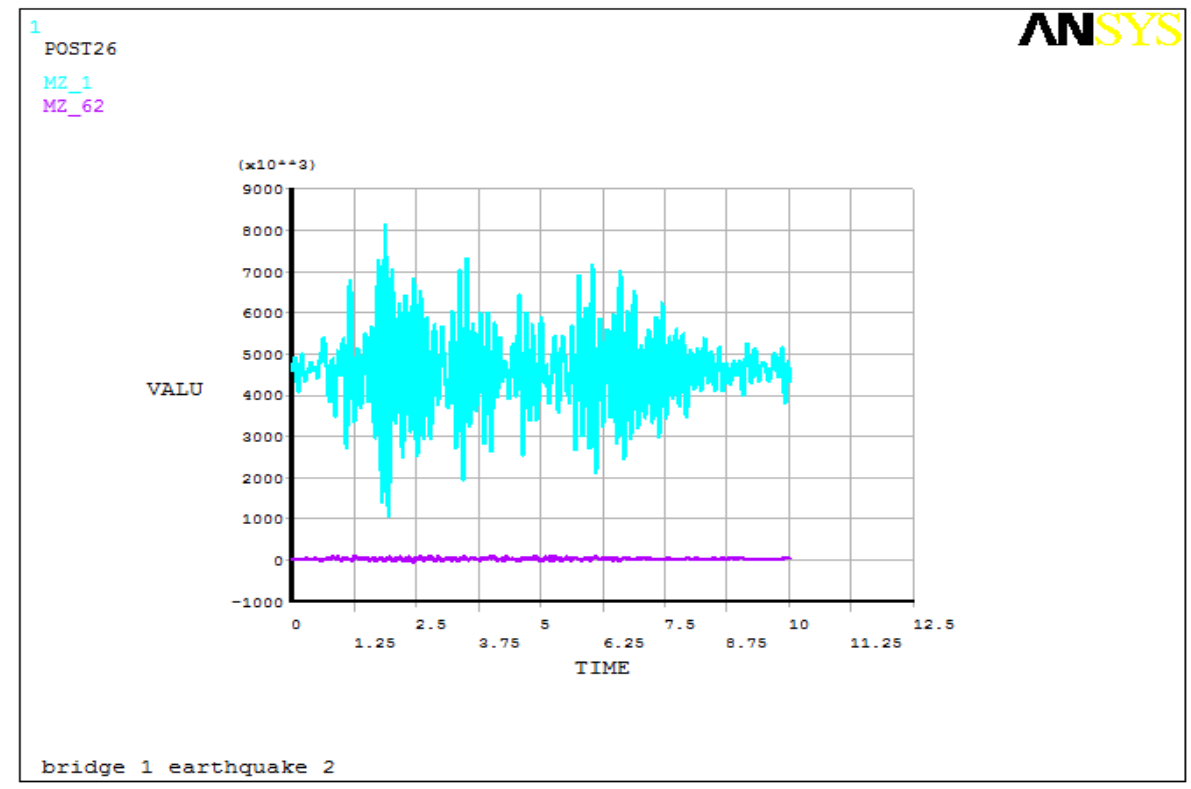

Figure VIII-1: Dynamic bending moment in node 1(deck) and node 62 (piers)

The second question to be answered is the contribution of relative displacement at the bridge supports compared to the overall moment distribution in the bridge. One of the more significant findings to emerge from this study is that a bridge which has relative displacement is subject to greater bending moments than a bridge which has dynamic loading only (without relative displacement). It can be seen from the results (i.e. from Figures VI-23 and VI-24) that the dynamic bending moments which occur in the bridge without relative displacement are smaller than those in a bridge with relative displacement.

The results of this investigation also show that for an integral bridge with this particular configuration, the values of static and dynamic moments are small in the vicinity of the middle of the spans. Hence, static relative displacement moments are not significant in these areas. Instead, the highest moment values occur at joints in the structure. By way of explanation, the results of this study indicate that the relative displacement tends to have a dominant effect at joints within the structure. Therefore, it can also be stated that a structure with relative displacement results in a larger dynamic moment than those caused by dynamic response effects alone.

The same conclusion can be drawn for the piers, because the results show that moment values are greater in magnitude in the vicinity of the supports. The closer the element to the relative displacement node applied, the higher is the moment magnitude in that area of the structure. The comparison of dynamic moment and static relative displacement, 
concludes that relative displacements of the supports have more significant effects on the structure than does the dynamic moment. These findings suggest that in general terms, compared to dynamic loads, the relative displacement that might accompany the dynamic load has more significant effects, especially in the regions of the supports and piers. This is an important problem to be considered in bridge engineering, since when an earthquake occurs, it will cause the structure to experience relative displacement between the supports in addition to dynamic loads.

The static dynamic bending moment ratio $(S D R)$ is used in this research to define the relation of the static moment to the dynamic moment. There are two specific terms in the static dynamic bending moment ratio, namely static bending moment and dynamic bending moment. The former occurs in a given node with static relative displacement analysis, and the latter is due to dynamic analysis. In static relative displacement analysis, moments are due to relative displacement and the gravity load of the bridge, but in dynamic analysis, they occur as a result of dynamic load, gravity and relative displacement. By using this ratio, the contribution of static relative displacement to the overall moment created by dynamic analysis can be obtained.

In other words, $S D R$ describes the composition of the static moment due to gravity load and static relative displacement in a total bending moment due to the gravity loads, dynamic loads and relative displacement loads. The results for both fixed and spring based bridges show that the ratio is more than $50 \%$ for horizontal earthquake ground motion. This means, for the moment created by the earthquake horizontal acceleration, that more than $50 \%$ comes from relative displacement. Piers appear to exhibit a larger ratio than the deck, and the lowest ratio occurs at mid-span. This means that relative displacement has the least effect at mid-span. Therefore piers should be given particular consideration in designing a bridge in accordance with relative displacement. However, for vertical ground motion acceleration, piers show a lower relative displacement ratio to overall dynamic moment. Higher ratios occur in the piers for horizontal relative displacements. On the other hand, the piers have a lower ratio for vertical relative displacement in the mid-spans.

The third research question is to observe the responses of this kind of bridge under different soil conditions, and the influence of differential displacement of supports. Under different soil stiffness conditions, the bridge responds at different natural frequencies. For magnitudes of bending moments are increased generally by increasing the stiffness. 
However, for certain nodes, such as nodes 1 and 61 for horizontal relative displacement, increasing the stiffness reduces the moment values.

Finally, the stiffness effects of the bridge system are questioned. The fixed and spring base conditions cannot be separated from the stiffness of the structure. This study provides an analysis of fixed base support conditions in order to compare the behaviour of the structure when founded on a very stiff soil or, in other words, infinite stiffness as compared to finite stiffness.

It can be seen from the results that a fixed base support gives a higher frequency than the spring base support structure. It has also has been shown that fixed base supports have a higher percentage of relative displacement ratios for piers, but a lower percentage of relative displacement in the deck supports. Therefore, it can be concluded that springs reduce the ratio of relative moment contribution in piers. Further, applying springs to the supports can also change the relative displacements occurring in the supports, and this can increase the bending moments resulting in relative displacement. The reason for this behaviour is the change in the global stiffness of the system.

Considering of relative displacements also yields significant results for the bending moment trend. Different case analyses have produced different patterns of bending moment. These findings enhance the understanding of the correlation between dynamic relative displacement and dynamic bending moments, indicating. a positive correlation between them. The patterns of dynamic bending moments are similar to the patterns of dynamic relative displacements occurring in the structure.

The present study confirms previous findings and contributes additional evidence that suggests the importance of soil or support movements on bending moments compared to the dynamic bending response, confirming the conclusions of other researchers. For example, Wei et al. (2008) stated that when large soil displacement occurs, bridge piles bear horizontal stresses, which include dynamic stress induced by inertial forces of superstructure as well as additional stress induced by lateral soil movement. Wei et al. also confirmed that, in most cases, the latter plays a more important role than the former. The results of analyses by Wang et al. (2009) also show that non-uniform earthquake ground motions significantly influence the response of long bridges both with and without expansion joints. 
As a result of this investigation, it is possible to establish guidelines for designers to simplify the design of earthquake-resistant bridge structures. A suggested hierarchy in the design procedure is as follows:

a. Carry out a finite element analysis under gravity loading and additional service / loading.

b. Carry out relative displacement analysis under static loading to determine the capacity of the structure to suffer static relative displacements.

c. Carry out modal analysis to determine the dynamic response characteristics.

d. Combine b. and c. in a new design rule.

The new design rule is the dynamic moment of the structure that can be achieved by multiplying a moment caused by the static relative displacement with a constant ' $k$ '. However, because this study was conducted with many simplifications and idealizations, more intensive research is needed to provide a definitive value for the constant.

The ' $k$ ' factors which have been obtained in this study as can be seen in the Table VIII-1, are based on the analysis in Chapter VII.

Table VIII-1: Multiplying factors ' $k$ ’ obtained from the study.

\begin{tabular}{||c||c||c||}
\hline Areas & Deck & Piers \\
\hline \hline Joint & $1.5-2.0$ & $3.0-5.0$ \\
\hline Mid span & $2.0-2.5$ & $4.0-5.0$ \\
\hline & & \\
\hline
\end{tabular}

Designers usually welcome a methodology which simplifies the amount and expense of the analysis required. If the designer can predict dynamic behaviour on the results of a static analysis (albeit with the incorporation of a conservative 'factor') then there will be savings intime and cost. Thus, this is where the direction in which further research should lie.

A number of caveats need to be noted regarding the present study, because the project was limited in several ways. Firstly, the project used a convenient existing bridge in India which was then idealized in $2 D$ modelling to fulfil the research conditions and limitations. The use of $3 D$ effects was dismissed as the results show $2 D$ modelling is adequate. 
Secondly, since the focus of this study was on structural rather than geo-technical responses, soil behaviour and characteristics were not given significant consideration. Thirdly, limitation is that other structural responses such as shear force, axial force and torsion were not discussed in this research, since the behaviour of these internal forces is similar to bending moments. This is supported by Alvarez et al. (2012), who said that during an earthquake, axial load values in structural elements continually change and element capacities also fluctuate.

\subsection{Recommendations and Future Work}

\subsubsection{Recommendations}

The findings of this study have a number of important implications for future practice:

- In terms of earthquake dynamic loading, relative displacement plays a significant role in the response of this particular bridge, especially for supports and piers. In other words, designers should consider the structural response in piers and joints for structures under dynamic loadings.

- The integral bridge is more suitable for construction in a stiffer and more stable foundation.

- Basic guidance on obtaining dynamic moments by considering static bending moments is presented in this study. By using this simple method, the correlation between relative displacement and bending moments can be calculated.

\subsubsection{Future Work}

As a basic method and guidance have been achieved in this research, more intensive study is needed in order to obtain more valuable results. The research presented in this thesis suggests that an amplification factor could usefully be developed for dynamic analysis in rigid structures. This should encourage interest in the development of a simple guide. There are several lines of research arising from this work which should be pursued. It is recommended that further research be undertaken in the following areas:

1. The interesting problem that has not been fully addressed in this research but could be a subject study for future is the problem of determining a proper constant function for 
dynamic bending moment characterization. Since there are so many variables involved in determining the value ' $k$ ', there needs to be more research to find a value for this constant which is related to correlation of the static and dynamic bending moments of the bridge.

2. As the research is only concerned with one bridge configuration, further investigation is required with regard to the stiffness of the bridge members, i.e. deck length, pier length, section sizes, and modified cracked section. Overload capacity or ductility and the ability of the structure to distribute the moments should also be considered in future research.

3. A wider range of soil conditions should be considered, to gain more powerful results and conclusions.

4. This current research is based on bending moment only; however, it is predicted that other structural responses, such as shear, axial, and torsion, might have a similar performance to bending moment. Therefore, more research should be conducted to observe these effects more deeply.

5. Further experimental investigations are needed to estimate the accuracy of this simulation. Experiments might be conducted on small-scale bridges to predict the behaviour of the integral bridge under different relative displacements. Artificial earthquake ground motion could also be attached to the small-scale bridge by using a shaking table. 


\section{References}

Alizadeh, M. H., Khalim, A. R., Chik, Z. \& Hosseiny, S. M. M. M., (2010). Full Height Frame Integral Bridges Abutment-Backfill Interaction on Loose Granule Backfill. Journal of Applied Science. Volume 10, pp 1588-1595

Alvarez, J. J., Aparicio, A. C., Jara, J. M. \& Jara, M., (2012). Seismic Assessment of A Long Span Arch Bridge Considering the Variation in Axial Forces Induced by Earthquakes. Engineering Structures, Volume 34, pp. 69-80.

Ansary, M. A., Noor, M. A. \& Seraj, S. M., (2000). Response Spectra based on Simulated Earthquakes: Its Application and Assesment. Journal of Civil Engineering, 28(1).

Arnold, C. \& Reitherman, R., (1982). Building Configuration and Seismic Design. New York: John Wiley.

Arsoy, S., Barker, R. M. \& Duncan, J. M., (1999). The Behaviour of Integral Bridges, Virginia, USA: Virginia Transportation Research Council.

Attewel P.B. \& Taylor, R.K, (1984). Ground Movements \& Their Effects on Structures, Surrey University Press.

Austin, M., 1996. Earthquake response Spectrum Analysis for 4 Story Shear Building. [Online] Available at: http://www.isr.umd.edu/ austin/aladdin.d/matrix-eq-spectra.html [Accessed 27 July 2012]. 
Baker, J. W. \& Corner, C. A., (2006). Which Spectral Acceleration are You Using. Spectra Bulletin, 22(2), pp. 293-312.

Berenev, I. A., Nightengale, A. M. \& Silva, W. J., (2002). Properties of Vertical Ground Motions. Bulletin of the Seismological Society of America, 92(8), pp. 3152-3164.

Bisch, P. et al., (2012). Eurocode 8: Seismic Design of Buildings Worked Example, Lisbon: JRC European Commision.

Bommer, J. J. \& Elnashai, A. S., (1999). Displacement Spectra for Seismic Design. Journal of Earthquake Engineering, 3(1), pp. 1-32. 193

Bore, D. M., (2001). Effect of Baseline Correction on Displacements and Response Spectra for Several Recordings of the 1999 Chi-Chi, Taiwan Earthquake. Bulletin of Seismological Society of America, 91(5), pp. 1199-1211.

Bozorgni, Y. \& Campbell, K. W., (2004). Engineering Characterization of Ground Motion. In: Y. Bozorgnia \& V. V. Bertero, eds. Earthquake Engineering from Engineering Seismology to Performance Based Engineering. Florida: CRC Press.

Burdette, N. J. \& Elnashai, A. S., (2011). Effect of Asynchoronous Earthquake Motion on Complex Bridges. II: Results and Implications on Assesment. Journal of Bridge Engineering, 12(2), pp. 166-172.

Burker, M. P., (2009). Integral and Semi Integral Bridge. $1^{\text {st }}$ ed. Singapore: Wiley Blackwell. 
Cai, Z. \& Bathrust, R. J., (1996). Determining Sliding Block Methods for Estimating Seismic Displacements of Earth Structures. Soil Dynamic and Earthquake Engineering, 15(4), pp. 255268.

Chandra, S., Madhira, R. M., Iyengar N.G.R., (1987). A New Model for Non Linear Subgrades. Mathematical Modelling, Volume 8, pp 513-518

Clough, R. W. \& Penzien, J., (2003). Dynamics of Structures. $3^{\text {rd }}$ ed. Berkeley: Computers \& Structures Inc..

Collier, C. J. \& Elnashai, A. S., (2001). A Procedure for Combining Vertical and Horizontal Seismic Action Effects. Journal of Earthquake Engineering, 5(4), pp. 521-539.

Connal, J., 2005. Integral Bridges - Australia and US Practice. Tasmania, Austroads.

David, T. K. \& Forth, J. P., (2011). Modelling of Soil Structure Interaction of Integral Bridge. s.l., World Academy of Science, Engineering and Tehcnology.

Elnashai, A. S., He, L. \& Elgamal, A., (2004). Spectra for Vertical Earthquake Ground Motion. Proceeding of $13^{\text {rd }}$ World Conference on Earthquake Engineering. (WCEE). Vancouver, Canada.

Fardis, M. N. et al., (2005). Eurocode 8: Design of Structure for Earthquake Resistance. $1^{\text {st }}$ ed. London: Thomas Telford. 194

Filippou, F. C. \& Fenves, G. L., (2004). Methods of Analysis for Earthquake Resistant Structures. In: Y. Bozorgnia \& V. V. Bertero, eds. Earthquake Engineering from Engineering Seismology to Performance Based Engineering. London.:CRC Press. 
Flener, E. B., (2004). Soil Structure Interaction for Integral Bridge and Culvert. Stockholm: Royal Institute of Technology.

Freeman, S. A., (2007). Response Spectra as Useful Design and Analysis Tool for Practicing Structural Engineers. Journal of Earthquake Technology, 44(1), pp. 25-37.

Frosch, R. J., Kreger, M. E. \& Talbott, A. M., (2009). Earthquake Resistance of Integral Abutment Bridges, West Lafayette, Indiana: Department of Transportation and Purdue University.

Frosch, R. J. \& Lovell, M. D., (2011). Long Term Behaviour of Integral Abutment Bridges, Purdue University, Indiana: FHWA Joint Transportation Research Program.

Goel, R. K., (1997). Earthquake Characteristics of Bridges with Integral Abutments. Journal of Structural Engineering, 123(11), pp. 1453-1443.

Graizer, V. \& E, K., (2009). Prediction of Response Spectral Acceleration Ordinates Based on PGA Attenuation. Earthquake Spectra, 25(1), pp. 36-69.

Iles, D. C., (2006). Integral Bridge in UK. In: P. Collin, M. Veljkvic \& H. Petursson, eds. Technical Report: International Workshop on the Bridges with Integral Abutments. Lulea: Lulea University of Technology, pp. 13-24.

Indoria, R., (2010). Indian Road Congress. [Online] Available at:

http://irc.org.in/ENU/knowledge/editorial/june 2010.pdf [Accessed 12 June 2012]. 
Issa, M. A. \& Shahawy, M. A., (1993). Dynamic and Static Tests of Prestressed Concrete Girder in Florida, Florida: Florida Department of Transportation.

Karantzikis, M. \& Spyrakos, A., (2000). Seismic Analysis of Bridges Including Soil Abutment Interaction. Proceeding of $12^{\text {nd }}$ World Conference on Earthquake Engineering. (WCEE). Auckland, New Zealand.

Kasai, K., Liu, W. D. \& Jeng, V., (1994). Effect of Relative Displacements Between Adjacent Bridge Segments, SMIP92: Seminar on Seismological and Engineering Implications of Recent Strong Motion Data. Washington DC: National Academy Press.

Khunnath, S. K., (2008). Response of Ordinary Highway Bridges to Combined Horizontal and Vertical ground Motions. 24th US-Japan Bridge Engineering Workshop, September 22-24.

Khunnath, S. K. \& Huiling Zhao, H., n.d. Effects of Near-Fault Vertical Accelerations on Highway Bridge Columns. [Online] Available at: http://www.pwri.go.jp/eng/ujnr/tc/g/pdf/25/62.pdf [Accessed 3 June 2010].

Kim, S. J., (2008). Analytical and Experimental Investigations of Effect of Vertical Ground Motions on RC Bridge Piers. Proceeding of $14^{\text {rd }}$ World Conference on Earthquake Engineering. (WCEE). Beijing, China.

Kim, S. J., Holub, C. J. \& Elnashai, A. S., (2011). Analytical Assessment of the Effect of Vertical Earthquake Motion on RC Bridge Piers. Analytical Assessment of the effect, 137(2), pp. 252-260. 
Kim, S. J., Holub, C. J. \& Elnashai, A. S., February (2011). Analytical Assessment of the Effect of Vertical Earthquake Motion on RC Bridges Piers. Journal of Structural Engineering, 137(2), pp. 252-260.

Kolias, B., (2008). Eurocode 8 - Part 2. Seismic Design of Bridges. [Online] Available at: http://eurocodes.jrc.ec.europa.eu/doc/WS2008/Kolias_2008.pdf [Accessed 7 Augustus 2010].

Larsen, R. W., (2001). Introduction to Mathcad 2000. 1st ed. New Jersey: Prentice Hall.

Lavelle, F. H., (1969). Curved Girder Analysis, Computer Program, Structures Design and Subdivisions, New York Department of Transportation.

Legeron, F. \& Sheikh, M. N., (2009). Bridge Support Elastic Reactions under Vertical Earthquake Ground Motion. Engineering Structures, Volume 31, pp. 2317-2326.

Ling, H. I. \& Leshchinsky, D., (1998). Effects of Vertical Acceleration on Seismic Design of Geosyntheticreinforced Soil Structures. Geotechnique, 48(3), pp. 347-373.

Lumantarna, E., Wilson, J. \& Lam, N., (2012). Bi-Linear Displacement Response Spectrum Model for Engineering Applications in Low and Moderate Seismicity Regions. Soil Dynamics and Earthquake Engineering, Volume 43, pp. 85-96.

Millais, M., (1997) Building Structures, From Concepts to Design, $2^{\text {rd }}$ Ed, London, Spon Press.

Mitoulis, S. A. \& Tegos, I. A., (2011). Two New Earthquake Resistant Integral Abutments for Medium to Long Span Bridge, Structural Engineering International. DOI: 10.2749/101686611X12994961034011 
Mourad, S. \& Tabsh, S. W., (1999). Deck Slab Stresses in Integral Abutment Bridges. Journal of Bridge Engineering, 4(2), pp. 125-130.

Mylonakis, G., Gazetas, G. Nikolaou, S., \& Michaelides, O., (2000), The Role of Soil on the Collapse of 18 Piers of Hanshin Express Way in Kobe Earthquake, Proceeding of $12^{\text {th }}$ World Conference on Earthquake Engineering (WCEE), Auckland, New Zealand, 1074

NEHRP (2004). Recommendation Provisions for Seismic Regulations for New Buildings and Other Structures. Washington DC

Papazoglou, A. J. \& Elnashai A, S., (1996). Analytical and Field Evidence of Damaging Effect of Vertical Earthquake Ground Motion. Journal of Earthquake Engineering and Structural Dynamic, Volume 25, pp. 1109-1137.

Pekcan, G., Monzon, E. V. \& Itani, A. M., (2011). Seismic Performance of Steel Plate Girder Bridges with Integral Abutments, Nevada: FHWA.

Priestley, (2000). Performance Based Seismic Design, Proceeding of $12^{\text {th }}$ World Conference on Earthquake Engineering (WCEE), Auckland, New Zealand, 2831

Publications Committe of ICSMFEE, (1999). Proceedings of the Fourteenth International Conference on Soil Mechanics and Foundation Engineering. Rotterdam, A.A. Balkema.

Rahai, A., (2004). Effect of Earthquake Vertical Motion on RC Bridge Piers. Proceeding of $13^{\text {th }}$ World Conference on Earthquake Engineering (WCEE), Vancouver, Canada. 
Rodriguez, J., Martinez, F. \& Marti, J., (2011). Integral Brigde for High Speed Railway. [Online] Available at: http://oa.upm.es/11242/2/INVE_MEM_2011_102837.pdf [Accessed 121 2012].

Saadeghvariri, M. A. \& Foutch, D. A., (1991). Dynamic Behaviour of R/C Highway Bridges under the Combined Effect of Vertical Earthquake Motions. Earthquake Engineering \& Structural Dynamic, 20(6), pp. 535-549.

Schott, C. \& Schwarz, J., (2004). Reliability of Eurocode 8 Spectra and The Problems of Their Application to Central European Earthquake Regions, Proceeding of $12^{\text {th }}$ World Conference on Earthquake Engineering (WCEE), Vancouver, Canada.

Sextos, A., Kappos, A. J. \& Mergos, P., (2004). Effect of Soil Structure Interaction and Spatial Variability of Ground Motion on Irregular Bridges: The Case of The Krystallopigi Bridge. Proceeding of $13^{\text {th }}$ World Conference on Earthquake Engineering (WCEE), Vancouver, Canada.

Shrestha, B., (2009). Vertical Ground Motions and Its Effects on Engineering Structures: A State of the Art. Katmandu, India, International Seminar on Hazard Management for Sustainable Development.

Siddharthan, R., Ara, S. \& Norris, G., (1992). Simple Rigid Plastic Model for Seismic Tilting of Rigid Walls. ASCE Journal of Structural Engineering, 118(2).

Si, H. \& Midorikawa, (2000). New Attenuation Relations for Peak Ground Acceleration and Velocity Considering Effects of Fault Type and Site Condition. Proceeding of $12^{\text {th }}$ World Conference on Earthquake Engineering (WCEE), Auckland, Newzealand. 
Silva, W. J., (1997). Characteristics of Vertical Strong Ground Motions for Applications to Engineering Design, Technical Report NCEER 97-0010: I.M. Friedland, M.S Power and R. L. Mayes.

Spyrakos, C. \& Loannidis, G., (2003). Seismic Behaviour of a Post-Tensioned Integral Bridge Including Soil-Structure Interaction (SSI). Soil Dynamics and Earthquake Engineering, Volume 23, pp. 53-63.

Srbulov, M., (2011). Practical Soil Dynamics: Case Studies in Earthquake \& Geotechnical Engineering. 1st ed. London: Springer.

Sucuoglu, H. \& Akkar, S., (2011). Introduction to Earthquake Engineering, Ankara: http://www2.ce.metu.edu.tr/ ce490/documents/CE\%20490\%20LECTURE\%20NOTESCOMBINED.pdf.

Tandon, M., (2005). Economical Design of Earthquake Resistant Bridges. Journal of Earthquake Technology, 42(1), pp. 13-20.

Tandon, M., (2005). Recent Integral Bridge. Winnipeg, Canada, International Workshop on Innovative Bridge Deck Technologies 14-15 April.

Terzaghi, S. \& Sisk, G., (2009). Integral Bridge - Derivation of the Spring Constant for Modelling the Soil Structure Interaction. Auckland: Convention Management.

Veletzos, M. J. \& Restrepo, J. I., (2008). The Influence of Vertical Earthquake Motion and PreEarthquake Stress State on Seismic Response of Precast Segmental Bridge Superstructures. 
Proceeding of $6^{\text {th }}$ National Seismic Conference on Bridges \& Highways, 27-30 July, Charleston, South Carolina.

Wang, J., \& Carr, A.J., (2009). The Response of a 344 m Long Bridge to Non-Uniform Earthquake Ground Motions. Engineering Structures, Volume 31, pp. 2554-2567.

Warn, G. P. \& Whittaker, A. S., (2008). Vertical Earthquake Loads on Seismic Isolation Systems in Bridges. Journal of Structural Engineering, 134(11), pp. 1696-1704.

Wei, X., Wang, Q. \& Wang, J., (2008). Damage Patterns and Failure Mechanisms of Bridge Pile Foundation under Earthquake. Proceeding of $14^{\text {th }}$ World Conference on Earthquake Engineering (WCEE). Beijing, China.

White, H., (2007). Integral Abutment Bridges: Comparison of Current Practice Between European Countries and the United States of America, New York: Transportation Research and Development Bureau.

Wilson, E. L., (1995). Three Dimensional Static and Dynamic Analysis of Structures. Berkeley: Computer \& Structures. Inc.

Wilson, E. L., (2002). Three-Dimensional Static and Dynamic Analysis of Structures A phisical Approach With Emphasis on Earthquake Engineering. 3rd ed. Berkeley: Computers and Structures, Inc.

Wolf, J.P., (1985). Dynamic Soil Structure Interaction. New Jersey: Prentice - Hall, Inc. 
Wu, H. C. \& Clarke, C., (1966). Normal Stresses in Beams Due to Non Uniform Torsion. Engineering Bulletin, Volume 10.

Yang, J., Sato, T., Savidis, S. \& Li, X., (2002). Horizontal and Vertical Components of Earthquake Ground Motion at Liquefiable Sites. Soil Dynamics and Earthquake Engineering, Volume 22, pp. 229-240.

Zanardo, G., Hao, H. \& Modena, C., (2002). Seismic Response of Multi-span Simply Suported Bridges to a Spatially Varying Earthquake Ground Motion. Earthquake Engineering and Structural Dynamics, Volume 31, pp. 1325-1345 


\section{APPENDIX 1}

\section{Section Properties}

1. Composition of girder and slab.

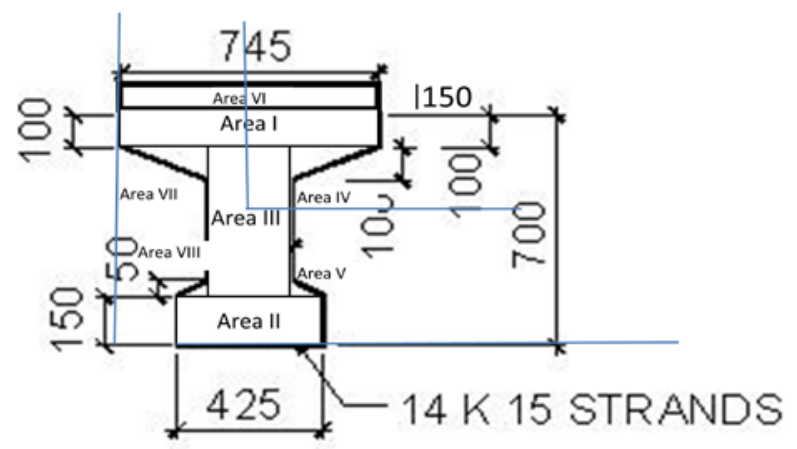
a. $\quad \mathrm{I}_{\mathrm{xx}}=0.30767 \mathrm{~m}^{4}$
b. $\quad \mathrm{l}_{\mathrm{yy}}=0.1375 \mathrm{~m}^{4}$
c. $\quad A=4.62825 \mathrm{~m}^{2}$

2. Pier caps:

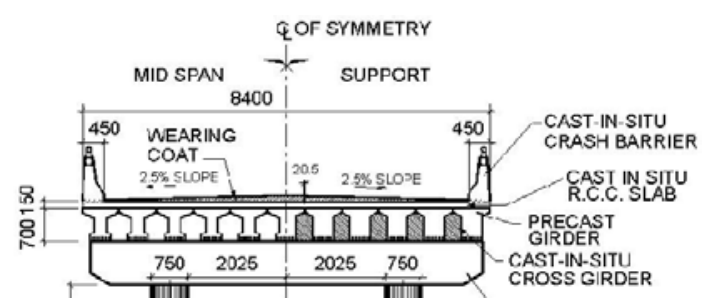

$b=8 m$

$\mathrm{h}=1 \mathrm{~m}$

$I_{x}=(1 / 12) b h^{3} \quad I_{y}=(1 / 12) b^{3} h$

$\begin{array}{ll}\text { a. } & \mathrm{I}_{\mathrm{x}}=0.6667 \mathrm{~m}^{4} \\ \text { b. } & \mathrm{l} y=42.6667 \mathrm{~m}^{4}\end{array}$

$A=8 m^{2}$ 
3. Piers

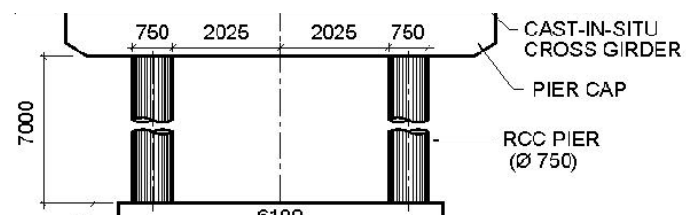

Radius of piers $=0.375 \mathrm{~m}$

$\mathrm{I}_{\mathrm{x}}=\mathrm{I}_{\mathrm{y}}=\pi \mathrm{r}^{4} / 4$

$A=\pi r^{2}$

a. $\mathrm{I}_{\mathrm{x}}=0.2484 \mathrm{~m}^{4}$

b. $\quad \mathrm{I}_{\mathrm{y}}=0.2484 \mathrm{~m}^{4}$

c. $\quad A=0.8831 \mathrm{~m}^{2}$

For all sections the material properties of the structure are considered as follows:

- $\quad$ E concrete $=30 \mathrm{Gpa}=3 \times 10^{10} \mathrm{~Pa}=3 \times 10^{7} \mathrm{kN} / \mathrm{m}^{2}$ (high strength)

- $\quad \gamma($ Poisson ratio $)=0.3$ 


\section{Modelling the Structure and Validation}

Support reactions for a simple model of the bridge are as follows:

a. From ANSYS analysis:

\begin{tabular}{|l|l|l|l|}
\hline \multicolumn{1}{|c|}{ NODE } & \multicolumn{1}{|c|}{ FX (N) } & \multicolumn{1}{|c|}{$\mathrm{FY}(\mathrm{N})$} & \multicolumn{1}{c|}{$\mathrm{MZ}(\mathrm{Nm})$} \\
\hline 1 & 3576.9 & $1.35 \mathrm{E}+06$ & $4.60 \mathrm{E}+06$ \\
\hline 61 & -3576.9 & $1.35 \mathrm{E}+06$ & $-4.60 \mathrm{E}+06$ \\
\hline 62 & -10731 & $2.86 \mathrm{E}+06$ & 27843 \\
\hline 70 & 10731 & $2.86 \mathrm{E}+06$ & -27843 \\
\hline
\end{tabular}

Figures below are the description of bending moment and shear diagram of the idealization of the bridge.

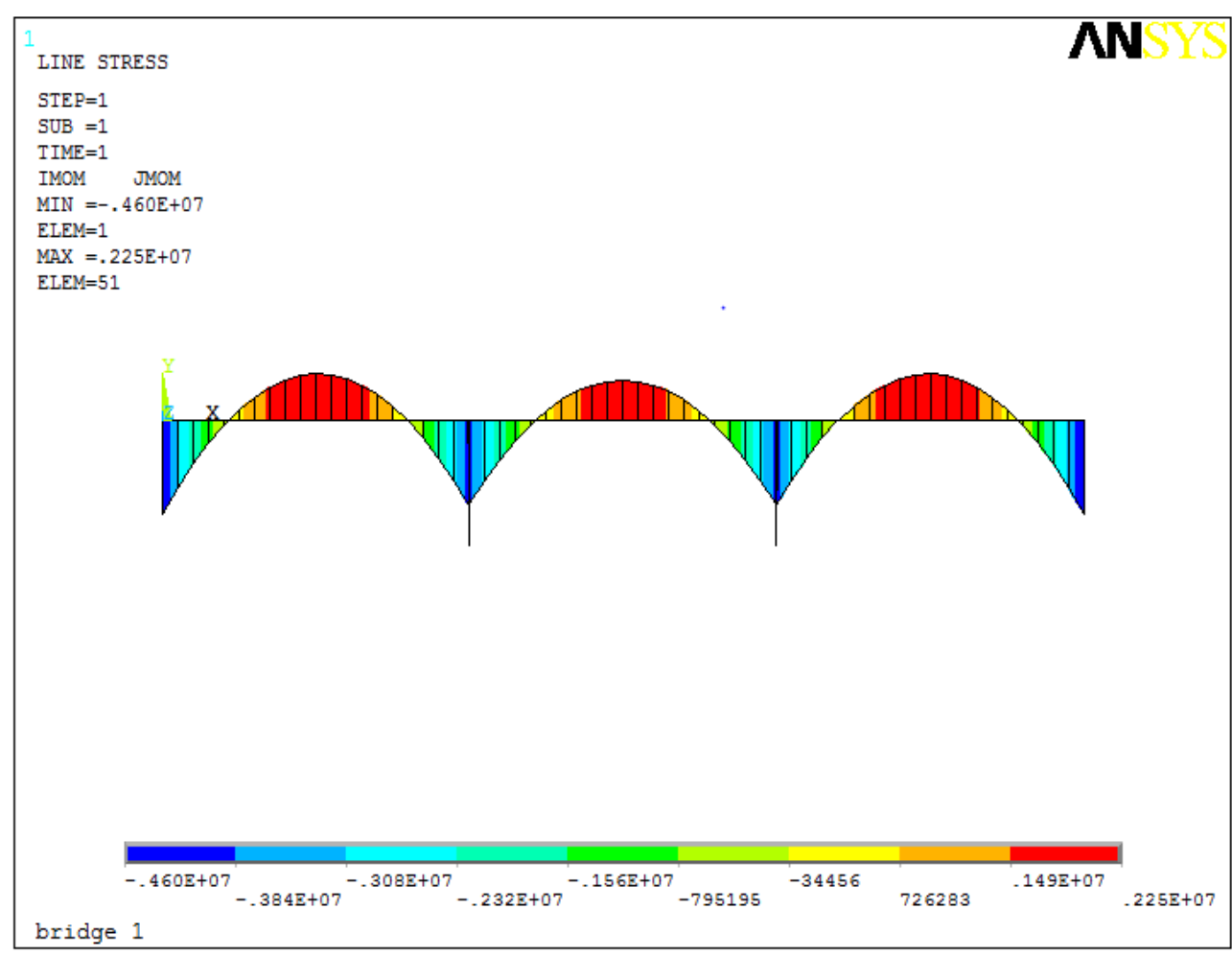

Figure II-1: Moment diagram 


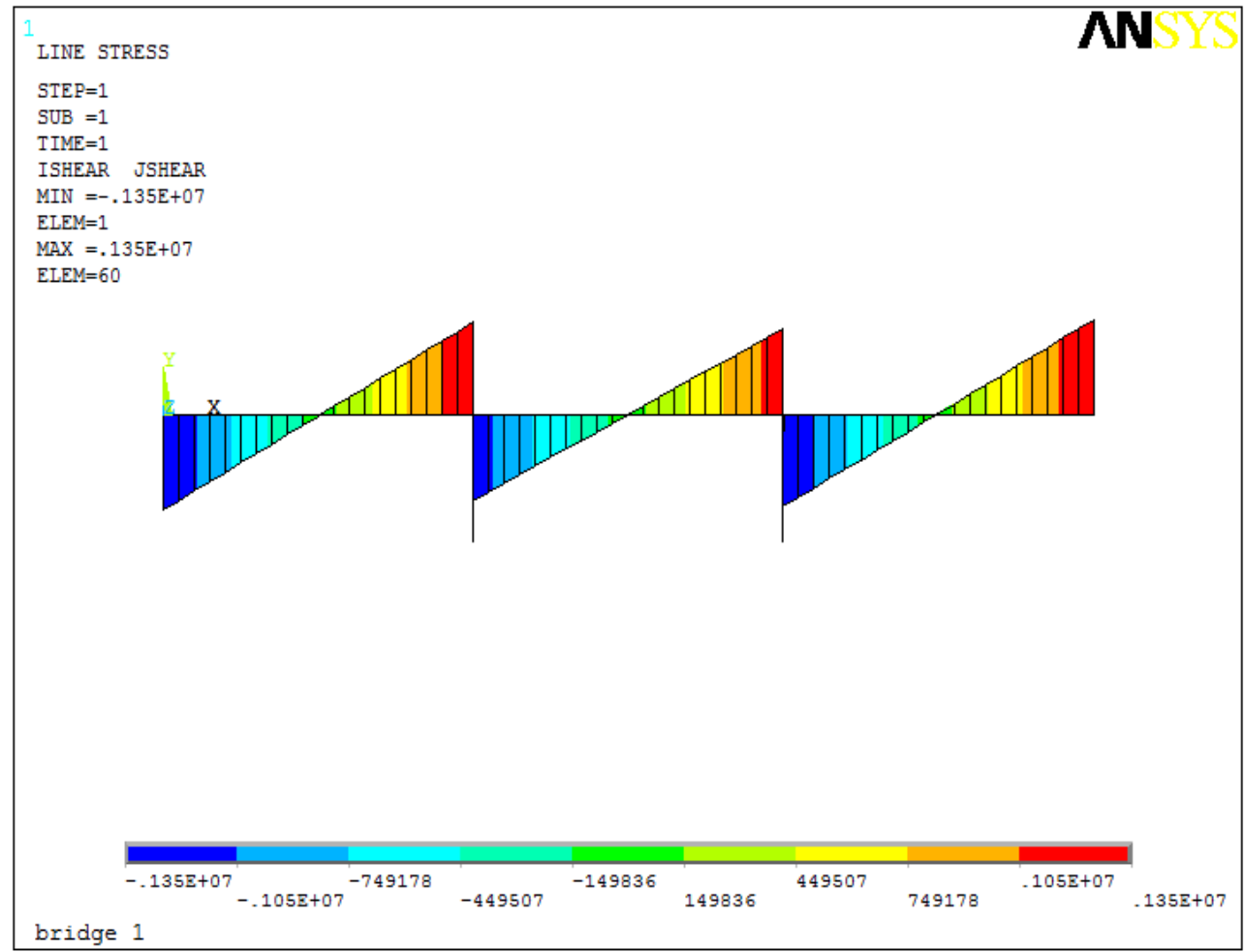

Figure II-2: Shear force diagram

b. Validation

For validation, support reactions are calculated using moment distribution (cross method).

For simplicity, the model of the structure can be seen in Error! Reference source not found.

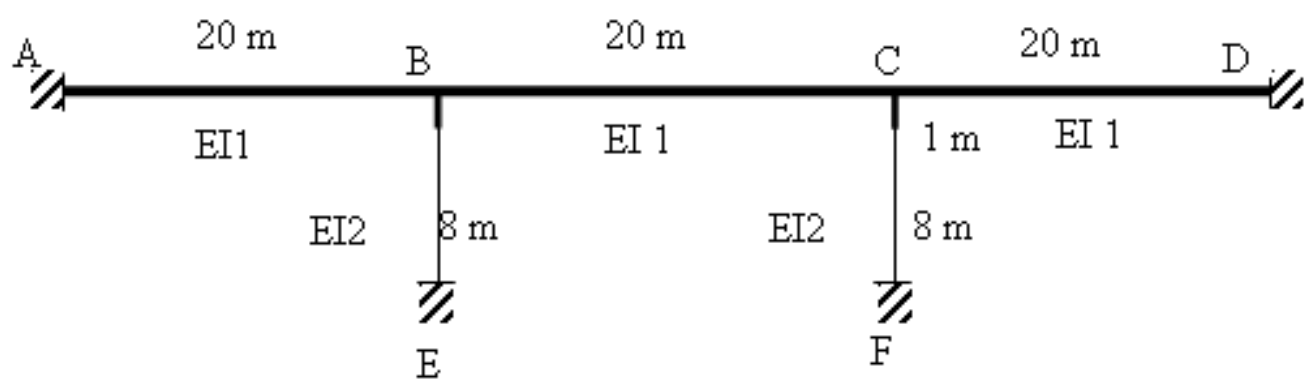

The results are obtained as follows:

$$
\begin{aligned}
& A_{\mathrm{AB}}=A_{\mathrm{BC}}=A_{\mathrm{CB}}=4.6282 \mathrm{~m}^{2} \\
& A_{\mathrm{BE}}=A_{\mathrm{CF}}=0.883 \mathrm{~m}^{2} \\
& I_{\mathrm{AB}}=I_{\mathrm{BC}}=I_{\mathrm{CB}}=0.3076 \mathrm{~m}^{4} \\
& I_{\mathrm{BE}}=I_{\mathrm{CF}}=0.0031 \mathrm{~m}^{4}
\end{aligned}
$$


The density of the total deck plus superimposed load is $2679.276 \mathrm{~kg} / \mathrm{m}^{3}$, when converted to distributed load this becomes $113.0667 \mathrm{kN} / \mathrm{m}$. Meanwhile, the density of the total deck plus superimposed load is $2924.304 \mathrm{~kg} / \mathrm{m}^{3}$.

From moment distribution, the results are resumed in Table below:

\begin{tabular}{|c|c|c|c|c|c|c|c|c|c|c|c|}
\hline \multirow{2}{*}{ ITEM } & $\mathrm{A}$ & \multicolumn{3}{|c|}{$B$} & \multicolumn{3}{|c|}{ C } & \multirow{2}{*}{$\begin{array}{ll}\mathrm{D} & \\
\mathrm{DC}\end{array}$} & \multirow{2}{*}{$\begin{array}{c}E \\
E B \\
\end{array}$} & \multirow{2}{*}{\begin{tabular}{|l|}
$F$ \\
$F C$
\end{tabular}} & \\
\hline & $A B$ & BA & $B C$ & $\mathrm{BE}$ & $\mathrm{CB}$ & $\mathrm{CD}$ & $\mathrm{CF}$ & & & & \\
\hline \multirow{2}{*}{$\mathrm{SF}$} & \multicolumn{2}{|c|}{$4 \mathrm{EI} / \mathrm{L}$} & $4 \mathrm{EI} / \mathrm{L}$ & $4 \mathrm{EI} / \mathrm{L}$ & $4 \mathrm{EI} / \mathrm{L}$ & $4 \mathrm{EI} / \mathrm{L}$ & $4 \mathrm{EI} / \mathrm{L}$ & $4 \mathrm{EI} / \mathrm{L}$ & $4 \mathrm{EI} / \mathrm{L}$ & $4 \mathrm{EI} / \mathrm{L}$ & \\
\hline & $1.85 \mathrm{E}+09$ & $1.85 \mathrm{E}+09$ & $1.85 \mathrm{E}+09$ & $4.66 \mathrm{E}+08$ & $1.85 \mathrm{E}+09$ & $1.85 E+09$ & $4.66 \mathrm{E}+08$ & $1.85 \mathrm{E}+09$ & $4.66 \mathrm{E}+08$ & $4.66 \mathrm{E}+08$ & \\
\hline DF ratio & \begin{tabular}{l|l}
0 \\
\end{tabular} & $4.44 \mathrm{E}-01$ & $4.44 \mathrm{E}-01$ & $1.12 \mathrm{E}-01$ & $4.44 \mathrm{E}-01$ & $4.44 \mathrm{E}-01$ & $1.12 \mathrm{E}-01$ & 0 & 0 & 0 & \\
\hline FEM & 4425.70 & -4425.70 & 4054.87 & 0.00 & -4054.87 & 4425.70 & 0.00 & -4425.70 & 0.00 & 0.00 & siklus 1 \\
\hline Dist 1 & & 164.65 & 164.65 & 41.54 & -164.65 & -164.65 & -41.54 & 0.00 & & & SIKIUS 1 \\
\hline $\mathrm{CO} 2$ & 82.32 & 0.00 & -82.32 & 0.00 & 82.32 & 0.00 & 0.00 & -82.32 & 20.77 & -20.77 & siklus 2 \\
\hline Dist. 2 & & 36.55 & 36.55 & 9.22 & -36.55 & -36.55 & -9.22 & 0.00 & & & \\
\hline $\mathrm{CO} 3$ & 18.28 & 0.00 & -18.28 & 0.00 & 18.28 & 0.00 & 0.00 & -18.28 & 4.61 & -4.61 & siklus 3 \\
\hline Dist. 3 & & 8.11 & 8.11 & 2.05 & -8.11 & -8.11 & -2.05 & 0.00 & & & \\
\hline $\mathrm{CO} 4$ & 4.06 & 0.00 & -4.06 & 0.00 & 4.06 & 0.00 & 0.00 & -4.06 & 1.02 & -1.02 & siklus 4 \\
\hline Dist. 4 & & 1.80 & 1.80 & 0.45 & -1.80 & -1.80 & -0.45 & 0.00 & & & \\
\hline $\mathrm{CO} 5$ & 0.90 & 0.00 & -0.90 & 0.00 & 0.90 & 0.00 & 0.00 & -0.90 & 0.23 & -0.23 & siklus \\
\hline Dist. 5 & & 0.40 & 0.40 & 0.10 & -0.40 & -0.40 & -0.10 & 0.00 & & & SIKIUS S \\
\hline $\mathrm{CO} 6$ & 0.20 & 0.00 & -0.20 & 0.00 & 0.20 & 0.00 & 0.00 & -0.20 & 0.05 & -0.05 & siklus \\
\hline Dist. 6 & & 0.09 & 0.09 & 0.02 & -0.09 & -0.09 & -0.02 & 0.00 & & & SIKIUS o \\
\hline $\mathrm{CO} 7$ & 0.04 & 0.00 & -0.04 & 0.00 & 0.04 & 0.00 & 0.00 & -0.04 & 0.01 & -0.01 & ciklus 7 \\
\hline Dist. 7 & & 0.02 & 0.02 & 0.00 & -0.02 & -0.02 & 0.00 & 0.00 & & & SIKIUS $/$ \\
\hline TEM & 4531.51 & -4214.08 & 4160.70 & 53.39 & -4160.70 & 4214.08 & -53.39 & -4531.51 & 26.69 & -26.69 & \\
\hline
\end{tabular}

$$
\begin{aligned}
& \text { Moment } A=4.531 \mathrm{MNm} \\
& \text { Moment } D=-4.531 \mathrm{MNm} \\
& \text { Moment } E=26.7 \mathrm{kNm} \\
& \text { Moment } F=-26.7 \mathrm{kNm}
\end{aligned}
$$

The results are close to the ANSYS results, with variations in assumptions (i.e. joint translation) accounting for any discrepancies.

Support reactions for shear actions can be obtained by:

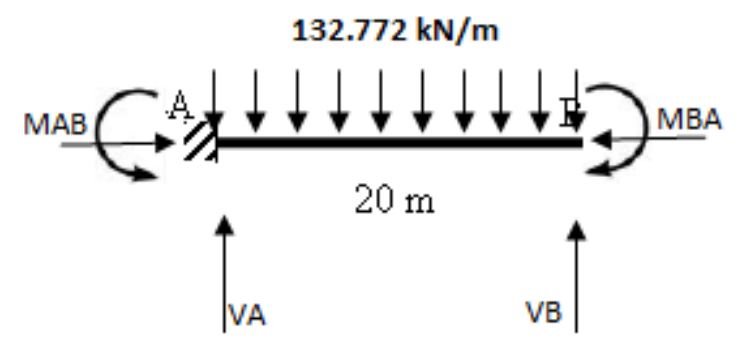

$$
\begin{aligned}
& V_{a x} L-M_{a b}-0.5 q L^{2}+M_{b a}=0 \\
& M_{A}=M_{E} \\
& V_{A}=V_{E}=132.772 \mathrm{kN} / \mathrm{m} * 20 / 2 \mathrm{~m}+\left(\mathrm{M}_{\mathrm{AB}}-\mathrm{M}_{\mathrm{BA}}\right) / \mathrm{L} \\
& =1343.58 \mathrm{kN} \\
& V_{E}=V_{F}=V_{A}+V_{B}
\end{aligned}
$$




$$
\begin{aligned}
& =1343.58+132.772 * 20 / 2+\left(M_{A B}-M_{B A}\right) / L \\
& =2560 \mathrm{kN}
\end{aligned}
$$

\section{Moment Capacity of the Deck}

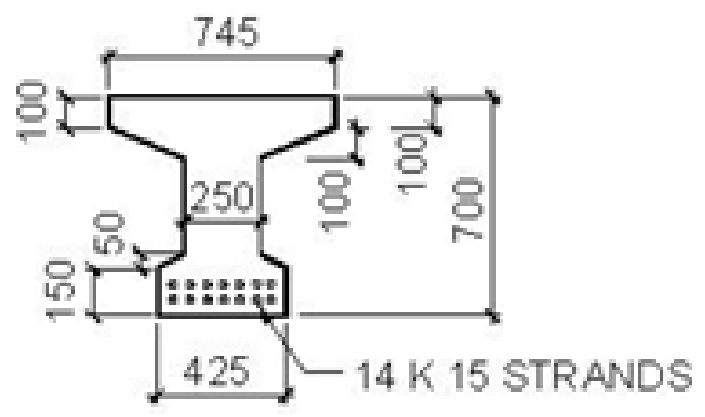

From the figure above and the data about pre-stressed, it is known that

$\begin{array}{ll}\text { Fult }_{\text {ult }} & : 3649 \mathrm{Nmm}^{2} \\ \mathrm{f}_{\text {ck }}^{\prime} & : 60 \mathrm{Nmm}^{2} \\ \mathrm{~A}_{\text {strands }} & : 140 \mathrm{~mm}^{2} \\ \text { Number of strands } & : 14 \\ \mathrm{~A}_{\text {strands }} \text { total } & : 1.96 \times 10^{3} \mathrm{~mm}^{2} \\ \mathrm{~h}_{\text {beam }} & : 700 \mathrm{~mm} \\ \mathrm{~b}_{1} & : 745 \mathrm{~mm} \\ \mathrm{t}_{\mathrm{f}} & : 150 \mathrm{~mm} \\ \mathrm{~b}_{2} & : 425 \mathrm{~mm} \\ \mathrm{~d}_{1} & : \mathrm{t}_{\mathrm{f}} / 2=75 \mathrm{~mm} \\ \mathrm{~d} & : \mathrm{h}_{\text {beam }}-\mathrm{d}_{1}=625 \mathrm{~mm}\end{array}$

The moment capacity of a pre-stressed beam can be calculated by considering the compression and tension area on their section property.

From the figure above $\mathrm{M}=\mathrm{Cxz}$, where $\mathrm{C}$ is compression area of the beam and $\mathrm{z}$ is the arm moment of the section.

$$
\begin{gathered}
C=0.8 \times x \times 0.57 f_{c k} \times b_{1} \\
T=0.78 F_{u l t} \times A_{p s}=5.565 \times 10^{6} \mathrm{~N} \\
x=\frac{T}{0.8 \times 0.57 f_{c k} \times b_{1}}=273.011 \mathrm{~mm} \\
z=d-0.4 x=515.796 \mathrm{~mm}
\end{gathered}
$$

Therefore,

$$
M=C \times z=5.565 \times 10^{6} \mathrm{~N} \times 515.796 \mathrm{~mm}=2.87 \times 10^{9} \mathrm{Nmm}
$$


So the moment capacity of the each girder is $2.87 \times 10^{9} \mathrm{Nmm}$. For whole girders, the moment capacity becomes $11 \times 2.87 \times 10^{9} \mathrm{Nmm}=3.157 \times 10^{10} \mathrm{Nmm}$, or $31.6 \mathrm{MNm}$. The calculations are referring to Eurocode 2 for concrete design structure.

\section{IV.Response Spectrum}

Horizontal
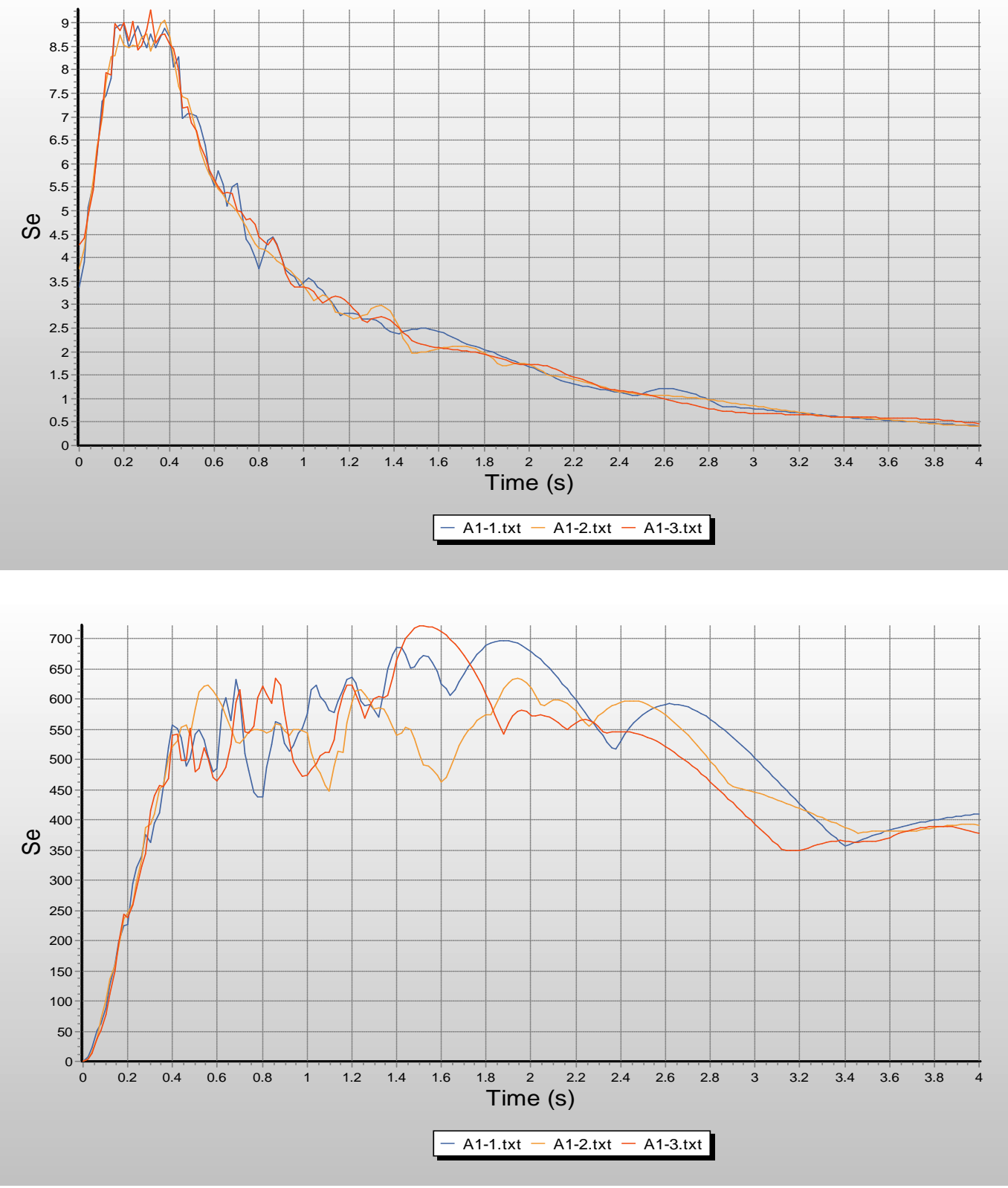


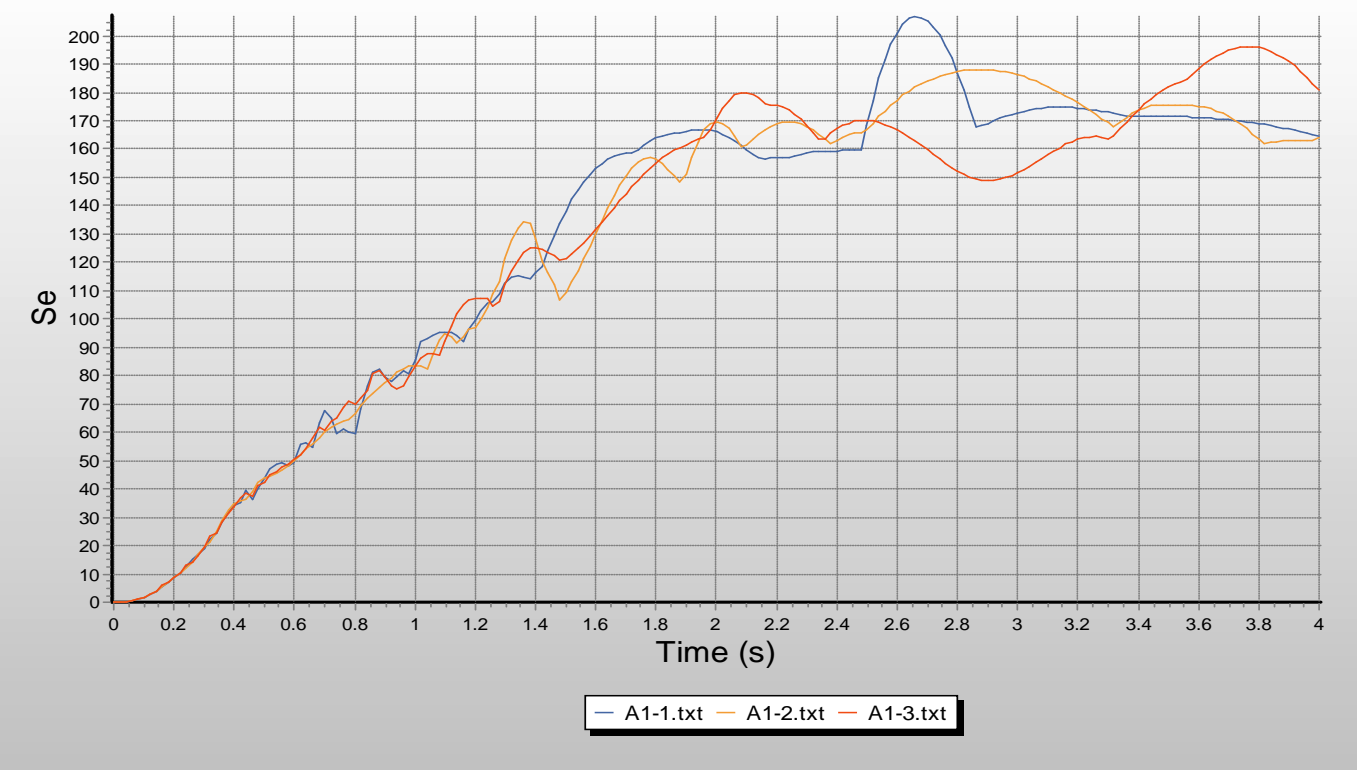

\section{Vertical}

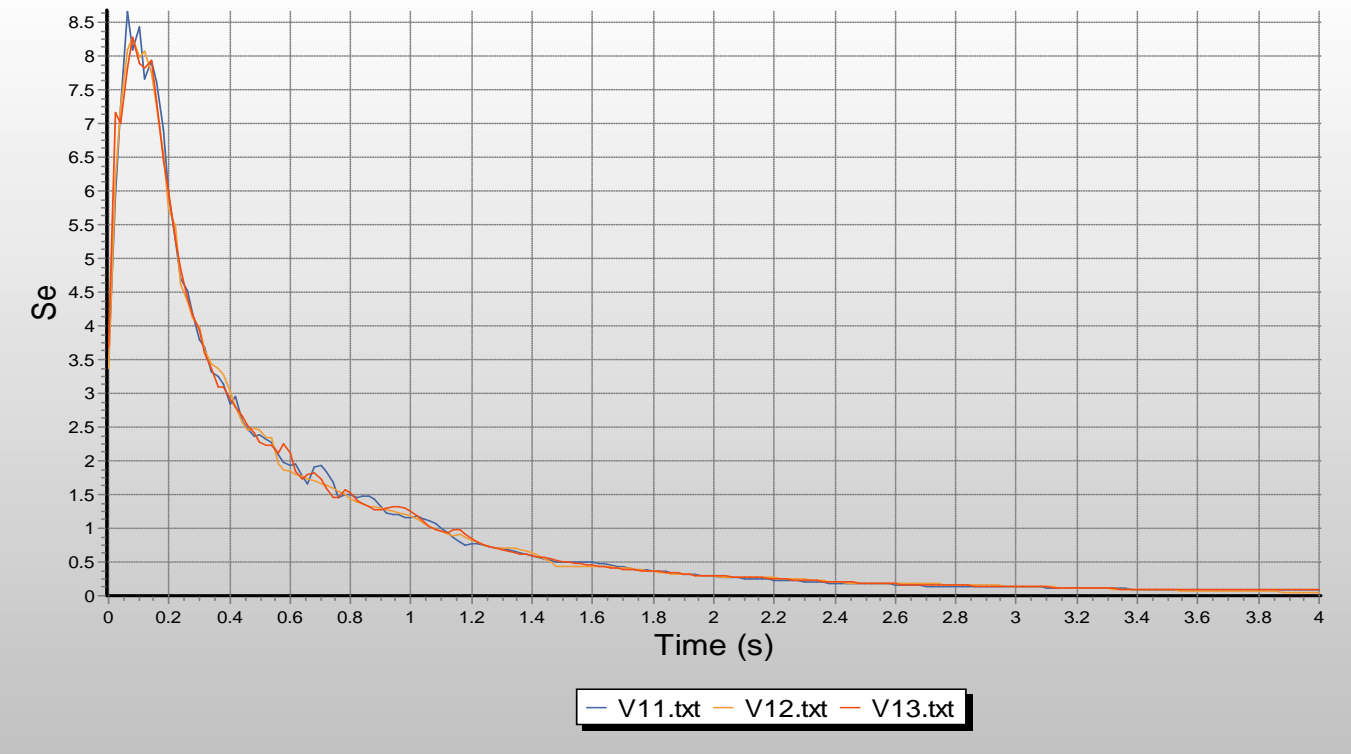



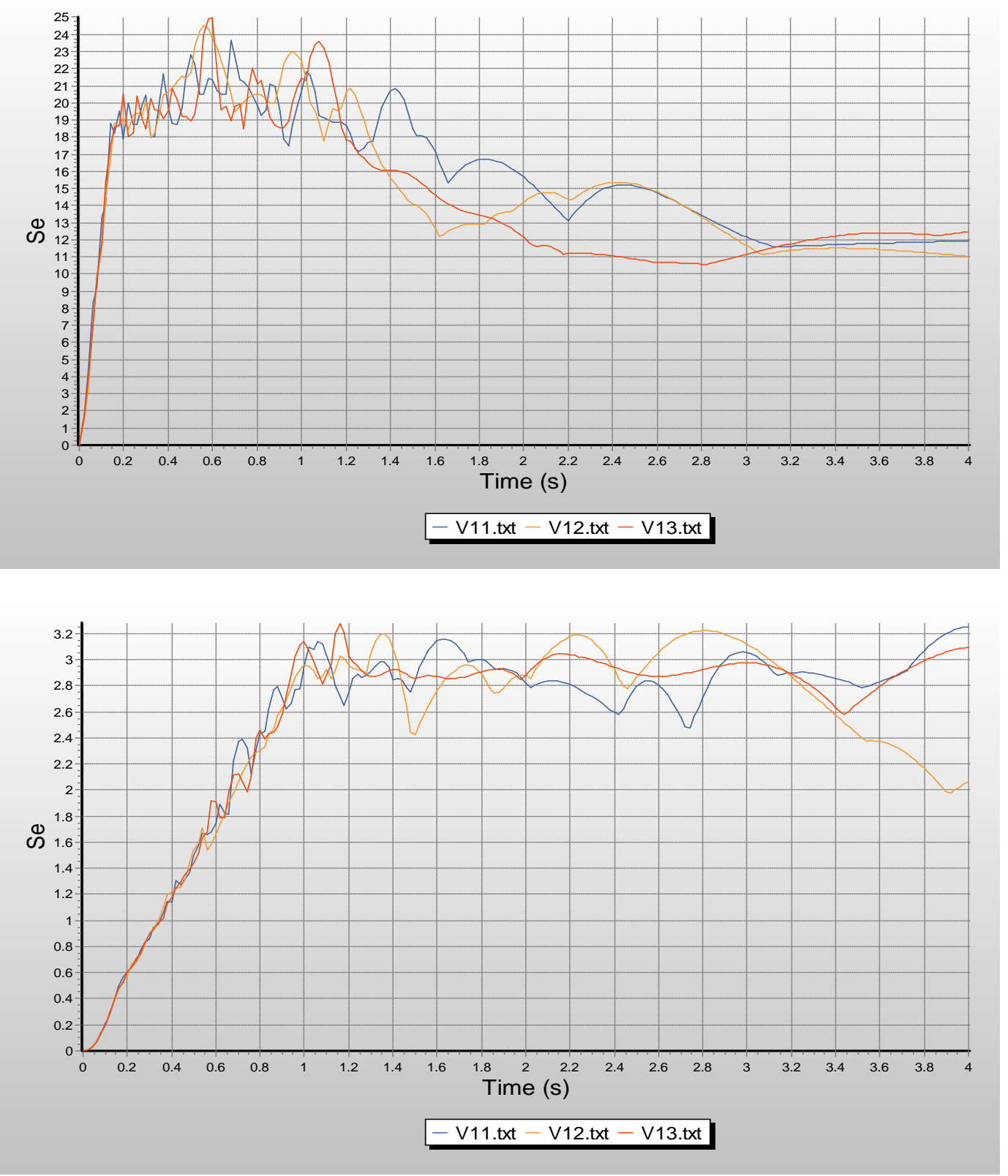


\title{
V. ANSYS Parametric Design Languange
}

\author{
Time History
}

Modelling the Bridge

\section{Fixed Base Support}

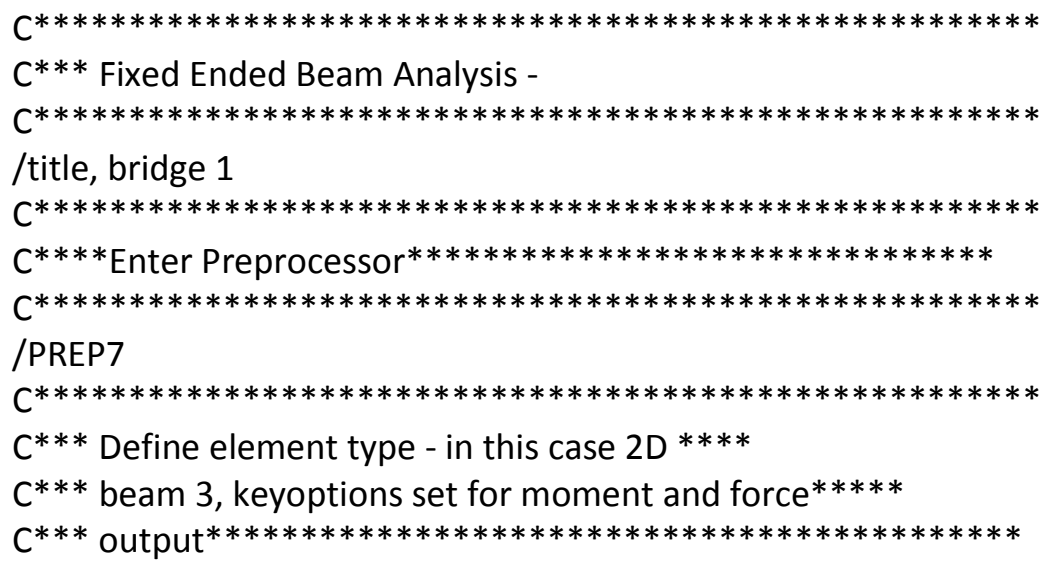

$\mathrm{ET}, 1, \mathrm{BEAM} 3$

$\mathrm{C} * * * * * * * * * * * * * * * * * * * * * * * * * * * * * * * * * * * * * * * * * * * * * * * * * * * * * *$

C*** Define real constants (geometry)*****************

$\mathrm{C}^{* * *}$ Area, Second moment of Area, Height $* * * * * * * * * * * * * *$

$R, 1,4.6282,0.30767,0.57853$,

$R, 2,8.0,0.6667,1.0$,

$\mathrm{R}, 3,0.88313,0.31047 \mathrm{e}-01,0.75$

$\mathrm{C} * * * * * * * * * * * * * * * * * * * * * * * * * * * * * * * * * * * * * * * * * * * * * * * * * * * * *$

C $* * *$ Define Material Properties $* * * * * * * * * * * * * * * * * * * * * * *$

$\mathrm{C} * * * * * * * * * * * * * * * * * * * * * * * * * * * * * * * * * * * * * * * * * * * * * * * * * * * * *$

$\mathrm{MP}, \mathrm{EX}, 1,0.3 \mathrm{e} 11$

$M P, P R X Y, 1,0.3$

MP,DENS, 1,2400

MP,EX,2,0.3e11

$M P, P R X Y, 2,0.3$

MP,DENS,2,2679.2765

$\mathrm{MP}, \mathrm{EX}, 3,0.3 \mathrm{e} 11$

MP,PRXY, 3, 0.3

MP,DENS,3,2924.304

$\mathrm{C} * * * * * * * * * * * * * * * * * * * * * * * * * * * * * * * * * * * * * * * * * * * * * * * * * * * * * *$

$C^{* * *}$ Generate Nodes (nodes 1-first and 13-last in*****

$\mathrm{C}^{* * *}$ this case with fill command used for those*******

$\mathrm{C}^{* * *}$ in between b $^{*} * * * * * * * * * * * * * * * * * * * * * * * * * * * * * * * * * * * * *$

C $* * * * * * * * * * * * * * * * * * * * * * * * * * * * * * * * * * * * * * * * * * * * * * * * * * * * * *$

$\mathrm{n}, 1,0,0,0,0,0,0$

$\mathrm{n}, 2,1,0,0,0,0,0$

$\mathrm{n}, 3,2,0,0,0,0,0$

$\mathrm{n}, 4,3,0,0,0,0,0$

$\mathrm{n}, 5,4,0,0,0,0,0$

$\mathrm{n}, 6,5,0,0,0,0,0$ 

$\mathrm{n}, 7,6,0,0,0,0,0$
$\mathrm{n}, 8,7,0,0,0,0,0$
$\mathrm{n}, 9,8,0,0,0,0,0$
n, $10,9,0,0,0,0,0$
$\mathrm{n}, 11,10,0,0,0,0,0$
$\mathrm{n}, 12,11,0,0,0,0,0$
n, $13,12,0,0,0,0,0$
$\mathrm{n}, 14,13,0,0,0,0,0$
$\mathrm{n}, 15,14,0,0,0,0,0$
$\mathrm{n}, 16,15,0,0,0,0,0$
n, $17,16,0,0,0,0,0$
$\mathrm{n}, 18,17,0,0,0,0,0$
$\mathrm{n}, 19,18,0,0,0,0,0$
$\mathrm{n}, 20,19,0,0,0,0,0$
$\mathrm{n}, 21,20,0,0,0,0,0$
$\mathrm{n}, 22,21,0,0,0,0,0$
$\mathrm{n}, 23,22,0,0,0,0,0$
$\mathrm{n}, 24,23,0,0,0,0,0$
n, $25,24,0,0,0,0,0$
$\mathrm{n}, 26,25,0,0,0,0,0$
$\mathrm{n}, 27,26,0,0,0,0,0$
$\mathrm{n}, 28,27,0,0,0,0,0$
$\mathrm{n}, 29,28,0,0,0,0,0$
n, 30,29,0,0,0,0,0
n, 31,30,0,0,0,0,0
$\mathrm{n}, 32,31,0,0,0,0,0$
n, $33,32,0,0,0,0,0$
$\mathrm{n}, 34,33,0,0,0,0,0$
$\mathrm{n}, 35,34,0,0,0,0,0$
n, $36,35,0,0,0,0,0$
$\mathrm{n}, 37,36,0,0,0,0,0$
$\mathrm{n}, 38,37,0,0,0,0,0$
n, $39,38,0,0,0,0,0$
$\mathrm{n}, 40,39,0,0,0,0,0$
$\mathrm{n}, 41,40,0,0,0,0,0$
$\mathrm{n}, 42,41,0,0,0,0,0$
$\mathrm{n}, 43,42,0,0,0,0,0$
$\mathrm{n}, 44,43,0,0,0,0,0$
$\mathrm{n}, 45,44,0,0,0,0,0$
$\mathrm{n}, 46,45,0,0,0,0,0$
$\mathrm{n}, 47,46,0,0,0,0,0$
$\mathrm{n}, 48,47,0,0,0,0,0$
$\mathrm{n}, 49,48,0,0,0,0,0$
n, $50,49,0,0,0,0,0$
$\mathrm{n}, 51,50,0,0,0,0,0$
$\mathrm{n}, 52,51,0,0,0,0,0$
$\mathrm{n}, 53,52,0,0,0,0,0$
$\mathrm{n}, 54,53,0,0,0,0,0$
$\mathrm{n}, 55,54,0,0,0,0,0$
$\mathrm{n}, 56,55,0,0,0,0,0$
$\mathrm{n}, 57,56,0,0,0,0,0$ 
$\mathrm{n}, 58,57,0,0,0,0,0$

$\mathrm{n}, 59,58,0,0,0,0,0$

$\mathrm{n}, 60,59,0,0,0,0,0$

$\mathrm{n}, 61,60,0,0,0,0,0$

$\mathrm{n}, 62,20,-8,0,0,0,0$

$\mathrm{n}, 63,20,-7,0,0,0,0$

$\mathrm{n}, 64,20,-6,0,0,0,0$

$\mathrm{n}, 65,20,-5,0,0,0,0$

$\mathrm{n}, 66,20,-4,0,0,0,0$

$\mathrm{n}, 67,20,-3,0,0,0,0$

$\mathrm{n}, 68,20,-2,0,0,0,0$

$\mathrm{n}, 69,20,-1,0,0,0,0$

$\mathrm{n}, 70,40,-8,0,0,0,0$

$\mathrm{n}, 71,40,-7,0,0,0,0$

$\mathrm{n}, 72,40,-6,0,0,0,0$

$\mathrm{n}, 73,40,-5,0,0,0,0$

$\mathrm{n}, 74,40,-4,0,0,0,0$

$\mathrm{n}, 75,40,-3,0,0,0,0$

$\mathrm{n}, 76,40,-2,0,0,0,0$

$\mathrm{n}, 77,40,-1,0,0,0,0$

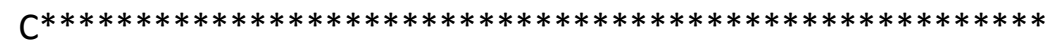

$\mathrm{C}^{* * *}$ Before generating elements set relevant element**

$\mathrm{C}^{* * *}$ type, real constant set and material active with*

$\mathrm{C}^{* * *}$ the following commands $* * * * * * * * * * * * * * * * * * * * * * * * * * *$

$\mathrm{C}^{* * * * * * * * * * * * * * * * * * * * * * * * * * * * * * * * * * * * * * * * * * * * * * * * * * * * * * * *}$

type,1

real,1

mat,3

$\mathrm{C} * * * * * * * * * * * * * * * * * * * * * * * * * * * * * * * * * * * * * * * * * * * * * * * * * * * * *$

$\mathrm{C}^{* * *}$ Generate elements $* * * * * * * * * * * * * * * * * * * * * * * * * * * * * * * *$

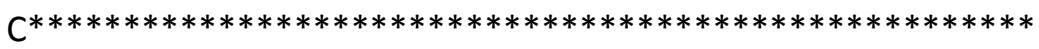

en, $1,1,2$

en, $2,2,3$

en, $3,3,4$

en, $4,4,5$

en, $5,5,6$

en, $6,6,7$

en, $7,7,8$

en, $8,8,9$

en, $9,9,10$

en, $10,10,11$

en, $11,11,12$

en, $12,12,13$

en, $13,13,14$

en, $14,14,15$

en, $15,15,16$

en, $16,16,17$

en, $17,17,18$

en, $18,18,19$

en, $19,19,20$

en, $20,20,21$ 


$$
\begin{aligned}
& \text { type,1 } \\
& \text { real,1 } \\
& \text { mat,2 } \\
& \text { en,21,21,22 } \\
& \text { en,22,22,23 } \\
& \text { en,23,23,24 } \\
& \text { en,24,24,25 } \\
& \text { en,25,25,26 } \\
& \text { en,26,26,27 } \\
& \text { en,27,27,28 } \\
& \text { en,28,28,29 } \\
& \text { en,29,29,30 } \\
& \text { en,30,30,31 } \\
& \text { en,31,31,32 } \\
& \text { en,32,32,33 } \\
& \text { en,33,33,34 } \\
& \text { en,34,34,35 } \\
& \text { en,35,35,36 } \\
& \text { en,36,36,37 } \\
& \text { en,37,37,38 } \\
& \text { en,38,38,39 } \\
& \text { en,39,39,40 } \\
& \text { en,40,40,41 } \\
& \text { type,1 } \\
& \text { real,1 } \\
& \text { mat,3 } \\
& \text { en,41,41,42 } \\
& \text { en, } 42,42,43 \\
& \text { en,43,43,44 } \\
& \text { en,44,44,45 } \\
& \text { en,45,45,46 } \\
& \text { en,46,46,47 } \\
& \text { en,47,47,48 } \\
& \text { en,48,48,49 } \\
& \text { en,49,49,50 } \\
& \text { en,50,50,51 } \\
& \text { en,51,51,52 } \\
& \text { en,52,52,53 } \\
& \text { en,53,53,54 } \\
& \text { en, }, 1 \\
& \text { en,54,54,55 } \\
& \text { en,55,55,56 } \\
& \text { en,69, } 61,76,56,57 \\
& \text { en,57,57,58 } \\
& \text { en,58,58,59 } \\
& \text { en,59,59,60 } \\
& \text { en,60,60,61 } \\
& \hline
\end{aligned}
$$




\section{type, 1}

real, 3

mat,1

en, $62,62,63$

en, $63,63,64$

en, $64,64,65$

en, $65,65,66$

en, $66,66,67$

en, $67,67,68$

en, $68,68,69$

en, $70,70,71$

en, $71,71,72$

en, $72,72,73$

en, $73,73,74$

en, $74,74,75$

en, $75,75,76$

en, $76,76,77$

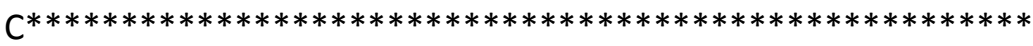

C C $^{* * *}$ Exit preprocessor $* * * * * * * * * * * * * * * * * * * * * * * * * * * * * * * * * *$

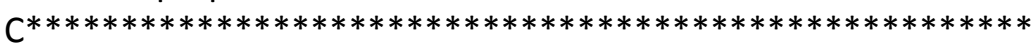

FINISH

C $* * * * * * * * * * * * * * * * * * * * * * * * * * * * * * * * * * * * * * * * * * * * * * * * * * * * * * * *$

C***Enter Solution Phase $* * * * * * * * * * * * * * * * * * * * * * * * * * * * * * *$

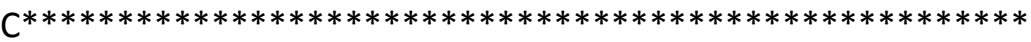

/SOLU

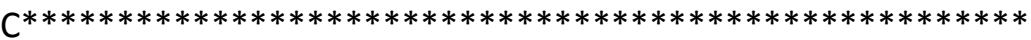

$\mathrm{C}^{* * *}$ Fix the ends of the beams by specifying $* * * * * * * * * *$

$\mathrm{C}^{* * *}$ zero dispacement for all degrees of freedom******

C*** i.e. UX $=$ UY $=\mathrm{ROTZ}=0 * * * * * * * * * * * * * * * * * * * * * * * * *$

C $* * * * * * * * * * * * * * * * * * * * * * * * * * * * * * * * * * * * * * * * * * * * * * * * * * * * * *$

$\mathrm{D}, 1, \mathrm{UX}, 0,0,0$

$\mathrm{D}, 1, \mathrm{UY}, 0,0,0$

$\mathrm{D}, 1, \mathrm{ROTZ}, 0,0,0$

$D, 61, U X, 0,0,0$

$\mathrm{D}, 61, \mathrm{UY}, 0,0,0$

D,61,ROTZ,0,0,0

$D, 62, U X, 0,0,0$

$\mathrm{D}, 62, \mathrm{UY}, 0,0,0$

D, 62, ROTZ,0,0,0

$\mathrm{D}, 70, \mathrm{UX}, 0,0,0$

$\mathrm{D}, 70, \mathrm{UY}, 0,0,0$

D, 70,ROTZ,0,0,0 


\section{For Springs Base Support}

C $* * * * * * * * * * * * * * * * * * * * * * * * * * * * * * * * * * * * * * * * * * * * * * * * * * * * * *$

C*** Fixed Ended Beam Analysis - Material Nonlinearity

$\mathrm{C} * * * * * * * * * * * * * * * * * * * * * * * * * * * * * * * * * * * * * * * * * * * * * * * * * * * * *$

/title, bridge 2

$\mathrm{C} * * * * * * * * * * * * * * * * * * * * * * * * * * * * * * * * * * * * * * * * * * * * * * * * * * * * * * * *$

C $* * * *$ Enter Preprocessor $* * * * * * * * * * * * * * * * * * * * * * * * * * * * * * *$

$\mathrm{C} * * * * * * * * * * * * * * * * * * * * * * * * * * * * * * * * * * * * * * * * * * * * * * * * * * * * *$

/PREP7

$\mathrm{C} * * * * * * * * * * * * * * * * * * * * * * * * * * * * * * * * * * * * * * * * * * * * * * * * * * * * * * *$

C*** Define element type - in this case $* * * *$

C*** beam 3 , keyoptions set for moment and force $* * * * *$

C*** output $* * * * * * * * * * * * * * * * * * * * * * * * * * * * * * * * * * * * * * * * * * *$

ET,1,BEAM3

$\mathrm{ET}, 2, \mathrm{COMBIN} 14$

C $* * * * * * * * * * * * * * * * * * * * * * * * * * * * * * * * * * * * * * * * * * * * * * * * * * * * *$

$\mathrm{C}^{* * *}$ Define real constants (geometry) $* * * * * * * * * * * * * * * * *$

C*** Area, Second moment of Area, Height**************

$\mathrm{R}, 1,4.6282,0.30767,0.57853$,

$\mathrm{R}, 2,8.0,0.6667,1.0$,

$\mathrm{R}, 3,0.88313,0.31047 \mathrm{e}-01,0.75$

$\mathrm{R}, 4,400000000$

$\mathrm{R}, 5,150000000$

$\mathrm{C} * * * * * * * * * * * * * * * * * * * * * * * * * * * * * * * * * * * * * * * * * * * * * * * * * * * * * * * *$

C $* * *$ Define Material Properties $* * * * * * * * * * * * * * * * * * * * * * *$

$\mathrm{C} * * * * * * * * * * * * * * * * * * * * * * * * * * * * * * * * * * * * * * * * * * * * * * * * * * * * *$

$\mathrm{MP}, \mathrm{EX}, 1,0.3 \mathrm{e} 11$

$M P, P R X Y, 1,0.3$

MP,DENS,1,2400

$\mathrm{MP}, \mathrm{EX}, 2,0.3 \mathrm{e} 11$

$M P, P R X Y, 2,0.3$

MP,DENS,2,2679.2765

$M P, E X, 3,0.3 e 11$

$M P, P R X Y, 3,0.3$

MP,DENS,3,2924.304

C $* * * * * * * * * * * * * * * * * * * * * * * * * * * * * * * * * * * * * * * * * * * * * * * * * * * * *$

C*** Generate Nodes $* * * * *$

C*** this case with fill command used for those $* * * * * * *$

C*** in between $* * * * * * * * * * * * * * * * * * * * * * * * * * * * * * * * * * * * * * *$

C*** NODE, X,Y,Z, THXY, THYZ, THZX ******

$\mathrm{C} * * * * * * * * * * * * * * * * * * * * * * * * * * * * * * * * * * * * * * * * * * * * * * * * * * * * *$

$\mathrm{n}, 1,0,0,0,0,0,0$

$\mathrm{n}, 2,1,0,0,0,0,0$ 


$$
\begin{aligned}
& \mathrm{n}, 3,2,0,0,0,0,0 \\
& \mathrm{n}, 4,3,0,0,0,0,0 \\
& \mathrm{n}, 5,4,0,0,0,0,0 \\
& \mathrm{n}, 6,5,0,0,0,0,0 \\
& \mathrm{n}, 7,6,0,0,0,0,0 \\
& \mathrm{n}, 8,7,0,0,0,0,0 \\
& \mathrm{n}, 9,8,0,0,0,0,0 \\
& \text { n, } 10,9,0,0,0,0,0 \\
& \mathrm{n}, 11,10,0,0,0,0,0 \\
& \mathrm{n}, 12,11,0,0,0,0,0 \\
& \mathrm{n}, 13,12,0,0,0,0,0 \\
& \mathrm{n}, 14,13,0,0,0,0,0 \\
& \mathrm{n}, 15,14,0,0,0,0,0 \\
& \mathrm{n}, 16,15,0,0,0,0,0 \\
& \mathrm{n}, 17,16,0,0,0,0,0 \\
& \mathrm{n}, 18,17,0,0,0,0,0 \\
& \mathrm{n}, 19,18,0,0,0,0,0 \\
& \mathrm{n}, 20,19,0,0,0,0,0 \\
& \mathrm{n}, 21,20,0,0,0,0,0 \\
& \mathrm{n}, 22,21,0,0,0,0,0 \\
& \mathrm{n}, 23,22,0,0,0,0,0 \\
& \mathrm{n}, 24,23,0,0,0,0,0 \\
& \mathrm{n}, 25,24,0,0,0,0,0 \\
& \mathrm{n}, 26,25,0,0,0,0,0 \\
& \mathrm{n}, 27,26,0,0,0,0,0 \\
& \mathrm{n}, 28,27,0,0,0,0,0 \\
& \text { n, 29,28,0,0,0,0,0 } \\
& \mathrm{n}, 30,29,0,0,0,0,0 \\
& \mathrm{n}, 31,30,0,0,0,0,0 \\
& \text { n, 32,31,0,0,0,0,0 } \\
& \mathrm{n}, 33,32,0,0,0,0,0 \\
& \mathrm{n}, 34,33,0,0,0,0,0 \\
& \mathrm{n}, 35,34,0,0,0,0,0 \\
& \mathrm{n}, 36,35,0,0,0,0,0 \\
& \mathrm{n}, 37,36,0,0,0,0,0 \\
& \text { n, } 38,37,0,0,0,0,0 \\
& \mathrm{n}, 39,38,0,0,0,0,0 \\
& \mathrm{n}, 40,39,0,0,0,0,0 \\
& \mathrm{n}, 41,40,0,0,0,0,0 \\
& \mathrm{n}, 42,41,0,0,0,0,0 \\
& \mathrm{n}, 43,42,0,0,0,0,0 \\
& \mathrm{n}, 44,43,0,0,0,0,0 \\
& \mathrm{n}, 45,44,0,0,0,0,0 \\
& \mathrm{n}, 46,45,0,0,0,0,0 \\
& \mathrm{n}, 47,46,0,0,0,0,0
\end{aligned}
$$




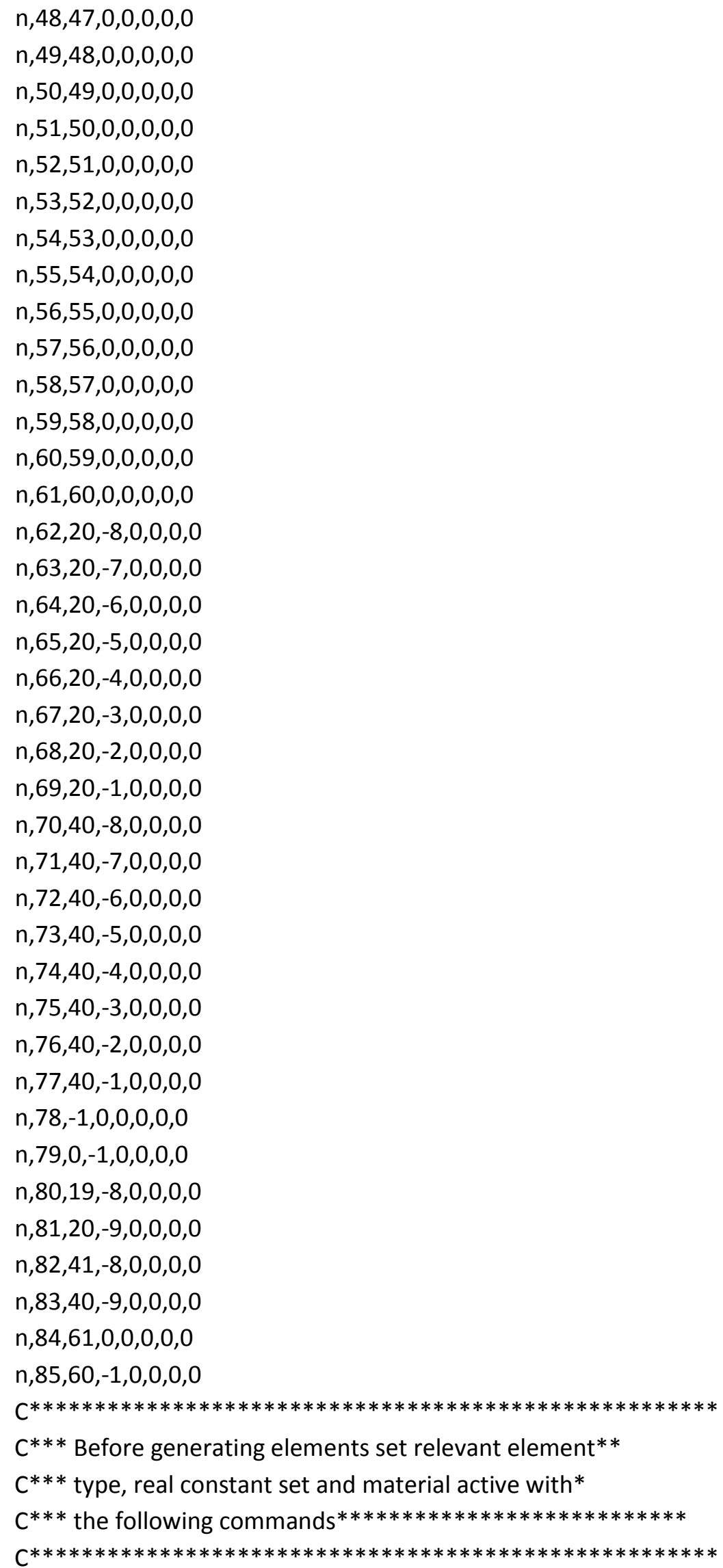

real,1 
mat,3

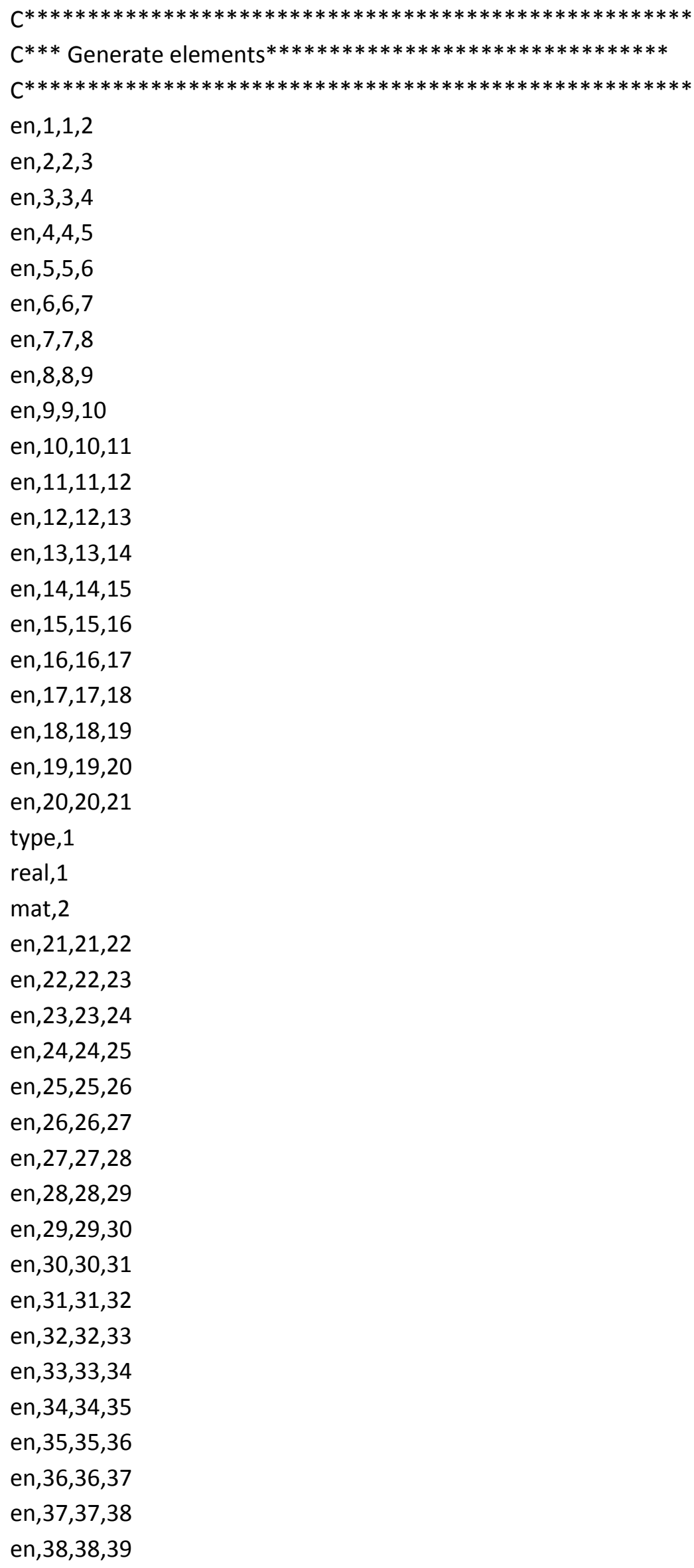




$$
\begin{aligned}
& \text { en, } 39,39,40 \\
& \text { en, } 40,40,41 \\
& \text { type, } 1 \\
& \text { real,1 } \\
& \text { mat, } 3 \\
& \text { en, } 41,41,42 \\
& \text { en, } 42,42,43 \\
& \text { en, } 43,43,44 \\
& \text { en, } 44,44,45 \\
& \text { en, } 45,45,46 \\
& \text { en, } 46,46,47 \\
& \text { en, } 47,47,48 \\
& \text { en, } 48,48,49 \\
& \text { en, } 49,49,50 \\
& \text { en, } 50,50,51 \\
& \text { en, } 51,51,52 \\
& \text { en, } 52,52,53 \\
& \text { en, } 53,53,54 \\
& \text { en, } 54,54,55 \\
& \text { en, } 55,55,56 \\
& \text { en, } 56,56,57 \\
& \text { en, } 57,57,58 \\
& \text { en, } 58,58,59 \\
& \text { en, } 59,59,60 \\
& \text { en, } 60,60,61 \\
& \text { type, } 1 \\
& \text { real,2 } \\
& \text { mat,1 } \\
& \text { en, } 61,21,69 \\
& \text { en, } 69,41,77 \\
& \text { type, } 1 \\
& \text { real,3 } \\
& \text { mat,1 } \\
& \text { en, } 62,62,63 \\
& \text { en, } 63,63,64 \\
& \text { en, } 64,64,65 \\
& \text { en, } 65,65,66 \\
& \text { en,66,66,67 } \\
& \text { en, } 67,67,68 \\
& \text { en, } 68,68,69 \\
& \text { en, } 70,70,71 \\
& \text { en, } 71,71,72 \\
& \text { en, } 72,72,73 \\
& \text { en, } 73,73,74 \\
& \text { en, } 74,74,75
\end{aligned}
$$


en, $75,75,76$

en, $76,76,77$

type, 2

real,4

mat, 1

en, $77,1,78$

en, $78,1,79$

en, $83,61,84$

en, $84,61,85$

type, 2

real,5

mat,1

en, $79,62,80$

en, $80,62,81$

en, $81,70,82$

en, $82,70,83$

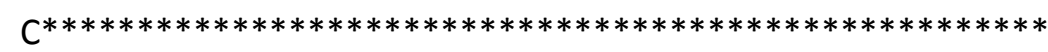

$\mathrm{C}^{* * *}$ Exit preprocessor $\mathrm{C}^{* * * * * * * * * * * * * * * * * * * * * * * * * * * * * * * * *}$

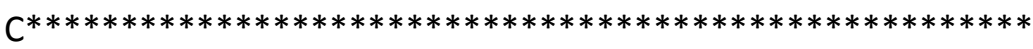

FINISH

$\mathrm{C}^{* * * * * * * * * * * * * * * * * * * * * * * * * * * * * * * * * * * * * * * * * * * * * * * * * * * * *}$

C ${ }^{* * *}$ Enter Solution Phase $\mathrm{C}^{* * * * * * * * * * * * * * * * * * * * * * * * * * * * * * * *}$

$\mathrm{C}^{* * * * * * * * * * * * * * * * * * * * * * * * * * * * * * * * * * * * * * * * * * * * * * * * * * * * *}$

/SOLU

C $* * * * * * * * * * * * * * * * * * * * * * * * * * * * * * * * * * * * * * * * * * * * * * * * * * * * *$

$\mathrm{C}^{* * *}$ Fix the ends of the beams by specifying**********

$\mathrm{C}^{* * *}$ zero displacement for all degrees of freedom $* * * * * *$

$\mathrm{C}^{* * *}$ i.e. UX $=\mathrm{UY}=\mathrm{ROTZ}=0 * * * * * * * * * * * * * * * * * * * * * * * * *$

$\mathrm{C}^{* * * * * * * * * * * * * * * * * * * * * * * * * * * * * * * * * * * * * * * * * * * * * * * * * * * * *}$

$\mathrm{D}, 78, \mathrm{UX}, 0,0,0$

$\mathrm{D}, 78, \mathrm{UY}, 0,0,0$

$\mathrm{D}, 78, \mathrm{ROTZ}, 0,0,0$

$\mathrm{D}, 79, \mathrm{UX}, 0,0,0$

$\mathrm{D}, 79, \mathrm{UY}, 0,0,0$

$\mathrm{D}, 79, \mathrm{ROTZ}, 0,0,0$

$\mathrm{D}, 80, \mathrm{UX}, 0,0,0$

$\mathrm{D}, 80, \mathrm{UY}, 0,0,0$

D, 80,ROTZ,0,0,0

$\mathrm{D}, 81, \mathrm{UX}, 0,0,0$

$\mathrm{D}, 81, \mathrm{UY}, 0,0,0$

D, 81, ROTZ,0,0,0

$\mathrm{D}, 82, \mathrm{UX}, 0,0,0$

$\mathrm{D}, 82, \mathrm{UY}, 0,0,0$

D, 82, ROTZ,0,0,0

$D, 83, U X, 0,0,0$ 

$D, 83, U Y, 0,0,0$
D, 83, ROTZ, 0,0,0
$\mathrm{D}, 84, \mathrm{UX}, 0,0,0$
$D, 84, U Y, 0,0,0$
$\mathrm{D}, 84, \mathrm{ROTZ}, 0,0,0$
$D, 85, U X, 0,0,0$
$D, 85, U Y, 0,0,0$
$\mathrm{D}, 85, \mathrm{ROTZ}, 0,0,0$
D,1,ROTZ,0,0,0
$\mathrm{D}, 61, \mathrm{ROTZ}, 0,0,0$
$\mathrm{D}, 62, \mathrm{ROTZ}, 0,0,0$
$\mathrm{D}, 70, \mathrm{ROTZ}, 0,0,0$

\section{APDL for Time History Analysis}

For Fixed Base Support

Case 1 (Same Time History)

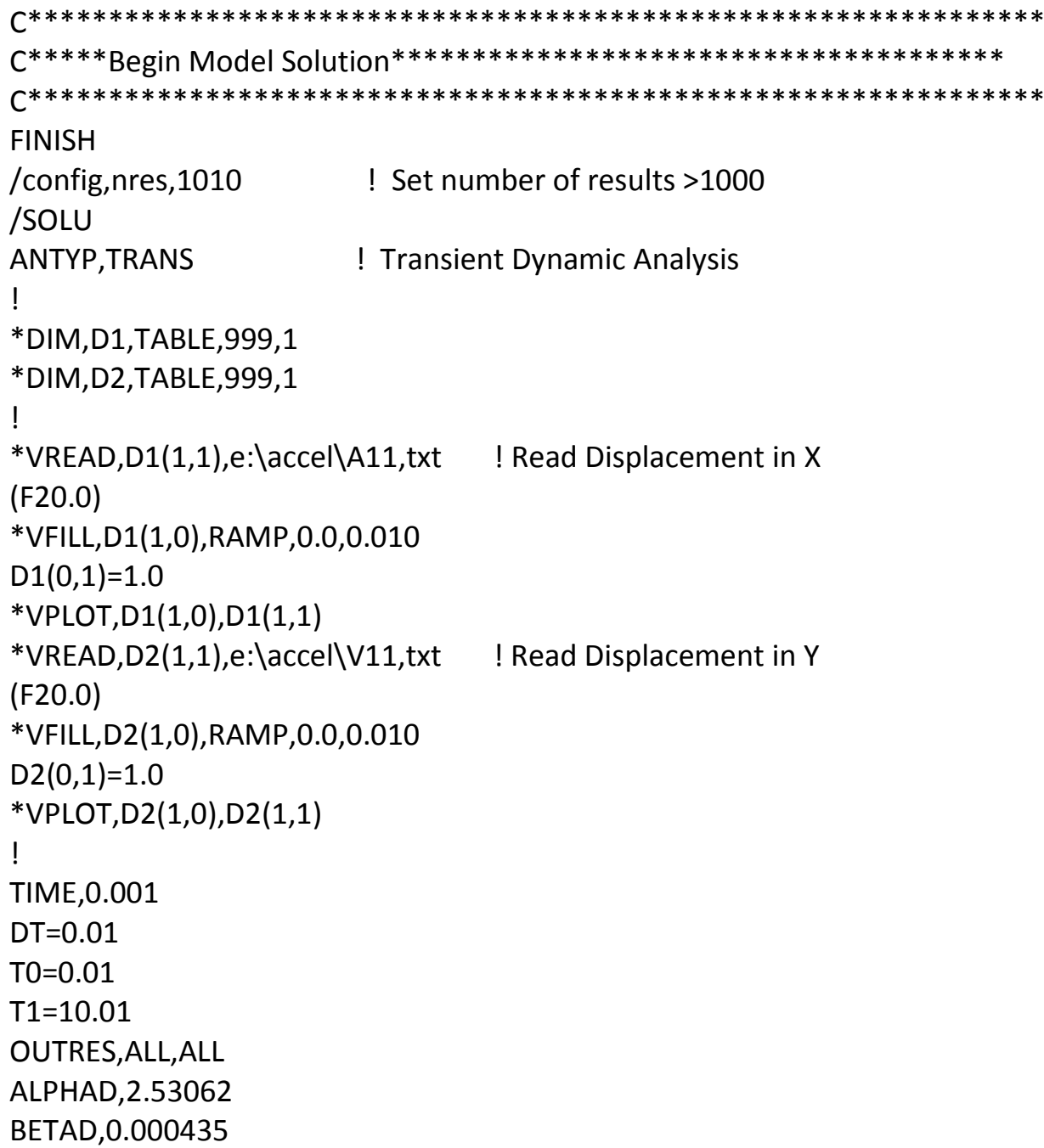




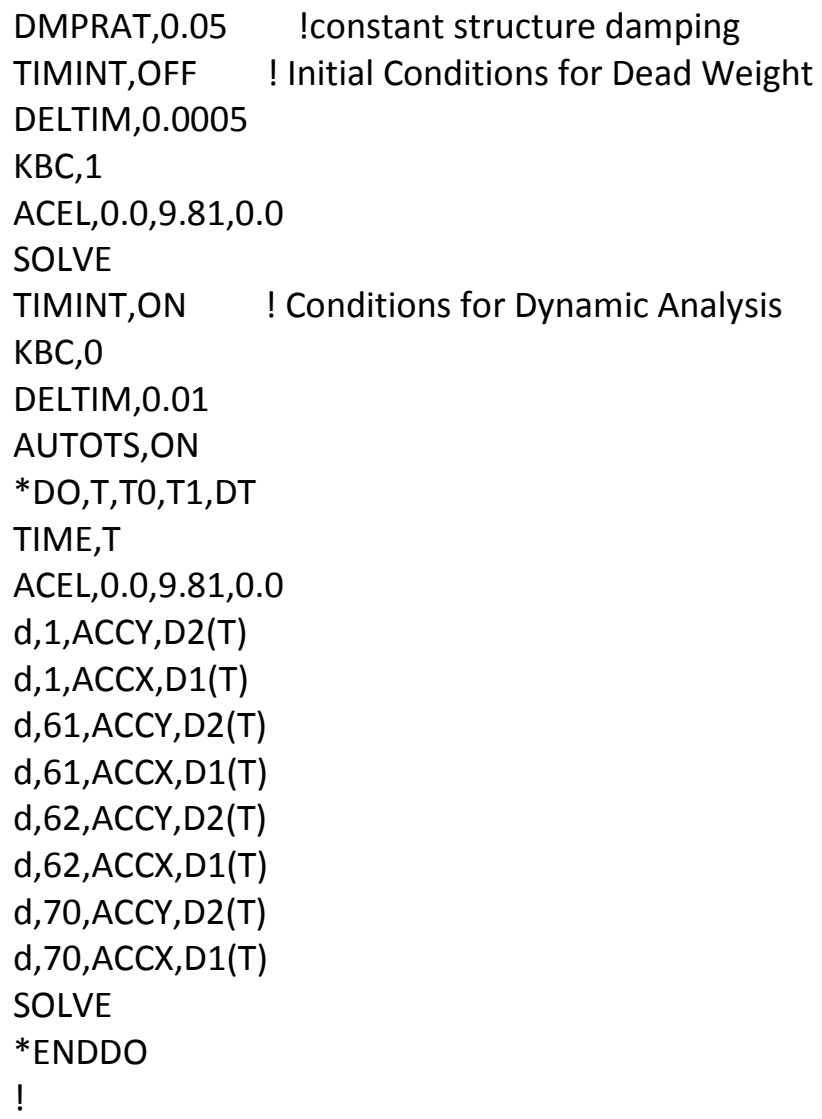

Case 2: Different Time Histories in X Direction

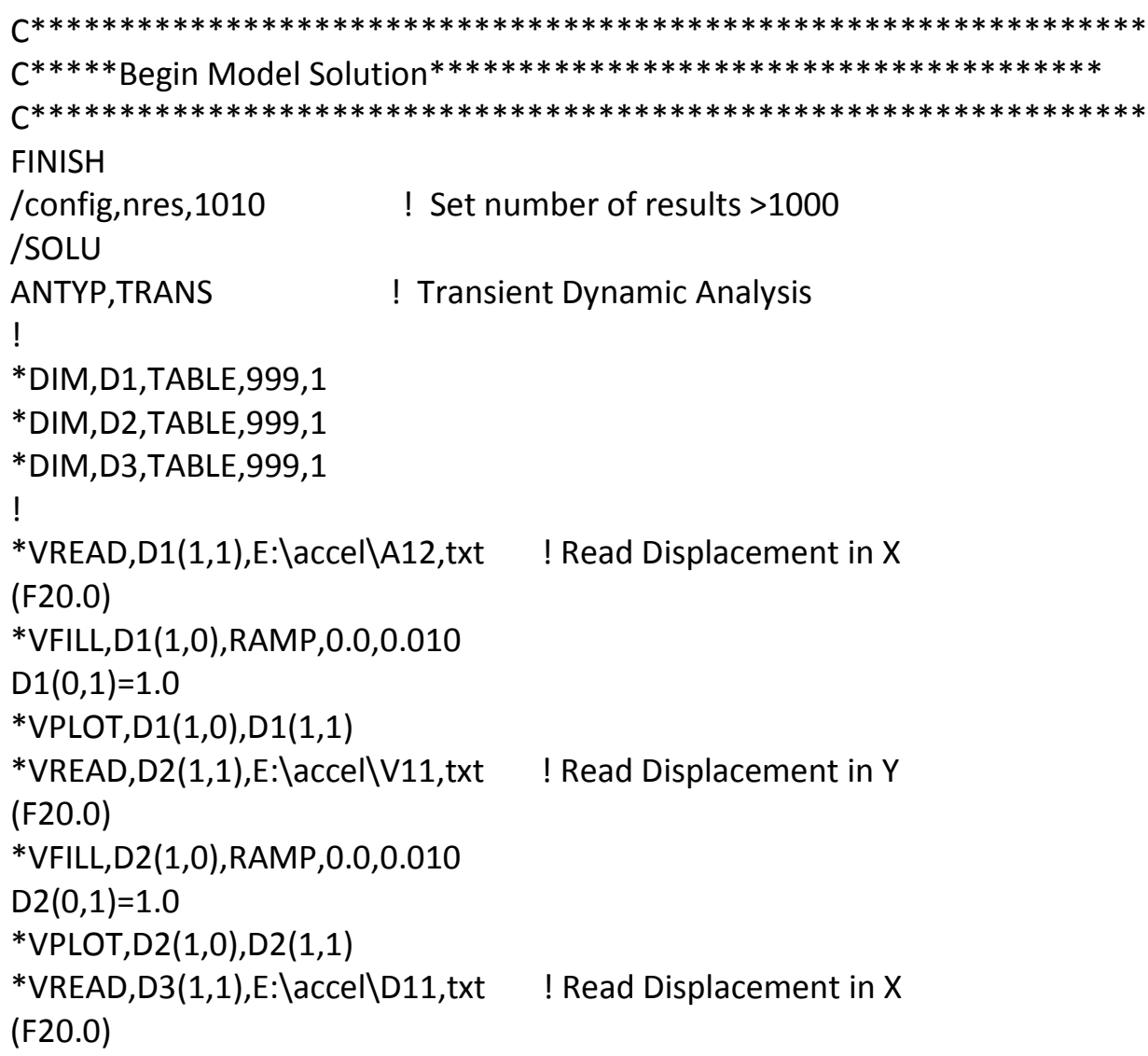




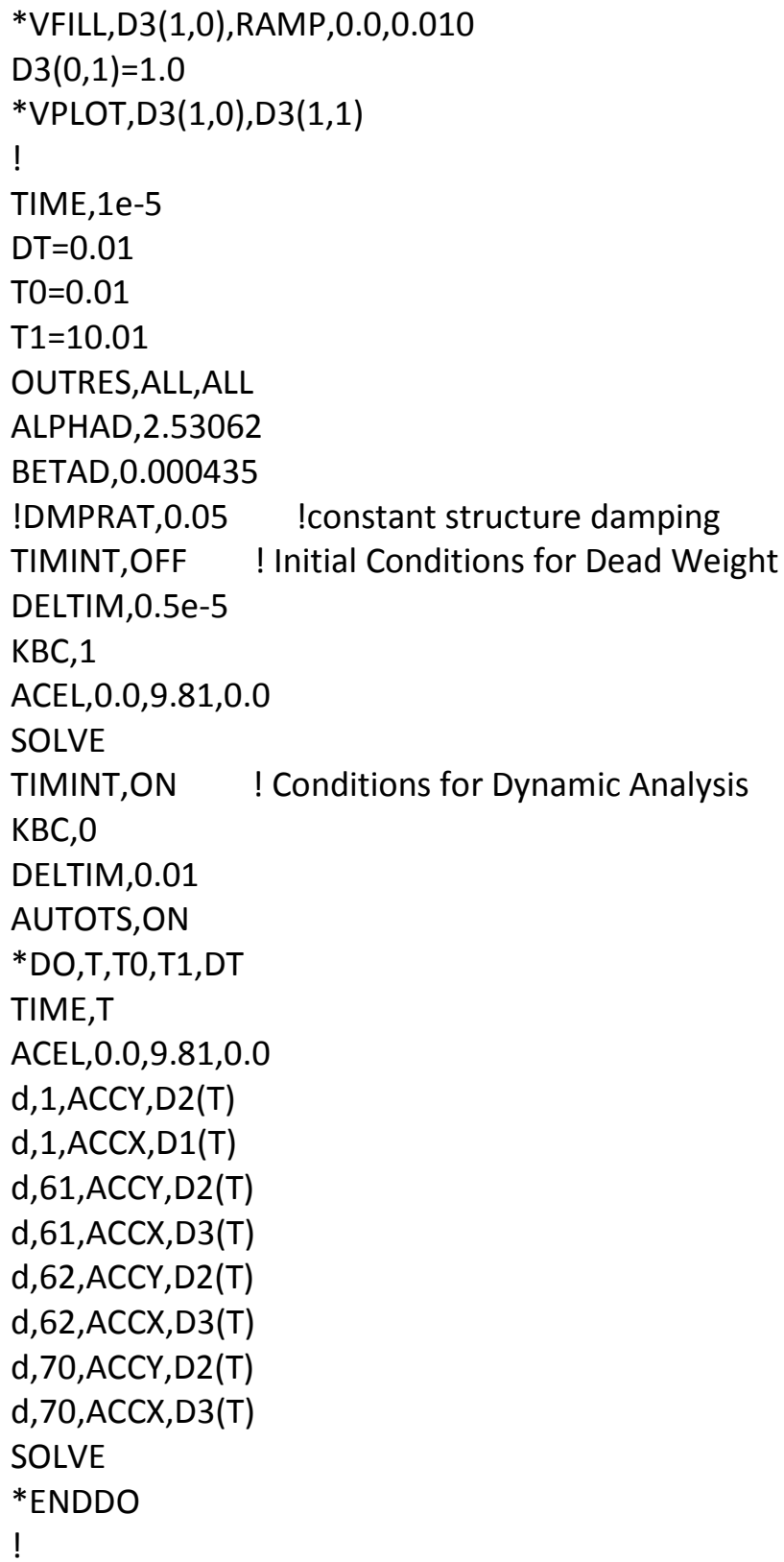

Case 3: Different Time Histories in Y Direction

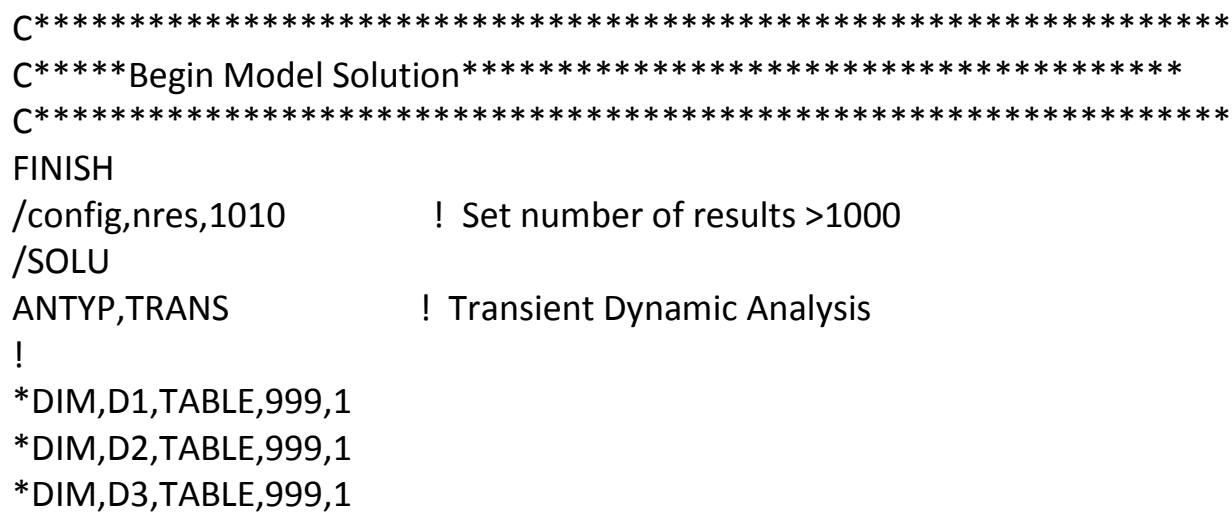




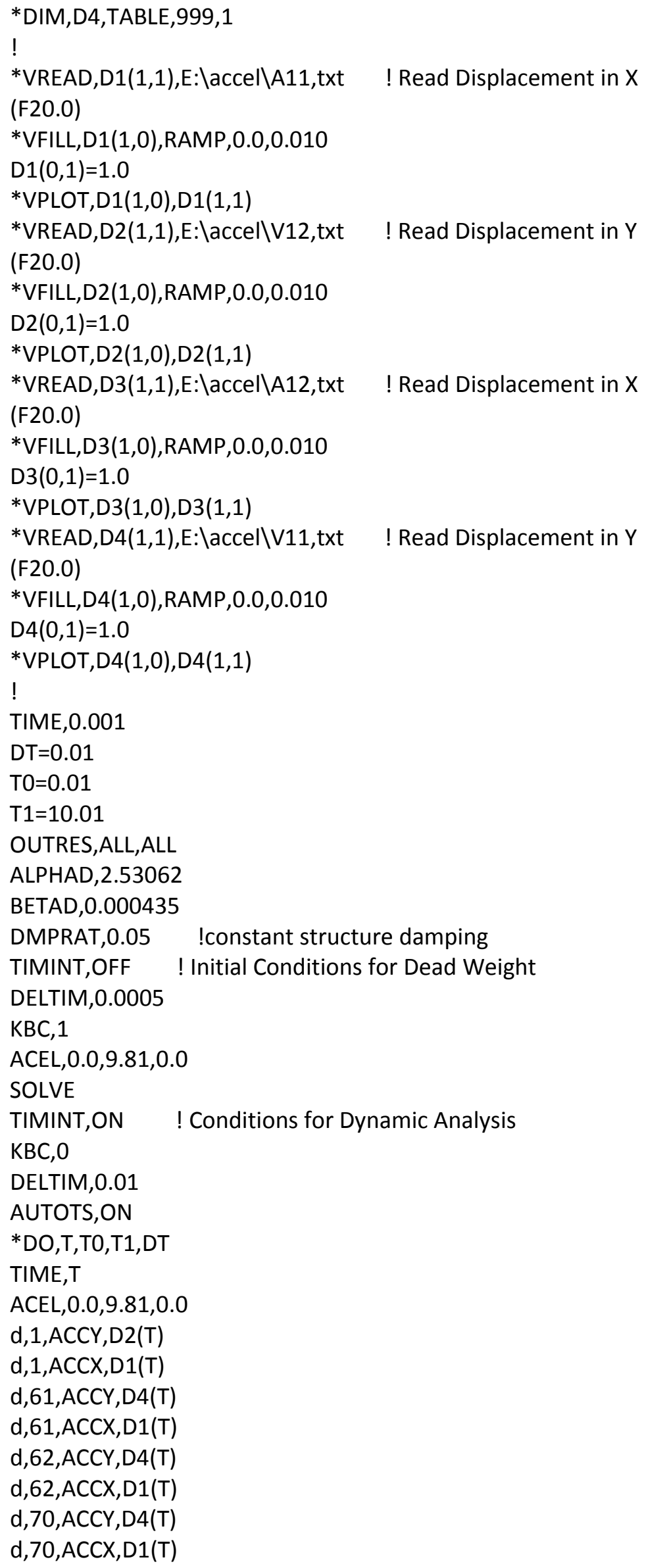


SOLVE

*ENDDO

!

For Spring Base Support Condition

Case 1: Same Time History

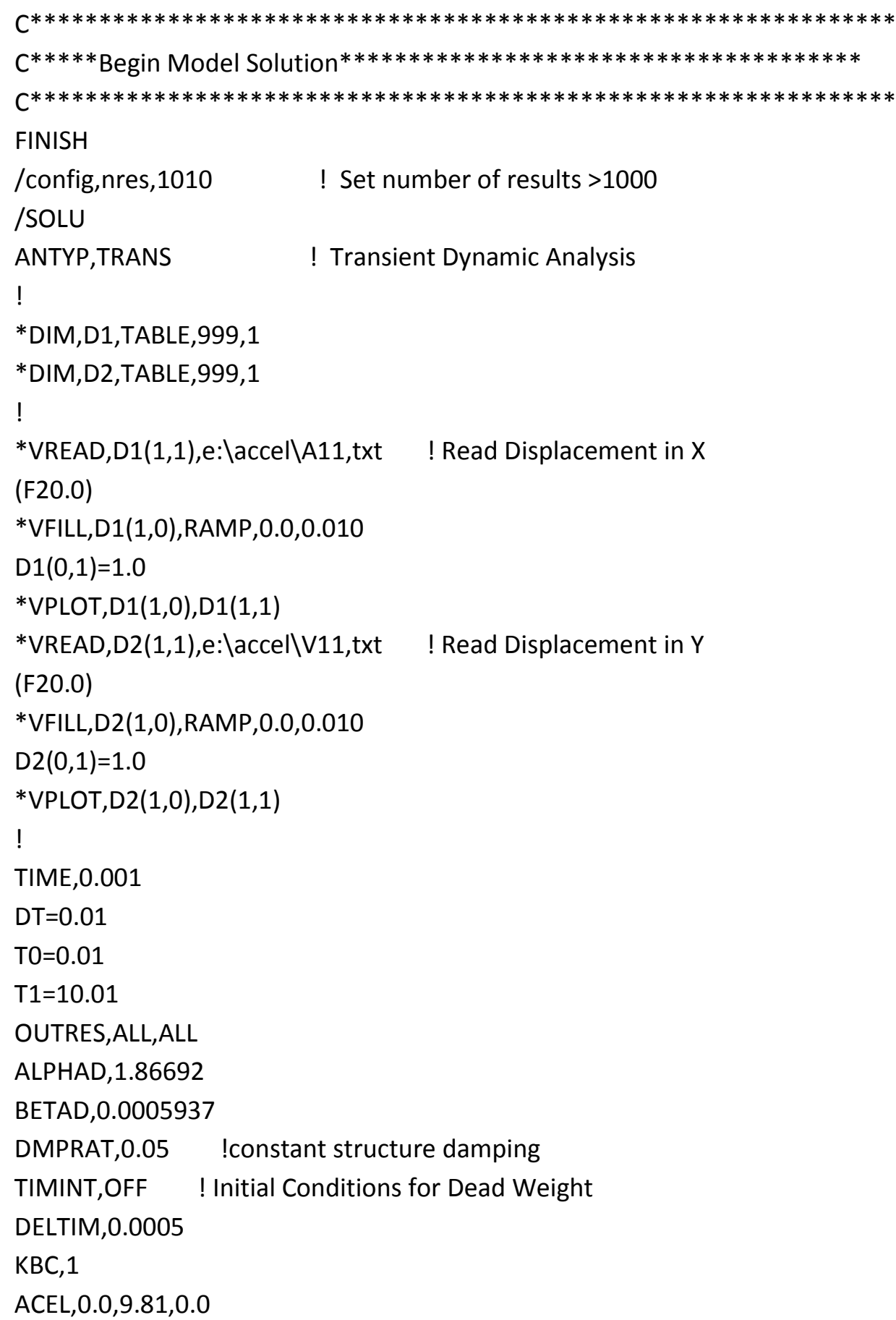




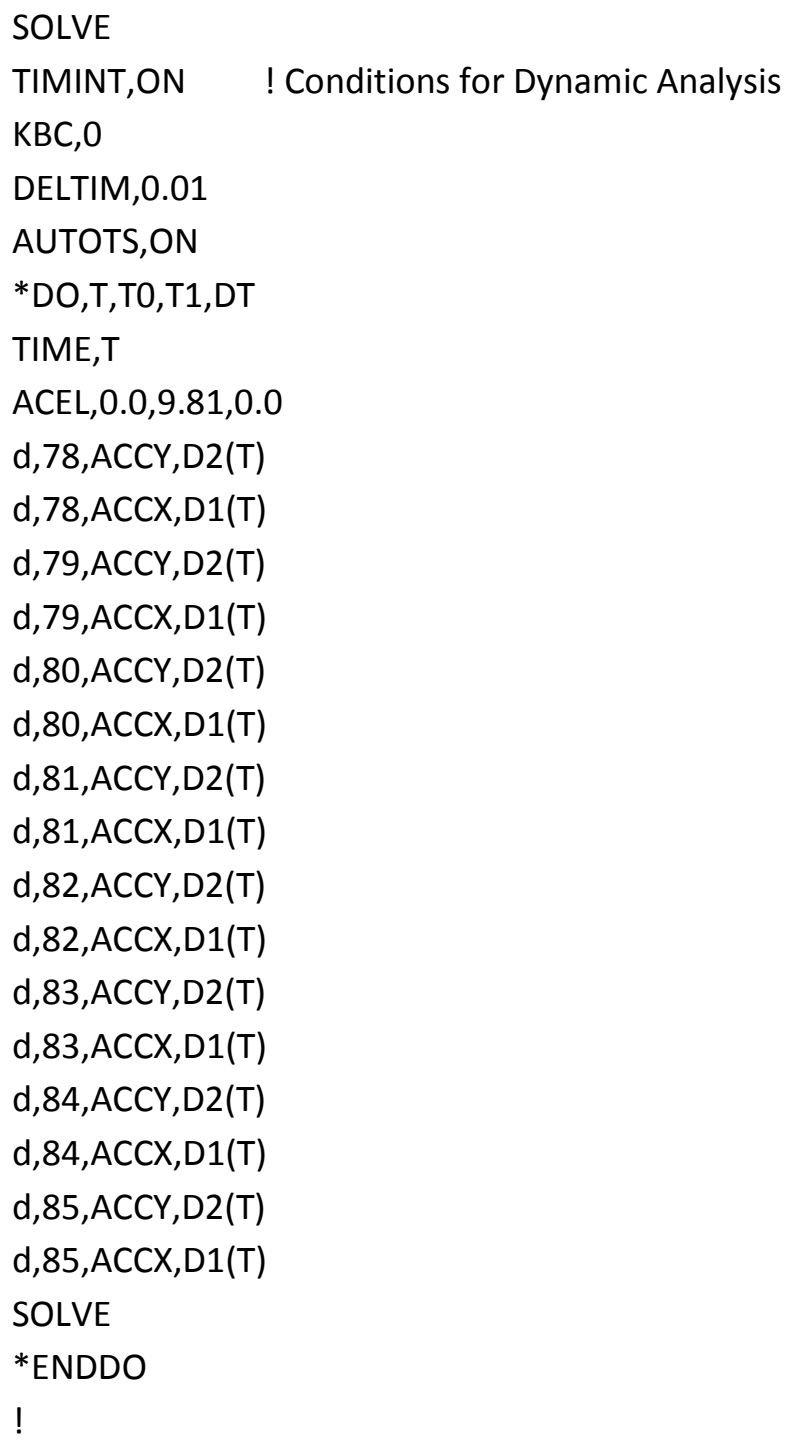

Case 2: Different Time Histories in X Direction

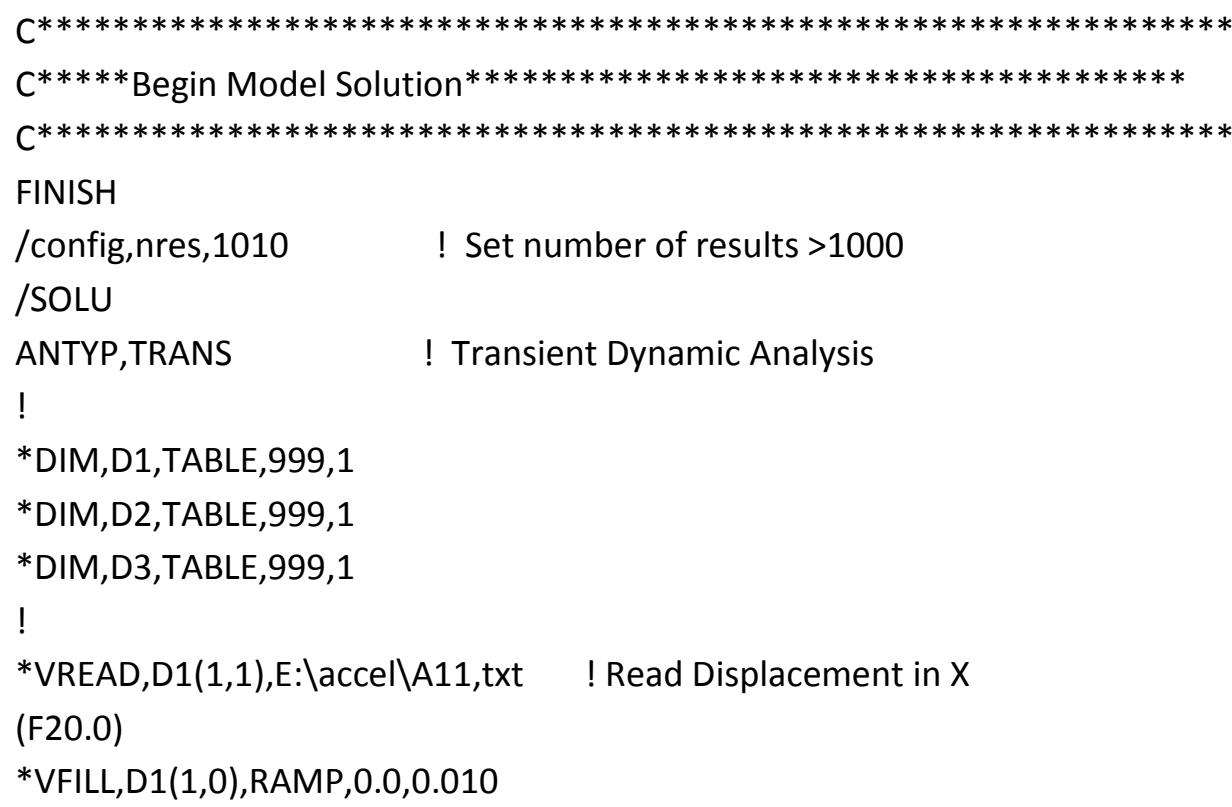


$\mathrm{D} 1(0,1)=1.0$

*VPLOT,D1(1,0),D1(1,1)

*VREAD,D2(1,1),E: \accel \V11,txt ! Read Displacement in Y

(F20.0)

*VFILL,D2(1,0),RAMP, 0.0,0.010

$\mathrm{D} 2(0,1)=1.0$

*VPLOT,D2(1,0),D2(1,1)

*VREAD,D3(1,1),E: \accel\A12,txt ! Read Displacement in X (F20.0)

*VFILL,D3(1,0),RAMP,0.0,0.010

$\mathrm{D} 3(0,1)=1.0$

*VPLOT,D3(1,0),D3(1,1)

!

TIME,1e-5

$\mathrm{DT}=0.01$

$\mathrm{T} 0=0.01$

$\mathrm{T} 1=10.01$

OUTRES,ALL,ALL

ALPHAD, 1.86692

BETAD, 0.0005937

!DMPRAT,0.05 !constant structure damping

TIMINT,OFF ! Initial Conditions for Dead Weight

DELTIM,0.5e-5

$\mathrm{KBC}, 1$

ACEL,0.0,9.81,0.0

SOLVE

TIMINT,ON ! Conditions for Dynamic Analysis

$\mathrm{KBC}, 0$

DELTIM,0.01

AUTOTS,ON

*DO,T,T0,T1,DT

TIME,T

ACEL,0.0,9.81,0.0

$\mathrm{d}, 78, \mathrm{ACCY}, \mathrm{D} 2(\mathrm{~T})$

d,78,ACCX,D1(T)

d,79,ACCY,D2(T)

d,79,ACCX,D1(T)

d,80,ACCY,D2(T)

d,80,ACCX,D3(T)

d,81,ACCY,D2(T)

d,81,ACCX,D3(T)

d, 82,ACCY,D2(T)

d,82,ACCX,D3(T)

d,83,ACCY,D2(T)

d,83,ACCX,D3(T) 


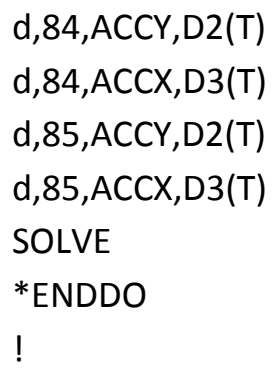

Case 3: Different Time Histories in Y Direction

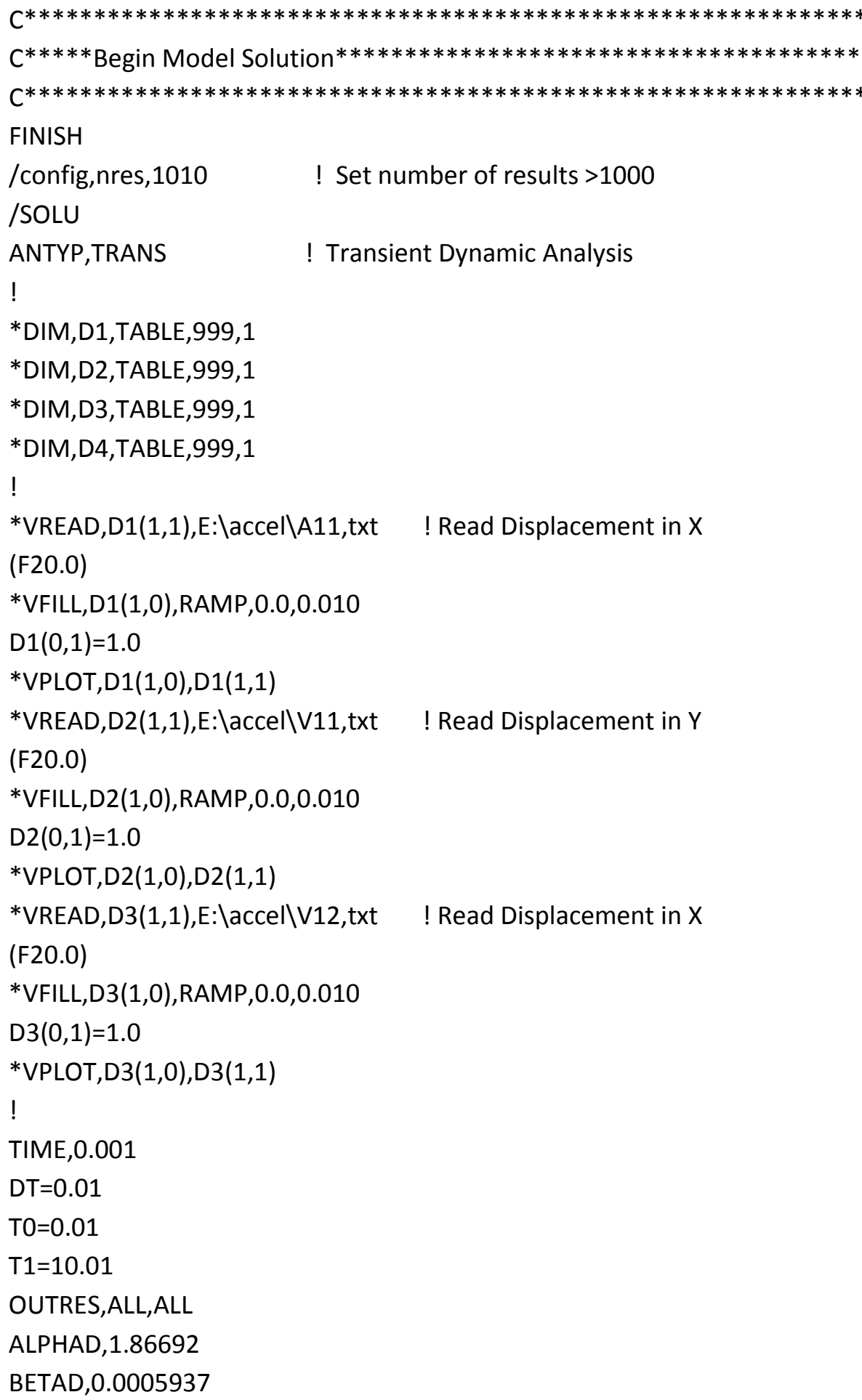




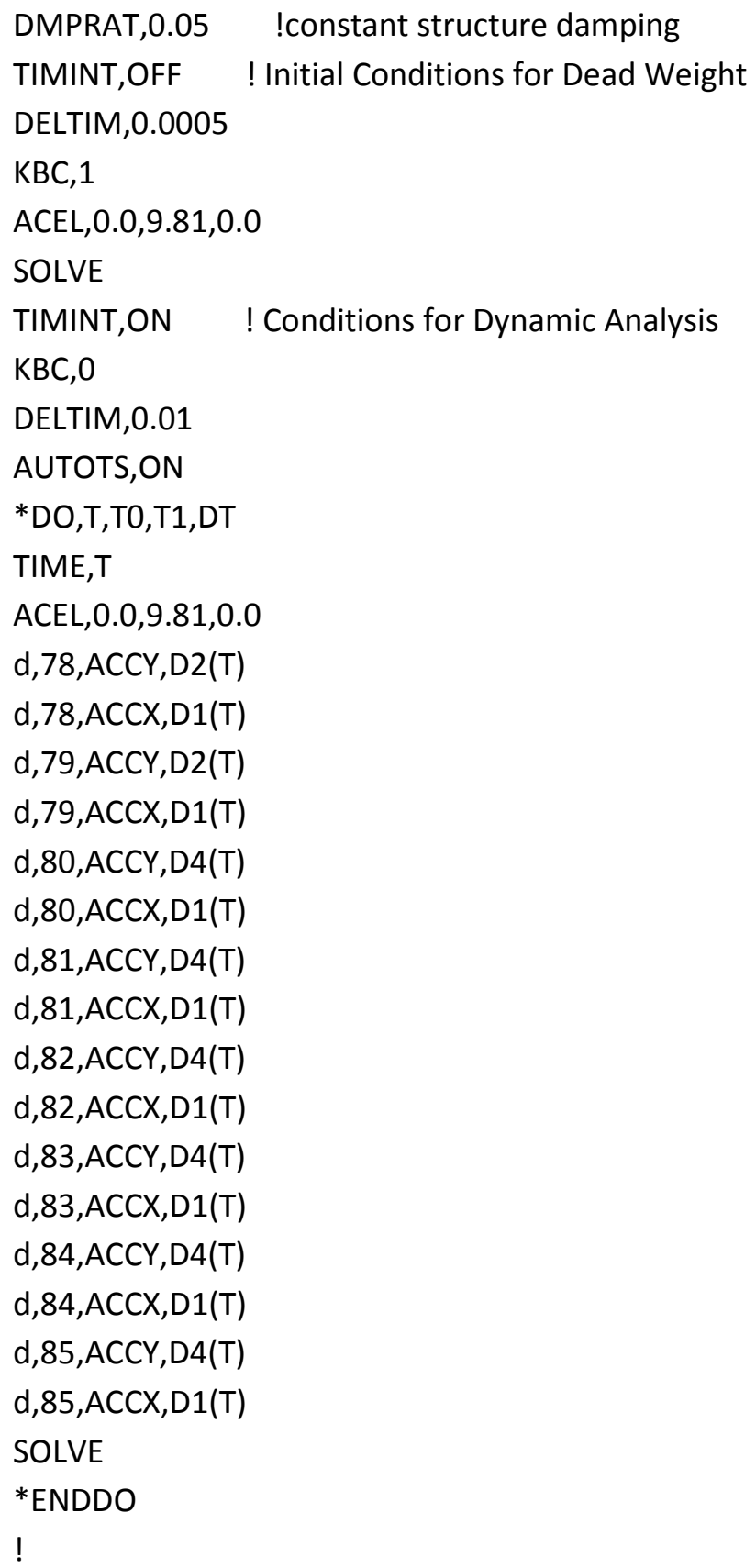




\section{VI.Synthetic Earthquake Properties}

For Horizontal earthquake properties

A. Soil type A Acceleration

a. acceleration

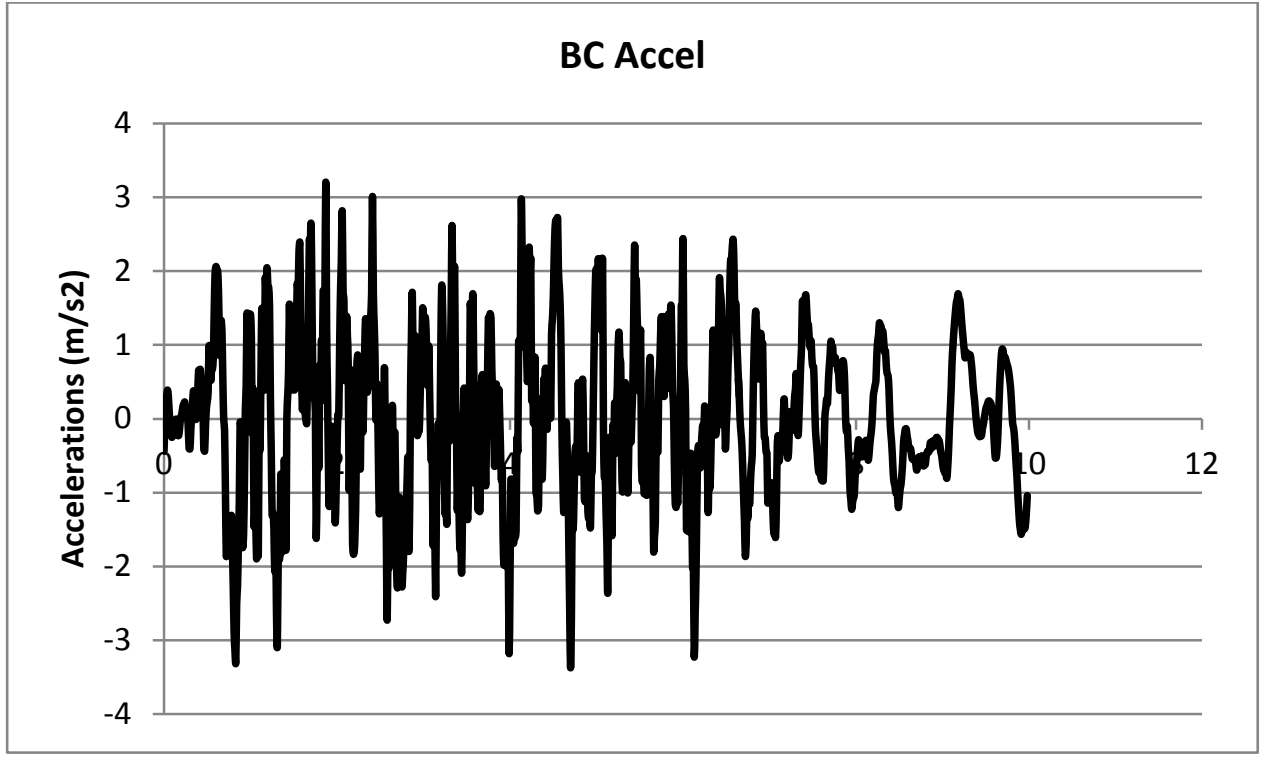

b. Velocity

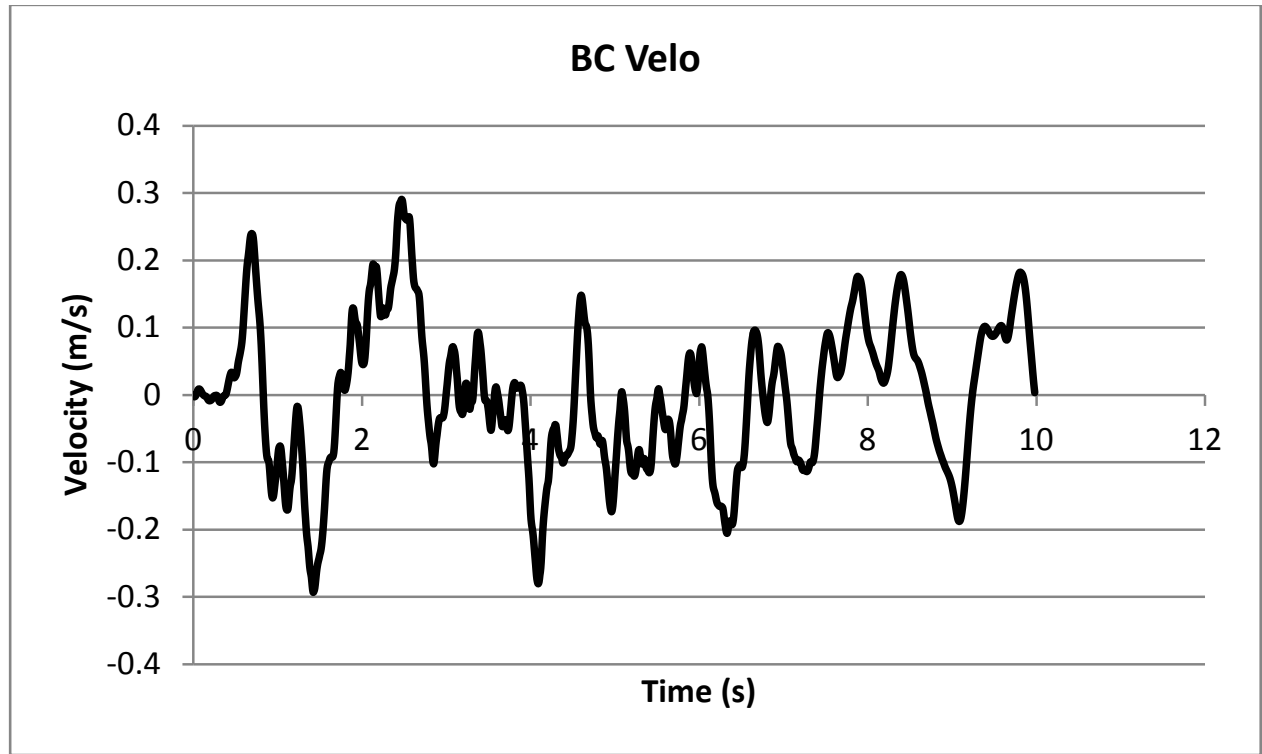


c. Displacement

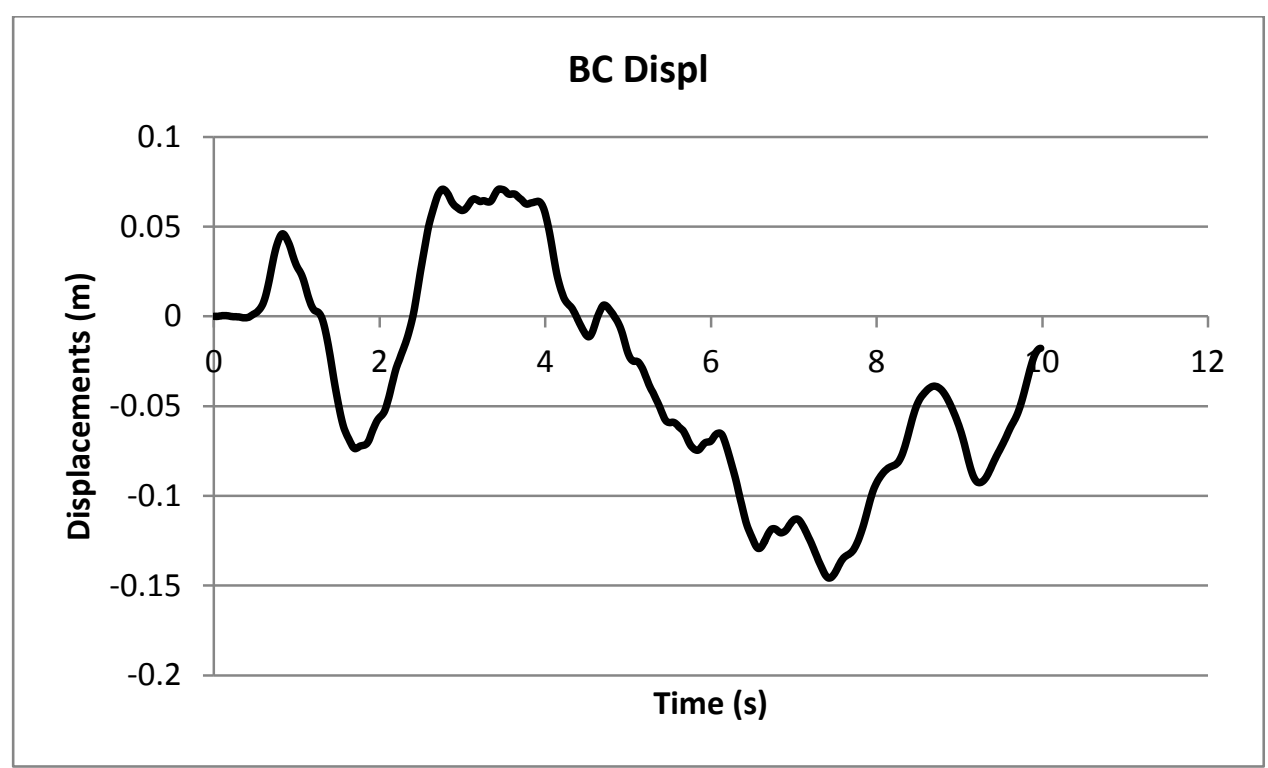

\section{B. Soil type B}

a. Acceleration

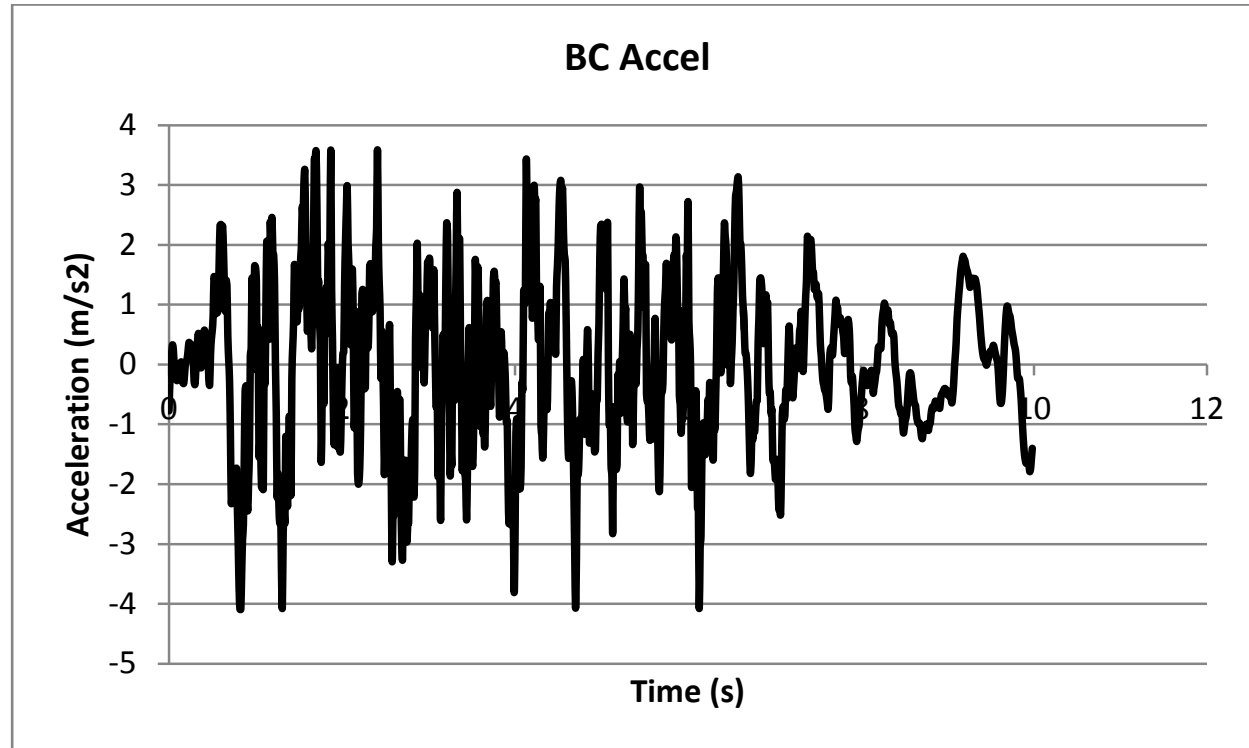


d. Velocity

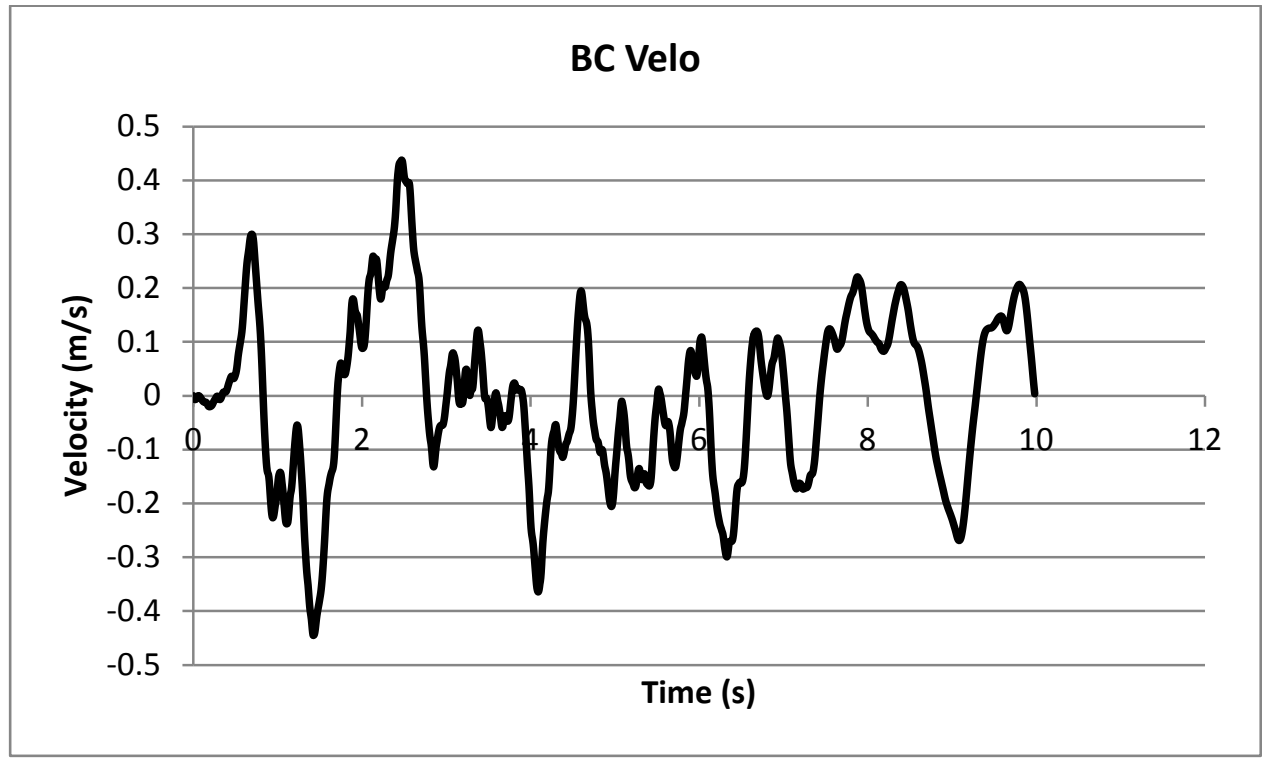

e. Displacement

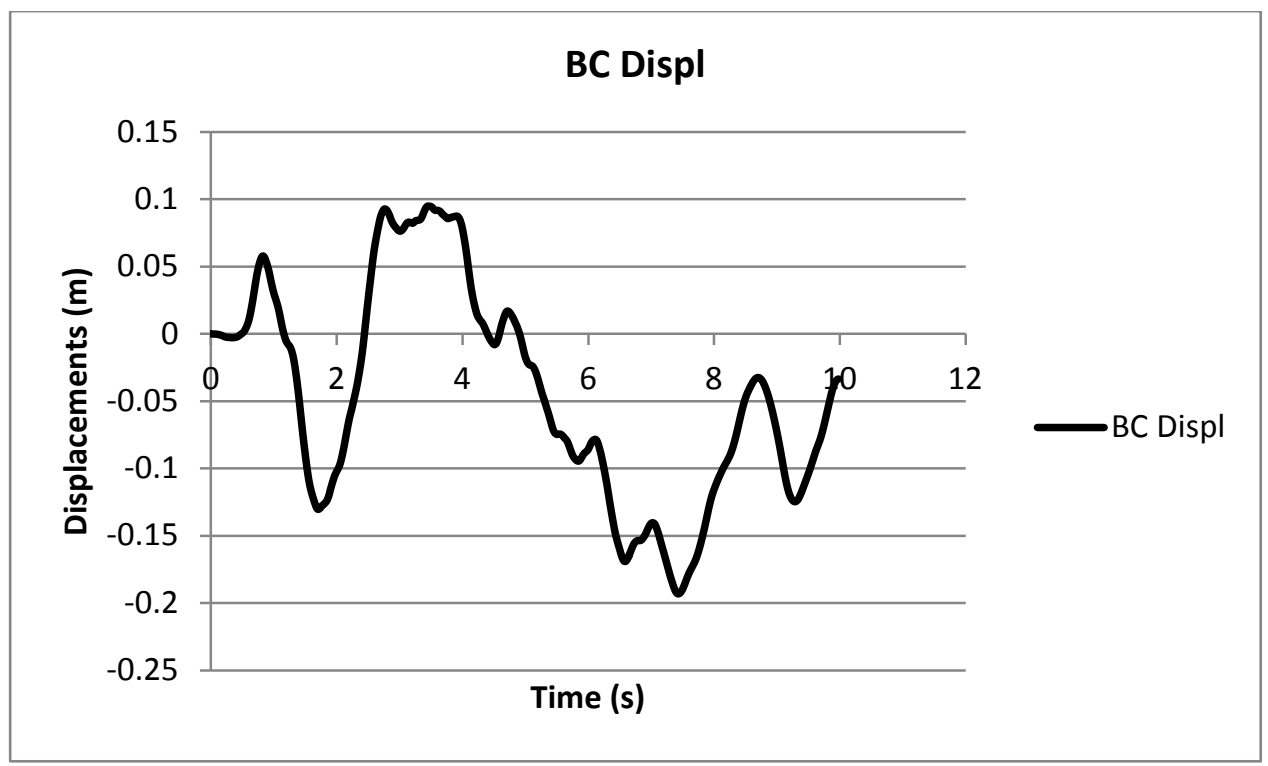




\section{Soil type C}

a. Acceleration

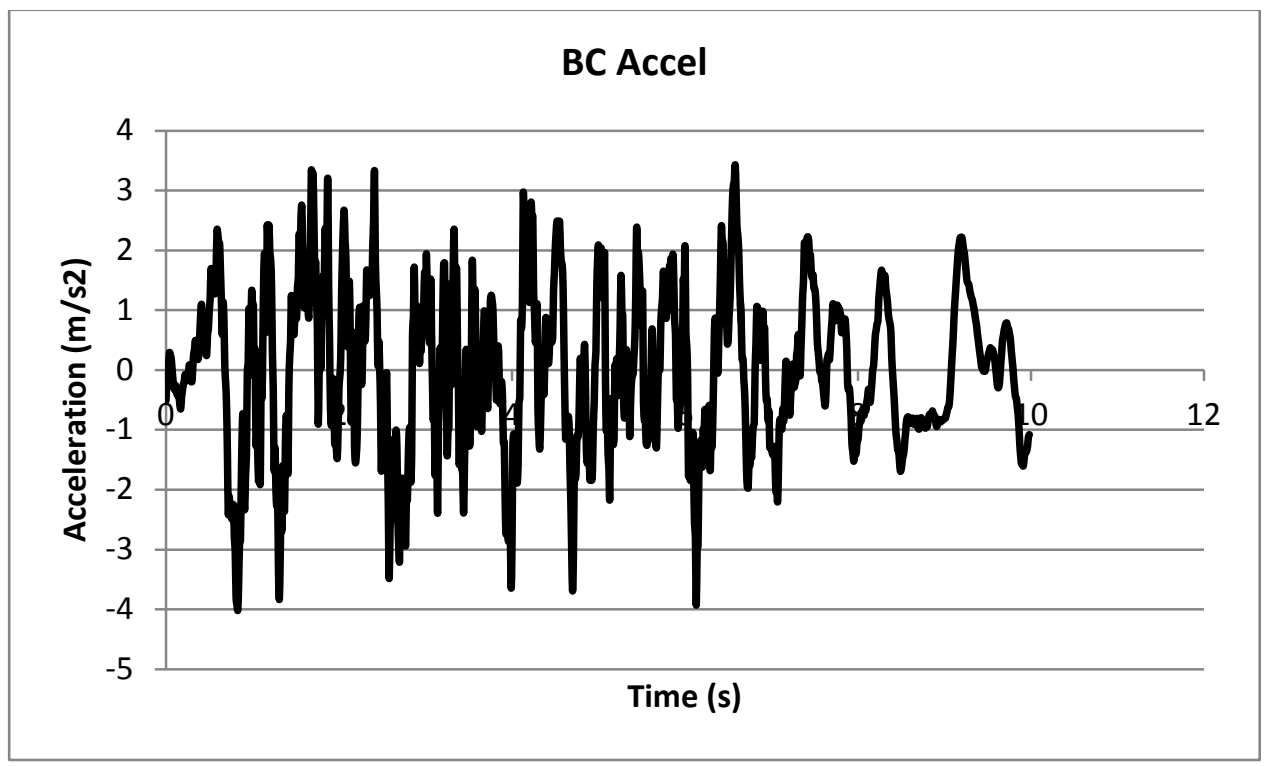

b. Velocity

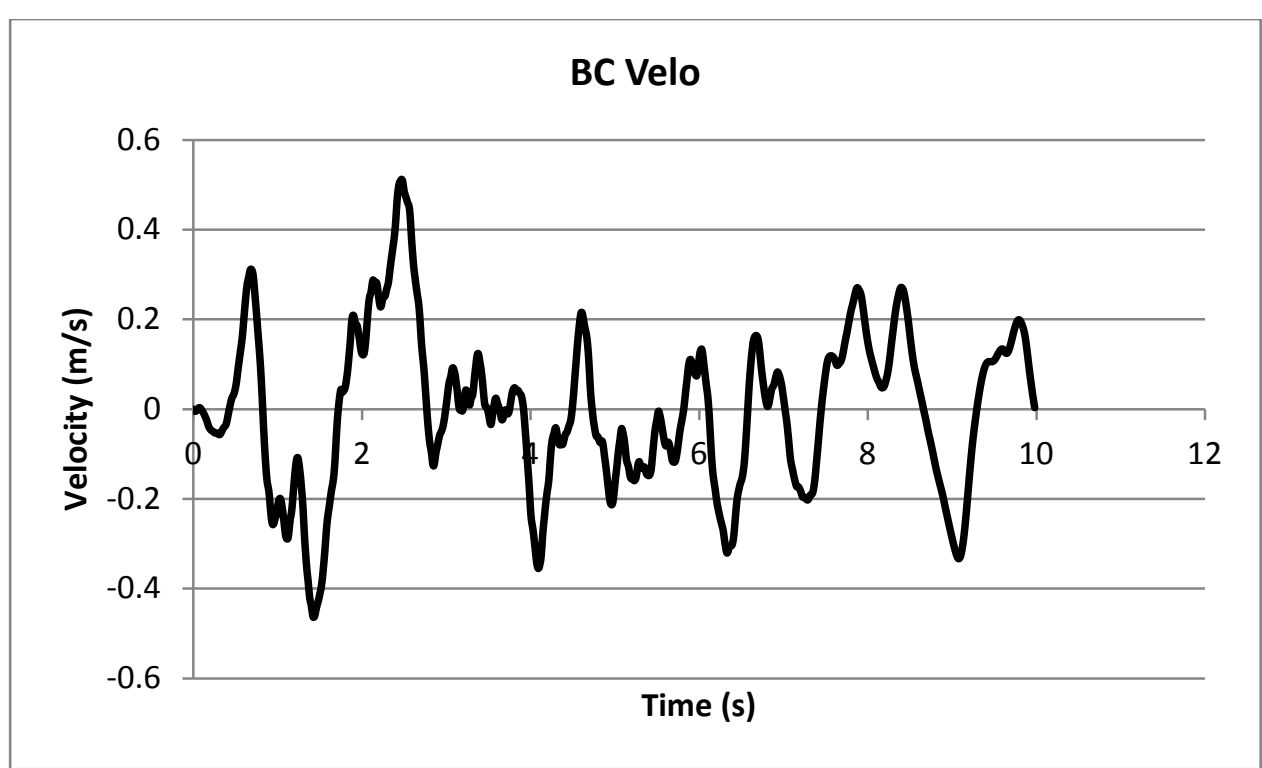


c. Displacement

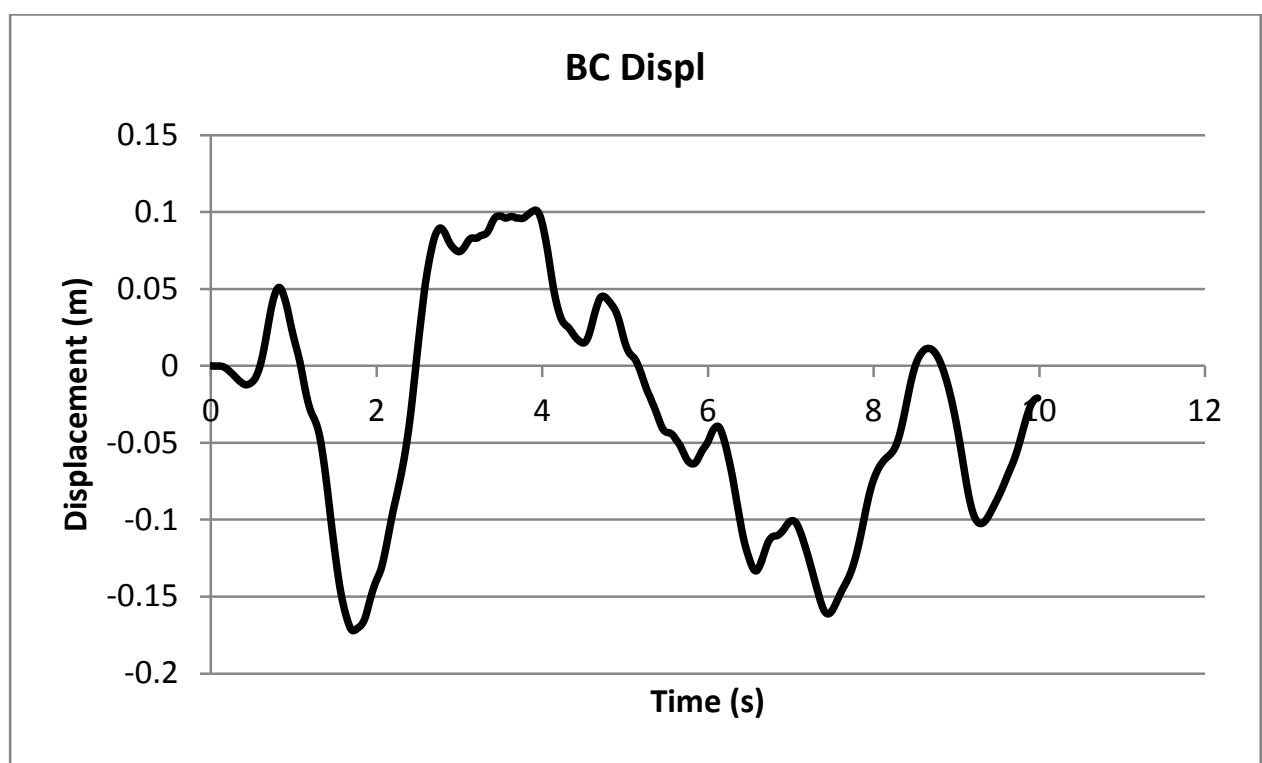

D. Soil type D

a. Acceleration

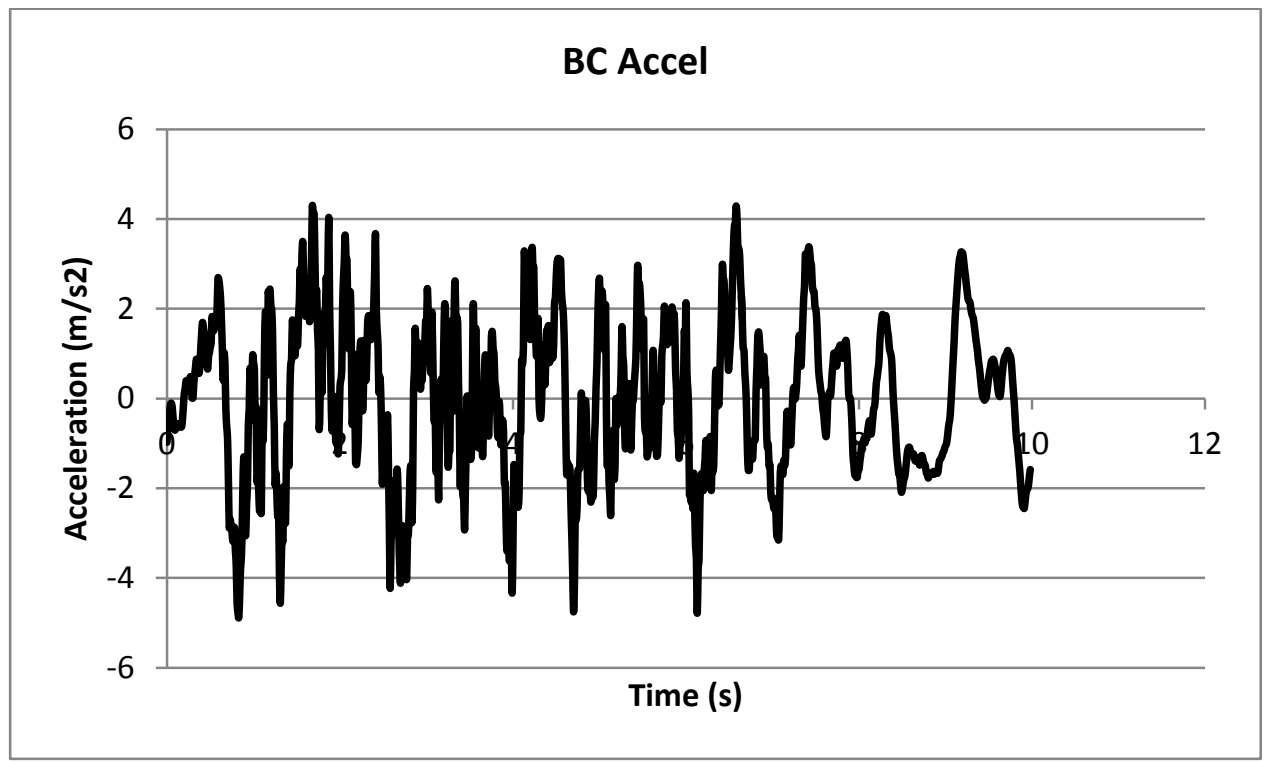


b. Velocity

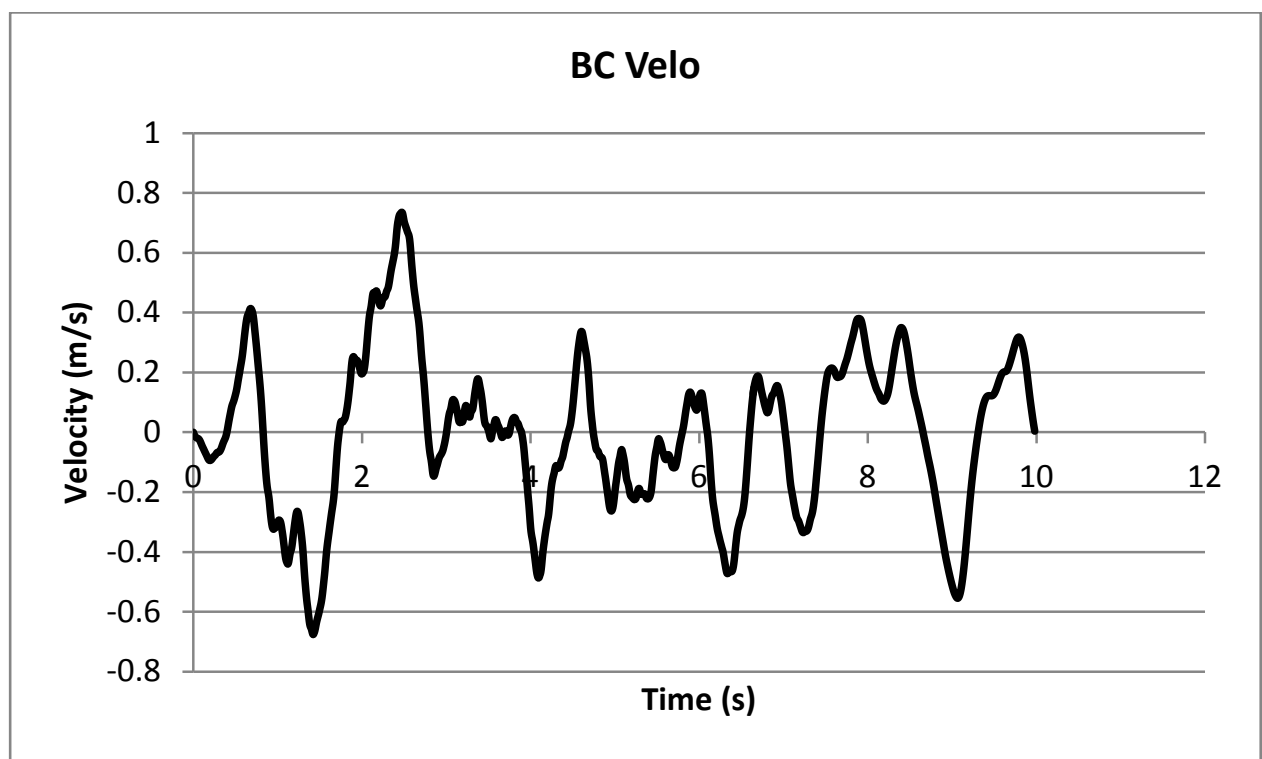

c. Displacement

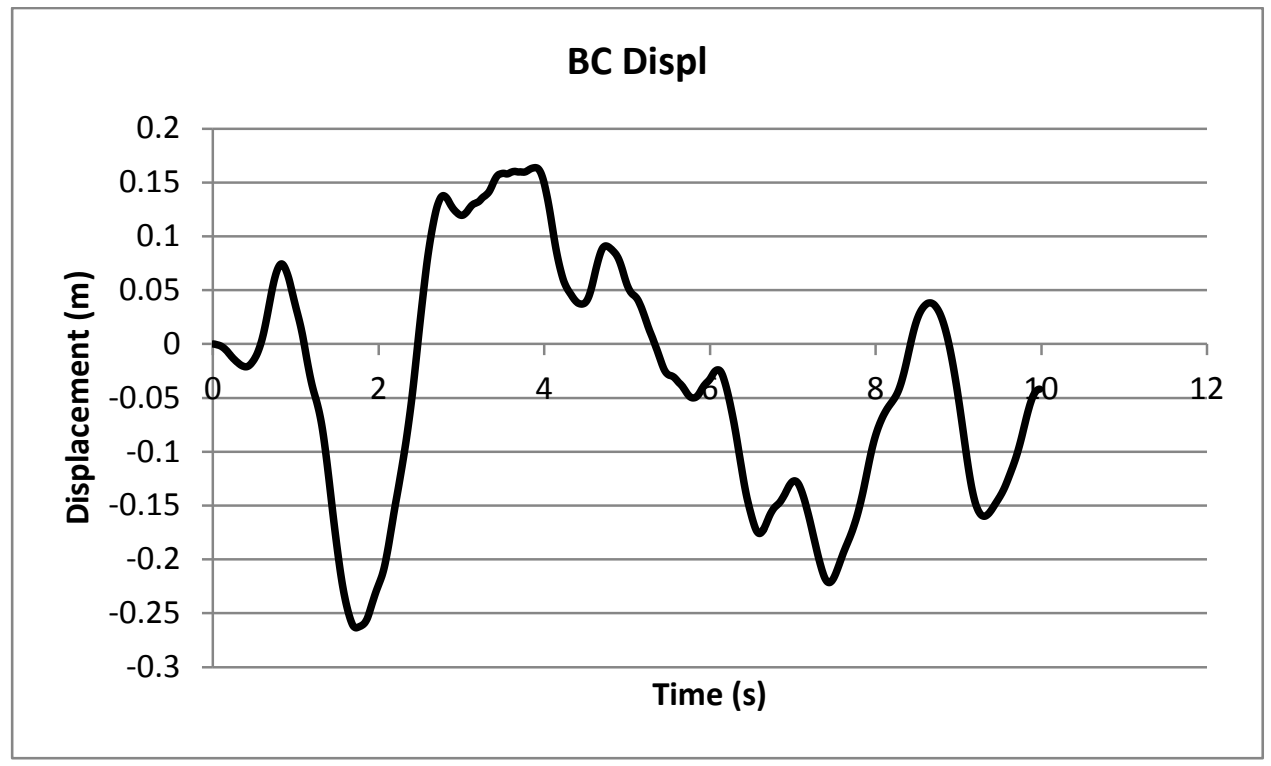




\section{E. Soil type E}

a. Acceleration

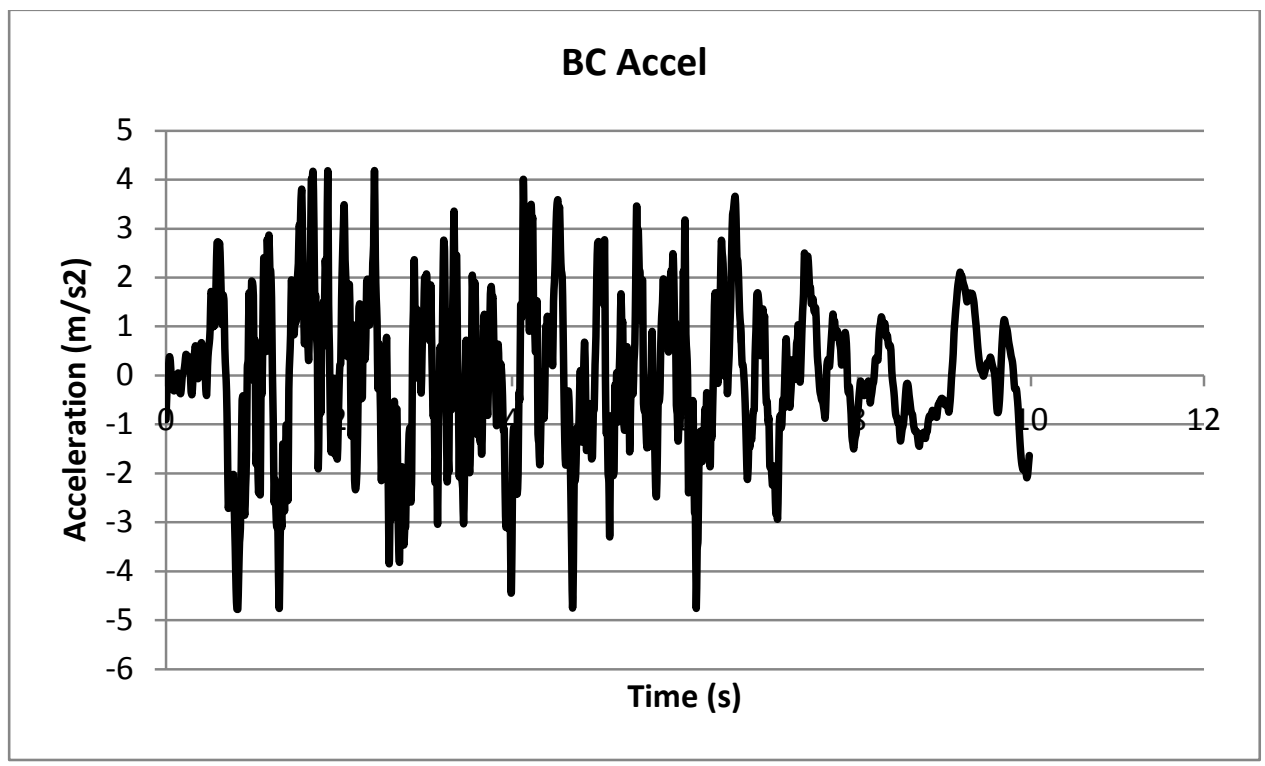

b. Velocity

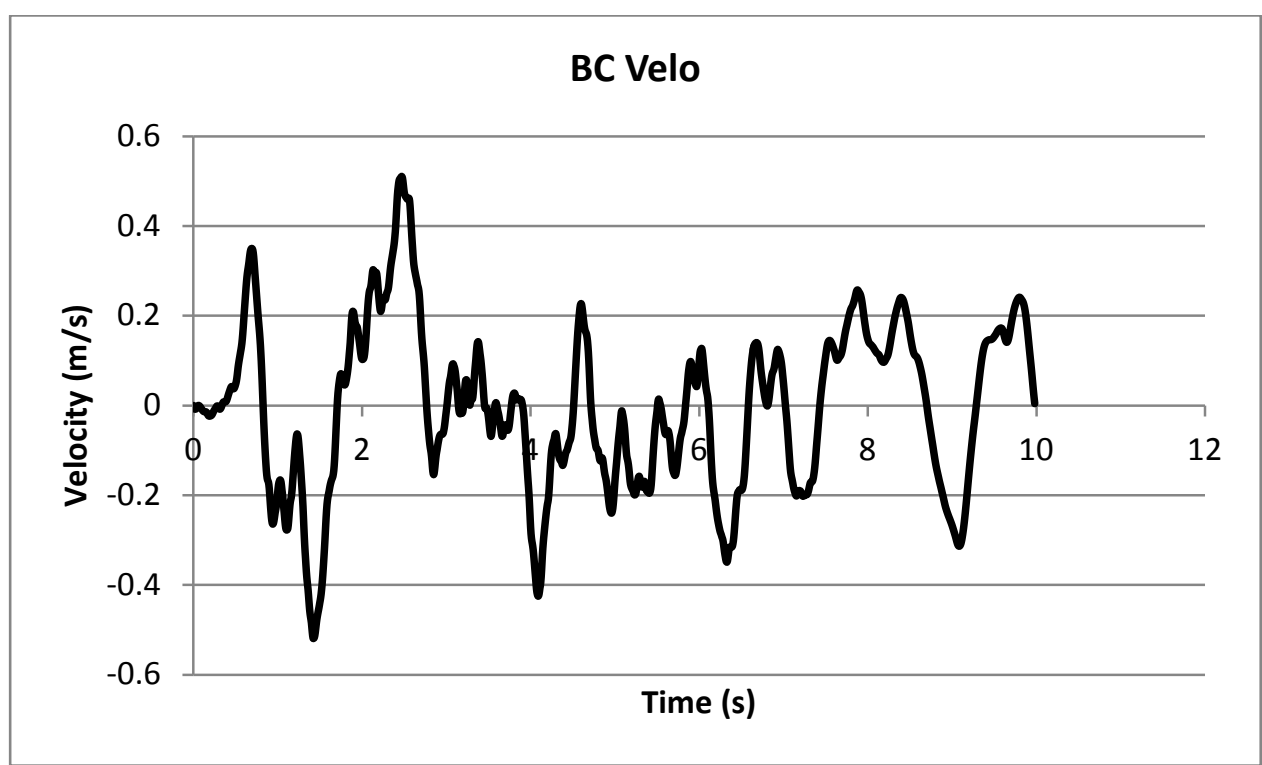


c. Displacement

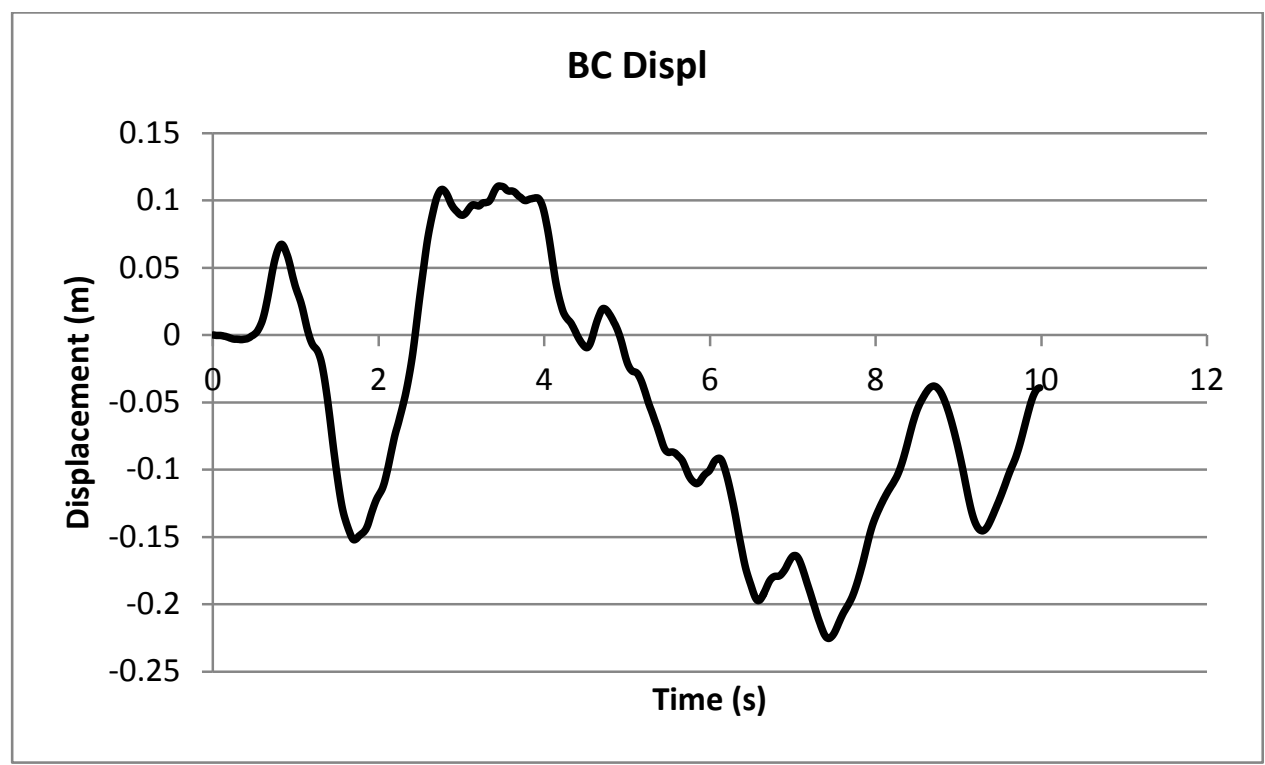

For vertical earthquake properties

The properties of earthquake for vertical actions are same for all types of the soil.

a. Acceleration

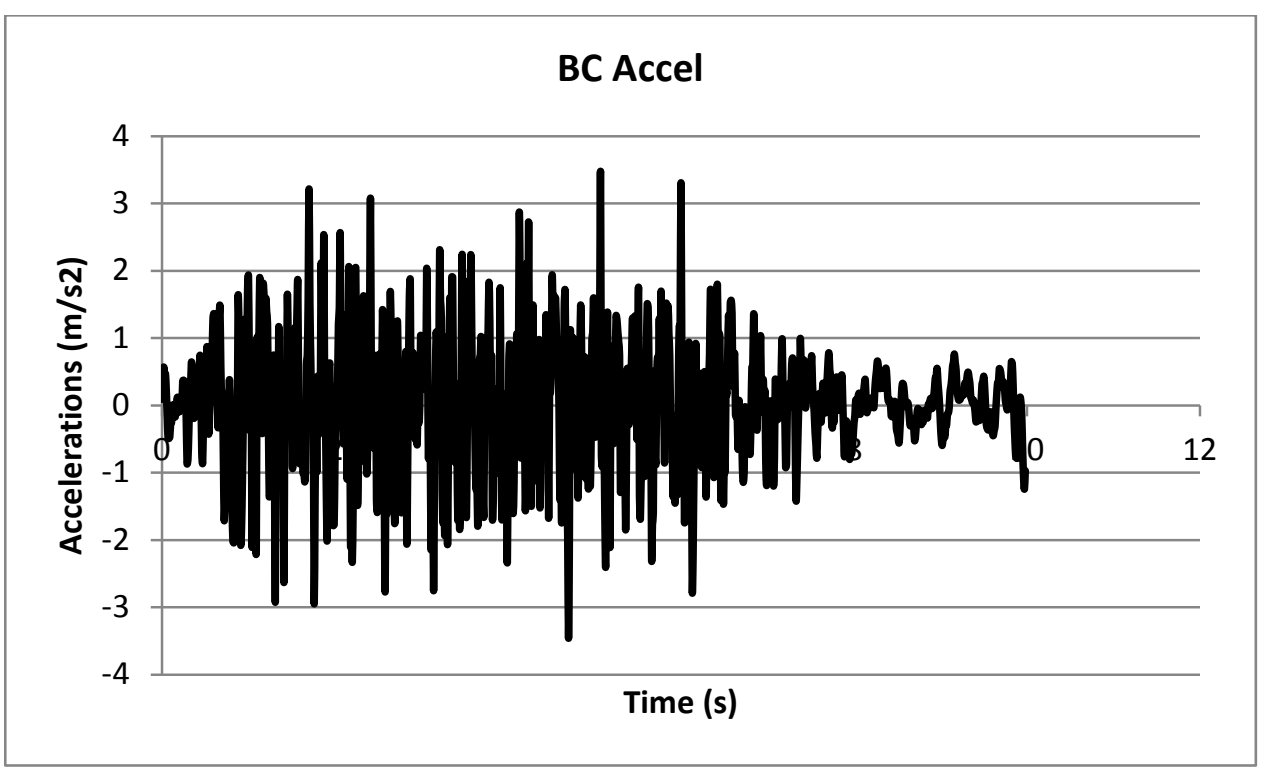


b. Velocity

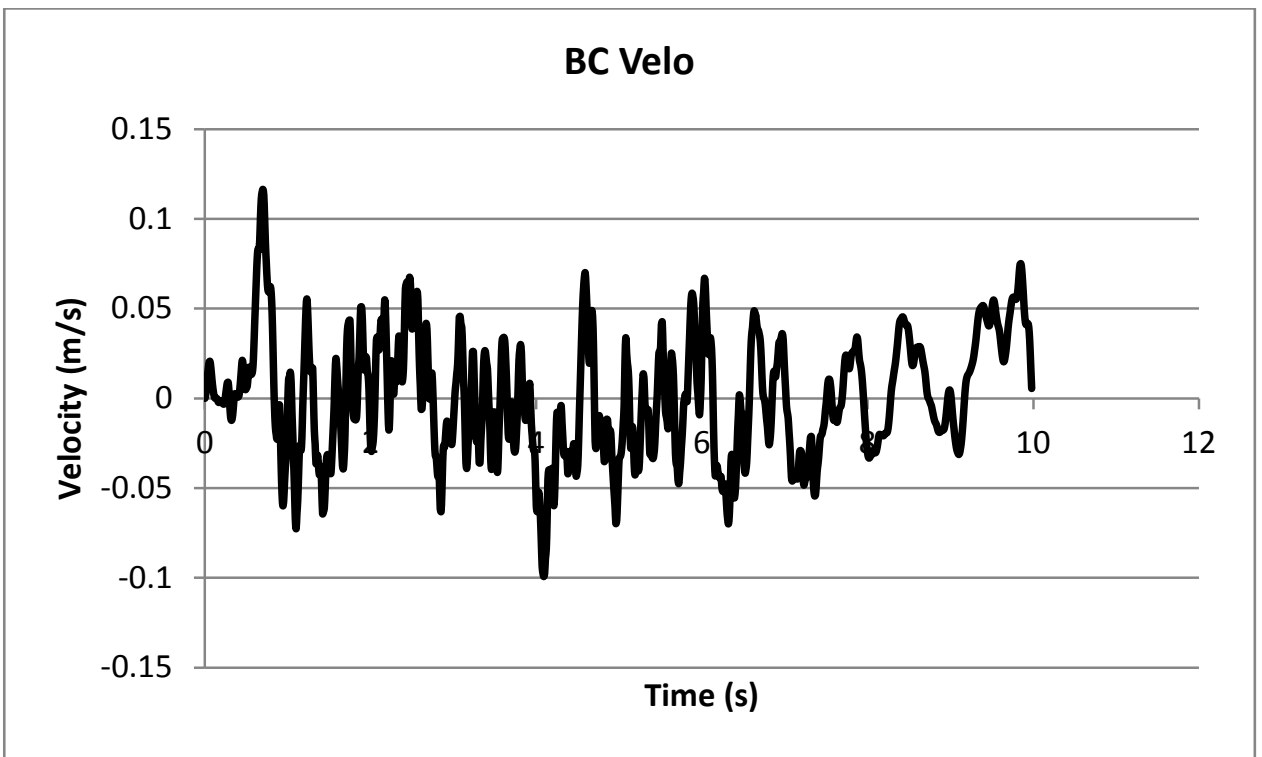

c. Displacement

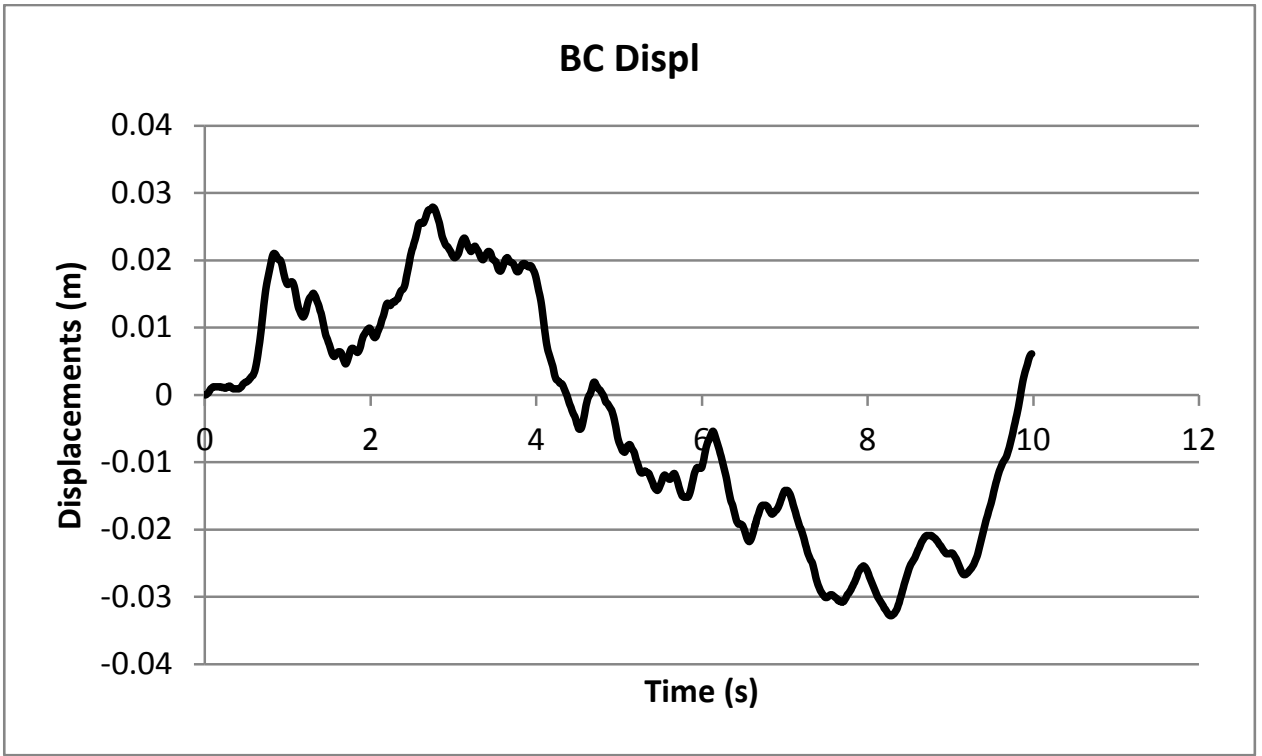




\section{Relative Displacements for Analysis}

1. Fixed Base Supports

\section{Horizontal}

\section{Soil A}

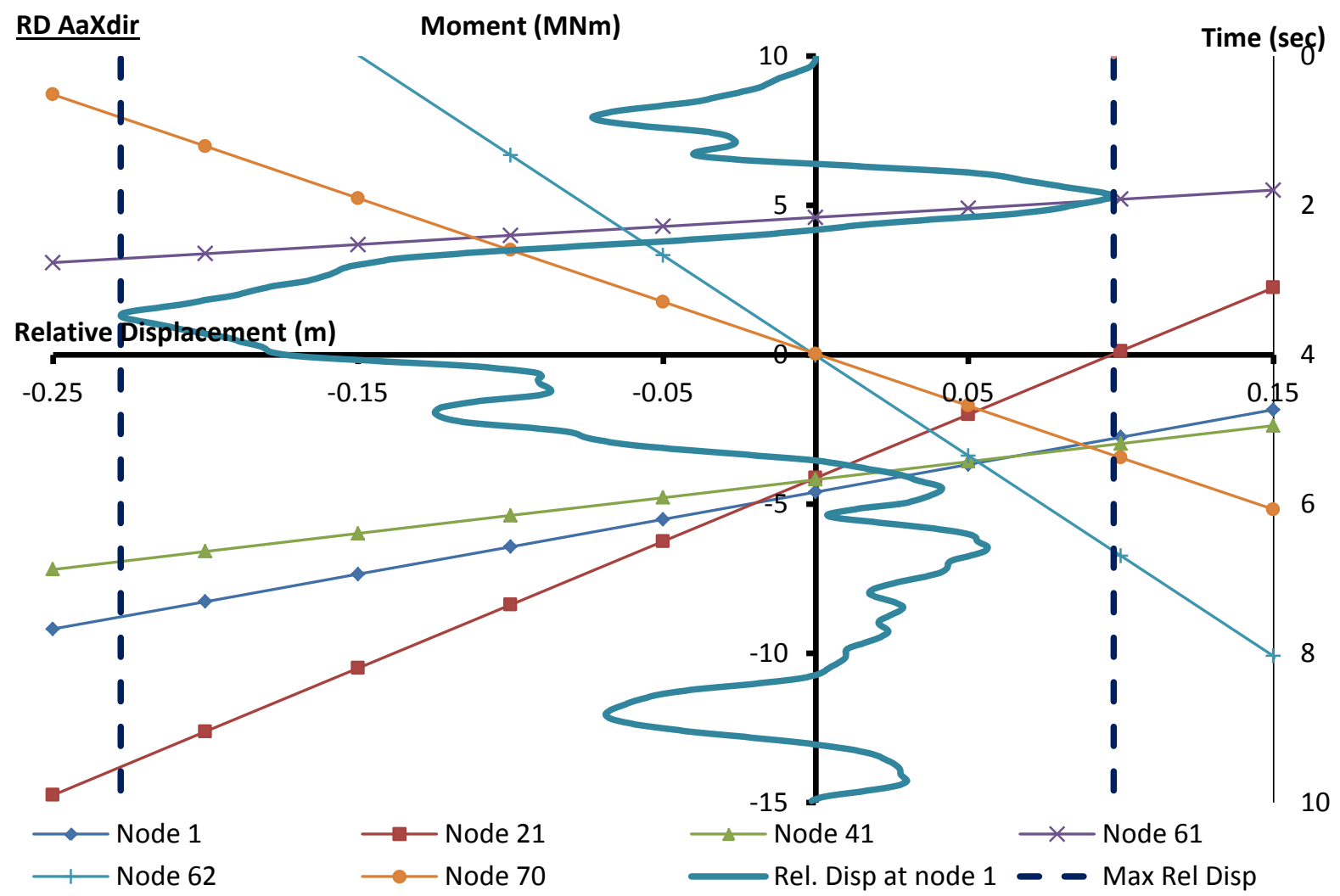




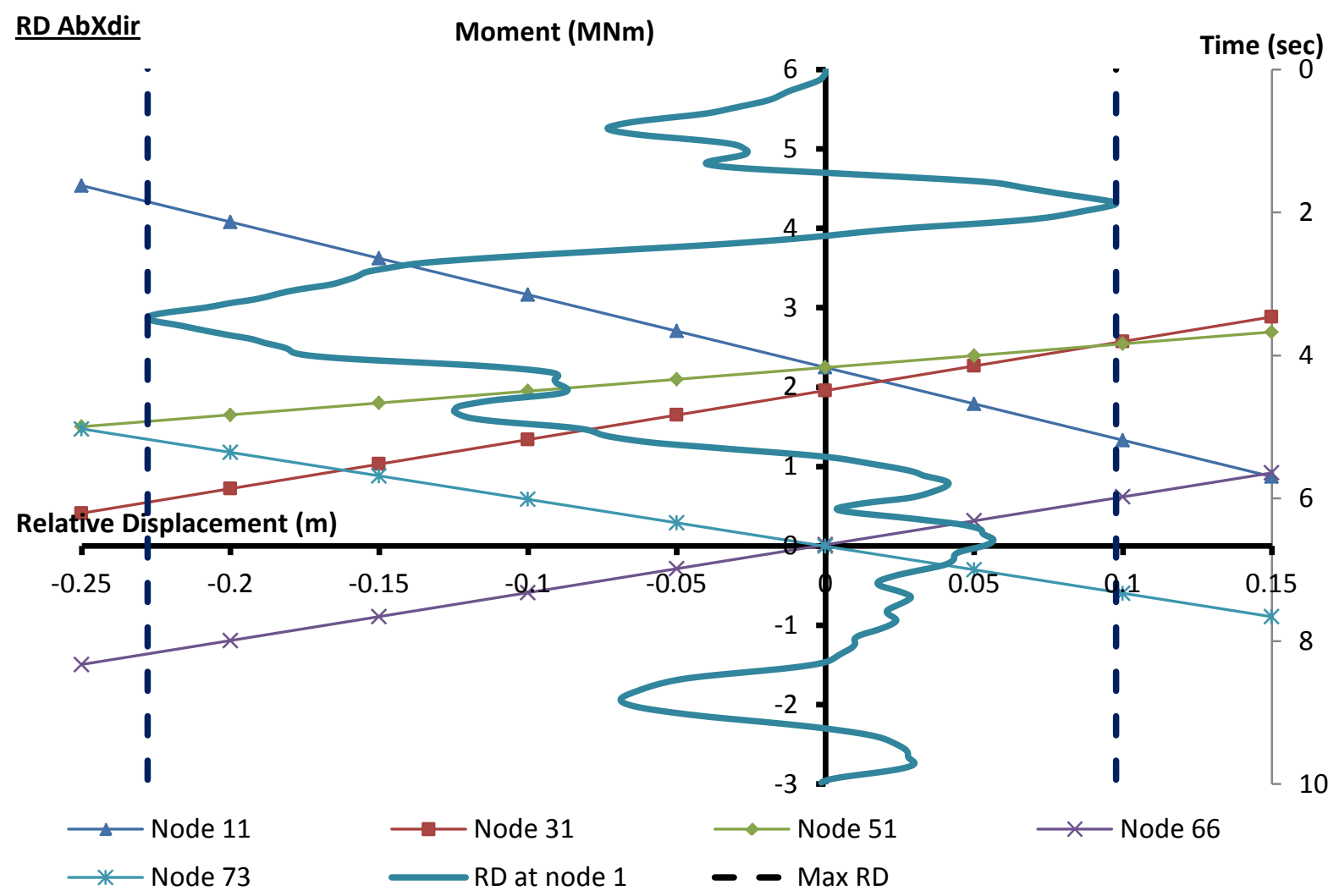

\section{Soil B}

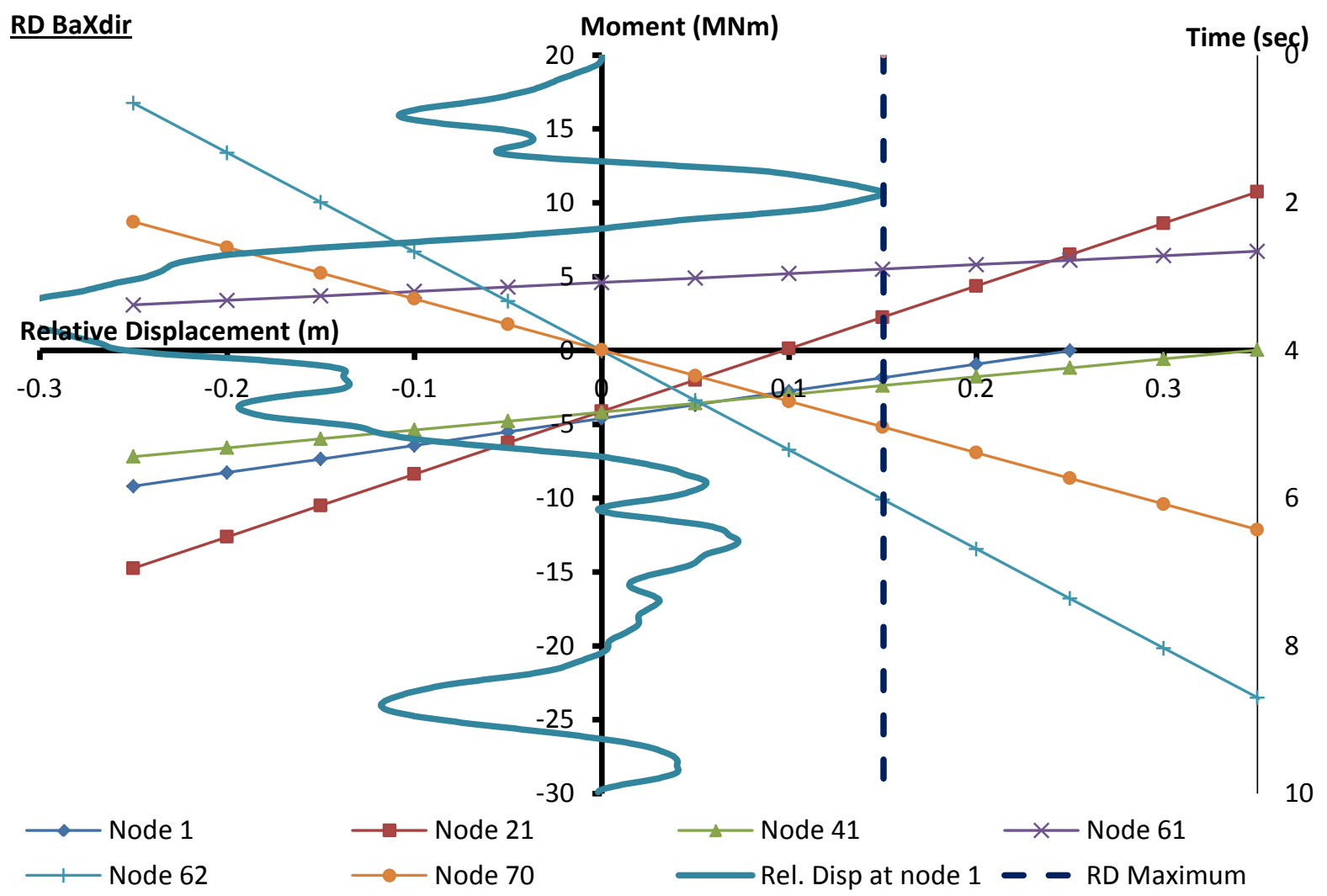




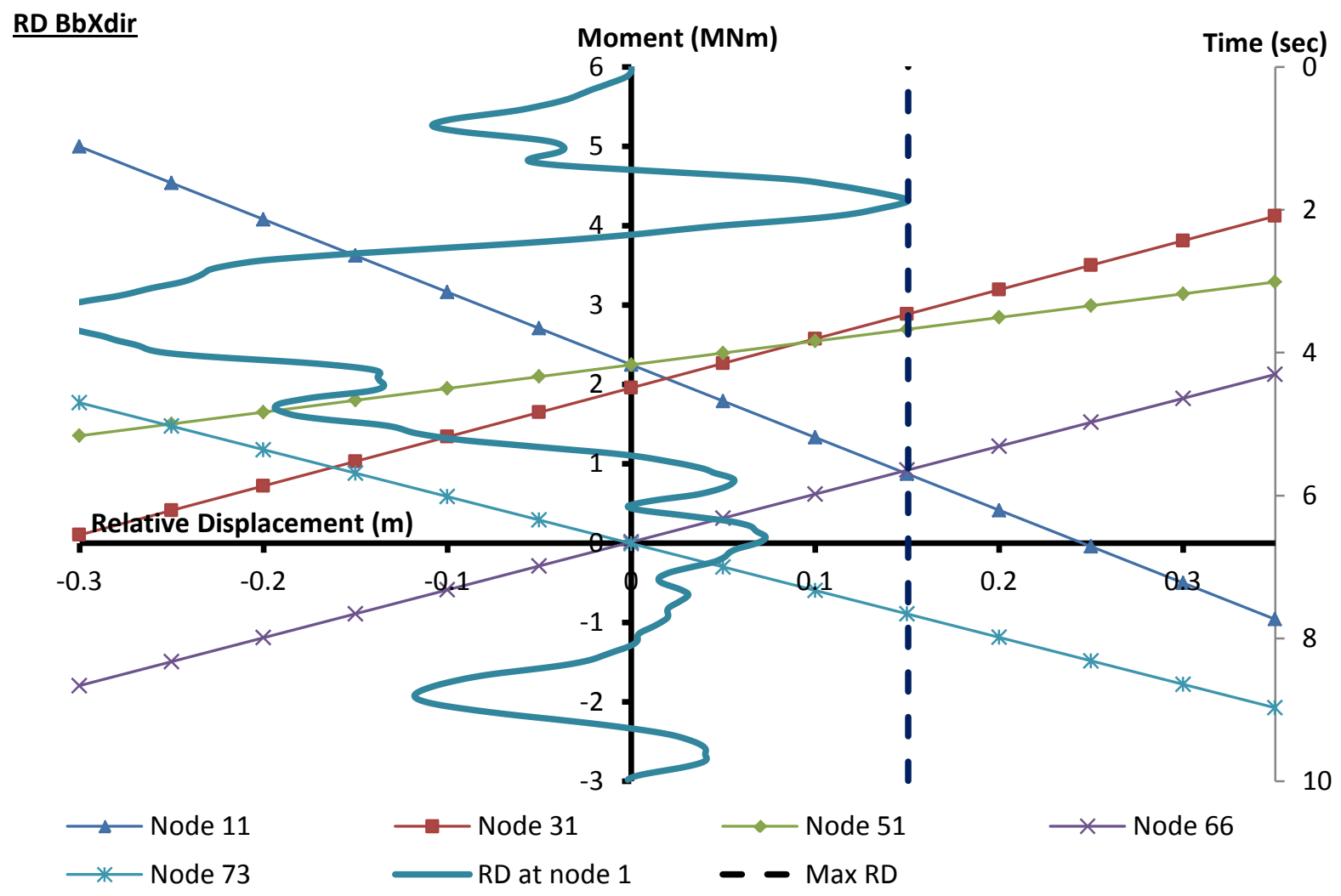

Soil C

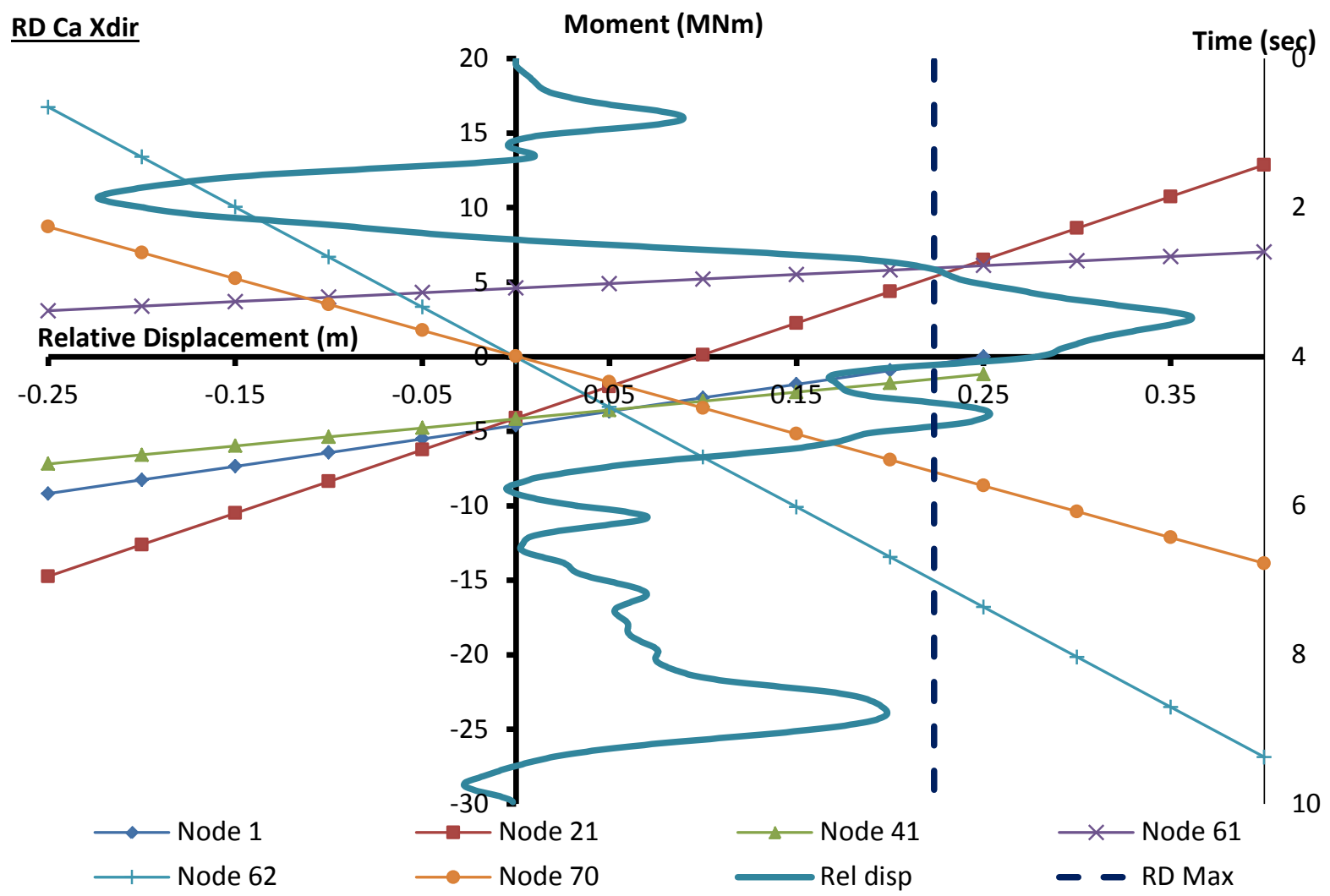




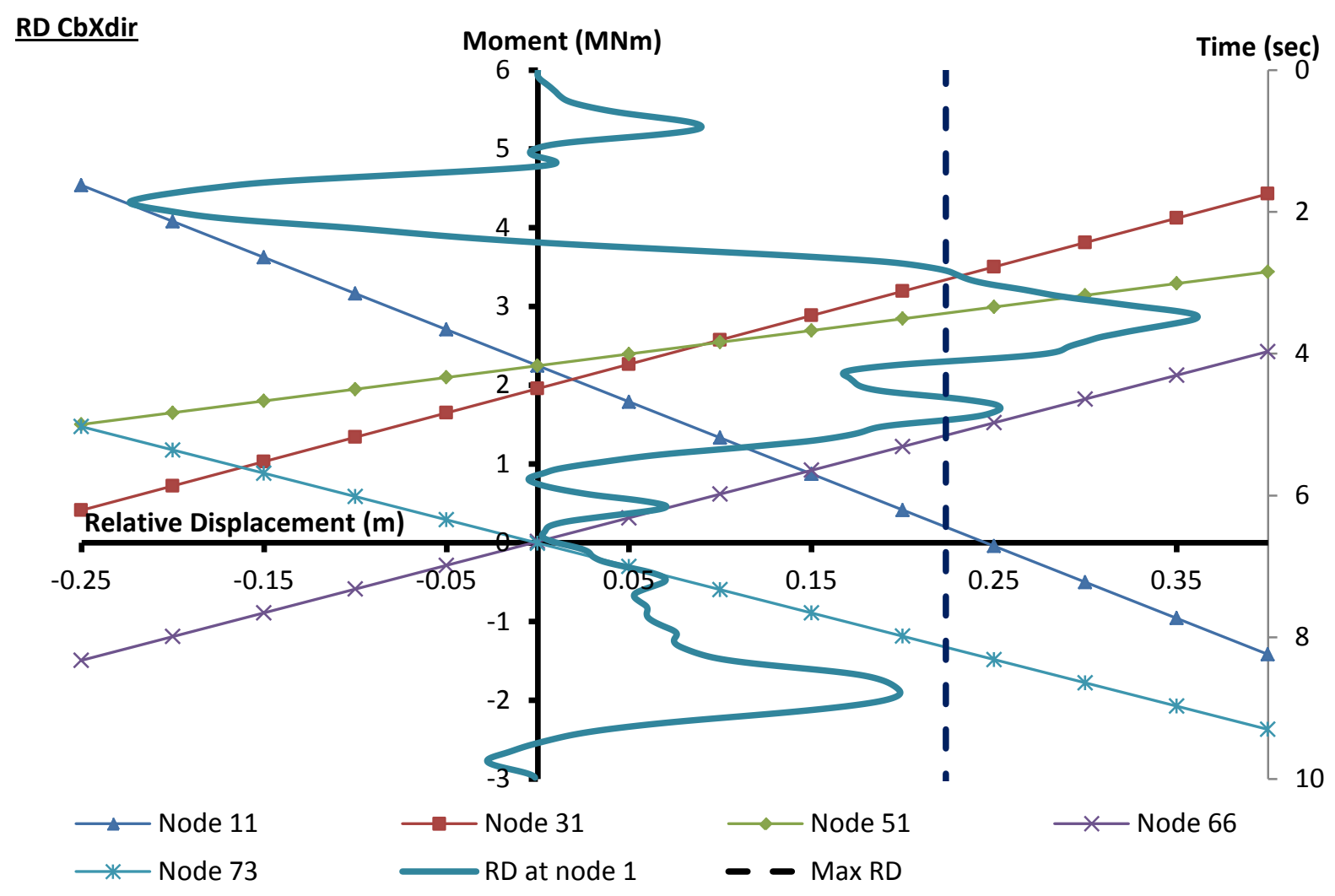

\section{Soil D}

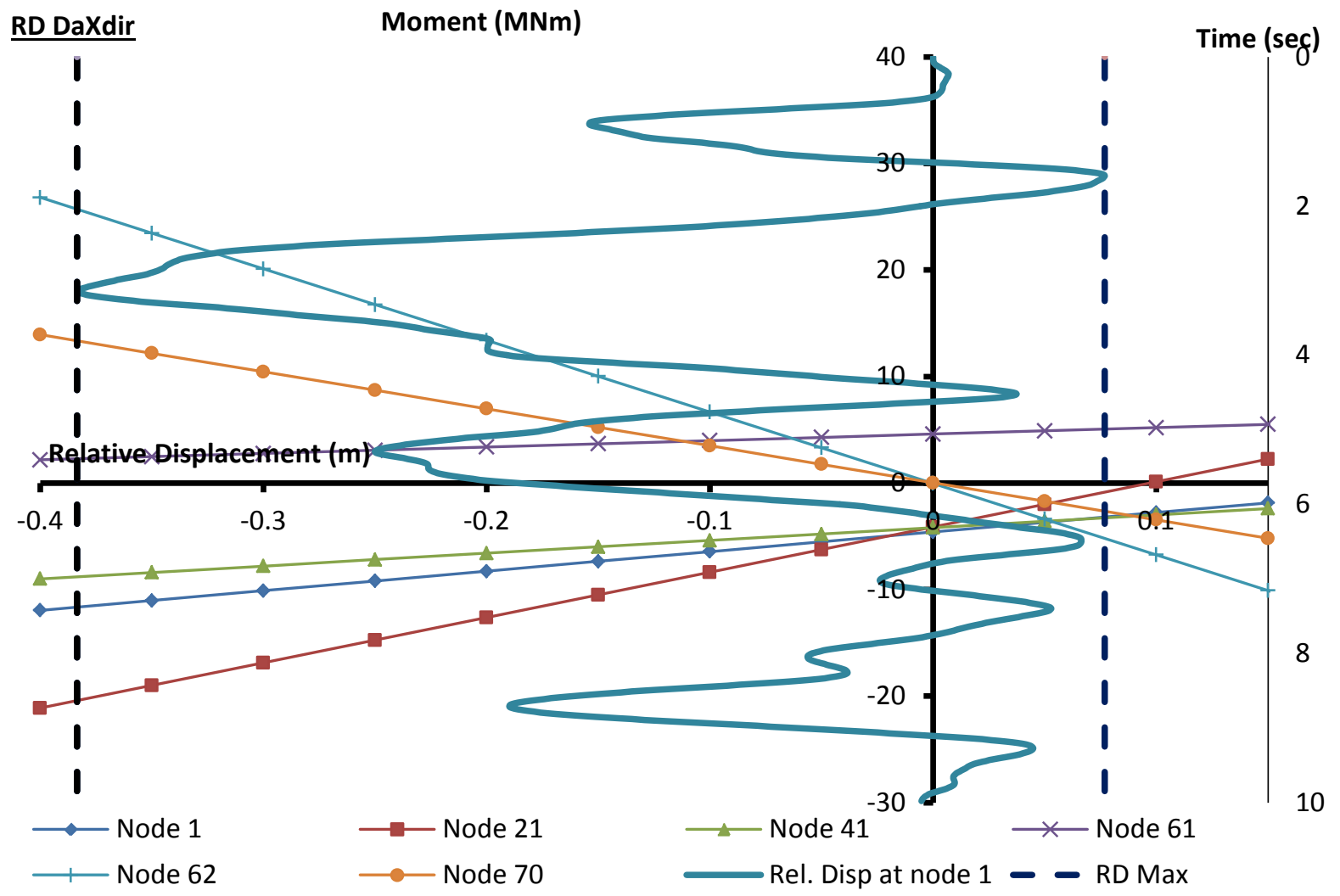




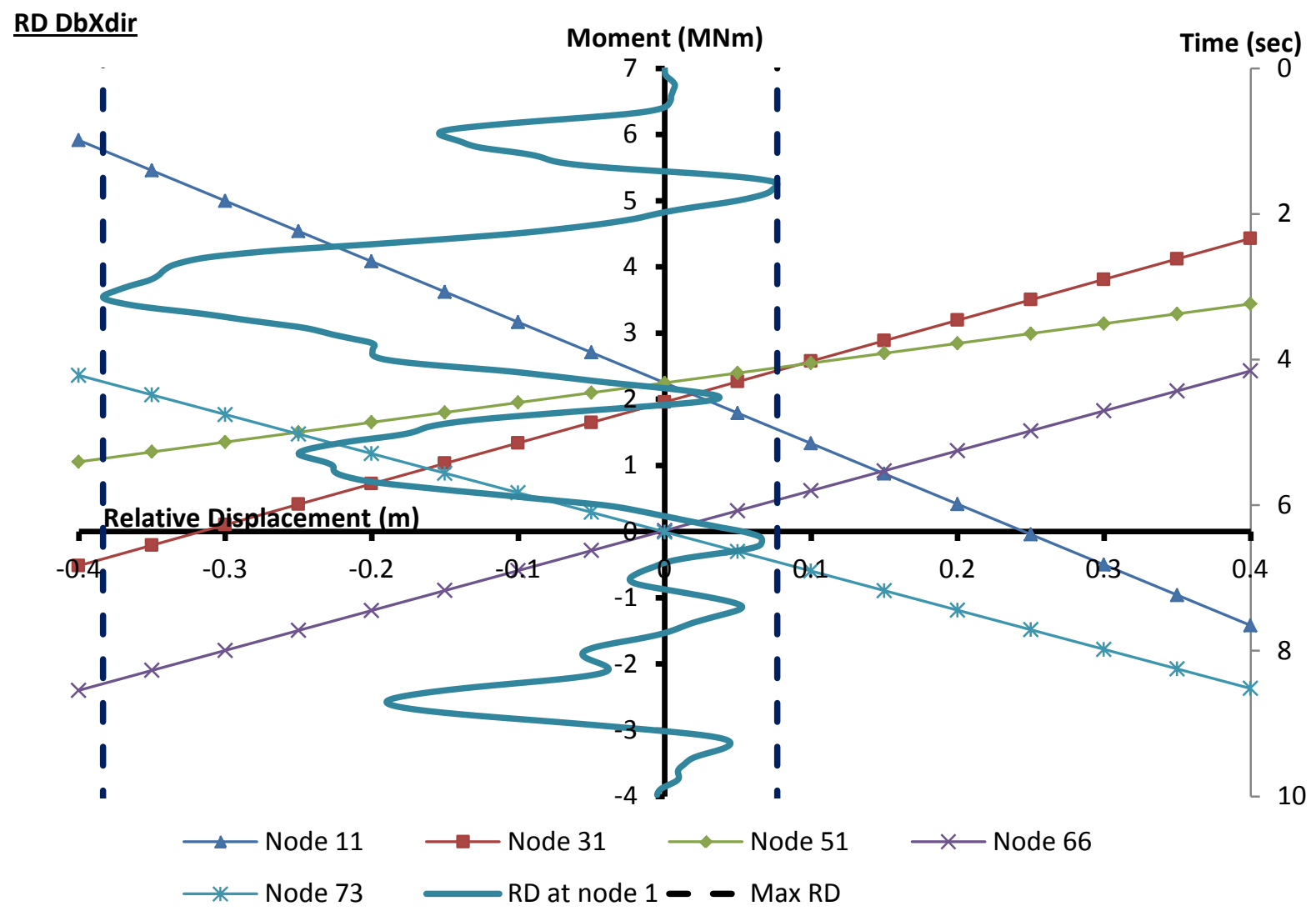




\section{Vertical}
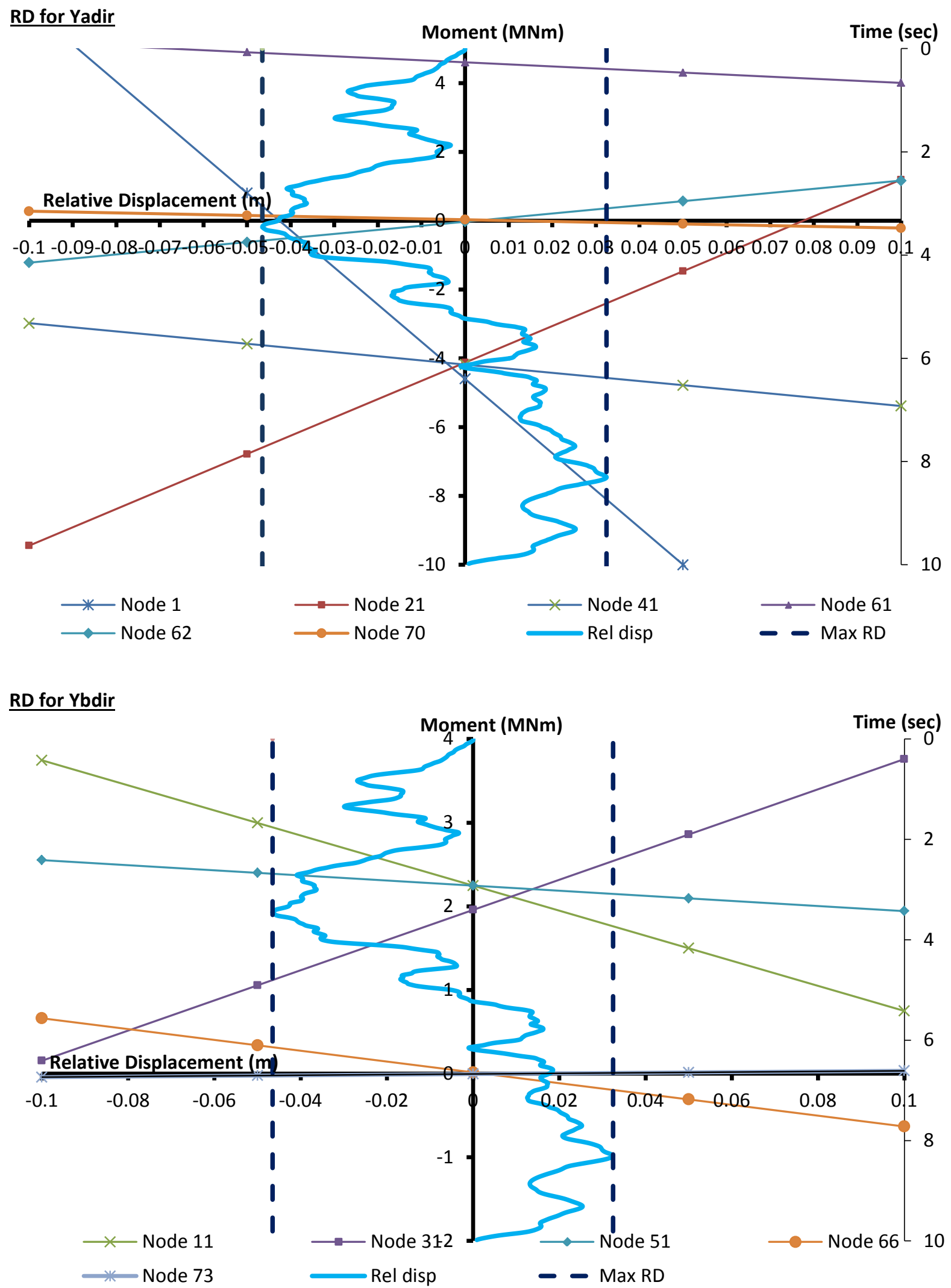


\section{Springs Base Supports}

\section{Horizontal}

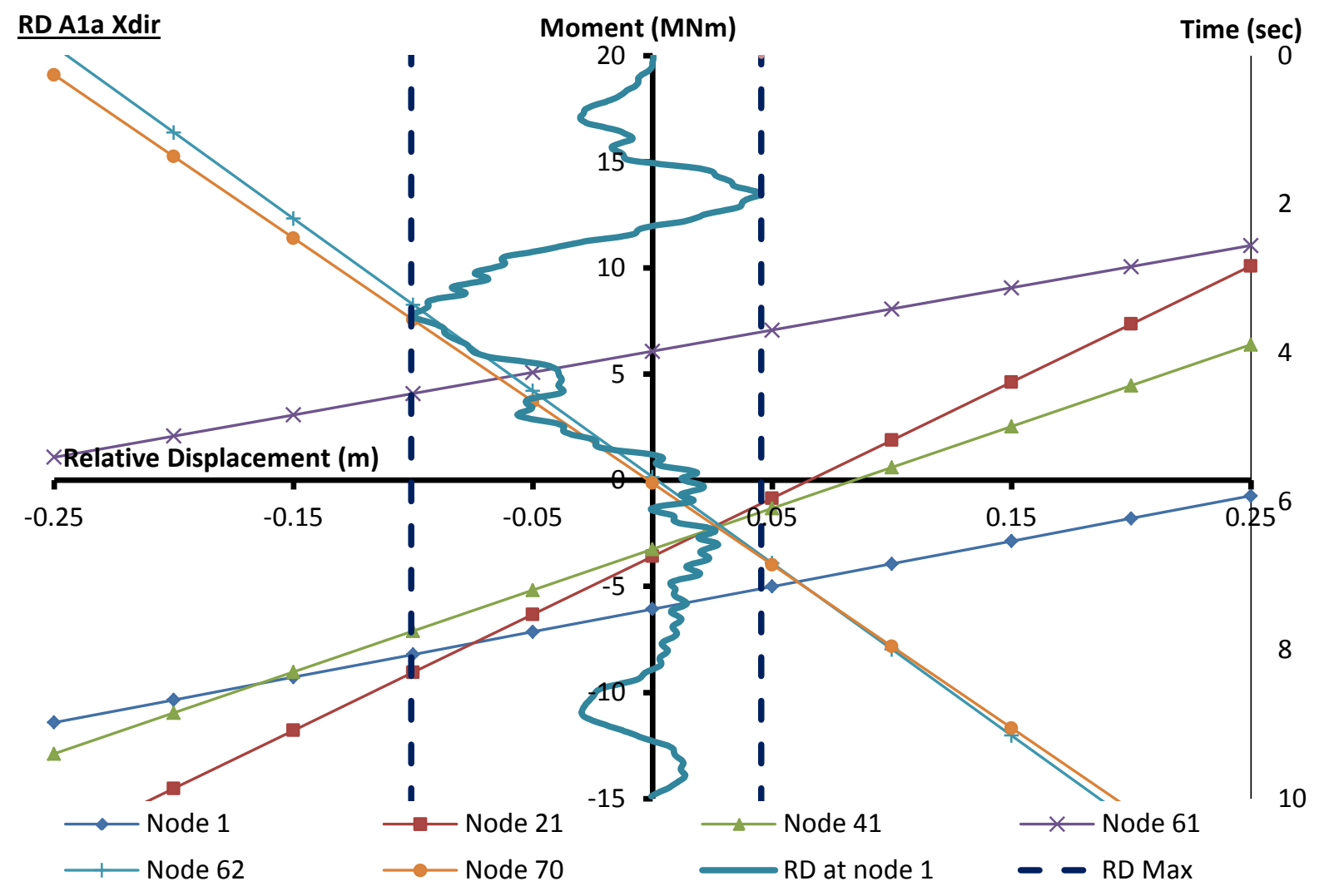



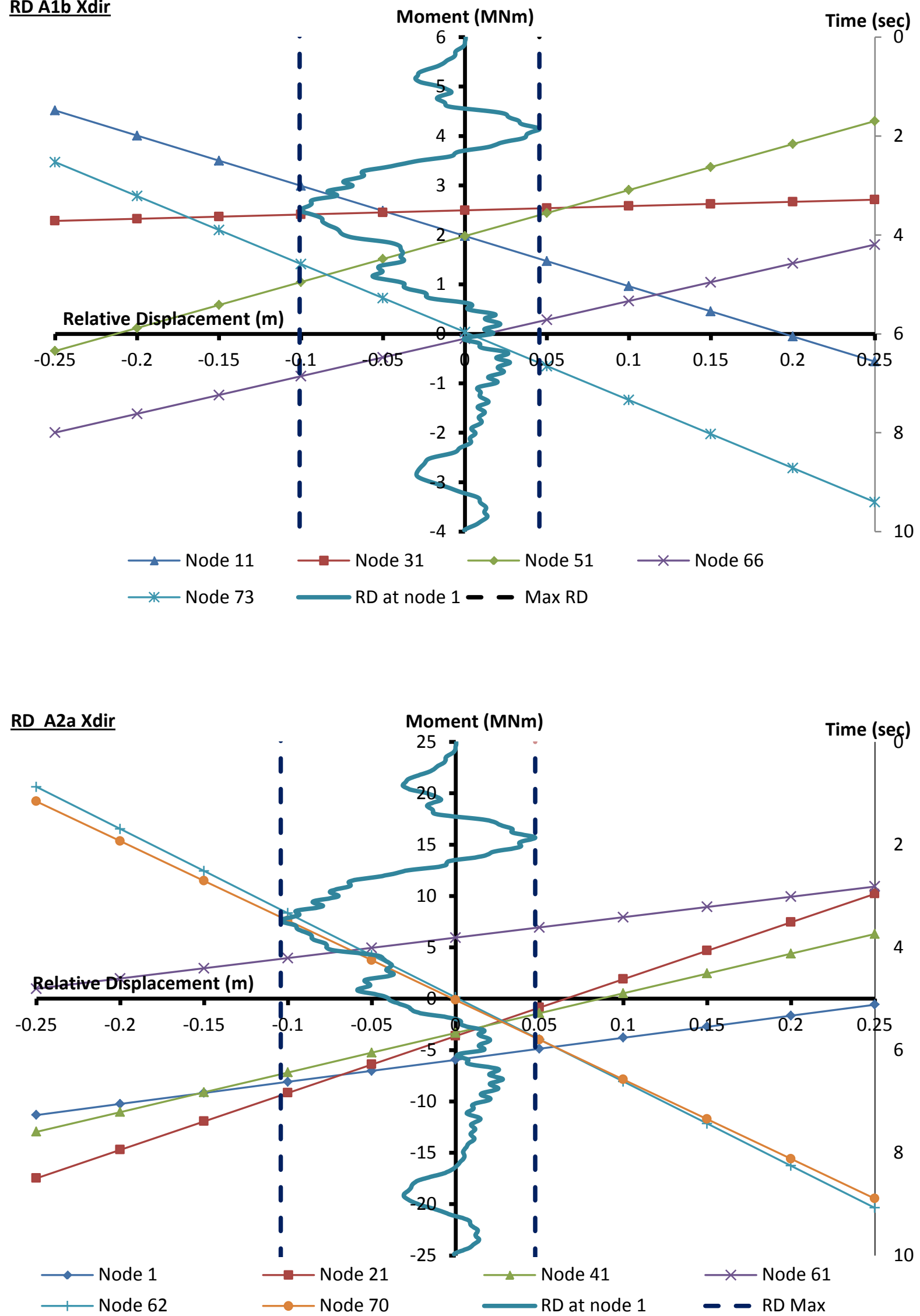

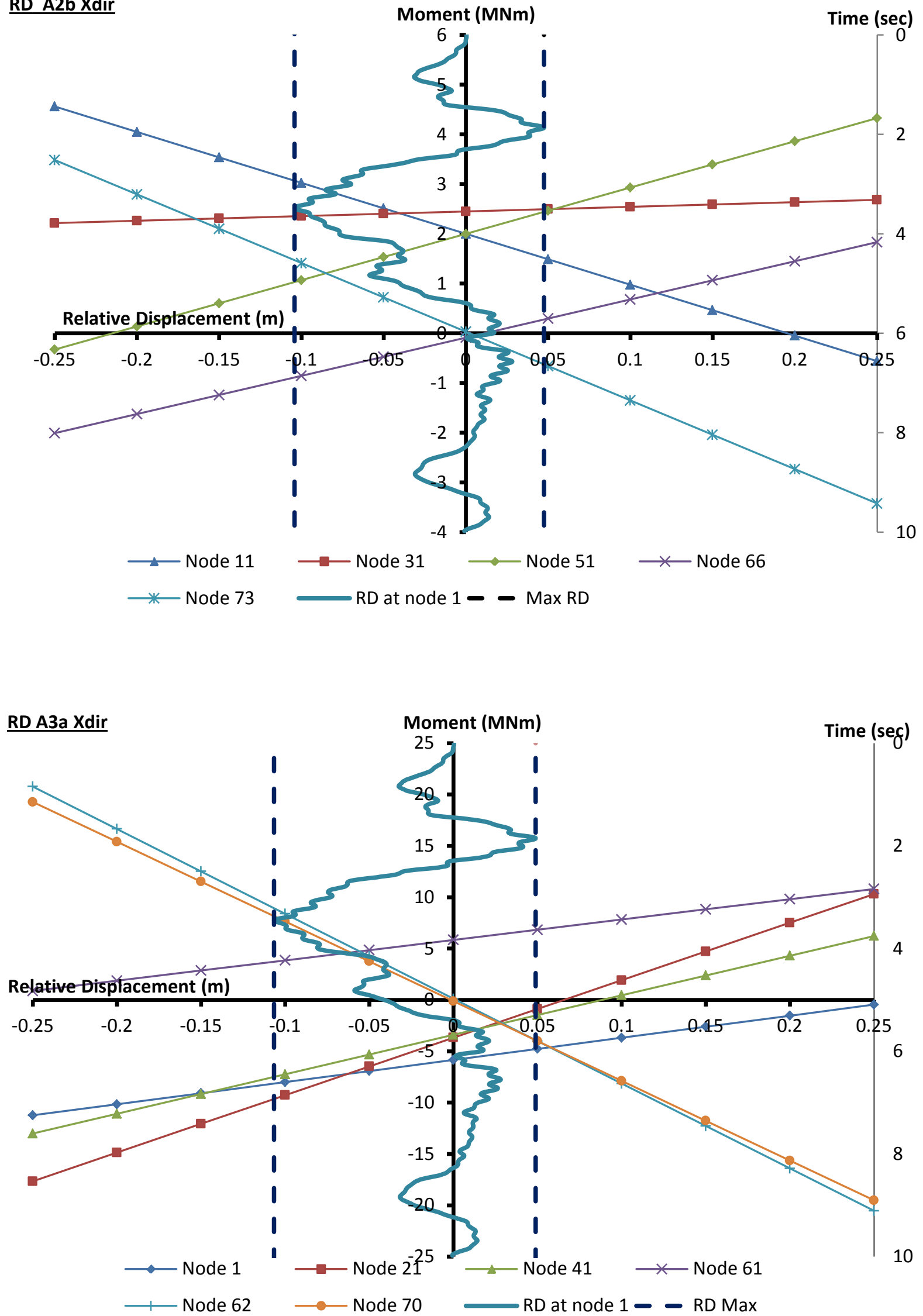

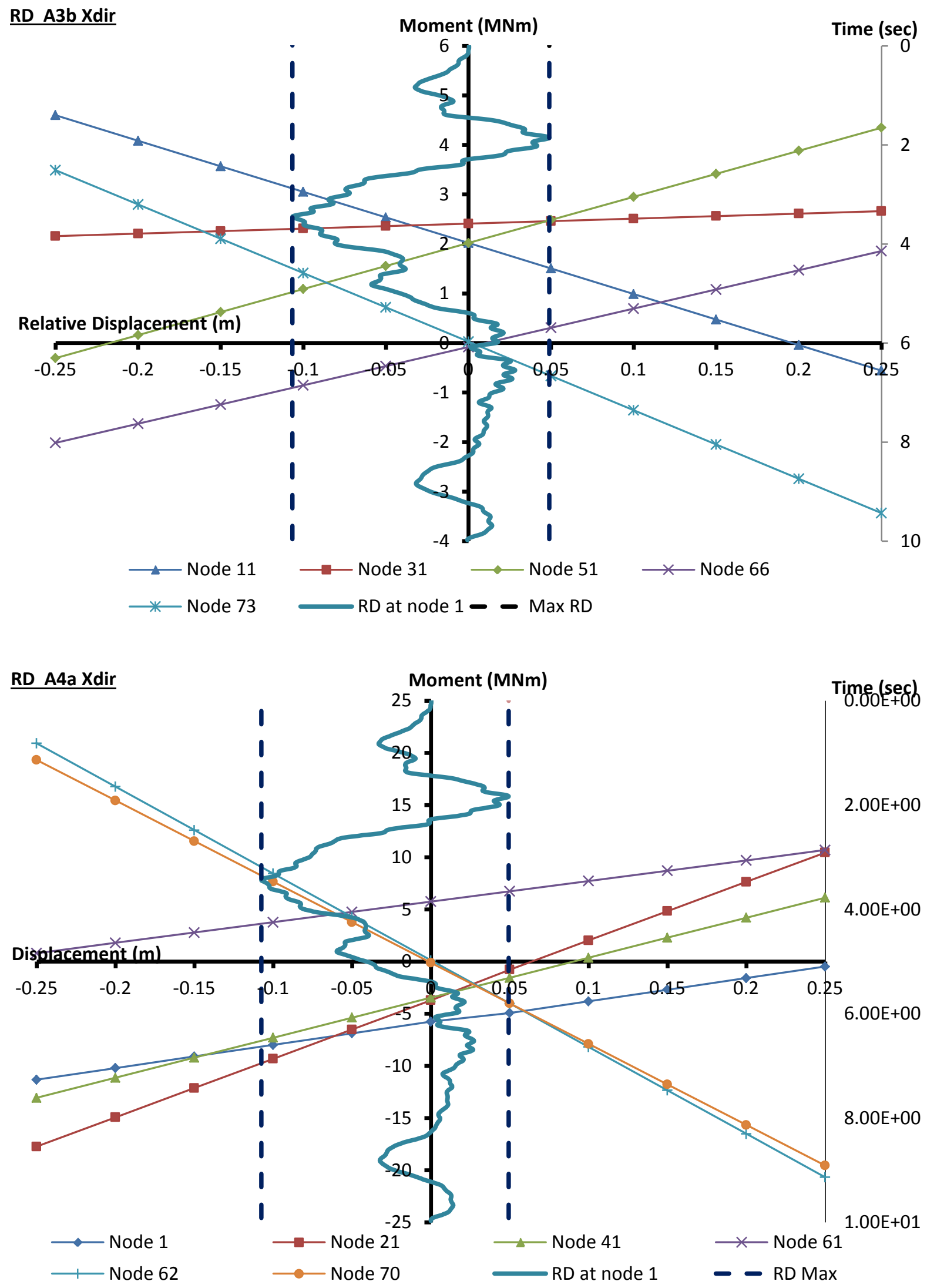

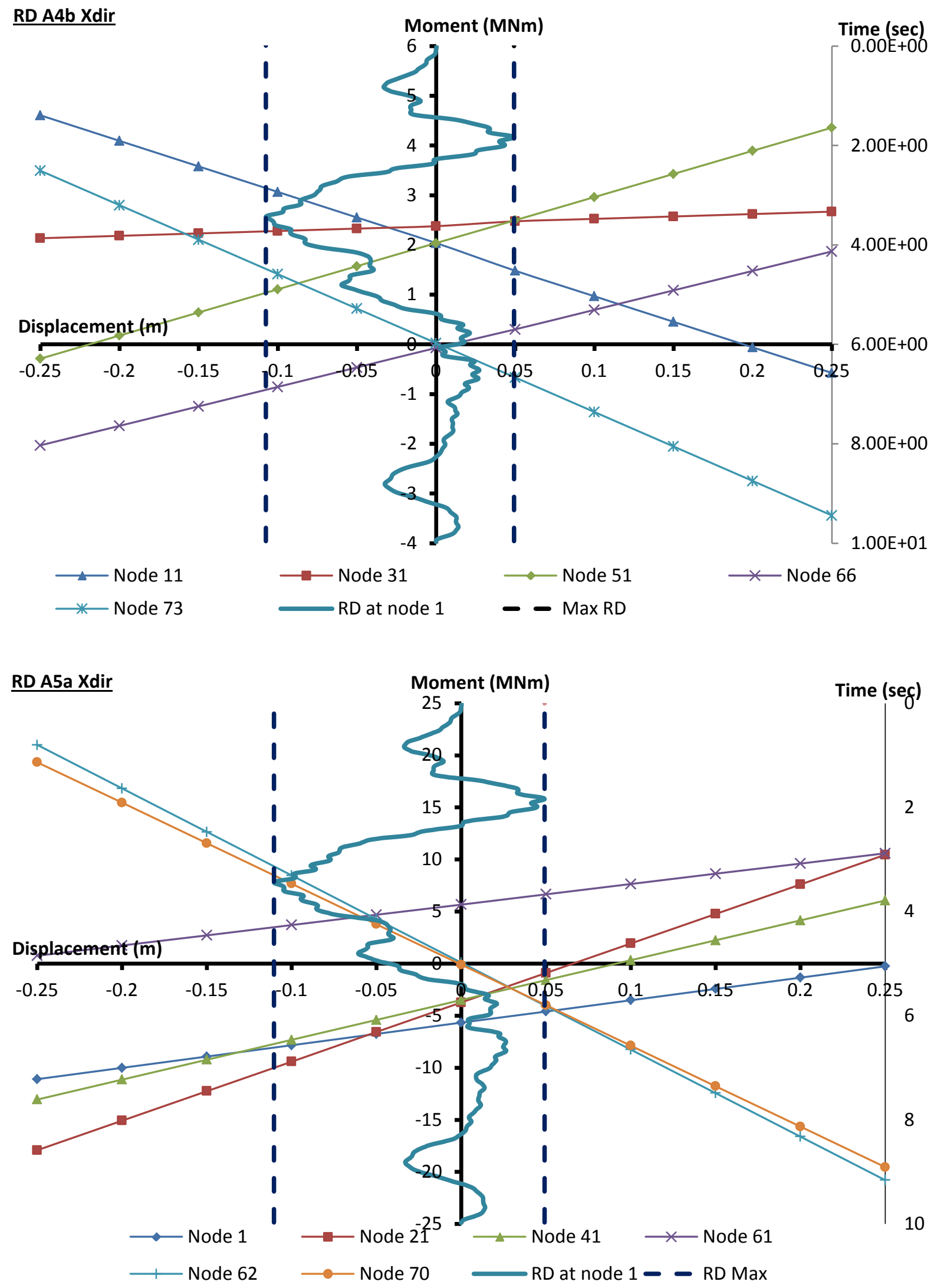

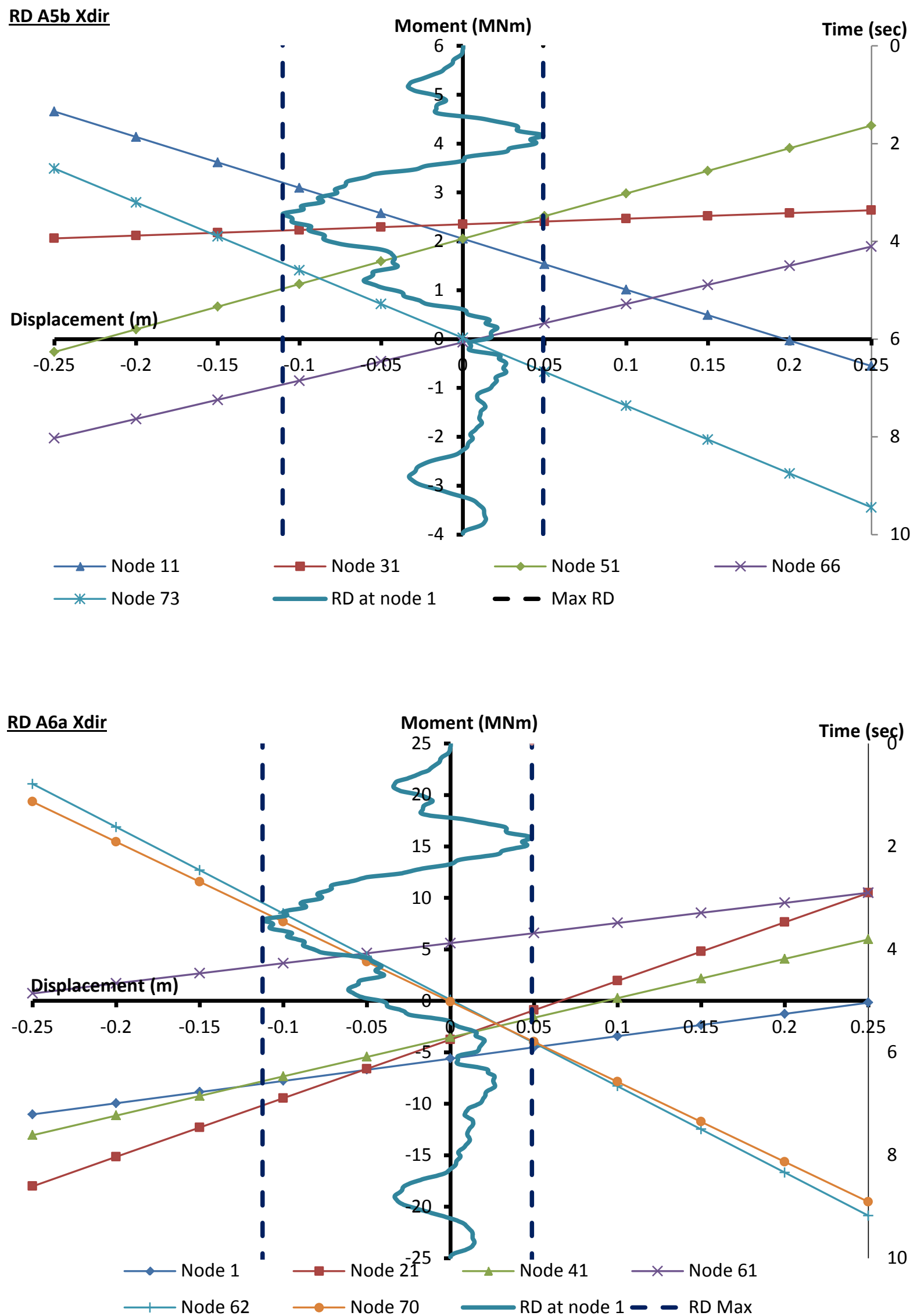


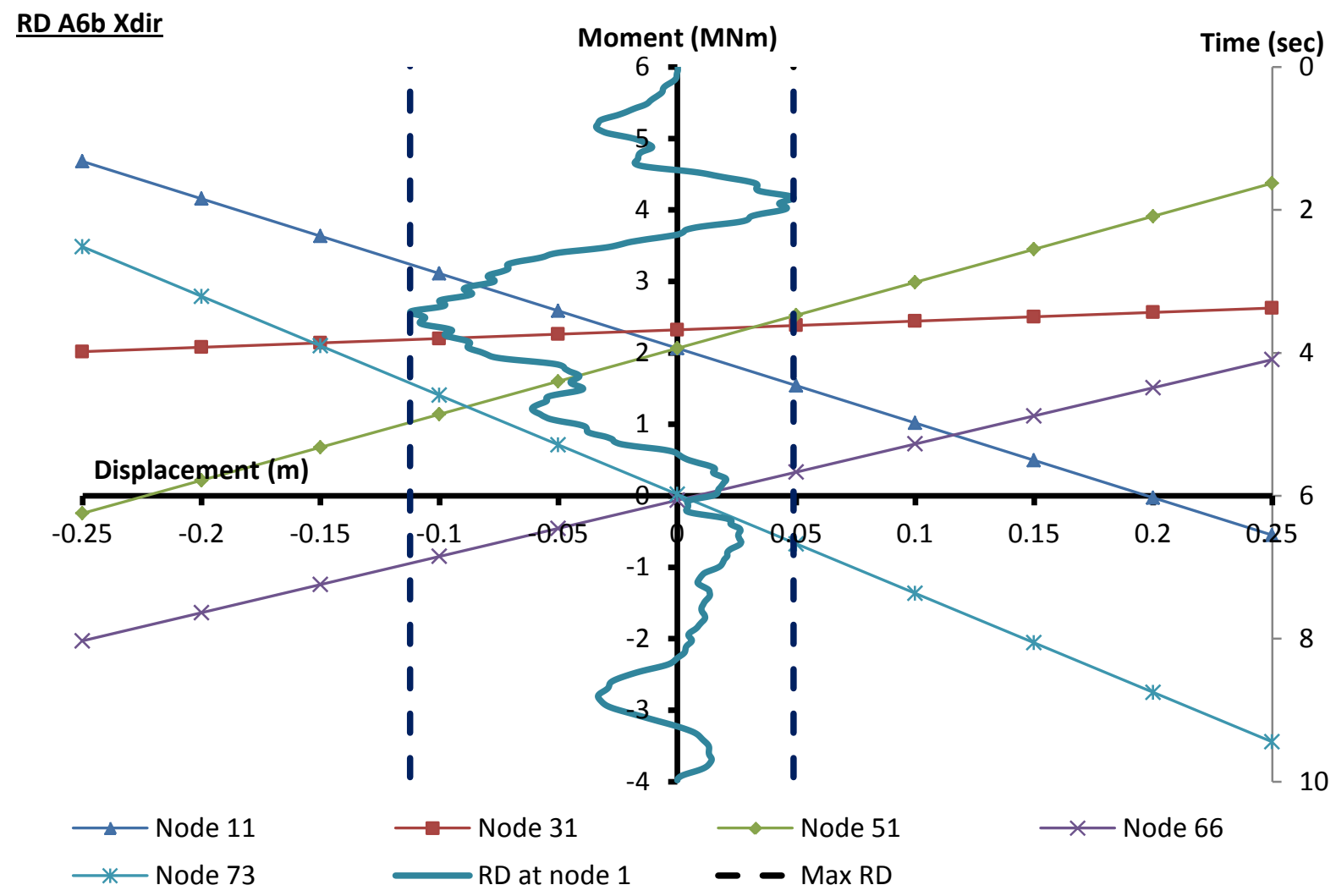

\section{Vertical}

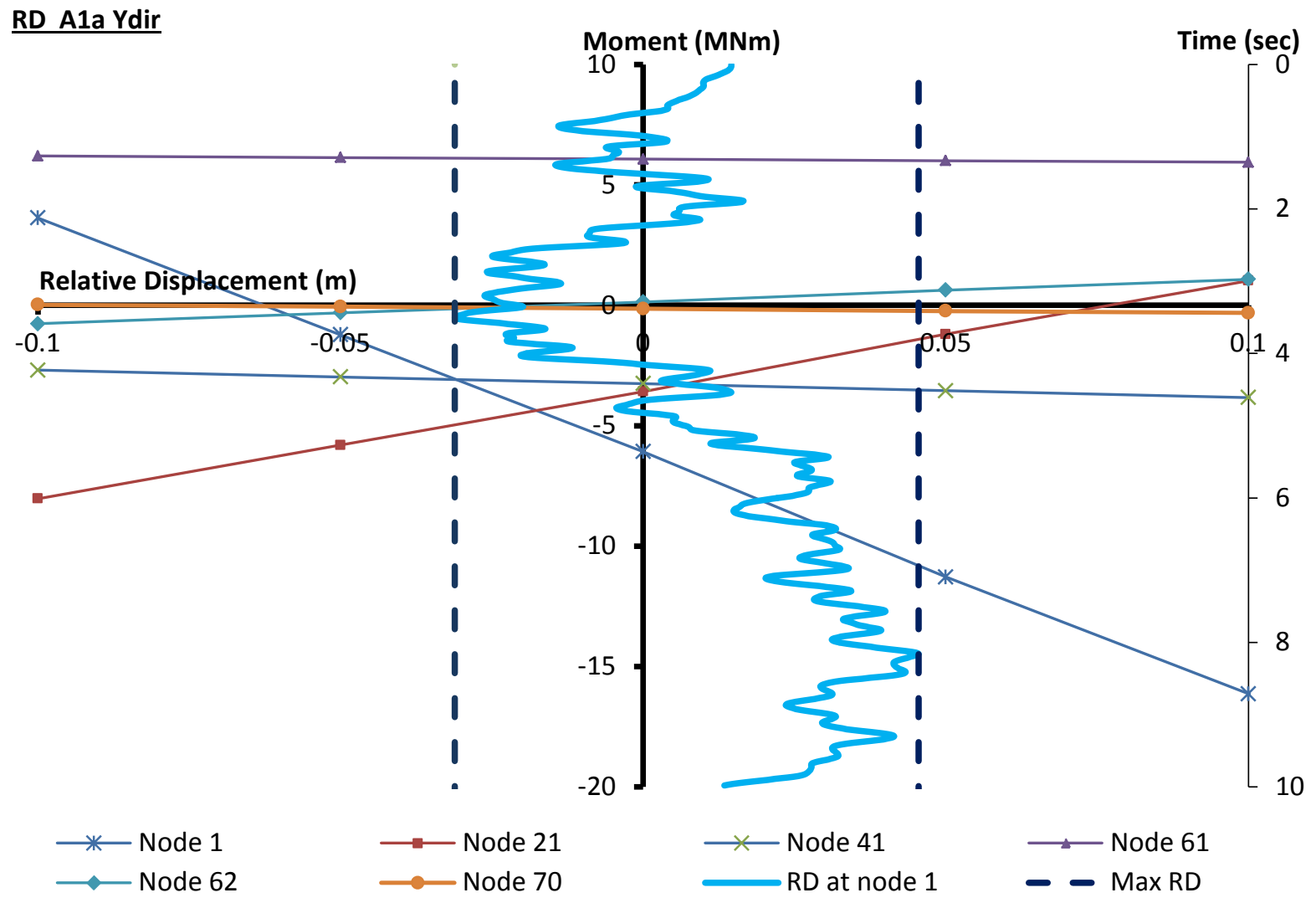




\section{RD A1b Ydir}

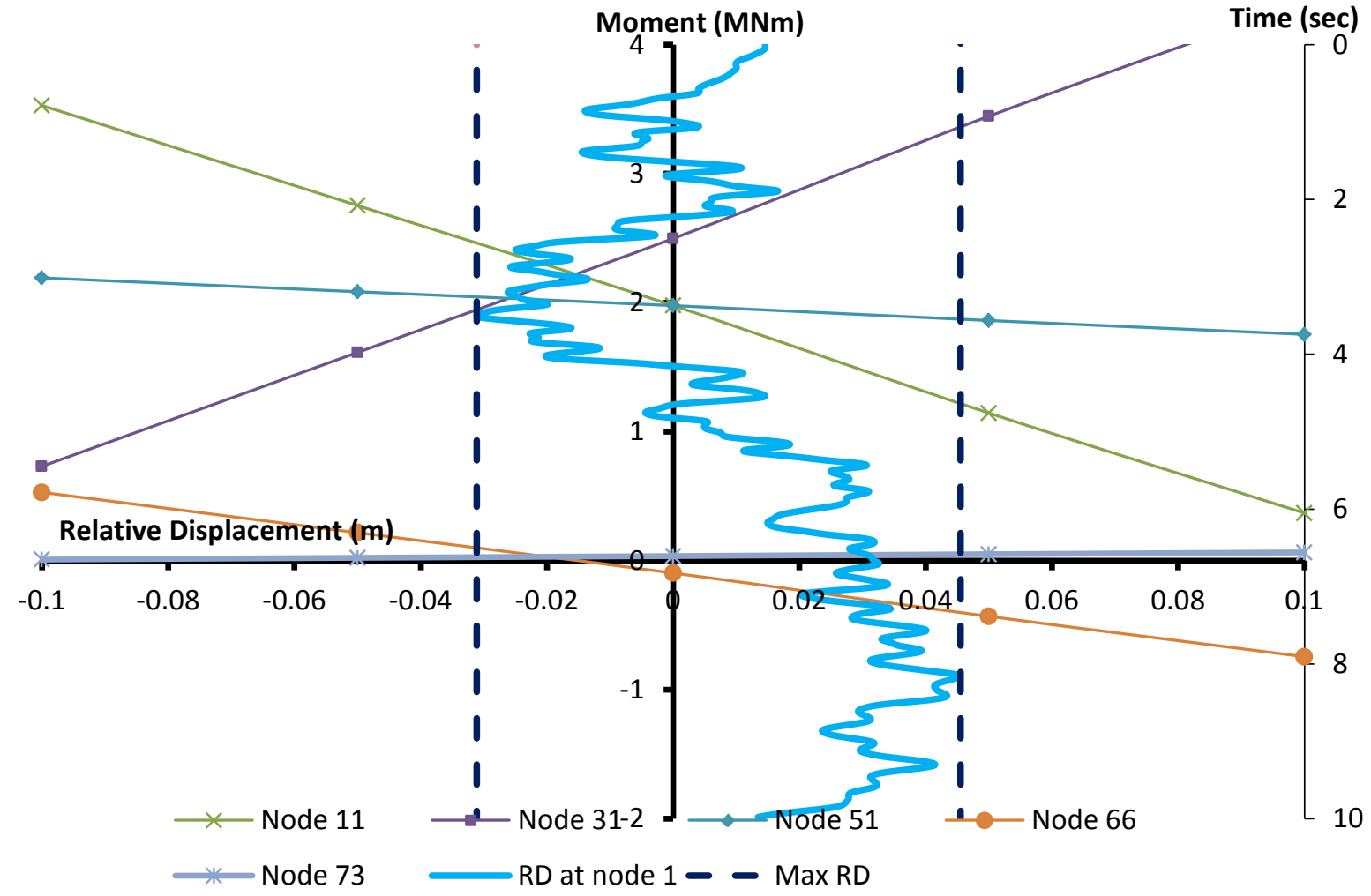

\section{RD A2a Ydir}
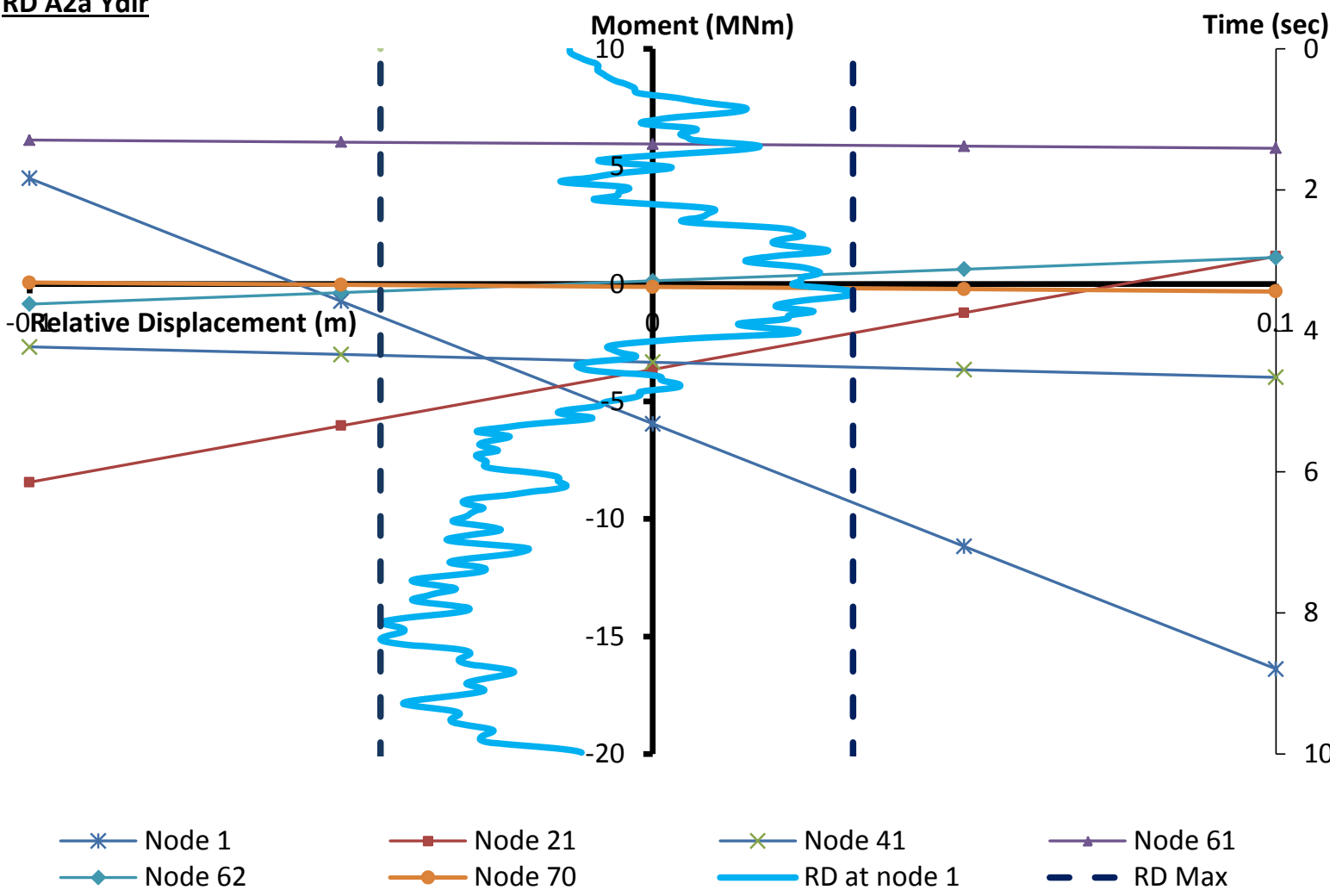


\section{RD A2b Ydir}

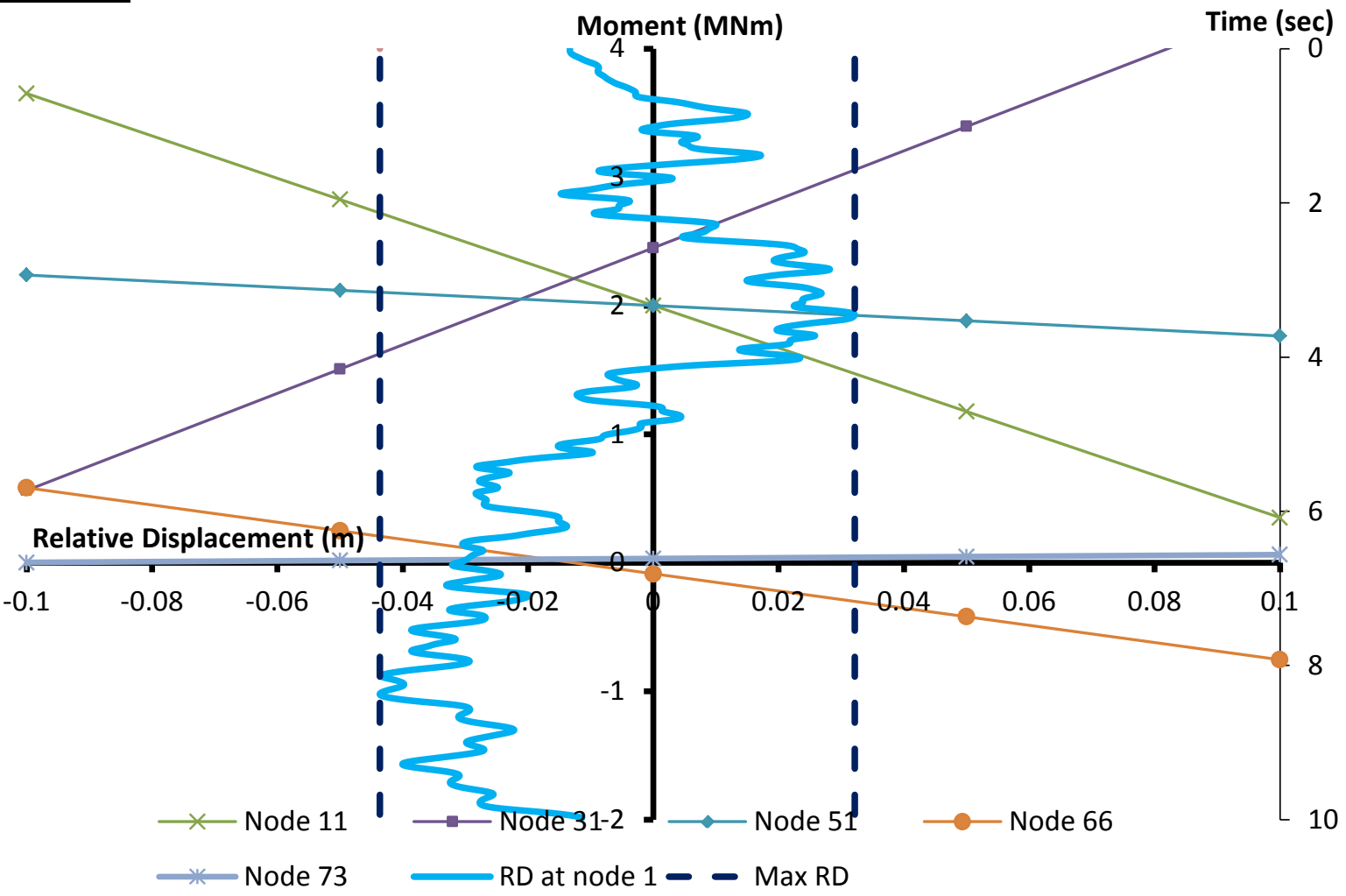

\section{$\underline{\text { RD A3a Ydir }}$}
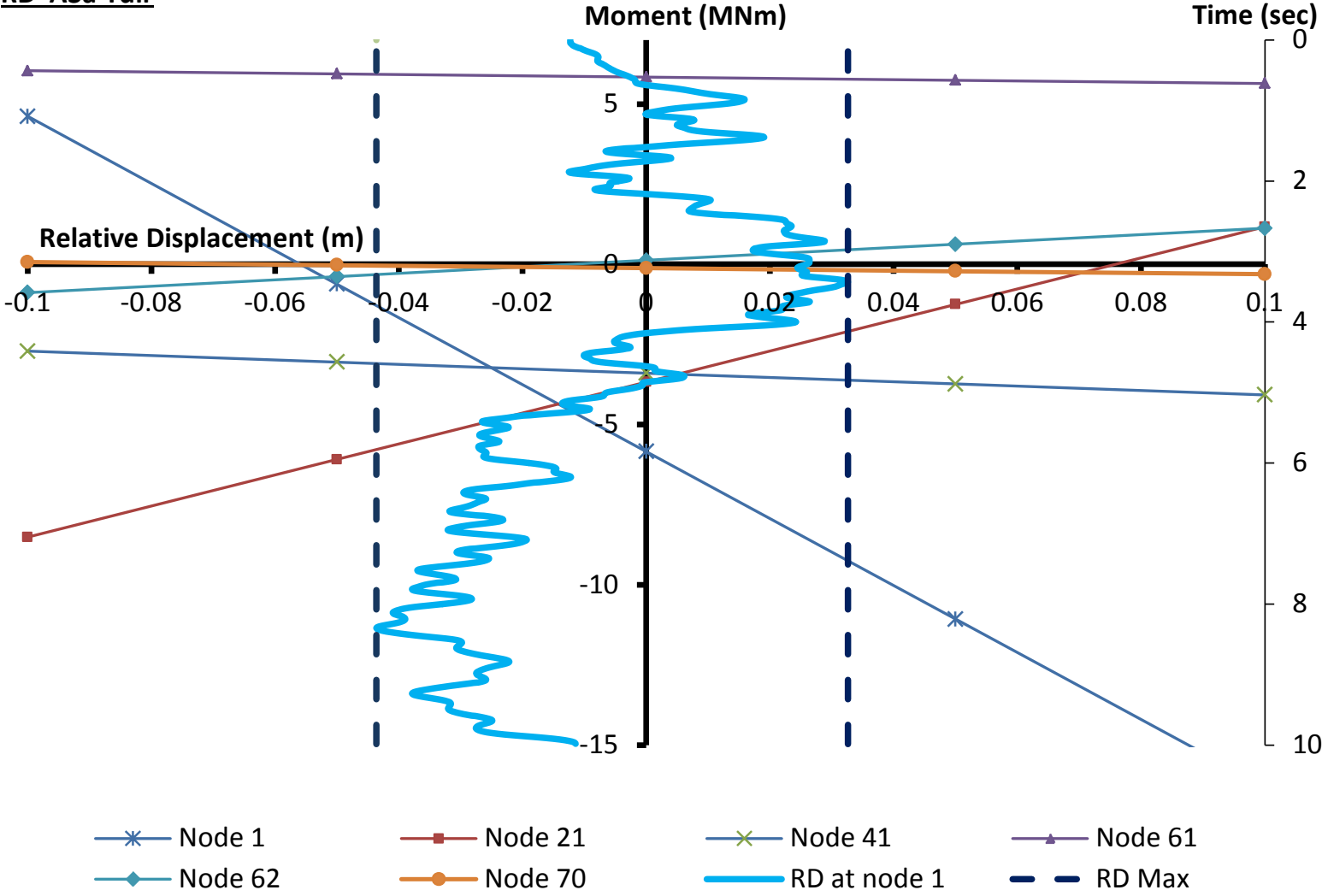


\section{RD A3b Ydir}

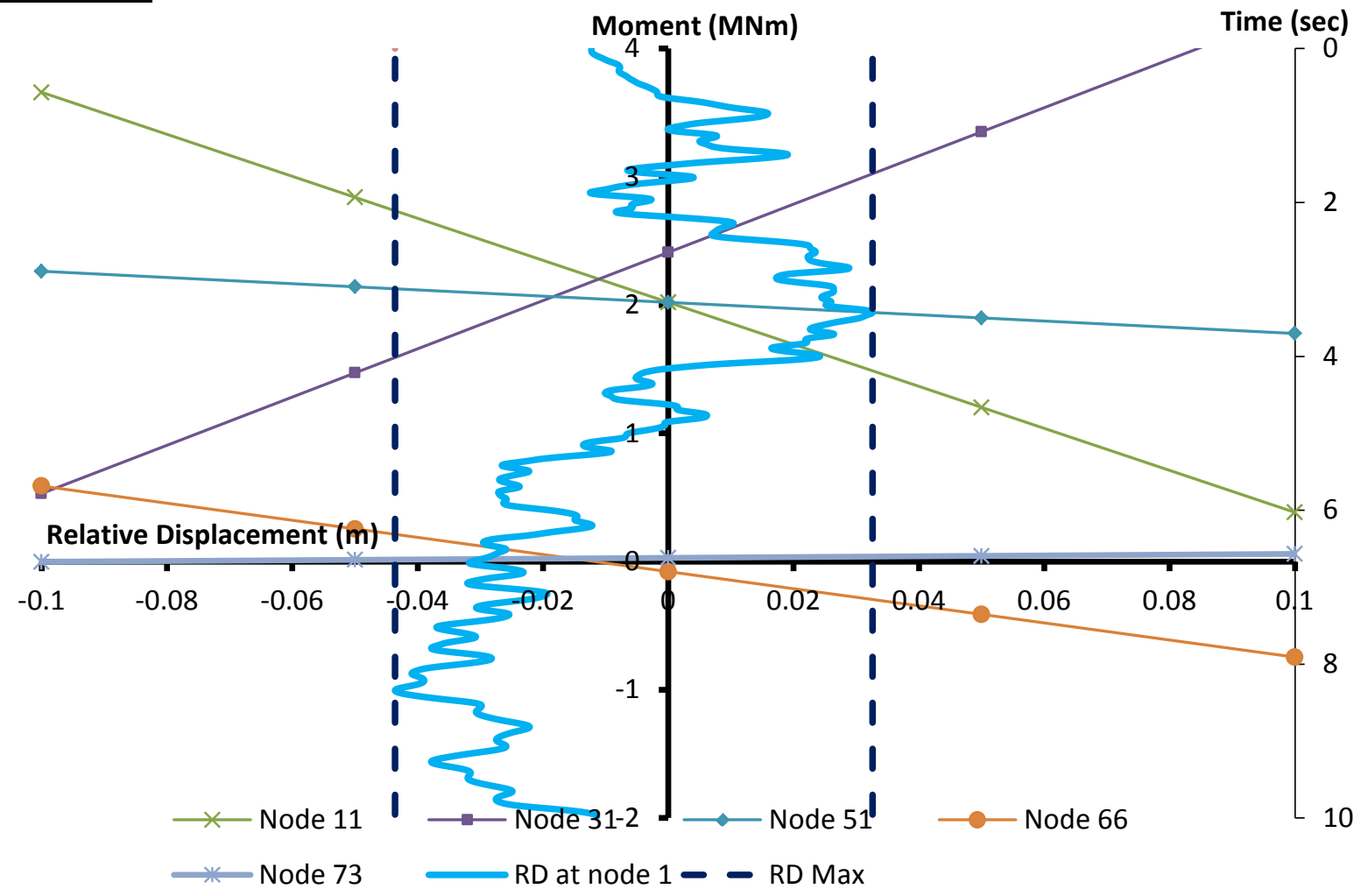

RD A4a Ydir
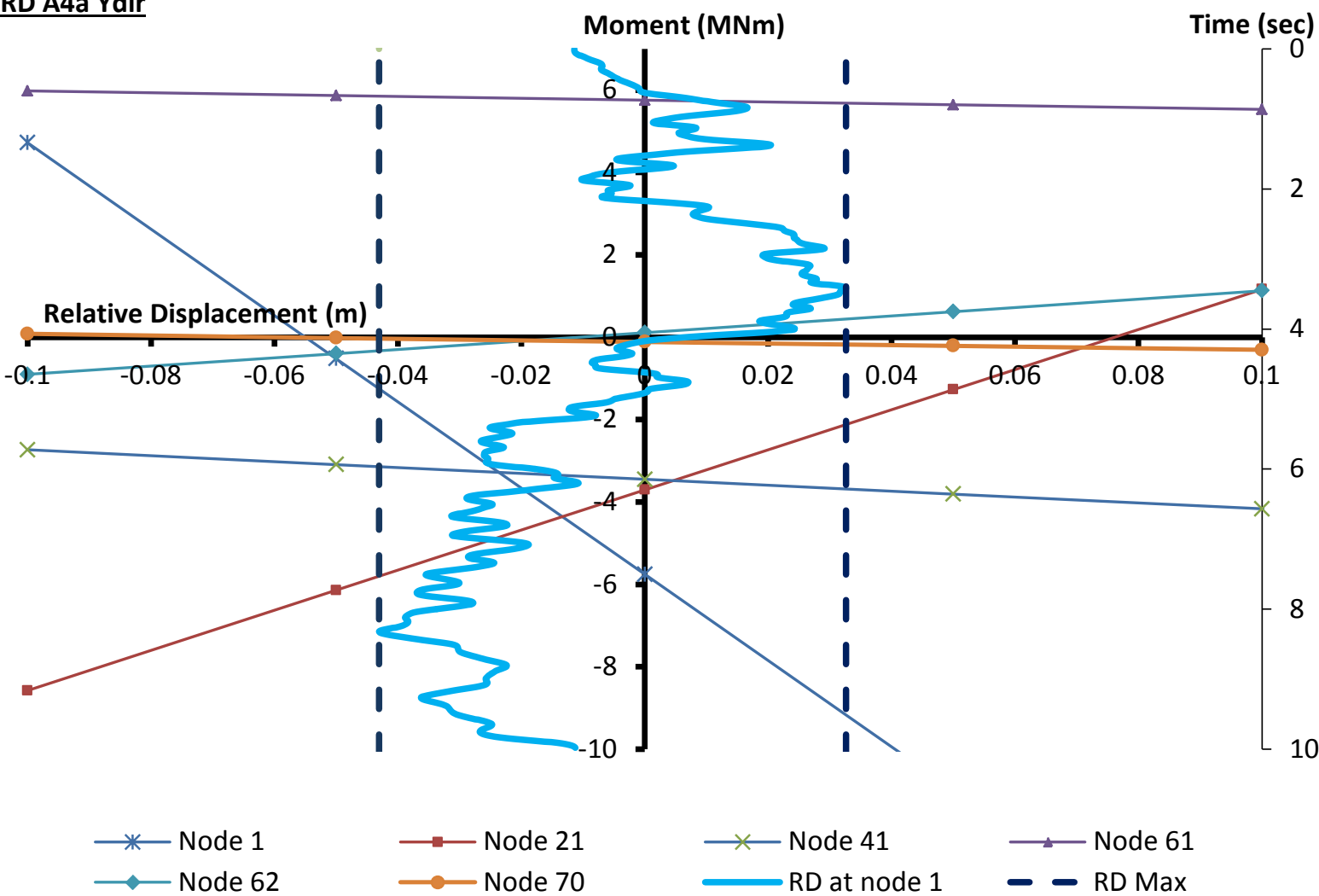


\section{$\underline{\text { RD A4b Ydir }}$}

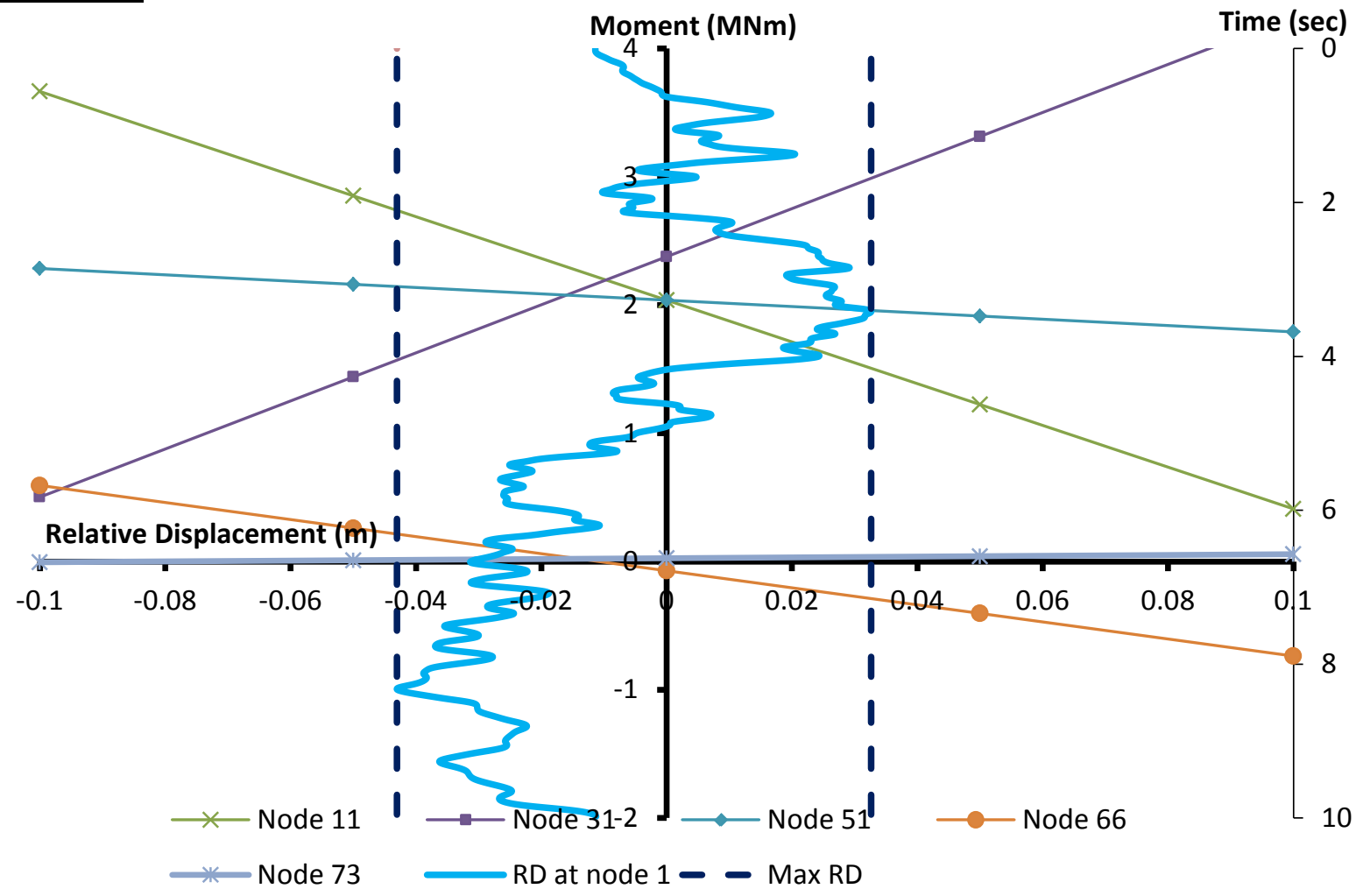

\section{RD A5a Ydir}

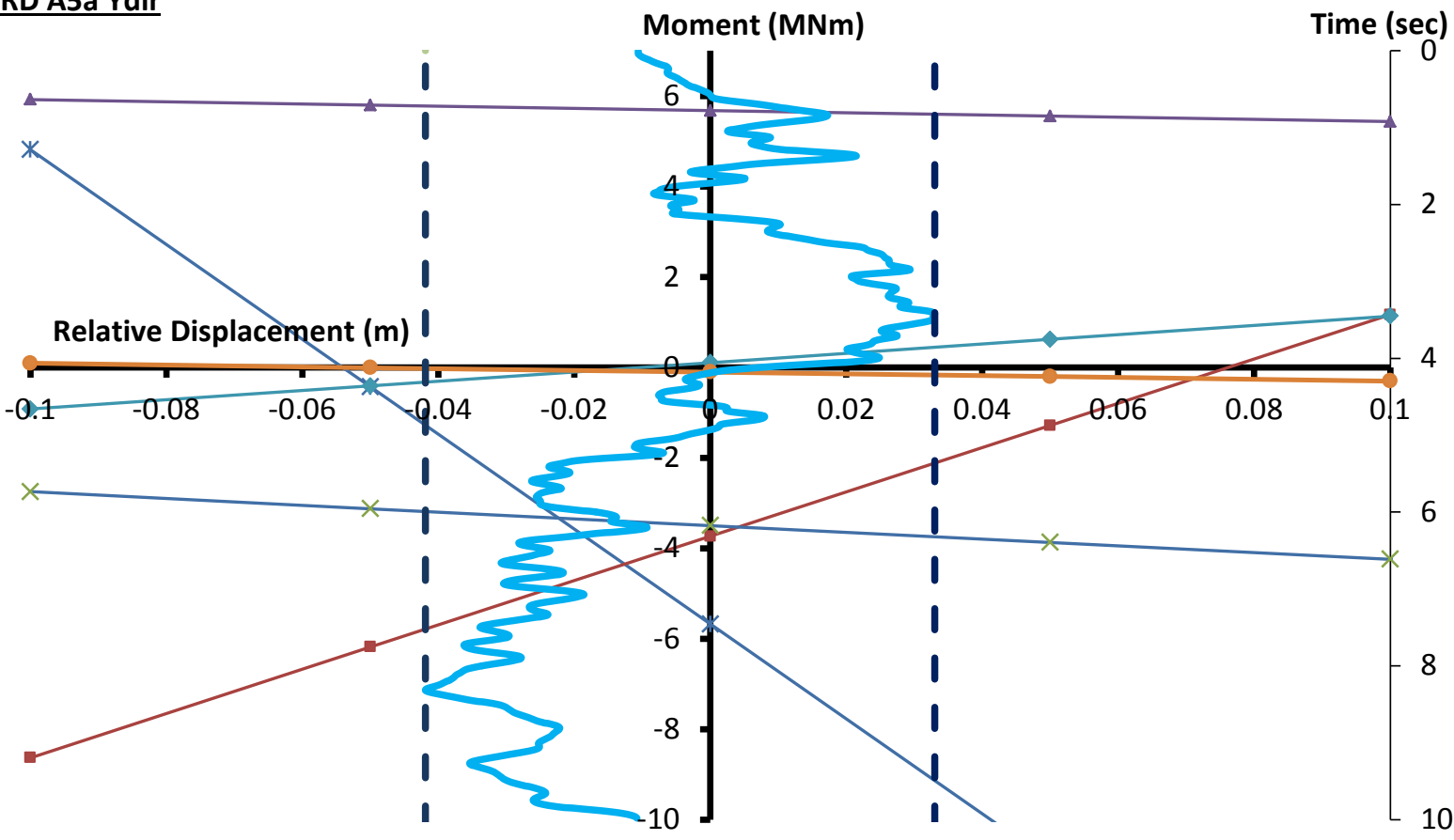

$*$ Node 1
$\longrightarrow$ Node 62 Node 21

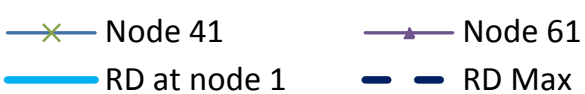




\section{$\underline{\text { RD A5b Ydir }}$}

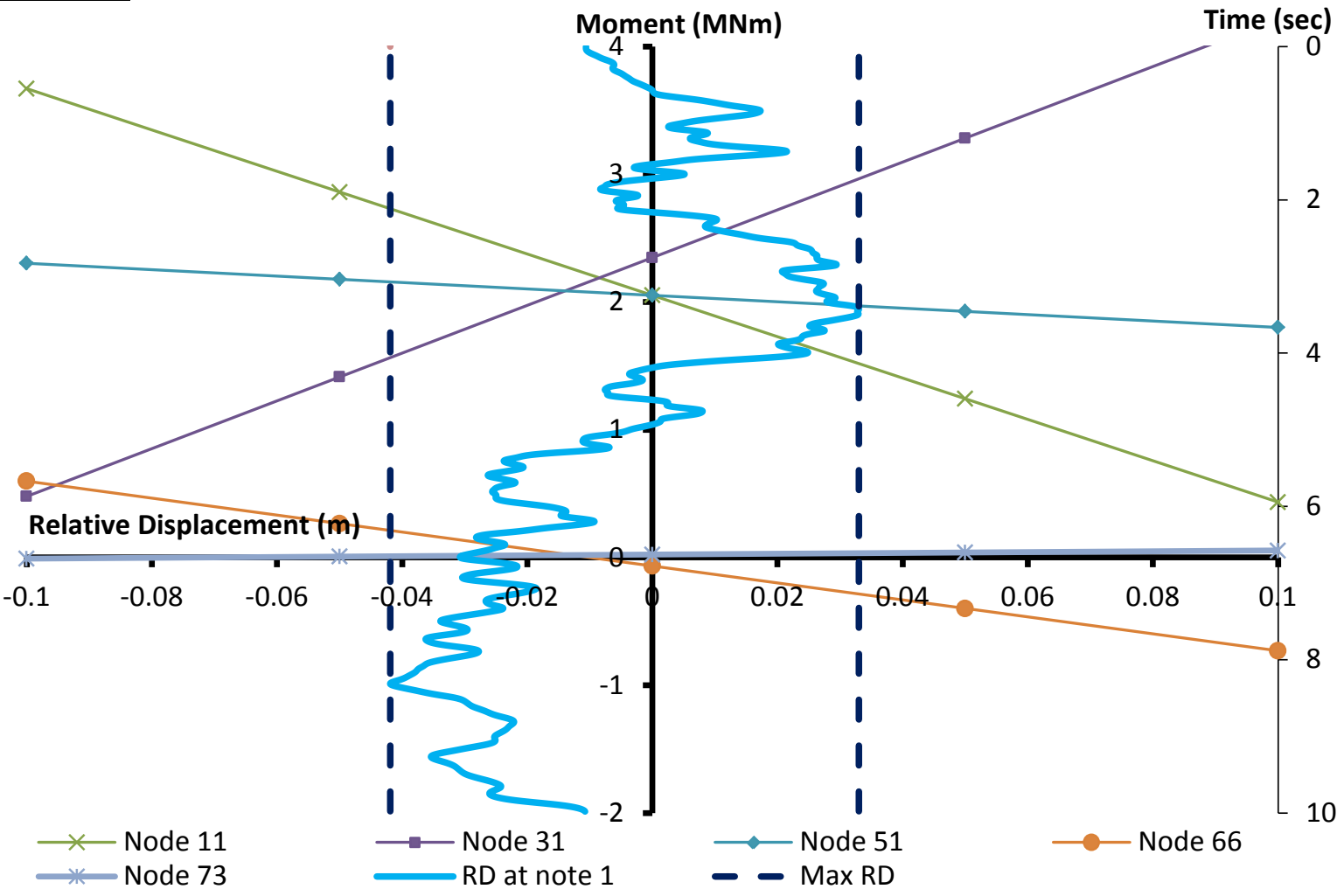

$\underline{\text { RD A6a Ydir }}$

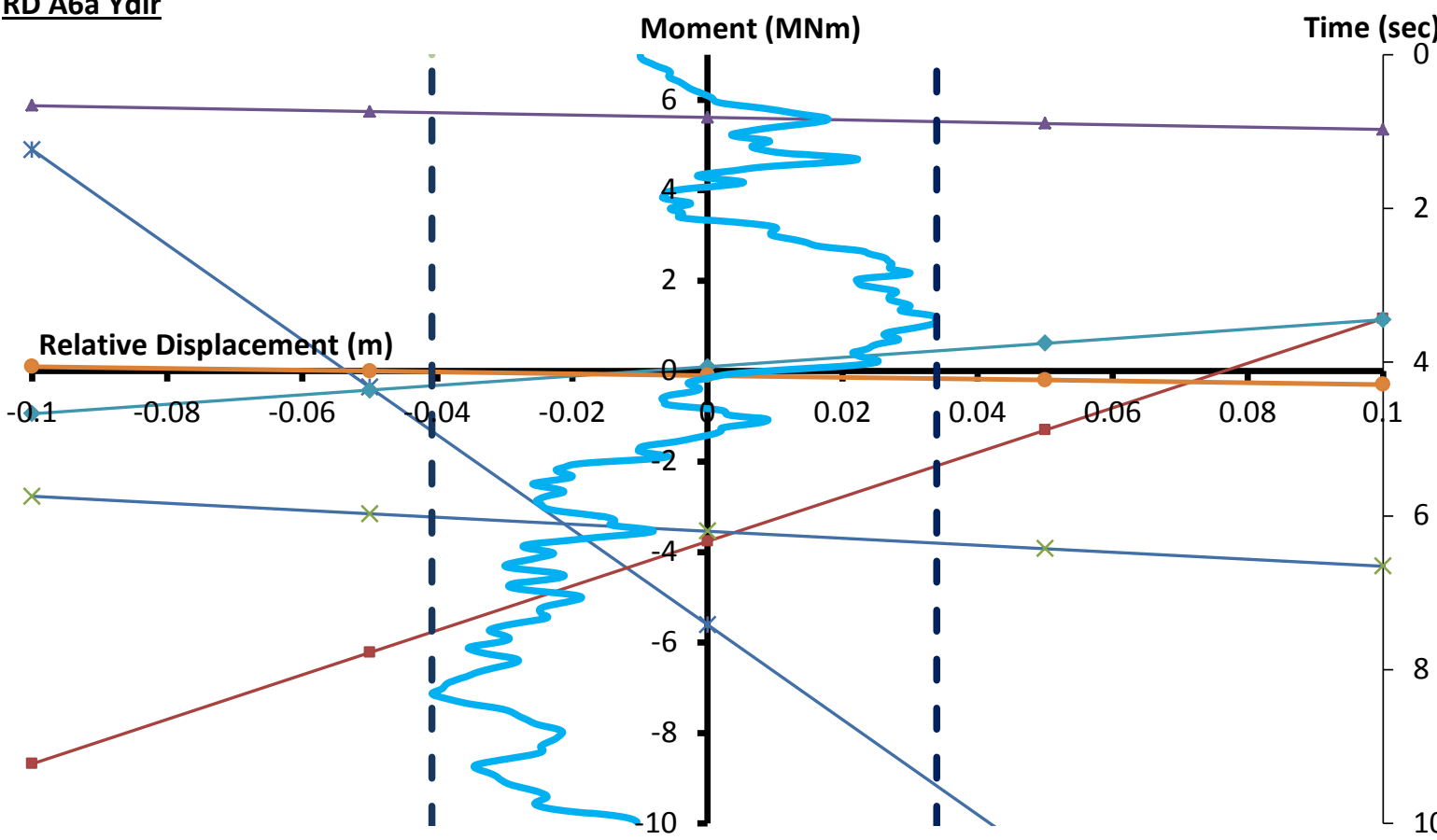

$\begin{array}{llll}* \text { Node } 1 & \longrightarrow \text { Node } 21 & \text { Node } 41 & \text { Node } 61 \\ \longrightarrow \text { Node } 62 & \text { RD at node } 1 \quad \text { Node } 70 & \text { RD Max }\end{array}$ 


\section{RD A6b Ydir}

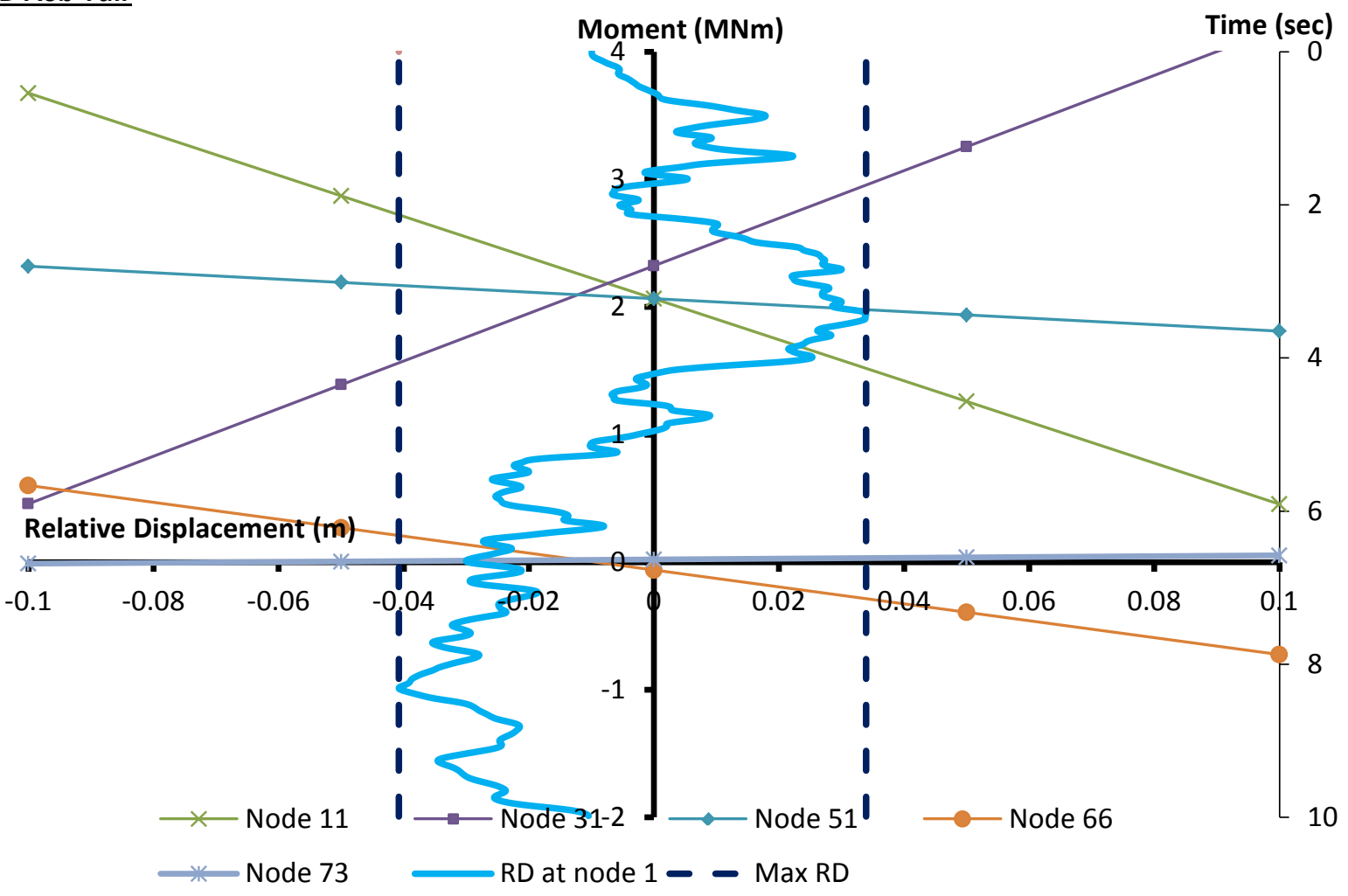


$\underline{\text { RD D1a Xdir }}$

Moment (MNm)

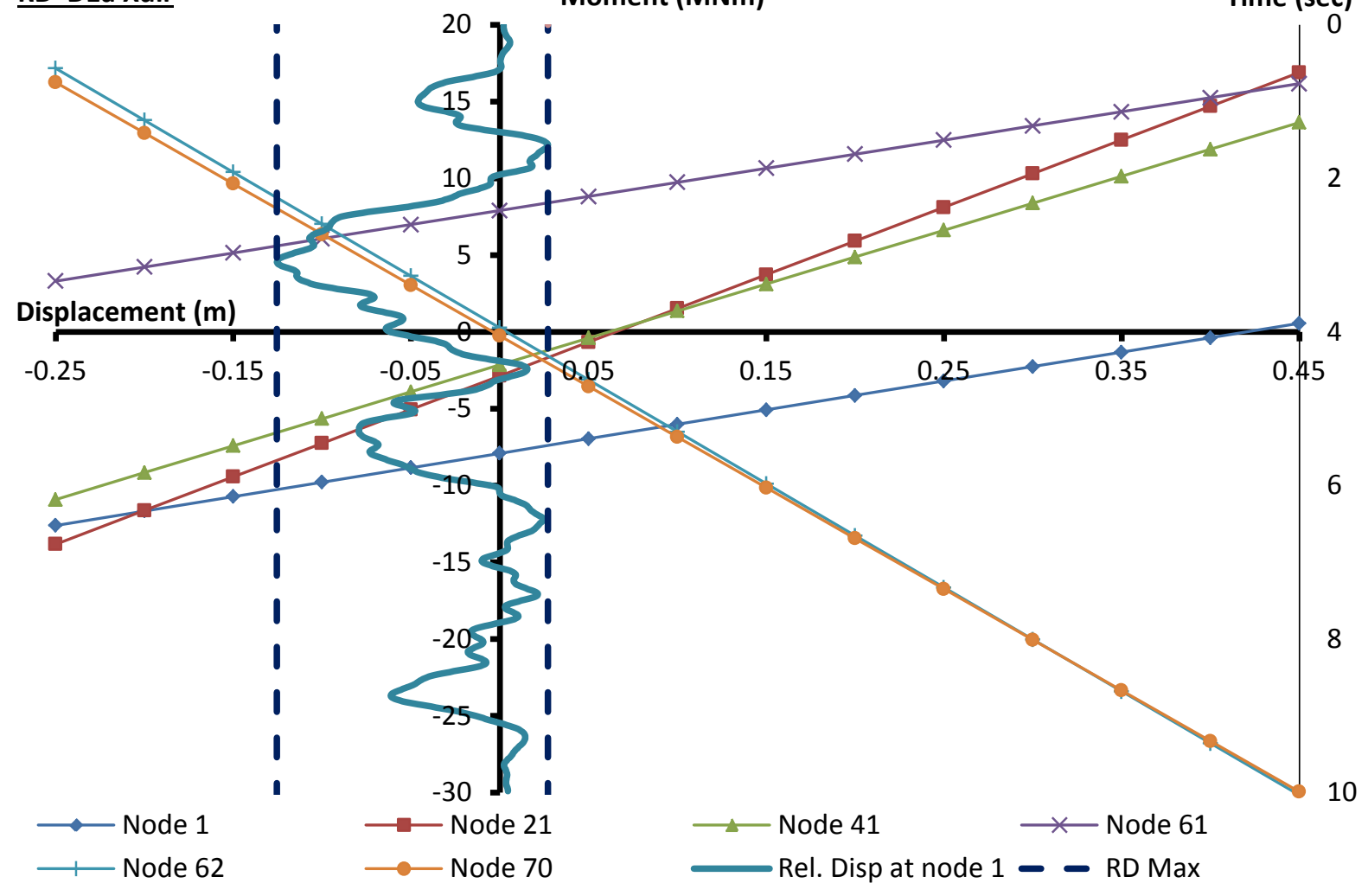

\section{$\underline{\text { RD D1b Xdir }}$}

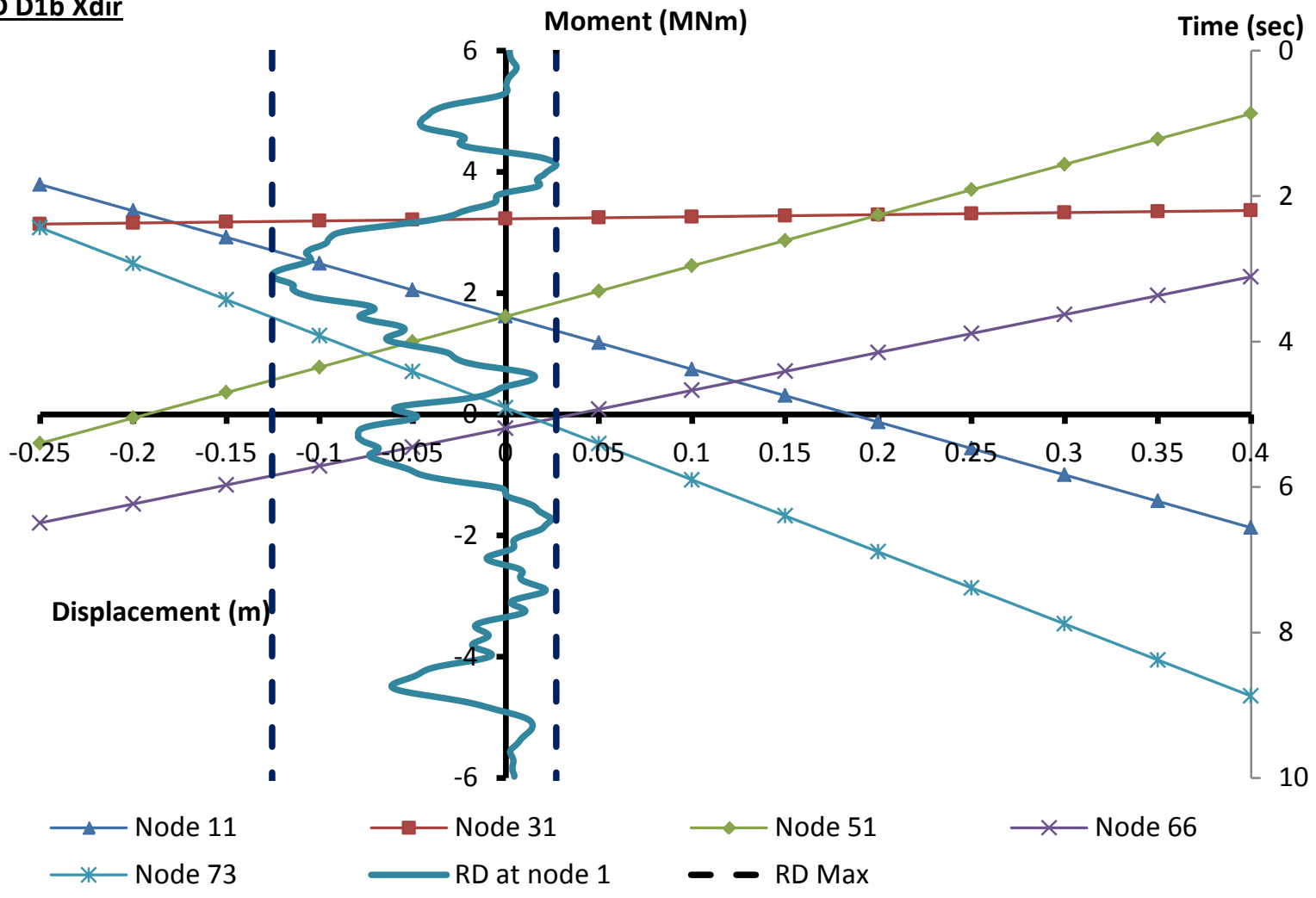



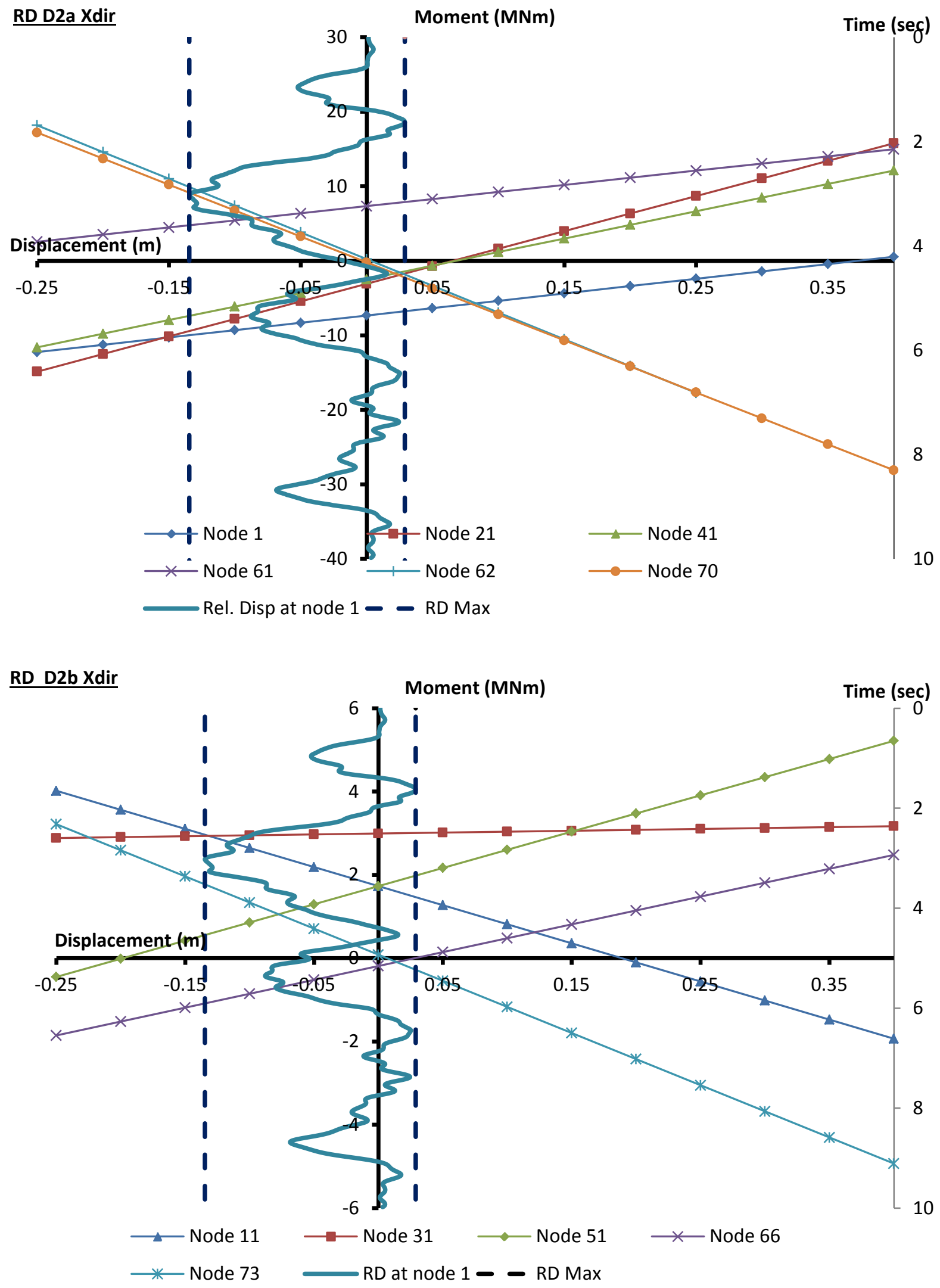
Relative Displacement-Moment relationships

Fixed Based Support
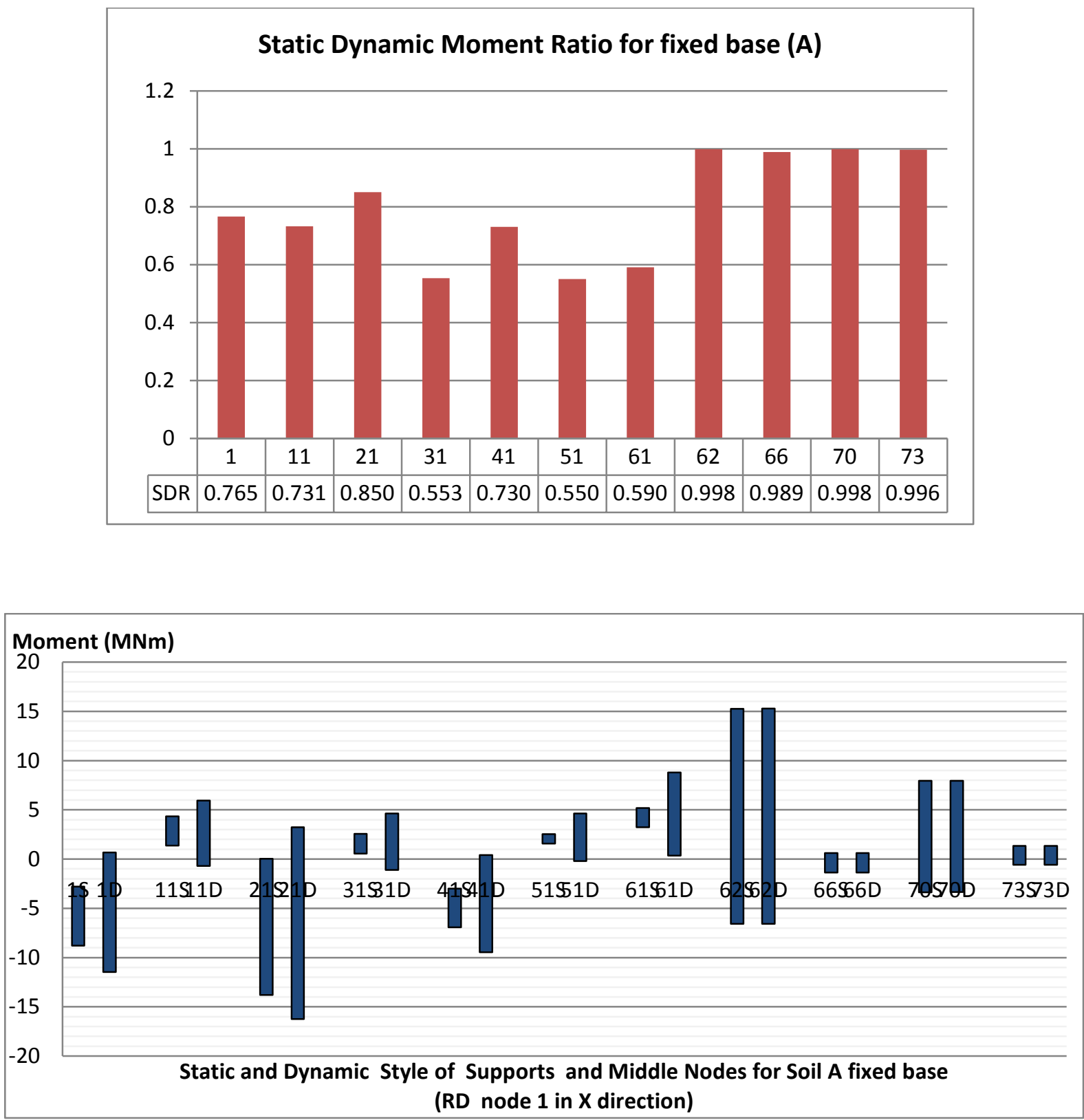

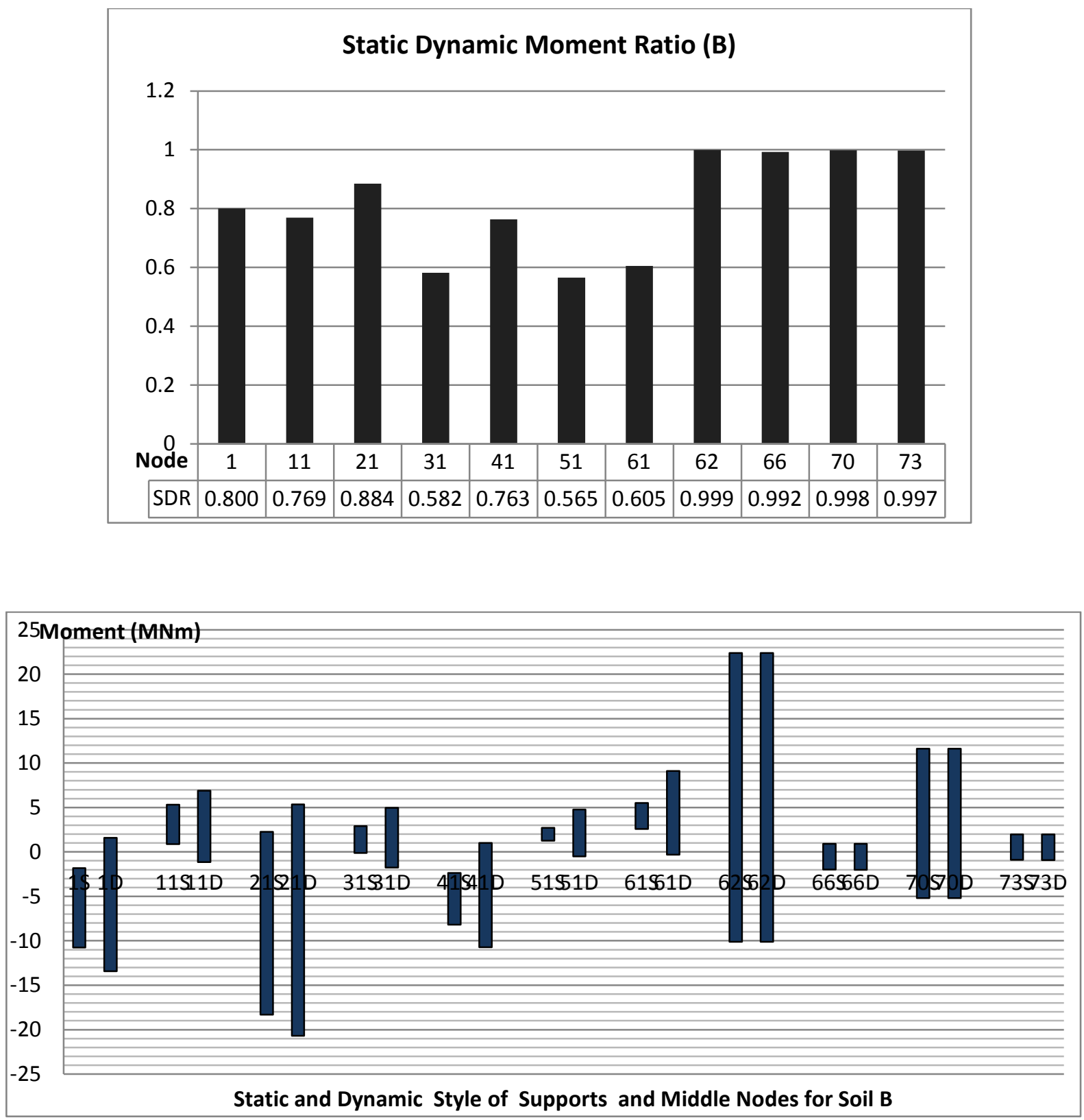

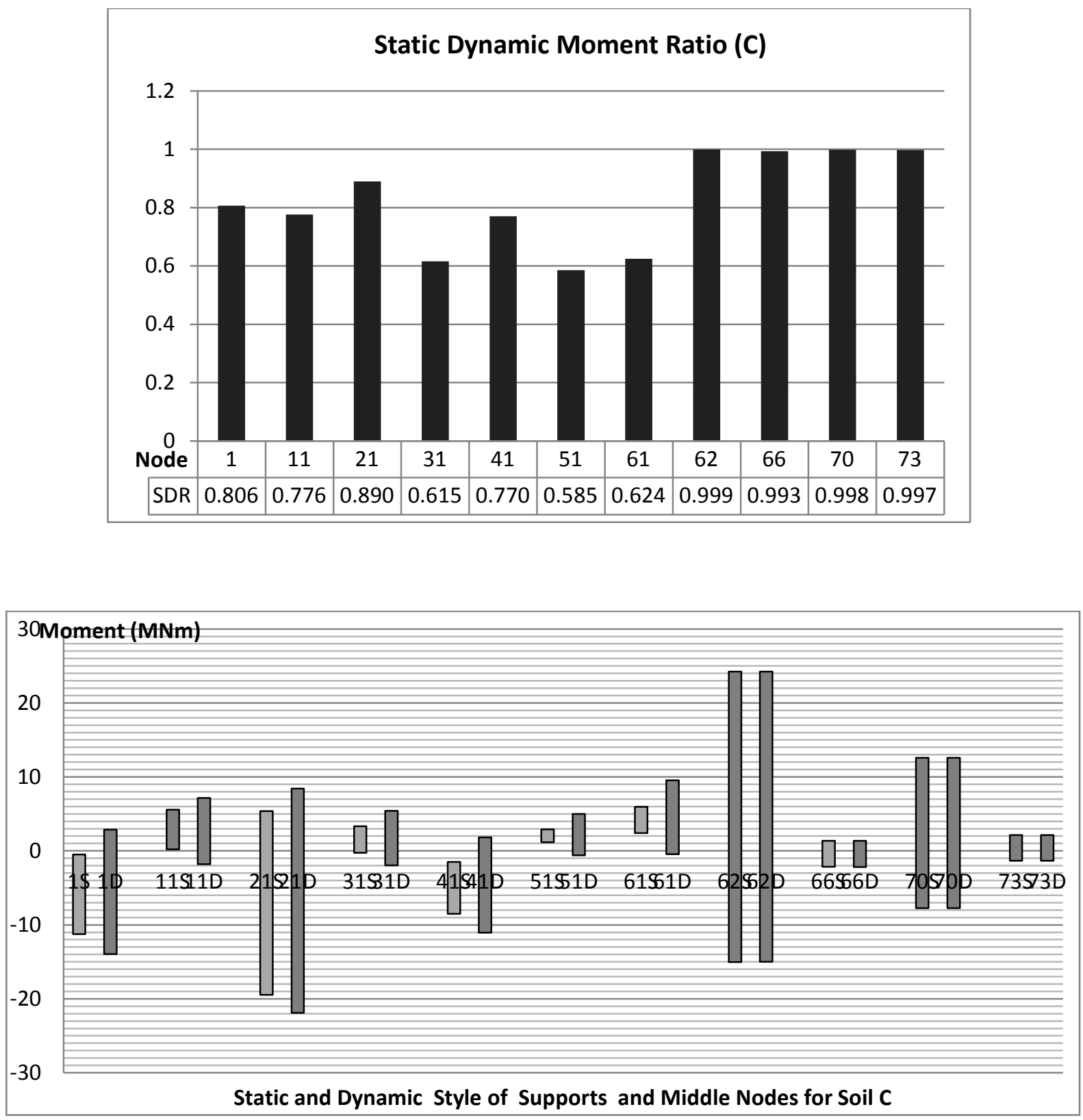

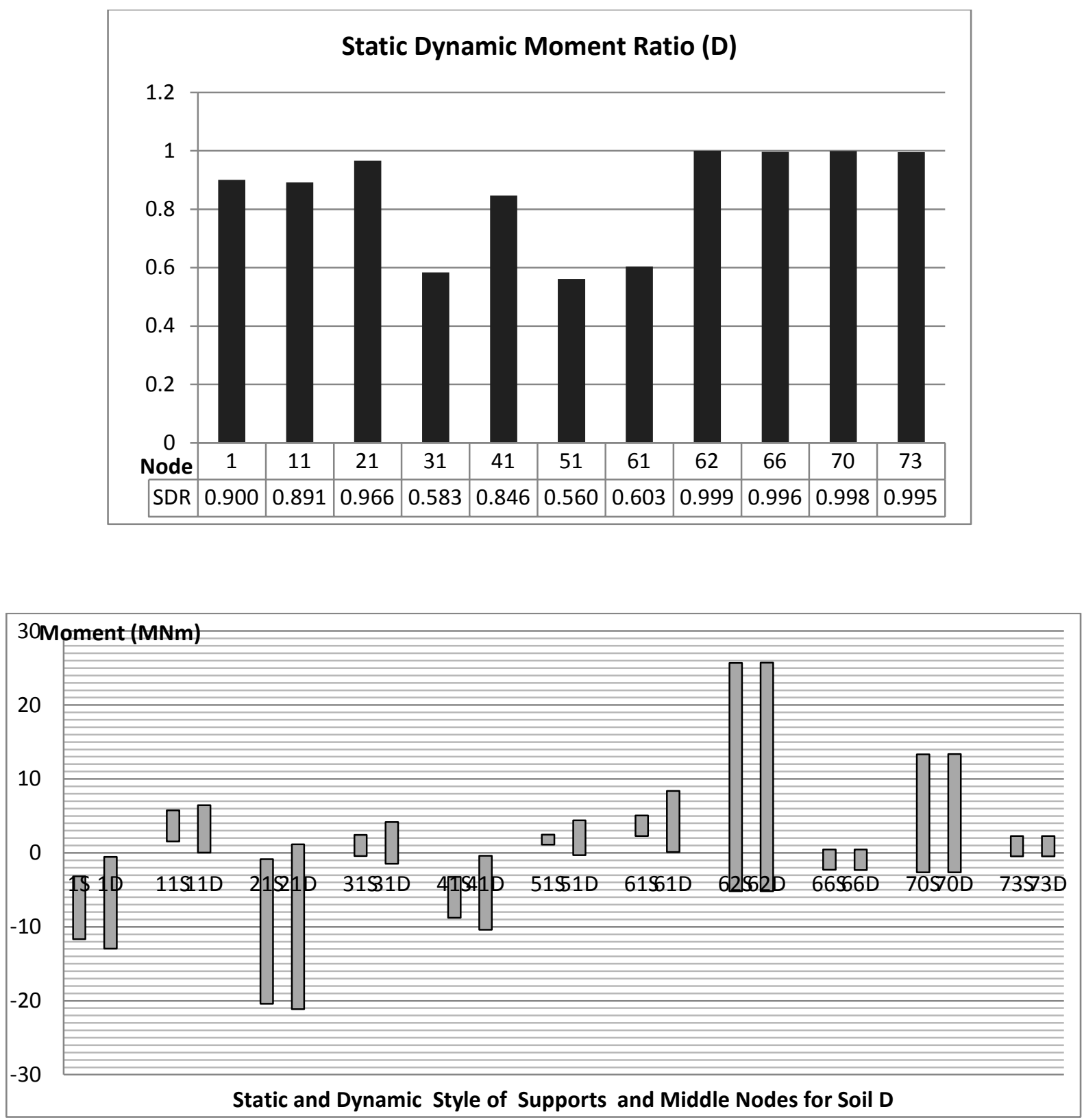


\section{For Vertical Direction}
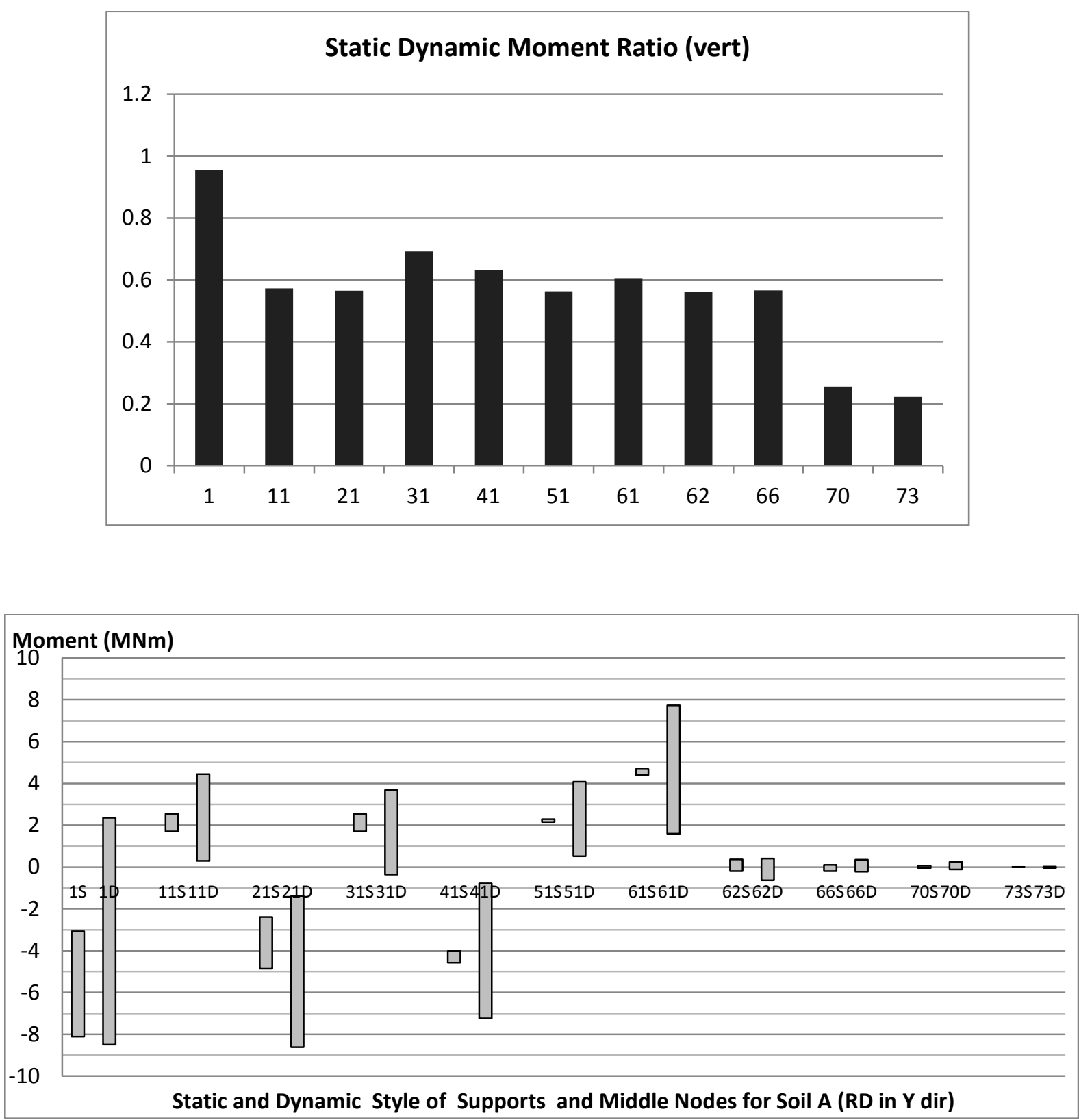
Springs based supports
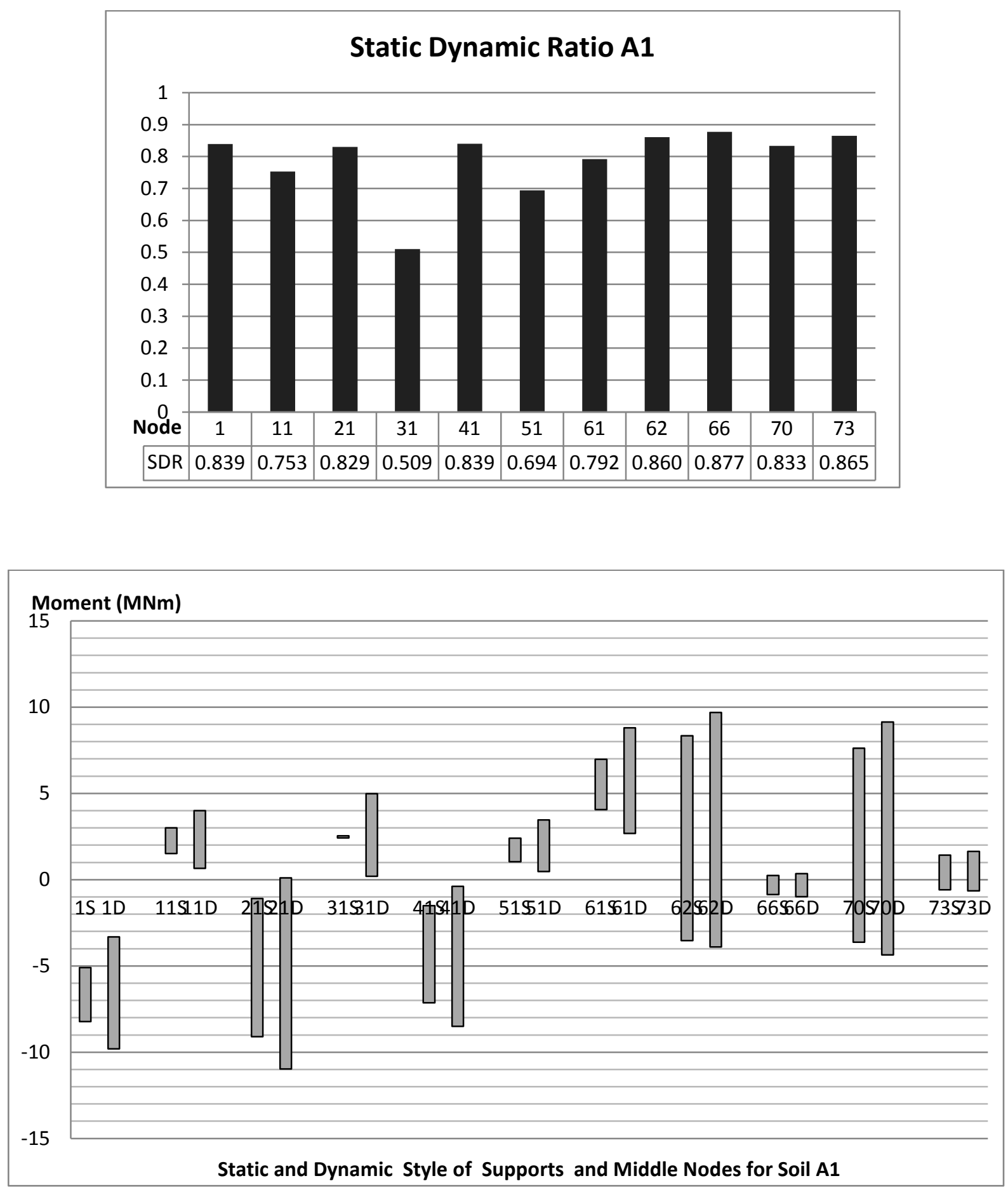

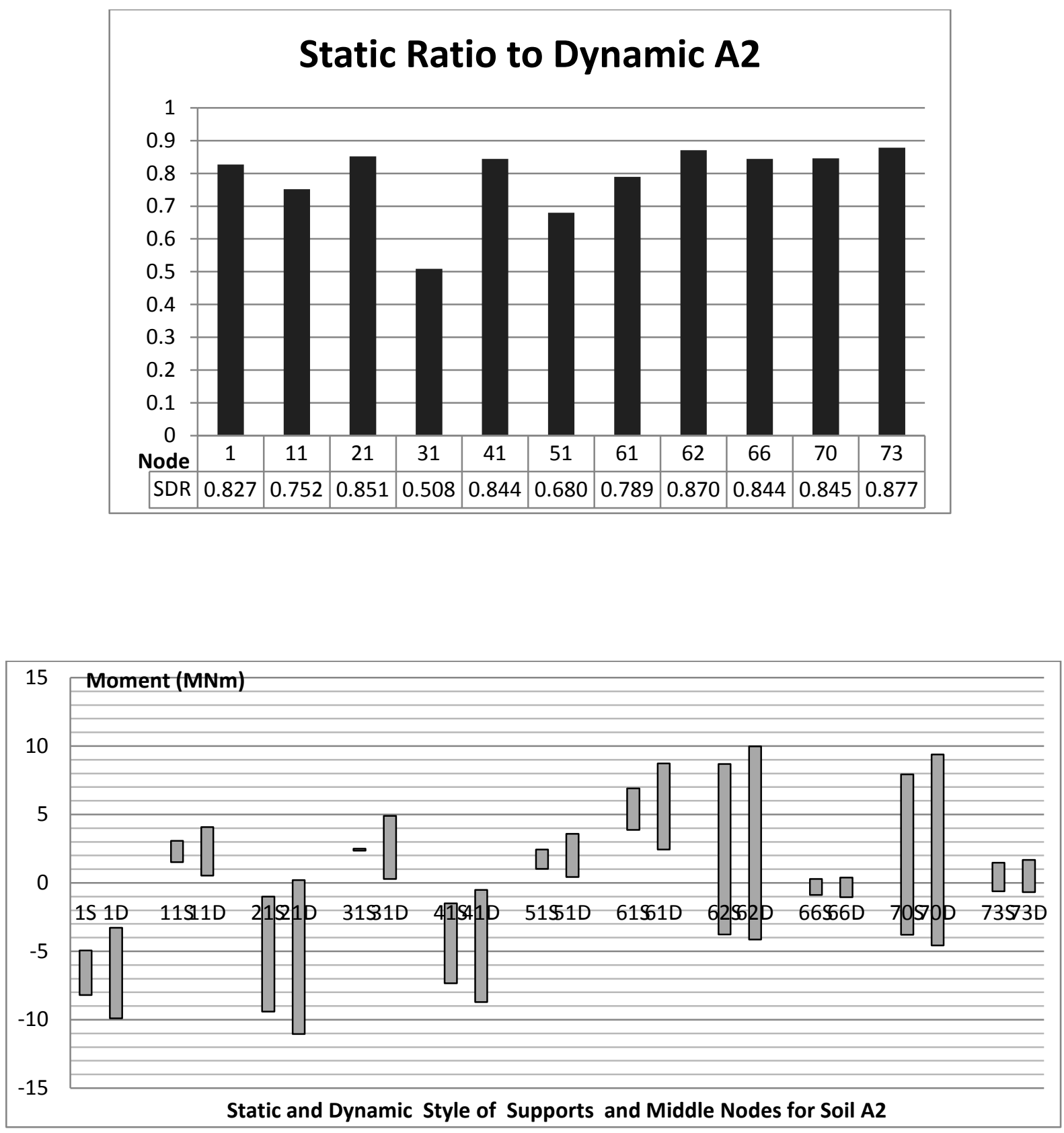

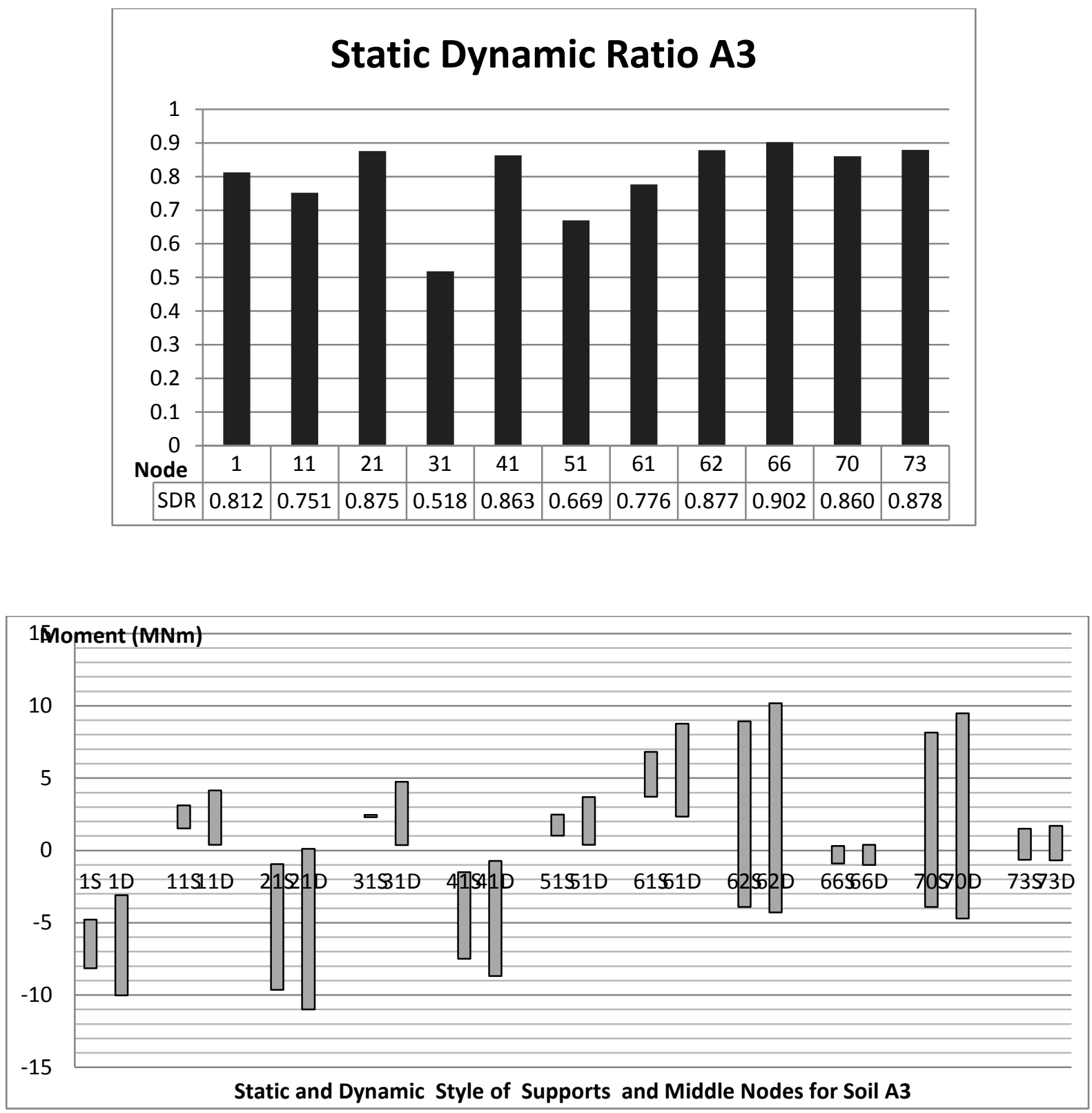

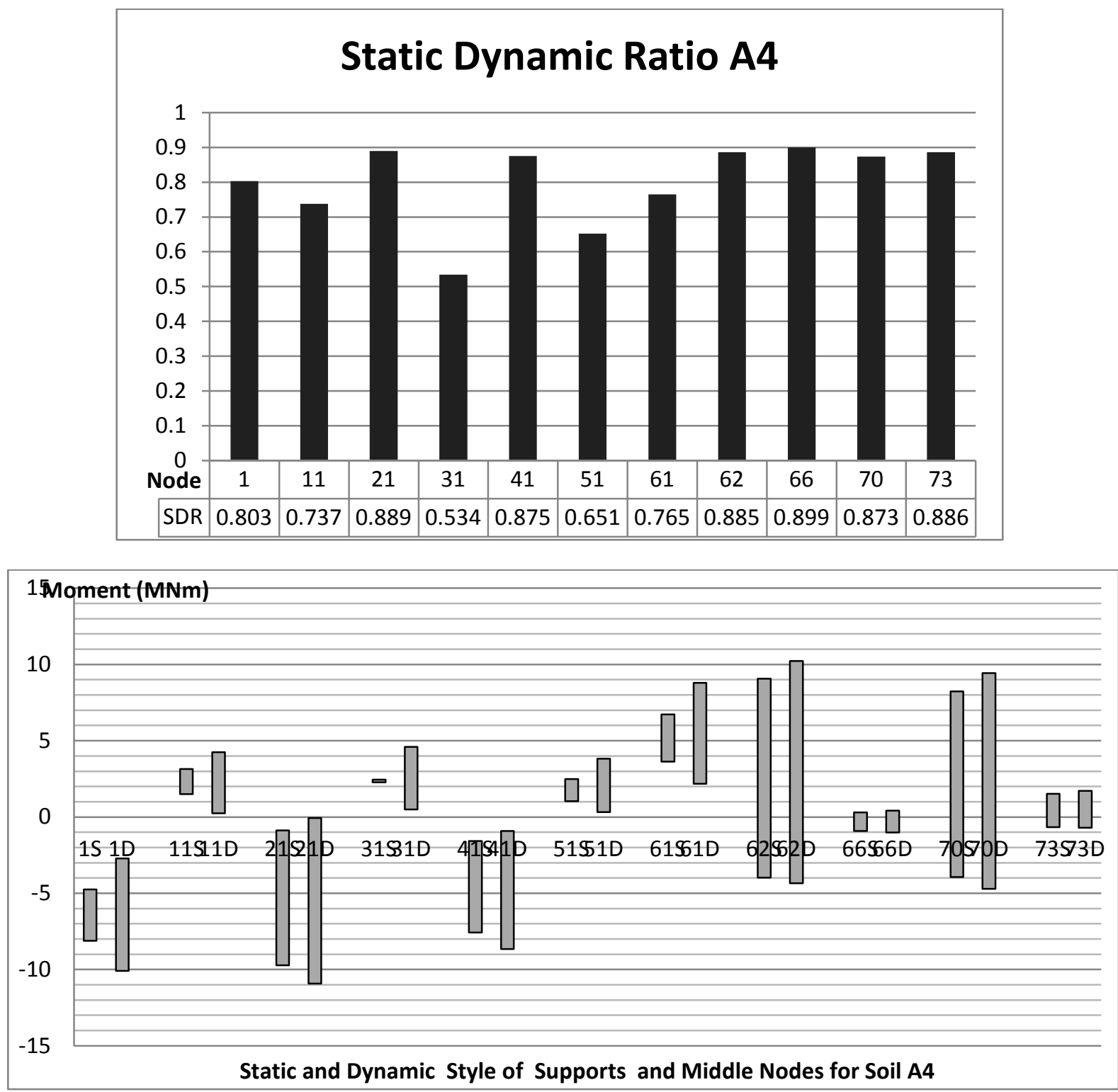

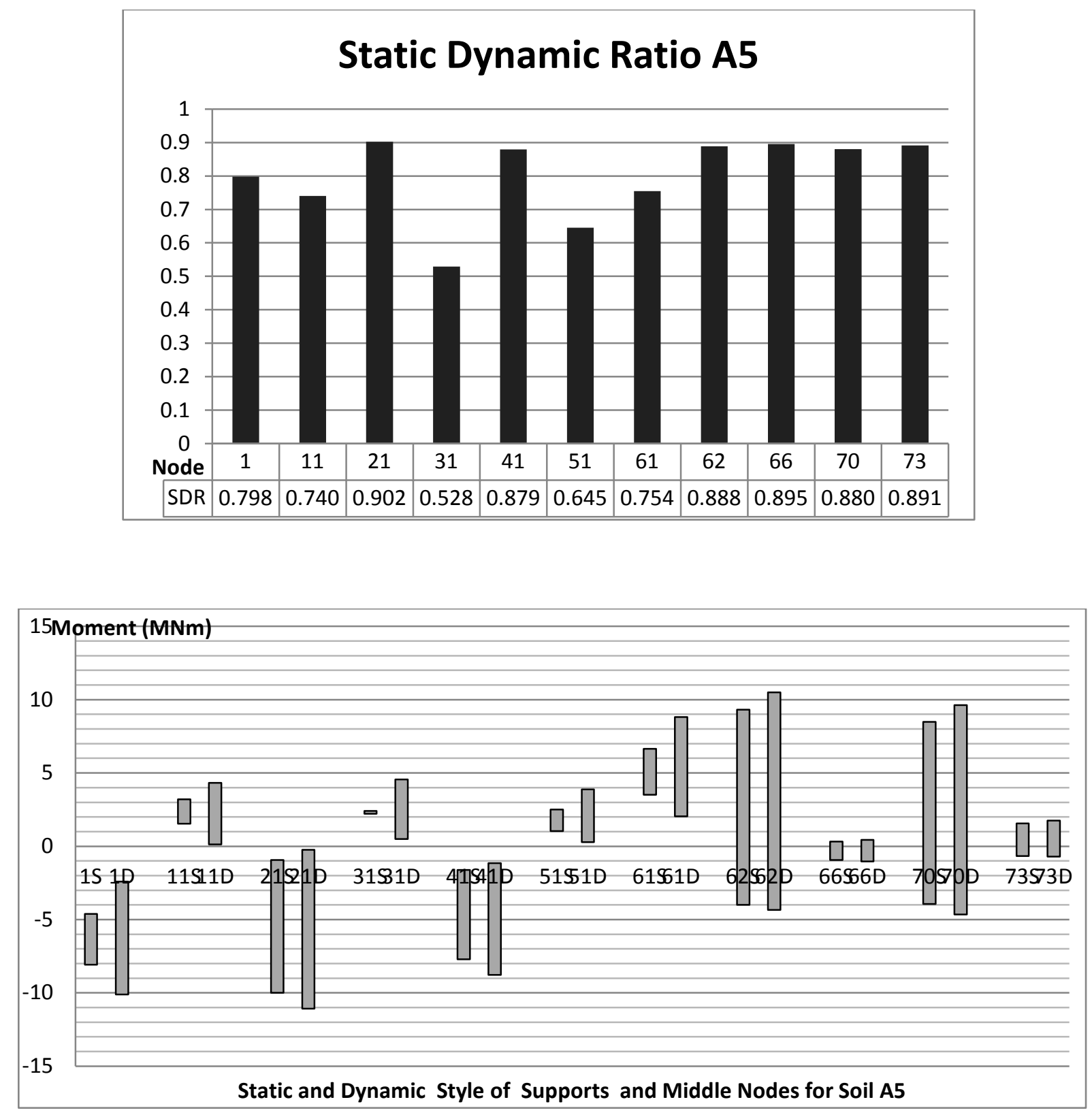

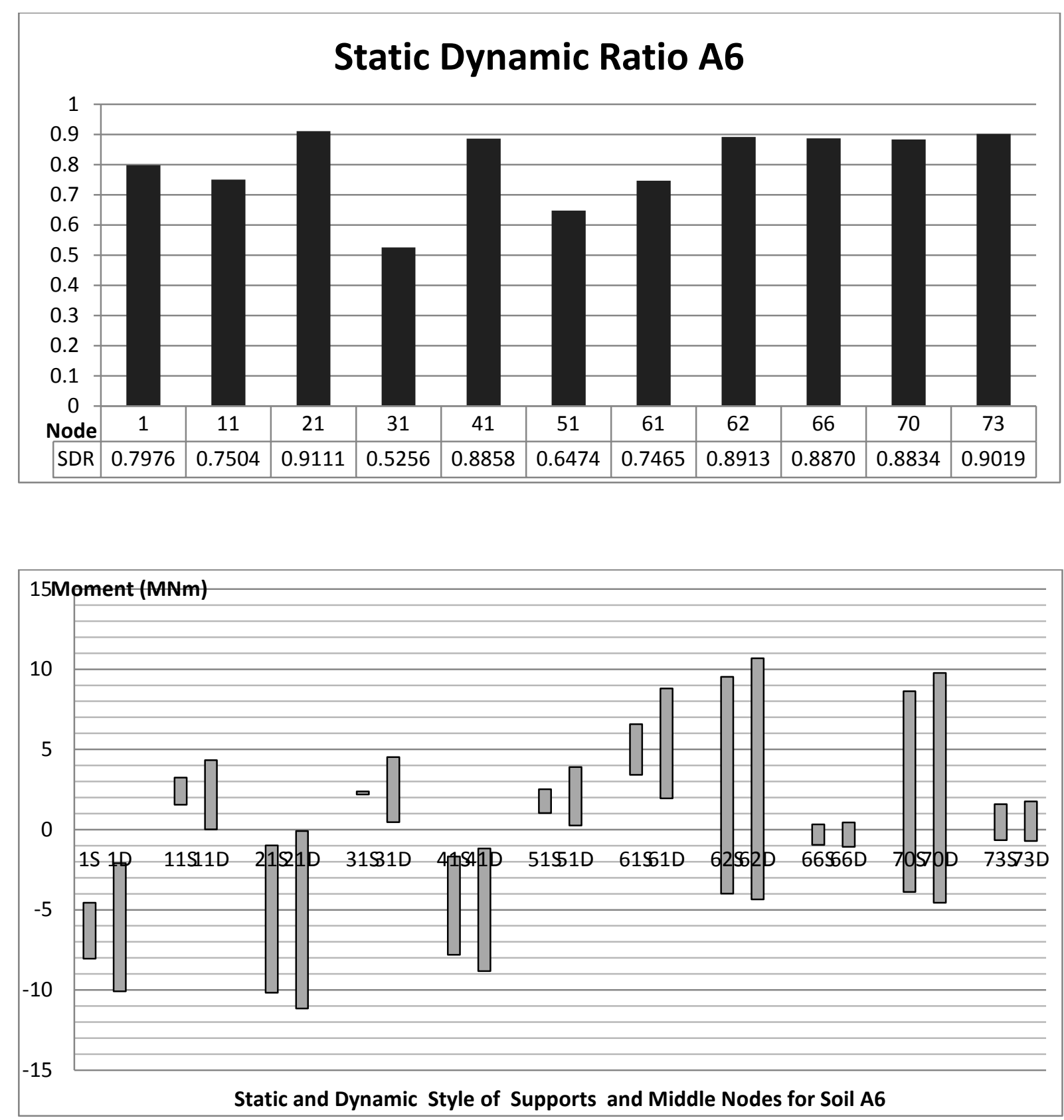


\section{Data of Acceleration Response Spectrum of 5 Soil Types}

\begin{tabular}{|c|c|c|c|c|c|c|c|c|c|c|c|c|c|c|c|}
\hline \multirow{2}{*}{ Time } & \multicolumn{15}{|c|}{ Response Spectrum (Acceleration) } \\
\hline & A1 & $A 2$ & A3 & B1 & B2 & B3 & C1 & $\mathrm{C} 2$ & C3 & D1 & D2 & D3 & E1 & $\mathrm{E} 2$ & E3 \\
\hline 0 & 3.368 & 3.761 & 4.277 & 4.088 & 5.083 & 5.260 & 3.971 & 5.083 & 4.483 & 4.820 & 5.336 & 5.841 & 4.770 & 5.240 & 5.496 \\
\hline 0.02 & 3.912 & 4.192 & 4.416 & 4.344 & 5.238 & 5.307 & 4.237 & 5.178 & 4.701 & 5.018 & 5.571 & 5.925 & 5.068 & 8.173 & 5.978 \\
\hline 0.04 & 5.077 & 4.938 & 4.906 & 6.048 & 5.828 & 5.872 & 5.279 & 5.276 & 5.004 & 6.274 & 5.943 & 5.760 & 7.056 & 7.086 & 6.431 \\
\hline 0.06 & 5.557 & 5.651 & 5.442 & 6.787 & 6.808 & 6.593 & 5.861 & 5.937 & 5.764 & 6.724 & 6.739 & 6.267 & 7.918 & 7.795 & 7.864 \\
\hline 0.08 & 6.187 & 6.360 & 6.258 & 7.472 & 7.376 & 7.493 & 6.469 & 6.470 & 6.384 & 7.594 & 7.443 & 7.195 & 8.717 & 9.141 & 8.621 \\
\hline 0.1 & 7.325 & 6.967 & 7.084 & 8.723 & 8.127 & 8.452 & 7.269 & 6.961 & 6.907 & 8.554 & 7.880 & 8.297 & 10.177 & 9.930 & 9.366 \\
\hline 0.12 & 7.449 & 7.735 & 7.930 & 8.837 & 9.103 & 9.390 & 7.316 & 7.266 & 7.653 & 8.644 & 8.946 & 8.980 & 10.310 & 10.367 & 10.746 \\
\hline 0.14 & 7.807 & 8.284 & 7.883 & 9.300 & 9.978 & 9.471 & 7.679 & 8.284 & 8.324 & 9.023 & 9.778 & 9.416 & 10.850 & 11.984 & 11.907 \\
\hline 0.16 & 8.887 & 8.308 & 8.977 & 10.703 & 10.044 & 10.808 & 8.959 & 8.678 & 9.029 & 10.429 & 10.584 & 10.233 & 12.487 & 12.263 & 12.173 \\
\hline 0.18 & 8.948 & 8.745 & 8.829 & 10.388 & 10.439 & 10.200 & 9.217 & 9.466 & 9.448 & 11.111 & 10.929 & 11.236 & 12.120 & 12.398 & 12.669 \\
\hline 0.2 & 8.954 & 8.516 & 8.997 & 10.724 & 10.660 & 10.725 & 10.042 & 10.005 & 9.688 & 11.864 & 11.286 & 10.919 & 12.512 & 11.317 & 12.003 \\
\hline 0.22 & 8.459 & 8.461 & 8.606 & 10.190 & 10.219 & 10.323 & 9.775 & 9.849 & 10.102 & 11.579 & 11.935 & 11.733 & 11.888 & 12.626 & 11.612 \\
\hline 0.24 & 8.698 & 8.518 & 9.017 & 10.408 & 10.195 & 10.711 & 9.913 & 9.771 & 10.023 & 11.679 & 11.876 & 11.248 & 12.143 & 12.520 & 11.801 \\
\hline 0.26 & 8.936 & 8.486 & 8.416 & 10.564 & 10.206 & 10.028 & 10.211 & 9.734 & 9.949 & 11.908 & 11.585 & 11.695 & 12.324 & 12.374 & 11.825 \\
\hline 0.28 & 8.725 & 8.690 & 8.512 & 10.379 & 10.192 & 10.400 & 9.956 & 9.677 & 9.639 & 11.832 & 11.347 & 11.599 & 12.109 & 11.593 & 12.316 \\
\hline 0.3 & 8.468 & 8.769 & 8.857 & 10.018 & 10.405 & 10.542 & 9.516 & 9.942 & 10.115 & 11.190 & 11.917 & 12.775 & 11.687 & 12.065 & 11.837 \\
\hline 0.32 & 8.750 & 8.395 & 9.271 & 10.424 & 10.036 & 11.040 & 9.983 & 9.823 & 9.816 & 11.815 & 11.512 & 11.368 & 12.161 & 12.327 & 12.234 \\
\hline 0.34 & 8.473 & 8.677 & 8.565 & 10.129 & 10.415 & 10.076 & 9.726 & 9.868 & 10.081 & 11.467 & 11.945 & 11.195 & 11.817 & 12.595 & 12.166 \\
\hline 0.36 & 8.714 & 8.977 & 8.749 & 10.440 & 10.881 & 10.684 & 10.002 & 10.485 & 9.960 & 11.755 & 11.778 & 11.134 & 12.180 & 12.117 & 12.532 \\
\hline 0.38 & 8.887 & 9.047 & 8.768 & 10.654 & 10.838 & 10.428 & 10.214 & 10.539 & 10.145 & 12.003 & 11.906 & 11.183 & 12.430 & 11.882 & 12.232 \\
\hline 0.4 & 8.683 & 8.705 & 8.540 & 10.387 & 10.493 & 10.196 & 10.203 & 10.255 & 10.051 & 11.980 & 11.830 & 12.587 & 12.118 & 12.114 & 12.203 \\
\hline 0.42 & 8.058 & 8.256 & 8.450 & 10.146 & 10.289 & 10.482 & 9.482 & 9.941 & 9.805 & 11.159 & 11.617 & 10.954 & 11.837 & 11.826 & 12.632 \\
\hline 0.44 & 8.287 & 7.635 & 7.981 & 10.519 & 9.994 & 10.315 & 10.564 & 9.464 & 10.136 & 12.242 & 11.605 & 11.356 & 12.272 & 11.886 & 12.100 \\
\hline 0.46 & 6.963 & 7.428 & 7.192 & 10.124 & 10.317 & 10.208 & 9.190 & 9.877 & 9.945 & 11.845 & 11.817 & 11.830 & 11.811 & 12.298 & 11.866 \\
\hline 0.48 & 7.059 & 7.378 & 7.201 & 10.250 & 10.577 & 10.168 & 9.764 & 10.147 & 10.033 & 11.327 & 11.765 & 11.457 & 11.958 & 11.875 & 12.505 \\
\hline 0.5 & 7.071 & 7.097 & 6.859 & 10.262 & 10.417 & 10.216 & 10.212 & 10.099 & 9.807 & 11.813 & 11.517 & 11.459 & 11.973 & 12.344 & 11.963 \\
\hline 0.52 & 7.014 & 6.672 & 6.708 & 10.171 & 9.968 & 9.973 & 10.475 & 10.055 & 9.671 & 12.143 & 11.359 & 12.014 & 11.866 & 11.683 & 11.495 \\
\hline 0.54 & 6.795 & 6.281 & 6.372 & 9.888 & 9.553 & 9.635 & 10.494 & 9.926 & 9.794 & 12.235 & 11.555 & 12.282 & 11.535 & 11.461 & 11.069 \\
\hline 0.56 & 6.372 & 5.965 & 6.136 & 9.361 & 9.188 & 9.270 & 10.186 & 9.757 & 10.159 & 12.032 & 11.853 & 11.678 & 10.921 & 10.818 & 10.672 \\
\hline 0.58 & 5.818 & 5.768 & 5.873 & 8.657 & 8.840 & 8.860 & 9.634 & 9.582 & 10.938 & 11.521 & 12.485 & 13.049 & 10.100 & 10.834 & 10.039 \\
\hline 0.6 & 5.542 & 5.603 & 5.647 & 7.928 & 8.513 & 8.520 & 9.564 & 9.411 & 10.351 & 10.870 & 11.966 & 12.896 & 9.250 & 10.397 & 10.332 \\
\hline 0.62 & 5.847 & 5.459 & 5.502 & 8.507 & 8.227 & 8.289 & 10.028 & 9.241 & 9.228 & 11.677 & 11.008 & 11.522 & 9.924 & 10.047 & 9.985 \\
\hline 0.64 & 5.553 & 5.329 & 5.375 & 8.268 & 7.978 & 8.089 & 9.645 & 9.066 & 9.343 & 11.759 & 11.695 & 11.784 & 9.646 & 9.596 & 9.476 \\
\hline 0.66 & 5.086 & 5.205 & 5.397 & 7.759 & 7.756 & 8.120 & 8.919 & 8.880 & 9.511 & 11.670 & 12.279 & 12.084 & 9.052 & 9.043 & 8.745 \\
\hline 0.68 & 5.520 & 5.089 & 5.371 & 7.747 & 7.546 & 8.121 & 9.201 & 8.677 & 9.390 & 11.721 & 12.506 & 11.424 & 9.038 & 8.409 & 7.564 \\
\hline 0.7 & 5.575 & 4.965 & 5.003 & 7.946 & 7.344 & 7.646 & 9.356 & 8.452 & 8.872 & 12.170 & 12.165 & 11.275 & 9.271 & 8.121 & 7.693 \\
\hline 0.72 & 5.059 & 4.824 & 4.970 & 7.208 & 7.142 & 7.199 & 8.555 & 8.203 & 8.104 & 11.374 & 11.334 & 11.748 & 8.409 & 8.473 & 8.546 \\
\hline 0.74 & 4.405 & 4.665 & 4.801 & 6.863 & 6.921 & 6.995 & 7.591 & 7.934 & 7.276 & 11.818 & 10.155 & 11.866 & 8.007 & 8.808 & 8.382 \\
\hline 0.76 & 4.279 & 4.497 & 4.824 & 6.609 & 6.672 & 7.091 & 7.426 & 7.683 & 7.373 & 11.669 & 11.015 & 12.116 & 7.710 & 8.827 & 8.208 \\
\hline
\end{tabular}




\begin{tabular}{|c|c|c|c|c|c|c|c|c|c|c|c|c|c|c|c|}
\hline 0.78 & 4.002 & 4.305 & 4.707 & 6.242 & 6.413 & 6.924 & 7.023 & 7.670 & 8.003 & 11.060 & 12.056 & 11.209 & 7.283 & 8.673 & 7.799 \\
\hline 0.8 & 3.753 & 4.199 & 4.436 & 5.919 & 6.381 & 6.556 & 6.619 & 7.608 & 7.914 & 10.315 & 12.073 & 11.919 & 6.905 & 8.342 & 7.478 \\
\hline 0.82 & 4.111 & 4.180 & 4.339 & 5.926 & 6.342 & 6.212 & 7.009 & 7.494 & 7.341 & 10.797 & 11.417 & 12.128 & 6.914 & 7.795 & 7.183 \\
\hline 0.84 & 4.368 & 4.128 & 4.267 & 6.418 & 6.264 & 6.382 & 7.470 & 7.345 & 6.810 & 11.495 & 10.526 & 11.801 & 7.488 & 7.078 & 7.283 \\
\hline 0.86 & 4.452 & 4.034 & 4.412 & 6.614 & 6.139 & 6.623 & 7.653 & 7.161 & 6.604 & 11.816 & 10.167 & 11.014 & 7.717 & 6.638 & 7.278 \\
\hline 0.88 & 4.291 & 3.937 & 4.275 & 6.422 & 5.996 & 6.429 & 7.410 & 6.969 & 6.516 & 11.497 & 10.103 & 9.879 & 7.492 & 6.248 & 7.062 \\
\hline 0.9 & 3.987 & 3.856 & 3.971 & 5.988 & 5.866 & 5.982 & 6.873 & 6.780 & 6.575 & 10.694 & 10.257 & 9.645 & 6.986 & 6.382 & 6.711 \\
\hline 0.92 & 3.735 & 3.783 & 3.657 & 5.603 & 5.736 & 5.509 & 6.382 & 6.587 & 6.714 & 9.923 & 10.541 & 10.422 & 6.537 & 6.389 & 6.373 \\
\hline 0.94 & 3.636 & 3.712 & 3.444 & 5.441 & 5.602 & 5.191 & 6.155 & 6.388 & 6.865 & 9.536 & 10.811 & 10.636 & 6.348 & 6.517 & 6.285 \\
\hline 0.96 & 3.582 & 3.621 & 3.364 & 5.376 & 5.450 & 5.067 & 6.056 & 6.167 & 6.946 & 9.364 & 10.958 & 10.480 & 6.272 & 6.617 & 6.118 \\
\hline 0.98 & 3.408 & 3.515 & 3.366 & 5.165 & 5.267 & 5.069 & 5.807 & 5.928 & 6.917 & 8.988 & 10.932 & 10.080 & 6.026 & 6.671 & 5.898 \\
\hline 1 & 3.473 & 3.390 & 3.378 & 5.135 & 5.059 & 5.089 & 5.977 & 5.666 & 6.759 & 9.276 & 10.693 & 9.562 & 5.991 & 6.679 & 5.958 \\
\hline 1.02 & 3.577 & 3.248 & 3.352 & 5.318 & 4.834 & 5.050 & 6.125 & 5.390 & 6.493 & 9.590 & 10.337 & 9.079 & 6.204 & 6.631 & 6.028 \\
\hline 1.04 & 3.485 & 3.091 & 3.276 & 5.318 & 4.590 & 4.925 & 6.087 & 5.351 & 6.323 & 9.576 & 10.013 & 8.704 & 6.205 & 6.519 & 6.057 \\
\hline 1.06 & 3.378 & 3.135 & 3.165 & 5.186 & 4.728 & 4.742 & 5.969 & 5.614 & 6.043 & 9.331 & 9.535 & 8.484 & 6.050 & 6.342 & 6.022 \\
\hline 1.08 & 3.294 & 3.209 & 3.030 & 4.994 & 4.838 & 4.519 & 5.784 & 5.681 & 5.693 & 8.987 & 8.955 & 8.408 & 5.826 & 6.113 & 5.924 \\
\hline 1.1 & 3.187 & 3.169 & 3.082 & 4.783 & 4.773 & 4.632 & 5.564 & 5.566 & 5.330 & 8.616 & 8.363 & 8.432 & 5.581 & 5.846 & 5.779 \\
\hline 1.12 & 3.068 & 3.035 & 3.151 & 4.564 & 4.565 & 4.721 & 5.334 & 5.304 & 4.985 & 8.245 & 7.907 & 8.483 & 5.325 & 5.544 & 5.599 \\
\hline 1.14 & 2.929 & 2.844 & 3.174 & 4.370 & 4.286 & 4.749 & 5.077 & 4.938 & 5.224 & 7.951 & 8.216 & 8.515 & 5.098 & 5.215 & 5.378 \\
\hline 1.16 & 2.767 & 2.818 & 3.159 & 4.272 & 4.234 & 4.726 & 4.750 & 4.530 & 5.238 & 7.787 & 8.189 & 8.485 & 4.984 & 4.972 & 5.141 \\
\hline 1.18 & 2.806 & 2.790 & 3.101 & 4.319 & 4.196 & 4.652 & 4.765 & 4.301 & 5.080 & 7.853 & 8.006 & 8.374 & 5.038 & 4.919 & 4.888 \\
\hline 1.2 & 2.809 & 2.730 & 3.014 & 4.306 & 4.105 & 4.534 & 4.727 & 4.573 & 5.083 & 7.806 & 8.001 & 8.174 & 5.024 & 5.250 & 4.634 \\
\hline 1.22 & 2.810 & 2.705 & 2.907 & 4.198 & 4.077 & 4.377 & 4.804 & 4.726 & 5.027 & 7.591 & 7.896 & 7.891 & 4.898 & 5.278 & 4.553 \\
\hline 1.24 & 2.781 & 2.724 & 2.820 & 4.159 & 4.089 & 4.229 & 4.778 & 4.793 & 4.936 & 7.484 & 7.739 & 7.538 & 4.852 & 5.042 & 4.747 \\
\hline 1.26 & 2.701 & 2.768 & 2.659 & 4.057 & 4.152 & 4.002 & 4.651 & 4.785 & 4.832 & 7.298 & 7.564 & 7.393 & 4.733 & 4.634 & 4.873 \\
\hline 1.28 & 2.695 & 2.783 & 2.628 & 3.894 & 4.287 & 3.925 & 4.421 & 4.714 & 4.729 & 6.953 & 7.390 & 7.550 & 4.543 & 4.318 & 4.951 \\
\hline 1.3 & 2.703 & 2.906 & 2.690 & 3.899 & 4.454 & 4.030 & 4.315 & 4.741 & 4.635 & 6.874 & 7.233 & 7.628 & 4.549 & 4.335 & 4.975 \\
\hline 1.32 & 2.668 & 2.969 & 2.725 & 3.848 & 4.528 & 4.091 & 4.255 & 4.893 & 4.553 & 6.783 & 7.099 & 7.628 & 4.489 & 4.312 & 4.945 \\
\hline 1.34 & 2.599 & 2.978 & 2.733 & 3.745 & 4.522 & 4.107 & 4.142 & 4.961 & 4.477 & 6.603 & 6.979 & 7.554 & 4.369 & 4.253 & 4.871 \\
\hline 1.36 & 2.505 & 2.943 & 2.712 & 3.601 & 4.448 & 4.079 & 4.055 & 4.954 & 4.407 & 6.354 & 6.865 & 7.412 & 4.202 & 4.162 & 4.765 \\
\hline 1.38 & 2.433 & 2.866 & 2.664 & 3.577 & 4.318 & 4.009 & 4.062 & 4.869 & 4.335 & 6.324 & 6.755 & 7.211 & 4.173 & 4.021 & 4.632 \\
\hline 1.4 & 2.412 & 2.721 & 2.594 & 3.545 & 4.094 & 3.903 & 4.042 & 4.648 & 4.257 & 6.303 & 6.635 & 6.956 & 4.136 & 4.126 & 4.476 \\
\hline 1.42 & 2.389 & 2.512 & 2.508 & 3.562 & 3.771 & 3.777 & 4.121 & 4.308 & 4.173 & 6.430 & 6.507 & 6.643 & 4.156 & 4.231 & 4.294 \\
\hline 1.44 & 2.425 & 2.277 & 2.412 & 3.622 & 3.420 & 3.637 & 4.181 & 3.877 & 4.083 & 6.527 & 6.404 & 6.273 & 4.226 & 4.127 & 4.083 \\
\hline 1.46 & 2.452 & 2.128 & 2.320 & 3.666 & 3.210 & 3.494 & 4.225 & 3.581 & 3.990 & 6.599 & 6.320 & 5.852 & 4.277 & 4.023 & 3.841 \\
\hline 1.48 & 2.472 & 1.967 & 2.228 & 3.701 & 2.977 & 3.347 & 4.256 & 3.386 & 3.924 & 6.650 & 6.231 & 5.711 & 4.318 & 4.143 & 3.564 \\
\hline 1.5 & 2.486 & 1.966 & 2.183 & 3.724 & 2.968 & 3.252 & 4.272 & 3.422 & 3.865 & 6.678 & 6.140 & 5.825 & 4.345 & 4.180 & 3.424 \\
\hline 1.52 & 2.492 & 1.981 & 2.154 & 3.735 & 2.983 & 3.217 & 4.275 & 3.458 & 3.812 & 6.684 & 6.052 & 5.926 & 4.358 & 4.165 & 3.480 \\
\hline 1.54 & 2.488 & 1.998 & 2.128 & 3.733 & 3.000 & 3.181 & 4.264 & 3.492 & 3.763 & 6.673 & 5.966 & 6.011 & 4.355 & 4.221 & 3.528 \\
\hline 1.56 & 2.477 & 2.016 & 2.104 & 3.719 & 3.022 & 3.158 & 4.241 & 3.524 & 3.715 & 6.642 & 5.883 & 6.080 & 4.339 & 4.206 & 3.567 \\
\hline 1.58 & 2.456 & 2.036 & 2.091 & 3.694 & 3.046 & 3.135 & 4.207 & 3.553 & 3.671 & 6.595 & 5.806 & 6.131 & 4.310 & 4.132 & 3.594 \\
\hline 1.6 & 2.428 & 2.056 & 2.078 & 3.660 & 3.070 & 3.113 & 4.164 & 3.580 & 3.630 & 6.532 & 5.732 & 6.164 & 4.270 & 4.015 & 3.609 \\
\hline 1.62 & 2.395 & 2.078 & 2.067 & 3.617 & 3.096 & 3.095 & 4.111 & 3.601 & 3.594 & 6.454 & 5.665 & 6.176 & 4.219 & 3.875 & 3.616 \\
\hline 1.64 & 2.356 & 2.097 & 2.057 & 3.566 & 3.121 & 3.076 & 4.051 & 3.618 & 3.563 & 6.366 & 5.608 & 6.168 & 4.160 & 3.751 & 3.608 \\
\hline 1.66 & 2.312 & 2.110 & 2.045 & 3.510 & 3.140 & 3.061 & 3.988 & 3.629 & 3.538 & 6.275 & 5.559 & 6.137 & 4.095 & 3.623 & 3.583 \\
\hline
\end{tabular}




\begin{tabular}{|c|c|c|c|c|c|c|c|c|c|c|c|c|c|c|c|}
\hline 1.68 & 2.267 & 2.117 & 2.033 & 3.451 & 3.151 & 3.044 & 3.921 & 3.629 & 3.516 & 6.175 & 5.516 & 6.084 & 4.026 & 3.502 & 3.610 \\
\hline 1.7 & 2.219 & 2.117 & 2.020 & 3.386 & 3.150 & 3.027 & 3.849 & 3.617 & 3.497 & 6.066 & 5.478 & 6.009 & 3.950 & 3.386 & 3.611 \\
\hline 1.72 & 2.170 & 2.106 & 2.007 & 3.319 & 3.137 & 3.011 & 3.776 & 3.591 & 3.479 & 5.960 & 5.445 & 5.913 & 3.873 & 3.274 & 3.586 \\
\hline 1.74 & 2.131 & 2.087 & 1.994 & 3.262 & 3.112 & 2.995 & 3.711 & 3.551 & 3.461 & 5.860 & 5.413 & 5.800 & 3.806 & 3.171 & 3.534 \\
\hline 1.76 & 2.105 & 2.057 & 1.980 & 3.219 & 3.072 & 2.977 & 3.656 & 3.496 & 3.444 & 5.765 & 5.381 & 5.672 & 3.756 & 3.079 & 3.457 \\
\hline 1.78 & 2.077 & 2.018 & 1.964 & 3.174 & 3.017 & 2.958 & 3.604 & 3.426 & 3.424 & 5.674 & 5.348 & 5.533 & 3.703 & 3.004 & 3.361 \\
\hline 1.8 & 2.048 & 1.968 & 1.946 & 3.124 & 2.945 & 2.936 & 3.548 & 3.340 & 3.401 & 5.576 & 5.311 & 5.382 & 3.645 & 2.946 & 3.247 \\
\hline 1.82 & 2.016 & 1.907 & 1.924 & 3.072 & 2.859 & 2.909 & 3.491 & 3.240 & 3.376 & 5.477 & 5.271 & 5.220 & 3.584 & 2.906 & 3.114 \\
\hline 1.84 & 1.981 & 1.836 & 1.901 & 3.019 & 2.760 & 2.877 & 3.433 & 3.130 & 3.346 & 5.377 & 5.225 & 5.048 & 3.523 & 2.883 & 2.967 \\
\hline 1.86 & 1.942 & 1.758 & 1.874 & 2.962 & 2.653 & 2.841 & 3.371 & 3.020 & 3.312 & 5.274 & 5.171 & 4.869 & 3.456 & 2.874 & 3.065 \\
\hline 1.88 & 1.900 & 1.694 & 1.844 & 2.899 & 2.555 & 2.799 & 3.304 & 2.931 & 3.273 & 5.164 & 5.112 & 4.688 & 3.383 & 2.877 & 3.144 \\
\hline 1.9 & 1.860 & 1.689 & 1.811 & 2.834 & 2.532 & 2.754 & 3.234 & 2.910 & 3.231 & 5.051 & 5.045 & 4.508 & 3.307 & 2.889 & 3.188 \\
\hline 1.92 & 1.829 & 1.722 & 1.779 & 2.772 & 2.561 & 2.705 & 3.168 & 2.916 & 3.186 & 4.946 & 4.975 & 4.328 & 3.234 & 2.908 & 3.201 \\
\hline 1.94 & 1.796 & 1.749 & 1.756 & 2.721 & 2.610 & 2.660 & 3.112 & 2.968 & 3.137 & 4.850 & 4.898 & 4.444 & 3.175 & 2.929 & 3.185 \\
\hline 1.96 & 1.758 & 1.756 & 1.730 & 2.669 & 2.627 & 2.618 & 3.054 & 2.990 & 3.083 & 4.753 & 4.815 & 4.544 & 3.114 & 2.951 & 3.143 \\
\hline 1.98 & 1.723 & 1.744 & 1.723 & 2.612 & 2.616 & 2.585 & 2.993 & 2.986 & 3.025 & 4.653 & 4.726 & 4.613 & 3.048 & 2.972 & 3.080 \\
\hline 2 & 1.686 & 1.717 & 1.727 & 2.558 & 2.577 & 2.596 & 2.929 & 2.957 & 2.964 & 4.551 & 4.631 & 4.654 & 2.984 & 2.991 & 3.002 \\
\hline 2.02 & 1.646 & 1.675 & 1.728 & 2.502 & 2.515 & 2.603 & 2.868 & 2.908 & 2.902 & 4.452 & 4.534 & 4.672 & 2.919 & 3.010 & 2.919 \\
\hline 2.04 & 1.606 & 1.625 & 1.725 & 2.444 & 2.436 & 2.599 & 2.805 & 2.844 & 2.836 & 4.350 & 4.433 & 4.678 & 2.851 & 3.023 & 2.833 \\
\hline 2.06 & 1.563 & 1.570 & 1.711 & 2.383 & 2.345 & 2.579 & 2.740 & 2.760 & 2.770 & 4.246 & 4.331 & 4.678 & 2.781 & 3.029 & 2.741 \\
\hline 2.08 & 1.519 & 1.506 & 1.688 & 2.320 & 2.253 & 2.546 & 2.673 & 2.652 & 2.702 & 4.141 & 4.225 & 4.666 & 2.707 & 3.027 & 2.646 \\
\hline 2.1 & 1.475 & 1.485 & 1.656 & 2.258 & 2.245 & 2.499 & 2.606 & 2.540 & 2.632 & 4.036 & 4.118 & 4.635 & 2.634 & 3.019 & 2.550 \\
\hline 2.12 & 1.430 & 1.478 & 1.617 & 2.194 & 2.231 & 2.442 & 2.538 & 2.518 & 2.560 & 3.932 & 4.009 & 4.586 & 2.560 & 3.006 & 2.457 \\
\hline 2.14 & 1.385 & 1.466 & 1.573 & 2.132 & 2.211 & 2.377 & 2.472 & 2.493 & 2.501 & 3.833 & 3.898 & 4.519 & 2.487 & 2.986 & 2.370 \\
\hline 2.16 & 1.358 & 1.451 & 1.527 & 2.084 & 2.185 & 2.307 & 2.409 & 2.463 & 2.470 & 3.754 & 3.821 & 4.438 & 2.432 & 2.958 & 2.292 \\
\hline 2.18 & 1.331 & 1.431 & 1.491 & 2.046 & 2.154 & 2.261 & 2.367 & 2.428 & 2.437 & 3.685 & 3.776 & 4.343 & 2.387 & 2.921 & 2.224 \\
\hline 2.2 & 1.311 & 1.413 & 1.466 & 2.005 & 2.119 & 2.224 & 2.325 & 2.389 & 2.404 & 3.615 & 3.727 & 4.237 & 2.340 & 2.877 & 2.166 \\
\hline 2.22 & 1.291 & 1.393 & 1.438 & 1.974 & 2.087 & 2.180 & 2.281 & 2.346 & 2.369 & 3.545 & 3.677 & 4.120 & 2.303 & 2.829 & 2.119 \\
\hline 2.24 & 1.270 & 1.371 & 1.405 & 1.943 & 2.052 & 2.129 & 2.241 & 2.300 & 2.333 & 3.481 & 3.625 & 3.994 & 2.267 & 2.808 & 2.083 \\
\hline 2.26 & 1.252 & 1.346 & 1.368 & 1.910 & 2.013 & 2.072 & 2.205 & 2.256 & 2.296 & 3.421 & 3.573 & 3.863 & 2.229 & 2.780 & 2.056 \\
\hline 2.28 & 1.233 & 1.321 & 1.328 & 1.881 & 1.973 & 2.011 & 2.168 & 2.209 & 2.260 & 3.369 & 3.519 & 3.729 & 2.194 & 2.744 & 2.039 \\
\hline 2.3 & 1.215 & 1.293 & 1.286 & 1.853 & 1.929 & 1.952 & 2.129 & 2.160 & 2.223 & 3.325 & 3.466 & 3.651 & 2.161 & 2.700 & 2.029 \\
\hline 2.32 & 1.197 & 1.262 & 1.246 & 1.823 & 1.881 & 1.892 & 2.088 & 2.108 & 2.187 & 3.276 & 3.412 & 3.603 & 2.127 & 2.645 & 2.026 \\
\hline 2.34 & 1.180 & 1.230 & 1.207 & 1.798 & 1.831 & 1.830 & 2.057 & 2.054 & 2.152 & 3.224 & 3.359 & 3.548 & 2.098 & 2.581 & 2.025 \\
\hline 2.36 & 1.163 & 1.196 & 1.189 & 1.775 & 1.778 & 1.788 & 2.033 & 2.025 & 2.117 & 3.175 & 3.308 & 3.487 & 2.071 & 2.508 & 2.026 \\
\hline 2.38 & 1.146 & 1.163 & 1.186 & 1.752 & 1.741 & 1.785 & 2.008 & 2.009 & 2.083 & 3.121 & 3.256 & 3.421 & 2.043 & 2.430 & 2.026 \\
\hline 2.4 & 1.130 & 1.152 & 1.177 & 1.728 & 1.725 & 1.773 & 1.984 & 1.993 & 2.049 & 3.080 & 3.206 & 3.350 & 2.016 & 2.348 & 2.022 \\
\hline 2.42 & 1.112 & 1.141 & 1.162 & 1.705 & 1.707 & 1.753 & 1.958 & 1.974 & 2.017 & 3.039 & 3.157 & 3.277 & 1.989 & 2.263 & 2.012 \\
\hline 2.44 & 1.095 & 1.128 & 1.147 & 1.681 & 1.688 & 1.732 & 1.933 & 1.955 & 1.986 & 2.998 & 3.110 & 3.200 & 1.961 & 2.178 & 1.994 \\
\hline 2.46 & 1.078 & 1.115 & 1.134 & 1.657 & 1.667 & 1.710 & 1.908 & 1.933 & 1.956 & 2.982 & 3.064 & 3.122 & 1.933 & 2.092 & 1.966 \\
\hline 2.48 & 1.061 & 1.101 & 1.119 & 1.633 & 1.644 & 1.683 & 1.884 & 1.911 & 1.928 & 2.954 & 3.021 & 3.043 & 1.905 & 2.008 & 1.929 \\
\hline 2.5 & 1.094 & 1.086 & 1.101 & 1.611 & 1.632 & 1.652 & 1.859 & 1.887 & 1.900 & 2.926 & 2.978 & 2.963 & 1.880 & 1.925 & 1.915 \\
\hline 2.52 & 1.134 & 1.079 & 1.081 & 1.676 & 1.628 & 1.618 & 1.833 & 1.862 & 1.874 & 3.046 & 2.937 & 2.885 & 1.955 & 1.843 & 1.920 \\
\hline 2.54 & 1.165 & 1.077 & 1.060 & 1.729 & 1.622 & 1.583 & 1.896 & 1.836 & 1.849 & 3.145 & 2.899 & 2.807 & 2.017 & 1.763 & 1.922 \\
\hline 2.56 & 1.189 & 1.074 & 1.039 & 1.769 & 1.615 & 1.550 & 1.948 & 1.823 & 1.825 & 3.223 & 2.861 & 2.731 & 2.064 & 1.686 & 1.920 \\
\hline
\end{tabular}




\begin{tabular}{|c|c|c|c|c|c|c|c|c|c|c|c|c|c|c|c|}
\hline 2.58 & 1.204 & 1.071 & 1.017 & 1.798 & 1.606 & 1.520 & 1.986 & 1.815 & 1.802 & 3.280 & 2.824 & 2.658 & 2.098 & 1.611 & 1.912 \\
\hline 2.6 & 1.211 & 1.066 & 0.996 & 1.816 & 1.596 & 1.490 & 2.012 & 1.805 & 1.780 & 3.316 & 2.789 & 2.587 & 2.119 & 1.540 & 1.900 \\
\hline 2.62 & 1.211 & 1.060 & 0.975 & 1.823 & 1.586 & 1.460 & 2.025 & 1.794 & 1.758 & 3.333 & 2.755 & 2.519 & 2.127 & 1.472 & 1.881 \\
\hline 2.64 & 1.204 & 1.054 & 0.954 & 1.819 & 1.574 & 1.429 & 2.025 & 1.782 & 1.737 & 3.331 & 2.721 & 2.454 & 2.122 & 1.429 & 1.858 \\
\hline 2.66 & 1.190 & 1.046 & 0.931 & 1.806 & 1.561 & 1.397 & 2.014 & 1.769 & 1.717 & 3.311 & 2.689 & 2.393 & 2.107 & 1.390 & 1.829 \\
\hline 2.68 & 1.170 & 1.038 & 0.909 & 1.784 & 1.547 & 1.365 & 1.993 & 1.755 & 1.698 & 3.275 & 2.657 & 2.335 & 2.081 & 1.362 & 1.795 \\
\hline 2.7 & 1.145 & 1.029 & 0.887 & 1.755 & 1.532 & 1.333 & 1.963 & 1.740 & 1.679 & 3.225 & 2.626 & 2.281 & 2.047 & 1.347 & 1.757 \\
\hline 2.72 & 1.116 & 1.019 & 0.865 & 1.718 & 1.515 & 1.301 & 1.925 & 1.725 & 1.660 & 3.162 & 2.596 & 2.230 & 2.004 & 1.334 & 1.715 \\
\hline 2.74 & 1.083 & 1.008 & 0.843 & 1.675 & 1.498 & 1.270 & 1.879 & 1.708 & 1.641 & 3.088 & 2.566 & 2.183 & 1.955 & 1.322 & 1.669 \\
\hline 2.76 & 1.048 & 0.997 & 0.823 & 1.627 & 1.480 & 1.241 & 1.827 & 1.690 & 1.623 & 3.003 & 2.537 & 2.140 & 1.898 & 1.311 & 1.631 \\
\hline 2.78 & 1.009 & 0.985 & 0.803 & 1.573 & 1.462 & 1.213 & 1.769 & 1.672 & 1.605 & 2.910 & 2.508 & 2.100 & 1.836 & 1.301 & 1.635 \\
\hline 2.8 & 0.968 & 0.973 & 0.785 & 1.515 & 1.443 & 1.187 & 1.706 & 1.653 & 1.587 & 2.810 & 2.480 & 2.064 & 1.768 & 1.293 & 1.636 \\
\hline 2.82 & 0.925 & 0.961 & 0.768 & 1.453 & 1.423 & 1.163 & 1.639 & 1.633 & 1.569 & 2.702 & 2.451 & 2.031 & 1.695 & 1.286 & 1.633 \\
\hline 2.84 & 0.880 & 0.948 & 0.753 & 1.388 & 1.403 & 1.142 & 1.568 & 1.613 & 1.551 & 2.590 & 2.423 & 2.001 & 1.619 & 1.279 & 1.627 \\
\hline 2.86 & 0.836 & 0.934 & 0.739 & 1.320 & 1.384 & 1.122 & 1.495 & 1.592 & 1.534 & 2.473 & 2.394 & 1.973 & 1.540 & 1.274 & 1.616 \\
\hline 2.88 & 0.830 & 0.922 & 0.727 & 1.257 & 1.365 & 1.105 & 1.420 & 1.570 & 1.515 & 2.353 & 2.365 & 1.949 & 1.466 & 1.270 & 1.603 \\
\hline 2.9 & 0.824 & 0.910 & 0.717 & 1.248 & 1.345 & 1.090 & 1.408 & 1.548 & 1.497 & 2.232 & 2.337 & 1.926 & 1.456 & 1.266 & 1.586 \\
\hline 2.92 & 0.817 & 0.897 & 0.707 & 1.239 & 1.325 & 1.077 & 1.399 & 1.527 & 1.479 & 2.175 & 2.308 & 1.906 & 1.446 & 1.262 & 1.565 \\
\hline 2.94 & 0.809 & 0.884 & 0.699 & 1.229 & 1.305 & 1.066 & 1.391 & 1.505 & 1.461 & 2.160 & 2.279 & 1.888 & 1.434 & 1.259 & 1.541 \\
\hline 2.96 & 0.801 & 0.870 & 0.693 & 1.219 & 1.285 & 1.057 & 1.381 & 1.484 & 1.442 & 2.144 & 2.249 & 1.872 & 1.422 & 1.256 & 1.513 \\
\hline 2.98 & 0.793 & 0.857 & 0.687 & 1.207 & 1.264 & 1.049 & 1.370 & 1.462 & 1.423 & 2.126 & 2.219 & 1.858 & 1.408 & 1.253 & 1.483 \\
\hline 3 & 0.784 & 0.843 & 0.683 & 1.195 & 1.243 & 1.042 & 1.359 & 1.440 & 1.404 & 2.108 & 2.188 & 1.844 & 1.394 & 1.250 & 1.450 \\
\hline 3.02 & 0.775 & 0.830 & 0.679 & 1.183 & 1.222 & 1.037 & 1.346 & 1.419 & 1.384 & 2.090 & 2.158 & 1.832 & 1.380 & 1.247 & 1.415 \\
\hline 3.04 & 0.766 & 0.816 & 0.676 & 1.171 & 1.201 & 1.032 & 1.334 & 1.396 & 1.364 & 2.071 & 2.126 & 1.821 & 1.366 & 1.243 & 1.378 \\
\hline 3.06 & 0.758 & 0.802 & 0.674 & 1.158 & 1.180 & 1.028 & 1.321 & 1.374 & 1.344 & 2.052 & 2.094 & 1.810 & 1.352 & 1.238 & 1.338 \\
\hline 3.08 & 0.749 & 0.788 & 0.672 & 1.146 & 1.159 & 1.024 & 1.308 & 1.352 & 1.324 & 2.032 & 2.062 & 1.800 & 1.337 & 1.233 & 1.297 \\
\hline 3.1 & 0.740 & 0.775 & 0.670 & 1.133 & 1.137 & 1.020 & 1.295 & 1.329 & 1.303 & 2.012 & 2.030 & 1.791 & 1.322 & 1.227 & 1.254 \\
\hline 3.12 & 0.731 & 0.761 & 0.669 & 1.121 & 1.116 & 1.016 & 1.281 & 1.307 & 1.281 & 1.992 & 1.998 & 1.782 & 1.307 & 1.220 & 1.210 \\
\hline 3.14 & 0.722 & 0.747 & 0.667 & 1.108 & 1.095 & 1.013 & 1.267 & 1.284 & 1.260 & 1.971 & 1.967 & 1.773 & 1.292 & 1.212 & 1.200 \\
\hline 3.16 & 0.714 & 0.733 & 0.665 & 1.095 & 1.074 & 1.008 & 1.253 & 1.262 & 1.238 & 1.951 & 1.936 & 1.764 & 1.278 & 1.204 & 1.190 \\
\hline 3.18 & 0.705 & 0.720 & 0.662 & 1.082 & 1.053 & 1.003 & 1.239 & 1.239 & 1.216 & 1.930 & 1.904 & 1.755 & 1.263 & 1.194 & 1.179 \\
\hline 3.2 & 0.697 & 0.706 & 0.659 & 1.070 & 1.033 & 0.997 & 1.225 & 1.217 & 1.195 & 1.908 & 1.872 & 1.745 & 1.248 & 1.184 & 1.168 \\
\hline 3.22 & 0.688 & 0.692 & 0.655 & 1.056 & 1.012 & 0.990 & 1.211 & 1.194 & 1.174 & 1.886 & 1.840 & 1.736 & 1.232 & 1.172 & 1.156 \\
\hline 3.24 & 0.679 & 0.679 & 0.650 & 1.043 & 0.992 & 0.981 & 1.196 & 1.172 & 1.153 & 1.863 & 1.808 & 1.726 & 1.217 & 1.159 & 1.142 \\
\hline 3.26 & 0.669 & 0.665 & 0.645 & 1.029 & 0.972 & 0.971 & 1.182 & 1.150 & 1.133 & 1.840 & 1.776 & 1.716 & 1.201 & 1.145 & 1.129 \\
\hline 3.28 & 0.660 & 0.652 & 0.638 & 1.015 & 0.952 & 0.959 & 1.167 & 1.128 & 1.112 & 1.817 & 1.744 & 1.705 & 1.184 & 1.130 & 1.114 \\
\hline 3.3 & 0.651 & 0.639 & 0.630 & 1.001 & 0.932 & 0.946 & 1.151 & 1.106 & 1.092 & 1.793 & 1.711 & 1.694 & 1.168 & 1.114 & 1.099 \\
\hline 3.32 & 0.642 & 0.626 & 0.621 & 0.987 & 0.930 & 0.931 & 1.136 & 1.084 & 1.071 & 1.769 & 1.679 & 1.682 & 1.152 & 1.097 & 1.082 \\
\hline 3.34 & 0.632 & 0.619 & 0.612 & 0.973 & 0.929 & 0.922 & 1.120 & 1.068 & 1.052 & 1.744 & 1.646 & 1.670 & 1.135 & 1.079 & 1.065 \\
\hline 3.36 & 0.623 & 0.617 & 0.612 & 0.959 & 0.926 & 0.923 & 1.105 & 1.068 & 1.033 & 1.720 & 1.614 & 1.657 & 1.119 & 1.059 & 1.048 \\
\hline 3.38 & 0.614 & 0.615 & 0.613 & 0.945 & 0.922 & 0.923 & 1.089 & 1.065 & 1.014 & 1.696 & 1.581 & 1.643 & 1.103 & 1.042 & 1.030 \\
\hline 3.4 & 0.605 & 0.612 & 0.614 & 0.931 & 0.917 & 0.923 & 1.073 & 1.061 & 0.994 & 1.674 & 1.549 & 1.629 & 1.086 & 1.039 & 1.016 \\
\hline 3.42 & 0.596 & 0.608 & 0.613 & 0.917 & 0.910 & 0.921 & 1.057 & 1.055 & 0.974 & 1.651 & 1.517 & 1.614 & 1.070 & 1.035 & 1.005 \\
\hline 3.44 & 0.587 & 0.603 & 0.612 & 0.903 & 0.902 & 0.918 & 1.041 & 1.047 & 0.963 & 1.629 & 1.490 & 1.598 & 1.053 & 1.031 & 0.993 \\
\hline 3.46 & 0.580 & 0.597 & 0.611 & 0.889 & 0.893 & 0.914 & 1.025 & 1.038 & 0.963 & 1.608 & 1.489 & 1.591 & 1.037 & 1.026 & 0.981 \\
\hline
\end{tabular}




\begin{tabular}{|c|c|c|c|c|c|c|c|c|c|c|c|c|c|c|c|}
\hline 3.48 & 0.574 & 0.590 & 0.609 & 0.877 & 0.883 & 0.909 & 1.011 & 1.027 & 0.962 & 1.588 & 1.487 & 1.589 & 1.023 & 1.020 & 0.969 \\
\hline 3.5 & 0.568 & 0.583 & 0.606 & 0.867 & 0.872 & 0.904 & 0.999 & 1.015 & 0.961 & 1.567 & 1.485 & 1.585 & 1.011 & 1.014 & 0.960 \\
\hline 3.52 & 0.561 & 0.575 & 0.602 & 0.857 & 0.860 & 0.897 & 0.986 & 1.001 & 0.960 & 1.547 & 1.482 & 1.580 & 1.000 & 1.007 & 0.954 \\
\hline 3.54 & 0.555 & 0.566 & 0.598 & 0.847 & 0.847 & 0.889 & 0.974 & 0.986 & 0.958 & 1.527 & 1.479 & 1.574 & 0.988 & 0.999 & 0.947 \\
\hline 3.56 & 0.549 & 0.558 & 0.593 & 0.837 & 0.836 & 0.885 & 0.962 & 0.970 & 0.956 & 1.506 & 1.476 & 1.565 & 0.976 & 0.991 & 0.940 \\
\hline 3.58 & 0.542 & 0.552 & 0.588 & 0.826 & 0.828 & 0.884 & 0.950 & 0.954 & 0.954 & 1.486 & 1.472 & 1.556 & 0.964 & 0.983 & 0.932 \\
\hline 3.6 & 0.536 & 0.547 & 0.586 & 0.816 & 0.819 & 0.883 & 0.938 & 0.937 & 0.951 & 1.466 & 1.468 & 1.545 & 0.952 & 0.973 & 0.924 \\
\hline 3.62 & 0.529 & 0.540 & 0.586 & 0.806 & 0.809 & 0.880 & 0.926 & 0.921 & 0.949 & 1.445 & 1.463 & 1.532 & 0.940 & 0.963 & 0.915 \\
\hline 3.64 & 0.523 & 0.533 & 0.585 & 0.796 & 0.798 & 0.877 & 0.914 & 0.906 & 0.946 & 1.425 & 1.458 & 1.519 & 0.928 & 0.953 & 0.905 \\
\hline 3.66 & 0.517 & 0.526 & 0.583 & 0.785 & 0.787 & 0.873 & 0.902 & 0.892 & 0.942 & 1.406 & 1.452 & 1.507 & 0.916 & 0.942 & 0.895 \\
\hline 3.68 & 0.511 & 0.518 & 0.581 & 0.775 & 0.775 & 0.868 & 0.890 & 0.877 & 0.939 & 1.386 & 1.447 & 1.498 & 0.904 & 0.931 & 0.884 \\
\hline 3.7 & 0.505 & 0.509 & 0.578 & 0.765 & 0.762 & 0.862 & 0.878 & 0.862 & 0.935 & 1.367 & 1.440 & 1.489 & 0.892 & 0.919 & 0.873 \\
\hline 3.72 & 0.499 & 0.500 & 0.574 & 0.755 & 0.749 & 0.855 & 0.865 & 0.846 & 0.931 & 1.347 & 1.434 & 1.479 & 0.880 & 0.907 & 0.862 \\
\hline 3.74 & 0.493 & 0.491 & 0.570 & 0.744 & 0.735 & 0.847 & 0.853 & 0.836 & 0.926 & 1.328 & 1.427 & 1.468 & 0.868 & 0.895 & 0.850 \\
\hline 3.76 & 0.487 & 0.481 & 0.565 & 0.734 & 0.721 & 0.838 & 0.841 & 0.826 & 0.922 & 1.308 & 1.420 & 1.456 & 0.857 & 0.882 & 0.838 \\
\hline 3.78 & 0.482 & 0.471 & 0.560 & 0.725 & 0.706 & 0.829 & 0.830 & 0.816 & 0.917 & 1.289 & 1.412 & 1.444 & 0.846 & 0.869 & 0.826 \\
\hline 3.8 & 0.476 & 0.461 & 0.553 & 0.715 & 0.691 & 0.818 & 0.819 & 0.805 & 0.912 & 1.270 & 1.404 & 1.430 & 0.834 & 0.856 & 0.814 \\
\hline 3.82 & 0.470 & 0.452 & 0.547 & 0.706 & 0.676 & 0.807 & 0.808 & 0.795 & 0.907 & 1.251 & 1.396 & 1.416 & 0.823 & 0.843 & 0.801 \\
\hline 3.84 & 0.465 & 0.445 & 0.540 & 0.696 & 0.661 & 0.795 & 0.796 & 0.783 & 0.901 & 1.232 & 1.388 & 1.400 & 0.812 & 0.831 & 0.788 \\
\hline 3.86 & 0.459 & 0.442 & 0.532 & 0.687 & 0.655 & 0.783 & 0.785 & 0.772 & 0.896 & 1.213 & 1.379 & 1.383 & 0.801 & 0.821 & 0.775 \\
\hline 3.88 & 0.454 & 0.438 & 0.523 & 0.677 & 0.649 & 0.769 & 0.774 & 0.760 & 0.890 & 1.195 & 1.370 & 1.365 & 0.790 & 0.811 & 0.761 \\
\hline 3.9 & 0.448 & 0.434 & 0.515 & 0.668 & 0.643 & 0.755 & 0.763 & 0.752 & 0.884 & 1.177 & 1.361 & 1.346 & 0.780 & 0.801 & 0.748 \\
\hline 3.92 & 0.443 & 0.430 & 0.506 & 0.659 & 0.636 & 0.741 & 0.752 & 0.744 & 0.878 & 1.159 & 1.352 & 1.326 & 0.769 & 0.791 & 0.734 \\
\hline 3.94 & 0.437 & 0.426 & 0.496 & 0.650 & 0.629 & 0.726 & 0.741 & 0.736 & 0.872 & 1.142 & 1.342 & 1.305 & 0.759 & 0.782 & 0.721 \\
\hline 3.96 & 0.432 & 0.422 & 0.486 & 0.642 & 0.622 & 0.711 & 0.731 & 0.728 & 0.865 & 1.124 & 1.332 & 1.284 & 0.748 & 0.775 & 0.707 \\
\hline 3.98 & 0.426 & 0.418 & 0.476 & 0.633 & 0.616 & 0.695 & 0.720 & 0.720 & 0.859 & 1.107 & 1.322 & 1.262 & 0.738 & 0.769 & 0.693 \\
\hline 4 & 0.421 & 0.416 & 0.465 & 0.624 & 0.612 & 0.679 & 0.710 & 0.717 & 0.852 & 1.091 & 1.312 & 1.239 & 0.728 & 0.762 & 0.680 \\
\hline
\end{tabular}




\section{Dynamic Bending Moments}

Fixed Based Supports

Soil A
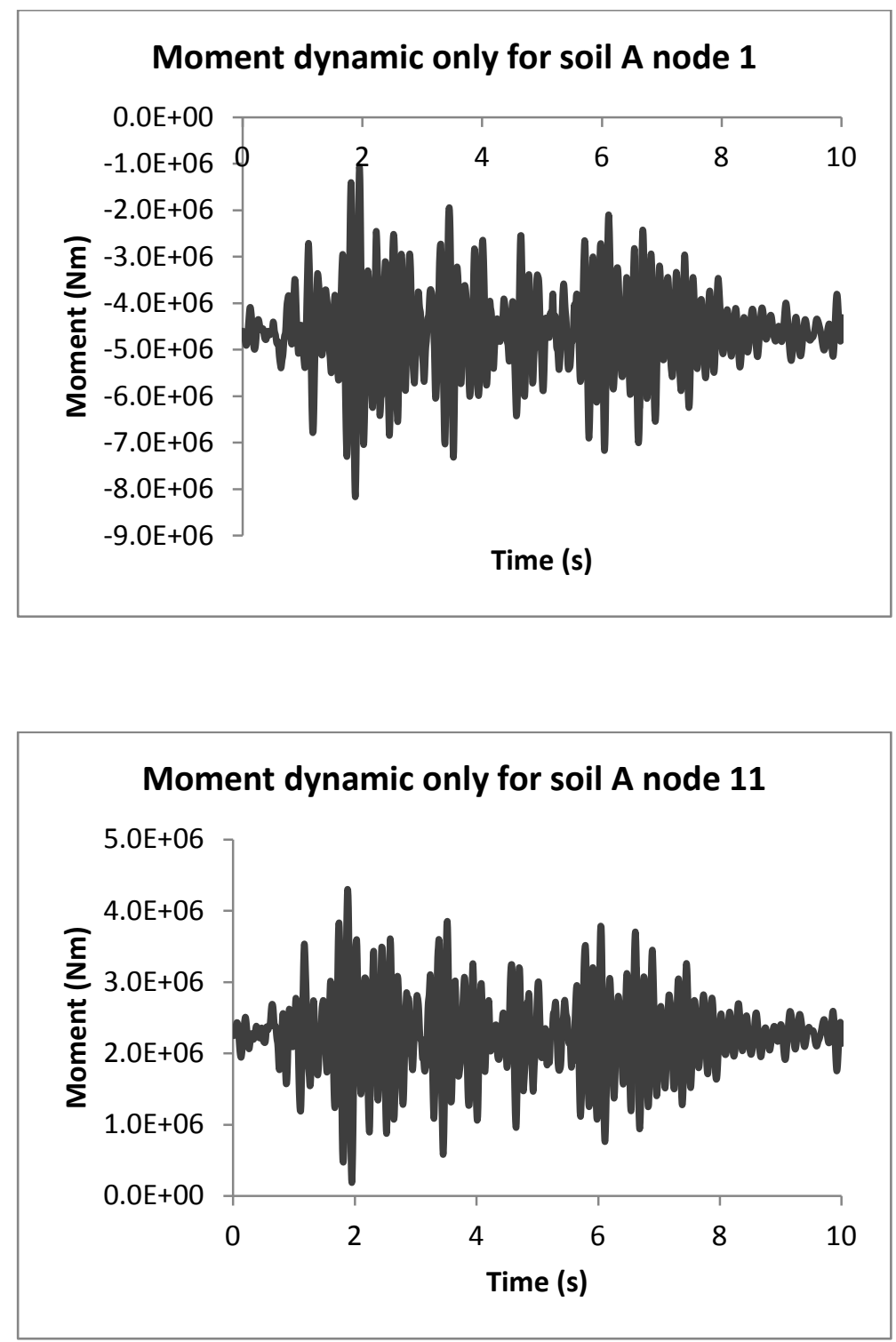
Moment dynamic only for soil A node 21
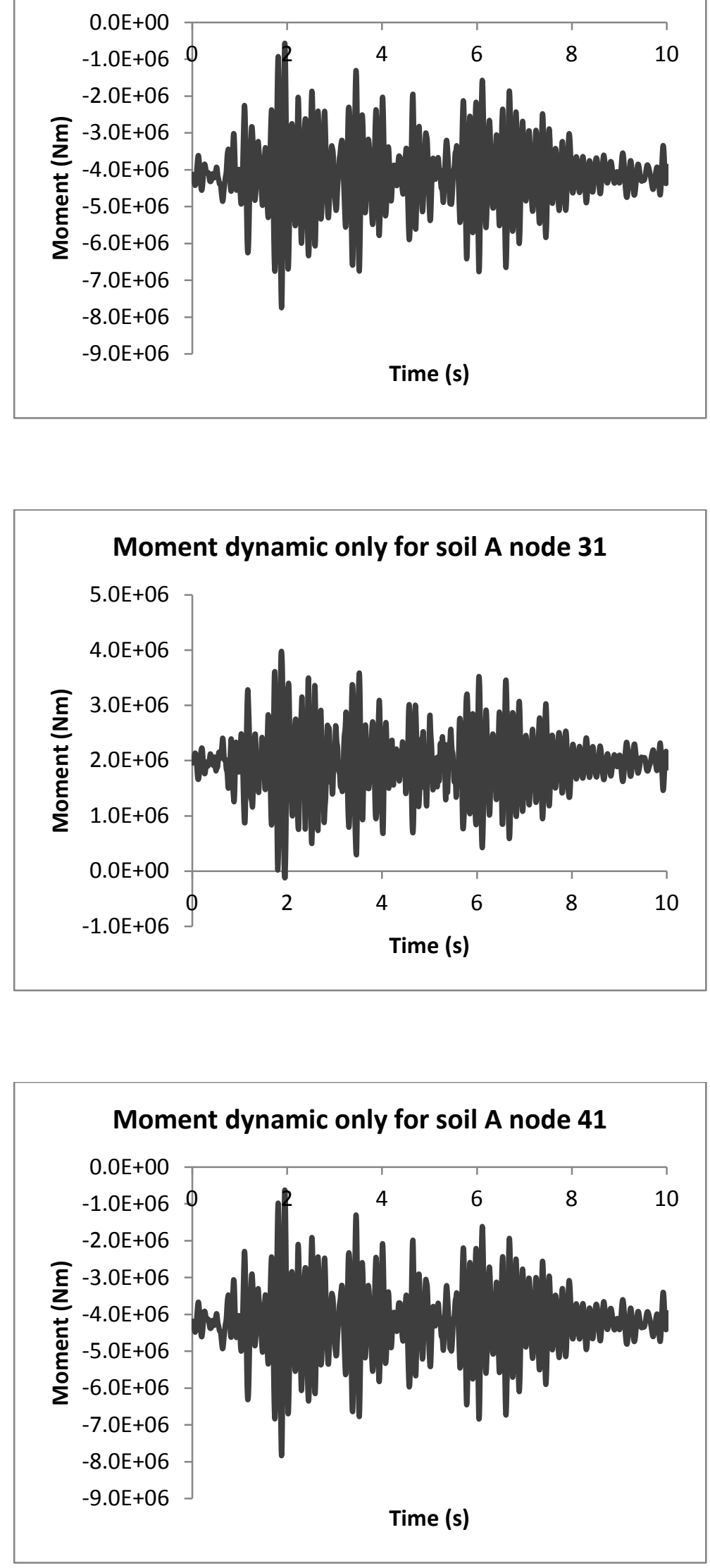

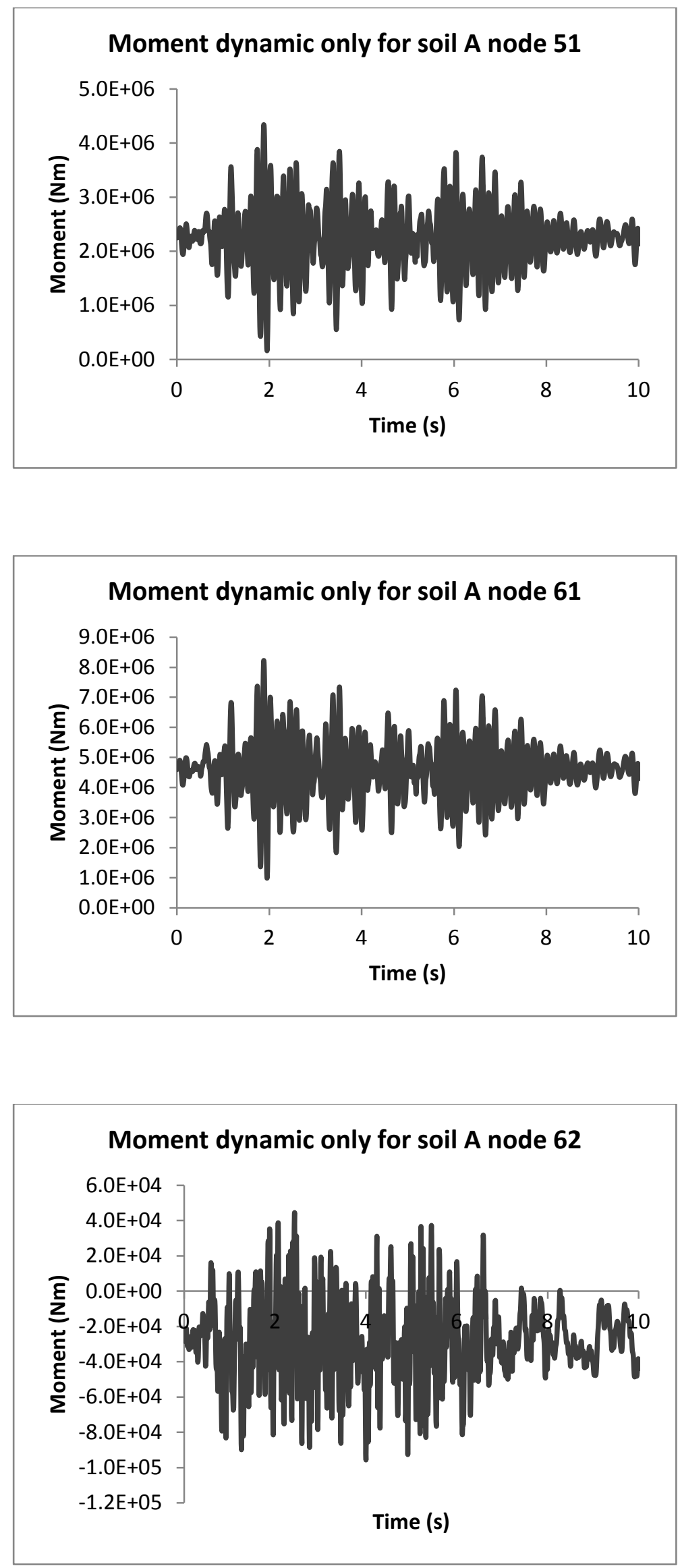

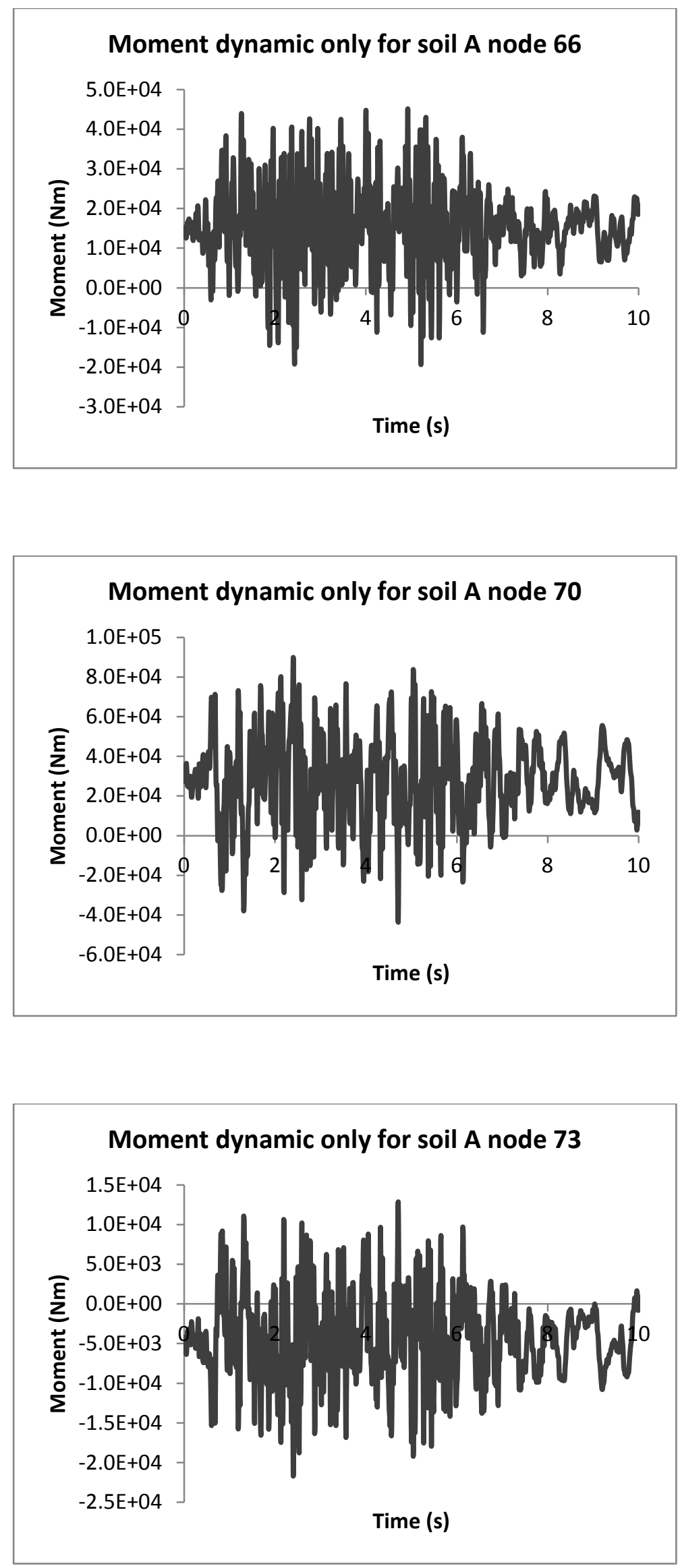
Horizontal RD
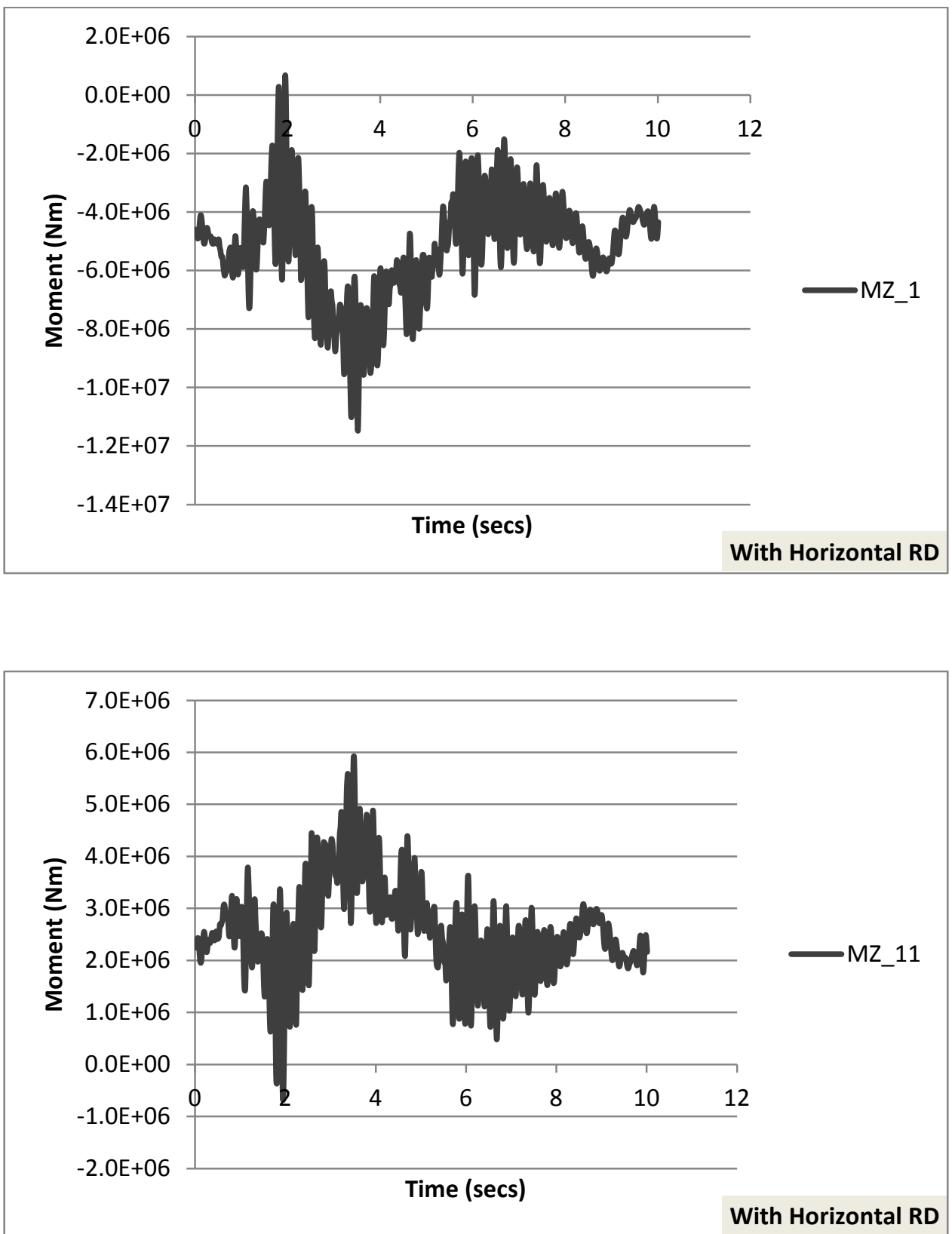

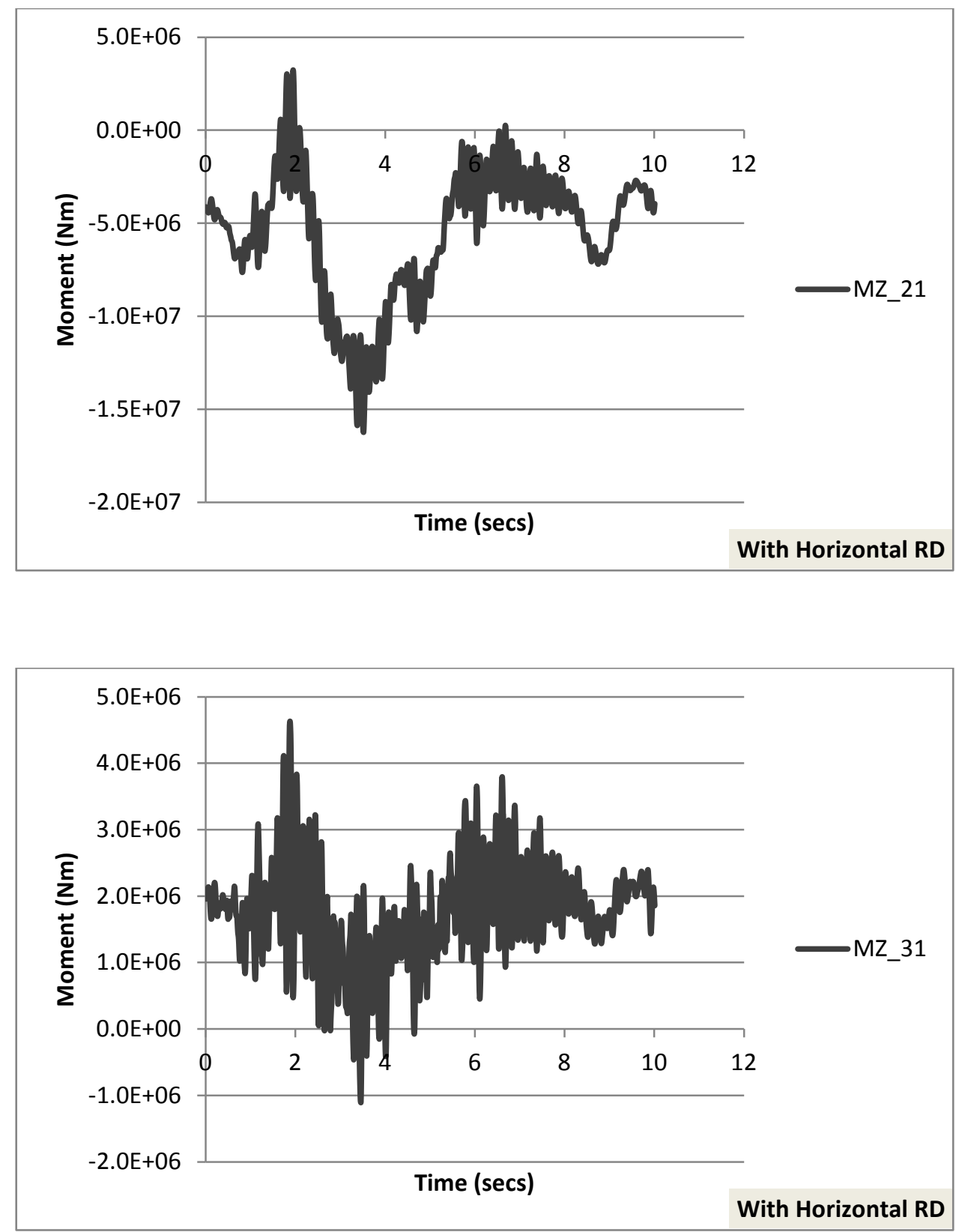

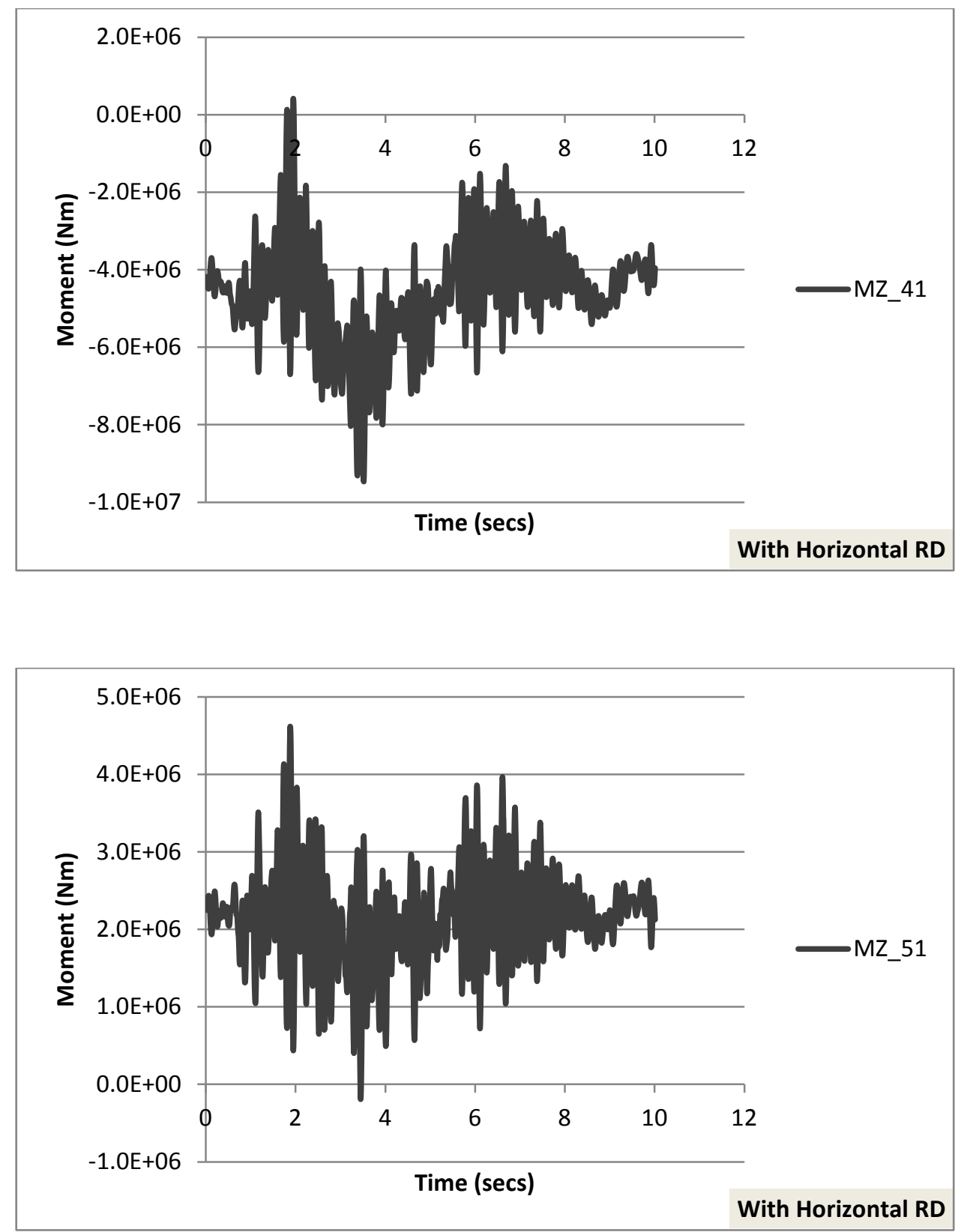

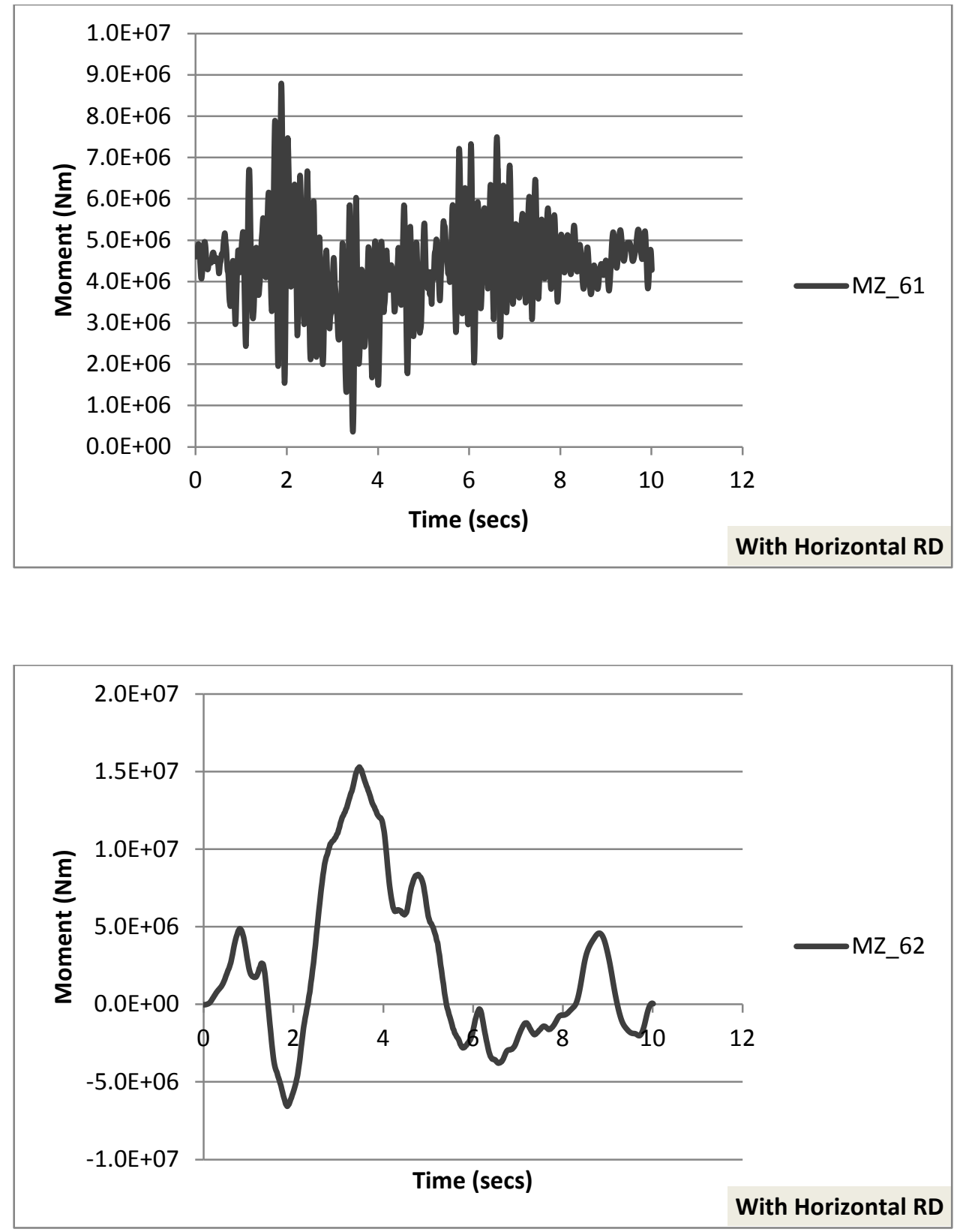

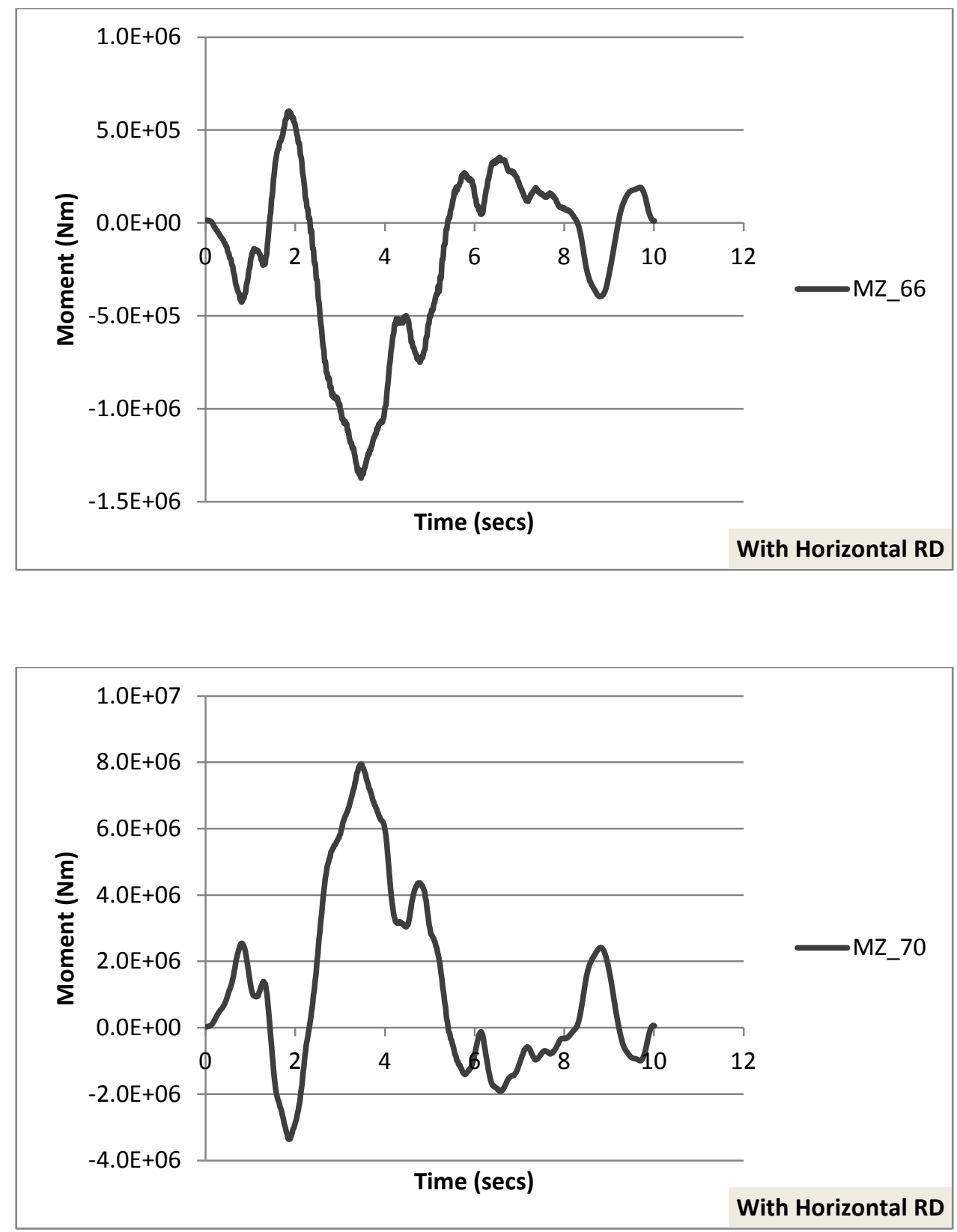


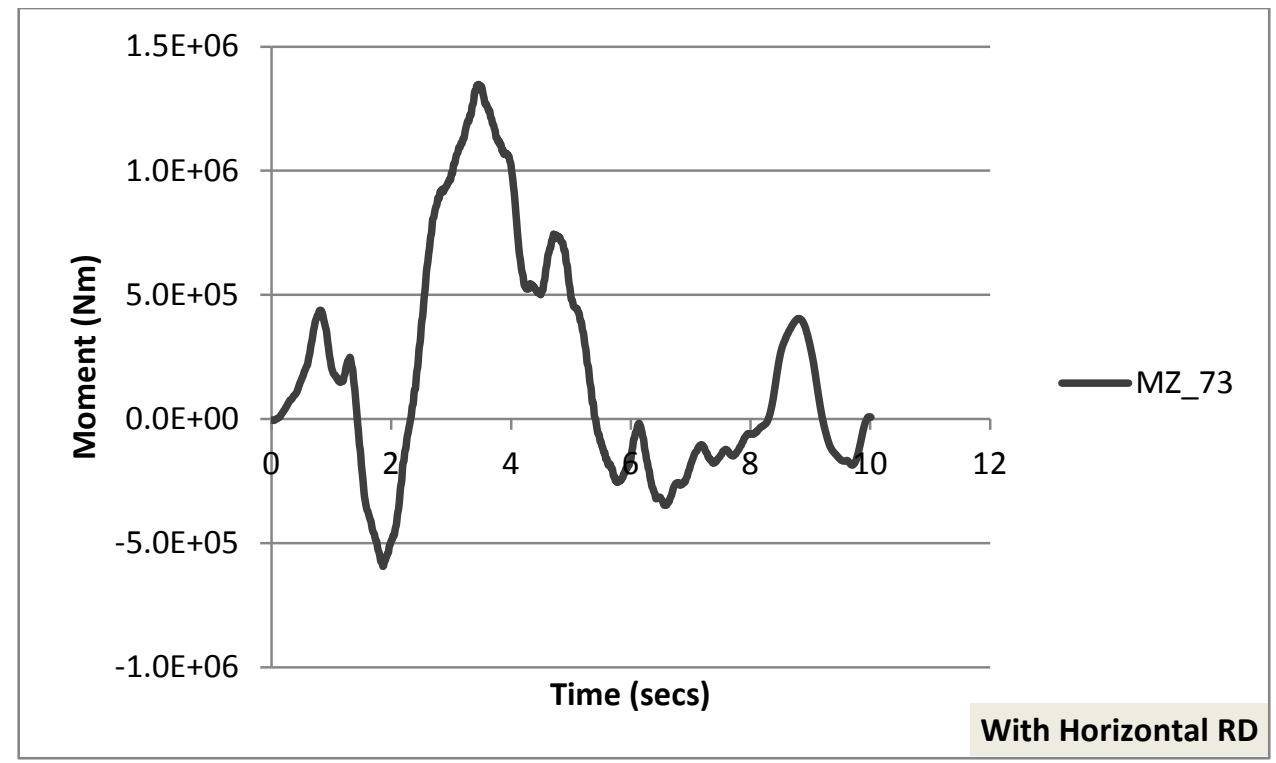

Vertical

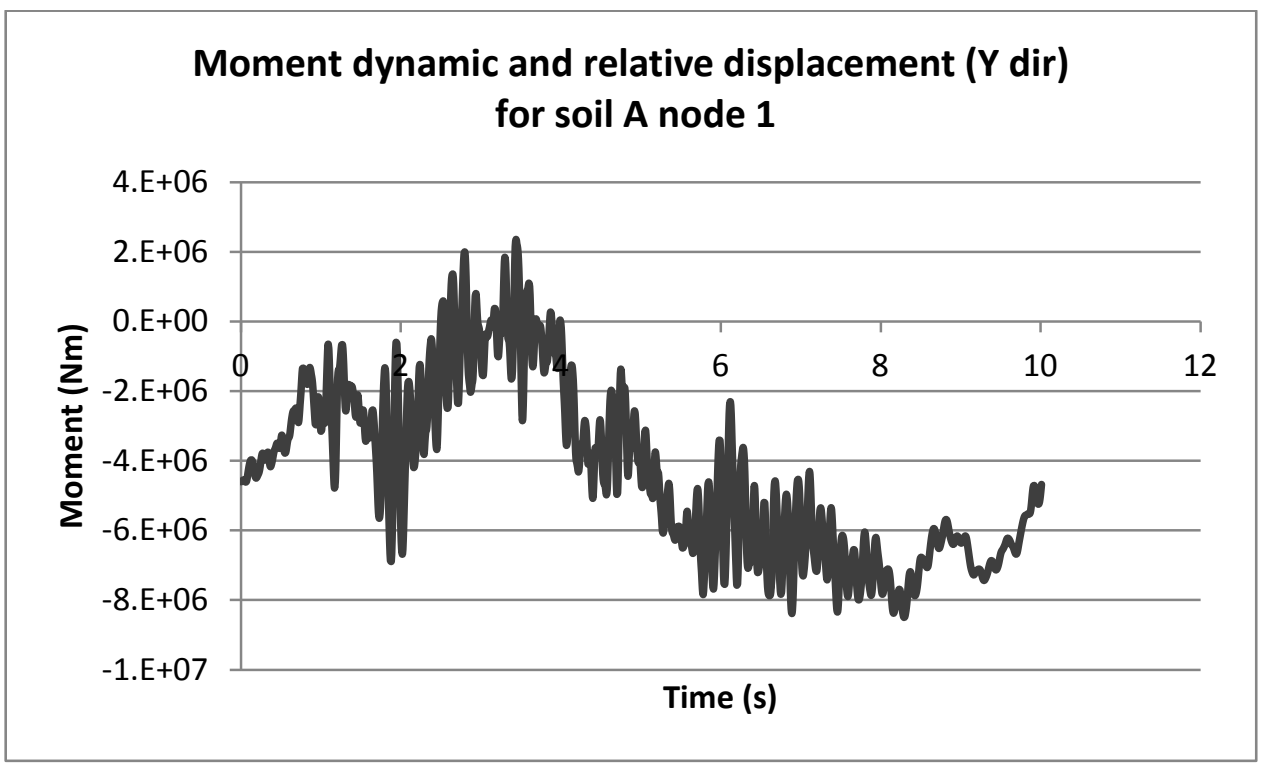



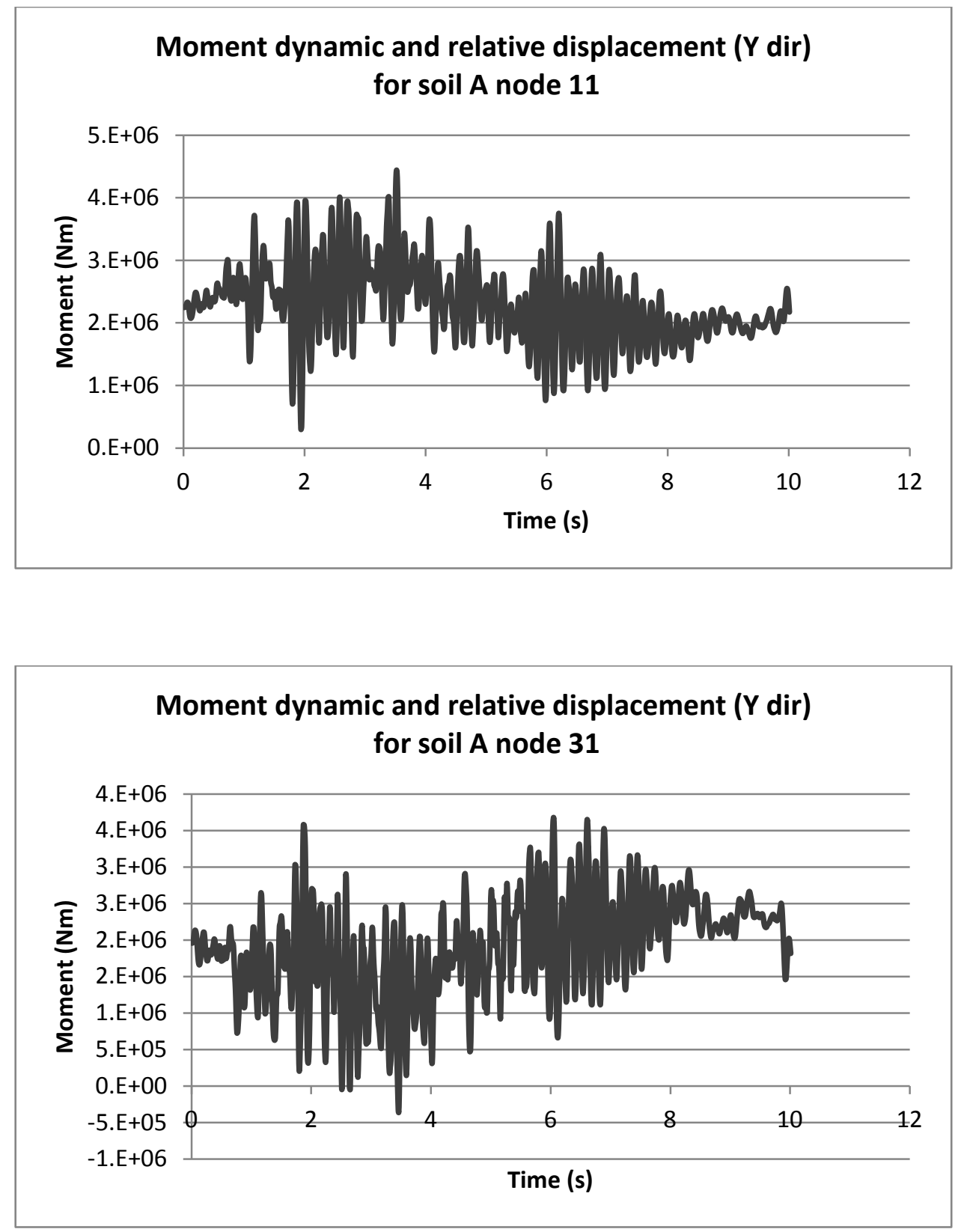

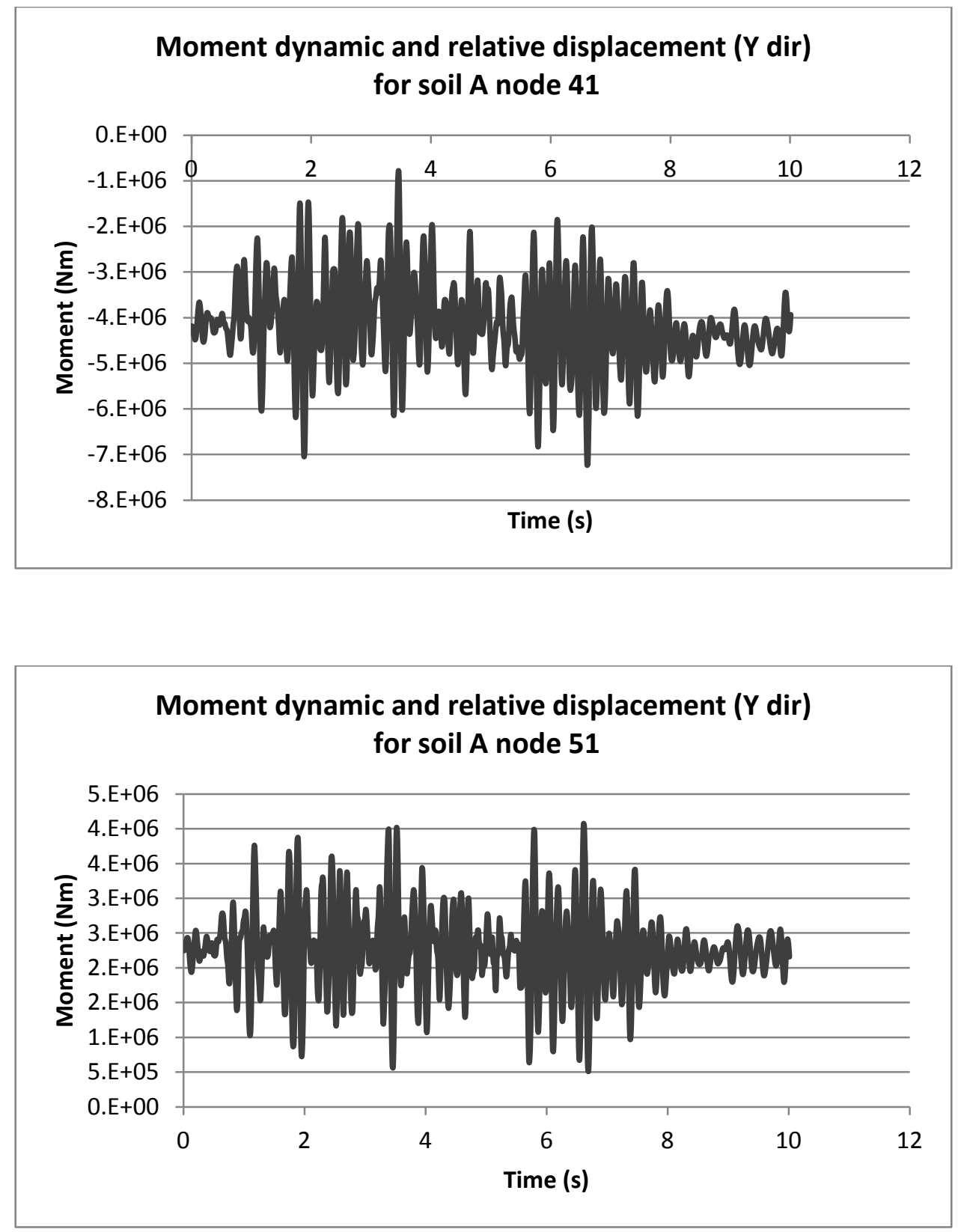

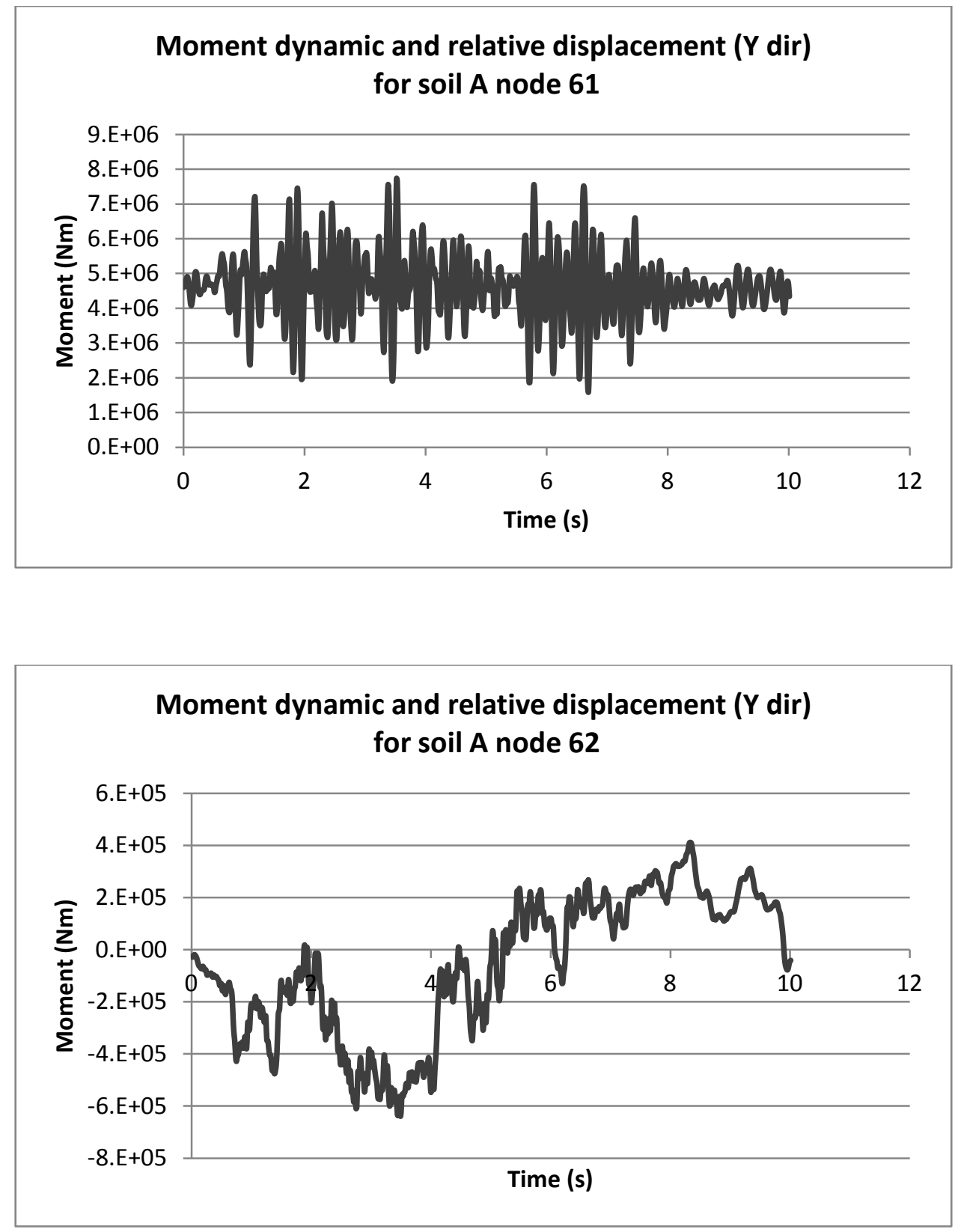

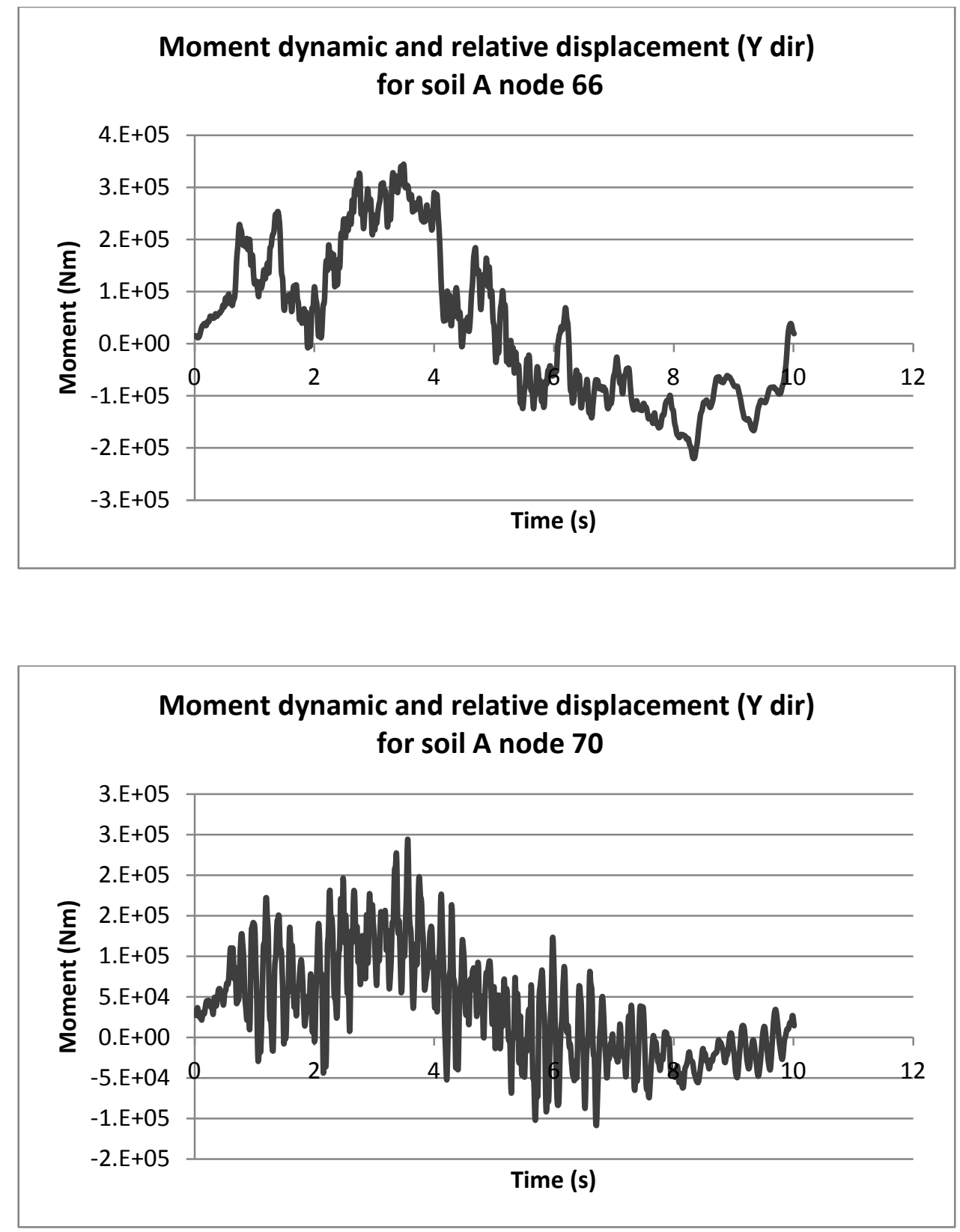

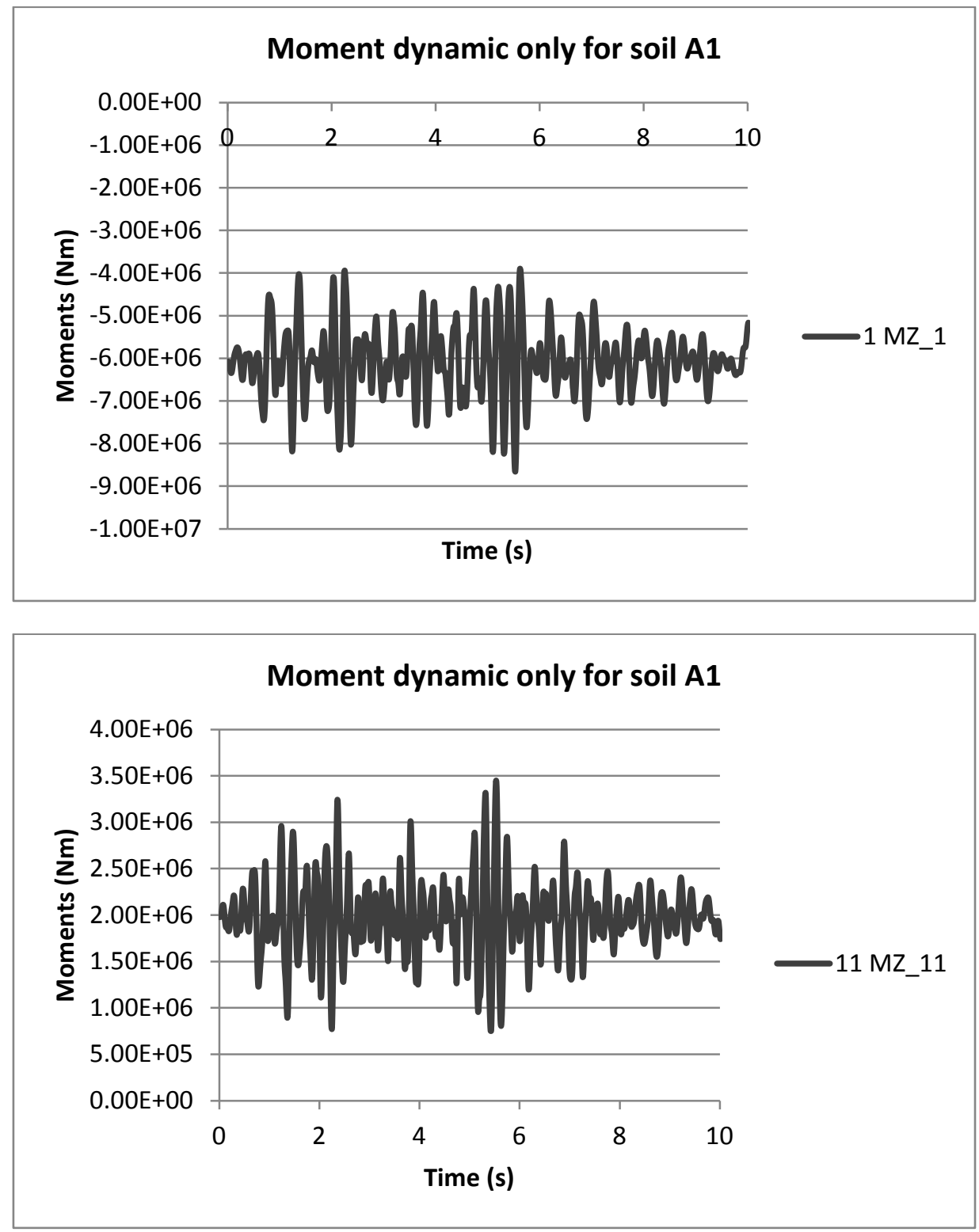

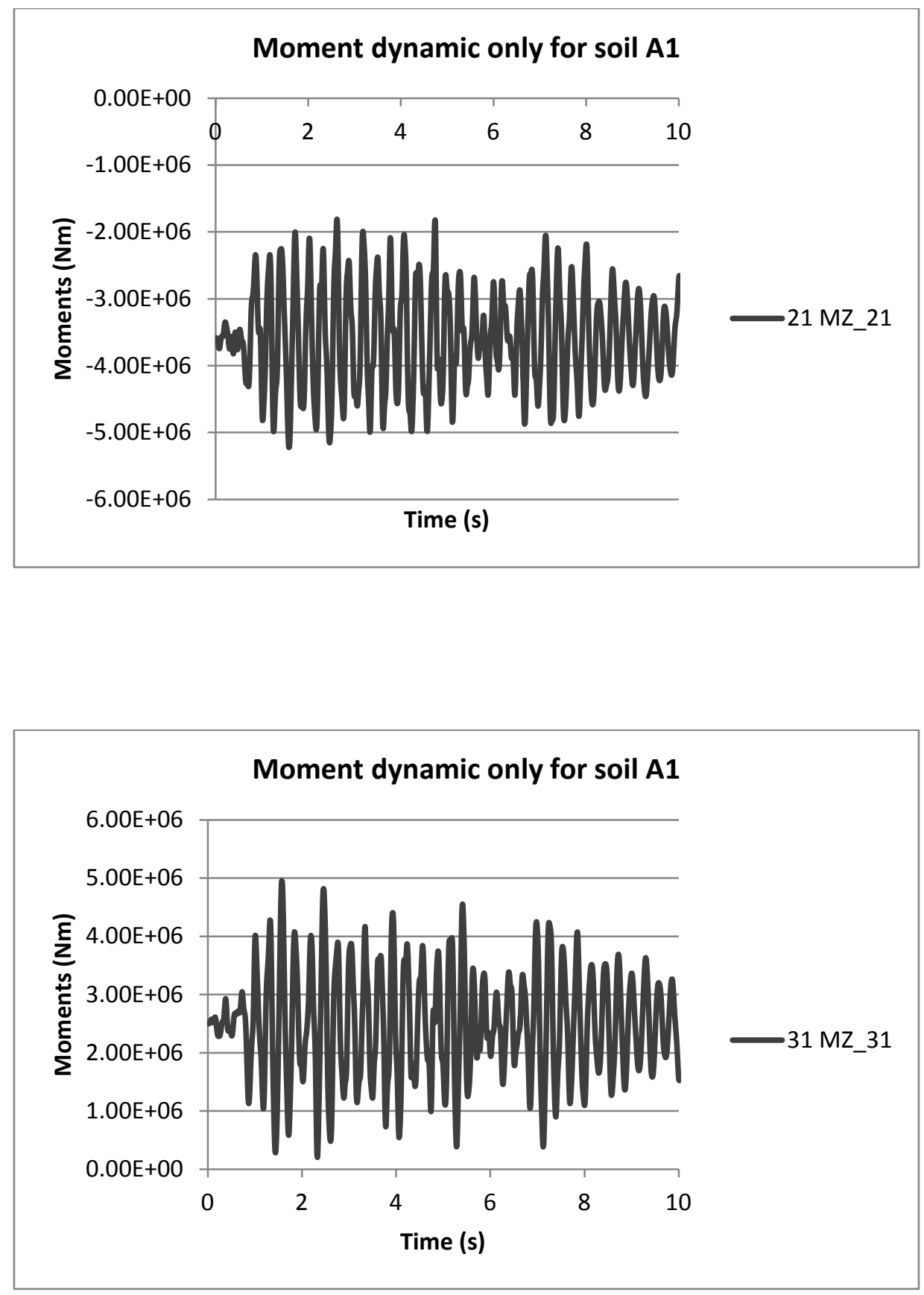

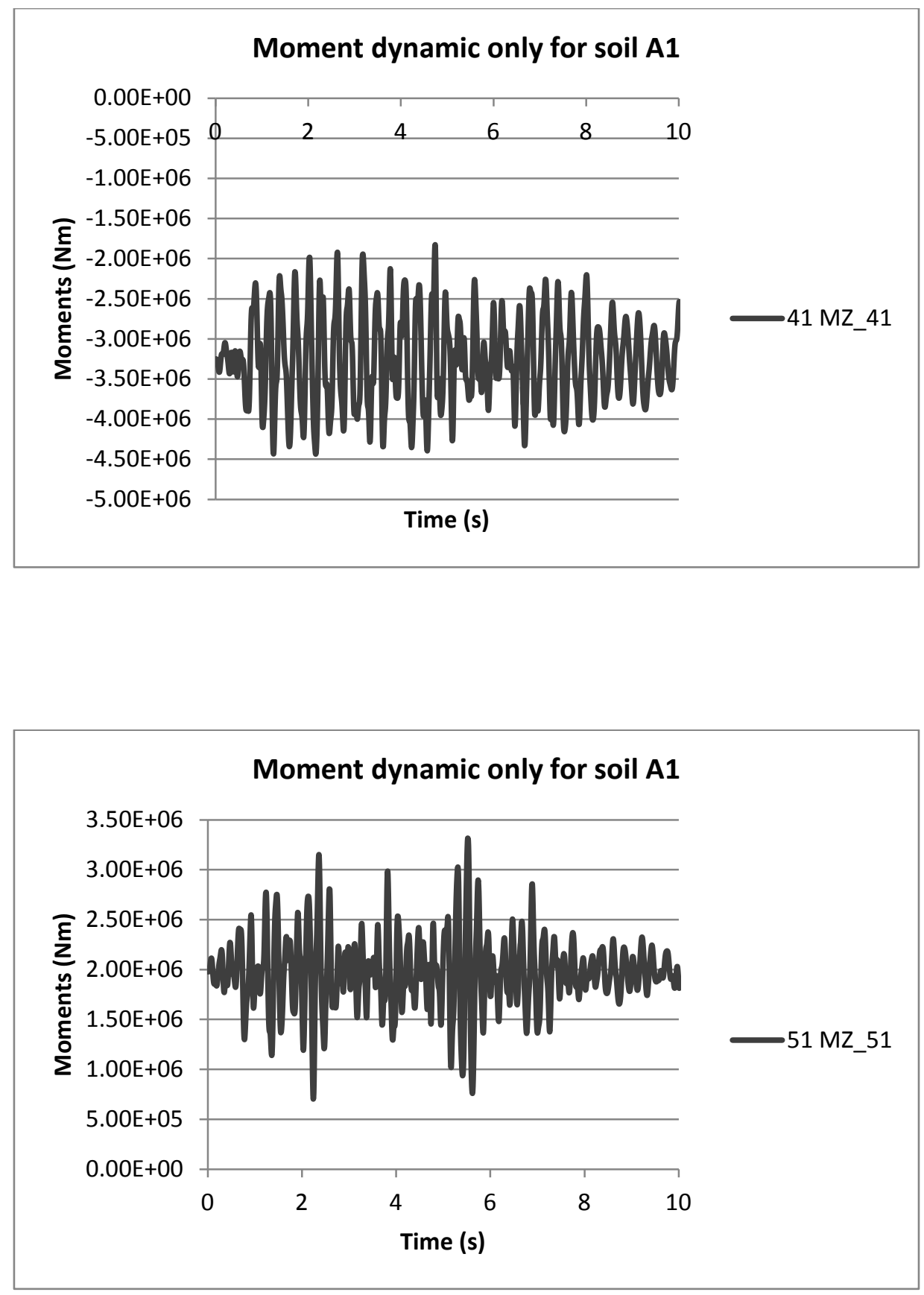

Horizontal RD 

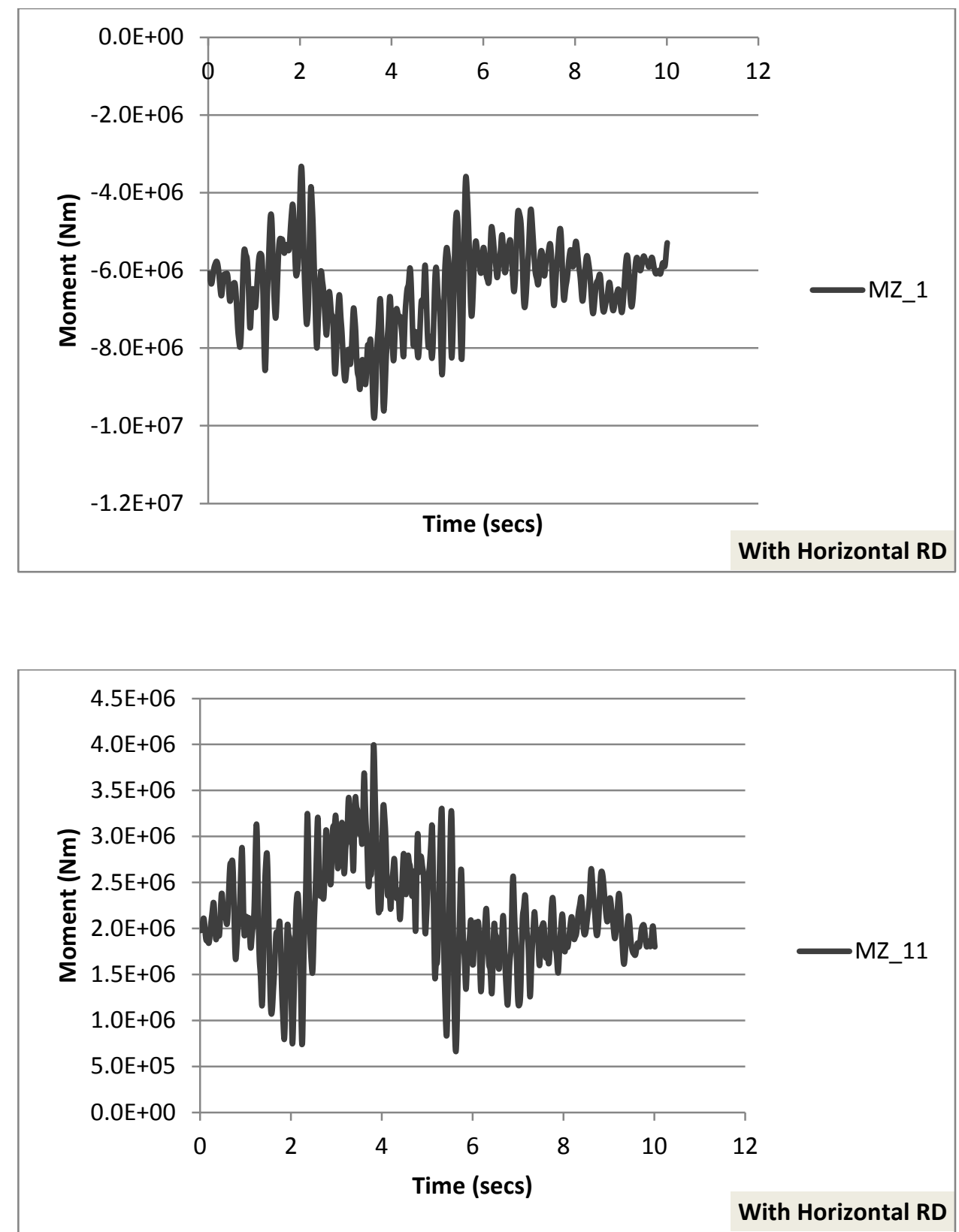

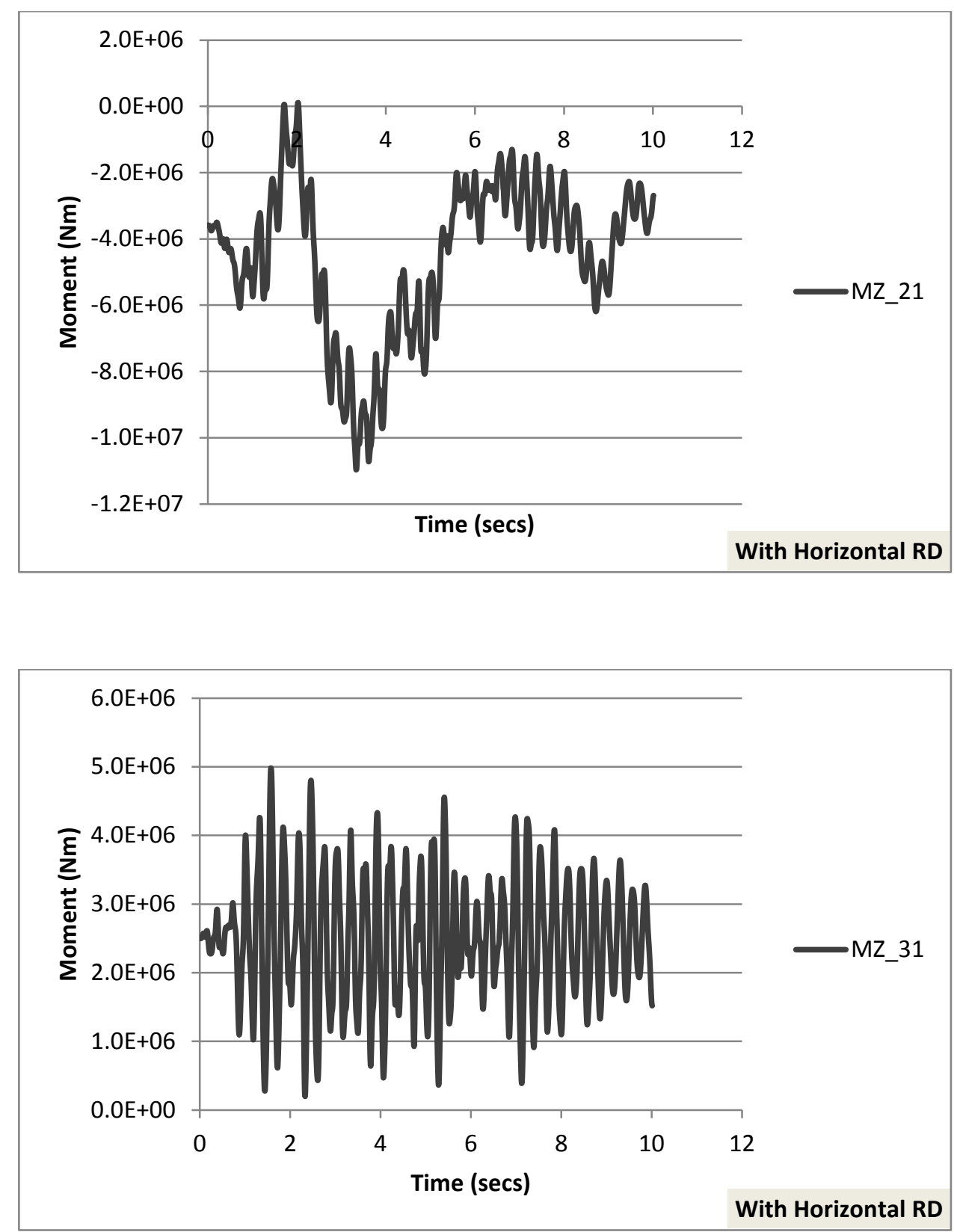

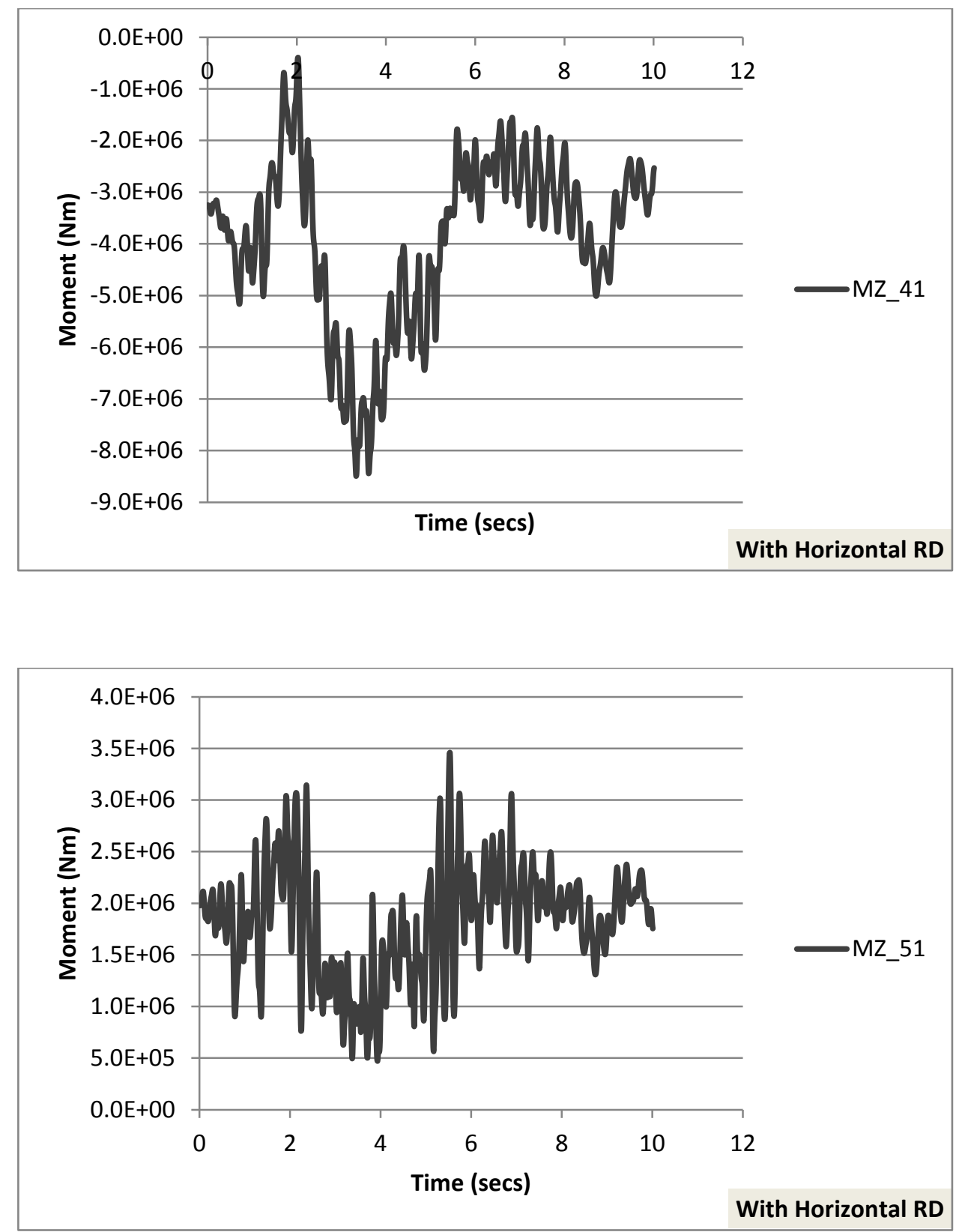

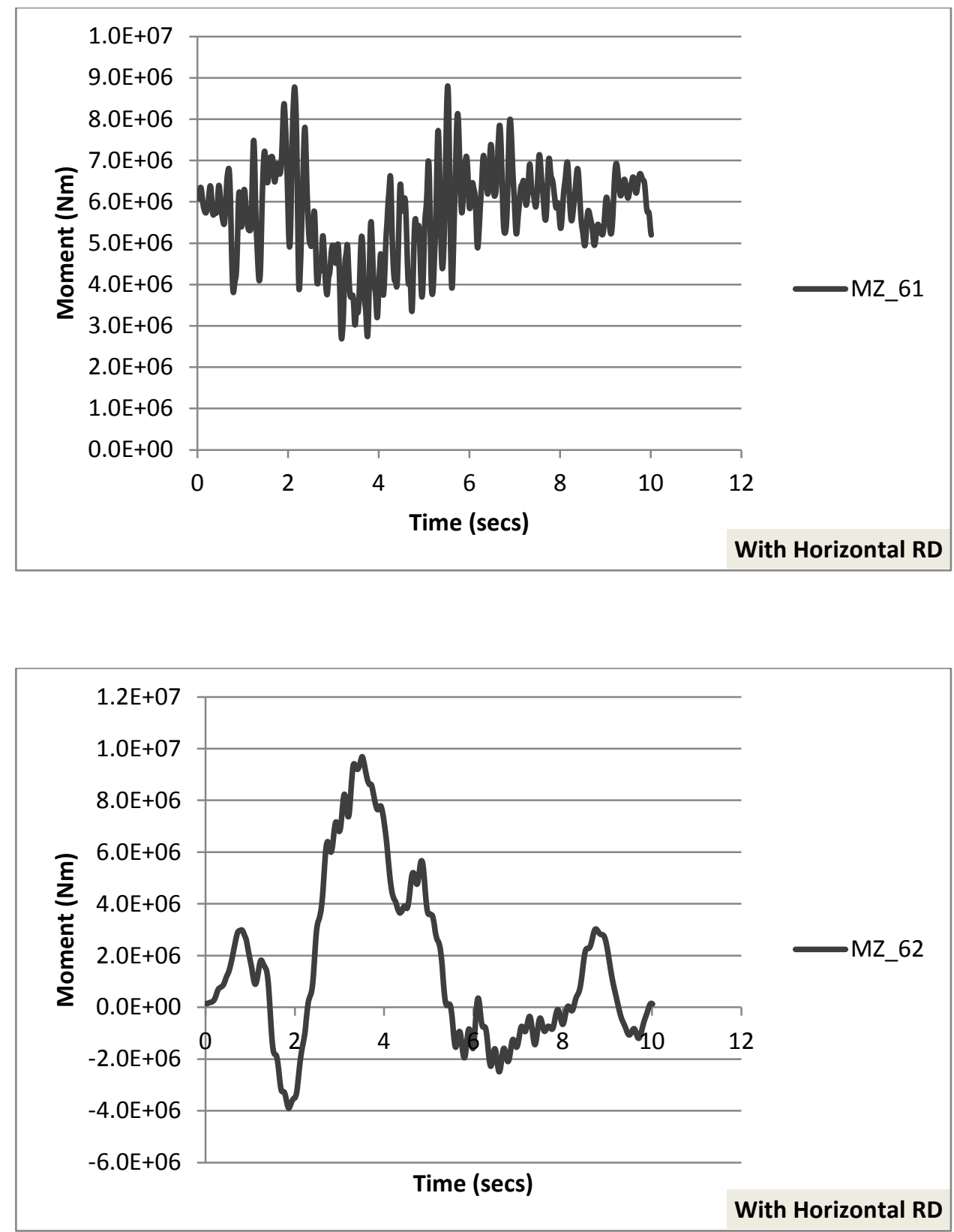

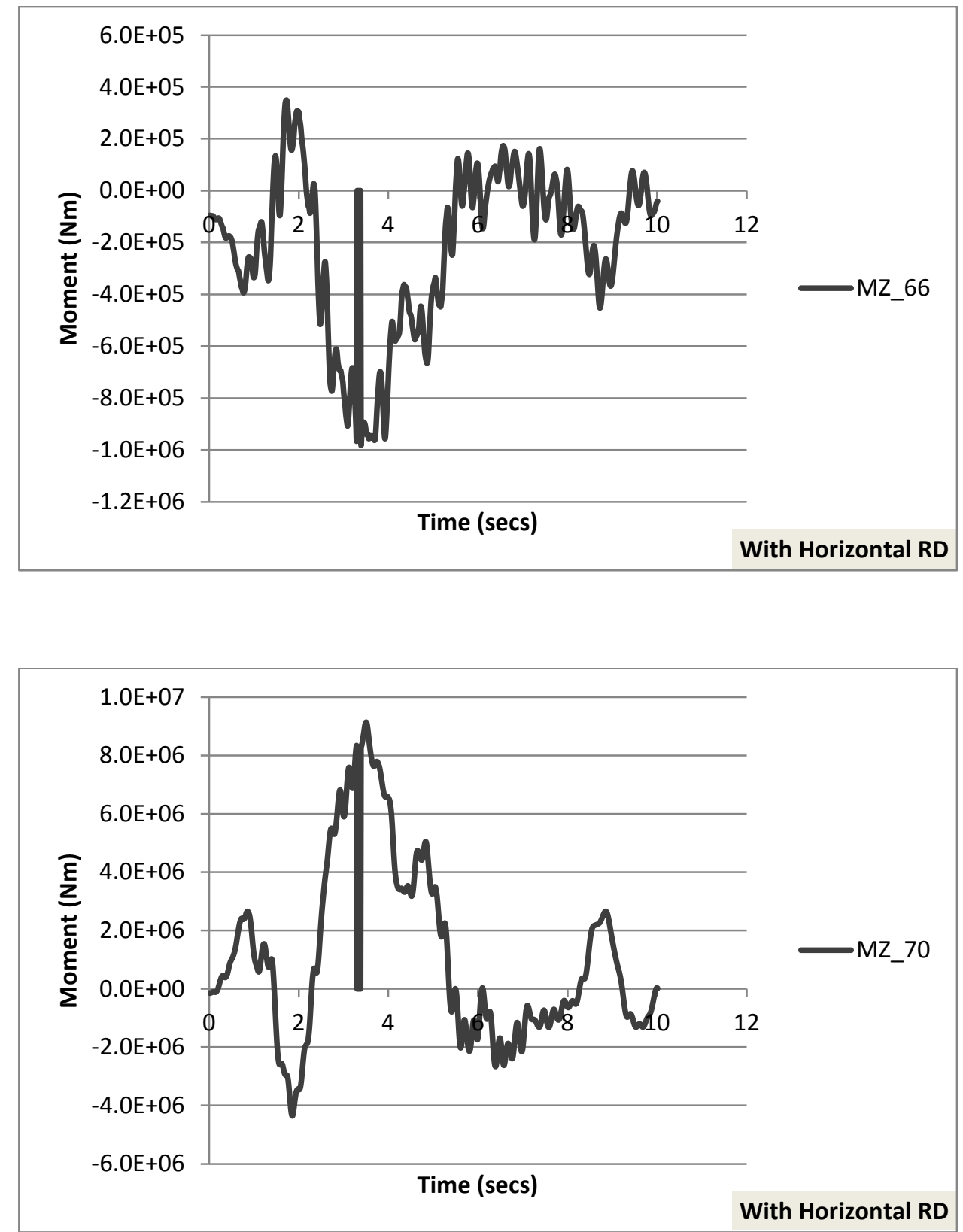


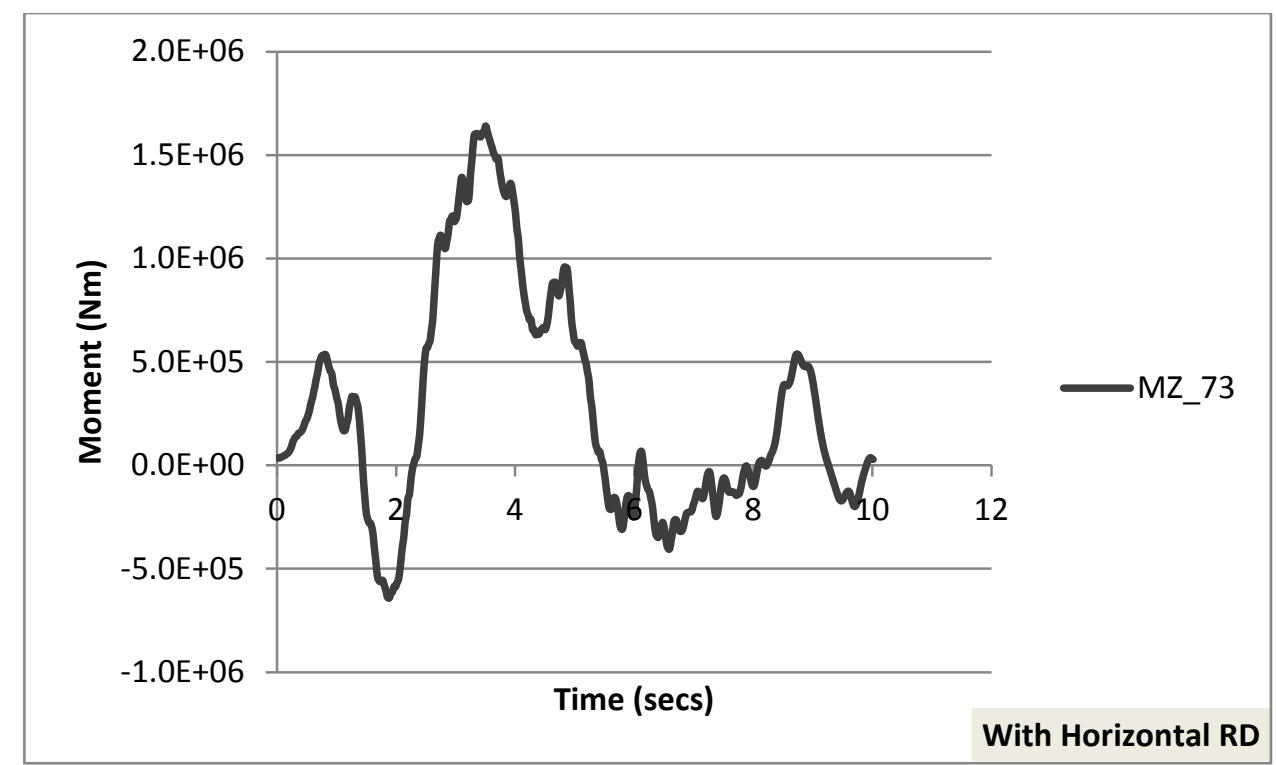

Vertical

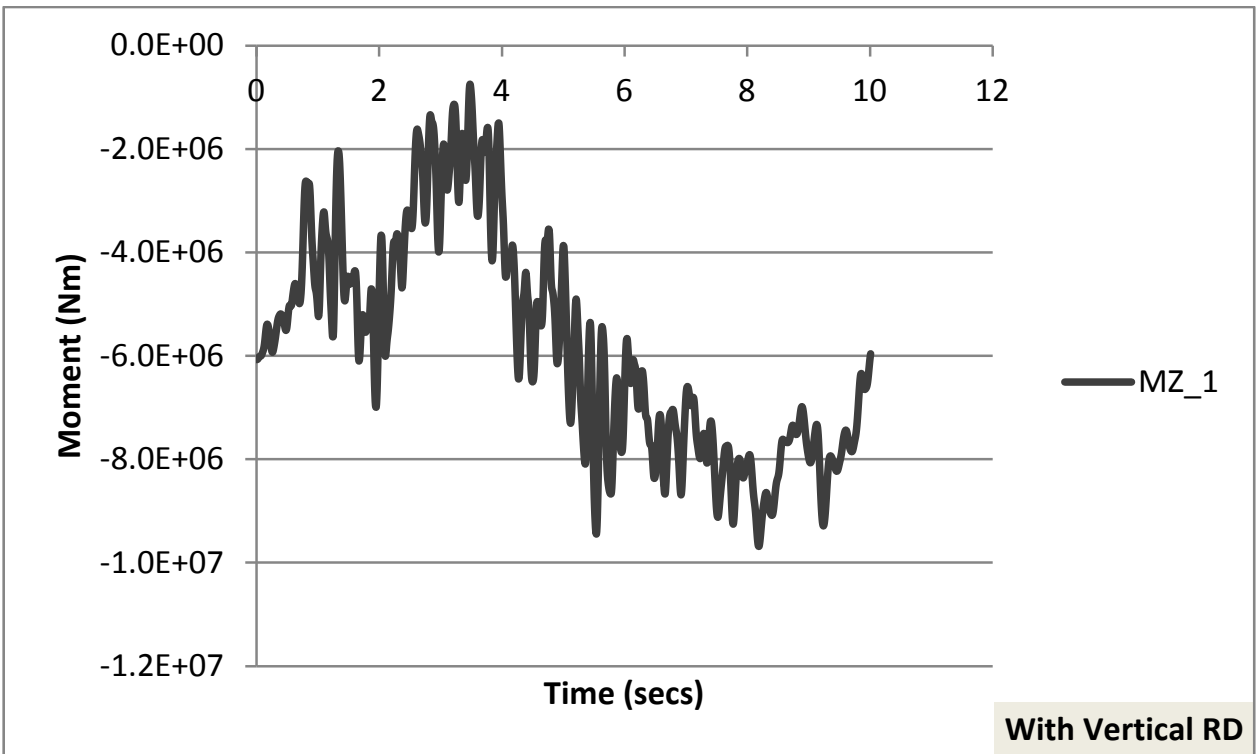



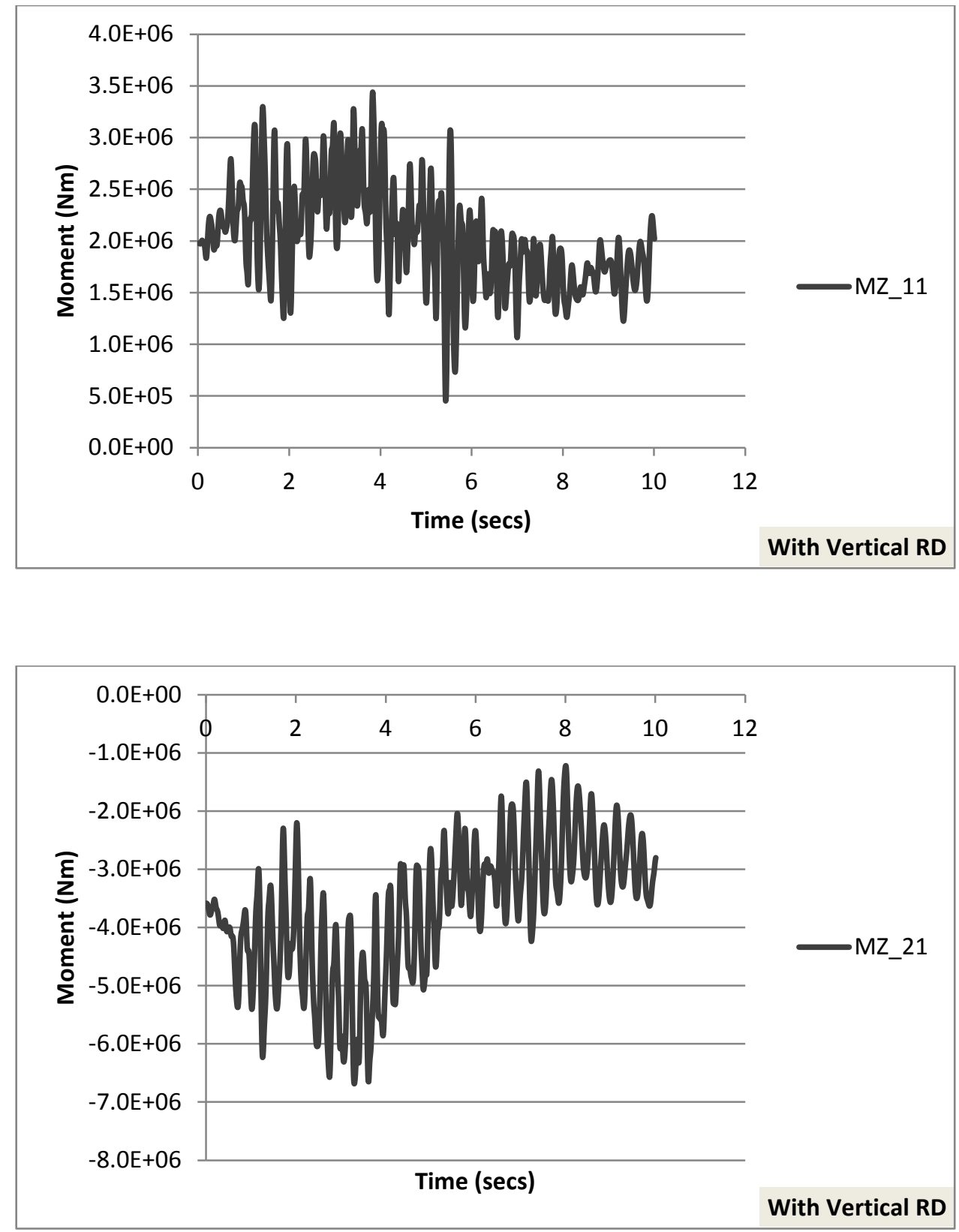

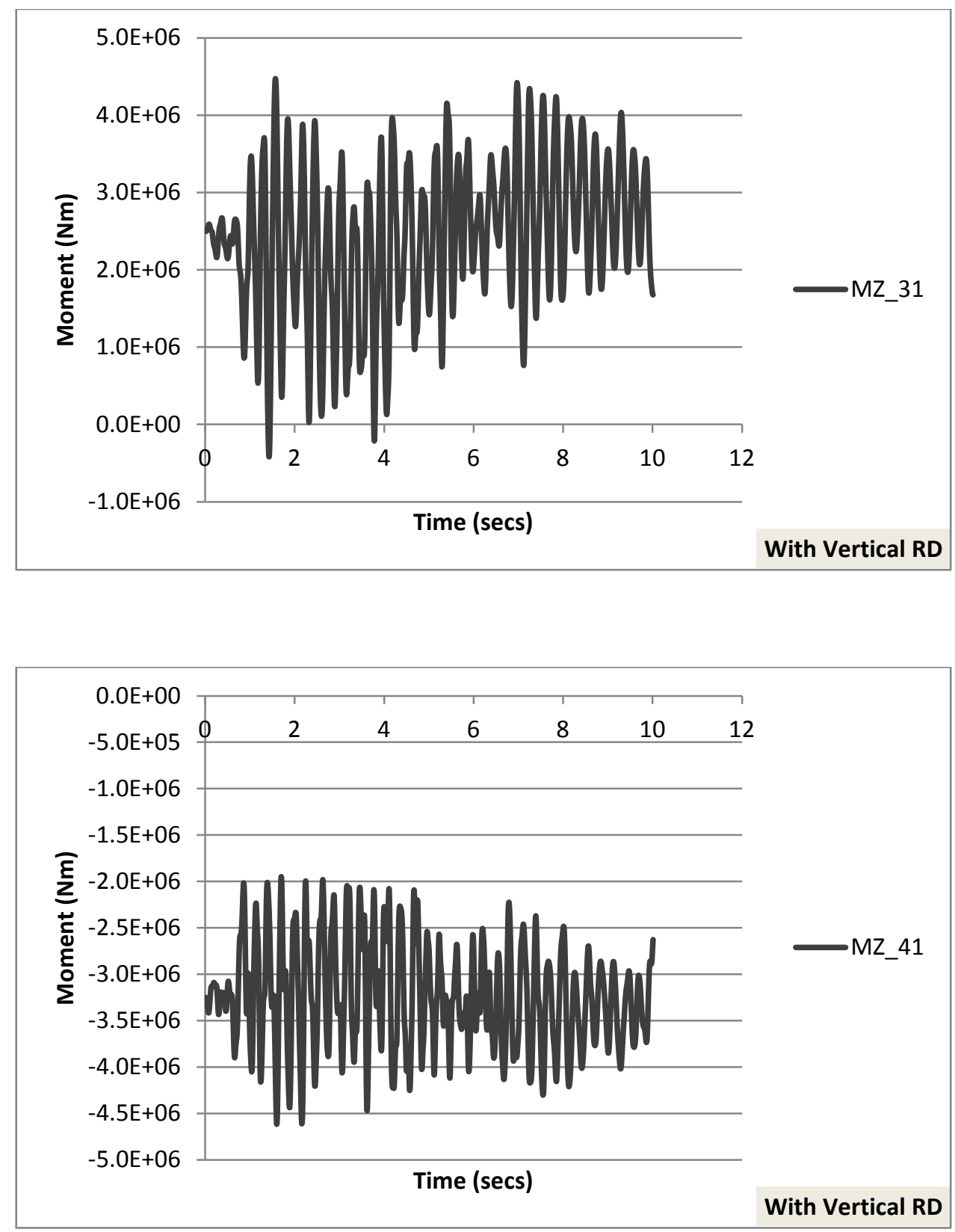

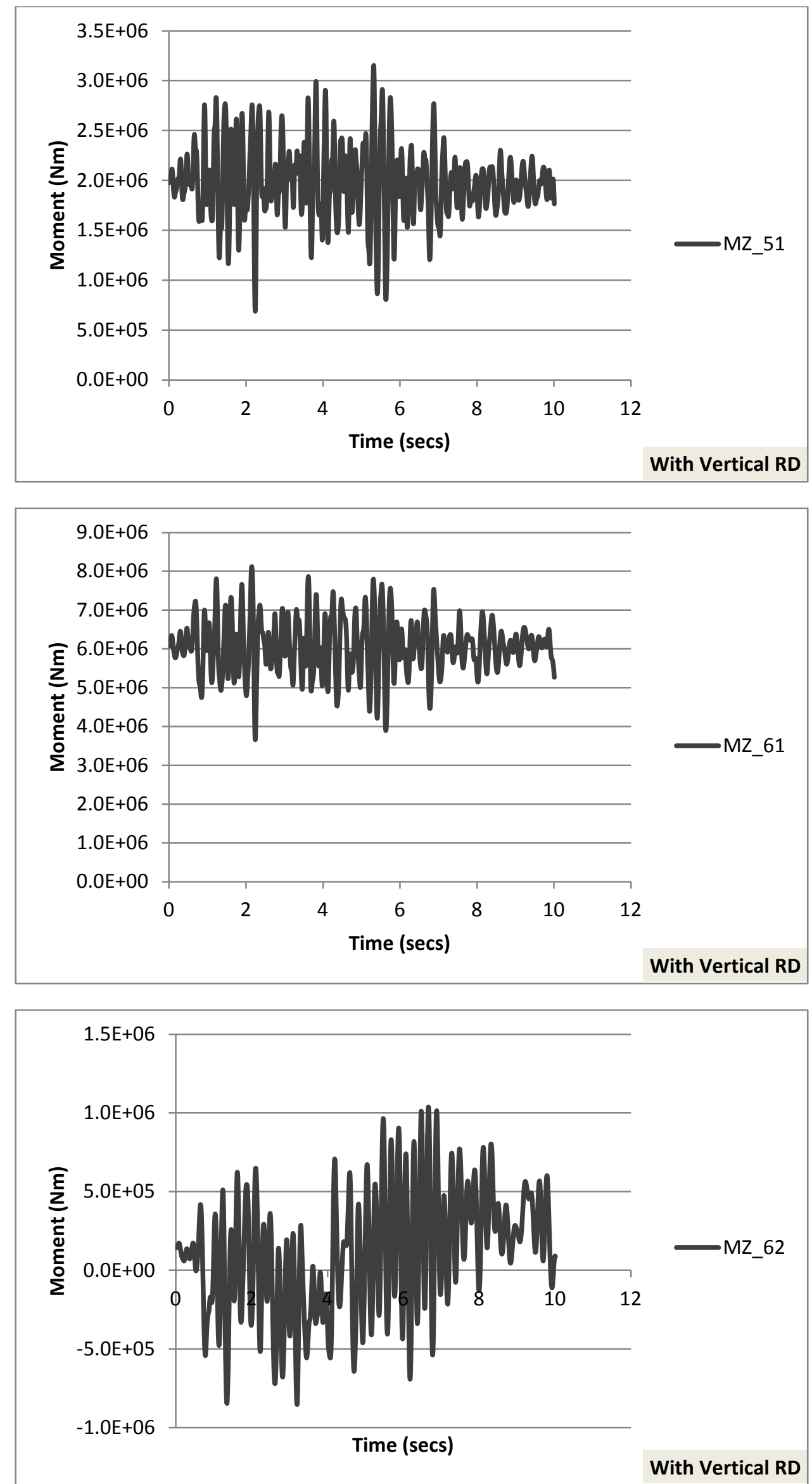

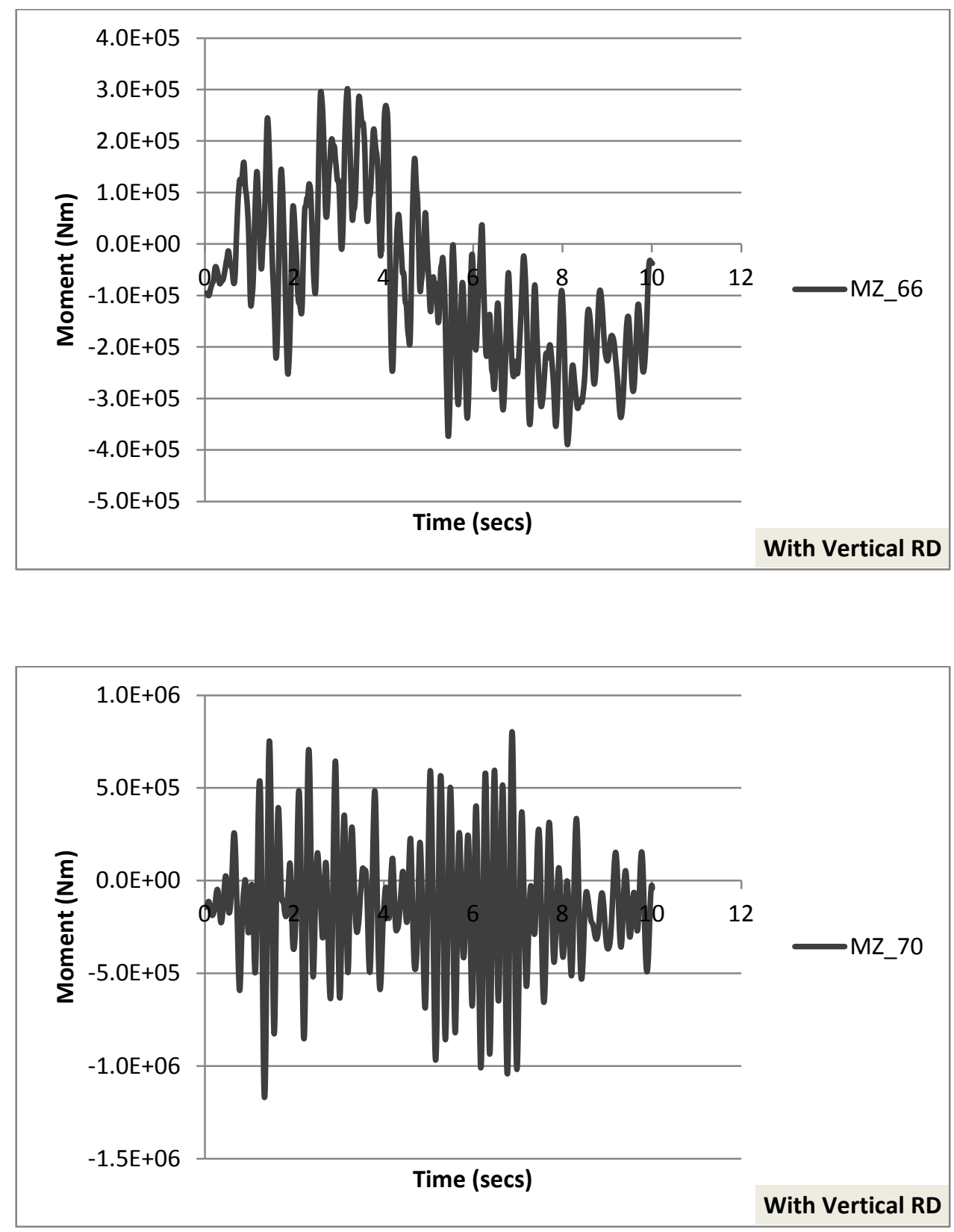


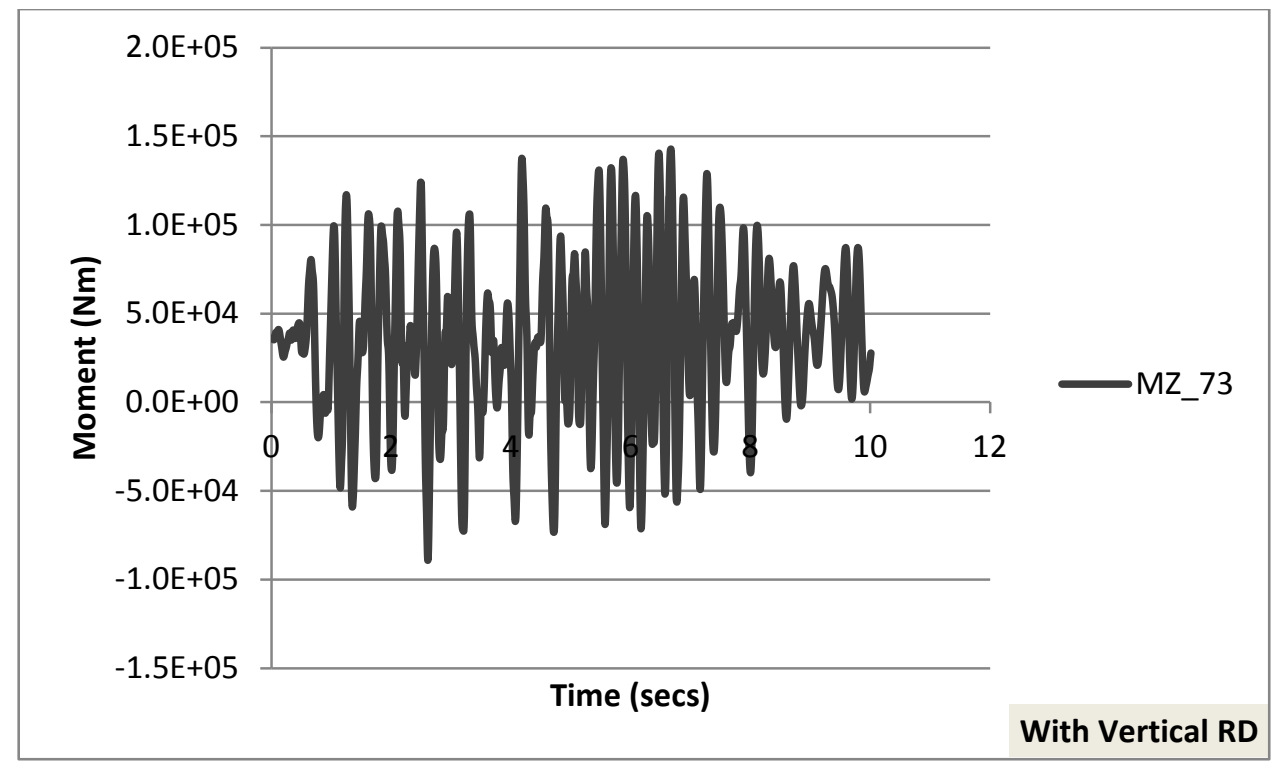

X. Analysis for different stiffness vertically and horizontally.

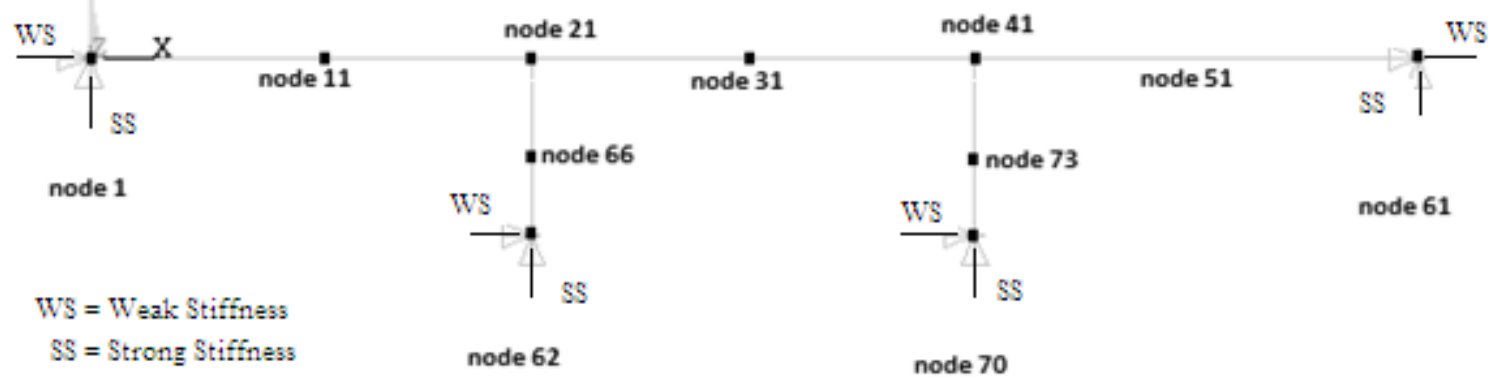

Figure 1: Configuration of soil stiffness for Bridge A 


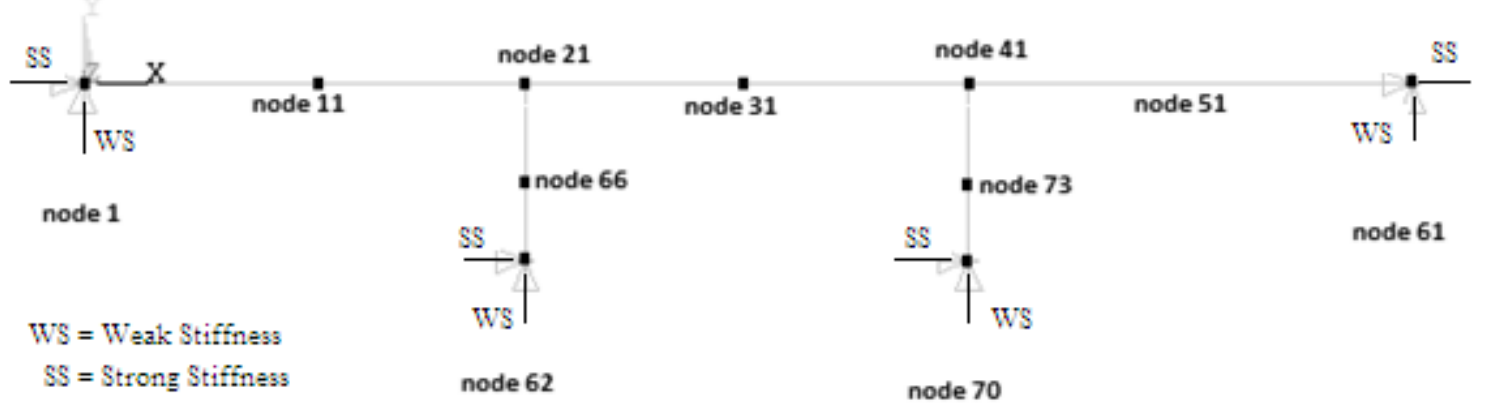

Figure 2: Configuration of soil stiffness for Bridge B

\section{Relative Displacements}

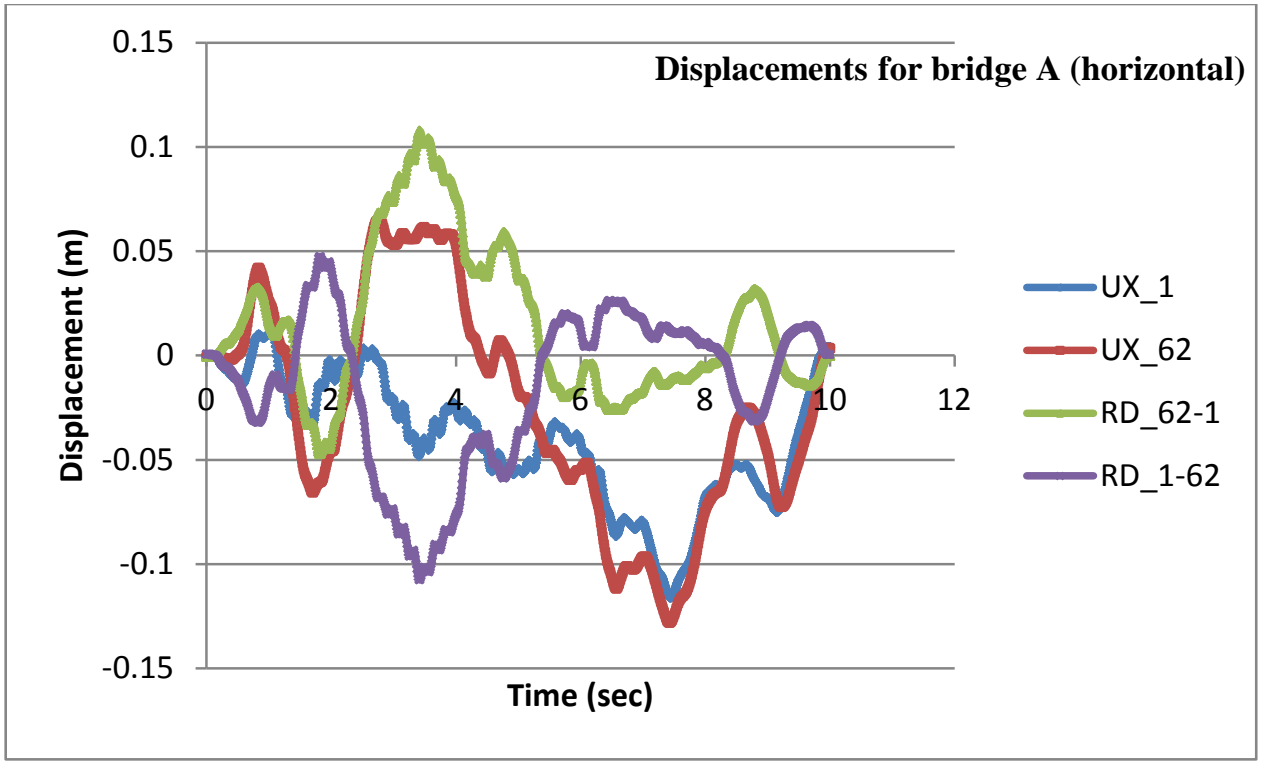

Displacements for bridge A 


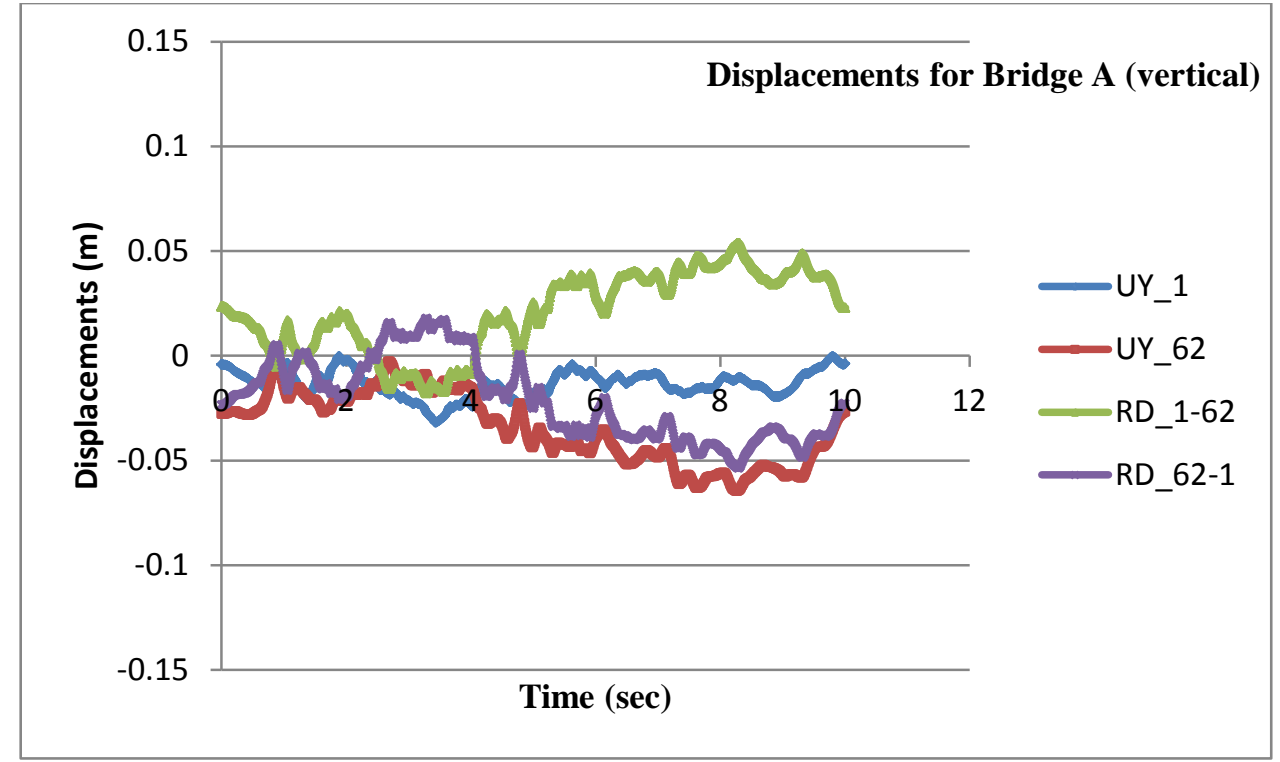

Displacements for bridge B

\section{Dynamic Bending Moment}

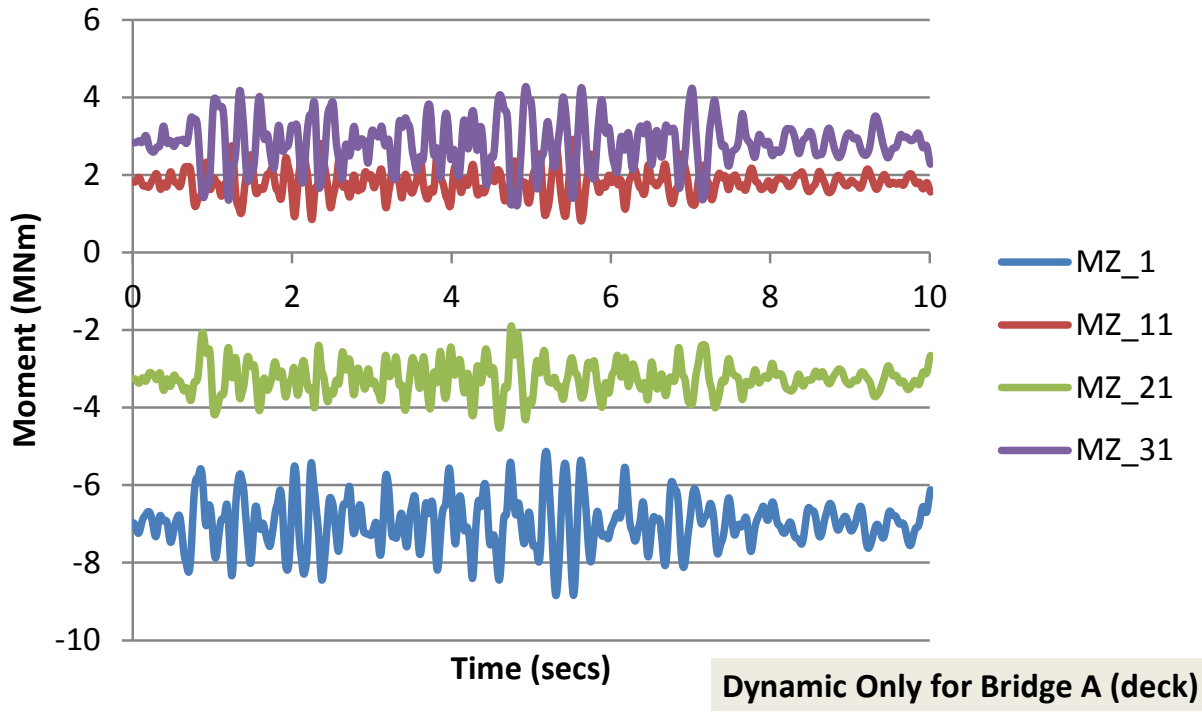



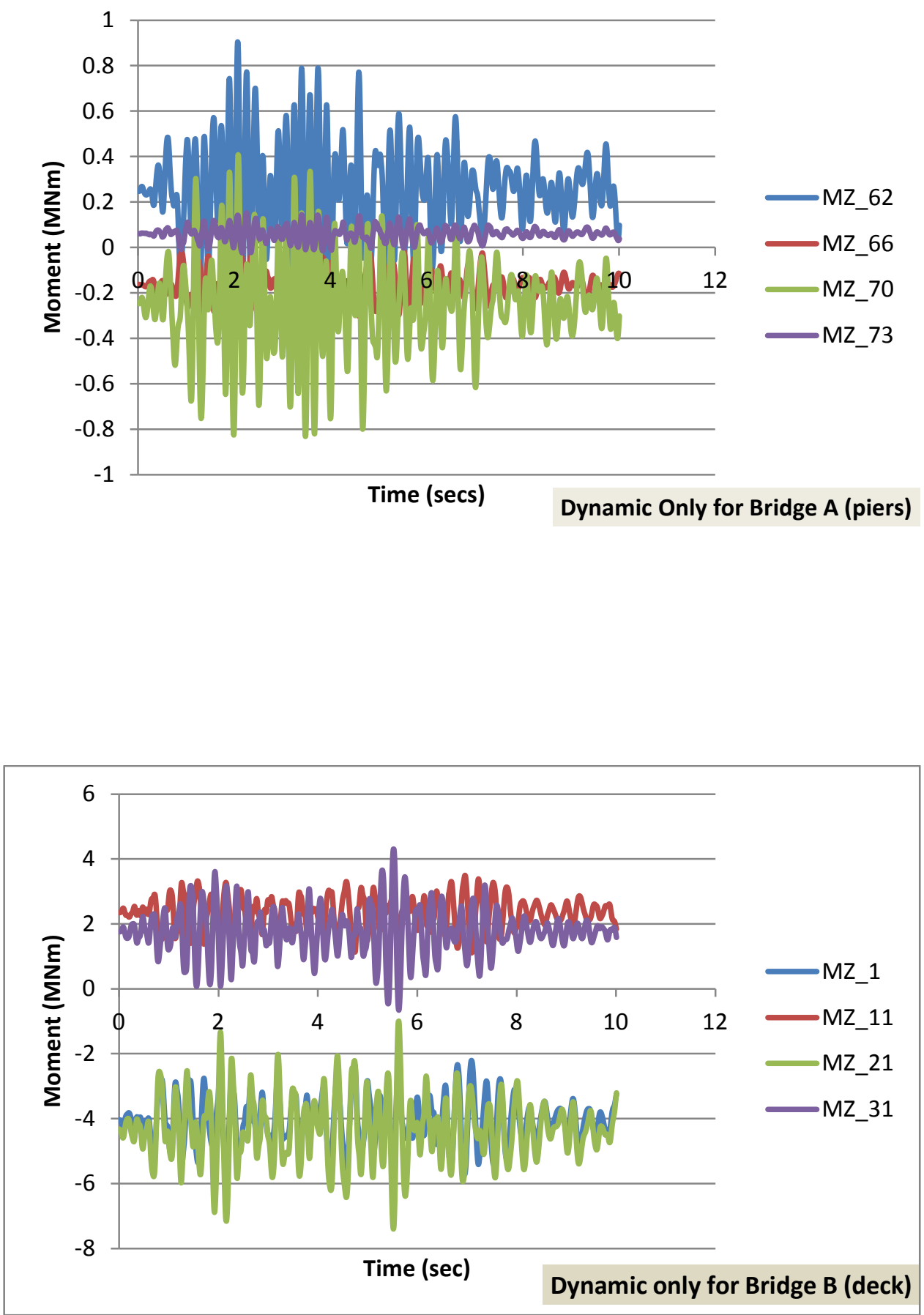


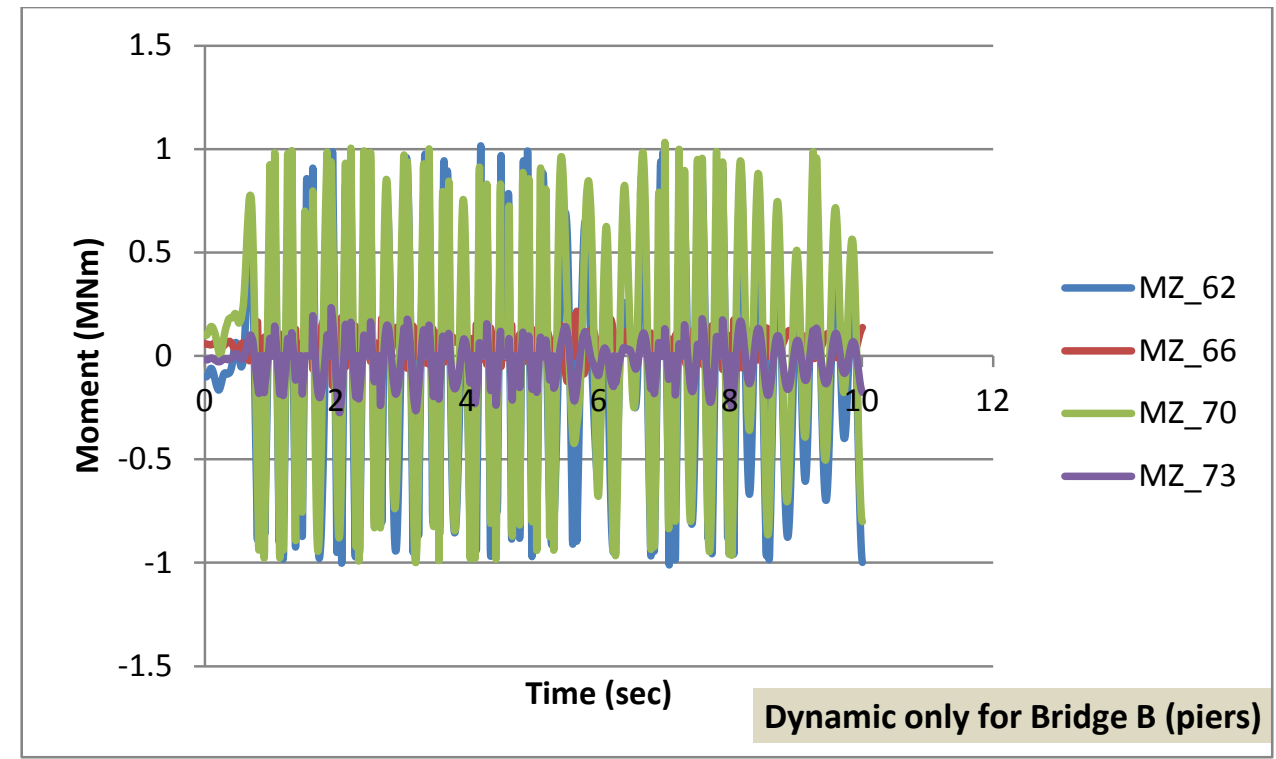

Relative Displacement (horizontal)

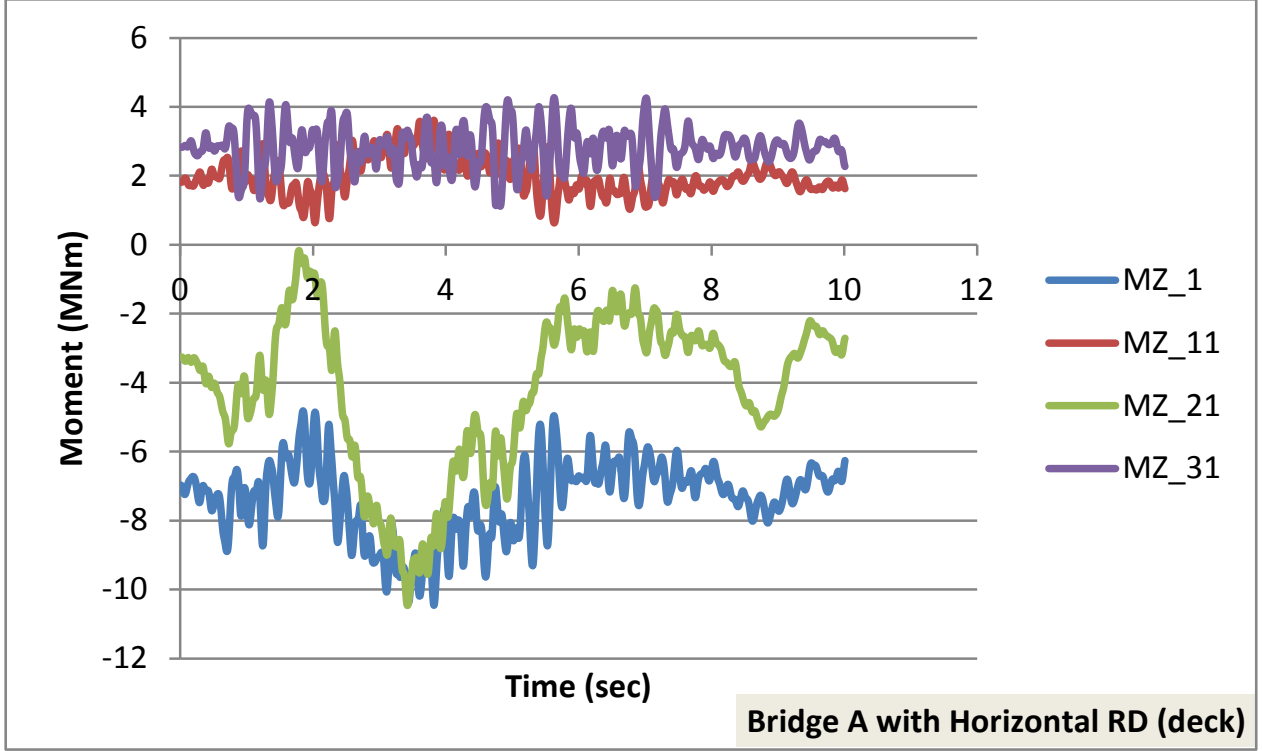



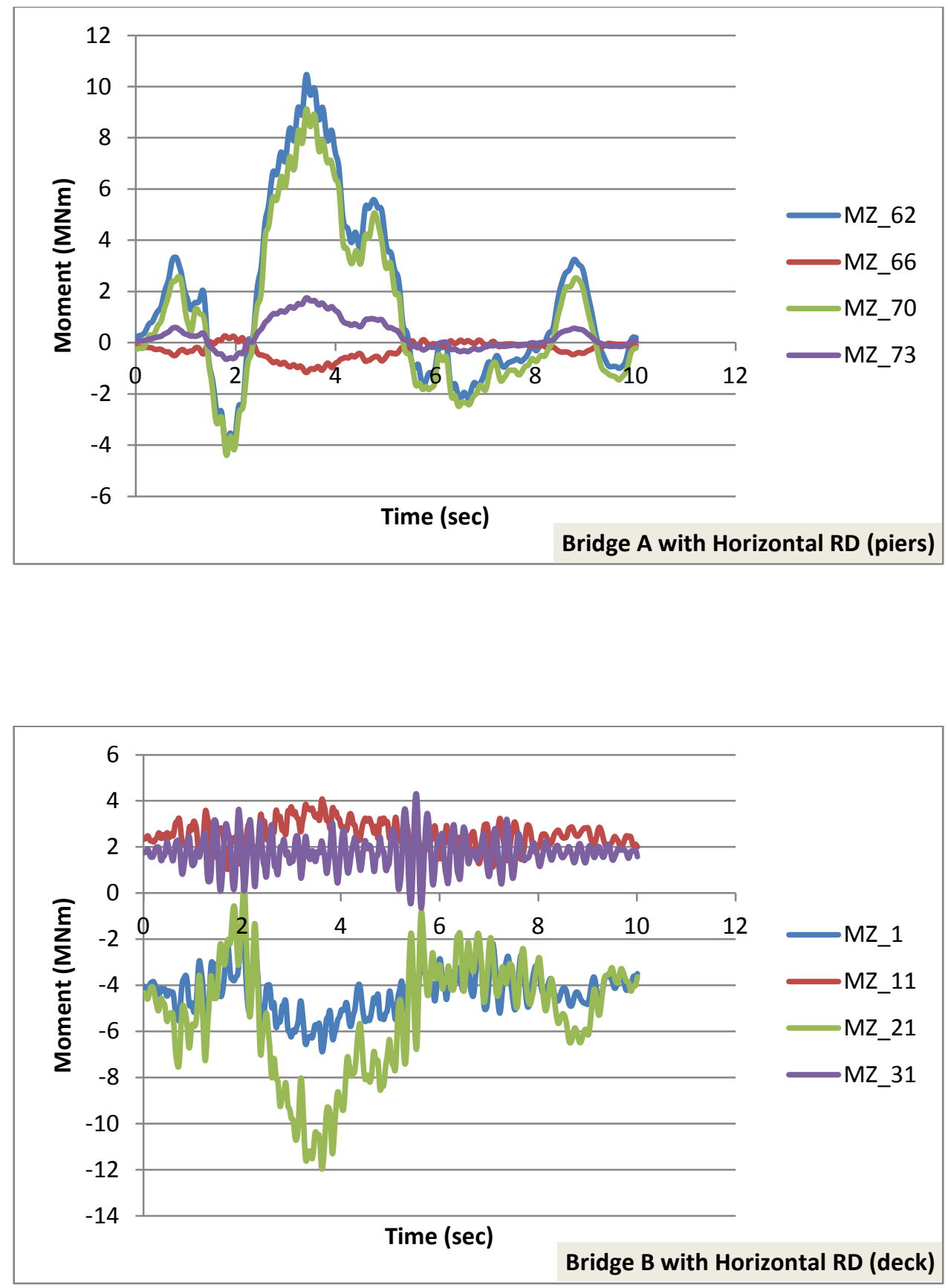

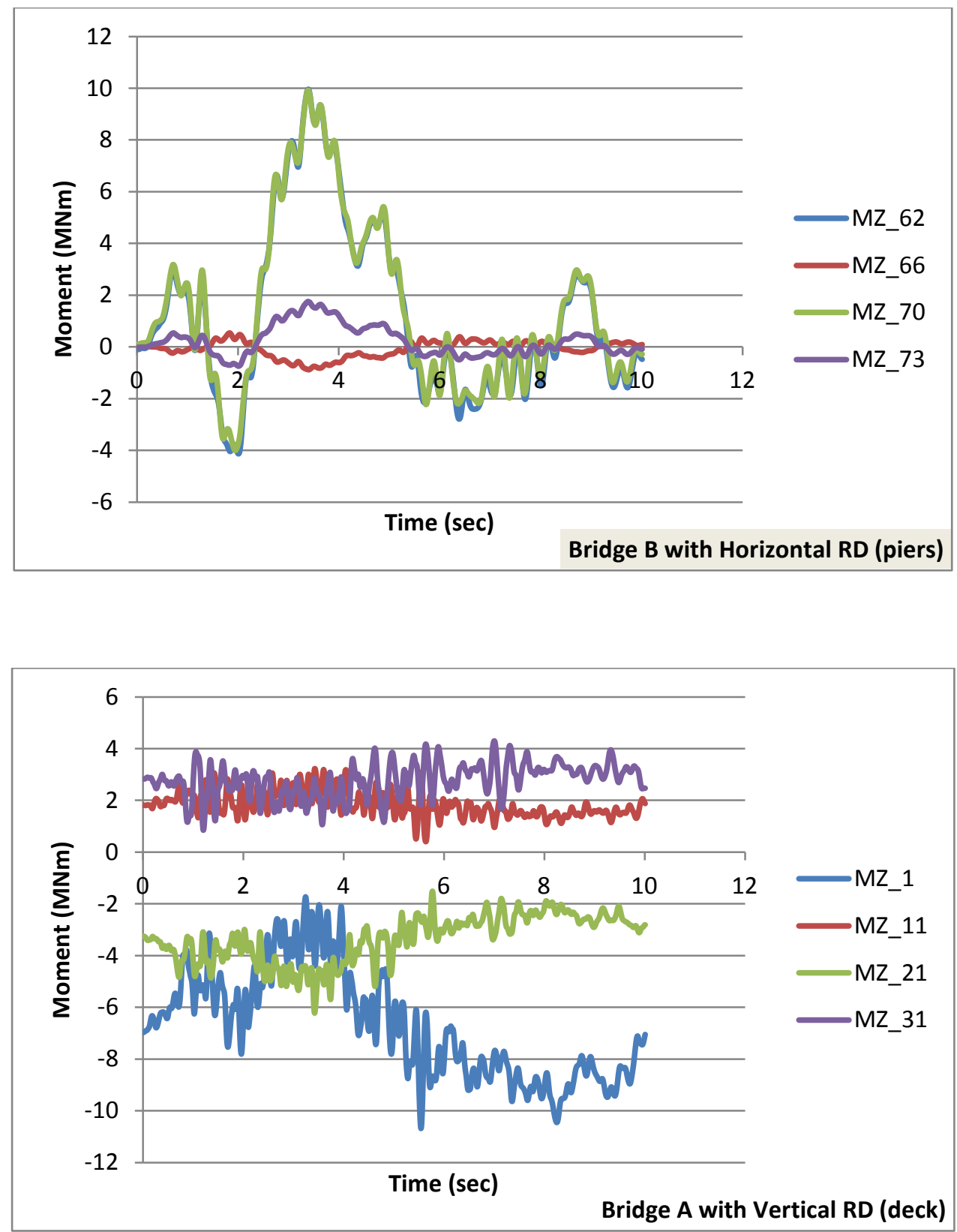

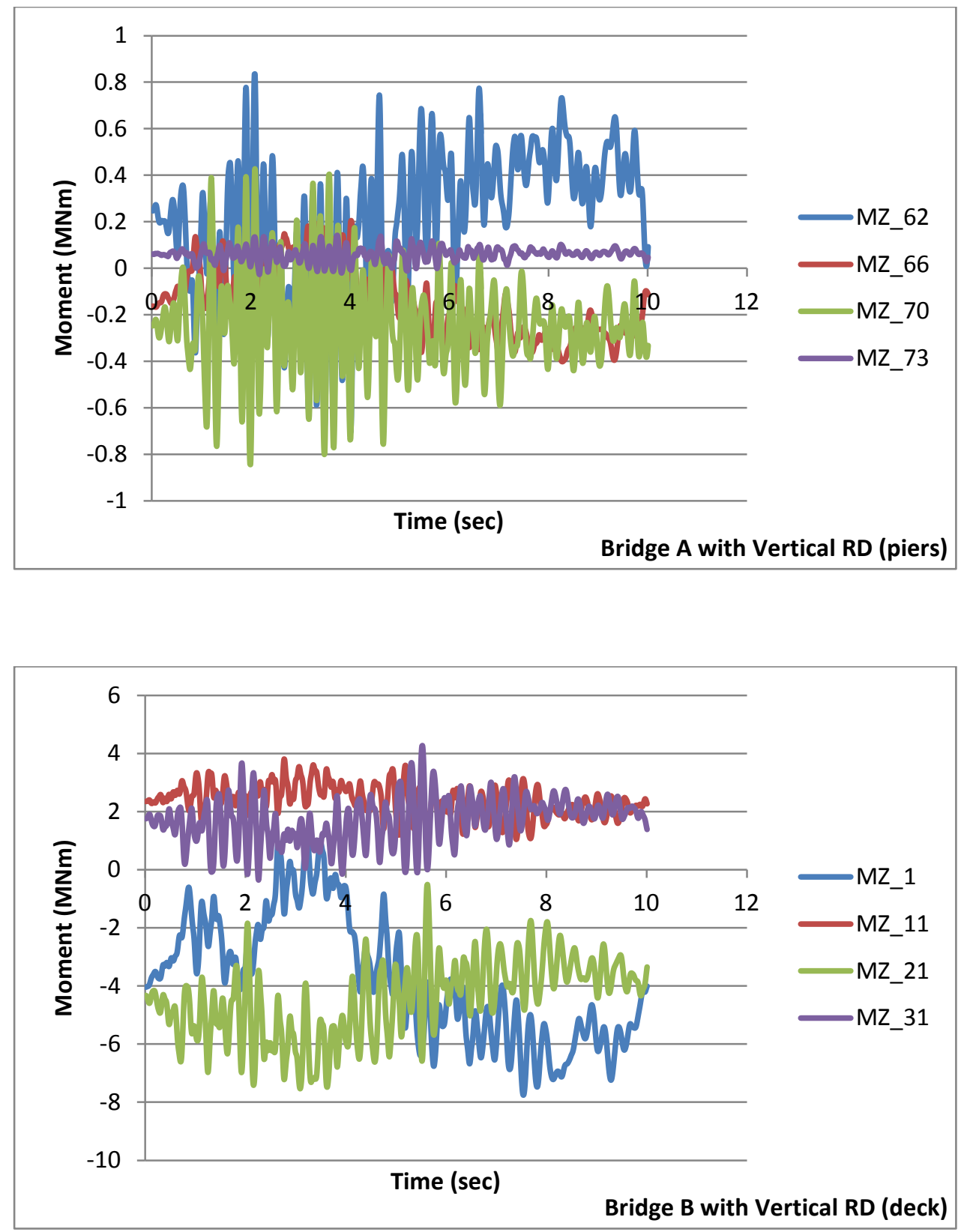


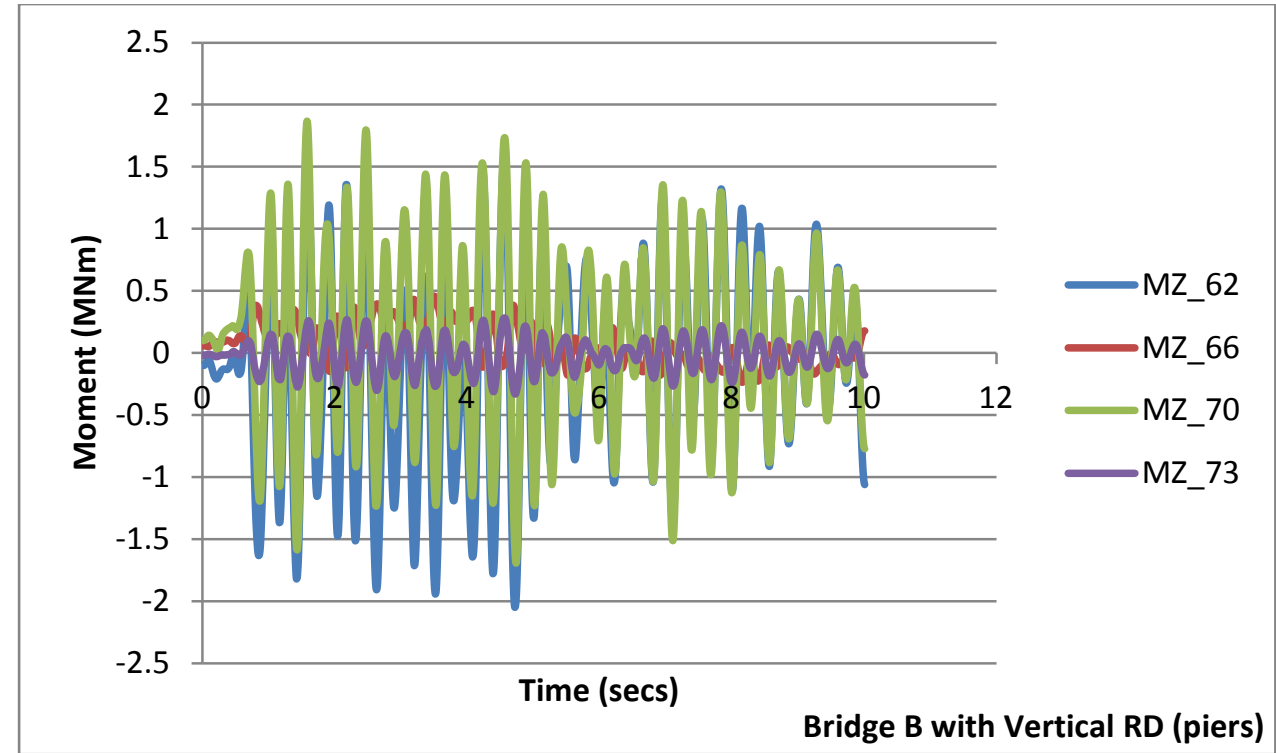




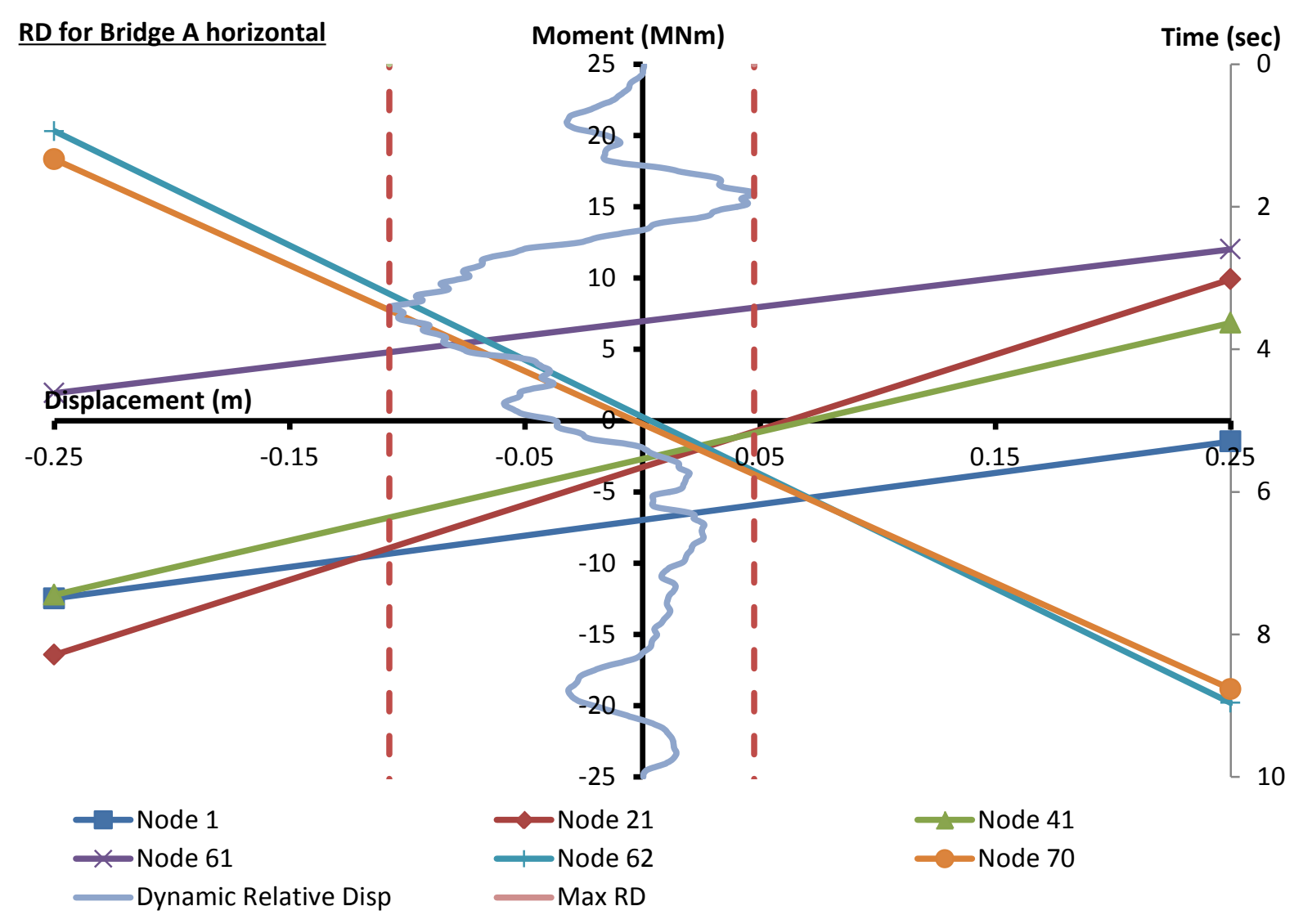




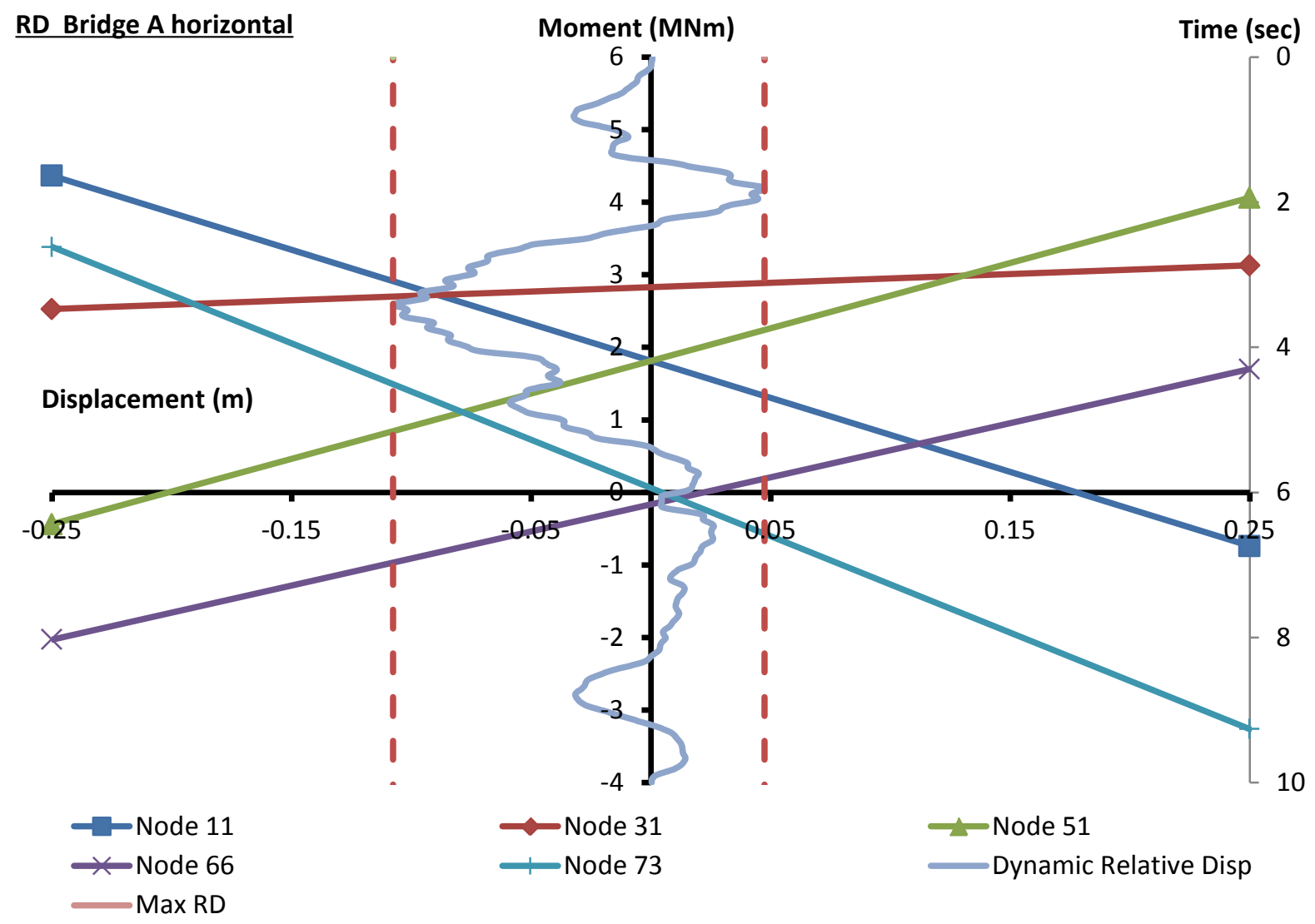

The equations for all lines in this case are as can be seen at below:

$\begin{array}{ll}1 & y=2.20 \mathrm{e}^{7} x-6.97 \mathrm{e}^{6} \\ 11 & y=-1.02 \mathrm{e}^{7} x+1.81 \mathrm{e}^{6} \\ 21 & y=5.27 \mathrm{e}^{7} x-3.25 \mathrm{e}^{6} \\ 31 & y=1.21 \mathrm{e}^{6} x+2.83 \mathrm{e}^{6} \\ 41 & y=3.82 \mathrm{e}^{7} x-2.68 \mathrm{e}^{6} \\ 51 & y=9.00 \mathrm{e}^{6} x+1.81 \mathrm{e}^{6} \\ 61 & y=2.02 \mathrm{e}^{7} x+6.97 \mathrm{e}^{6} \\ 62 & y=-8.02 \mathrm{e}^{7} x+2.48 \mathrm{e}^{5} \\ 66 & y=7.45 \mathrm{e}^{6} x-1.63 \mathrm{e}^{5} \\ 70 & y=-7.43 \mathrm{e}^{7} x-2.47 \mathrm{e}^{5} \\ 73 & y=-1.33 \mathrm{e}^{7} x+6.07 \mathrm{e}^{4}\end{array}$



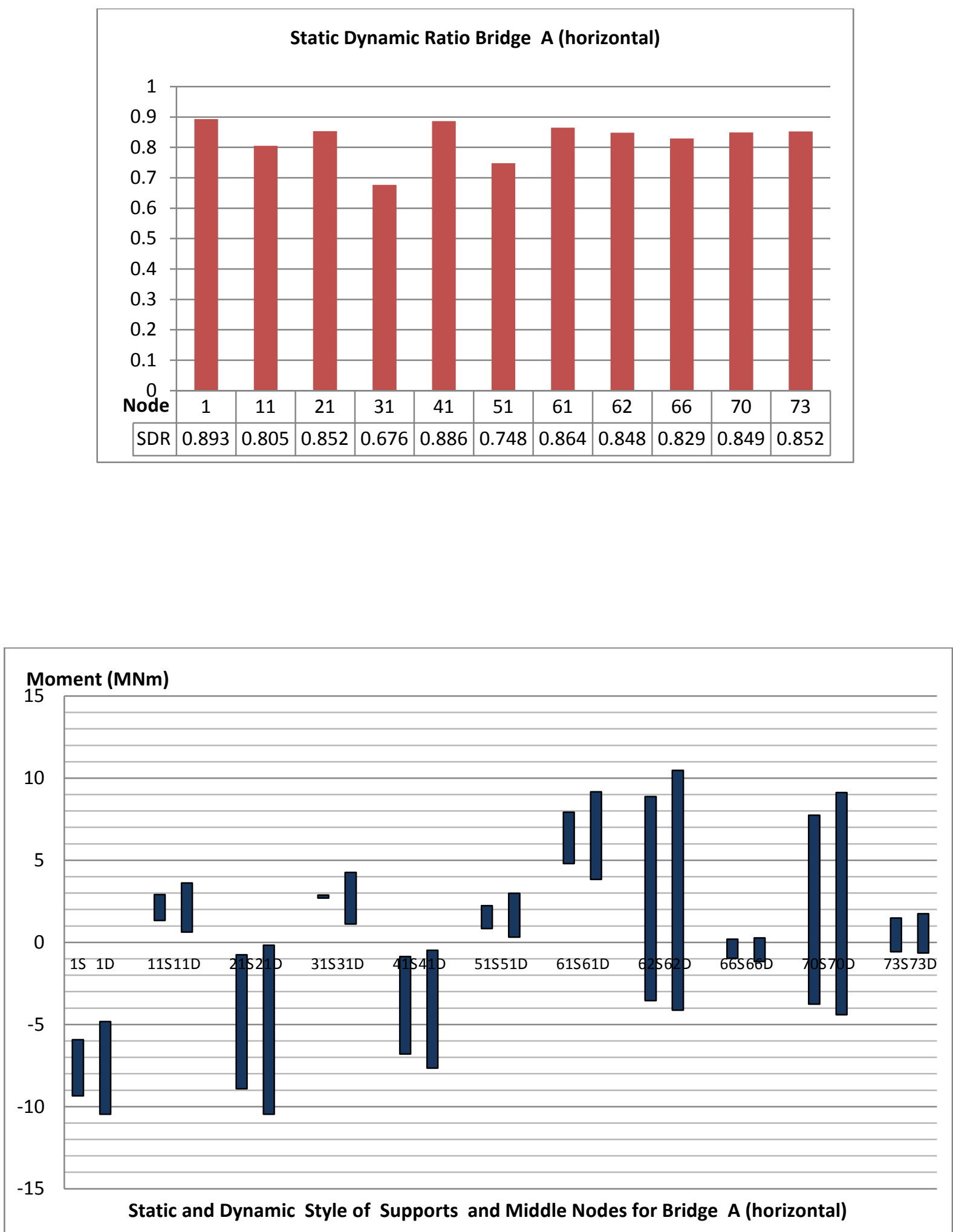

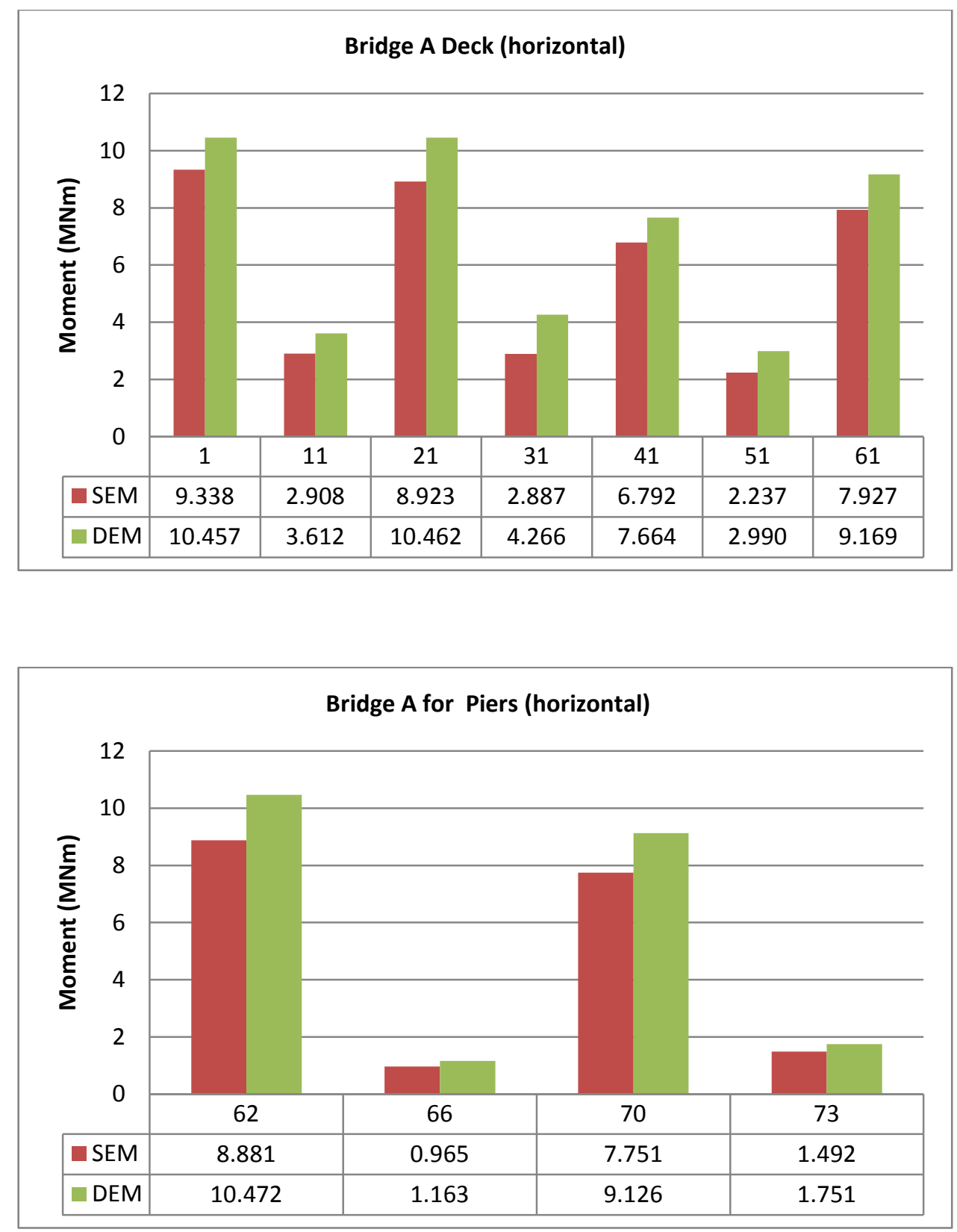


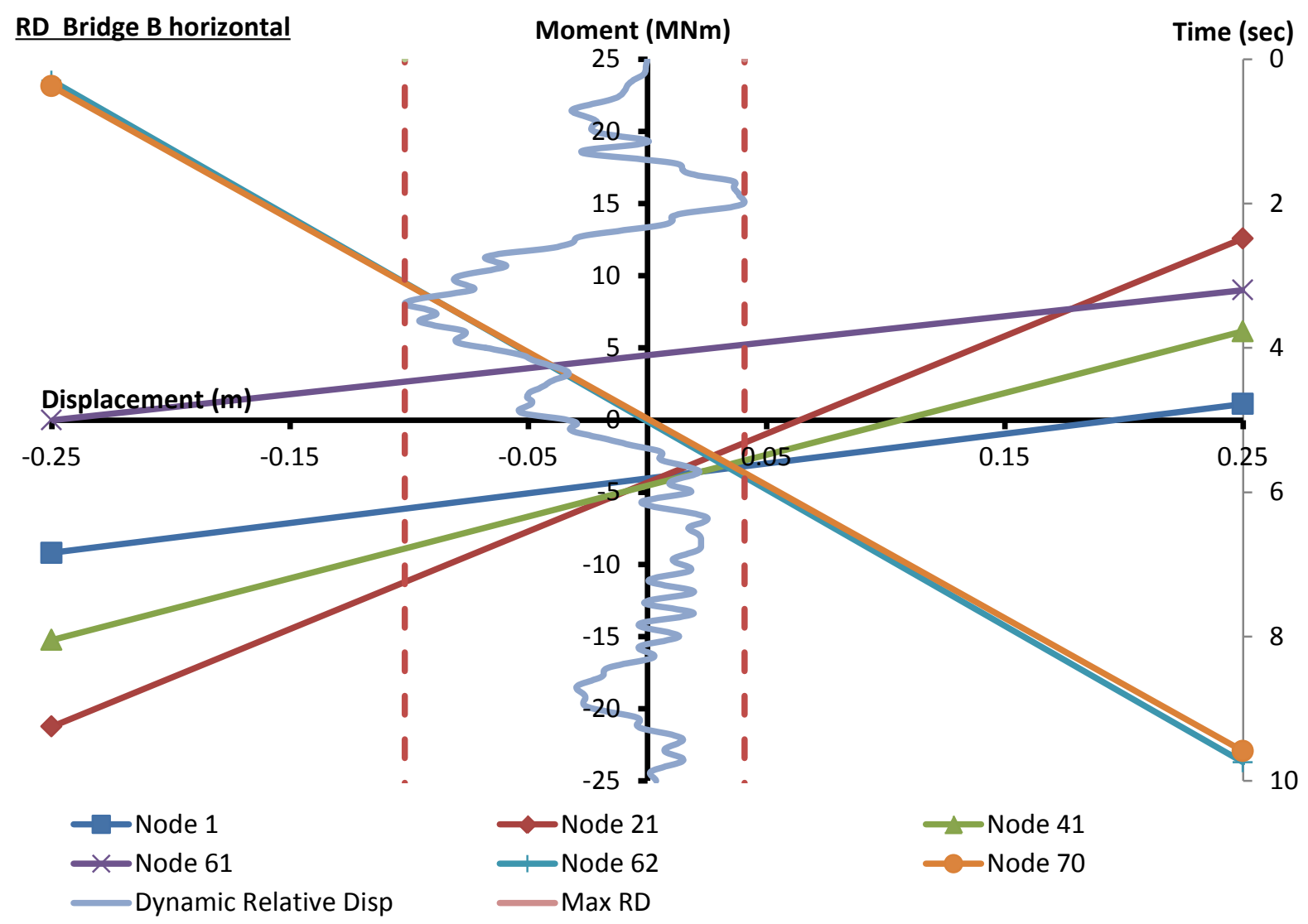

The equations for all lines in this case are as can be seen at below:

$\begin{array}{cc}1 & y=2.06 \mathrm{e}^{7} x-4.04 \mathrm{e}^{6} \\ 11 & y=-1.19 \mathrm{e}^{7} x+2.35 \mathrm{e}^{6} \\ 21 & y=6.76 \mathrm{e}^{7} x-4.33 \mathrm{e}^{6} \\ 31 & y=4.73 \mathrm{e}^{5} x+1.76 \mathrm{e}^{6} \\ 41 & y=4.28 \mathrm{e}^{7} x-4.54 \mathrm{e}^{6} \\ 51 & y=1.14 \mathrm{e}^{7} x+2.35 \mathrm{e}^{6} \\ 61 & y=1.80 \mathrm{e}^{7} x+4.51 \mathrm{e}^{6} \\ 62 & y=-9.44 \mathrm{e}^{7} x-1.01 \mathrm{e}^{5} \\ 66 & y=8.81 \mathrm{e}^{6} x+5.75 \mathrm{e}^{4} \\ 70 & y=-9.21 \mathrm{e}^{7} x+1.01 \mathrm{e}^{5} \\ 73 & y=-1.65 \mathrm{e}^{7} x-1.80 \mathrm{e}^{4}\end{array}$



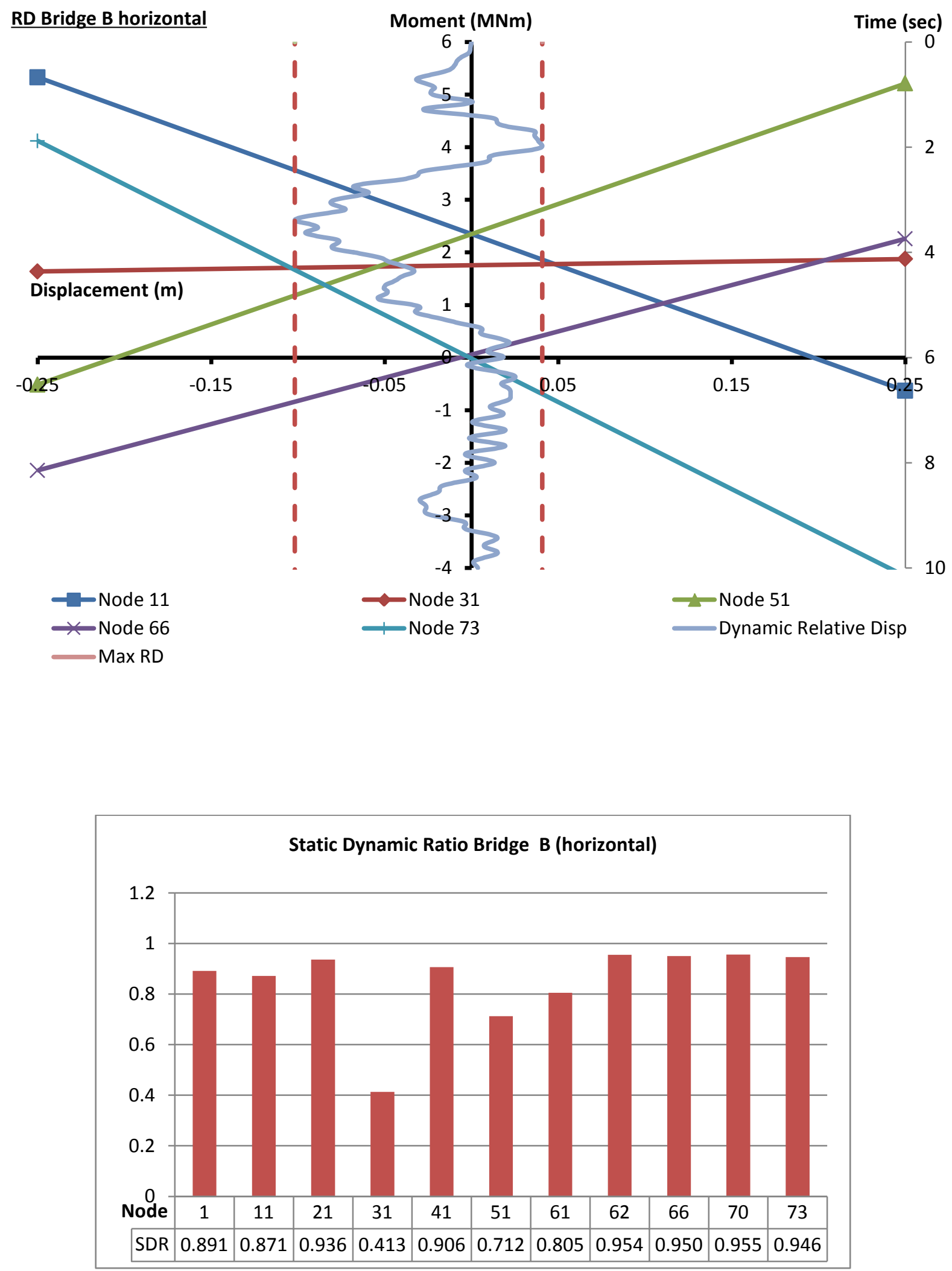

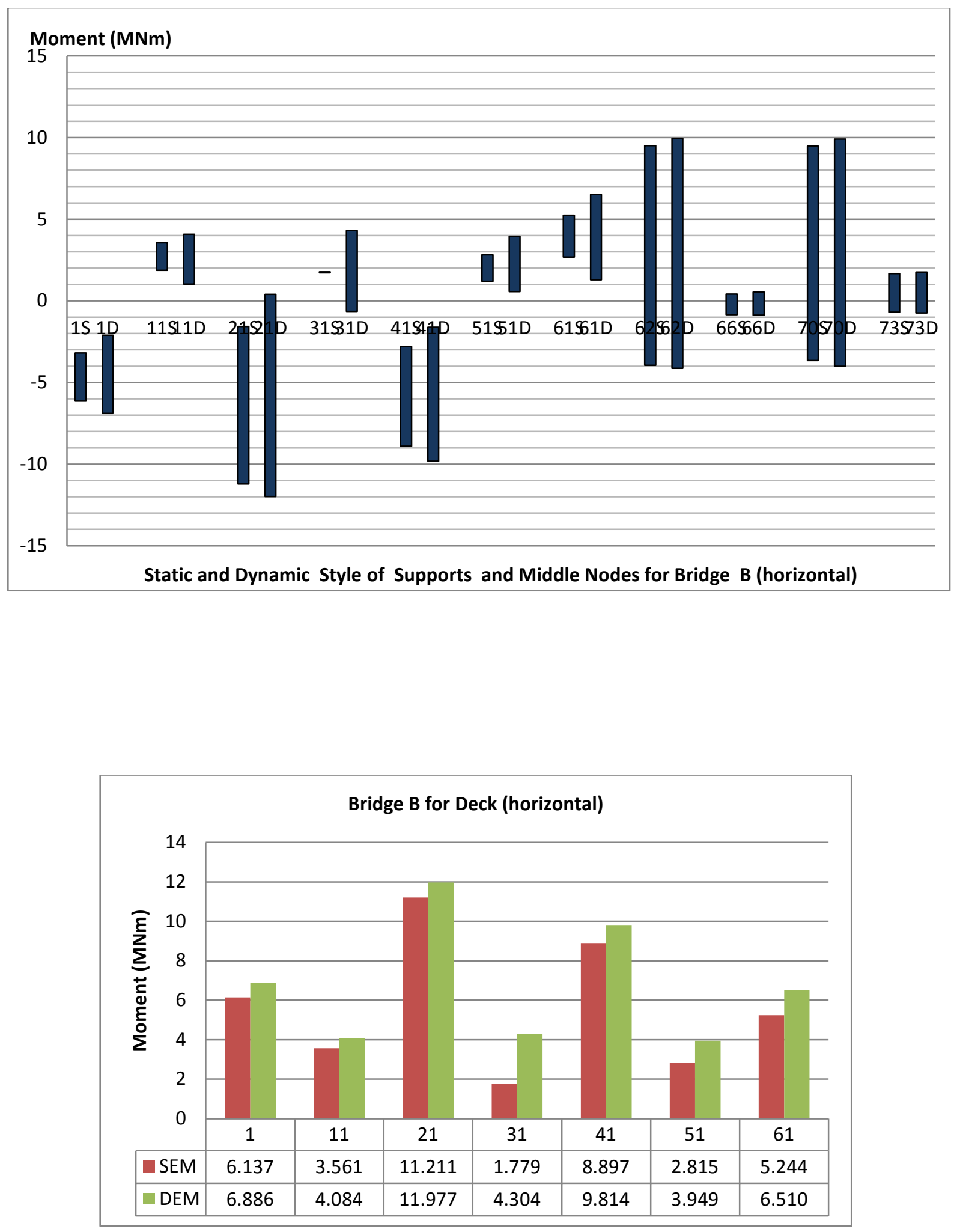

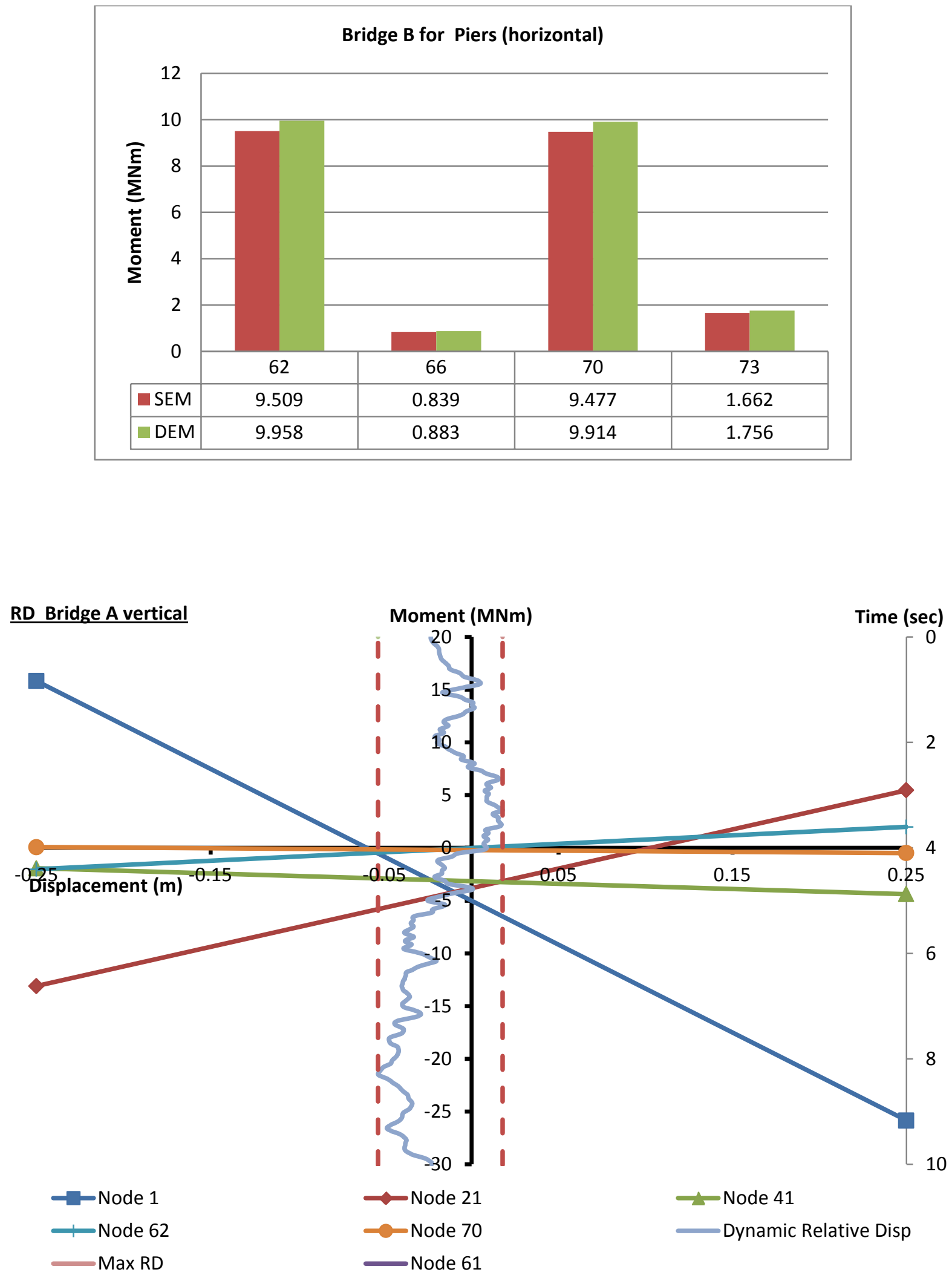


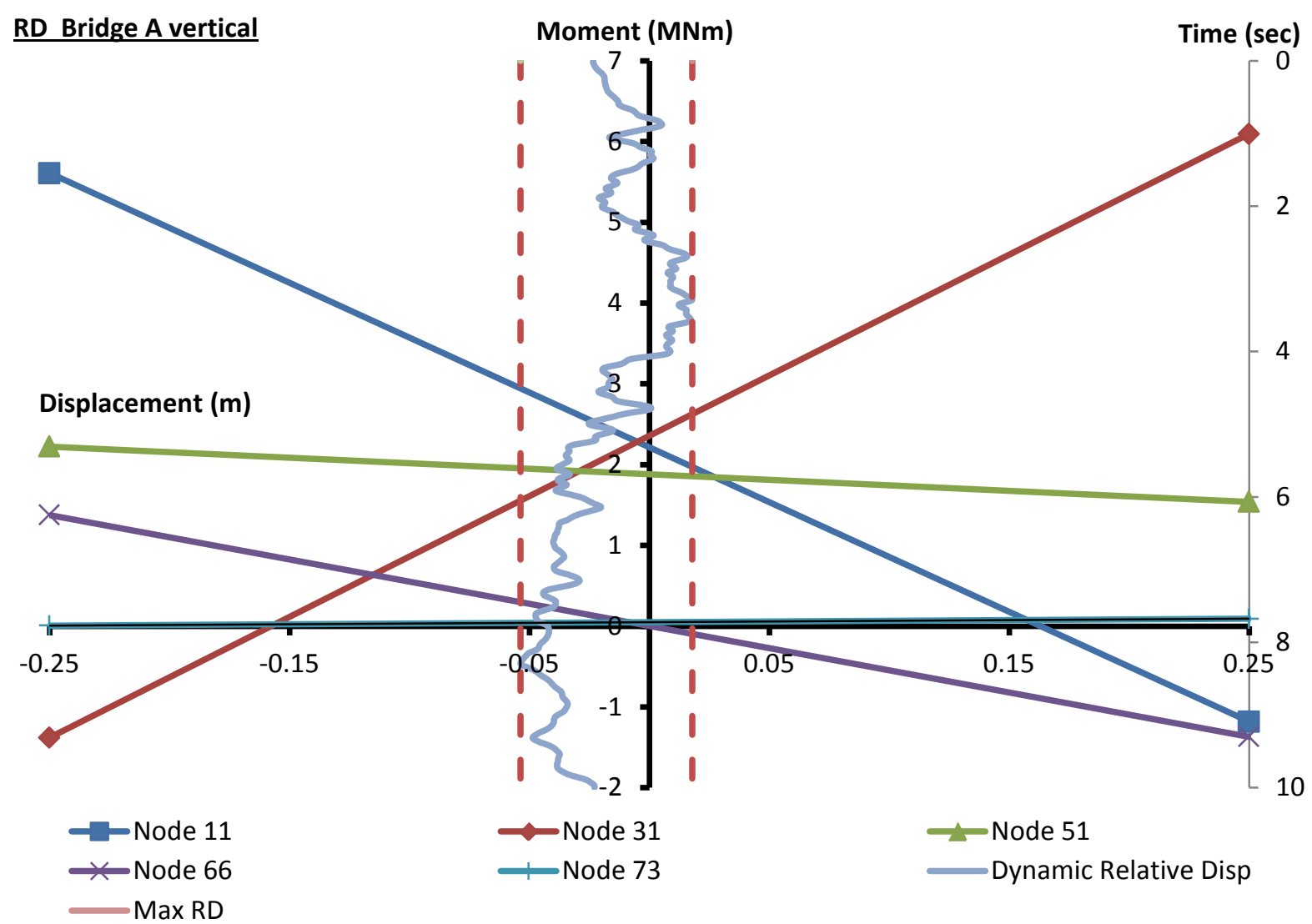

The equations for all lines in this case are as can be seen at below:

$$
\begin{aligned}
& y=-8.33 \mathrm{e}^{7} x-5.01 \mathrm{e}^{6} \\
& y=-1.36 \mathrm{e}^{7} x+2.21 \mathrm{e}^{6} \\
& y=3.71 \mathrm{e}^{7} x-3.81 \mathrm{e}^{6} \\
& y=1.50 \mathrm{e}^{7} x+2.36 \mathrm{e}^{6} \\
& y=-4.91 \mathrm{e}^{6} x-3.16 \mathrm{e}^{6} \\
& \mathrm{y}=-1.36 \mathrm{e}^{7} x+2.21 \mathrm{e}^{6} \\
& y=-2.17 \mathrm{e}^{6} x+6.35 \mathrm{e}^{6} \\
& \mathrm{y}=-1.12 \mathrm{e}^{6} x-2.06 \mathrm{e}^{5} \\
& y=-5.49 \mathrm{e}^{6} x+3.20 \mathrm{e}^{3} \\
& y=-1.12 \mathrm{e}^{6} \mathrm{x}-2.06 \mathrm{e}^{5} \\
& y=1.66 \mathrm{e}^{5} x+5.00 \mathrm{e}^{4}
\end{aligned}
$$



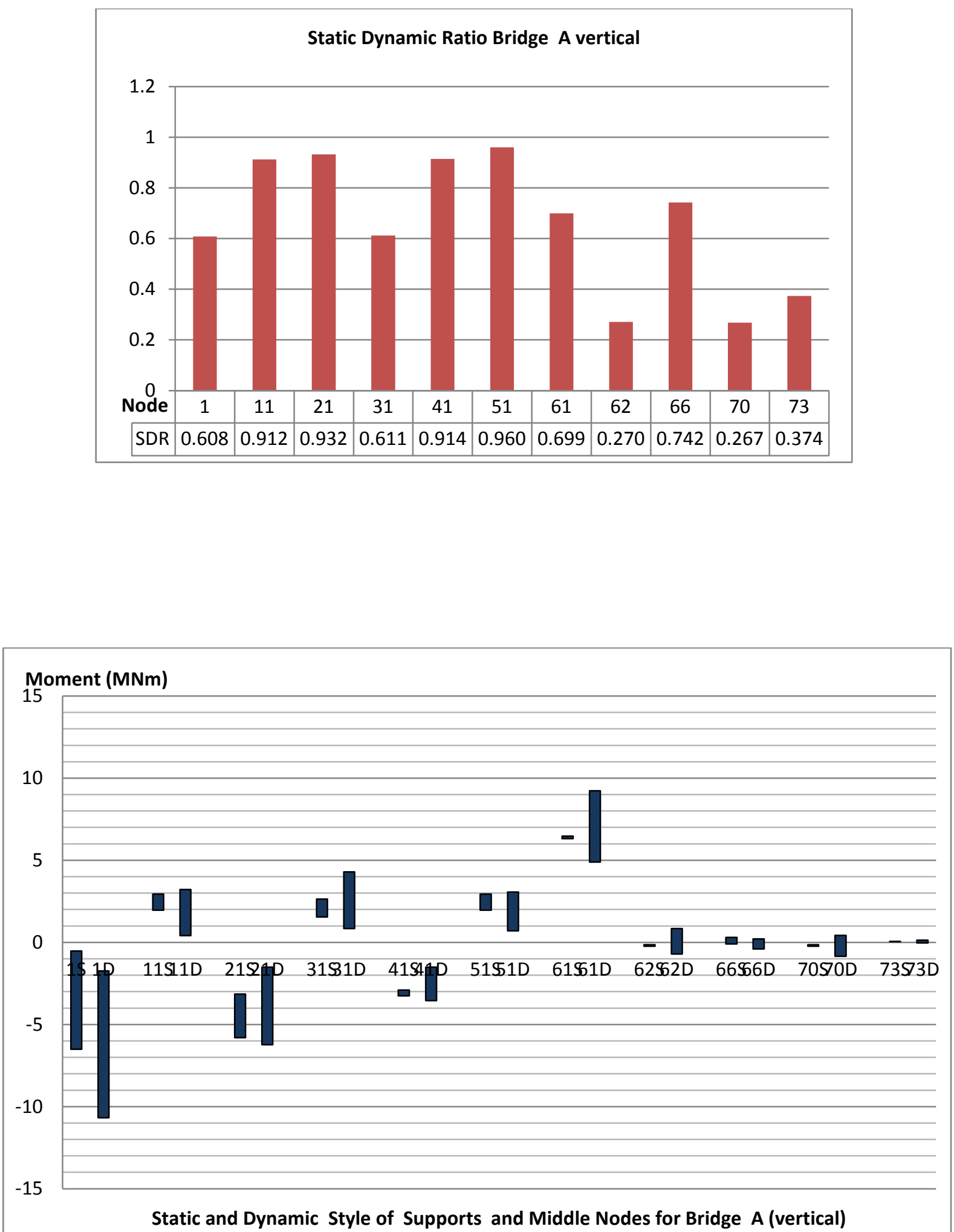

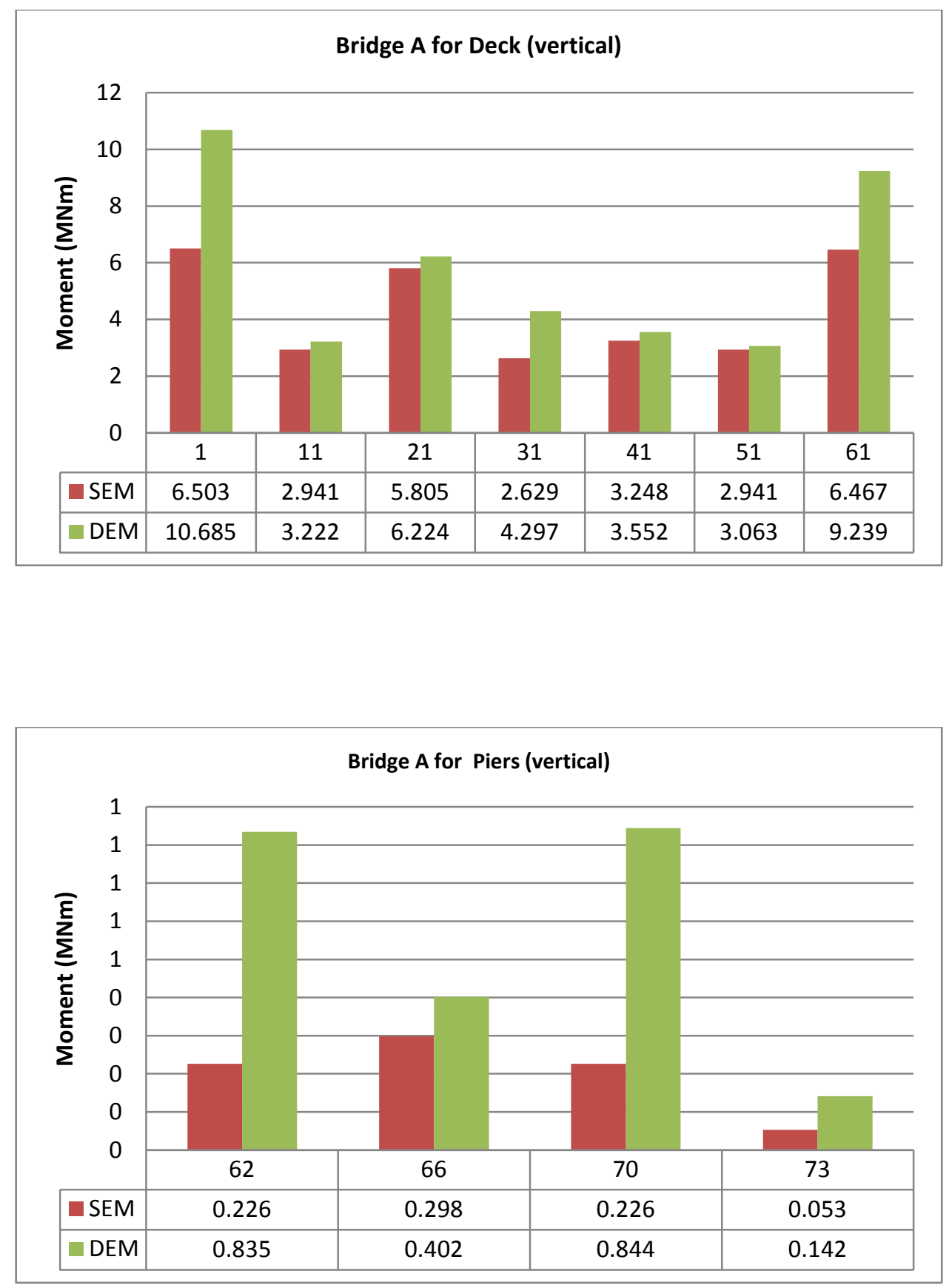


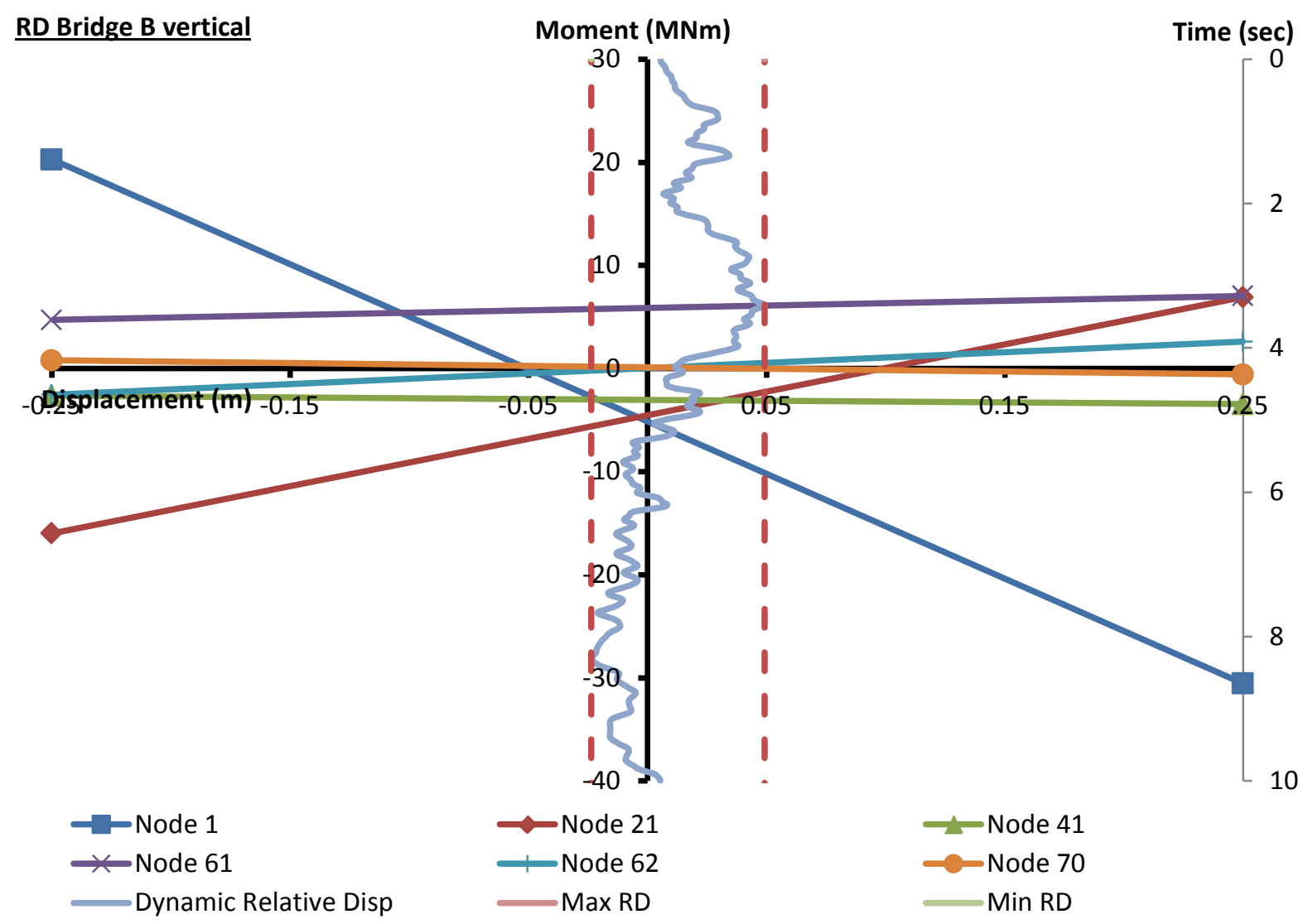

The equations for all lines in this case are as can be seen at below:

$$
\begin{aligned}
& y=-1.02 \mathrm{e}^{8} x-5.13 \mathrm{e}^{6} \\
& y=-1.62 \mathrm{e}^{7} x+1.92 \mathrm{e}^{6} \\
& y=4.58 \mathrm{e}^{7} x-4.53 \mathrm{e}^{6} \\
& y=1.93 \mathrm{e}^{7} x+2.35 \mathrm{e}^{6} \\
& y=-1.65 \mathrm{e}^{6} x-3.04 \mathrm{e}^{6} \\
& y=-3.15 \mathrm{e}^{6} x+2.18 \mathrm{e}^{6} \\
& \mathrm{y}=4.66 \mathrm{e}^{6} x+5.88 \mathrm{e}^{6} \\
& \mathrm{y}=1.03 \mathrm{e}^{7} x+3.41 \mathrm{e}^{4} \\
& y=-6.63 \mathrm{e}^{6} x-9.89 \mathrm{e}^{4} \\
& y=-2.77 \mathrm{e}^{6} x+1.12 \mathrm{e}^{5} \\
& y=3.55 \mathrm{e}^{5} x+2.58 \mathrm{e}^{4}
\end{aligned}
$$




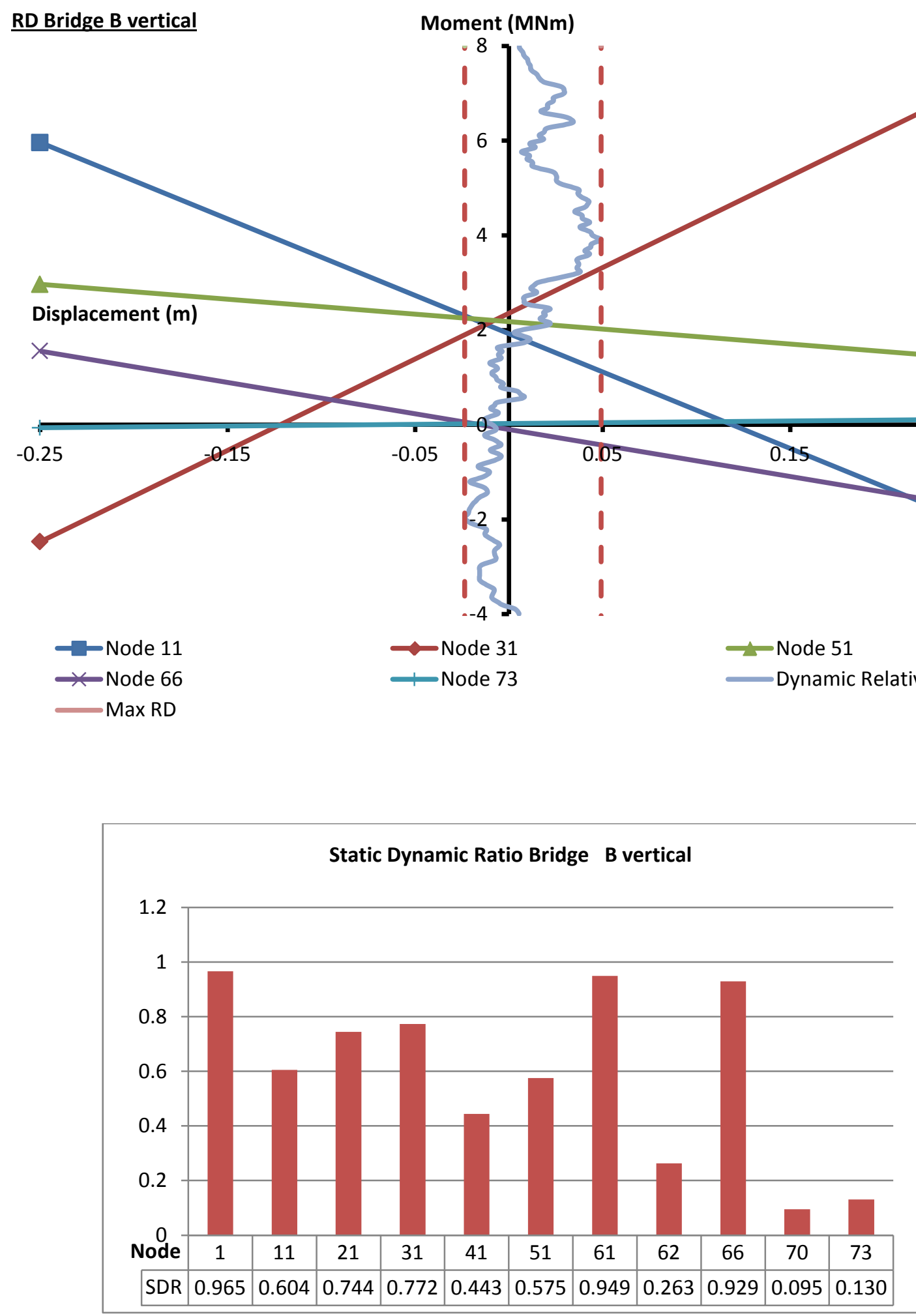



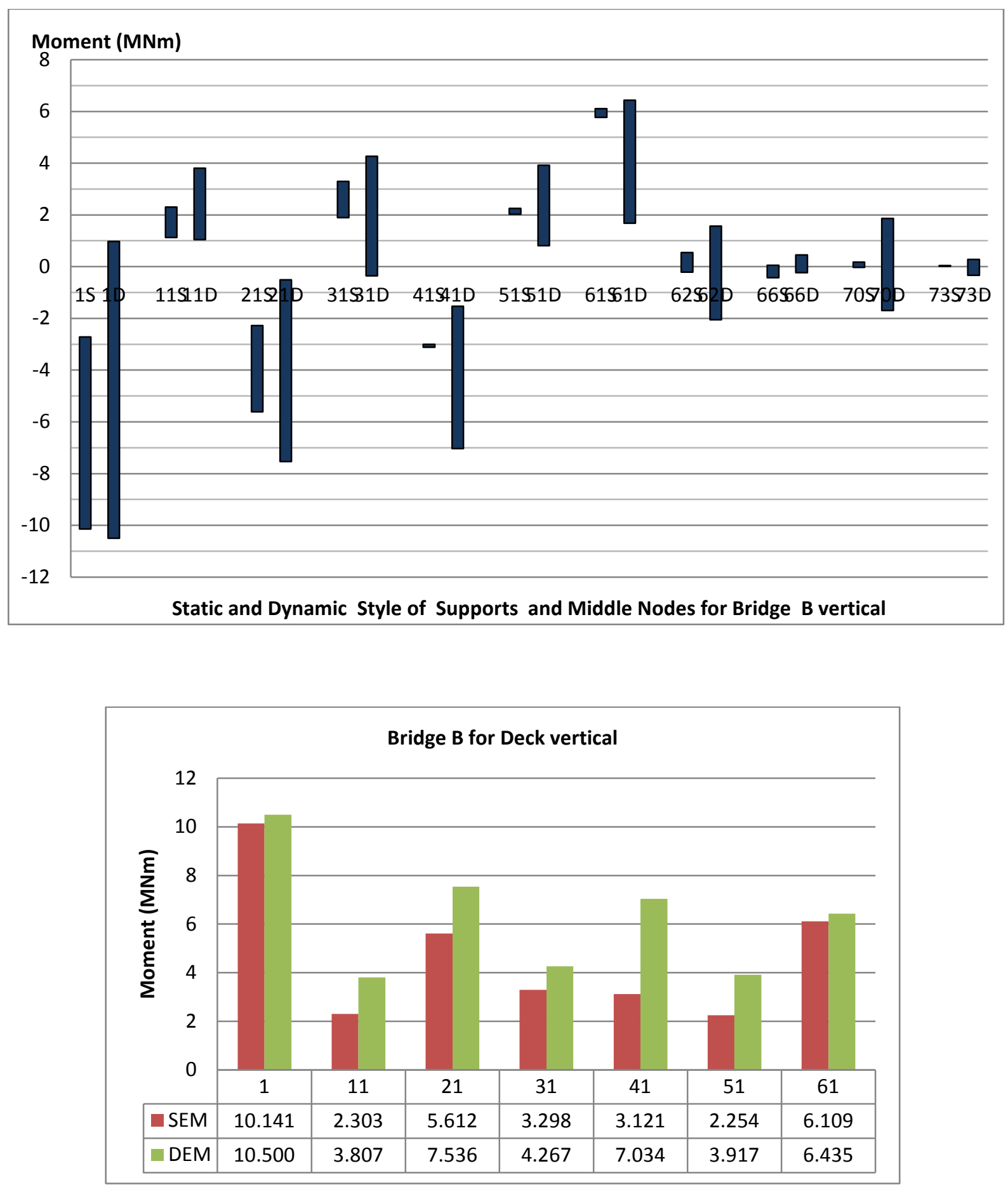


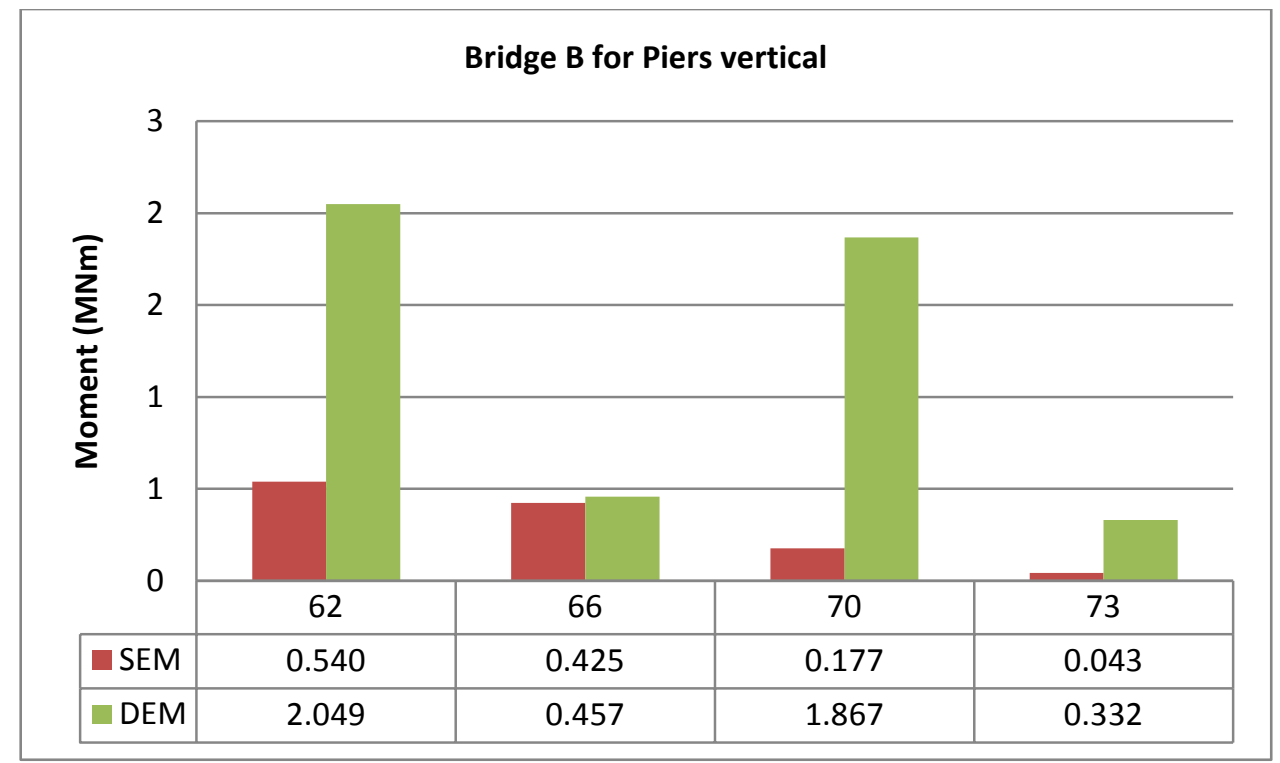

